\title{
An omics analysis of genetic sleep loss in C. elegans
}

\author{
Dissertation \\ for the award of the degree \\ "Doctor rerum naturalium" \\ (Dr. rer. nat.) \\ of the Georg-August-Universität Göttingen \\ within the doctoral program Systems Neuroscience \\ of the Georg-August University School of Science (GAUSS) \\ submitted by

\section{Anastasios Koutsoumparis} \\ from Thessaloniki, Greece
}

Göttingen 2020 



\section{Thesis Committee Members}

Prof. Dr. Henrik Bringmann

( $1^{\text {st }}$ Reviewer)

Prof. Dr. Ralf Heinrich

Prof. Dr. Henning Urlaub
Department of Animal Physiology/

Neurophysiology

Philipps-Universität Marburg

Department of Cellular Neurobiology

Georg-August-Universität Göttingen

Max Planck Institute for Biophysical Chemistry Bioanalytical Mass Spectrometry Group

Dept. of Molecular Neurobiology of Behaviour University of Göttingen

Dept. of Molecular Neurobiology

Max Planck Institute for Experimental Medicine

Neural Circuits and Cognition

European Neuroscience Institute

Date of oral examination: 09.11.2020 



\section{Affidavit}

I herewith declare that this thesis was produced entirely by myself and that I have only used sources and materials cited. The thesis has not been submitted to any other examination board for any other academic award.

Anastasios Koutsoumparis 



\section{Acknowledgements}

First and foremost, I would like to thank my supervisor Prof. Henrik Bringmann, for giving me the life-changing opportunity to do my Ph.D. in his laboratory. I am thankful for his advice, his support and for keeping his door always open to me. He has been a mentor, an exemplary scientist who helped shape my character and understanding of the world.

I would also like to thank my thesis committee members Prof. Ralf Heinrich and Prof. Henning Urlaub. Their contribution at our meetings was invaluable for the success of my project. Furthermore, I would like to thank the members of my examination board Prof. André Fiala, who was kind enough to be my $2^{\text {nd }}$ reviewer, Prof. Nils Brose and Dr. Caspar Schwiedrzik. I would also like to thank the GGNB, for offering unique learning and networking opportunities.

A lot of my results come from collaborations with other laboratories. I would like to thank:

1. Luisa Welp and the group of Prof. Henning Urlaub from MPI-BPC, who performed the proteome analysis (TMT) and guided me through the annotation process.

2. Dr. David Meierhofer from MPI-MG, who performed the proteome (LFQ) and the metabolome analyses and discussed with me the relevance of the results.

3. Prof. Bart Braeckman from the University of Ghent, who accommodated me in his laboratory and guided me through the isothermal microcalorimetry experiment.

4. Prof. Sebastian Leidel from the University of Bern, who performed the $U_{34}$ thiolation experiment and discussed with me the importance of the findings.

I would like to thank my student assistant Eva Naumann for her strong work ethic, her highquality work and her devotion to the project. She worked hard to create a lot of strains used in this study, making her contribution crucial for the success of this project.

Moreover, I would like to thank my students:

1. Gamze Naz Öztan, for her contribution to the sample preparation of the $\mathrm{U}_{34}$ thiolation experiment ("I feel nothing!") and to the characterization of $d g k-1$ (ok1462) phenotype

2. Lina Paola Baena Lozada, for her contribution to the RIS activity measurements in the $d g k-1$ (ok1462) and unc-13(s69) mutant backgrounds. 
I would like to thank our technicians for keeping the laboratory up and running: Silvia Gremmler, Sabine König, Sigrid Stöhr, Regina Löchel and Gábor Szerencsi.

Since my arrival in the lab, I had the good luck of working together with wonderful, supportive and accepting people, a group which came to be known as the Royal Worm Force: I would like to thank Dr. Jan Konietzka for his guidance, support and patience when I was just starting to learn my way around the lab. He has been a great colleague and a greater friend. I hope we will continue to share the same "scientific curiosity" for the world. I would like to thank Dr. Elisabeth Maluck for teaching me how to cross C. elegans and endlessly discussing with me about science and life. We learned a lot from each other, be it in the lab or in our "food group". She made significant contributions to discovering and assessing the role of $x b p-1$ in the aptfI (gk794) background. I would like to thank Dr. Judith Besseling for her valuable advice in the beginning of my Ph.D., her active support throughout and recently for providing corrections for the "Introduction" part of this thesis. I would like to thank Inka Busack for her attitude, help and advice throughout my studies. She provided corrections for the "Materials and methods" and "Results" parts of this thesis. She has been great company for many instances of fun, singing, tickling and drinking! I would like to thank Marina Sinner for her help and pleasant collaboration in the lab, for our endless talks about...everything (!) and for the carefree nights of clubbing and dancing in Göttingen. I would like to thank Dr. Yin Wu for her advice and discussions about the project, for our very interesting extracurricular activities and for introducing me to the magnificent Chinese culture. I would like to thank Yang $\mathrm{Hu}$ for her advice, the philosophical conversations and for all the funny moments we shared. Her genuine interest in my music and her melodic contributions, made me a better pianist and a better person. I would like to thank Byounjun Park for his stoic attitude and his help in the lab, that made the hectic and stressful last months of my Ph.D. tolerable.

I would like to thank Maximilian Fritz for his advice and patience during our in-depth scientific conversations which usually ended with him saying “...but Tasos, this is not how it works!”. I feel we was the best scientist of us all, even though he passed away so young...

I want to thank all my friends and relatives from Greece who were supporting me from far away. I would like to thank my beloved late grandfather Dimitrios and my beloved grandmother Styliani, who made every sacrifice possible for me to be where I am today. I owe them everything and I hope they are proud of who I have become. 
Finally, I would like to thank my brother Dimitrios for his support and advice during my studies in Germany. He took on himself more burden than he should have, so that I can pursue my dream. I hope I can pay him back one day. I would also like to dedicate this thesis to him and remind him the words of the Nobel prize laureate for literature in 1979, poet Odysseas Elytis:

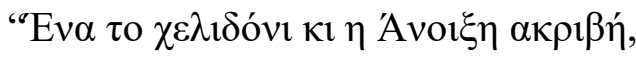

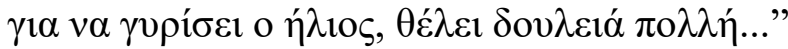





\section{Abbreviations}

EEG

REM

VLPO

GABA

$\mathrm{SCN}$

EGF

FDR

TGF- $\beta$

FOXO

ROS

DAG

AMP

ATP

TCA

UPR

gf
Electroencephalogram

Rapid eye movement

Ventrolateral preoptic nucleus

Gamma-amino butyric acid

Suprachiasmatic nucleus

Endothelial growth factor

False discovery rate

Transforming growth factor beta

Forkhead box (transcription factor) $\mathrm{O}$

Reactive oxygen species

Diacylglycerol

Adenosine monophosphate

Adenosine triphosphate

Tricarboxylic acid (cycle)

Unfolded protein response

Gain of function 



\begin{abstract}
Sleep is a state of consciousness that has persisted through evolution and has become a necessity for animal welfare. In humans sleep loss has been shown to be inextricably intertwined with poor health. The prevalence of sleep defects in elderly people not only associates sleep quality with aging, but also draws attention to an underrated problem that will continue to affect the life quality of the increasingly aging population of the western world.
\end{abstract}

Sleep regulates energy metabolism and higher brain functions. Little is known about the precise molecular mechanisms that control and are controlled by sleep, even though it has been extensively studied, due to its complex nature. In this thesis, I used the nematode C. elegans, which is a simple, yet complex enough model organism in order to discover the downstream processes that are regulated by sleep.

Although sleep is connected to development in C. elegans, I used sleep loss in L1 arrested worms, as a model that couples sleep, metabolism and aging phenotypes. This is more relevant to mammal physiology than developmental sleep. By genetically ablating the sleep-active neuron RIS and recruiting powerful omics techniques, I was able to identify those components of $C$. elegans physiology that are affected by sleep loss. I complemented the omics experiments with extensive genetic screening for sleep alterations in animals with defects in metabolic pathways, lifespan, egg-laying and other assays.

I was able to discover that sleep loss causes tissue-specific changes, reduces body size and increases the basal metabolic rate. Furthermore, I discovered that sleep loss upregulates the unfolded protein response in the endoplasmic reticulum by upregulating relevant transcription factors. Finally, I discovered that sleep loss causes oxidative stress and I characterized components of the life-prolonging antioxidant pathways that are directly regulated by the sleepactive neuron RIS. 



\section{Table of Contents}

1. Introduction ................................................................................................................................... 1

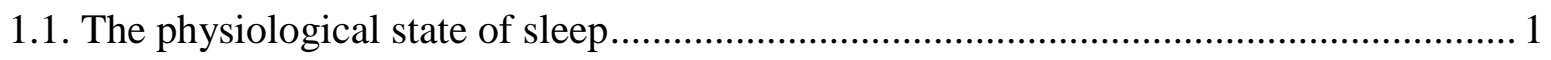

1.2. Sleep regulation in humans and other mammals ......................................................... 2

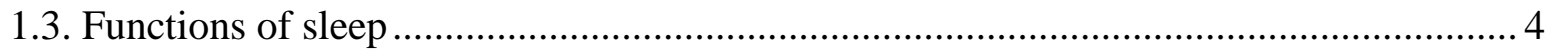

1.4. C. elegans as a model organism for sleep research ...................................................... 5

1.5. Sleep during starvation and the $\mathrm{L} 1$ arrest state ..................................................... 7

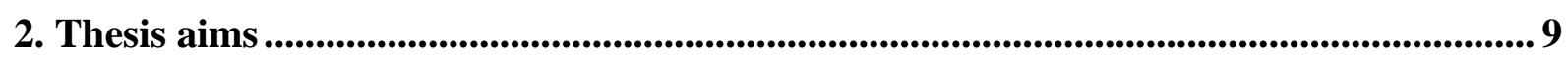

3. Materials and methods..................................................................................................................... 11

3.1. Worm maintenance and strain generation...................................................... 11

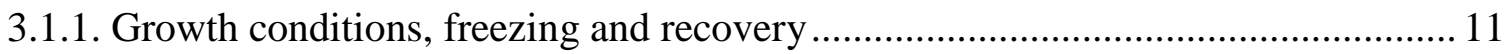

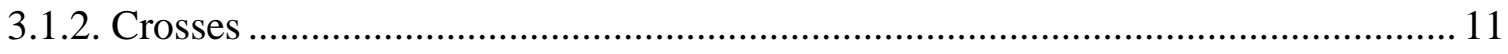

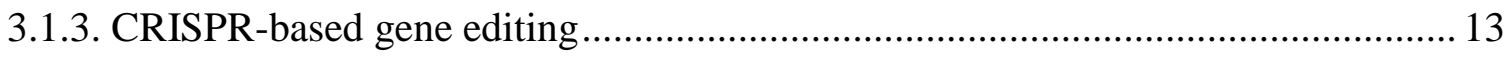

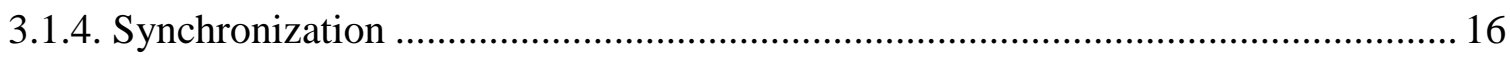

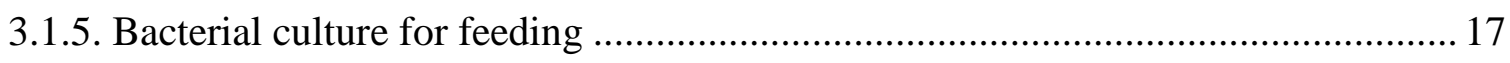

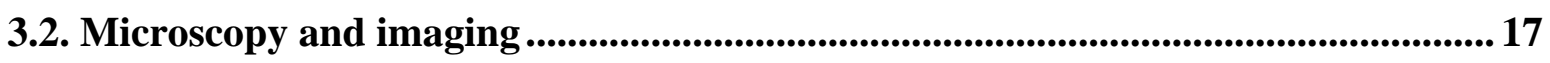

3.2.1. Long-term imaging using agarose hydrogel microchambers ............................... 17

3.2.2. DIC imaging for quiescence assessment in L1 arrest (48 hours)......................... 18

3.2.3. Fluorescence and calcium imaging in $\mathrm{L} 1$ arrest................................................. 19

3.3. Transcriptional profiling by RNA-sequencing ..................................................... 21

3.4. Agglomerative hierarchical clustering analysis of RNA-seq data......................... 22

3.5. Proteomic characterization of $C$. elegans .........................................................23

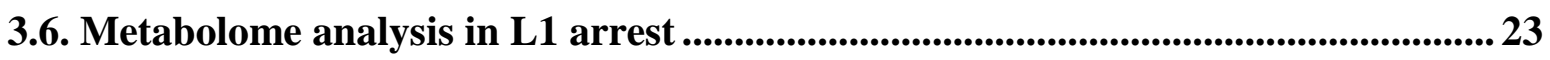

3.7. Survival and behavioral assays ................................................................................................. 24

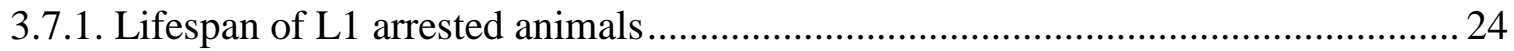

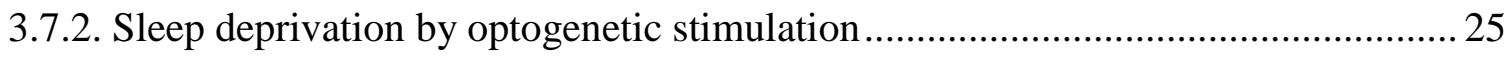

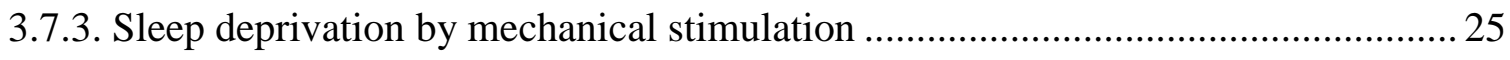

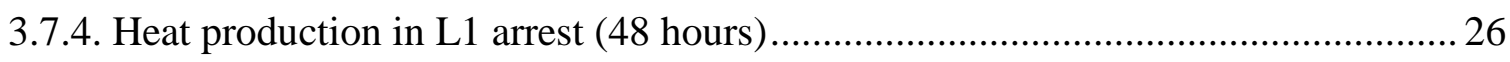

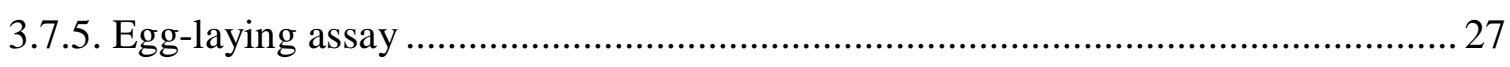

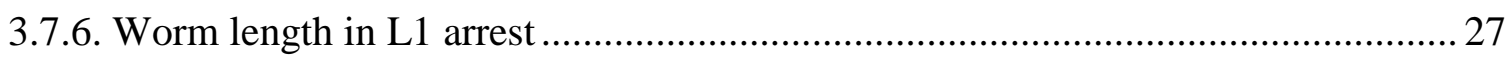

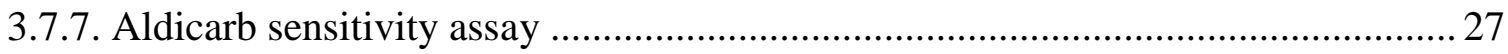

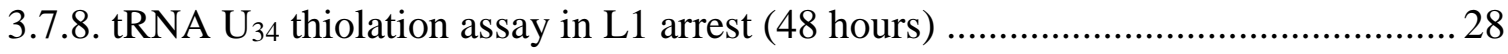

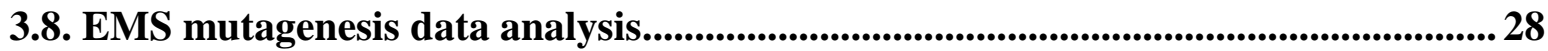


4.1. Sleep loss affects metabolism by regulating the starvation response in $\mathrm{L} 1$ arrested worms

4.2. Sleep loss in L1 arrest alters the physiology of C. elegans

4.2.1. Sleep loss upregulates hypodermis-specific gene expression and reduces body size

4.2.2. Sleep loss conditionally changes muscle-specific and mitochondrial gene expression

4.2.3. Sleep loss affects intestine-specific and FOXO gene expression 46

4.2.4. Sleep loss conditionally affects components of germline-specific gene expression

4.2.5. Sleep loss affects neuronal gene expression and neurotransmission 51

4.2.6. Sleep controls protein synthesis via $U_{34}$ thiolation in $\mathrm{L} 1$ arrest............................ 55

4.2.7. Sleep loss alters lysosomal function in $\mathrm{L} 1$ arrest ................................................... 57

4.3. Sleep loss induces specific metabolic changes in $L 1$ arrest and vice versa.............59

4.4. $d g k-1$ loss of function results in sleep loss by changing RIS physiology ................. 65

4.5. The transcription factor XBP-1 regulates aspects of $L 1$ arrest quiescence ........... 67

4.6. Sleep loss affects FOXO-dependent transcription in muscles .................................. 69

4.7. RIS and the nervous system control HSP-12.6 expression in muscles.....................70

4.8. The endoplasmic reticulum unfolded protein response and the hypoxia response regulate HSP-12.6 expression in $\mathrm{L1}$ arrest via $a p t f-1$............................................... 74

4.9. Heat-shock induces the expression of HSP-12.6 regardless of aptf-1 ...................... 75

4.10. $x b p-1$ partially rescues the survival of aptf-1 loss of function in L1 arrest........... 76

4.11. RIS de-polarization via mechanical stimulation increases HSP-12.6 expression and lifespan, contrary to optogenetic hyper-polarization of RIS ..................................... 78

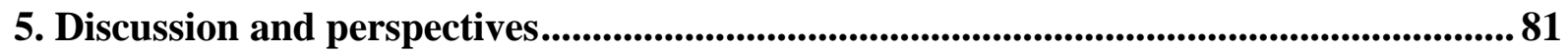

5.1. Sleep loss induces global metabolic changes in C. elegans ..................................... 81

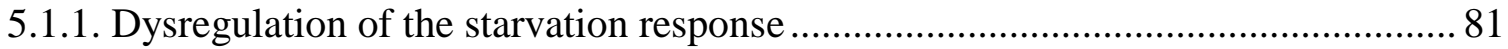

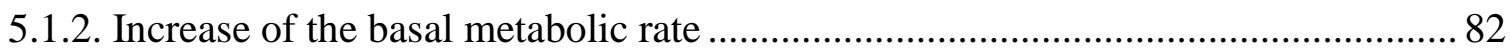

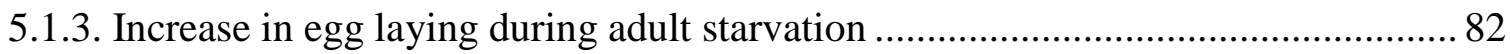

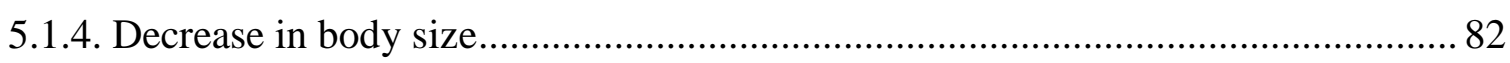

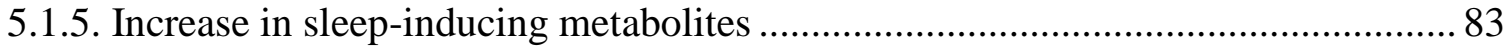

5.1.6. Increase of oxidative stress and decrease of life-prolonging antioxidant factors ... 83 
5.1.7. Increase of the unfolded protein response and dysregulation of proteostasis. .84

5.2. Changes in metabolism result in changes in sleep ..................................................... 85

5.3. Sleep loss induces RIS-dependent tissue-wide changes...............................................86

5.4. RIS controls the nuclear localization of FOXO homolog daf-16 ........................... 87

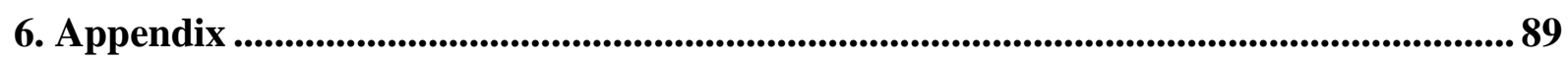

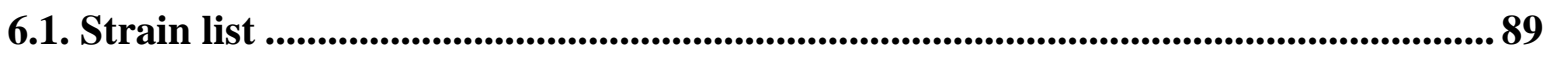

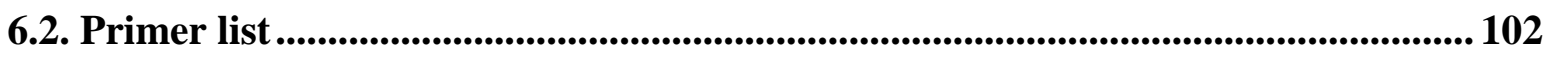

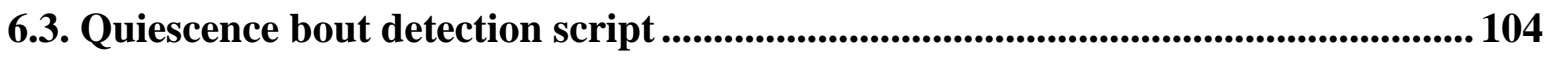

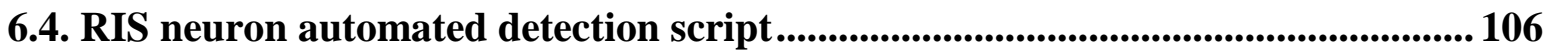

6.5. RNA-seq differential expression analysis script .............................................. 107

6.6. Proteomics and metabolomics differential expression analysis script.................. 108

6.7. RNA-seq (CeGaT) - N2 (wild-type) vs HBR227 (aptf-1) - 48h Starved ................ 109

6.8. RNA-seq (CeGaT) - N2 (wild-type) vs HBR227 (aptf-1) - 4h Fed ......................... 116

6.9. Proteome (TMT) - N2 (wild-type) vs HBR227 (aptf-1) - 48h Starved................... 122

6.10. Proteome (TMT) - N2 (wild-type) vs HBR227 (aptf-1) - 4h Fed ........................ 124

6.11. RNA-seq (CeGaT) - N2 (wild-type) vs HBR1777 (flp-11::EGL-1) - 48h Starved 125

6.12. RNA-seq (CeGaT) - N2 (wild-type) vs HBR1777 (flp-11::EGL-1) - 4h Fed...... 128

6.13. Proteome (TMT) - N2 (wild-type) vs HBR1777 (flp-11::EGL-1) - 48h Starved 138

6.14. Proteome (TMT) - N2 (wild-type) vs HBR1777 (flp-11::EGL-1) - 4h Fed ......... 140

6.15. RNA-seq (CeGaT) - 4h Fed vs 48h Starved - N2 (wild-type)............................. 141

6.16. RNA-seq (CeGaT) - 4h Fed vs 48h Starved - HBR227 (aptf-1) ............................. 161

6.17. RNA-seq (CeGaT) - 4h Fed vs 48h Starved - HBR1777 (flp-11::EGL-1)........... 172

6.18. Proteome (TMT) - 4h Fed vs 48h Starved - N2 (wild-type) .................................. 199

6.19. Proteome (TMT) - 4h Fed vs 48h Starved - HBR227 (aptf-1) ............................ 202

6.20. Proteome (TMT) - 4h Fed vs 48h Starved - HBR1777 (flp-11::EGL-1) ............ 204

6.21. RNA-seq (MPI-MG) - N2 (wild-type) vs HBR227 (aptf-1) - 48h Starved........... 205

6.22. RNA-seq (MPI-MG) - N2 (wild-type) vs HBR1777 (flp-11::EGL-1) - 48h Starved

6.23. Proteome (LFQ) - N2 (wild-type) vs HBR227 (aptf-1) - 48h Starved ................ 210

6.24. Proteome (LFQ) - N2 (wild-type) vs HBR1777 (flp-11::EGL-1) - 48h Starved . 210

6.25. Metabolome - N2 (wild-type) vs HBR227 (aptf-1) - 48h Starved ........................ 211

6.26. Metabolome - N2 (wild-type) vs HBR1777 (flp-11::EGL-1) - 48h Starved ........ 212

6.27. Metabolome - N2 (wild-type) vs HBR227 (aptf-1) - 6 days Starved ...................... 212 
6.28. Metabolome - N2 (wild-type) vs HBR1777 (flp-11::EGL-1) - 6 days Starved ... 213

6.29. Genetic screen for quiescence phenotypes....................................................... 215

6.30. Representative mobility traces and quiescence bouts ....................................... 218

6.31. Quiescence assessment of wild-type animals after supplementation with methionine, glycine and glutamate........................................................................................... 219

6.32. Impact of maternal growth conditions in the quiescence fraction of the offspring in the wild-type background ....................................................................................................... 219

6.33. Representative RIS intensity GCaMP traces ....................................................... 220

6.34. HSP-12.6 expression screening in L1 arrest ...................................................... 221

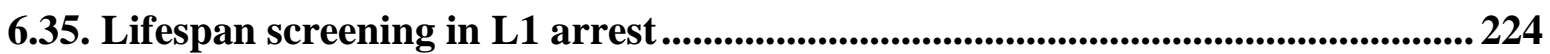

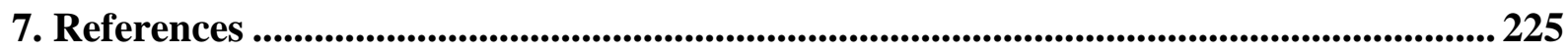




\section{Introduction}

\subsection{The physiological state of sleep}

Sleep is a state of consciousness that has persisted through evolution and has become a necessity for animal welfare. In humans (Homo sapiens) sleep loss has been shown to be inextricably intertwined with poor health. Insomnia and low sleep quality in general have been associated with susceptibility to infectious diseases and the untimely activation of proinflammatory immune responses that lead to the development of cardiovascular and metabolic diseases (e.g. type 2 diabetes) ${ }^{[1]}$. Furthermore, the comorbidity of insomnia and psychiatric disorders, such as depression and anxiety, has been well documented in the literature. Finally, the prevalence of sleep defects in elderly people not only associates sleep quality with aging, but also draws attention to an underrated problem that will continue to affect the life quality of the increasingly aging population of the western world ${ }^{[2]}$.

Sleep studies in humans are based on electroencephalogram (EEG) readings, which show the existence of two phases with distinct characteristics: an "active" phase accompanied by rapid eye movements (REM) and a "quiet" phase (non-REM), which alternate periodically to result, on average, in 8 hours of sleep for a healthy adult ${ }^{[3]}$. Moreover, several behavioral criteria have been implemented to define the physiological state of sleep:

1. Elevated arousal threshold in response to sensory stimulation, which differentiates sleep from tranquility during wakefulness.

2. Reversibility, in the sense that the individual can easily recover its motor functions, unlike being paralyzed or in a coma.

3. Specific posture, indicative of muscle relaxation.

4. Homeostatic regulation, which compensates for sleep loss by increasing the duration of the subsequent sleep interval.

These criteria extend the notion of the sleep state to animals, in which EEG recordings are not possible $^{[4]}$. 
So far, sleep states have been identified in all animals that possess a nervous system, the simplest ones being the jellyfish of the phylum Cnidaria ${ }^{[5]}$. The daily sleep fraction varies across species and depends on their environment, but a lower limit of $10 \%$ seems to exist (Figure 1) ${ }^{[6]}$.

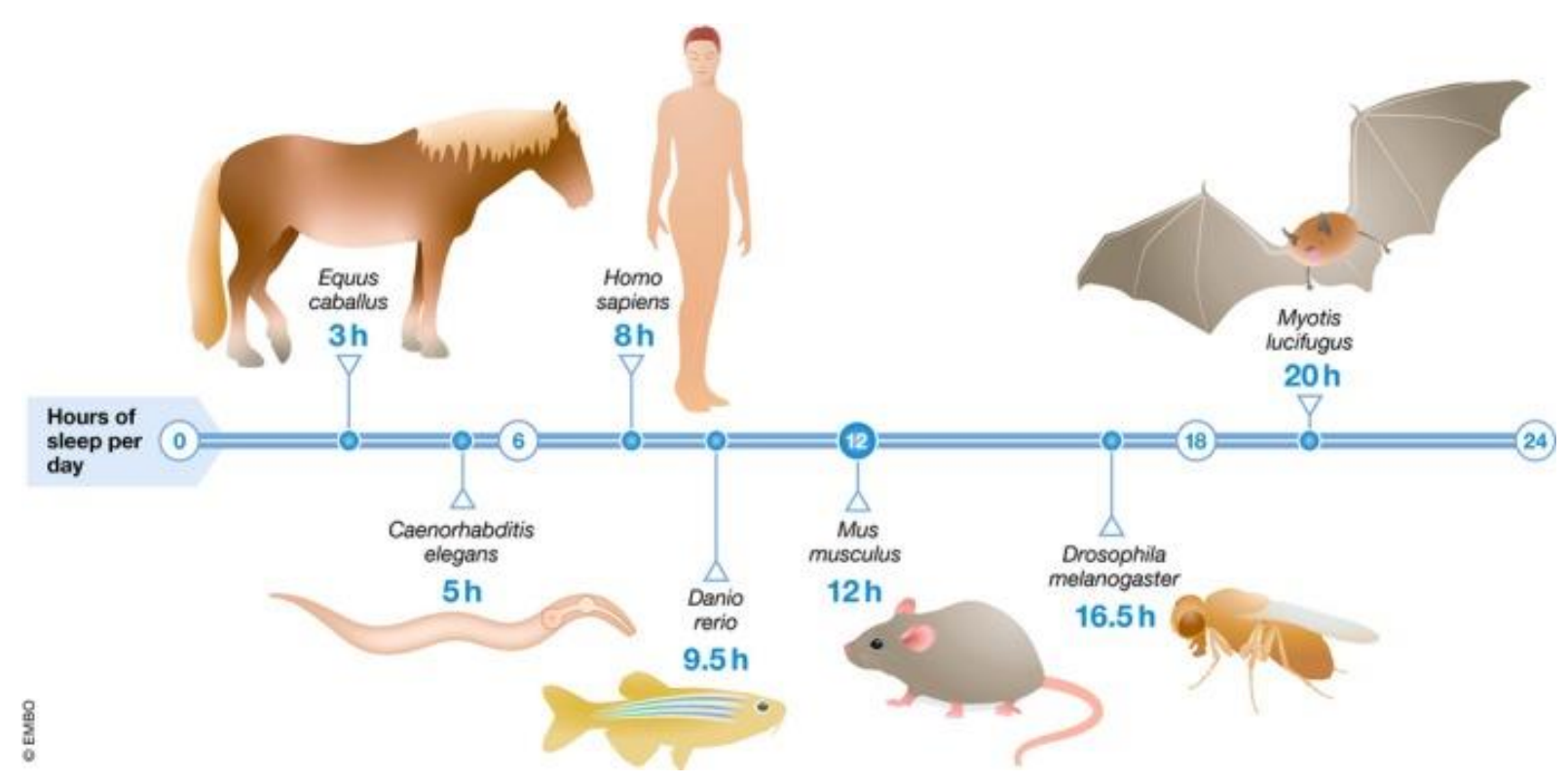

Figure 1. Daily sleep fraction variance among species

Figure adapted from Bringmann H. (2019) ${ }^{[6]}$.

\subsection{Sleep regulation in humans and other mammals}

In humans, the wake state is controlled by the ascending arousal system. Cholinergic signals that originate in the pedunculopontine and laterodorsal tegmental nuclei of the brainstem, activate the thalamic relay neurons which activate the cerebral cortex. Complementarily, monoaminergic signals from the brainstem activate the neurons at the lateral hypothalamus, the basal forebrain and the cerebral cortex in order to promote wakefulness. On the other hand, the sleep state is controlled by the ventrolateral preoptic nucleus (VLPO). As a cluster of sleepactive neurons, the VLPO sends inhibitory signals (e.g. GABA) to the aforementioned centers of arousal. The wake-active neuronal network can in turn inhibit the VLPO, creating a flip-flop circuit, which permits fast transitions between the two states (Figure 2) ${ }^{[7,8]}$. The sleepcontrolling center, VLPO, is also present in other mammals. In elderly populations, degeneration of the VLPO has been associated with poor sleep quality ${ }^{[9]}$. Additionally, other brain areas that have sleep-active neurons have been characterized in rats, such as the basal forebrain, the lateral hypothalamus and the brainstem ${ }^{[10]}$. 
Moreover, in studies done with mice, it was found that the appetite-promoting neuropeptide hormone orexin promotes wakefulness, establishing a link between nutritional status and sleep ${ }^{[11]}$. Human patients suffering from narcolepsy, reportedly have low levels of orexin in their cerebrospinal fluid ${ }^{[12]}$.
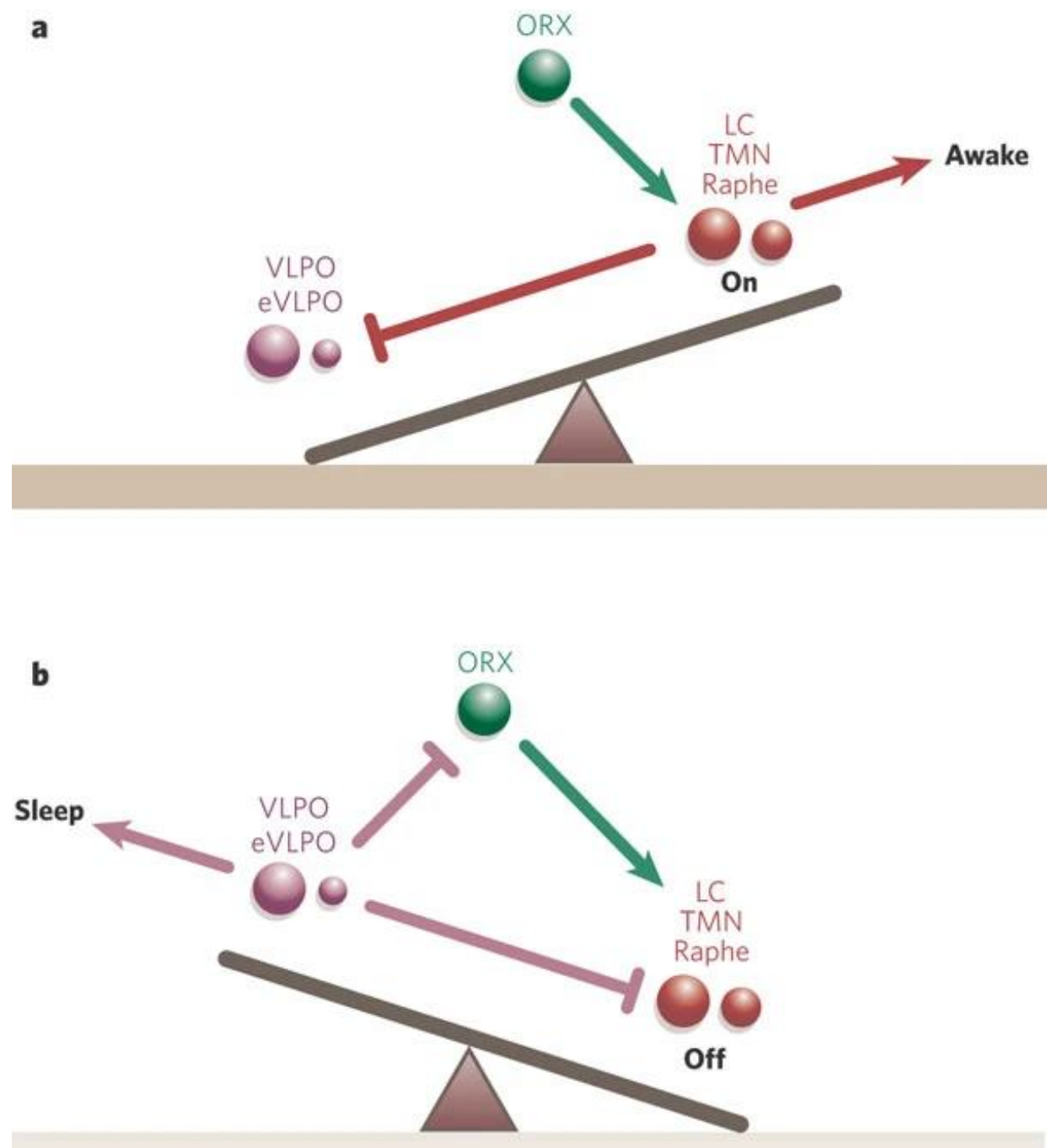

Figure 2. A flip-flop switch regulates sleep in mammals

(A) Wakefulness is established by cholinergic and monoaminergic nuclei (red) that inhibit the ventrolateral preoptic nucleus - VLPO (purple). This lifts the inhibition of the wake-promoting brainstem regions as well as that of the orexinergic neurons (green).

(B) Sleep is established by the activation of the sleep-promoting VLPO region (purple), which sends inhibitory signals to inactivate the centers of arousal (red) and orexinergic neurons (green). Crosstalk of these regions forms the sleep-regulating flip-flop switch.

ORX: orexinergic neurons, LC: locus coeruleus, TMN: tuberomammillary nucleus Figure adapted from Saper et al. (2005) ${ }^{[7]}$. 
Finally, the sleep-wake cycle is controlled by the circadian clock. Humans are diurnal, as the majority of their activity happens throughout the day while rodents are nocturnal. The suprachiasmatic nucleus ( $\mathrm{SCN}$ ) is the center for circadian rhythm control and thus sleep behavior ${ }^{[13]}$. Melatonin, a sleep-promoting hormone that is secreted by the pineal gland in a circadian manner, acts on receptors at the SCN to regulate sleep by inhibiting orexinergic neurons ${ }^{[14,15]}$.

\subsection{Functions of sleep}

Sleep is an ancient and conserved physiological process, despite the obvious disadvantage of leaving the organism open to attack by predators. It has been preserved through evolutionary pressure, indicating its importance and thus there are several hypotheses that address its purpose [16].

The most straightforward function of sleep seems to be energy conservation. However, this hypothesis has been challenged by the fact that REM sleep is a condition of increased brain energy metabolism and that, overall, the relative amount of energy saved is little compared to wakefulness. This has led to the "energy allocation" hypothesis, in which biological functions are differently regulated in a, wake- or sleep-, state-dependent manner. This allows the segregation of competing metabolic processes e.g. catabolism during wake and anabolism during sleep ${ }^{[17]}$.

Apart from metabolic regulation, sleep has been shown to play a role in higher brain functions. During sleep, synapses undergo remodeling in order to achieve optimal transmission of information by removing or de-phosphorylating glutamate receptors ${ }^{[18]}$. Sleep also introduces quantitative and qualitative effects on memory representations, aiding memory consolidation ${ }^{[19]}$. Finally, chronic sleep deprivation results in accumulation of amyloid- $\beta$ peptides in the brain, indicating a link to Alzheimer's disease ${ }^{[20]}$. 


\subsection{C. elegans as a model organism for sleep research}

The sleep state is driven by the de-polarization of "sleep active" neurons that release inhibitory neurotransmitters ${ }^{[21]}$. However, this process is intricate and not well understood at the molecular level. Therefore, there was a necessity for a simple, yet complex enough model organism to be used in sleep research.

Caenorhabditis elegans ( $C$. elegans) is a non-parasitic nematode that was introduced as a eukaryotic model organism by Sydney Brenner in 1974. It offers distinct advantages in biological research ${ }^{[22]}$ :

1. All adult individuals have an exact number of 959 somatic cells, whose lineage has been traced since the start of embryogenesis. 302 of these cells constitute a simple nervous system with its wiring already characterized in a functional connectome.

2. Its genome has been sequenced and a wide selection of mutant and transgenic strains exist or can easily be created, making it a valuable tool in genetic analysis. The animals are mostly hermaphrodite (two X chromosomes), thus they have the capability to self-fertilize, although they can also cross-breed with males (one $\mathrm{X}$ chromosome). Furthermore, C. elegans is transparent, which allows for easy assessment of fluorescent transgene expression.

3. Moreover, C. elegans is easy to work with in the lab, since adult worms have an approximate size of $1 \mathrm{~mm}$ and can be observed using a simple stereo microscope. It grows on agar plates feeding on non-pathogenic E. coli and has a short generation time of 3 days at $20{ }^{\circ} \mathrm{C}$. A single worm lays around 300 eggs and has an average lifespan of 18 days.

In addition to the aforementioned advantages, several quiescence states have been described in C. elegans that fulfill all the criteria that define the physiological state of sleep.

Sleep in C. elegans is most notably associated with development, through the 4 larval stages until adulthood (Figure 3). At the end of each larval stage there is a period of quiescence, characterized by reduced movement and cessation of feeding called lethargus ${ }^{[23]}$. When eggs hatch and there is no available food source, development comes to a halt and the worm repeatedly goes in and out of sleep bouts, a state termed L1 arrest quiescence ${ }^{[24]}$. Furthermore, 
in the presence of cellular stress e.g. in the form of heat-shock, worms enter a protective state that is also characterized by behavioral quiescence ${ }^{[25]}$. Finally, in adult individuals the fraction of sleep is dictated by the amount and the quality of the available food ${ }^{[24]}$.

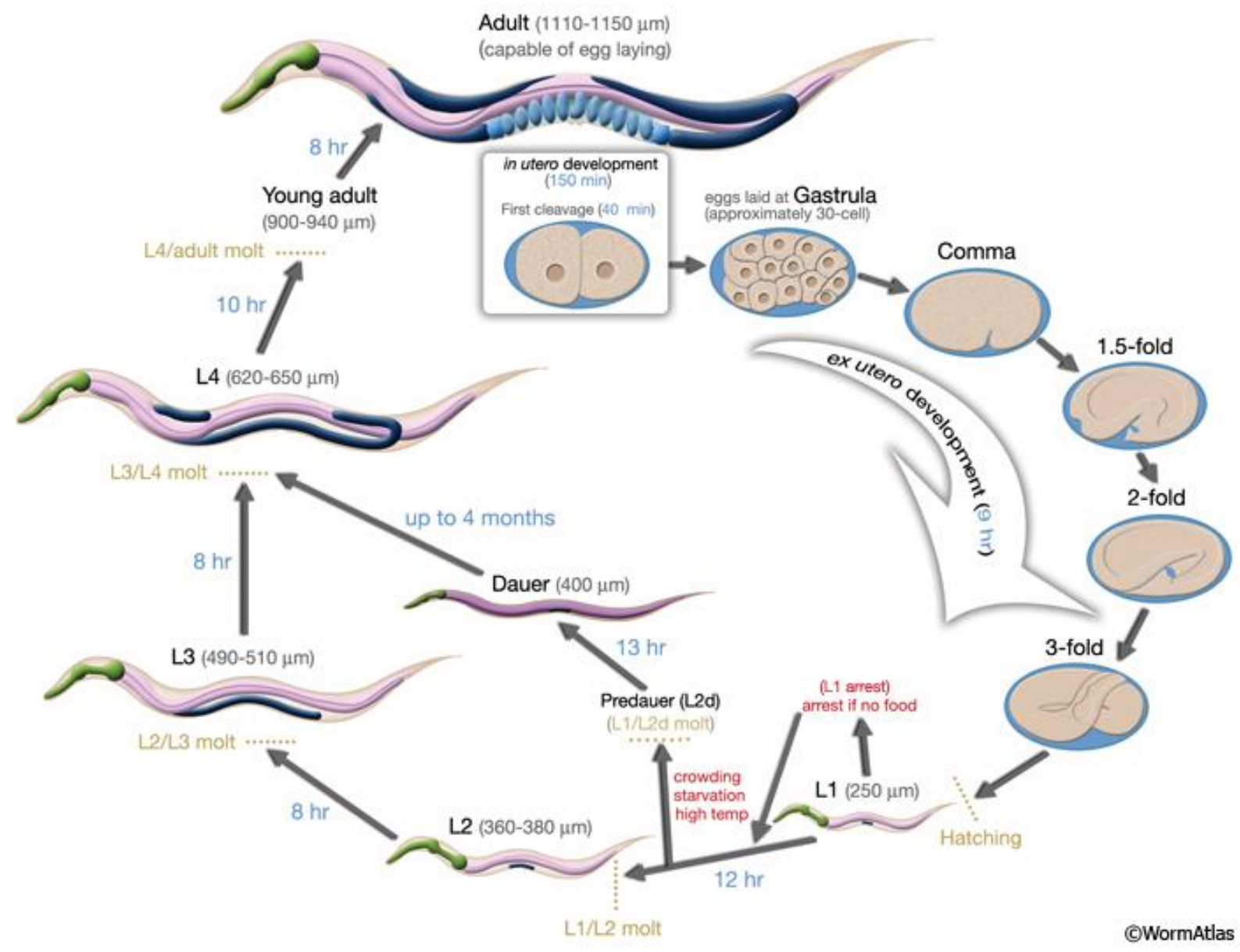

Figure 3. C. elegans life cycle

The molt of every larval stage is preceded by a quiescence interval (lethargus).

Starvation at the L1 stage induces sleep-bouts.

Figure adapted from Wormatlas.org

Sleep and its functions in $C$. elegans are under tight neuronal regulation. The protective response that follows a noxious stimulus, is carried out by the drowsiness-inducing neuron ALA which is activated by the EGF receptor. Genetic ablation of ALA (ceh-14 loss of function) leads to reduced quiescence ${ }^{[26]}$.

The neuron that governs all aspects of sleep behavior in C. elegans is the GABAergic and peptidergic head interneuron RIS. Sleep is induced by FLP-11, an RIS-specific, secreted neuropeptide. Gene expression in RIS is controlled by the AP2 transcription factor aptf-1, which is homologous to the human TFAP2 that has been linked to insomnia (Figure 4). 
Genetic ablation of RIS either by aptf- 1 or $f l p-11$ loss of function leads to significant loss of sleep ${ }^{[27]}$. In turn, RIS activity is regulated by the upstream neurons PVC (cholinergic) and RIM (glutamatergic and tyraminergic) that are part of the locomotion circuit ${ }^{[28]}$.
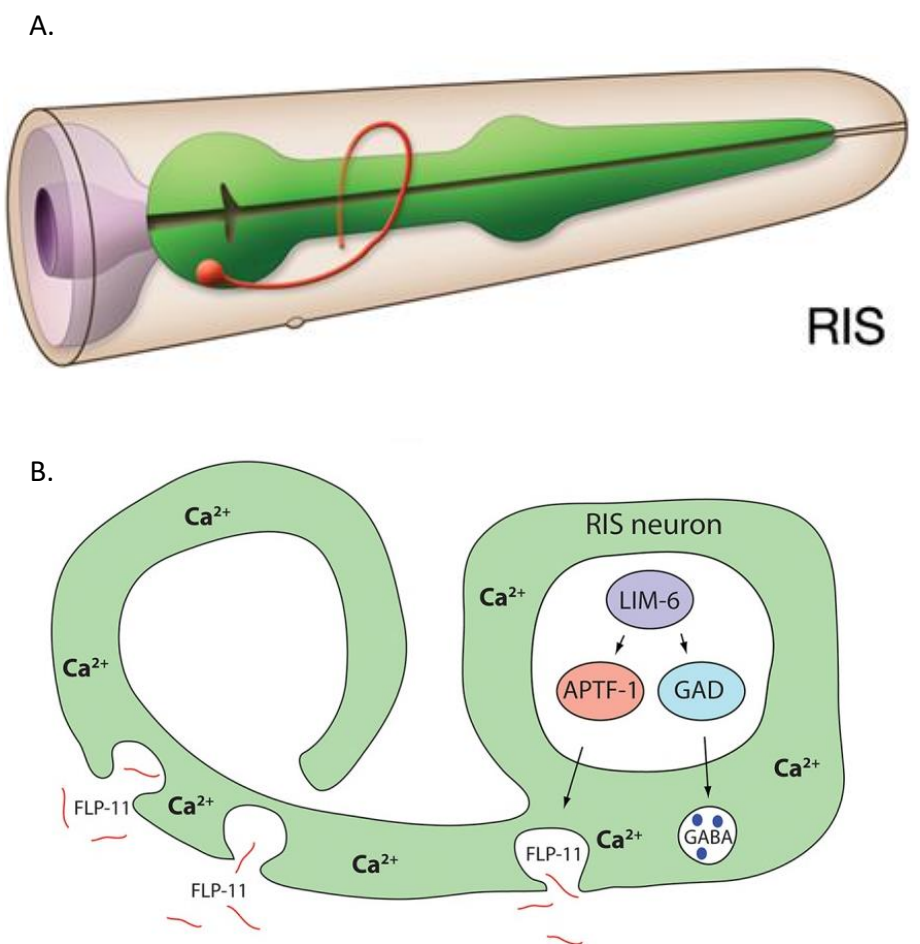

Figure 4. The sleep-active neuron RIS

(A) Anatomical position of the sleep-active RIS neuron in the head of C. elegans. Figure adapted from Wormatlas.org

(B) The AP2 transcription factor aptf-1, regulates the expression of the sleep-inducing neuropeptide FLP-11 in RIS.

Figure adapted from Turek et al. (2016) ${ }^{[27]}$.

\subsection{Sleep during starvation and the $\mathrm{L} 1$ arrest state}

Nutrient availability is a substantial concern for every living organism and for this reason they have developed protective mechanisms to survive, for as long as possible, the adversities of starvation. Sleep is one such mechanism, as it has been shown that in humans, starvation decreases the amount of active REM sleep while it increases the deep sleep phase (stage 3) of the quiet non-REM sleep ${ }^{[29]}$. Although there is an obvious trade-off during starvation between staying awake to search for food and sleeping to save energy, increased sleep during starvation is associated with greater lifespan in Drosophila melanogaster ${ }^{[30]}$.

In C. elegans, post-embryonic development is completely dependent on food intake. When the worms hatch and there is no readily accessible source of sustenance, they enter a state of 
developmental arrest, during the first larval stage, called L1 arrest. This is a manifestation of the starvation response, regulated by the energy-sensing enzyme AMP-dependent protein kinase (AMPK). Survival during L1 arrest is ensured by cessation of cell divisions, as all available energy resources (coming from yolk components and autophagy) are directed towards maintenance of fundamental functions via differential regulation of gene expression. Furthermore, the worm becomes more resistant to heat-shock and oxidative stress ${ }^{[31]}$.

The $\mathrm{L} 1$ arrest state is ultimately regulated by the FOXO transcription factor $d a f-16$, downstream of the insulin signaling pathway ${ }^{[32]}$. daf-16 was also shown to be responsible for RIS depolarization during L1 arrest, which initiates sleep behavior. Should sleep be ablated in this case (e.g. by aptf- 1 loss of function), not only survival is halved but upon reintroduction to food worms fail to recover and continue their development ${ }^{[24]}$. Finally, worms accumulate agingrelated cellular changes during L1 arrest, that can be reversed by feeding, provided that the unfolded protein response in the endoplasmic reticulum is not compromised ${ }^{[33]}$. 


\section{Thesis aims}

The complexity and inaccessibility of the human brain, gives rise to technical and ethical limitations in our quest to understand sleep on the molecular level. Efforts have been made to overcome this problem by using -omics techniques on blood samples (e.g. after sleep deprivation), which produced very general results that do not point specifically in one signaling pathway ${ }^{[34,35,36]}$.

On the other hand, by using $C$. elegans we can genetically ablate sleep without resulting in lethality, which allows us to complement classic sleep deprivation protocols (e.g. mechanical stimulation), in order to get a tissue-wide image of sleep-related changes. Even though sleep is coupled to larval development in growing C. elegans (lethargus), results from nematode research have already been translated in mammals ${ }^{[37]}$. However, the recently-characterized starvation-induced sleep state (L1 arrest) ${ }^{[24]}$ offers a more promising model, in which evolutionarily-conserved sleep-regulating pathways can be discovered.

Therefore, I have used the L1-arrested C. elegans to:

\section{Aim 1 - Discover how sleep affects metabolism and vice versa}

By using multiple -omics approaches (transcriptomics, proteomics and metabolomics), I wanted to determine how sleep loss affects metabolism globally (starvation response). I complemented these experiments with various other assays such as lifespan, isothermal microcalorimetry, egg-laying, size-measurement and aldicarb sensitivity assays. Also, in order to discover whether changes in metabolism alter sleep levels, I performed an extensive quiescence screening of metabolic deficient worms.

\section{Aim 2 - Discover specific components regulated by sleep}

Building on the results obtained in the experiments mentioned above, I used fluorescence quantification to measure how sleep loss affects the expression of FOXO homolog daf-16 and its downstream target HSP-12.6. Finally, I used mechanical stimulation and optogenetics to further strengthen the link of FOXO regulation and the sleep neuron RIS. 


\section{Materials and methods}

\subsection{Worm maintenance and strain generation}

\subsubsection{Growth conditions, freezing and recovery}

C. elegans strains were maintained on Nematode Growth Medium (NGM) seeded with OP50 E. coli bacteria, in medium-sized $(60 \times 15 \mathrm{~mm})$ Petri dishes and incubated at $20^{\circ} \mathrm{C}$ (unless otherwise specified) ${ }^{[38]}$.

Long-term storage of $C$. elegans can be achieved as follows:

L1-L2 stage worms can be collected from freshly starved and clean plates using $1 \mathrm{ml}$ of freezing buffer in $2 \mathrm{ml}$ cryovials. Then, they are put in a box made of expanded polystyrene at $-80^{\circ} \mathrm{C}$ overnight. The gradual freezing ensures that some worms survive the procedure. Older worms do not survive the freezing. It is imperative that new strains are frozen as soon as they are obtained, since they tend to accumulate random background mutations over a short period of time.

Recovery of frozen worms is achieved by transferring the cryovial at room temperature. When the content is thawed, it is pipetted on a fresh NGM plate.

A complete list of all strains used in this study can be found in section 6.1.

\subsubsection{Crosses}

Generation of male worms was achieved by transferring $10 \mathrm{~L} 4$ worms on a fresh plate, sealing it with Parafilm $\mathrm{M}$ and incubating it at $32^{\circ} \mathrm{C}$ for 4 hours. This process only works for strains that can survive the heat-shock and retain their fertility. Creating male worms with the "Roller" phenotype is not advised, because they do not mate effectively ${ }^{[39]}$.

Mating took place on small-sized $(35 \mathrm{x} 10 \mathrm{~mm})$ Petri dishes, optimally by picking 10 male and 5 hermaphrodite worms and then genotyping the offspring. 
Deletion mutations and transgenic knock-ins were genotyped by Duplex PCR of single worms ${ }^{[40]}$. Single nucleotide changes were genotyped by tetra-primer ARMS-PCR ${ }^{[41]}$. A complete list of primers used in this study, can be found in section 6.2 .

Isolation of genomic DNA for genotyping was done by digesting a single worm in $5 \mu \mathrm{l}$ of Proteinase K (New England BioLabs ${ }^{\circledR}$ ) solution:

\begin{tabular}{|c|c|c|}
\hline Reagent & Concentration & Volume $(\mu \mathrm{l})$ \\
\hline Proteinase K Buffer & $2 \mathrm{x}$ & 99 \\
\hline Proteinase K & $20 \mathrm{mg} / \mathrm{ml}$ & 1 \\
\hline
\end{tabular}

Digestion was done in the thermal cycler at $65^{\circ} \mathrm{C}$ for 1 hour, then $95^{\circ} \mathrm{C}$ for 15 minutes and finally $15^{\circ} \mathrm{C}$ for indefinite storage. The resulting lysate can be stored at $-20^{\circ} \mathrm{C}, 4^{\circ} \mathrm{C}$ or be used immediately for PCR by adding PCR mix.

The PCR mix for 10 samples was prepared with reagents from New England BioLabs ${ }^{\circledR}$ :

\begin{tabular}{|c|c|c|}
\hline Reagent & Concentration & Volume $(\mu \mathrm{l})$ \\
\hline Taq Buffer & $10 \mathrm{x}$ & 10 \\
\hline dNTPs & $10 \mathrm{mM}$ & 2 \\
\hline Primer mix & $20 \mu \mathrm{M}$ & 3 \\
\hline Taq polymerase & 5000 units/ml & 1 \\
\hline Sterile $\mathrm{H}_{2} \mathrm{O}$ & - & 84 \\
\hline
\end{tabular}

$10 \mu \mathrm{l}$ of PCR mix was mixed with 0,5 - $5 \mu$ l of worm lysate per sample.

PCR protocols were set up as follows:

\begin{tabular}{|c|c|c|}
\hline Step & Temperature $\left({ }^{\circ} \mathrm{C}\right)$ & Time \\
\hline 1. Initialization & 94 & $2 \mathrm{~min}$ \\
\hline 2. Denaturation & 94 & $30 \mathrm{sec}$ \\
\hline 3. Annealing & $\mathrm{T}_{\mathrm{a}}$ & $30 \mathrm{sec}$ \\
\hline 4. Elongation & 68 & $25 \mathrm{sec}$ \\
\hline 5. Final elongation & 68 & $5 \mathrm{~min}$ \\
\hline 6. Final hold & 15 & indefinite \\
\hline
\end{tabular}

Samples were then mixed with $5 \mu$ of Orange G dye (60\% Glycerol) for electrophoresis or stored at $4^{\circ} \mathrm{C}$ overnight. 
Gel preparation for electrophoresis was done by dissolving 0,3 $\mathrm{g}$ of UltraPure ${ }^{\circledR}$ Agarose (Thermo-Fisher Scientific) in $30 \mathrm{ml}$ of TBE buffer (1 x) for a final concentration of $1 \%$ and, after cooling down, adding 2,5 $\mu \mathrm{l}$ of SYBR Safe DNA Gel Stain (Thermo-Fisher Scientific). The agarose mix was poured in a custom-made plastic mold $(10 \times 7 \mathrm{~cm})$ and was left for 30 45 min to solidify. $3 \mu \mathrm{l}$ of DNA Ladder $100 \mathrm{bp}$ (Thermo-Fisher Scientific) were added, to identify the sizes of the PCR products. The gels were run at $120 \mathrm{~V}$ for $30 \mathrm{~min}$.

\subsubsection{CRISPR-based gene editing}

The strains PHX1364 (hsp-12.6 (syb1364) knock-in allele) and PHX1652 (cutl-17 (syb1652) deletion allele) were designed by Prof. Henrik Bringmann and created by Suny Biotech using CRISPR/Cas9. Briefly, PHX1364 contains an mKate2 sequence in the endogenous locus of hsp-12.6 on chromosome IV, while PHX1652 contains a 3900 bp deletion in the promoter of hsp-12.6 (syb1364) on chromosome IV.

>PHX1364: hsp-12.6 (coding sequence - exon 2) - Linker - mKate 2 - hsp-12.6 (end codon)

CTAGAAGCCCATAACTTCCTCCCAAAAGAGATTGAAGTCAAGAACATTGGAG AACTTCTTGAAATCCATATGGAGCACAATGTAAAGAAGGATTCATTTGGAGA TGTCTCTCGTAACATTACTCGTTGTTACAAACTTCCAAAGAATGTTGATATGA AGACAATCAAGAGCAACCTCGATTCACATGGAATTCTTCACATTGAAGCAAG AAAAATGCATGGATCCGGATCCGGAATGTCCGAGCTCATCAAGGAGAACATGCA CATGAAGCTCTACATGGAGGGAACCGTCAACAACCACCACTTCAAGTGCACCTCC GAGGGAGAGGGAAAGCCATACGAGGGAACCCAAACCATGCGTATCAAGgtaagtttaa acatatatatactaactaaccetgattatttaaattttcagGCCGTCGAGGGAGGACCACTCCCATTCGCCTT CGACATCCTCGCCACCTCCTTCATGTACGGATCCAAGACCTTCATCAACCACACC CAAGGAATCCCAGACTTCTTCAAGCAATCCTTCCCAGAGGGATTCACCTGGGAGC GTGTCACCACCTACGAGGACGGAGGAGTCCTCACCGCCACCCAAGACACCTCCCT CCAAGACGGATGCCTCATCTACAACGTCAAGATCCGTGGAGTCAACTTCCCATCC AACGGACCAGTCATGCAAAAGAAGACCCTCGGATGGGAGGCCTCCACCGAGACC CTCTACCCAGCCGACGGAGGACTCGAGGGACGTGCCGACATGGCCCTCAAGCTC GTCGGAGGAGGACACCTCATCTGCAACCTCAAGgtaagtttaaacatgattttactaactaactaatctgat ttaaattttcagACCACCTACCGTTCCAAGAAGCCAGCCAAGAACCTCAAGATGCCAGGA GTCTACTACGTCGACCGTCGTCTCGAGCGTATCAAGGAGGCCGACAAGGAGACCT 
ACGTCGAGCAACACGAGGTCGCCGTCGCCCGTTACTGCGACCTCCCATCCAAGCT CGGACACCGTTAA

\section{>PHX1652: $h s p-12.6$ (promoter) - Deletion - $h s p-12.6$ (promoter) - hsp-12.6 (start codon)}

tgatgaacataatgaaattctccaacagtagtgattccggcattaatcatttctttgaaagttgataagcagtactgaaataatattttctttga aacagcacaattattgaaattttcaaattctacgtttttctgaatttcctcattaacttgaattgagaagataataaaatttgaagttcaatttcac agtaggcacaaggatgttgaaggtaggcatgcaggcatcttaatgctcatttacctgataaatcttttccttcgtaacttcagcaaccaatc catacatattatctctccatttccaaaacgtatcagccgcagattttccaatttctgatttcctctcaaatgtcgatgaaatgcatgagaatga gtatttataaatcctggcagaagaacatggttgctcaaatgaacattctcatcgaatattttcttattaattccaacaatttttcccgtttcatcaa ctgatattgatacgttctcttctatttttccatcaatccaagcatatttgacatgaatggttttcccgcgaatgttcatctgaaattctattttttgag aacaaaaaataaaaaaaataattgttttaaaaacagtaaataacgtaattttccagttccaaactgccgatttatgtaaggttaaaacttgaat ggacgttgcaaaaaaagctttcaaaaaatcatgattgacctaacagtcaaaaatgtcatattttcagGTGATTTGTGATGAT TTGGAAATTCGAAATGTGGACAGTTTTGGCAGGAAAAAACGAAATTCAAATGTA GAAAAATGGTTGAAAGCTGGAgtaagtcttgaaattattatcactaacgatatgaaaagtgaactaatttggaagaat gtccctttctaaacttcaaaagttggaatccaaaagtaaaaataaataaattttggtttcaaccagaaataaataggttgaacattcttaaaat ttaaagaagagaacatattcttcgaaatatgcacttttcggggcgacgtcacatttctgtgacaagtgcatcaaaatgtagaatttcaatttg atgtgtctcatccatcctcataaattttgaaaatatttattcgtatattgcagAACAAAAAAGAGACAAGAAAACGT CGTATGGTGACAGGAACACTTACAATAACCGATGAAGAAGAAACGAATACAACG GTCAAACCTCGTCCAGgtatctatttctgttgatttctgcgtctcttcatttcttcattatctcttttcaacttaattacacggtttca cagaaagtgttgtgttttatggetttgttgttcaggttgatgcaagttgagaaaccatgtaatttacacttgcataaaacaaccgttgcaaagt gtttcgaaatttgaaattgggcgttgaaatccctctgttagttgataagttatctccaatgggtaatatattaaaaacaattgttatggtgctaa aaattttttaaatatcatagtaaagcttttcaatatttttatgtttgagtataccaaaaattattgaagtgttttattattataattcatcatgttagctt aacaatactccacagtttaaaaatatctaacaactagcaaatcagaaatatttgaaacaaaacattatcacaaagaattatgataactgactt actagttaggcaaggctatgtaggttttaactatcaaactagtccaacaatcgttcaacagccatcccattaatttctaaaattttttgacggc ttttaaatgaaaaatgcatataaaaaagaagaagaattgaaaatttattgcacatccaaaagctgtgggaaagtgttgacaagtggaagttt gggtgagagggttaggaattatgaatgtcccactggaatttctgccaccaaaaacgttttttcaaatattcaaaactcaactttttcgtttcac atcactcatcacttcagAGTTTTCAACATCCTCTTCTTCATCTTCTCAACAACAATATTGTGT TCAAAAGGATCGAATTGAATTTTTAATTGTGATTGTTGTACTTCTGATTCTACTTC AAATCGGATTGGCATTATTCTTCTATAGAAAATGTGTCAGCCGATCATCAATGGA AACATCAAGTGTTTATTCGGAAGGTAGTTCGAGATGCTCTGCCACGTCATCGGCC TATAATACATGTAATCGACAAATGGAACCACCTGTCTTACCGGTCAGACCTGCAA GGTTTTCAGATGGAGTTCAACCTCCATTCACGGAAAATCCATACAATCGATTACA TCATTTCACATAAttggaacttttaaatttgtttatgtaaatgcgttttagtgtgaattttaacttttttgaaaaccattttaaattaata 
tattggaaacatttatgagacacgacaaatattttcaacttttagtttcgcagtgaactcacatgaaacgtattaactccttgataaaatatttat cattagagatccgtcaaggatttttgctcactactttccacacttttttaatgtacatatcttgecgtttgaaatatttatttaatattttatttaattcc tttctatattattttgtattttggctttccaattcctctaatactgtgcctttgaacaataattgtgtgtgtctttttaaaatctctagtgatctatgtca ttttataaatctttttacttattctaattttgtatttatattttatattttgagtaattttacgaataataatcaatttttcacttactttcaaatgtgaatca gacttgaacctaccggaataaatctgctcccgaaagaacacaaaacgagatacctcaatttccattacaactgaaaaaggttaaaacatt gtttacaaattattataataacaattgatttttttctcttttaaatataaaaattgtttaaattcaaatcaaaaggtttttatttggtttcatttttatgttt attatatgaacaccacatgtcacaatgttgtttccctctcattctgaacttcgccctcttctatagatagaaattgtttttatttattattttagaac agaataaaataaaaaaacattcacacaaagaatgttctcgatgcaaaaataatgtttttaaaattgaaataagagtatgtgagaggaattttc agatttagtaggtcaacatgtttacgtggtttctggtttattacggcttcaggttatttcctaggtttctagaatttttcgaaaatatatcttcaga gcacgtgcgaagattgaacagaaatcgttttttcttaactttgttgtaatgttttcttttgttattaccttccgatgtctgctacttggggaaatct gaaaatagcttggttttgaaaaatccgtgttttcagcaaagaaaaaagaacaagttattaaaattgaaatgatattacgattgatttcaaata tagaaattcaattccaaagtcactatggcatgtattgacatgaattcagaaaatgcaaaaaaaatttaaaagacaagaaagccgatggga tcatgcttgacactttttttgccaaaaatacgttttaacaaaactgtgaaaatttgtttctgatttaatattttaaaagtgaaaaatttttttaaaatt aatttaaaaattataagacttccattgaaatatgggcatttgaaactataattttacattttctgtatacaaattaacatttcgaatattagtcgaat ttggtcatttttcagatagaaaaattagacttcgggctatttcctatcaggaaaaaatttcgcgagatcagaatccgagtcgcctgaaaactt ctttgtcagagttaaaacgtataacagtgtcaaaaaaaaattttggaagcattttaaaatttcaaaagctctggaaaaggctgtttcacaattt tctgataattaaaaaacacagtcttccccaaaaaaaagtacagattctgaaagcccaagatgacaatggacatgtttaaattttttaaaaca actttctagtgagatgatgatttatataaatttataaggtttttttttctgtttcagaacatttttttttaacttggtgtgatttattaaattatcttatgc atcttctttcaagaagttatcaaaattgattagccatatcaactttccataaaatagagaaaaattctacagatattaaatttaatatatataattt aaatcatttttttacagaaatttttataactaacagaattttcaaaatctcatgatttccagcatctaatatactggtttactgattgttttctgatttg aagactgttttcatgaaatcgacgtgacttctacaagatatcaaagcttgaccttttgtttgcacctgaaatgctcccaattatcttttctgtttt tcctctccagacacgtgtcccatgctttttctatcttcgaagctttcccatatcacacatatccacatacacatctgttcttttatttttatttttgtg cttcttttcttccatgtgatttgggacttgctgaccacccaggtggtccgtgcaaaaattcaaaacaaaaaagaaaagaagatagagatta agagagagaatgagaagaaaaaaaataaaatttgtaattttagaactcttaaaattcgatcccattgaaaacatcacatgtatctggttttct ctggaaaaatatgttctcaagtatgaaatttctctgttttctctaagtcttattaactctcatttttcaactatttgtttattcttcaggacaatatgg gcggagtctggatatgaaaaacaagtctgctagctgaaaatatgtgggcggagtcagtaactgtcatgcgacgaaagacatgaatctgt gcgcgaagtaacaatataaaaagggtgagcattctgtgaatggacaacaatttcctctctttctaatactaacttctgaaaatttcagaaaa aatccaaaaacttttgccaagATG 


\subsubsection{Synchronization}

Synchronization of C. elegans at the L1 stage is based on the fact that adult worms are sensitive to treatment with alkaline hypochlorite (bleaching), while their eggshell protects the embryos from it ${ }^{[42]}$. The following steps were performed:

1. Alkaline hypochlorite solution was prepared fresh and heated to $36^{\circ} \mathrm{C}$. The solution loses its potency over time, even though it can be stored at $4^{\circ} \mathrm{C}$ up to a week. Hypochlorite solution is more effective when it is warm. However, heating hypochlorite solutions at high temperatures for prolonged periods of time produces toxic phosgene gas. Handle with care, since hypochlorite is a whitening agent and will bleach fabrics. The solution was made by mixing $6 \mathrm{ml} \mathrm{NaOCl}(15 \% \mathrm{w} / \mathrm{v}), 12.5 \mathrm{ml} \mathrm{NaOH}(1 \mathrm{M})$ and $31.5 \mathrm{ml}$ sterile $\mathrm{H}_{2} \mathrm{O}$ for a final volume of $50 \mathrm{ml}$.

2. Worms were grown on NGM plates until they became gravid adults. Then they were washed off with M9 buffer and transferred to an Eppendorf or Falcon tube, depending on the volume of buffer used.

3. Afterwards, the tube was put in ice for $2-3$ min which allowed all the adult worms to sink to the bottom, while younger larval-stage worms were suspended in the supernatant. C. elegans larvae are particularly resistant to bleaching and their presence will cause over-exposure of the eggs to the bleach solution, killing them in the process. The supernatant was then discarded.

4. $500 \mu \mathrm{l}$ of hypochlorite solution was added per $100 \mu \mathrm{l}$ of adult worm pellet as well as an equal amount of M9. The tube was shaken vigorously for $1 \mathrm{~min}$ and then centrifuged at $4200 \mathrm{x}$ g for $30 \mathrm{sec}$. This killed the worms without dissolving them and a compact pellet was formed. The supernatant was then discarded.

5. $1000 \mu \mathrm{l}$ of hypochlorite solution was added per $100 \mu \mathrm{l}$ of adult worm pellet. The tube was shaken vigorously for $1 \mathrm{~min}$. Under the stereomicroscope it was observed that the cuticles had started to dissolve and the eggs were released. Then it was centrifuged at $4200 \mathrm{x}$ g for $30 \mathrm{sec}$. The pellet should have a yellow color. The supernatant was then discarded.

6. $1000 \mu \mathrm{l}$ of M9 was added per $100 \mu \mathrm{l}$ of adult worm pellet, the pellet was resuspended and centrifuged at $4200 \mathrm{x}$ g for $30 \mathrm{sec}$. The pellet should be white and nearly all of the adult cuticles should be dissolved. The supernatant was then discarded. 
7. 2 more rounds of washing with M9 (Step 6 above) were performed. The pellet was suspended in an appropriate amount of M9 (usually $1 \mathrm{ml} \mathrm{M} 9$ in a $2 \mathrm{ml}$ Eppendorf tube), which allowed enough oxygen for worm development. The final suspension should not have the characteristic hypochlorite smell.

8. The worms were let to hatch overnight while rotating $(20 \mathrm{rpm})$ at $20^{\circ} \mathrm{C}$. The next day, a healthy population L1 arrested worms should be swimming in the tube.

\subsubsection{Bacterial culture for feeding}

Bacteria were scraped off the surface of the frozen $\left(-80^{\circ} \mathrm{C}\right)$ glycerol stock with a sterile pipette tip in a laminar flow hood. Then, the tip was discarded in an Erlenmeyer flask filled with 200 $\mathrm{ml} \mathrm{LB}$ medium. The flask was incubated at $36^{\circ} \mathrm{C}$ overnight, rotating at $60 \mathrm{rpm}$. If the bacteria were alive after thawing, the LB medium should turn opaque after incubation. The next day NGM plates were seeded with $200 \mu \mathrm{l}(60 \times 15 \mathrm{~mm}$ plates $)$ or $500 \mu \mathrm{l}(100 \times 20 \mathrm{~mm}$ plates $)$ of bacteria.

The following strains of bacteria were used in this study:

1. OP50: E. coli (Uracil auxotroph, standard C. elegans food source).

2. Na22: E. coli (Prototroph, grows in thick layers, abundant $C$. elegans food source).

3. HT115: E. coli (RNase III deficient, resistant to Tetracycline)

4. DL41: E. coli (Methionine auxotroph)

\subsection{Microscopy and imaging}

\subsubsection{Long-term imaging using agarose hydrogel microchambers}

Long-term imaging of L1 arrested worms was performed in agarose hydrogel microchambers as previously described ${ }^{[43]}$. Briefly, a poly-dimethyl-siloxane (PDMS) mold, activated by air plasma exposure for $30 \mathrm{sec}$, was used to create microcompartments from $1 \mathrm{ml}$ of $3 \%$ (quiescence assessment and calcium imaging) or 5\% (fluorescent imaging) high gelling temperature agarose (Sigma-Aldrich) dissolved in M9 buffer. The chamber size was 110 x 110 x $10 \mu \mathrm{m}$. The chambers were filled with 3-fold stage eggs without food, sealed with a glass coverslip and glued with double-sided adhesive tape into a square $(2 \times 2 \mathrm{~cm})$ opening, milled 
in a $35 \times 10 \mathrm{~mm}$ plate. The plate was filled with $2 \mathrm{ml}$ of agarose, which would act as a moisture reservoir, and sealed with Parafilm M (Sigma-Aldrich) to prevent moisture loss. Chambers were incubated at $20^{\circ} \mathrm{C}$ until use.

\subsubsection{DIC imaging for quiescence assessment in $L 1$ arrest ( 48 hours)}

Strains were assessed for quiescence in L1 arrest via continuous differential interference contrast (DIC) imaging. An "Eclipse Ti" microscope (Nikon) with an automated XY stage (Nikon), a digital DS-Qi2 camera (SLR, FX-format CMOS sensor, Nikon) and a 10x/0.45 Plan Apo $\lambda$ objective lens (Nikon) were used. A red-light filter (BrightLine HC 785/62, $45 \mathrm{~mm}$ diameter, Semrock) was used in order to minimize behavioral changes due to illumination. A custom-made heating lid was used to keep the temperature constant at $25.5^{\circ} \mathrm{C}$ to avoid condensation (sample temperature was measured at $23.5^{\circ} \mathrm{C}$ ). The software used for image acquisition and microscope control was "NIS elements" (Nikon). The length of the movies was 4 hours and the frame rate 0.2 FPS ( 1 frame $/ 5 \mathrm{sec}$ ). Exposure time was set to $50 \mathrm{~ms}$ and resolution was 14-bit $808 \times 808$.

Mean pixel intensity values (that correspond to the magnitude of the displacement) were extracted for individual worms via image subtraction ${ }^{[44]}$, smoothed at $1 \%$ of total points (robust LOESS) and divided by the time interval to obtain speed values. Quiescence bouts were detected by applying a threshold of "speed $<40 \%$ of maximum speed continuously for a duration of time $>2$ minutes", with the custom-written MATLAB script found in section 6.3. This threshold was selected as it most accurately describes the wild-type worn quiescence in the $110 \times 110 \times 10 \mu \mathrm{m}$ chamber that was used. Then, the fraction of quiescence was calculated for each worm. Eggs that did not hatch or worms that died before or during the measurement were excluded from the analysis. Each strain was imaged in at least 2 chambers (biological replicates). A median number of 15 animals was screened per strain (minimum $=7$ ). The Effect Size (Cohen's $d$ ), as well as $95 \%$ confidence intervals, were calculated by comparing each mutant strain to the wild-type. Precision was calculated as the reciprocal of the Standard Error of the Mean. Statistical significance was assessed by the 2-tailed Welch's $t$-test (assuming unequal variances) and the resulting p-values were corrected for false discovery rate by the Benjamini-Hochberg method. 
For a hit to be considered significant in this study, it has to satisfy the following criteria:

a. $\mid$ Cohen's $d \mid>1.2$ "Very large" [45]

b. $\mid 95 \%$ Confidence Interval of mutant $|>| 95 \%$ Confidence Interval of wild-type $\mid$

The above criteria are stricter than the regular FDR $<0.05$ threshold, but are justified by the extent of the study and the magnitude of the biological effect observed.

In the special cases of determination of the impact of 1 . Maternal growth conditions and 2. Amino-acid supplementation (Figure 32) in L1 arrest quiescence of wild-type worms, only a threshold of FDR $<0.05$ was applied because the expected biological effects are very small.

\subsubsection{Fluorescence and calcium imaging in $L 1$ arrest}

Fluorescence-tagged transgene expression was quantified by fluorescence microscopy, and neuronal activity with calcium imaging. The same setup was used as above, with the following modifications: a 40x/0.75 Plan Fluor Oil objective lens was used combined with an additional built-in 1.5x lens for a total magnification of 60x. For GFP and mKate2 excitation, an LED system (CoolLED) provided light at $490 \mathrm{~nm}$ and $565 \mathrm{~nm}$, respectively. Exposure time was set to $100 \mathrm{~ms}$ and EM Gain to 1.0x. Light intensity was measured to be $1.75 \mathrm{~mW} / \mathrm{mm}^{2}$ for $490 \mathrm{~nm}$ and $0.517 \mathrm{~mW} / \mathrm{mm}^{2}$ for $565 \mathrm{~nm}$ illumination, using an optical power meter (PM100A, Thorlabs). A standard set of GFP and Texas Red filters were used for light filtering.

Reporter transgene expression was measured either continuously for 3 days ( 1 frame every 15 or $30 \mathrm{~min}$ ) or once every 24 hours for up to 12 days. Average worm fluorescence intensity was quantified per worm after background subtraction, assuming that the size of the worm is $10 \%$ of the total image size.

For the continuous 3-day imaging experiments, frames were extracted from the movies and aligned to the point of hatching. Frames before the hatching point were excluded from downstream analysis. At least 8 animals were imaged per strain in at least 2 chambers (biological replicates).

For the daily measurement experiments, chambers were made in the evening of the day prior to the start of the experiment (Day 0). That gave the eggs a time interval of 10-12 hours to 
complete embryogenesis and hatch but not yet starve. At least 20 animals were imaged per strain in 1 to 3 chambers (biological replicates). The chambers were kept at the $20{ }^{\circ} \mathrm{C}$ incubator when not being imaged.

In both cases, worms that died before or during the measurement period were omitted from downstream analysis. Single worms with transgene intensities $>\mathrm{Q} 3+1.5 *$ IQR or transgene intensities < Q1 + $1.5 *$ IQR for every time point of the analysis, were scored as outliers and omitted from further analysis $\left(\mathrm{Q} 1=25^{\text {th }}\right.$ percentile, $\mathrm{Q} 3=75^{\text {th }}$ percentile, IQR $=$ Interquartile range). Significance was assessed at every time point by the 2-tailed Welch's $t$-test (assuming unequal variances), the resulting p-values were corrected for false discovery rate by the Benjamini-Hochberg method and FDR $<0.05$ was selected as the significance threshold. In every case, error bars denote the standard error of the mean.

Neuronal activity was quantified by expressing the calcium-sensitive probe GCaMP 3.35 (codon-optimized for use in C. elegans) under a specific promoter and selecting only the neuron of interest (RIS neuron) or by average total fluorescence intensity (mechanosensory, dopaminergic and tyraminergic neurons). Specifically, for RIS, it was automatically detected by identifying the maximum intensity value of the image, selecting a 12 x 12 pixels region around it (size of RIS), subtracting the lowest pixel intensity as background and averaging the remaining intensities, with the custom-written MATLAB script found in section 6.4. (Figure $5)$.

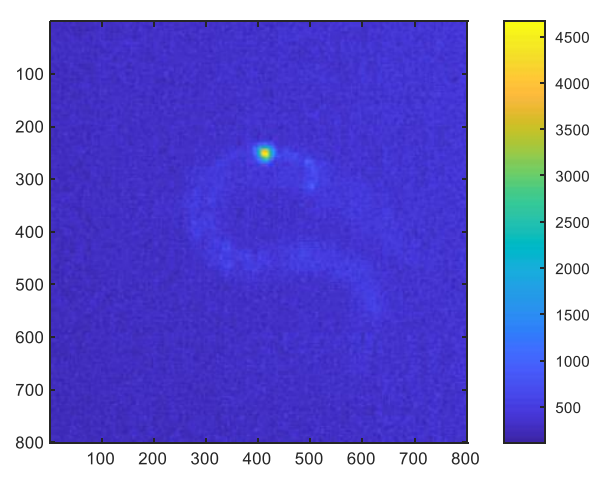

\section{Figure 5. RIS detection by an automated MATLAB script}

The absence of a background signal and the high fluorescence intensity of the flp-11::GCaMP transgene allows for easy automated tracking of RIS. These conditions ensure, that in all cases the maximum intensity value will be part of RIS. Averaging the intensities in the $12 \times 12$ pixels window centered at the maximum value gives a robust estimation of RIS intensity. Here, a representative worm of the wild-type HBR1361 strain is shown in L1 arrest (48 hours). 
Images were taken once every 5 seconds for RIS neuron measurements or 10 seconds for the other neurons, for a total duration of 4 hours. In either case the intensity of GCaMP was normalized to the co-expressed mKate 2 signal, which is not affected by the changes in calcium levels. For RIS at least 12 worms were imaged in 3 chambers (biological replicates), while for the other neurons at least 8 animals were imaged in 2 chambers. Significance was assessed at every time point by the 2-tailed Welch's $t$-test (assuming unequal variances), the resulting $\mathrm{p}$ values were corrected for false discovery rate by the Benjamini-Hochberg method and FDR < 0.05 was selected as the significance threshold.

\subsection{Transcriptional profiling by RNA-sequencing}

Bulk sequencing of 3 C. elegans strains (N2, HBR227 and HBR1777) was performed by growing the worms on Nystatin-supplemented plates (NEP), seeded with $\mathrm{Na} 22$ bacteria, at $20^{\circ} \mathrm{C}$ until they reach adulthood. The population was then synchronized as described in section 3.1.4. and L1 arrested worms were harvested after 48 hours ("Starved"), at a final concentration of 20.000 worms/sample. The "Fed" samples were treated as described above, but were supplemented with OP50 for 4 hours after hatching. Then they were washed 3 times with M9 to remove the bacteria. In either case, the supernatant was aspirated from every sample and the pellet was either flash frozen in liquid $\mathrm{N}_{2}$ or suspended in $1 \mathrm{ml}$ RNA-later ICE (Thermo-Fisher Scientific) as requested. Then samples were stored at $-80^{\circ} \mathrm{C}$ overnight and shipped on dry ice for downstream processing.

In the first case (CeGaT transcriptome) the project was outsourced to the company CeGaT. RNA extraction, library preparation and quality control were performed and the samples were sequenced with an Illumina HiSeq 2500 platform at $30 \mathrm{M}$ reads/sample, single-end (1x50bp). An Excel file with genes and the corresponding counts was received.

In the second case (MPI-MG transcriptome) the project was outsourced to the Max Planck Institute for Molecular Genetics. The same processes as above were carried out and the samples were sequenced with an Illumina HiSeq 4000 platform at $50 \mathrm{M}$ reads/sample, paired-end (2x50bp). FASTQ files were received and they were annotated by Jik Nijssen (Ph.D.). An Excel file with genes and the corresponding counts was received.

In both cases, the differential expression analysis was carried out through the custom-written MATLAB script found in 6.5. Briefly, principal component analysis was performed to detect 
any outlier samples, which were omitted from downstream analysis. Then, raw counts were normalized to a pseudo-reference sample and lowly expressed genes $(\mathrm{CPM}<0.5)$ as well as genes with highly variant counts among replicates $\left(\mathrm{CV}>97.5^{\text {th }}\right.$ percentile) were removed. The rest of the genes were fitted in a negative binomial model (mean-variance link was determined by local regression), assessed for statistical significance and the p-values were corrected for false discovery rate by the Benjamini-Hochberg method. Significance thresholds were set as follows: $\left|\log _{2}(\mathrm{FC})\right|>1$ and FDR $<0.05$. This was implemented in order to account for biological significance. However, genes with smaller fold changes were also considered for downstream Gene Set Enrichment analysis, performed with the online tool "g:Profiler", using the default settings and the Bonferonni correction to get the adjusted p-values $\left(\mathrm{p}_{\text {adj }}\right)^{[46]}$.

\subsection{Agglomerative hierarchical clustering analysis of RNA-seq data}

Normalized counts of the RNA-seq data (from MPI-MG) were analyzed with the "clustergram" MATLAB function which produces a dendrogram and the associated heatmap. Each row of the heatmap corresponds to one gene and each column to one sample.

The following algorithm was implemented:

1. Data were transformed to their base 2 logarithm values and standardization (Z-score calculation) was done by row, to account for changes in gene expression.

2. Pairwise observation distance was calculated both in rows and columns with the Euclidean metric.

3. The linkage criterion to determine the distance between sets of observations was Ward's minimum variance method.

4. Data were clustered first among columns and then among rows and then optimal leaf ordering was applied to maximize the sum of similarities between adjacent leaves.

After that, enrichment analysis of selected pathways was carried out. The number of significantly regulated genes (either Up- or Down-) in a specific pathway, was statistically compared to the total number of significantly regulated genes in the whole transcriptome by using the right-sided Fisher's exact test, corrected with the Bonferroni method. The significance threshold was set to $\mathrm{p}_{\text {adj }}<0.05$. 


\subsection{Proteomic characterization of C. elegans}

C. elegans cultures of strains N2, HBR227 and HBR1777 were grown for proteome analysis as described in section 3.3. at a final concentration of 50.000 worms/sample. They were flash frozen in liquid $\mathrm{N}_{2}$ and shipped to different facilities for downstream processing.

Proteome analysis by TMT quantification was carried out by the laboratory of Prof. Henning Urlaub (MPI-BPC). The samples were ground in liquid $\mathrm{N}_{2}$ and soluble proteins were extracted. Proteins were labeled with TMTsixplex Isobaric Label Reagent (Thermo-Fisher Scientific) for quantitative analysis via mass spectrometry.

Proteome analysis by Label-Free Quantification (LFQ) was carried out by the laboratory of Dr. David Meierhofer (MPI-MG). Samples were digested, the soluble fraction was extracted and run through the mass spectrometer. The relative amount of proteins in each sample was then quantified and peptide intensities were corrected to remove systematic artefacts ${ }^{[47]}$.

In both cases, the differential expression analysis was carried out through the custom-written MATLAB script found in section 6.6. Briefly, principal component analysis was performed to detect any outlier samples, which were omitted from downstream analysis. Peptides not detected (intensity $=0$ ) in at least one sample were removed. Intensities were then quantile normalized and peptides with highly variant intensities among replicates $\left(\mathrm{CV}>97.5^{\text {th }}\right.$ percentile) were removed. The remaining peptides were assessed for statistical significance using the 2-tailed Welch's $t$-test (assuming unequal variances) and the p-values were corrected for false discovery rate by the Benjamini-Hochberg method. Significance thresholds were set to $\left|\log _{2}(\mathrm{FC})\right|>1$ and FDR $<0.05$, to account for biological significance.

\subsection{Metabolome analysis in $L 1$ arrest}

C. elegans cultures of strains N2, HBR227 and HBR1777 were grown for metabolome analysis as described in section 3.3. at a final concentration of 100.000 worms/sample.

Metabolome analysis by LC-MS was performed by the laboratory of Dr. David Meierhofer (MPI-MG) as previously described ${ }^{[48]}$. 
Differential expression analysis was carried out by the custom-written MATLAB script found in section 6.6. Briefly, principal component analysis was performed to detect any outlier samples, which were omitted from downstream analysis.

Metabolites not detected (intensity $=0$ ) in at least one sample were removed. Intensities were then quantile normalized and peptides with highly variant intensities among replicates (CV > $97.5^{\text {th }}$ percentile) were removed. The remaining peptides were assessed for statistical significance using the 2-tailed Welch's $t$-test (assuming unequal variances) and the p-values were corrected for false discovery rate by the Benjamini-Hochberg method. Significance thresholds were set as follows: $\left|\log _{2}(\mathrm{FC})\right|>0.5$ and FDR $<0.1$. This was implemented because metabolite levels are fluctuating fast and the changes in quantity are usually subtle (personal communication with Dr. Meierhofer).

\subsection{Survival and behavioral assays}

\subsubsection{Lifespan of $\mathrm{L} 1$ arrested animals}

Three different assays were used to evaluate survival in L1 arrest:

Chamber assay: 3-fold stage eggs were picked on 110 x 110 × $10 \mu \mathrm{m}$ microchambers without food, made of 3\% high gelling temperature agarose (Sigma-Aldrich) dissolved in M9 buffer. The chambers were stored in the $20^{\circ} \mathrm{C}$ incubator. Animals were scored as "dead", if they failed to move after exposure to blue light for 2 minutes. Measurements took place every day. Survival was estimated by using the Kaplan-Meier method, significance assessment was done by using the log-rank test and the significance threshold was set to $p<0.05$.

96-well plate assay: $100 \mu \mathrm{l}$ of 3\% high gelling temperature agarose (Sigma-Aldrich) dissolved in M9 buffer were pipetted at the bottom of each well. Worms were synchronized as described in section 3.1.4. and transferred to the 96-well plate in $100 \mu$ lof M9. Approximately 10 worms were transferred in each well, so that it is possible to keep track of individual worms.

The plates were stored in the $20^{\circ} \mathrm{C}$ incubator. Animals were scored as "dead", if they failed to start swimming after exposure to blue light for 2 minutes. Measurements took place every day. Survival was estimated by using the Kaplan-Meier method, significance assessment was done by using the log-rank test and the significance threshold was set to $\mathrm{p}<0.05$. 
Rotator assay: Worms were synchronized as described in section 3.1.4. and transferred to $2 \mathrm{ml}$ Eppendorf tubes, at a concentration of 10.000 worms in $1 \mathrm{ml}$ of M9 buffer. The tubes were strapped on a rotating device $(20 \mathrm{rpm})$ and put in the $20^{\circ} \mathrm{C}$ incubator. Then, $10 \mu \mathrm{l}$ of worm suspension was pipetted on NGM plates seeded with OP50 and the total number of worms was counted. One day later, worms were scored as "dead" if they have not shown any sign of movement (traces on the bacterial lawn) and were dried out. Measurements took place every day. Survival was estimated by using the Kaplan-Meier method, significance assessment was done by using Fisher's exact test for every time point, p-values were corrected for false discovery rate by the Benjamini-Hochberg method and the significance threshold was set to FDR $<0.05$. Finally, the point of $50 \%$ survival was determined by fitting a $6^{\text {th }}$ degree polynomial to the survival curve.

\subsubsection{Sleep deprivation by optogenetic stimulation}

Optogenetic stimulation of the worms was done in $110 \times 110 \times 10 \mu \mathrm{m}$ microchambers without food, made of 5\% high gelling temperature agarose (Sigma-Aldrich) dissolved in M9 buffer. The chambers were supplemented with $10 \mu \mathrm{l}$ of $10 \mathrm{mM}$ Retinal in 100\% ethanol every 3 days (+) or $10 \mu \mathrm{l}$ of $100 \%$ Ethanol (EtOH) or $10 \mu \mathrm{l}$ of water (-). Long-term optogenetic experiments were carried out with the OptoGenBox, designed by Inka Busack ${ }^{[49]}$. Temperature was set to $20^{\circ} \mathrm{C}$ and orange light intensity to $10 \mathrm{~mW}$. The "light" interval was set to 10 minutes and the "dark" interval was set to 20 minutes, alternating for 6 days until the end of the experiment. This setup was selected to avoid habituation. The samples were removed at the "dark" interval for daily transgene imaging and were promptly returned to the OptoGenBox.

\subsubsection{Sleep deprivation by mechanical stimulation}

Mechanical stimulation of the worms was done in $110 \times 110 \times 10 \mu \mathrm{m}$ microchambers without food, made of $5 \%$ high gelling temperature agarose (Sigma-Aldrich) dissolved in M9 buffer. A piston ( $2.5 \mathrm{~mm}$ in diameter) was driven into the chamber at the xy-plane, by the use of an electromagnet and the whole setup was fitted in a custom-made aluminum holder ${ }^{[50]}$. To avoid habituation, a set of 5 taps were delivered every 5 minutes and the time interval between taps was 1 second. This was achieved by the use of an Arbitrary Waveform Generator (Hewlett Packard, 33120A), which created square waves with amplitude $5 \mathrm{~V}$ and frequency $5.000 \mathrm{mHz}$. This signal was further amplified at $24 \mathrm{~V}$, by a TTL signal amplifier. A heating lid, set to $22^{\circ} \mathrm{C}$, 
was fit on the chamber to keep it from drying out (sample temperature: $20^{\circ} \mathrm{C}$ ). The chambers were imaged daily to assess transgene expression, as described above, and were then returned to the tapping device, taking care that a different spot will be tapped, since prolonged tapping at the same spot will destroy the plastic plate (Figure 6).

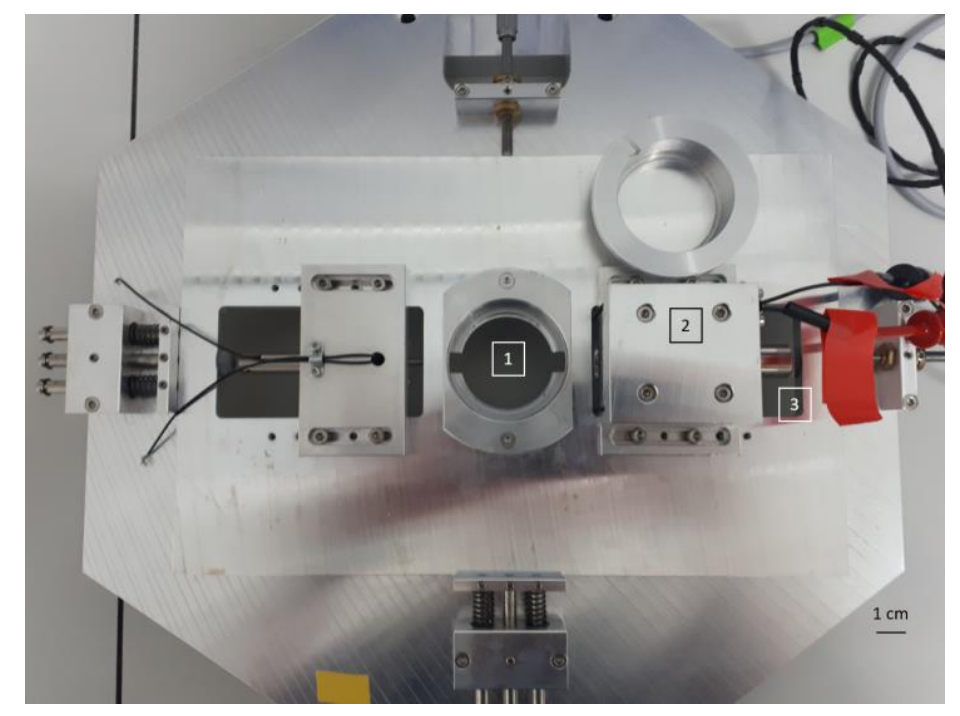

Figure 6. Tapping device for mechanical stimulation

The custom-made tapping device consists of a hexagonal aluminum frame which can also be mounted on a microscope and used as a stage. The chamber with the worms is fitted in the aluminum holder (1). An electrical current that passes through a coil, creates an electromagnet (2) which drives the tip of the piston (3) into the sample.

\subsubsection{Heat production in $L 1$ arrest ( 48 hours)}

The rate of heat production of the strains N2, HBR227 and HBR1777 was assessed at the laboratory of Prof. Bart Braeckman (University of Ghent). Worms were synchronized as described in section 3.1.4. and the worm suspension in M9 was transferred in cylindrical, sterile glass containers. Isothermal microcalorimetry was performed using two 2277 Thermal Activity Monitors and Digitam software (Thermometric AB, Jarfalla, Sweden). The temperature was set to $24^{\circ} \mathrm{C}$. A blank sample containing only M9 buffer was used to determine background heat production. Measurements were taken every 1 second for 4 hours (4 biological replicates). Only the last 5 minutes of measurements (equilibrium point) were used in the analysis, because at the start of the measurement there is increased heat production due to friction. Results were normalized to the total amount of worms per sample. Quantile normalization was used to remove technical variance, since the measurements are close to the detection limit. Statistical significance was assessed using the 2-tailed Welch's $t$-test (assuming unequal variances) and 
the p-values were corrected for false discovery rate by the Benjamini-Hochberg method. The significance threshold was set to FDR $<0.05$.

\subsubsection{Egg-laying assay}

For the egg-laying assay, L4 worms were picked on individual NGM plates with (OP50) and without food. Every day worms were transferred on fresh plates and the number of eggs was counted. Worms that died before the end of the experiment or escaped were removed from downstream analysis. Starved animals could not be assessed for more than 2 days, since they escaped the plate in search for food. Statistical significance was assessed using the 2-tailed Welch's $t$-test (assuming unequal variances) and the p-values were corrected for false discovery rate by the Benjamini-Hochberg method. The significance threshold was set to FDR $<0.05$. Although the number of eggs laid is always an integer and the corresponding distribution discrete, the variables considered here (genetic mutations) are independent and therefore the Central Limit Theorem applies, which permits the use of the $t$-test for statistical significance.

\subsubsection{Worm length in $L 1$ arrest}

Worm strains N2, HBR227 and HBR1777 were synchronized as described in section 3.1.4. The L1 arrested worms were put on NGM plates and fed with OP50. After reaching adulthood, 3fold stage eggs were picked and put on $190 \times 190 \times 15 \mu \mathrm{m}$ microchambers (without food) made of 3\% high gelling temperature agarose (Sigma-Aldrich) dissolved in M9 buffer. The eggs were filmed continuously using the DIC setup described in section 3.2.2., taking 1 image every 1 minute, to determine the exact time of hatching (Day 0). The worms were imaged again exactly 48 hours after hatching (Day 2). Worm length was determined using a custom MATLAB script. Statistical significance was assessed using the 2-tailed Welch's $t$-test (assuming unequal variances) and the p-values were corrected for false discovery rate by the Benjamini-Hochberg method. The significance threshold was set to FDR $<0.05$.

\subsubsection{Aldicarb sensitivity assay}

Small-sized (35 x $10 \mathrm{~mm}$ ) Petri dishes were filled with $250 \mu \mathrm{l}$ of $3 \%$ high gelling temperature agarose (Sigma-Aldrich) dissolved in M9 buffer, supplemented with $1 \mathrm{mM}$ aqueous solution of Aldicarb (Sigma-Aldrich). Worm strains N2 and HBR227 were synchronized as described in 
section 3.1.4. Approximately 100 worms were pipetted on each plate and they were scored every 10 minutes for total lack of movement. Percentage of moving animals was estimated by using the Kaplan-Meier method, significance assessment was done by using the log-rank test and the significance threshold was set to $\mathrm{p}<0.05$.

\subsection{8. tRNA $U_{34}$ thiolation assay in $L 1$ arrest ( 48 hours)}

Worm strains N2 and HBR227 were synchronized as described in section 3.1.4. Hatched L1 worms were left to starve for 48 hours in M9 buffer at a concentration of $5 * 10^{9}$ worms/sample. 3 biological replicates were made for each strain. The samples were flash frozen in liquid $\mathrm{N}_{2}$ and shipped to the laboratory of Prof. Sebastian Leidel (University of Bern). There, total RNA was isolated and Northern blots of APM ([(N-Acroyl-amino) phenyl] mercuric chloride - a molecule that reacts with thiolated biomolecules) gels, were quantified for Glutamine (UUG) and Lysine (UUU) tRNAs. Significance assessment was done by the 2-tailed Welch's $t$-test (assuming unequal variances). The significance threshold was set to $\mathrm{p}<0.05$.

\subsection{EMS mutagenesis data analysis}

An EMS mutagenesis screening was performed by Dr. Elisabeth Maluck, in order to isolate mutations that suppress the aptf-1 (-) phenotype. This resulted in 2 candidates that showed increased sleep fraction during lethargus in the aptf-1 (-) background. Those candidates were subsequently sequenced and a dataset containing NGS data for those candidates was received. Dr. Maluck had already confirmed that the mutation is homozygous, it does not lie on the X chromosome, it is different in each candidate and it is not the insertion in the rod-1 gene (personal communication with Dr. Elisabeth Maluck).

The following algorithm was implemented, to isolate the mutations of interest:

1. Discard all mutations common in both candidates.

2. Discard all heterozygous mutations (GENOTYPE: $1 / 1$ is homozygous for the mutant).

3. Discard all mutations with do not PASS the NGS generated filter.

4. Create a list of Mutations of Interest (MOI) resulting from the filtering steps above.

5. Separate the chromosomes in intervals, accounting for different chromosome sizes (10\% of total chromosome length was selected). 
6. Calculate total mutations per interval for each chromosome. Identify hotspots (intervals with an increased number of mutations) on each chromosome, by using the right-sided Fisher's exact test on the mutations per interval. Correct the resulting $\mathrm{p}$-values using the conservative Bonferroni method. The significance threshold was set to $\mathrm{p}_{\text {adj. }}<0.001$.

7. Create a list of MOI that lie in these hotspots.

8. Automatically map the list via WormBase to get a specific list of genes, introns and upstream/downstream loci that are altered by each MOI.

9. Calculate total mutations per interval around each GOI in the new list (density).

10. Employ biological criteria (EMS typical mutations $(\mathrm{G} \rightarrow \mathrm{A}, \mathrm{C} \rightarrow \mathrm{T})$, mutation density, position on the genome, effect on resulting protein etc.) to identify the most likely candidate.

\subsection{RHEB-1 depletion via Auxin-inducible degradation}

A deletion in the TOR regulator gene rheb-1, is lethal for C. elegans. For this reason, an Auxininducible protein degradation system was implemented, that eventually depletes the RHEB-1 protein from the organism. This is a reversible process that permits survival ${ }^{[51,52]}$. Briefly, a strain was obtained in which expression of the Auxin receptor of Arabidopsis thaliana TIR1 was driven under the ubiquitously expressed promoter eft-3. Then, a construct containing an Auxin Inducible Degron (AID) as well as an mKate2 fused to the endogenous rheb-1 was also crossed into the aforementioned strain. Addition of Auxin (3-Indoleatic acid, Sigma-Aldrich), causes polyubiquitination and rapid proteasomal degradation of the AID-fused protein, which here can be assessed by complete loss of the mKate 2 signal. For the lifespan experiment, $2 \mu 1$ of a $100 \mathrm{mM}$ solution (in DMSO) was added every 4 days, while for the HSP-12.6 expression experiment $10 \mu \mathrm{l}$ of a $200 \mu \mathrm{M}$ solution (in DMSO) was added every day on the chamber. Controls for these experiments were treated with the same volumes of DMSO. 


\section{Results}

\subsection{Sleep loss affects metabolism by regulating the starvation response in L1 arrested worms}

In order to further our understanding of the relationship between sleep and starvation, I performed transcriptomic and proteomic analyses on two C. elegans strains that show reduced quiescence in the L1 arrested state, the aptf-1(gk794) loss of function mutant and the RISablated flp-11::EGL-1 transgenic strain, which was created to induce apoptosis selectively in RIS (Figure 7).

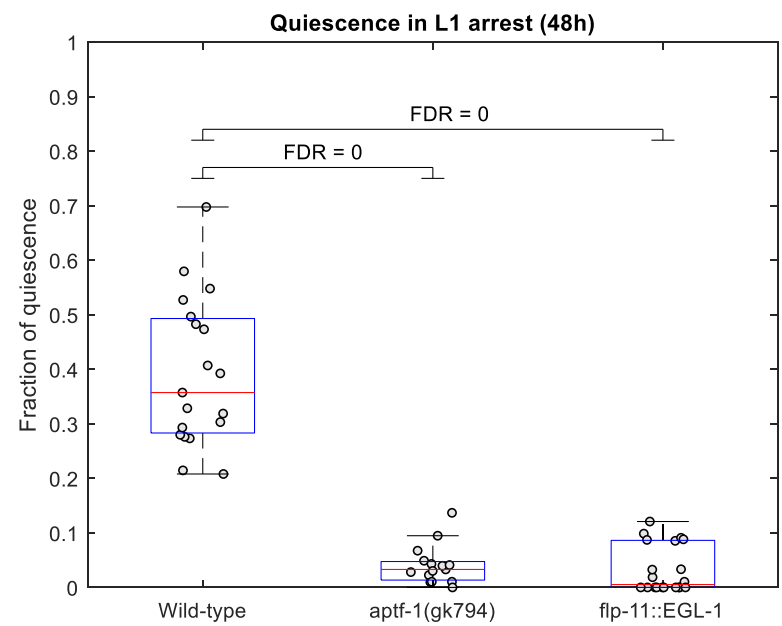

Figure 7. aptf-1 loss of function and RIS-ablation reduce quiescence in L1 arrest

Boxplot showing almost complete reduction in the quiescence fraction of aptf-1(gk794) and flp-11::EGL-1 worms, calculated at 48 hours of starvation. Average quiescence values are: wild-type $=0.3924$, aptf- $1(\mathrm{gk} 794)=0.0411(10,47 \%$ of wild-type $)$ and $f l p-11::$ EGL-1 $=0.0333$ (8.49\% of the wild-type) and the number of animals tested per condition is: 18, 14 and 19 respectively. Red horizontal line denotes the median value.

For the transcriptomic and proteomic analyses, I compared the "Starved" condition (48 hours in L1 arrest) with the "Fed" condition (4 hours in L1 development). In total, the expression of 12256 genes and 4066 proteins was quantified. The RNA-seq data (CeGaT) comes from 2 biological replicates, while the proteome data comes from 1 biological replicate processed with the TMT method. Although the low number of biological replicates in this experiment prohibits the identification of new regulatory genes/proteins, as well as downstream pathway enrichment analysis, it is nevertheless adequate to get a general overview of the changes induced by sleep loss and starvation. This is indeed the case both for aptf- 1 loss of function and RIS-ablation. In 
fact, in aptf- 1 loss of function we can observe the expected downregulation of $f l p-11$ since it is a downstream target of aptf-1 (FLP-11 was not detected in the proteome) ${ }^{[27]}$. Also, there is greater differential regulation of genes and proteins in starvation than in development, which points to a different and perhaps more significant role of sleep in the L1 arrested state (Figure 8 and sections $6.7-6.10)$.
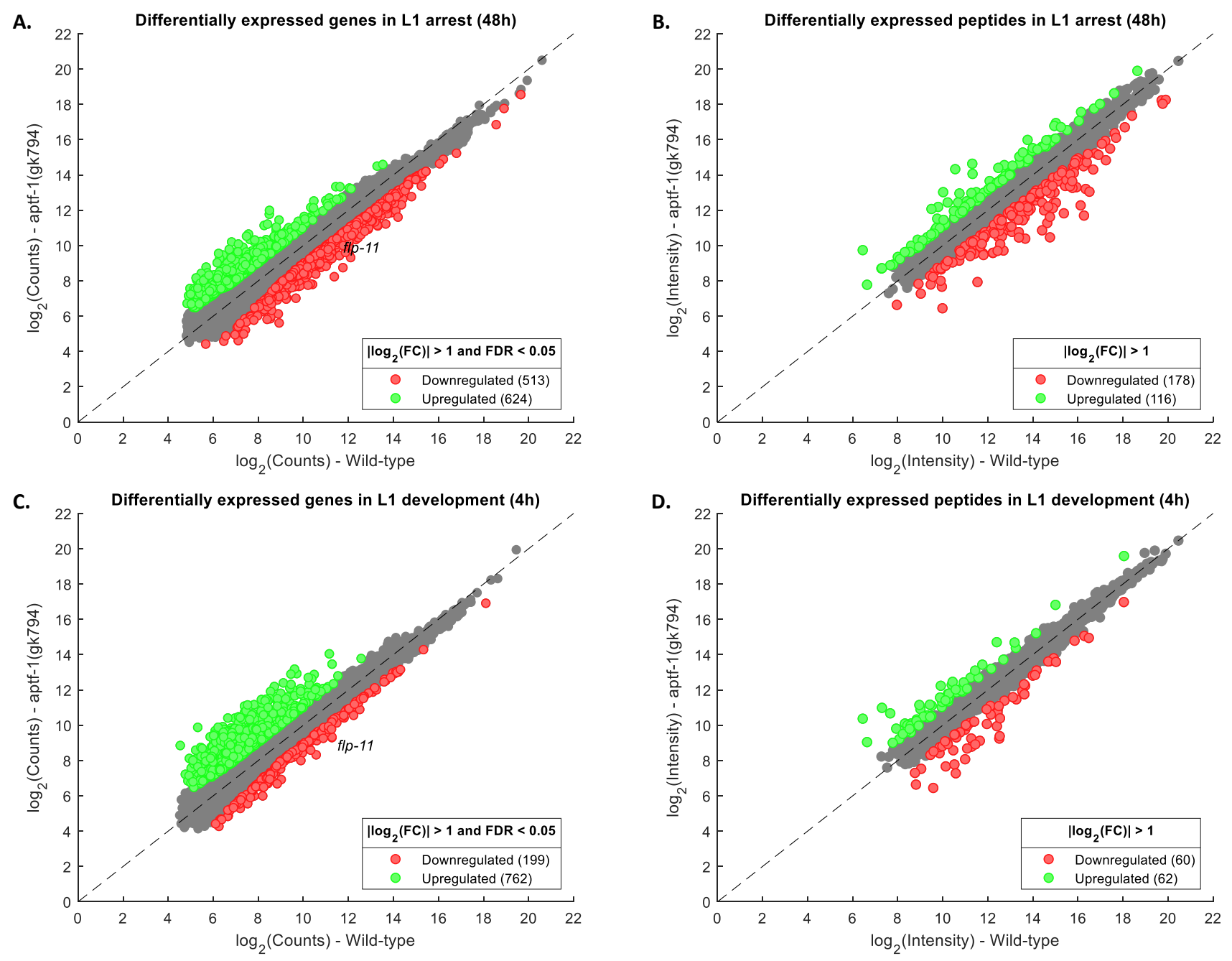

Figure 8. aptf-1 loss of function leads to transcriptomic and proteomic changes

Scatter plot overview of gene and protein expression.

Not significant entities according to the selected thresholds, are marked in grey.

(A-B) In L1 arrest $10.77 \%$ of the transcriptome and $7.23 \%$ of the proteome changes.

(C-D) In L1 development $7.84 \%$ of the transcriptome and 3\% of the proteome changes.

In the RIS-ablated strain, $f l p-11$ is, surprisingly, upregulated! This is was not expected from a strain that shows reduced quiescence, since FLP-11 is the major sleep-inducing neuropeptide [27]. With regard to egl-1, it is one of the most upregulated genes in the RIS-ablated condition. However, since there is no evidence of extensive apoptosis in this strain, it is logical to speculate that the differential regulation comes from constant expression of the transgene within RIS. Also, while differential regulation of protein levels remains similar to the aptf-1 
condition when comparing starvation and development, there is a reversal on the gene level which suggests that the role of RIS ablation is more important in L1 development (Figure 9 and sections $6.11-6.14)$.
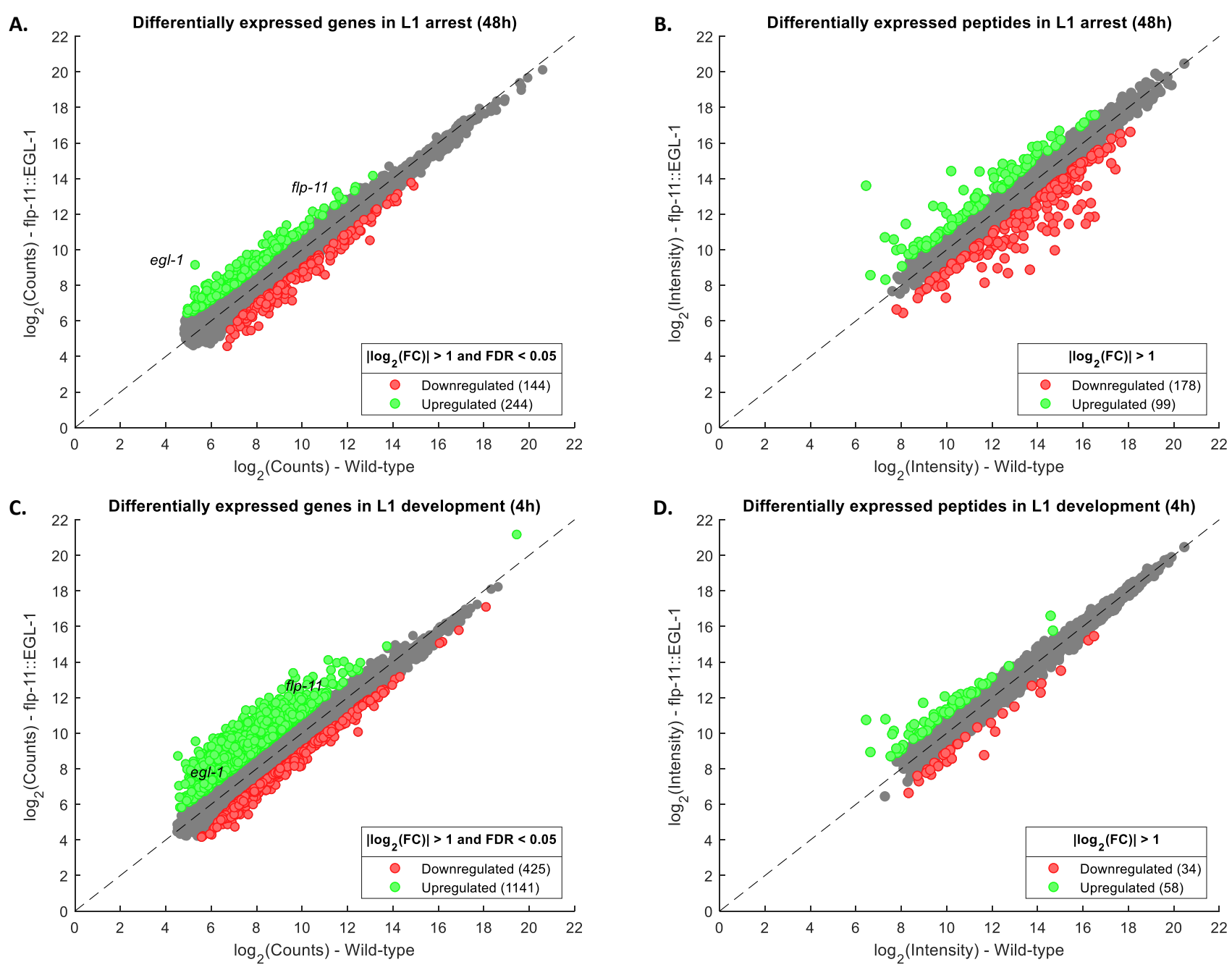

Figure 9. RIS ablation leads to transcriptomic and proteomic changes Scatter plot overview of gene and protein expression.

Not significant entities according to the selected thresholds, are marked in grey.

(A-B) In L1 arrest $3.17 \%$ of the transcriptome and $6.81 \%$ of the proteome changes.

(C-D) In L1 development $12.78 \%$ of the transcriptome and $2.26 \%$ of the proteome changes.

Having analyzed the impact of sleep loss in different nutritional conditions, I followed up with the complementary analysis, investigating the starvation response in animals with different capacities for sleep. In all conditions, $h s p-12.6$, a gene coding for a small heat-shock protein that is induced by fasting and plays a role in lifespan extension during caloric restriction in the adult worm ${ }^{[53]}$, was greatly upregulated (HSP-12.6 was not detected in the proteome) (Figure 10 and sections 6.15 - 6.20). 

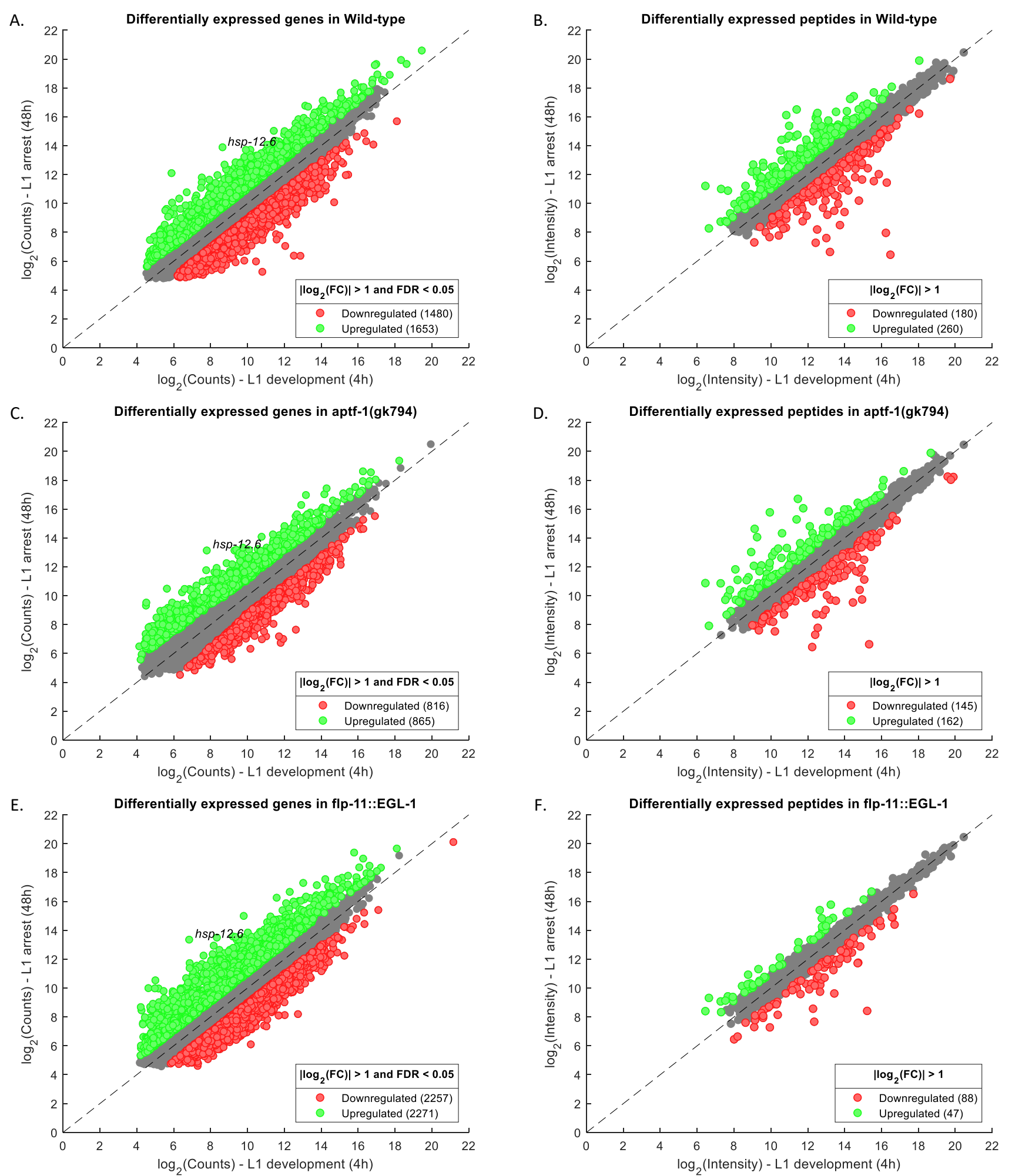

Figure 10. Starvation leads to transcriptomic and proteomic changes

Scatter plot overview of gene and protein expression.

Not significant entities according to the selected thresholds, are marked in grey.

(A-B) Starvation response in the wild-type, with $\log _{2}(\mathrm{FC})=5.24$ for $h s p-12.6$.

(C-D) Starvation response in the aptf-1 (gk794), with $\log _{2}(\mathrm{FC})=5.34$ for $h s p-12.6$.

(E-F) Starvation response in the $f l p-11:$ EGL-1, with $\log _{2}(\mathrm{FC})=6.51$ for $h s p-12.6$. 
RIS function and consequently sleep, affect both the quantity and the quality of the starvation response. aptf-1 loss of function reduces the number of genes and proteins that get differentially expressed in starvation compared to those in the wild-type, while RIS-ablation increases the number of genes but decreases the number of proteins (Figure $11 \mathrm{~A}, \mathrm{~B}$ ). This is the first indication that RIS might play a role in protein synthesis, which will be further investigated below. With regard to qualitative changes in the starvation response, gene and protein regulation in both sleep loss conditions negatively correlate with their regulation during wild-type starvation (Figure $11 \mathrm{C}-\mathrm{F}$ ).

To sum up, I have looked at the transcriptomic and proteomic profiles of two C. elegans strains which show reduced starvation-induced quiescence in the arrested L1 stage. The difference in the expression of the sleep-inducing neuropeptide FLP-11 and in the number of differentially expressed genes during starvation, signify that these strains are not equivalent in all of their aspects. However, in both sleepless strains, genes and proteins that should be upregulated to manifest a proper starvation response are downregulated and vice versa. This anti-correlation, provides direct evidence that sleep and RIS control one of the major metabolic processes, the starvation response. 
A.

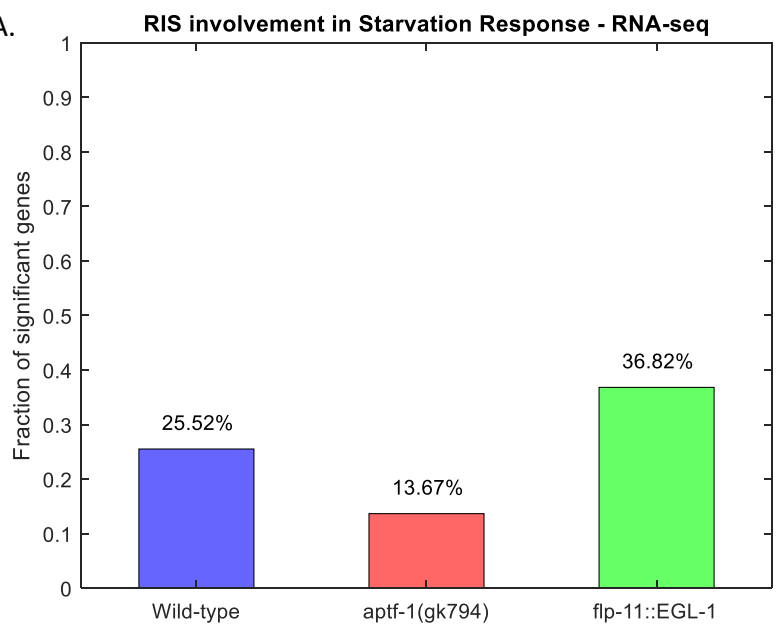

C.

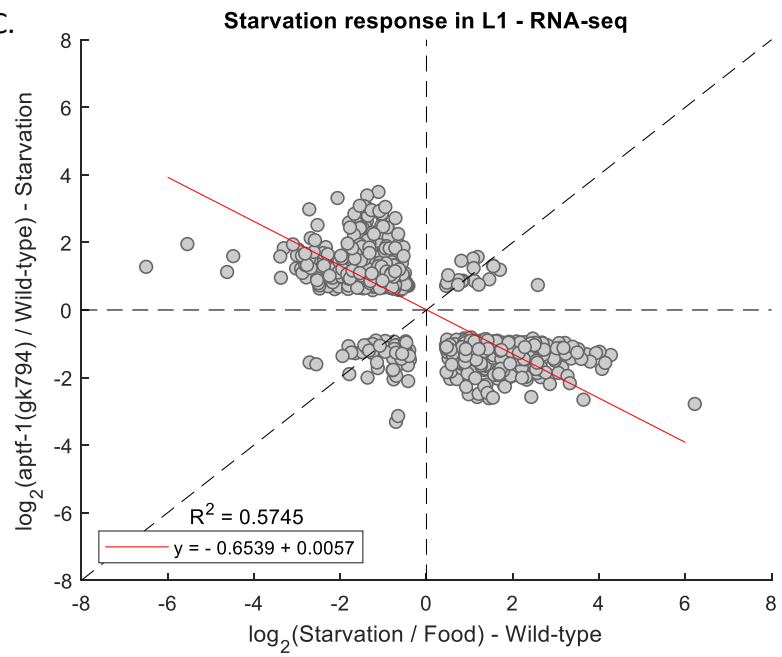

E.

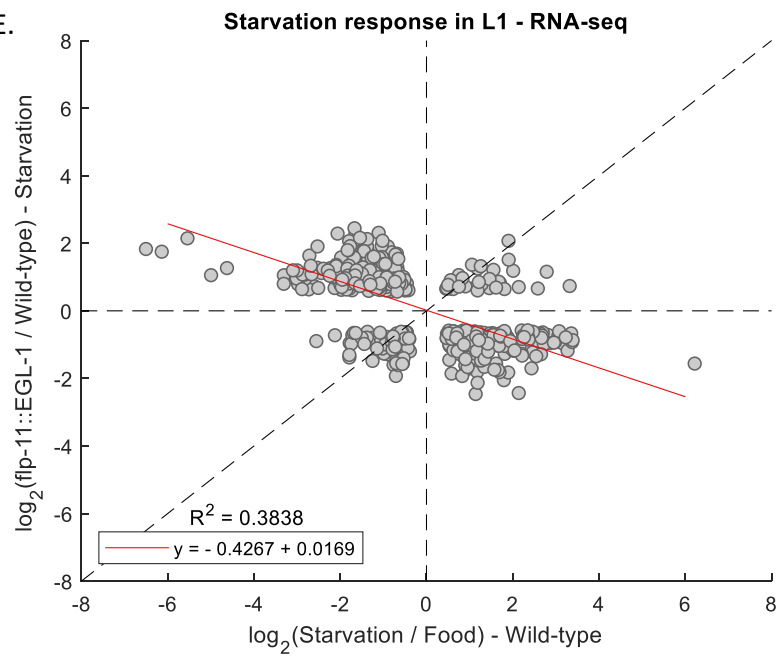

B.

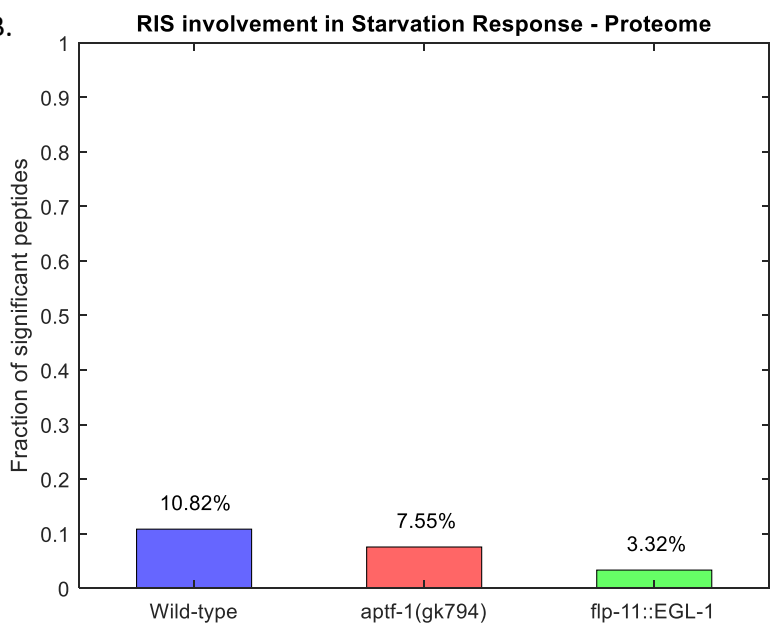

D.
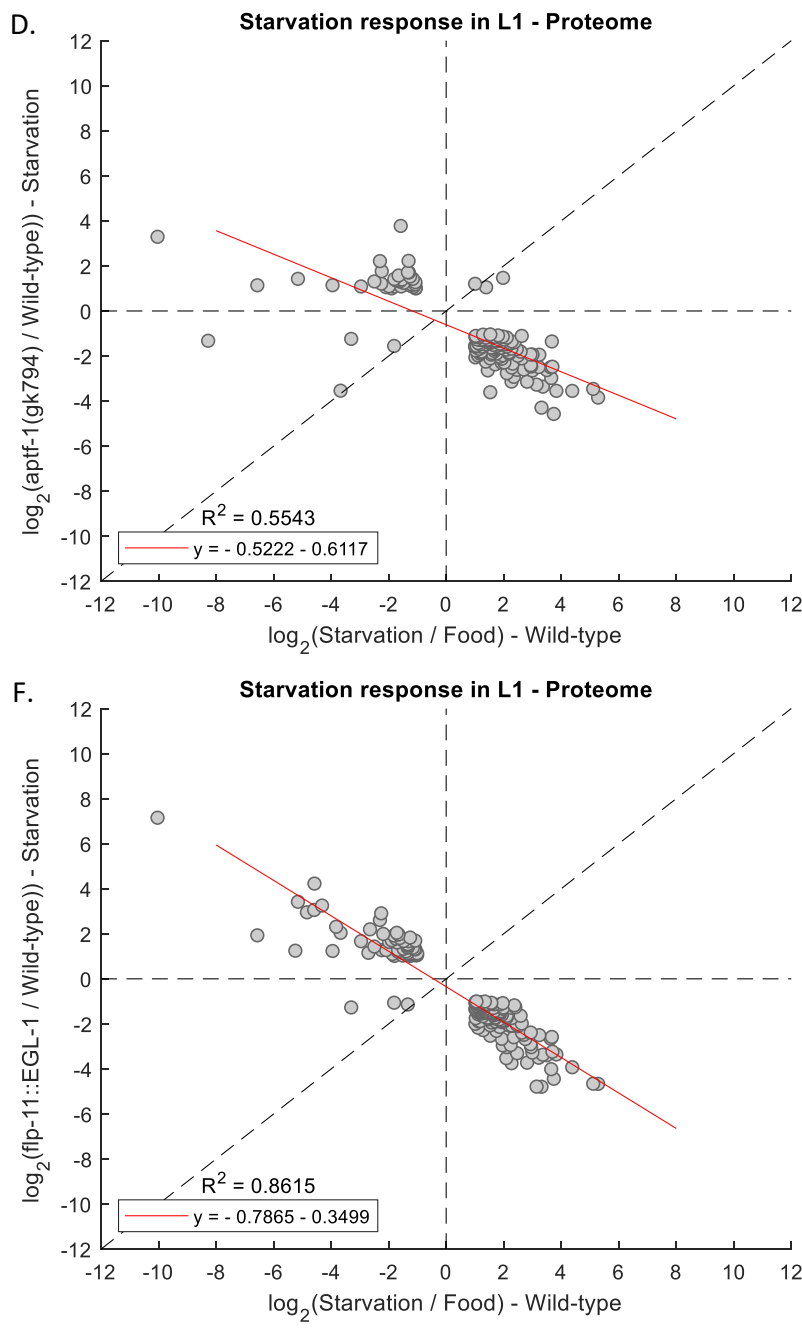

Figure 11. Starvation response depends on RIS function

(A-B) Bar plot showing the fraction of genes and proteins that globally change in starvation.

(C-D) Scatter plot showing the quality of the starvation response in aptf-1 (gk794).

(E-F) Scatter plot showing the quality of the starvation response in flp-11::EGL-1.

Linear fits for each case were produced by regression analysis using the least-squares method. 


\subsection{Sleep loss in $\mathrm{L} 1$ arrest alters the physiology of C. elegans}

In order to capitalize on the results produced by the initial transcriptomic and proteomic approaches, I repeated the analysis with more samples and increased sequencing depth. However, this time the "Fed" condition was omitted, because I had established the link between sleep and the starvation response previously. In total, 13849 genes and 1690 proteins were quantified. The RNA-seq data (MPI-MG) comes from 4 biological replicates for the wild-type and the $f l p-11:$ EGL-1 strains and 3 biological replicates for the aptf-1(gk794) strain, while the proteome data comes from 6 biological replicates processed with the LFQ method. This new analysis allows the detection of individual components that play a role in starvation-induced sleep, as well as downstream pathway enrichment analysis.

Although fewer genes and proteins were found to be differentially expressed in this new analysis, the "internal control" gene expression is reproducible with $f l p-11$ being downregulated in the aptf-1 loss of function condition and upregulated in the flp-11::EGL-1 condition $\left(\log _{2}(\mathrm{FC})=0.6377\right.$ and FDR $\left.=7 \times 10^{-20}\right)$. aptf- 1 is now detected and downregulated in both strains $\left(\log _{2}(\mathrm{FC})=-0.62\right.$ and FDR $=5.8 \times 10^{-5}$ in the $f l p-11::$ EGL-1 strain). Such disagreement in the expression of $f l p-11$ and its regulator aptf-1, has not been observed before. egl-1 is still the most upregulated gene in the flp-11::EGL-1 condition, while the most downregulated hit is $d m s r$-2, a predicted G-protein coupled neuropeptide receptor. This receptor is activated by the neuropeptide NLP-13 (personal communication with Prof. Isabel Beets, KU Leuven), which is one of the characteristic RIS neuropeptides according to the transcriptome published by Cao et al. (Enrichment in RIS vs all other neurons: $\log _{2}(\mathrm{FC})=2.22$ and FDR $\left.=5.7 \times 10^{-6}\right)^{[54]} \cdot n l p-13$ is not differentially expressed in any of the sleep loss conditions examined here. Finally, one of the few proteins detected in this analysis was the starvation-induced protein HSP-12.6, which was downregulated in both sleep loss conditions. The corresponding gene was found to be downregulated in the aptf-1 loss of function condition, but was upregulated in the $f l p-11:$ :EGL1 condition $\left(\log _{2}(\mathrm{FC})=0.47\right.$ and FDR $\left.=8.6 \times 10^{-11}\right)($ Figure 12 and sections $6.21-6.24)$.

The low number of differentially expressed proteins and the absence of significant results in downstream pathway enrichment analysis, has rendered the proteome of limited use. Of the detected proteins, only the role of HSP-12.6 will be extensively investigated, since it is the one component consistently detected and differentially expressed across all conditions and analyses. 

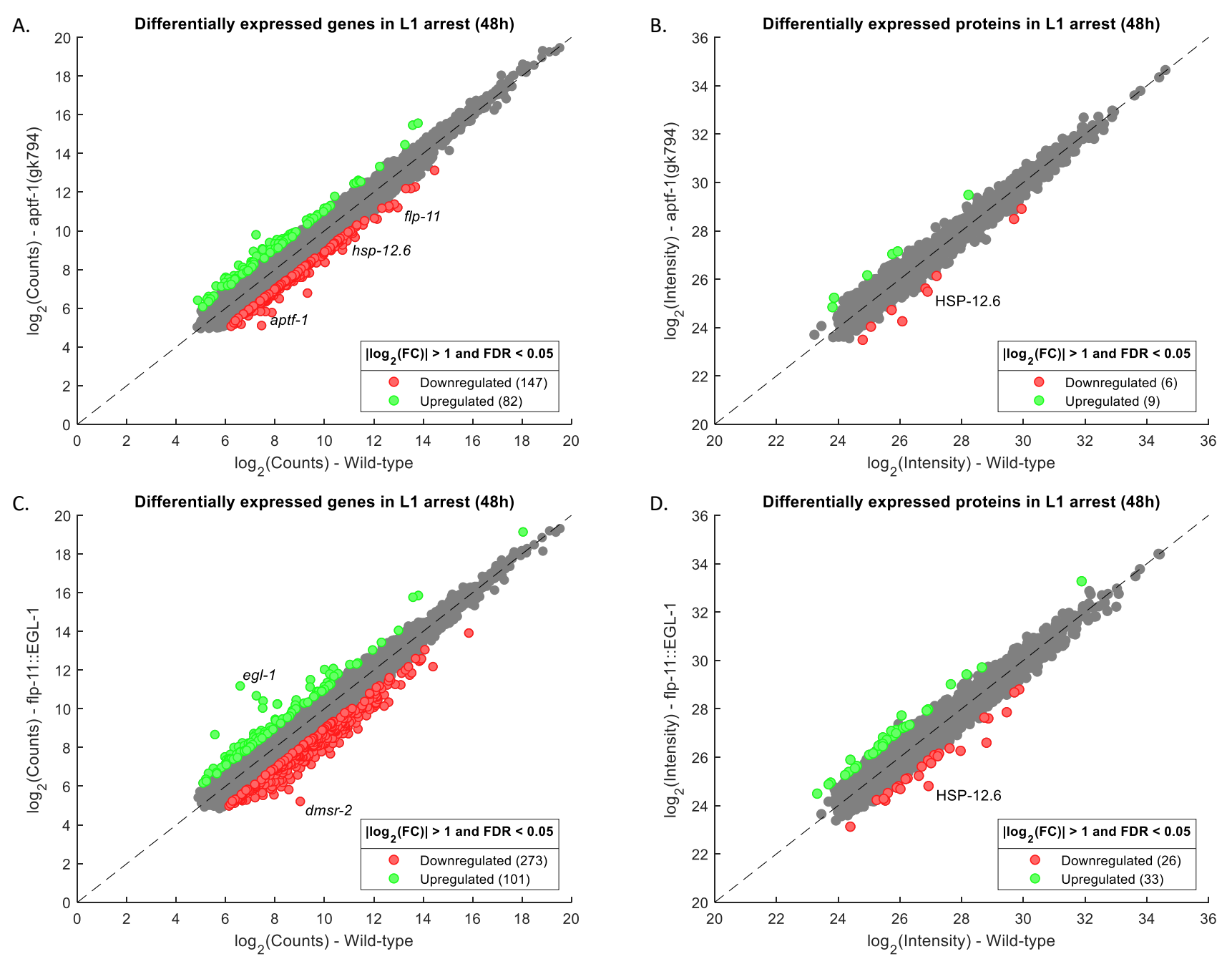

Figure 12. Sleep loss in L1 arrest leads to transcriptomic and proteomic changes

Scatter plot overview of gene and protein expression.

Not significant entities according to the selected thresholds, are marked in grey.

(A-B) In aptf-1 (gk794), $1.65 \%$ of the transcriptome and $0.89 \%$ of the proteome changes.

(C-D) In flp-11::EGL-1, 2.7\% of the transcriptome and 3.4\% of the proteome changes.

To ensure the validity of the transcriptome and its reliability for downstream analysis,

I selected individual genes, for which fluorescent transgenic lines already existed, crossed them into the aptf-1 loss of function background and imaged them continuously from hatching to a time point greater than 48 hours in L1 arrest (the flp-11::EGL-1 strain was not used in this type of analysis, since it requires further characterization due to its unexpected transcriptomic and proteomic profile). This approach would not only verify the differential expression at 48 hours of starvation, but would also reveal the expression pattern of each gene.

However, this is not the case, as 6 out of 8 total transgenic lines, failed to reproduce the transcriptomic expression of the corresponding gene at the 48-hour time point (Figure 13). 
A.

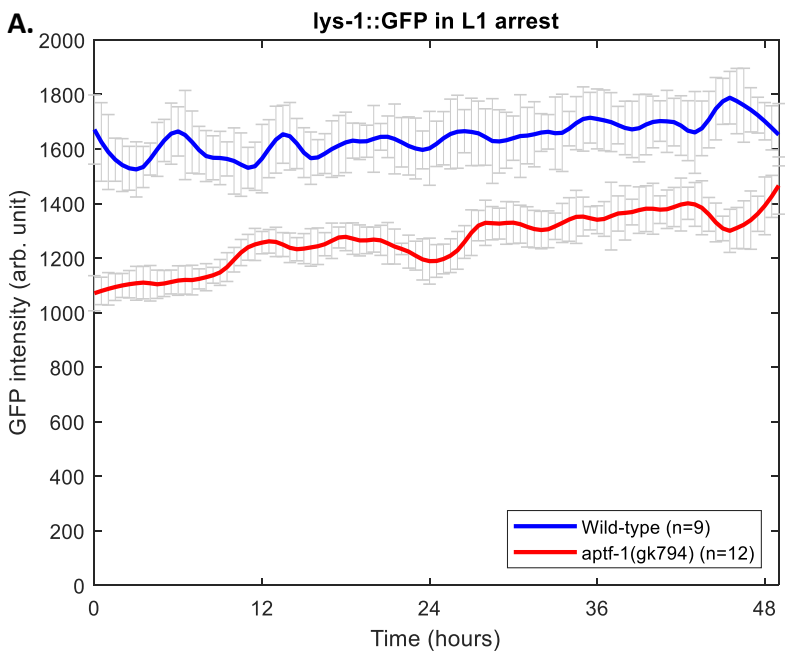

C.
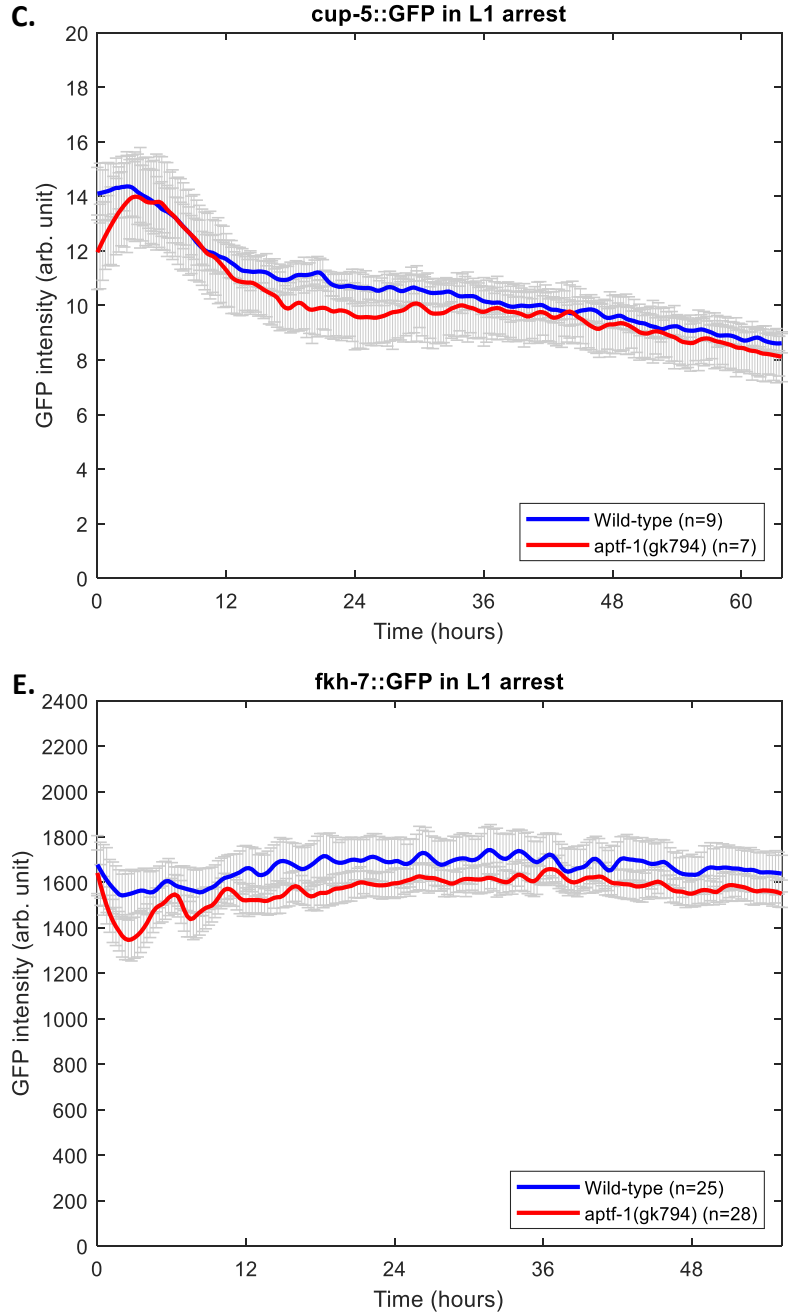

B.

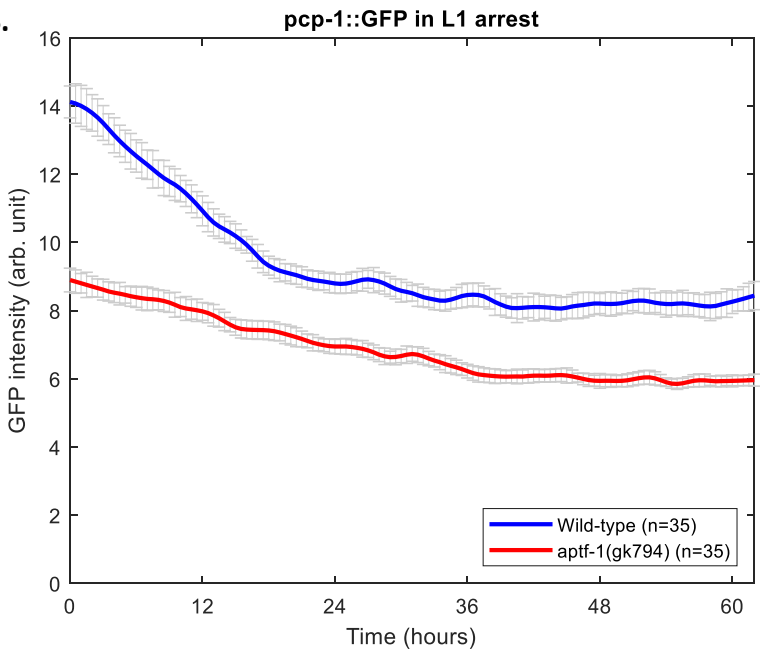

D.

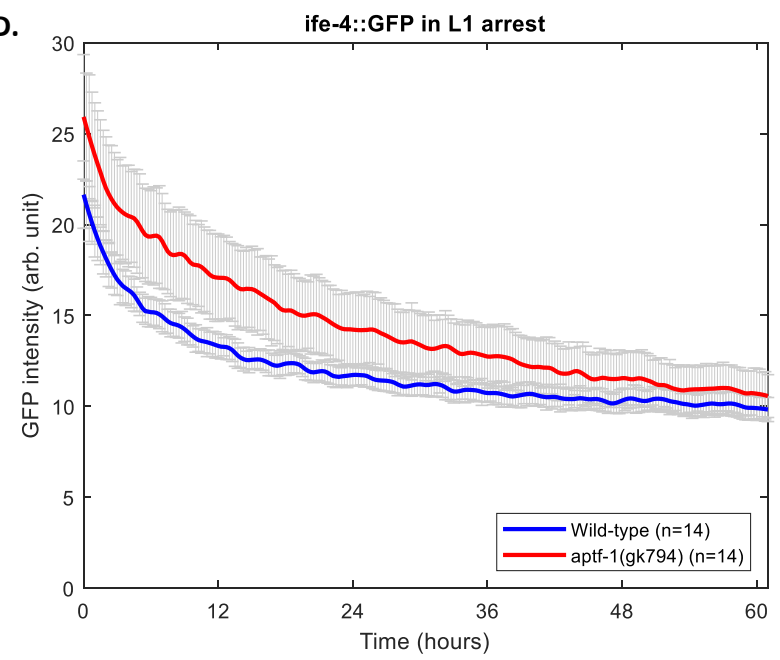

F.

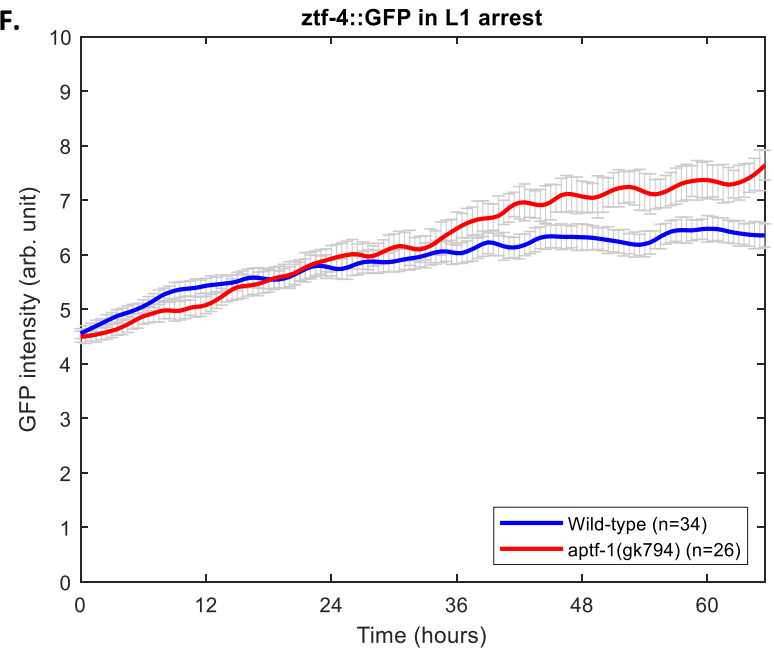

Figure 13. Transgene expression fails to verify the transcriptional changes

(A-B) The lysozyme homolog lys-1 (RNA-seq: $\log _{2}(\mathrm{FC})=0.69$ ) and the lysosomal prolylcarboxy-peptidase $p c p-1$ (RNA-seq: $\log _{2}(\mathrm{FC})=0.01$ ), are downregulated (FDR $<0.05$ for every time point) contrary to the transcriptome predictions.

(C-F) The mucolipin homolog cup-5, the translation initiation factor ife-4, and the transcription factors $f k h-7$ and $z t f-4$ (RNA-seq: $\log _{2}(\mathrm{FC})=0.20, \log _{2}(\mathrm{FC})=-0.39, \log _{2}(\mathrm{FC})=0.29$ and $\log _{2}(\mathrm{FC})=0.15$ respectively $)$, are not significantly changed. 
On the other hand, for 2 transgenic lines (hil-1::GFP and hsp-12.6::GFP) the transcriptomic expression can accurately be reproduced at the 48-hour time point (Figure 14 A, B). With regard to $h s p-12.6$, sleepless worms can also induce its expression but in a less efficient way, once again verifying the role of sleep in the starvation response. A shorter promoter region in the hsp-12.6::GFP transgene, leads to loss of this phenotype (Figure $14 \mathrm{C}$ ).
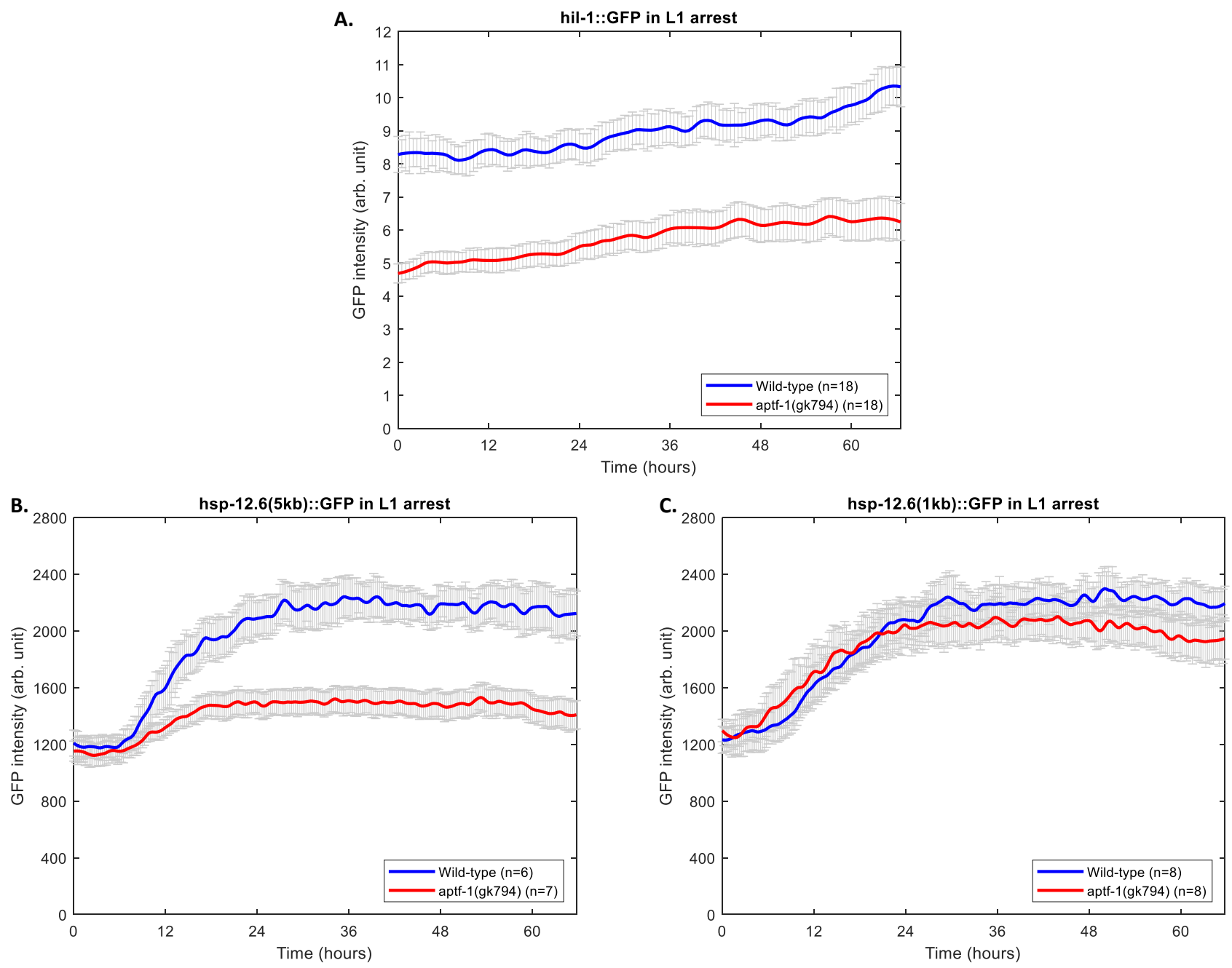

Figure 14. Transgene expression is in accordance to the transcriptional changes

(A) The histone hil-1, is significantly downregulated (FDR $<0.05$ for every time point) as expected from the transcriptome (RNA-seq: $\left.\log _{2}(\mathrm{FC})=-0.15\right)$.

(B-C) The small heat-shock protein $h s p-12.6$ is significantly downregulated (FDR $<0.05$ for every time point), as expected (RNA-seq: $\log _{2}(\mathrm{FC})=-1.43$ ). Expression of the transgene from a shorter promoter region ( $1 \mathrm{~kb}$ vs $5 \mathrm{~kb}$ ), abolishes the differential expression.

The limited success of the transgene screening can be attributed to a variety of factors related to the transgenic lines, their creation and GFP expression or to the quality of the transcriptome. The former is difficult to detect and not relevant for this project while the latter needs to be addressed. For this reason, I performed hierarchical clustering analysis on the 
RNA-seq samples, which showed that the samples of the same background cluster well together, ensuring the good quality of the dataset. It also showed that both RIS-manipulated strains are quite divergent in their gene expression patterns. This finding, taken together with the high expression levels of egl-1 in the flp-11::EGL-1 strain, support the notion of a strain in which RIS is constantly active. (Figure 15).

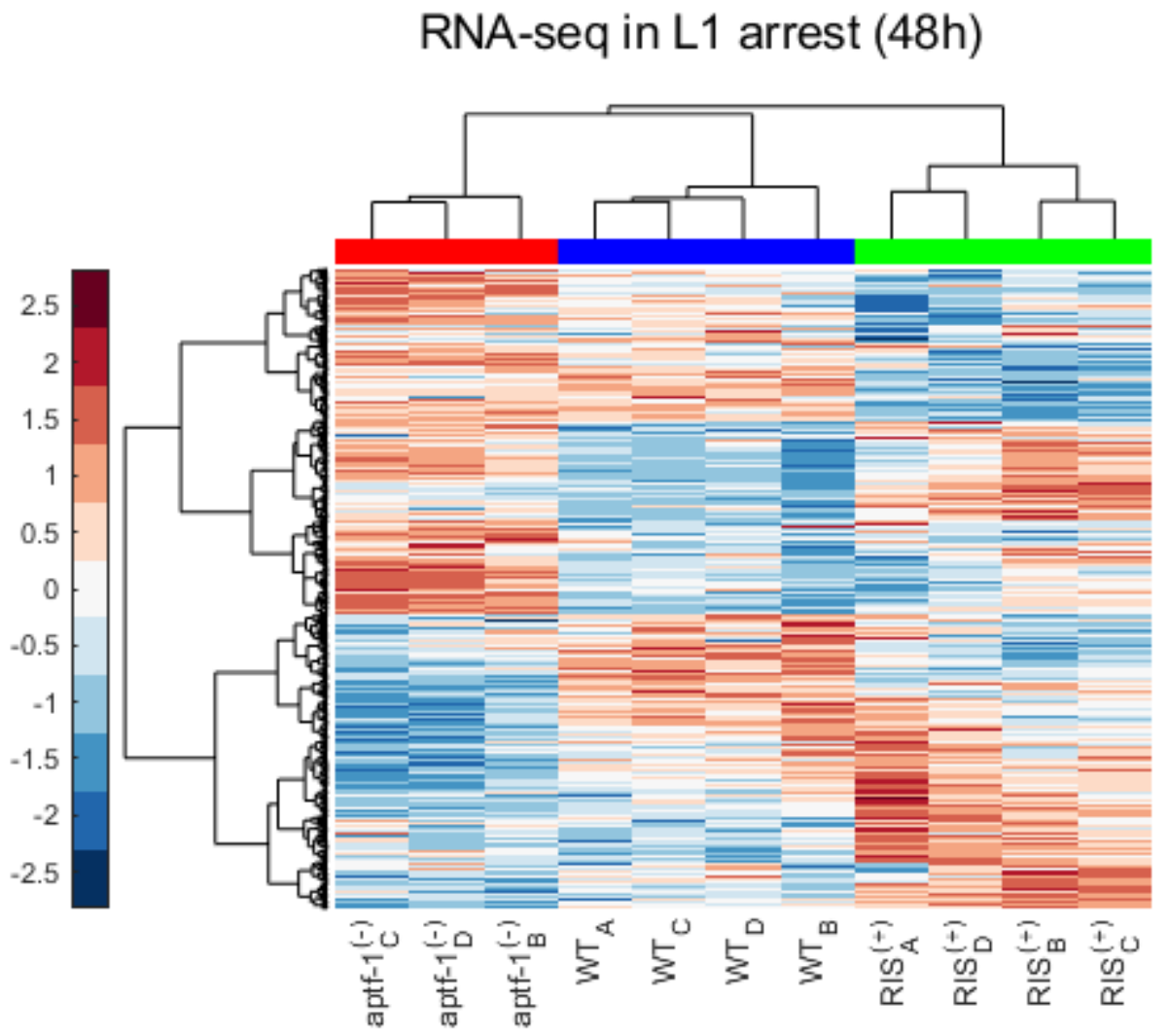

Figure 15. Hierarchical clustering analysis ensures the quality of the transcriptome

Dendrogram and heatmap representation of gene expression in sleep loss conditions. Every line of the heatmap corresponds to a single gene and every column to a single sample. Row Z-scores are color coded on the bar on the left side. aptf- $1^{(-)}$, WT and RIS ${ }^{(+)}$denote the different replicates of aptf-1(gk794), wild-type and flp-11::EGL-1 strains respectively.

After having ensured the quality of the transcriptome data, I performed Gene Ontology enrichment analysis. In the aptf-1 loss of function background on the one hand, there are 4 major pathways that are downregulated: 1. Mitochondrial function, which directly affects energy availability and metabolism, 2. Translation, which affects protein turnover and homeostasis, 3. Splicing, which affects the flow of genetic information and 4. Kinetochore and Centromere, which affect the progression of cell division. On the other hand, 3 major pathways are upregulated: 1. Neuropeptide signaling, which regulates the function of all tissues and affects many of the animal's behaviors (e.g. sleep, egg-laying, defecation etc.) ${ }^{[55]}$, 2. Defense response, which determines the ability to fight pathogens and 3. Unfolded protein response, 
which affects protein homeostasis and cellular stress levels (Figure $16 \mathrm{~A}$ ). The picture is very different in the flp-11::EGL-1 background, where the pathways downregulated are 1. Muscle tissue components, 2. Glutathione, which homeostatically regulates the cellular redox status and 3. Unfolded protein response. However, only 2 major pathways are upregulated: 1. Proteasome, which degrades damaged or unnecessary proteins and 2. Organism development (Figure 16 B).

A.

GO terms significantly enriched in aptf-1(gk794) - L1 arrest (48h)

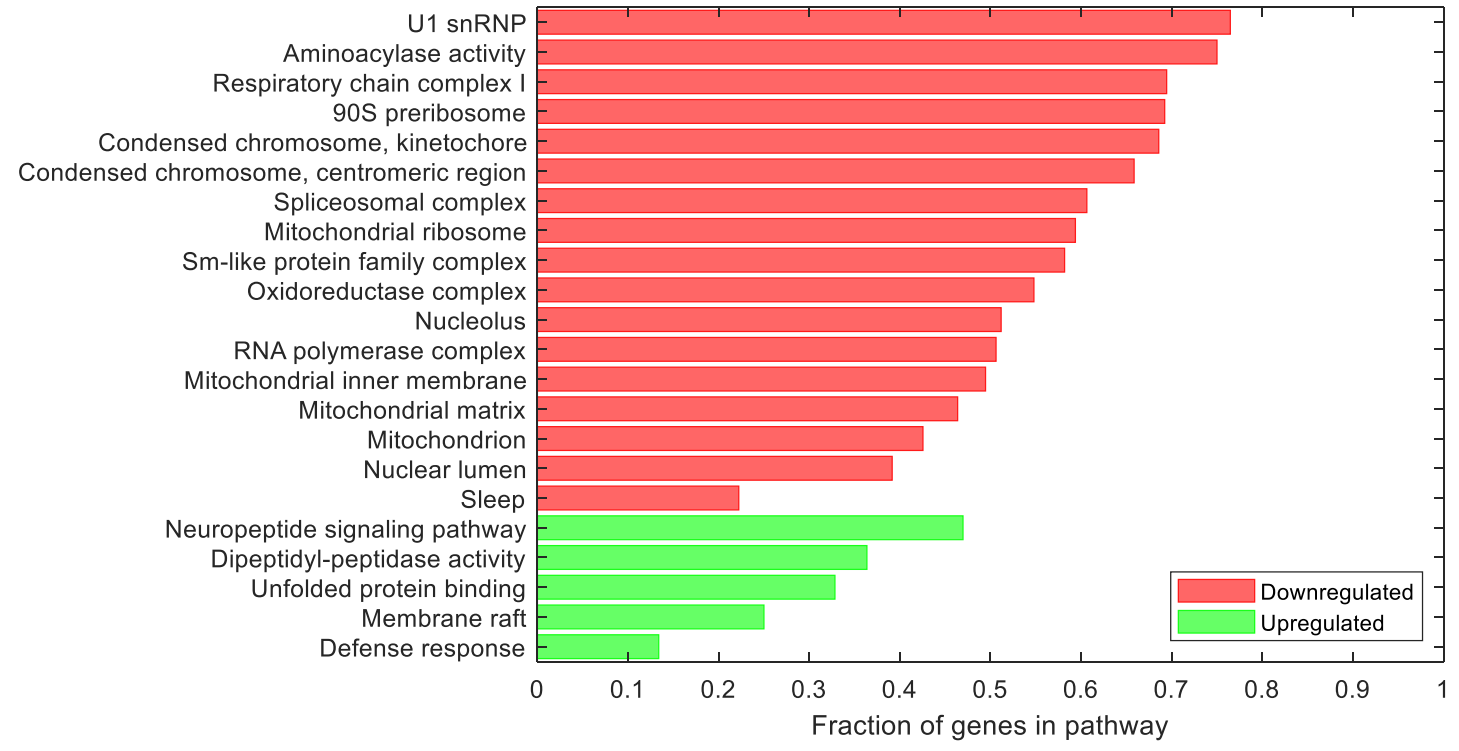

B.

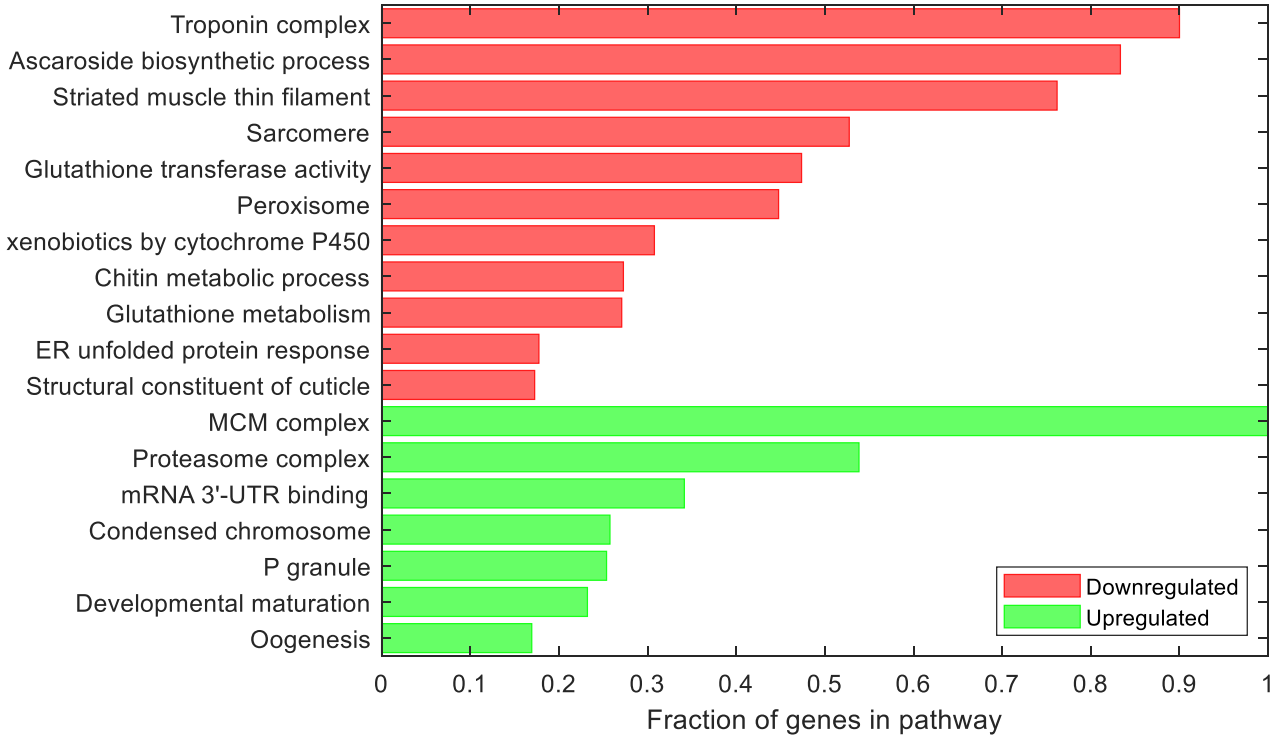

Figure 16. GO term enrichment analysis of the RNA-seq dataset

Horizontal bar chart representation of the GO terms that are affected by loss of sleep, at the transcript level. All of the shown pathways are significantly enriched with $p_{\text {adj }}<0.05$.
(A) Wild-type vs aptf-1(gk794)
(B) Wild-type vs flp-11::EGL-1 
The above results indicate that sleep loss induces organism-wide changes, in several major pathways necessary for life. I have now established that characterization of the flp-11::EGL-1 strain is incomplete. For this reason, it will be excluded from most downstream assays, which will focus on the well-characterized aptf- 1 loss of function strain. I have also showed that commercially available transgenic strains, might not be the optimal tool to assess gene expression with. In order to better understand and discover the origin of these sleep induced molecular changes, I performed tissue-specific enrichment analysis on the transcriptome data.

\subsubsection{Sleep loss upregulates hypodermis-specific gene expression and reduces body size}

The hypodermis is part of the epithelial system of C. elegans. It directs the development of the worm (molting), regulates cell fate and gives the worm its shape and its size ${ }^{[56]}$. I mined hypodermis-unique genes from the published dataset of Kaletsky et al. ${ }^{[57]}$ and calculated the significance of enrichment in my sleep loss dataset. I found significant upregulation of hypodermis-unique genes in both sleep loss conditions. This led me to analyze the components of the TGF- $\beta$ pathway (GO:0007179), which are mainly expressed in the hypodermis and control, among others, the body size of the worm ${ }^{[58]}$. Although there is significant upregulation of the pathway in the aptf- 1 loss of function background, there is no significant difference in the flp-11::EGL-1 strain (Figure 17 A, B). After that, I calculated the worm size at 2 time points in L1 arrest, immediately after hatching and at 48 hours of starvation. In the aptf-1 loss of function strain there is approximately $5 \%$ reduction in body size compared to the wild-type, which is not the case in the flp-11::EGL-1 strain. Also, in all cases, body size remains constant in the first 2 days of starvation, regardless of sleep capacity (Figure $17 \mathrm{C}, \mathrm{D}$ ). 

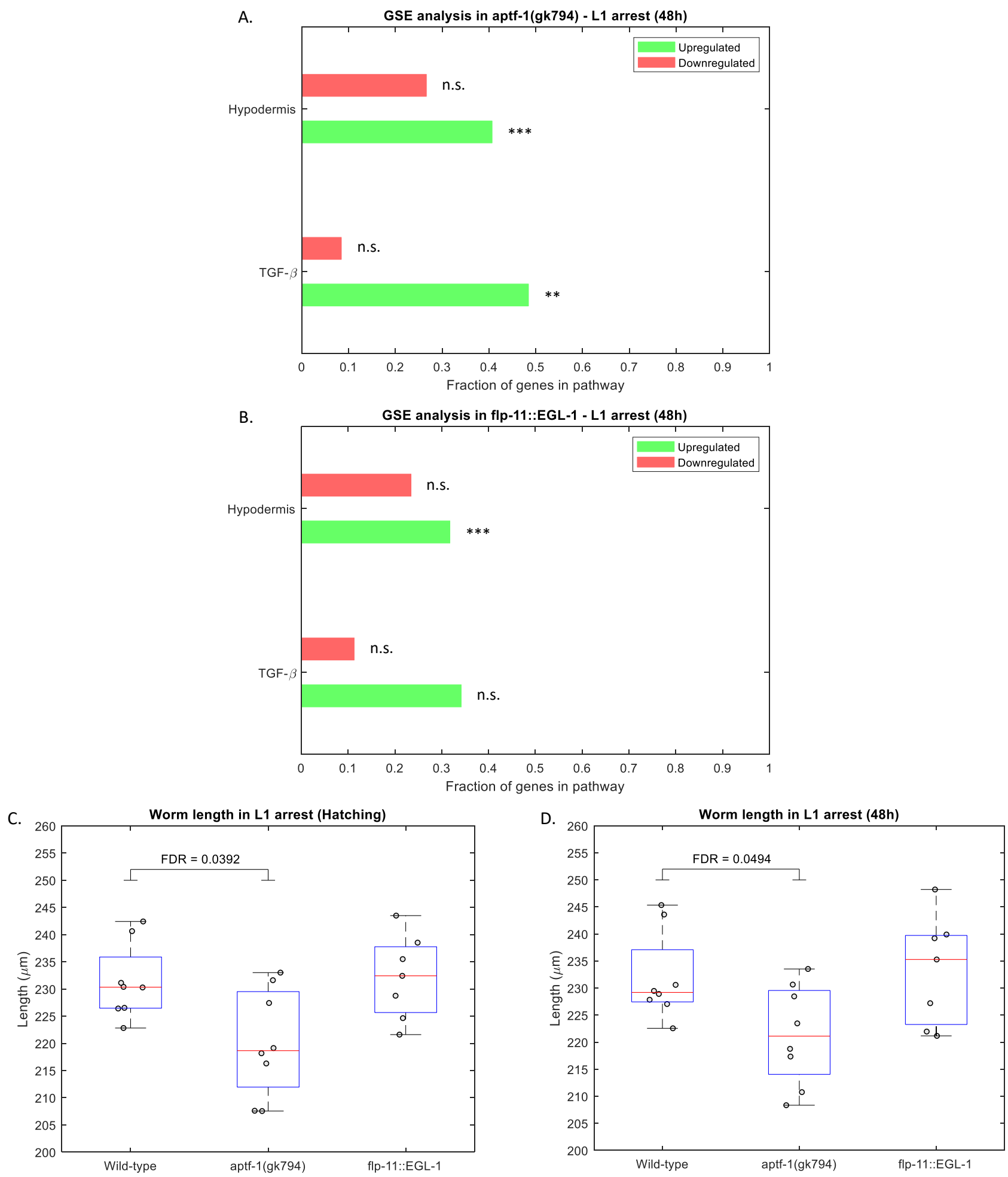

Figure 17. Sleep loss affects hypodermis-specific gene expression and body size

(A-B) Horizontal bar chart representation of hypodermis-related pathways that are affected by loss of sleep (***: $p_{\text {adj }}<0.001, * *$ : $p_{\text {adj }}<0.01, *$ : $p_{\text {adj }}<0.05$, n.s.: not significant).

(C-D) Boxplots indicating the worm length at hatching and at 2 days of starvation $(\mathrm{n}=8$ for wild-type and aptf-1(gk794) and n=7 for $f l p-11::$ EGL-1). 


\subsubsection{Sleep loss conditionally changes muscle-specific and mitochondrial gene expression}

In C. elegans, muscle tissue can be found in the body wall to control movement, in the pharynx to regulate the feeding behavior, in the vulva and uterus to facilitate reproduction and in the anus to regulate the defecation cycle ${ }^{[59]}$. I mined muscle-unique genes from the published dataset of Kaletsky et al. ${ }^{[57]}$ and calculated the significance of enrichment in my sleep loss dataset. I found significant downregulation of muscle-specific genes only in the $f l p-11:$ :EGL-1 condition. Since mitochondrial fragmentation in muscles is a consequence of sleep loss in the aptf-1 loss of function background as shown by Wu et al. ${ }^{[24]}$, I wanted to assess the enrichment of genes belonging to the Electron Transport Chain (GO:0022900) and the Mitochondrial Localization (GO:0070585) pathways. Both of these pathways are severely downregulated in the aptf-1 loss of function strain. This is not the case in the flp-11::EGL-1 strain (Figure 18).
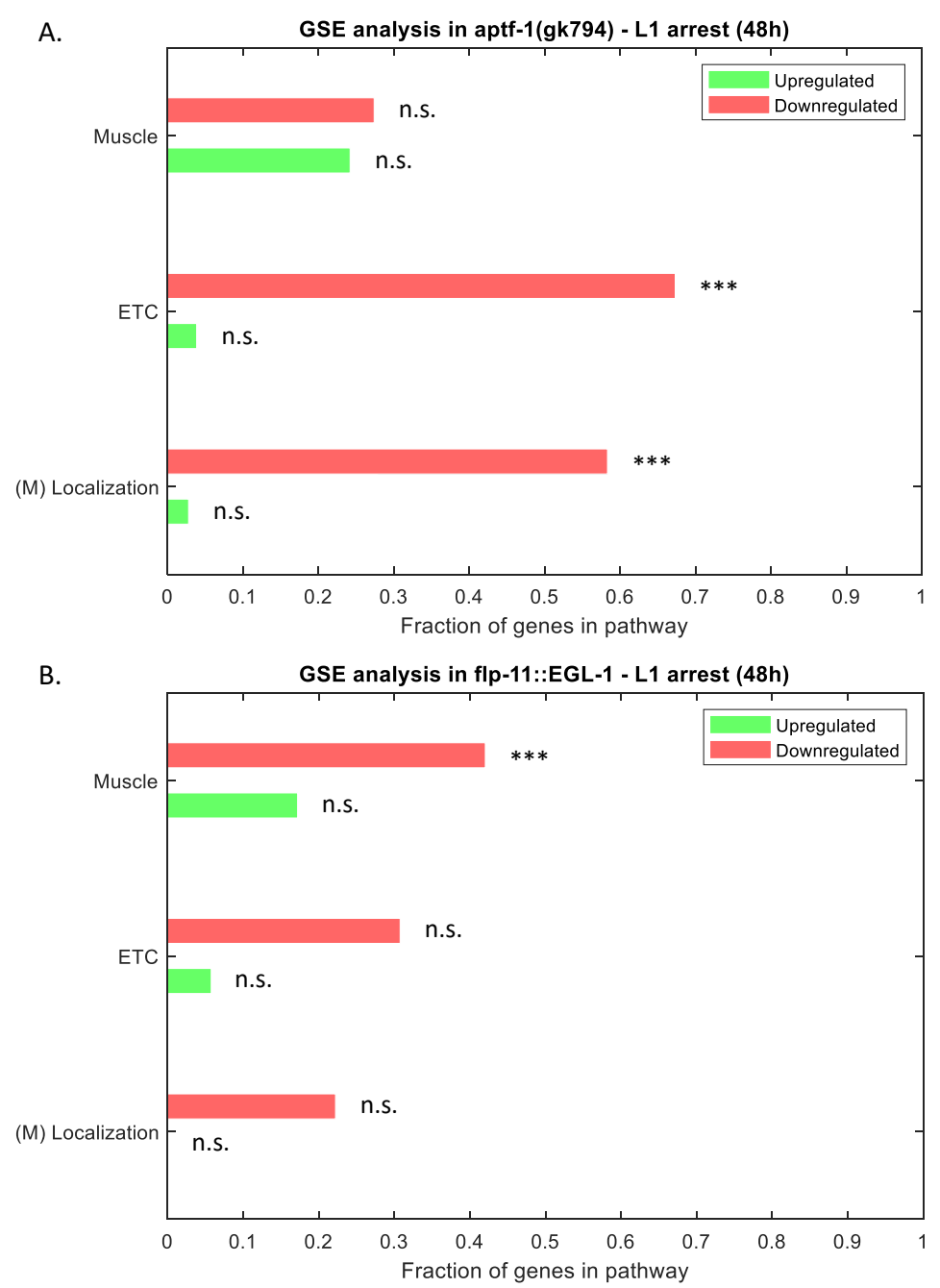

Figure 18. Sleep loss affects muscle-specific gene expression and mitochondrial function (A-B) Horizontal bar chart representation of muscle-related pathways that are affected by loss of sleep $\left(* * *: p_{\text {adj }}<0.001, * *: p_{\text {adj }}<0.01, *\right.$ : $p_{\text {adj }}<0.05$, n.s.: not significant). 


\subsubsection{Sleep loss affects intestine-specific and FOXO gene expression}

The intestine is a multi-functional tissue in C. elegans, that not only controls food digestion and nutrient absorption, but also macromolecule synthesis and storage, as well as the initiation of the immune response ${ }^{[60]}$. I mined muscle-unique genes from the published dataset of Kaletsky et al. ${ }^{[57]}$ and calculated the significance of enrichment in my sleep loss dataset. I found significant downregulation of intestine-specific genes in both sleep loss conditions and upregulation only in the aptf-1 loss of function. The insulin signaling pathway and the FOXO homolog daf-16 function in the intestinal cells non-autonomously to regulate the L1 arrested state ${ }^{[61]}$. For this reason, I mined the dataset of Murphy et. al ${ }^{[62]}$ that splits the FOXO-regulated genes in 2 categories: Class I - genes that are downregulated and Class II - genes that are upregulated in absence of $d a f-16$ and calculated their enrichment (Figure 19).
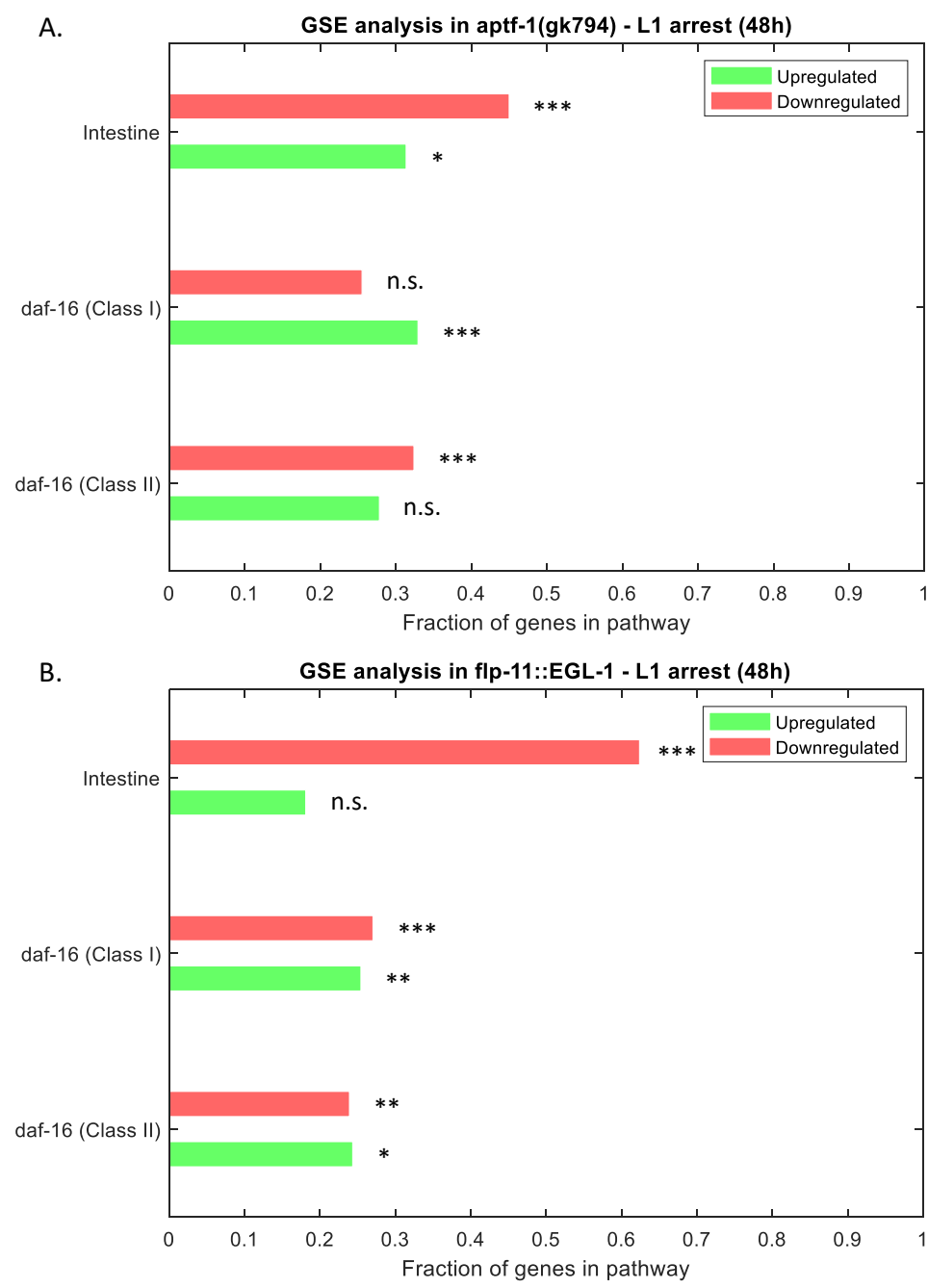

Figure 19. Sleep loss affects intestine-specific and FOXO target gene expression

(A-B) Horizontal bar chart representation of intestine-related pathways that are affected by loss of sleep $\left(* * *: p_{\text {adj }}<0.001, * *: p_{\text {adj }}<0.01, *\right.$ : padj $<0.05$, n.s.: not significant). 
Genes that are downregulated by loss of daf-16 (Class I) show a significantly enriched upregulated fraction in the aptf-1 loss of function background, while genes that are upregulated by loss of $d a f-16$ (Class II) show a significantly enriched downregulated fraction. This antipodal regulation of the insulin signaling pathway targets, may determine the reduced survival phenotype of the aptf-1(gk794) mutant shown by Wu et al. ${ }^{[24]}$. As for the $f l p-11::$ EGL-1 background, there is significant enrichment both in Class I and Class II genes, once again showing that although both the strains studied here show reduced quiescence, the underlying biochemical mechanisms are, at least in part, distinct.

To further address the role of $d a f-16$, I measured its expression using a transgenic line. In accordance with the transcriptome, DAF-16 expression is significantly upregulated in the aptf1 loss of function background at 48 hours (RNA-seq: $\log _{2}(\mathrm{FC})=0.55$ and FDR $=4 \times 10^{-24}$ ) (Figure $20 \mathrm{~A}$ ). However, this goes against the downregulation of $h s p-12.6$ (Class I) that was presented in the transgenic line screening above, as well as the downregulation of sod-3 (Class I) (RNA-seq: $\log _{2}(\mathrm{FC})=-0.58$ and FDR $\left.=0.0009\right)$ which encodes a superoxide dismutase, in the aptf-1 loss of function background (Figure $20 \mathrm{~B}$ ). DAF-16 is a transcription factor stationed in the cytoplasm and is subject to tight regulation via phosphorylation, which depending on the phosphorylated residue can permit or deny entry in the nucleus ${ }^{[63]}$. One possible explanation for the seemingly contradictory results (increased DAF-16 but decreased $h s p-12.6$ and sod-3) would be that nuclear localization of DAF-16 is affected in the sleep loss state. For this reason, I assessed the localization of DAF-16 in the intestinal nuclei of the aptf-1 loss of function mutant and of the low-quiescence, long-lived, RIS-hyperactive strain flp-11::UNC-58gf (characterized by my colleague Inka Busack). Although, DAF-16 is equally probable to be found in the nucleus of the L1 arrested worms in the first 2 days of starvation, the probability of nuclear localization drops dramatically for the non-sleeping strains at after the $3^{\text {rd }}$ day. This consists definitive evidence that sleep controls the starvation response, through DAF-16 (Figure 20 C-E). 

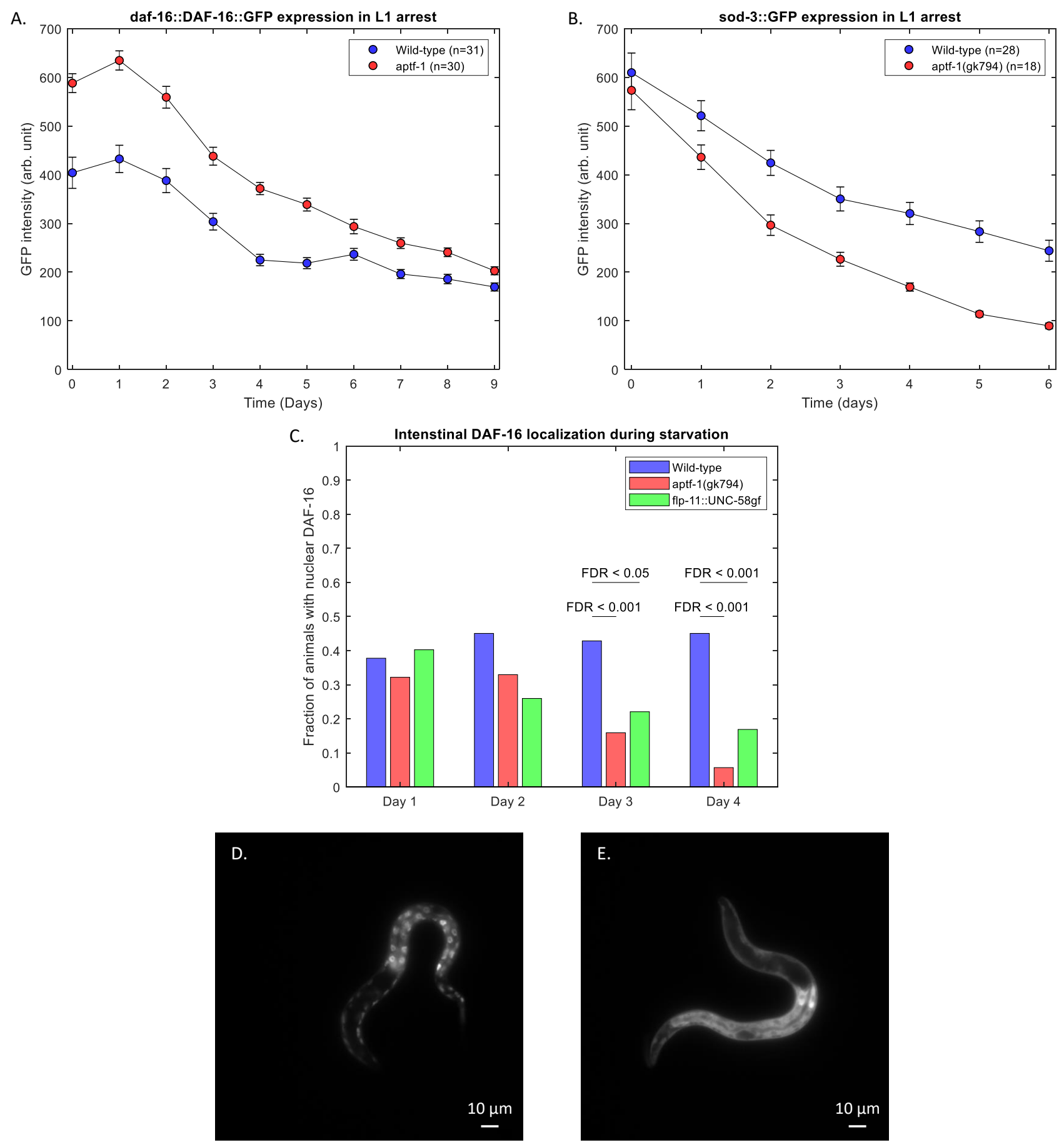

Figure 20. Sleep loss affects intestinal DAF-16 localization during L1 arrest

(A) DAF-16 is significantly upregulated in aptf-1 (gk794) (FDR $<0.05$ for all time points).

(B) sod-3 is significantly downregulated in aptf-1(gk794) (FDR < 0.05 for Time $>1$ days).

(C) Bar plot showing the fraction of animals with nuclear DAF-16 localization.

Results for days 1 and 2 are not significant (FDR > 0.05 for all comparisons).

Statistical significance was assessed with Fisher's exact test and the p-values were corrected for false discovery rate by the Benjamini-Hochberg method.

Wild-type: $\mathrm{n}=91$, aptf-1 (gk794): $\mathrm{n}=88$ and flp-11::UNC-58gf: $\mathrm{n}=77$

(D-E) During nuclear localization GFP tagged DAF-16 shows a spherical distribution pattern, while during cytoplasmic localization the distribution pattern is diffuse. 


\subsubsection{Sleep loss conditionally affects components of germline-specific gene expression}

The germline consists of 2 cells in the newly hatched L1 larvae, namely Z2 and Z3. These cells arrest during L1 starvation through the action of the PTEN homolog daf-18, which negatively regulates the insulin signaling pathway. However, daf-16 action is not required for germline arrest, unlike for somatic cell arrest ${ }^{[64]}$. I mined germline-unique genes from the published dataset of Han et al. ${ }^{[65]}$ and calculated the significance of enrichment in my sleep loss dataset. I found significant downregulation of germline-specific genes in the aptf-1 loss of function background, contrary to the flp-11::EGL-1 which shows significant upregulation. In the light of these results, I decided to investigate if sleep loss leads to a difference in the proliferation potential of germline cells. The major pathway controlling germline proliferation during development and maintenance during adulthood, is the Notch signaling pathway via its receptor $g l p-l^{[66]}$. Then, I mined the $g l p-1$ regulated genes from the published dataset of Greer et al. ${ }^{[67]}$, and calculated the significance of enrichment in my sleep loss dataset. I found significant downregulation of $g l p-1$ target genes in the aptf- 1 loss of function background, while in the $f l p$ $11::$ EGL-1 strain there is significant upregulation (Figure $21 \mathrm{~A}, \mathrm{~B}$ ). Also, $g l p-1$ gain of function, significantly increased the lifespan of L1 arrested worms in the microchamber assay in an aptf1 dependent manner (Figure $21 \mathrm{C}$ ). During the early stages of embryogenesis, germline blastomeres use 2 unique regulatory systems to determine their fate: 1 . Transcriptional silencing by pie- 1 and 2. control of RNA metabolism by RNA-rich granules, called P granules ${ }^{[68]}$. For this reason I assessed the enrichment of genes belonging to the P granules (GO:0043186) and I found significant upregulation only in the $f l p-11:$ :EGL-1 strain. Additionally, I quantified the expression of the transcription regulator pie-1 using a transgenic reporter line, which showed no difference between the wild-type and aptf- 1 loss of function as predicted by the transcriptome (RNA-seq: $\log _{2}(\mathrm{FC})=0.12$ and FDR $\left.=0.27\right)($ Figure $21 \mathrm{D})$. 

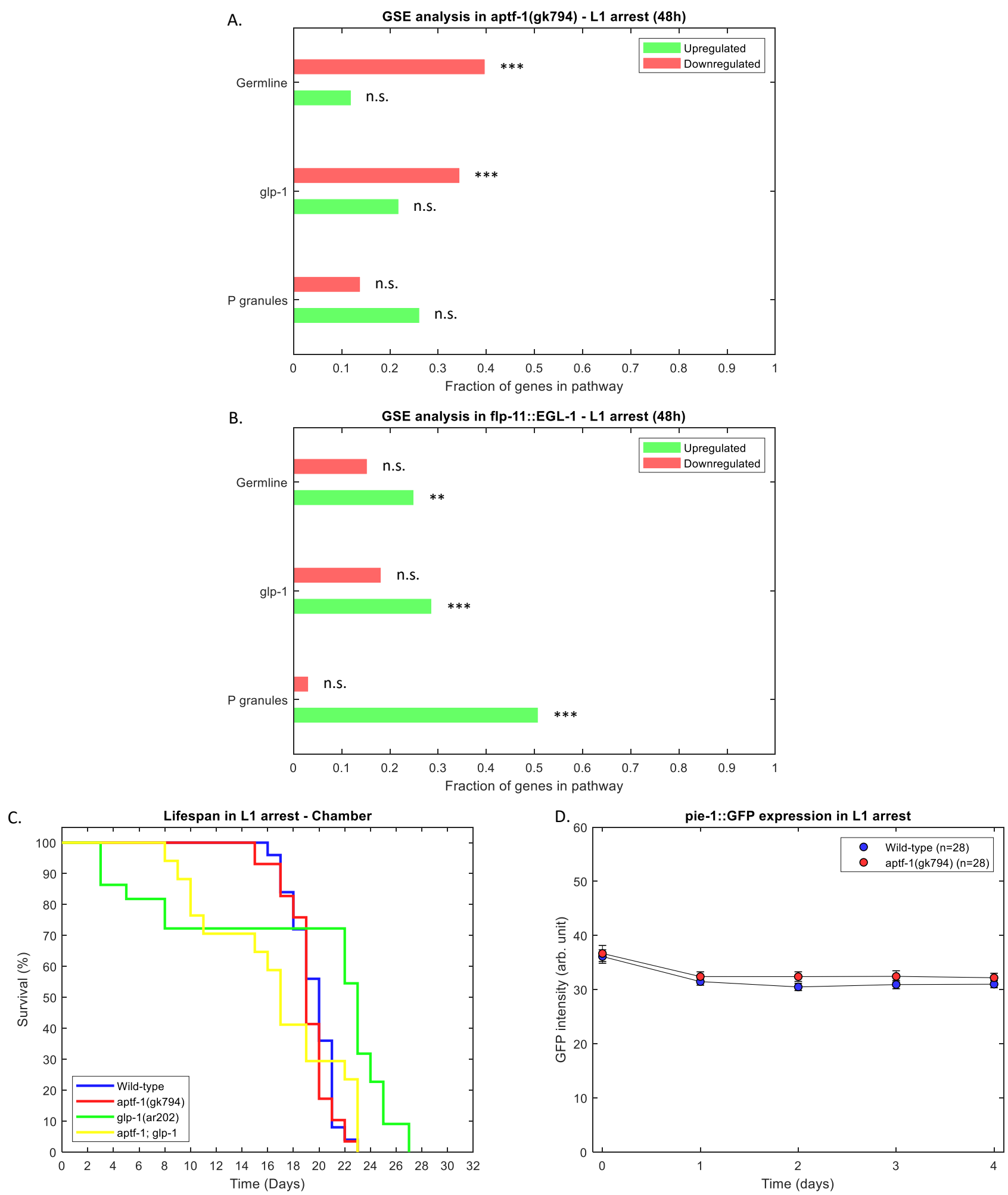

Figure 21. Sleep loss affects germline-specific gene expression and Notch signaling (A-B) Horizontal bar chart representation of intestine-related pathways that are affected by loss of sleep $\left(* * *: p_{\text {adj }}<0.001, * *: p_{\text {adj }}<0.01, *\right.$ : padj $<0.05$, n.s.: not significant).

(C) glp-1 gain of function significantly increases survival during L1 arrest in the wild type background, but not in the aptf-1 loss of function (wild-type vs $g l p-1(\operatorname{ar} 202)$ : $\mathrm{p}=0.0015$ and aptf-1(gk794) vs aptf-1; glp-1: $\mathrm{p}=0.9481)$.

(D) pie-1 expression is not changed in aptf-1(gk794) (FDR > 0.05 for all time points). 


\subsubsection{Sleep loss affects neuronal gene expression and neurotransmission}

The nervous system is the most complex tissue in C. elegans. Specialized functions are carried out by distinct types of neurons, such as motor neurons for locomotion, sensory neurons for environment perception and interneurons for information processing among others ${ }^{[69]}$. I mined neuron-unique genes from the published dataset of Kaletsky et al. ${ }^{[57]}$ and RIS, ALA and GABAergic neuron-unique genes $\left(\log _{2}(\mathrm{FC})>2\right.$ and FDR $\left.<0.05\right)$ from the published dataset of Cao et al. ${ }^{[54]}$ and calculated the significance of enrichment in my sleep loss dataset. In the aptf1 loss of function condition, neuron as well as RIS, ALA and GABAergic neuron specific genes are upregulated. On the other hand, neuron and GABAergic neuron specific genes are downregulated in the flp-11::EGL-1 strain (Figure 22).
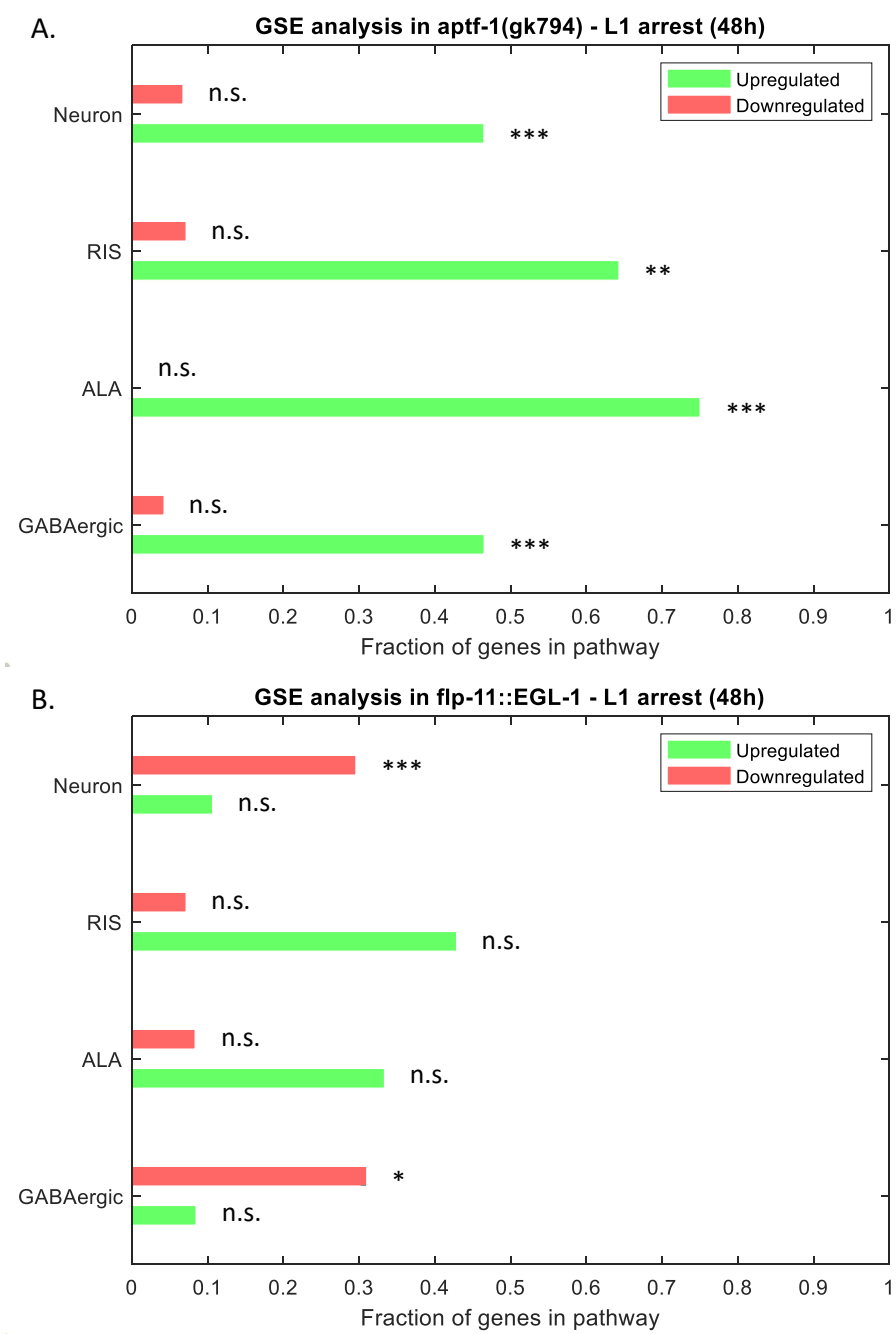

Figure 22. Sleep loss affects neuron-specific gene expression

(A-B) Horizontal bar chart representation of neuron-related pathways that are affected by loss of sleep $\left(* * *: p_{\text {adj }}<0.001, * *: p_{\text {adj }}<0.01, *: p_{\text {adj }}<0.05\right.$, n.s.: not significant). 
Apart from neuropeptides like FLP-11, neurotransmission in C. elegans employs several "classic" neurotransmitters. Glutamate, GABA, Acetylcholine and the biogenic amines Serotonin, Dopamine, Tyramine and Octopamine work in concert to modulate behavior, by binding to specific ionotropic or G-protein coupled receptors. Depending on their effect on the target cell, they can be inhibitory or excitatory ${ }^{[70]}$. In order to further investigate the impact of sleep loss in the nervous system functions, I compiled a list of all currently identified neurotransmitter receptor genes as they appear in WormBase (Version: WS277). These receptors receive neuronal input and their expression spans multiple tissues. Most of the neurotransmitter receptor classes follow the general transcriptome trend and are not significantly enriched. However, GABA receptors stand out because of their upregulation in the aptf-1 loss of function background and their downregulation in the flp-11::EGL-1, in which also glutamate receptors are downregulated (Figure 23).
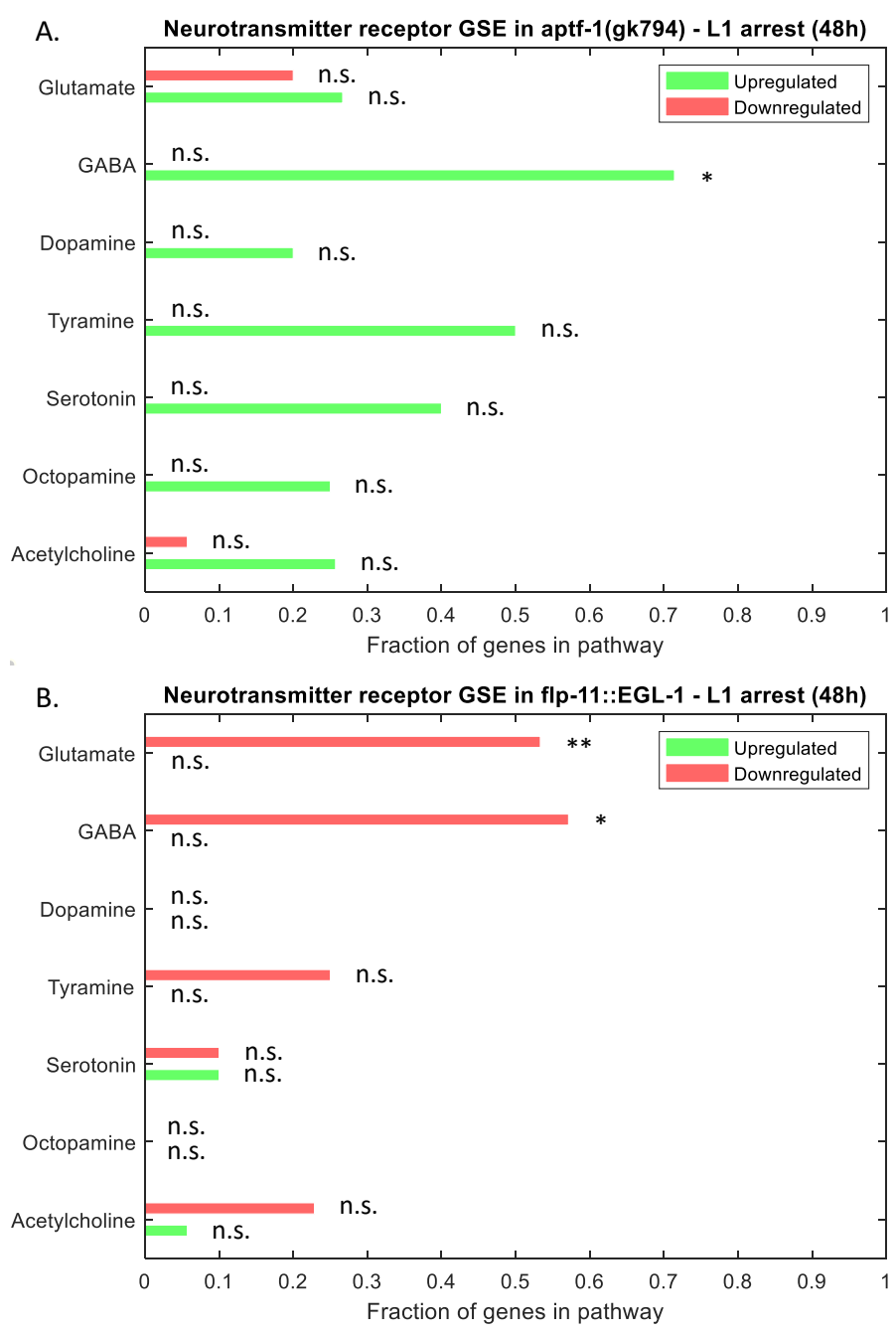

Figure 23. Sleep loss affects GABA and Glutamate receptor gene expression

(A-B) Horizontal bar chart representation of neurotransmitter receptor related pathways that are affected by loss of sleep $\left(* * *: p_{\text {adj }}<0.001, * *\right.$ : $p_{\text {adj }}<0.01, *: p_{\text {adj }}<0.05$, n.s.: not significant). 
Of the GABA receptor genes identified above, the ones that stand out are $g b b-1, g b b-2$ and $u n c-$ 49 , because they are regulated in opposite directions in the sleep loss conditions examined here. unc-49 is a $\mathrm{GABA}_{\mathrm{A}}$ gated chloride channel expressed in the muscles and its expression has been shown to be upregulated in lethargus ${ }^{[71]} . g b b-1$ and $g b b-2$ form a heterodimeric, inhibitory, G-protein coupled $\mathrm{GABA}_{\mathrm{B}}$ receptor that is expressed in cholinergic motor neurons. Loss of function of that $\mathrm{GABA}_{\mathrm{B}}$ receptor has been associated with hypersensitivity to aldicarb, an acetylcholinesterase inhibitor that causes accumulation of acetylcholine in the neuromuscular junction and subsequent paralysis ${ }^{[72]}$. For this reason, I tested the response of the aptf1 loss of function mutant to aldicarb. The response varies from resistance to hypersensitivity, depending on the time point of starvation (Figure 24).
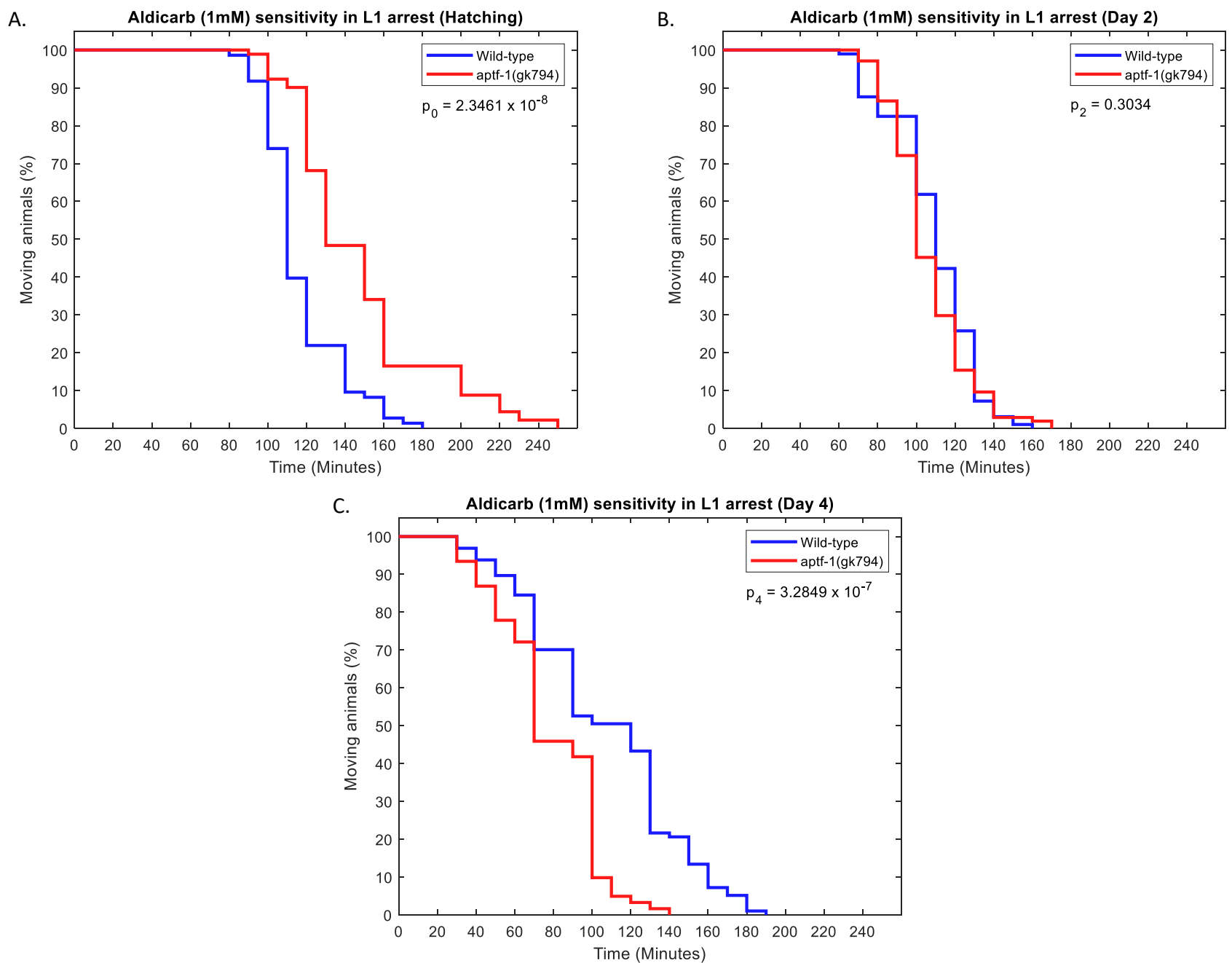

Figure 24. Sleep loss alters the sensitivity to aldicarb of C. elegans

(A-C) At hatching, aptf-1 loss of function results in resistance to aldicarb, which is lost by the $2^{\text {nd }}$ day of starvation. At day 4, the sleepless animals are hypersensitive to aldicarb.

Wild-type: $\mathrm{n}=73,97$ and 97 for days 0,2 and 4 respectively.

aptf-1(gk794): $\mathrm{n}=91,104$ and 122 for days 0,2 and 4 respectively. 
As an interneuron, RIS processes signals from a variety of other neurons. While the GABArelated results point to the motor neurons and the muscles to be at the receiving end of the consequences of sleeplessness, it is also important to address the function of the neurons that function upstream of RIS. These neurons are part of the locomotion circuit that also controls sleep behavior and can be mechanosensory (ASH), dopaminergic (CEP) and tyraminergic (RIM) among others ${ }^{[28]}$. While no difference in baseline activity was observed in mechanosensory and dopaminergic neurons, the tyraminergic RIM neurons show significantly increased activity in the aptf-1 loss of function background (Figure 25).
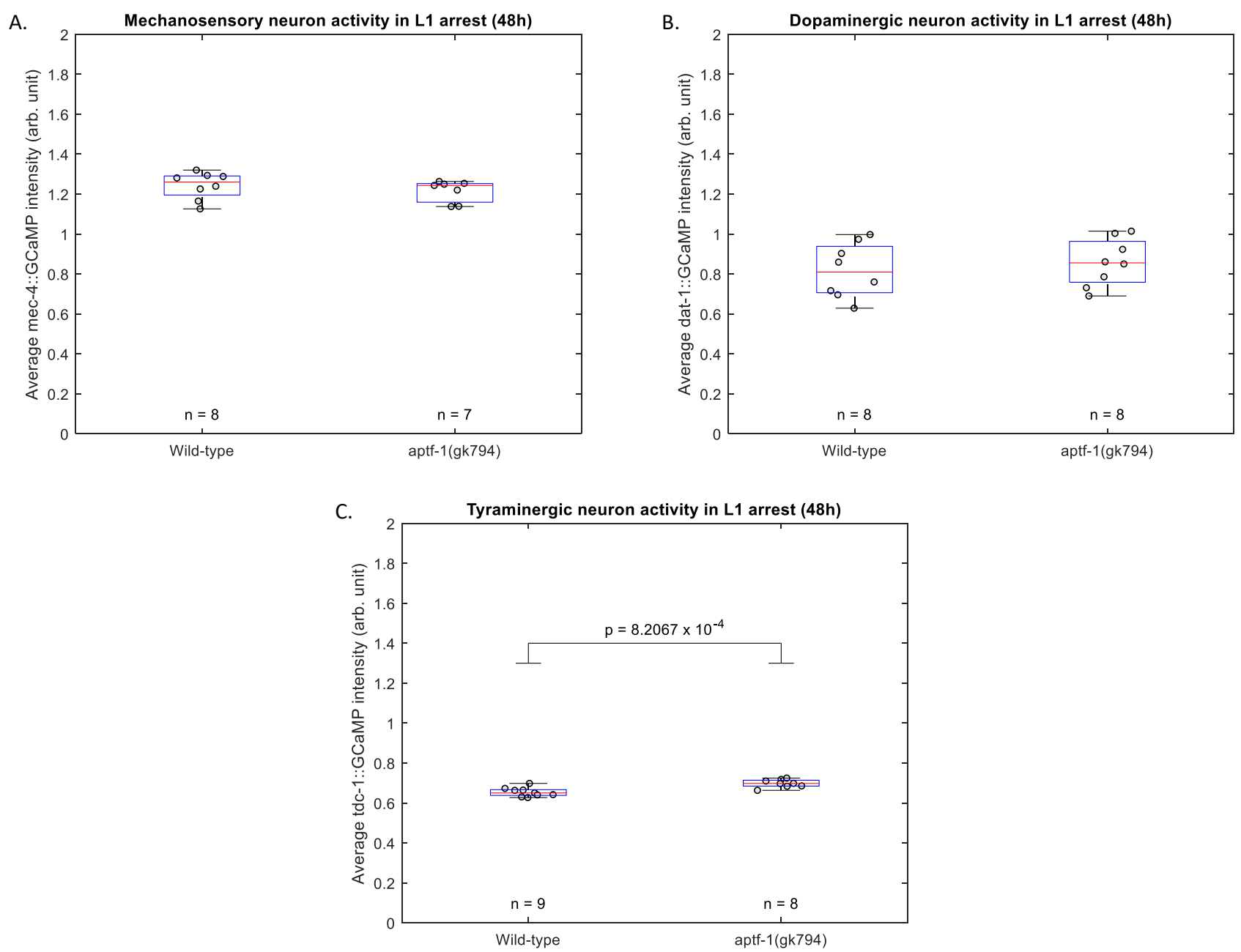

Figure 25. Sleep loss increases RIM neuron activity in $\mathrm{L} 1$ arrest

(A-C) Boxplots showing average GCaMP intensity under promoters specific for different neuron subgroups. Mechanosensory and dopaminergic neurons show no significant difference in the aptf- 1 (gk794) background ( $\mathrm{p}=0.4047$ and $\mathrm{p}=0.5365$ respectively). In tyraminergic neurons average baseline intensity increases by $6,6 \%$ in the aptf-1(gk794) background. Red horizontal line denotes the median value. 


\subsubsection{Sleep controls protein synthesis via $U_{34}$ thiolation in $L 1$ arrest}

I have shown above that sleep introduces imbalances to protein processing mechanisms, such as steps of the translation process and the unfolded protein response. In order to understand how gene expression translates to protein expression in sleep loss conditions, I performed a meta-analysis on the datasets by correlating gene and protein expression, keeping in mind that only approximately $10 \%$ of the genes had their corresponding proteins detected. There is almost zero correlation between the proteome and the transcriptome (Figure 26).
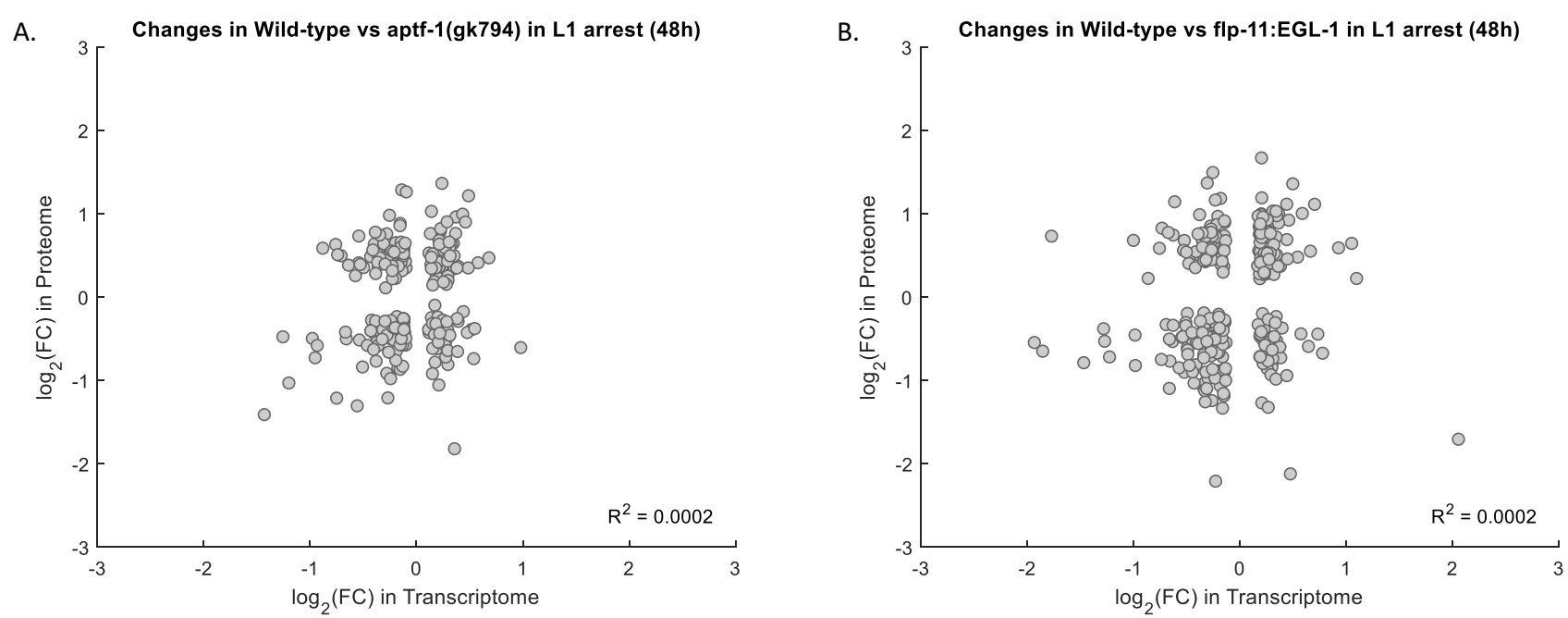

Figure 26. No correlation is found between gene and protein expression in L1 arrest (A-B) Scatter plot representation of transcriptomic and proteomic changes. The four groups that are formed (one in each quadrant) have approximately equal elements.

Protein homeostasis is regulated by a plethora of pathways, to ensure proper development and a healthy lifespan. A gene that stands out is tut- 1 (RNA-seq: $\log _{2}(\mathrm{FC})=0.22$ and FDR $=5.48$ $\mathrm{x} 10^{-5}$ in the aptf-1(gk794) background), which is responsible for the 2-thiolation of wobble uridine $\left(\mathrm{U}_{34}\right)$ in the tRNAs of Lysine, Glutamine and Glutamate. Changes in thiolation can affect the rate of mRNA translation and the equilibrium between protein synthesis and folding, thus creating proteotoxic stress ${ }^{[73]}$. Although Lysine-tRNA $U_{34}$ thiolation is not changed in the aptf-1 loss of function background, Glutamine-tRNA $\mathrm{U}_{34}$ thiolation is significantly increased, in symphony with tut-1 transcriptional expression. This is a significant finding, since changes in thiolation are very rare between different conditions (personal communication with Prof. Sebastian Leidel, University of Bern) (Figure 27 A, B). Also, loss of function of tut-1 reduces the lifespan of L1 arrested worms and aptf- 1 loss of function is able to rescue this phenotype (Figure 27 C, D). Loss of function of another gene in the same pathway, elpc-1, reduces lifespan as well but the phenotype cannot be rescued by aptf-1 loss of function (Figure $27 \mathrm{E}, \mathrm{F}$ ). 
A.

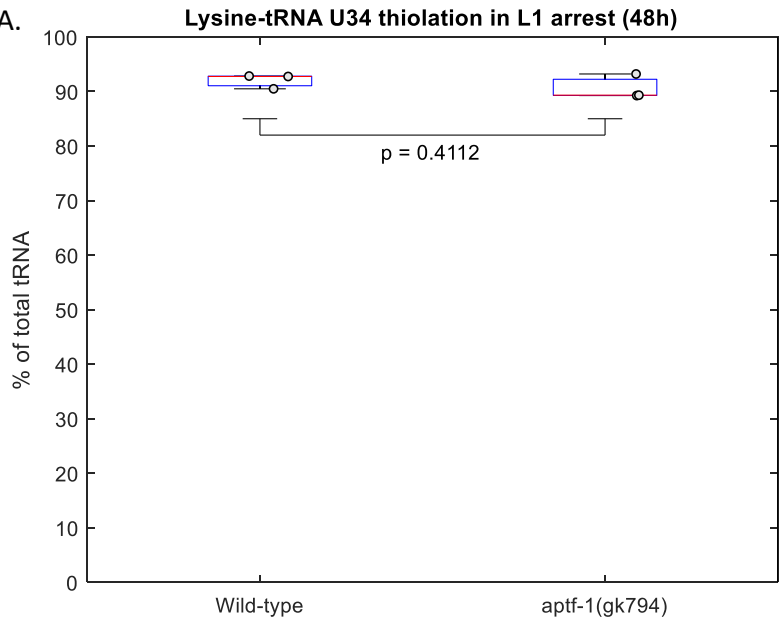

C.

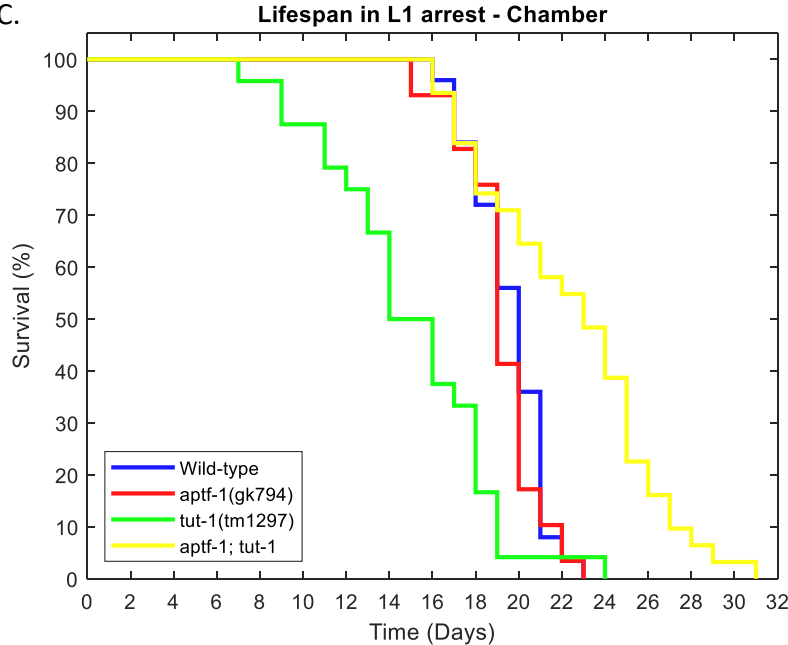

E.

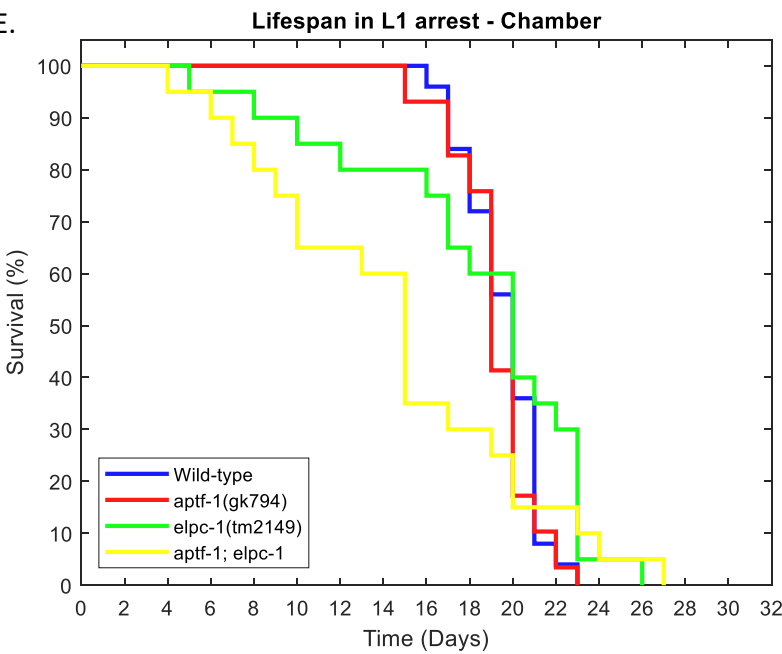

B.

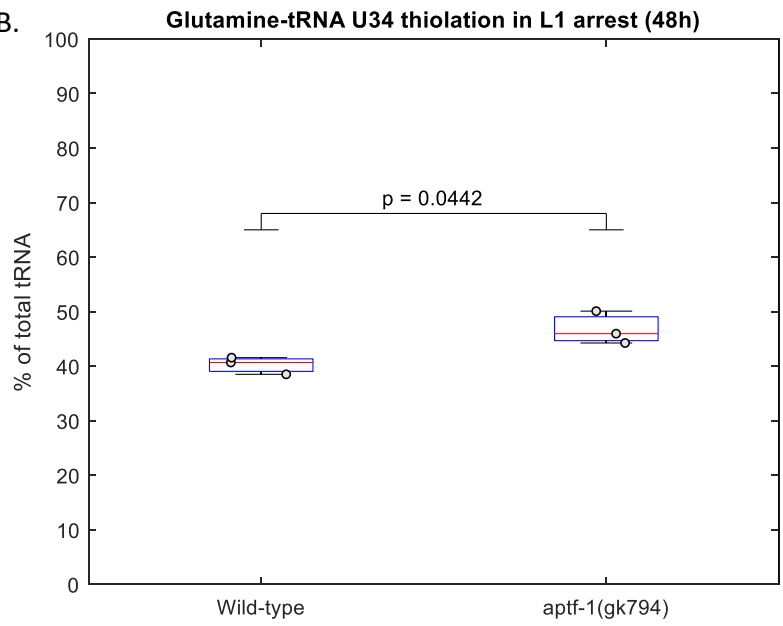

D.

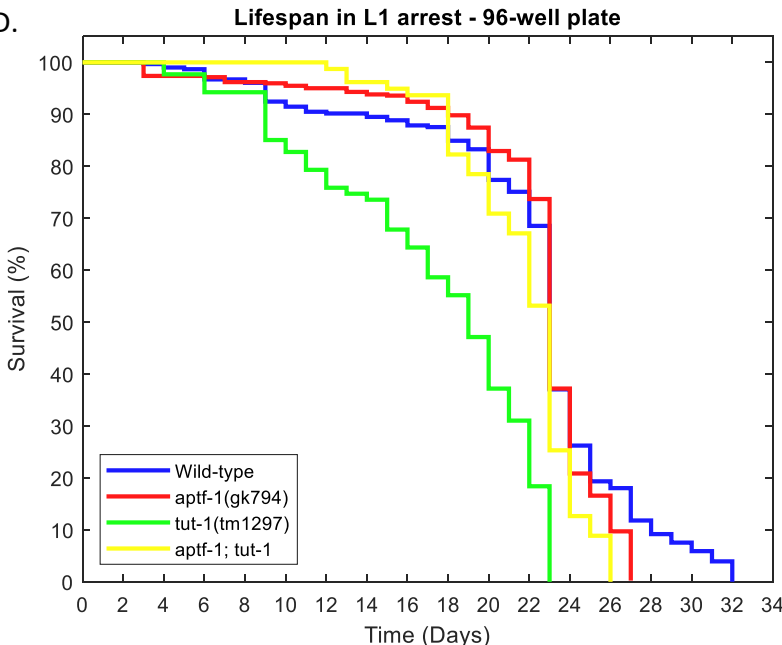

F.

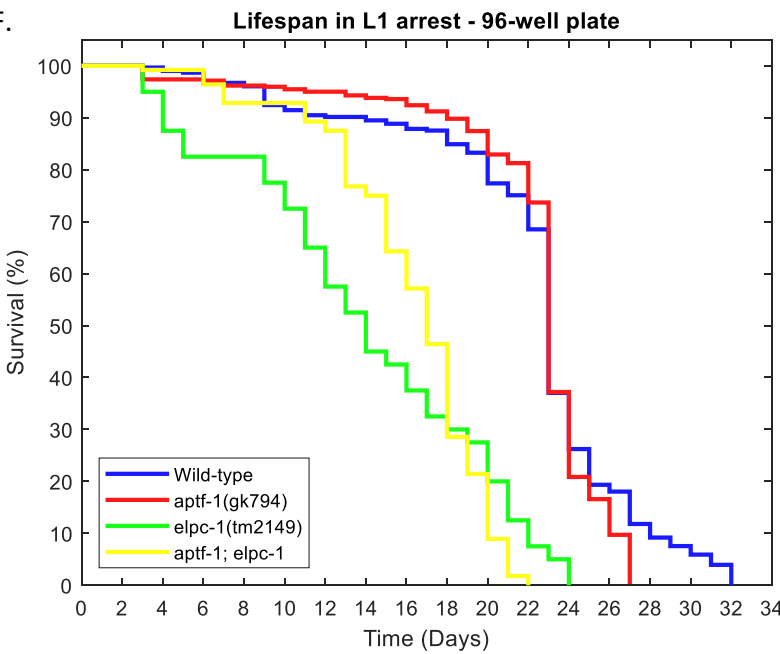

Figure 27. Sleep loss increases $\mathbf{U}_{34}$ thiolation in $L 1$ arrest

(A-B) Glutamate tRNA shows increased thiolation in the aptf-1(gk794) background, while Lysine tRNA does not (3 samples were used per condition).

(C-D) tut-1 loss of function shows significantly reduced lifespan compared to the wild-type in both the chamber and the 96-well plate assay $\left(\mathrm{p}=9.7 \times 10^{-4}\right.$ and $\mathrm{p}=0$ respectively), that can be rescued by aptf- 1 loss of function ( $\mathrm{p}=7 \times 10^{-4}$ and $\mathrm{p}=0.002$ respectively).

(E-F) elpc-1 loss of function produces inconsistent results between assays, both in the wildtype and in the aptf-1(gk794) background. 


\subsubsection{Sleep loss alters lysosomal function in $L 1$ arrest}

Organisms degrade cytoplasmic components, by delivering them to lysosomes in a process known as autophagy. Macro-autophagy, is the most well studied autophagy pathway in $C$. elegans, and involves compartmentalization of cytoplasmic material in vesicles, subsequent fusion with lysosomes and degradation by hydrolases ${ }^{[74]}$. In the aptf-1 loss of function background, macro-autophagy and lysosomal gene expression is upregulated (Figure $28 \mathrm{~A}$ ). However, the number of autophagic vesicles does not change, neither in the intestine nor in the nerve ring (Figure $28 \mathrm{~B}$ ). Genes coding for the $\mathrm{H}^{+}$pump v-ATPase, which acidifies the lumen of lysosomes, are downregulated, which might in turn affect the quality of autophagy.

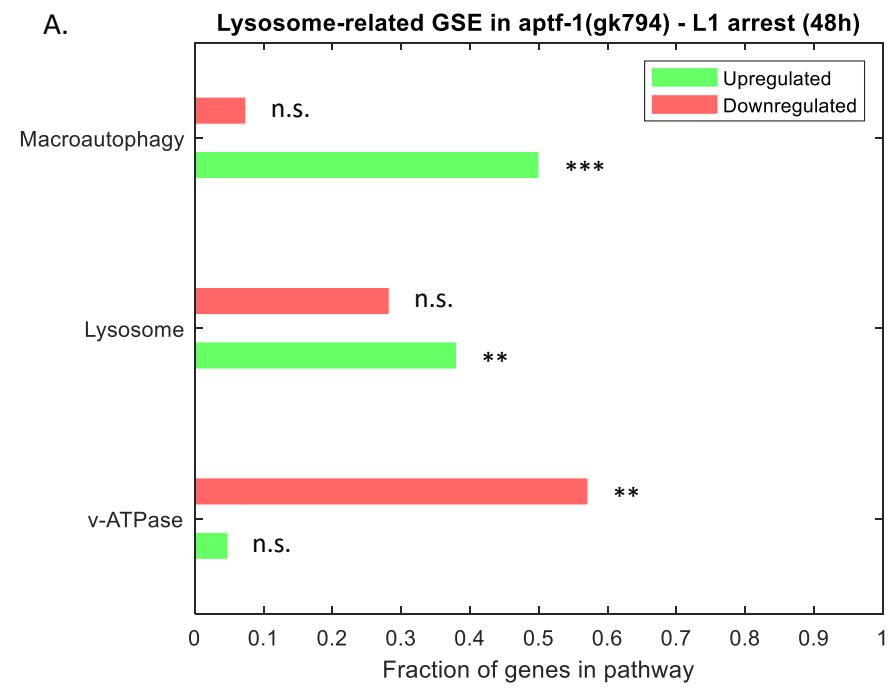

B.

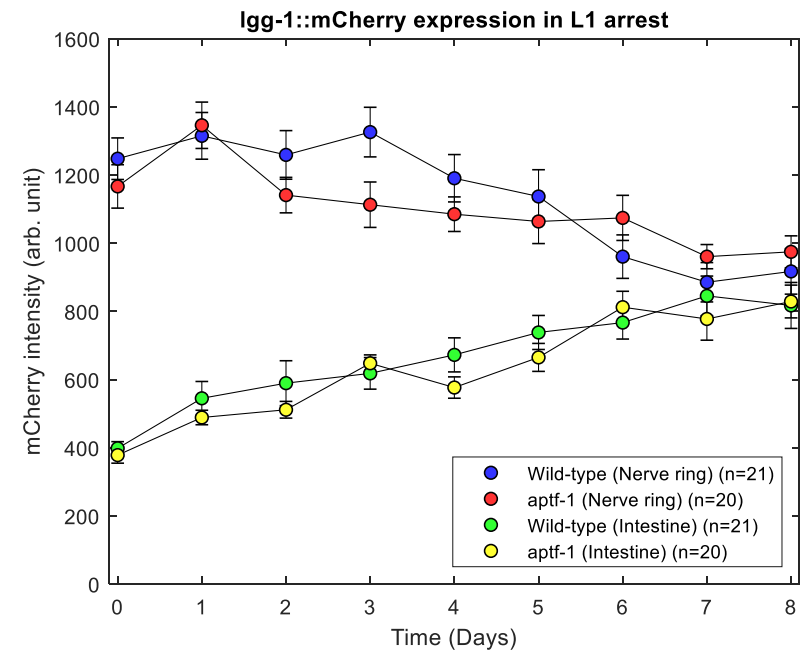

Figure 28. Sleep loss affects lysosomal gene expression in $\mathrm{L} 1$ arrest

(A) Horizontal bar chart representation of lysosomal related pathways that are affected by loss of sleep (***: $p_{\text {adj }}<0.001, * *$ : $p_{\text {adj }}<0.01, *$ : $p_{\text {adj }}<0.05$, n.s.: not significant).

(B) The number of autophagic vesicles in the nerve ring and the intestine does not change in the aptf- 1 (gk794) background (FDR > 0.05 for all time points). 
To sum up, in this chapter I have shown that sleep loss in L1 arrest induces several changes in important pathways that end up altering the physiology of C. elegans.

More specifically in the aptf-1 loss of function background:

Loss of sleep, decreases the expression of the starvation induced gene, hsp-12.6. Hypodermis and TGF- $\beta$ gene expression is upregulated, resulting in reduction of size. Although muscle gene expression seems to follow the global transcriptome trend, this is not the case for the mitochondrial electron transport chain genes and the genes coding for mitochondrial localization proteins, which are downregulated. Intestine-specific gene expression is generally downregulated and expression of many targets of the insulin signaling pathway is affected by sleep loss because FOXO homolog nuclear localization is compromised. Notch signaling and germline gene expression is downregulated and $g l p-1$ gain of function lifespan increase is aptf1 dependent. Neuronal and GABA receptor gene expression is upregulated, indicating the significance of this neurotransmitter in sleep loss. Acetylcholine signaling changes, as indicated by the aldicarb sensitivity assays. Also, tyraminergic (RIM) neuron activity levels are increased. Finally, sleep controls protein homeostasis via tRNA wobble $\mathrm{U}_{34}$ thiolation and by affecting aspects of macro-autophagy.

With regard to the $f l p-11::$ EGL-1 strain:

This seems to be a strain in which RIS is constantly active. Its gene expression patterns are generally very different from the ones in the aptf-1 loss of function strain. Neuropeptide receptor $d m s r-2$ expression, is greatly downregulated. Insulin signaling targets are affected as well, probably because of low FOXO nuclear localization. Finally, neuronal, GABA receptor and glutamate receptor gene expression is downregulated, contrary to the aptf-1 loss of function background. 


\subsection{Sleep loss induces specific metabolic changes in $L 1$ arrest and vice versa}

The transcriptomic and proteomic data collected so far, are an invaluable asset for identifying many components affected by sleep loss that were previously unknown. In order to get the complete image of the sleep loss condition, I performed 2 complementary metabolomic analyses at 48 hours and at 6 days of starvation. In total, 3 biological replicates per condition were measured, which led to the identification of 120 metabolites for the 48-hour time point and 141 metabolites for the 6-day time point.

At 48 hours of starvation, aptf-1 loss of function, upregulates metabolites related to oxidative stress, such as Allantoin and Glutathione (Figure 29 A). Allantoin is the last component of the nucleic acid degradation pathway and since $C$. elegans lacks the enzyme responsible for converting uric acid to allantoin, its presence can be attributed to non-enzymatic oxidation of uric acid by ROS ${ }^{[75]}$. Succinic acid, an intermediate of the TCA cycle and Complex II of the electron transport chain, is also upregulated, as well as Tryptophan, which is known to be upregulated during sleep deprivation in humans ${ }^{[36]}$. A big group of downregulated metabolites are Sphingomyelins, lipids that take part in the composition of the cell membrane and can act as signaling molecules. The most downregulated hit, both in aptf- 1 loss of function and flp11::EGL-1 is the amino-acid Methionine. Surprisingly, in the flp-11::EGL-1 strain there is no differential expression of any other metabolite (Figure 29 A, B and sections $6.25-6.26$ ). At 6 days of starvation the metabolite profile looks different, with no oxidative stress metabolites differentially expressed. The only one that stands out is Kynurenic acid, a neuroactive molecule and endogenous antagonist to the glutamate NMDA receptors, that is regulated by insulin signaling and its production in the RIM neuron has been linked to ageing phenotypes ${ }^{[76]}$. Kynurenic acid is upregulated in aptf- 1 loss of function and downregulated in the $f l p-11:$ EGL1 background (Figure 29 C, D and sections $6.27-6.28$ ). In addition to the metabolomic analyses, I performed isothermal microcalorimetry analysis to measure the heat output of sleepless worms as a readout of their metabolic status. Indeed, heat production is increased more than $100 \%$ in the sleepless strains. The largest part of this increase can be attributed to the difference in movement capacity between the wild-type and the non-sleeping worms. However, there is still a $20 \%$ extra heat production that can only be explained by a difference in metabolic rates (calculations by Prof. Bart Braeckman, University of Ghent, personal communication) (Figure $29 \mathrm{E}$ ). Finally, changes in the metabolic rate, introduced by incubation at $25{ }^{\circ} \mathrm{C}$ did not alter the difference in lifespans between wild-type and sleepless worms (Figure 29 F). 

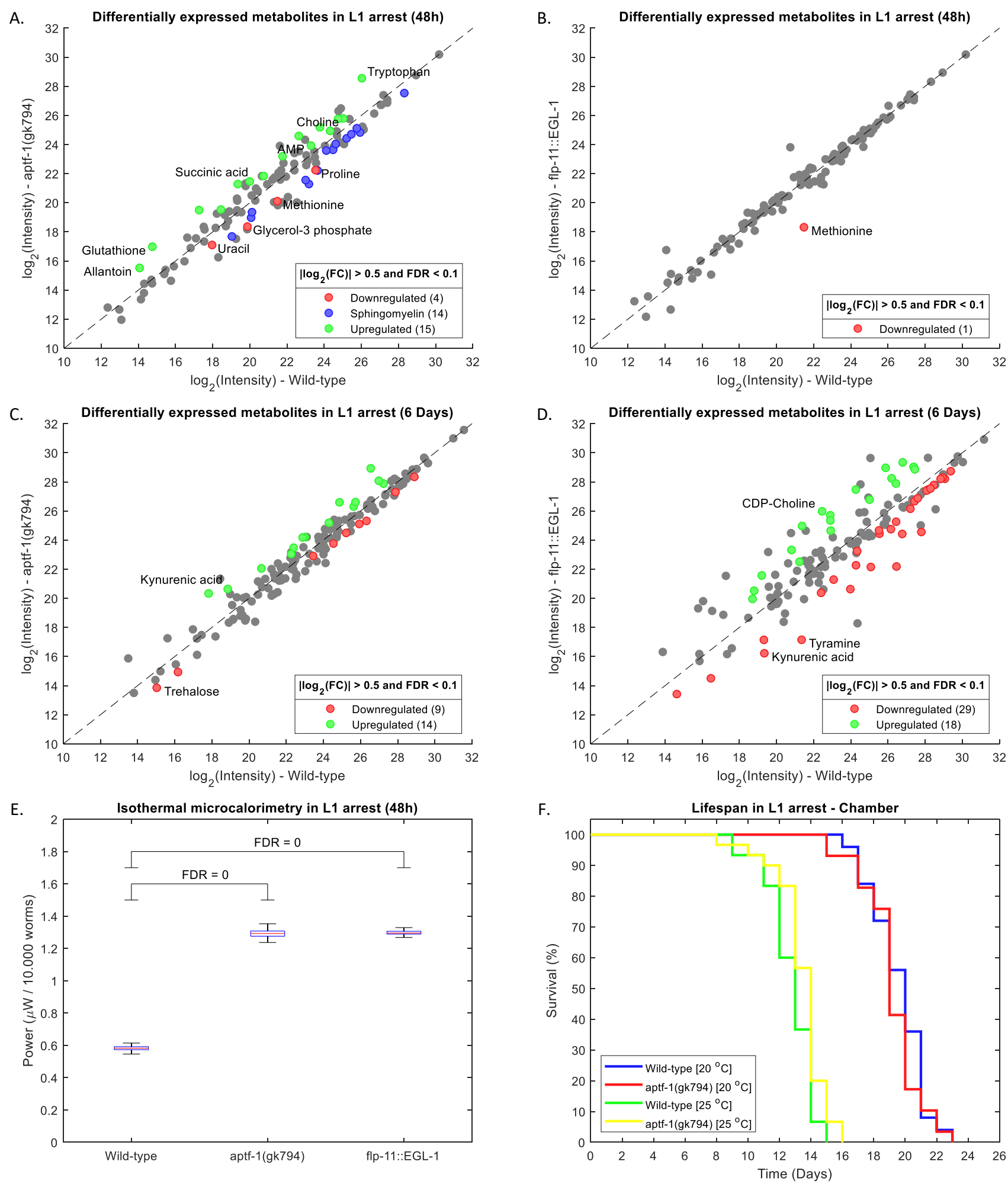

Figure 29. Sleep loss introduces metabolic changes in $\mathrm{L1}$ arrest

(A-D) Scatter plot overview of metabolite expression at 48 hours and at 6 days of starvation. Not significant metabolites according to the selected thresholds, are marked in grey.

(E) Boxplot showing the increased power output of sleepless worms.

(F) Incubation at higher temperatures reduces the lifespan of C. elegans. This change is not affected by sleep loss since the difference in wild-type vs aptf- 1 (gk794) is not significant in either case $\left(\mathrm{p}=0.5294\right.$ and $\mathrm{p}=0.1121$ at $20^{\circ} \mathrm{C}$ and $25^{\circ} \mathrm{C}$ respectively). 
Another aspect of the metabolic profile, is the creation of offspring. Reduced developmental quiescence has been associated with defects in egg-laying in the past ${ }^{[50]}$. In order to assess the ability of the worm to convert energy and nutrients into organized and functional tissue, I counted the number of eggs laid by the adult worm in well-fed and starved conditions. Fed worms lay the same number of eggs regardless of their sleep status. However, starved and sleepless worms lay 2 times more eggs than the wild-type ones (Figure 30).
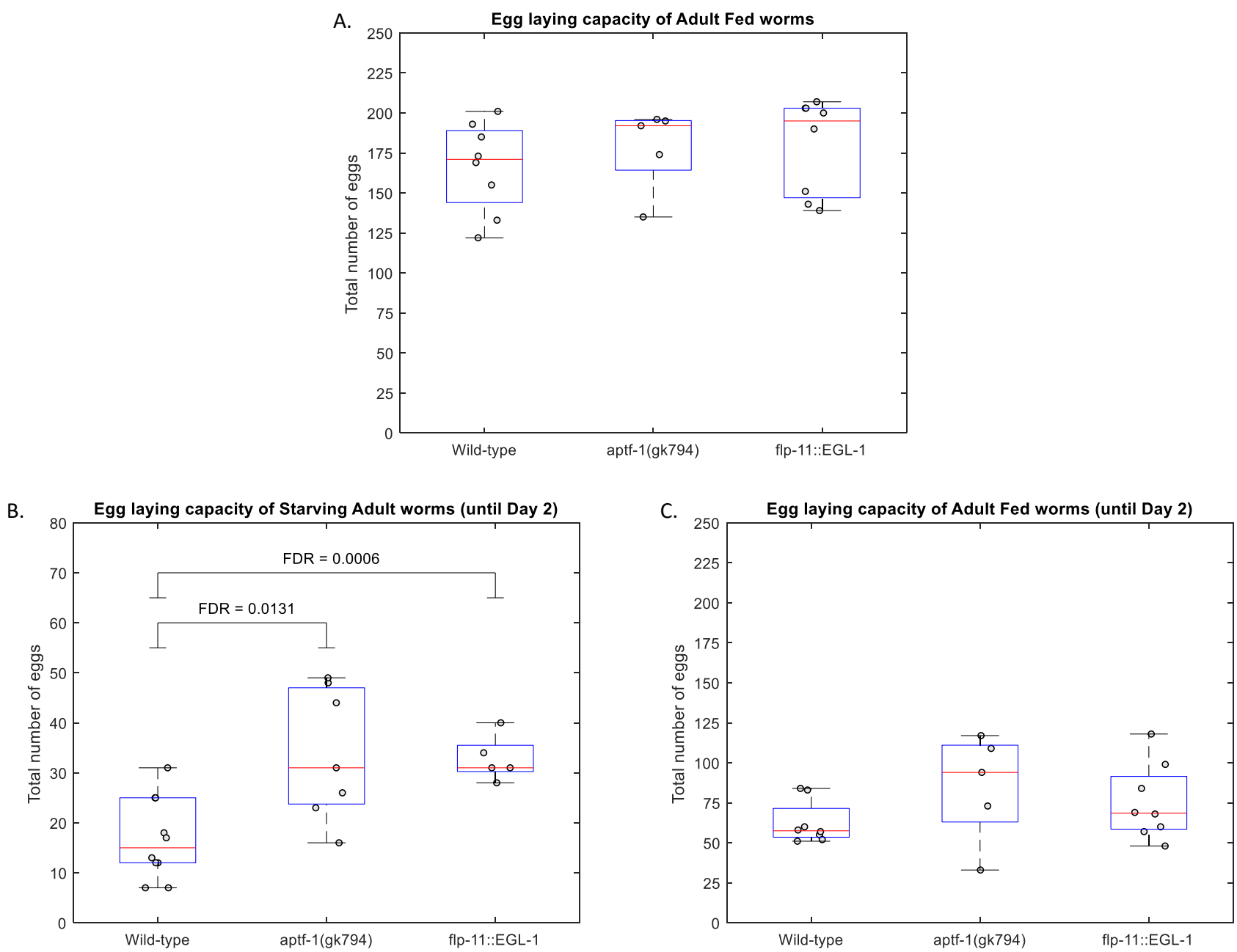

Figure 30. Sleep loss increases egg laying during adult starvation

(A) Sleep-deprived worms do not show egg laying defects (FDR $>0.05$ for all comparisons). (B-C) Starved and sleepless worms lay significantly more eggs. For the same time interval fed worms do not show differences in egg-laying (FDR >0.05).

Fed - Wild-type: $\mathrm{n}=8$, aptf-1 (gk794): $\mathrm{n}=5$, flp-11 ::EGL-1: $\mathrm{n}=8$

Starved - Wild-type: $\mathrm{n}=10$, aptf-1(gk794): $\mathrm{n}=7$, flp-11::EGL-1: $\mathrm{n}=5$

Red horizontal line denotes the median value. 
The discovery of these sleeplessness-induced metabolic changes, has prompted the question whether this is a 2-way phenomenon, that is whether genetic defects in metabolism induce changes in sleep. To answer this question, I performed an extensive genetic screening of every non-lethal metabolic mutant that is publicly available and assessed their sleep amount at 48 hours of L1 arrest. In total 546 strains were screened, 121 strains showed reduced quiescence and 8 strains showed increased quiescence (Figure $31 \mathrm{~A}$ and section 6.29).

With regard to the increased quiescence strains, the only one that stands out is the insulin receptor daf-2 loss of function mutant, with an increase of $59.72 \%$ in quiescence compared to the wild-type (Figure $31 \mathrm{~A}, \mathrm{~B}$ ). The other strains should be considered with caution, since many of them show movement defects that can wrongly be considered as sleep.

As for the low quiescence strains, one in particular stands out, the mammalian diacyl-glycerol kinase theta (DKG $\theta$ ) homolog $d g k-1$ loss of function, with a decrease of $93.56 \%$ in quiescence compared to the wild-type (Figure $31 \mathrm{~A}, \mathrm{~B}$ ). This mutation also resulted in a reduced lifespan phenotype in $\mathrm{L} 1$ arrest (Figure $31 \mathrm{D}, \mathrm{E}$ ). The strain that showed the lowest quiescence is the $t d c-1$; aptf- 1 loss of function double mutant (Figure $31 \mathrm{C}$ ). This is important for 2 reasons: 1. it significantly reduced the quiescence of the aptf-1 mutant, which is already low while $t d c-1$ loss of function did not show any difference from the wild-type and 2. tdc-1 codes for tyrosine decarboxylase, the enzyme responsible for the production of tyramine in RIM neurons. In the rest of the low quiescence strains there is an enrichment $\left(\mathrm{p}_{\mathrm{adj}}=10^{-6}\right)$ in sphingolipid metabolism genes (hyl-1, hyl-2, F33D4.4, sms-3, hpo-13, sptl-3, gba-1, lagr-1). This is interesting not only because of the connection to the metabolome, where sleep loss is shown to reduce sphingomyelins but also because the second messenger DAG, a product of sphingomyelin catabolism is phosphorylated by $d g k-1$ to transduce neuronal signals ${ }^{[77]}$. FOXO homolog daf-16, hypoxia response hif-1, vhl-1 and egl-9 and apoptosis regulator ced-3 loss of function, all result in low quiescence phenotypes. These genes should be considered for downstream screening and have their role in sleep regulation clarified. Representative mobility traces of wild-type, aptf-1(gk794), dgk-1(ok1462) and daf-2(e1370) can be found in section 6.30 .

Overall, the screening shows that metabolic manipulations are more likely to result in low quiescence and that mostly neurotransmission-related mutations produce the strongest phenotypes. 

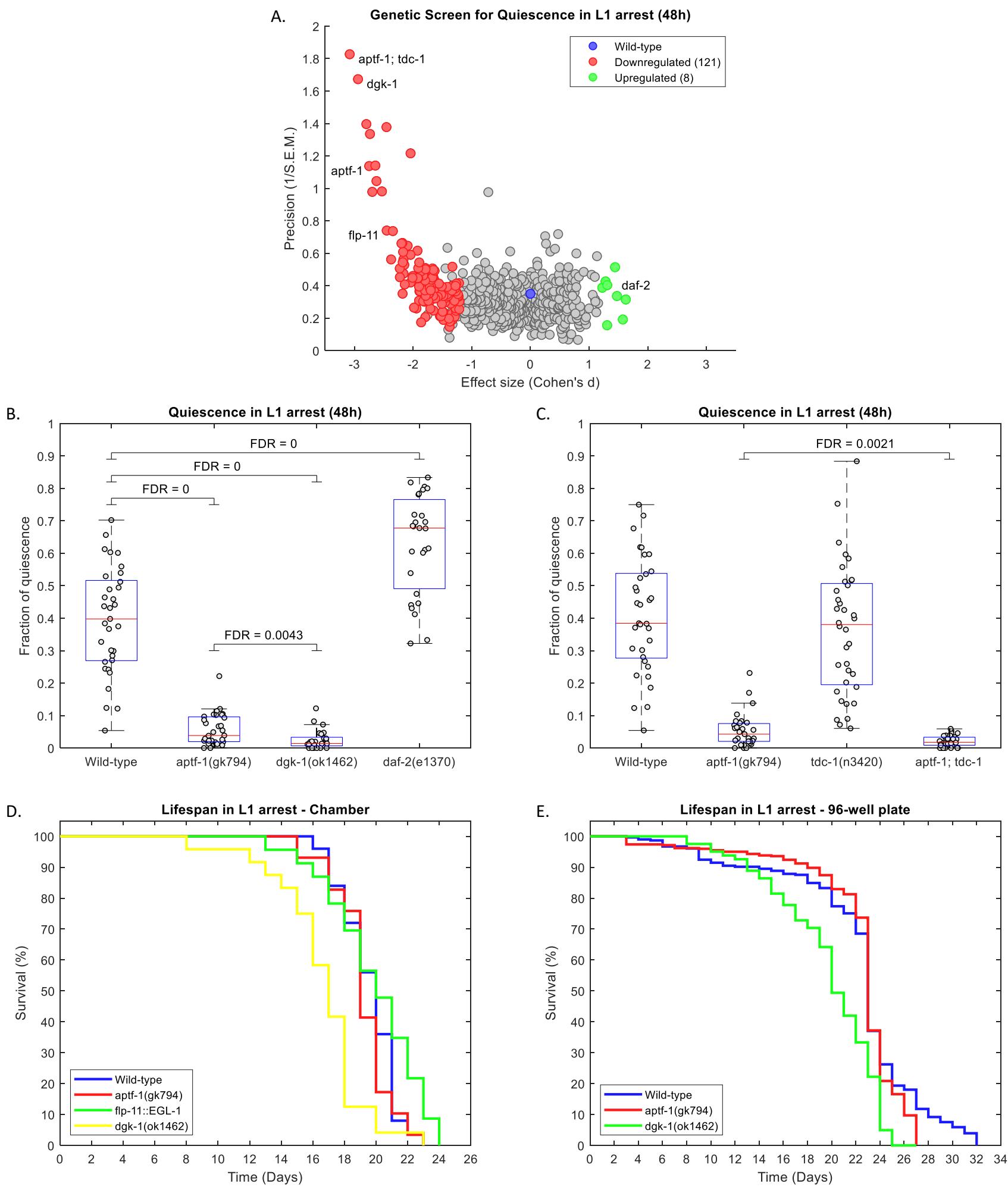

Figure 31. Metabolic defects cause changes in sleep and lifespan

(A) Genetic screening for quiescence assessment in L1 arrest.

(B-C) Boxplot representation of the fraction of quiescence of the most significant hits of the genetic screen. Wild-type: $\mathrm{n}=33$, aptf-1 (gk794): $\mathrm{n}=31, d g k-1(\mathrm{ok} 1462): \mathrm{n}=25$, daf-2(e1370): $\mathrm{n}=27, t d c-1(\mathrm{n} 3420): \mathrm{n}=36, t d c-1 ;$ aptf-1: $\mathrm{n}=34$. Red horizontal line denotes the median value. (D-E) Loss of function of $d g k-1$ reduces the lifespan in L1 arrest (Wild-type vs $d g k-1$ (ok1642): $\mathrm{p}=0.003$ and $\mathrm{p}=5.2 \times 10^{-8}$ respectively). 
In order to address the significant downregulation of methionine in the metabolome, I started another screening in the wild-type background. Initially I supplemented microchamber assays with various concentrations of methionine $(0.01-25 \mathrm{mM})$ and followed up with glycine and glutamate, to assess any changes in L1 arrest quiescence behavior. Indeed, there is a significant sleep fraction reduction after supplementation with $10 \mathrm{mM}$ of methionine, glycine and glutamate. Only methionine produces an effect large enough to be compared to the effects discovered in the genetic screening. However, $1 \mathrm{mM}$ of methionine produce the opposite result, a small increase in quiescence. Such contradicting results, did not favor an expansion of the screening to the aptf-1 loss of function mutant (Figure $32 \mathrm{~A}$ and section 6.31). Furthermore, in order to address the impact of maternal growth conditions in the arrested L1, I cultured the worms for 4 months on regular OP50 food source and various temperatures or at a constant temperature of $20^{\circ} \mathrm{C}$ and different food sources. High incubation temperatures $\left(25^{\circ} \mathrm{C}\right)$ and rich food sources $(\mathrm{Na} 22)$ reduce quiescence, while low incubation temperatures $\left(15^{\circ} \mathrm{C}\right)$ and poor food sources (DL42) increase quiescence in L1 arrest (Figure $32 \mathrm{~B}$ and section 6.32).
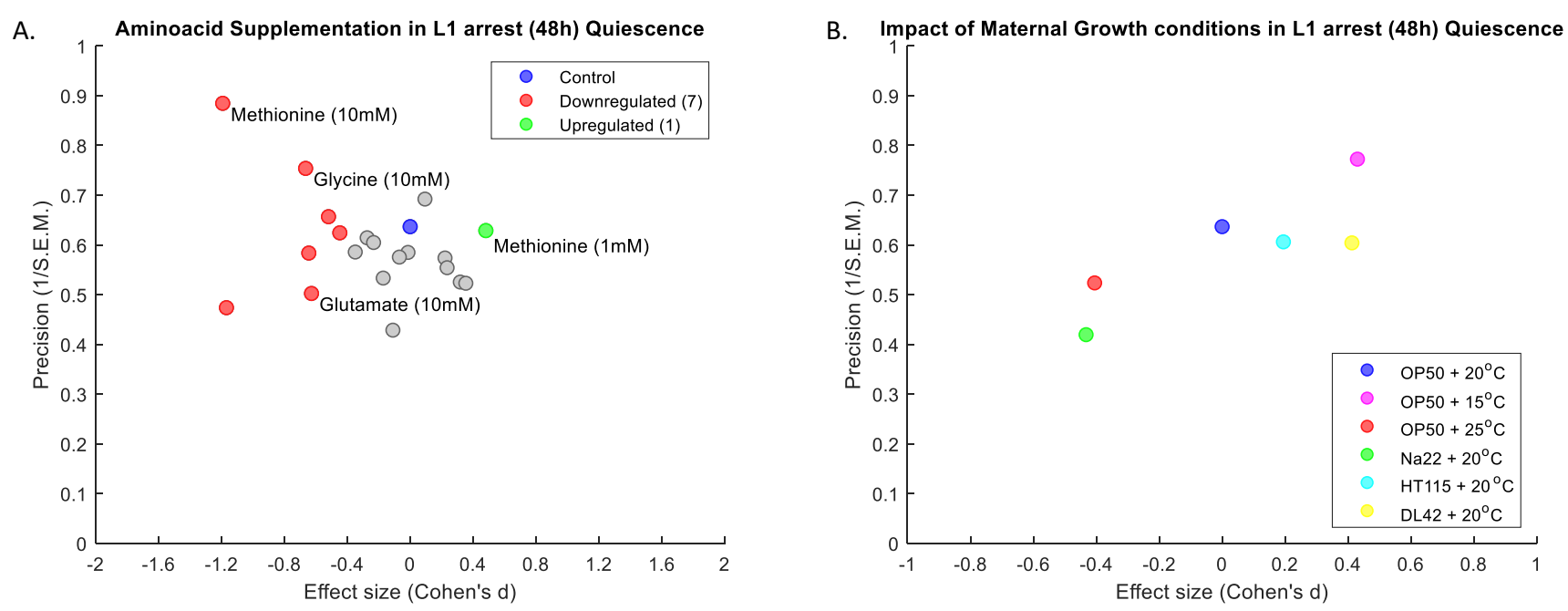

Figure 32. Maternal growth conditions and amino-acid supplementation affect sleep

(A) Methionine, Glycine and Glutamate supplementation at $10 \mathrm{mM}$ decreases quiescence, while $1 \mathrm{mM}$ of Methionine increases quiescence of wild-type animals.

(B) Growth temperature and nutritional status of the parent worm, affects offspring quiescence.

To sum up, sleep loss increases the metabolic rate of the worms. The key regulatory elements of L1 arrest quiescence are methionine metabolism, insulin signaling via daf-2 and daf-16, neuronal signaling mediated by RIM and the action of $d g k-1$ downstream of a G-protein coupled receptor probably regulated by sphingolipid metabolism. Maternal growth conditions play only a minor role in offspring quiescence. 


\section{4. $d g k-1$ loss of function results in sleep loss by changing RIS physiology}

In order to further investigate the $d g k-1$ loss of function that produces the low quiescence phenotype in the genetic screen, I decided to assess the behavior of RIS in that background. In addition to the wild type, I have included two more control strains that were designed (by Prof. Henrik Bringmann) to keep RIS constantly de-polarized (flp-11::UNC-58(gf)) or hyperpolarized (flp-11::TWK-18). Surprisingly, both these strains show reduced quiescence in L1 arrest as was shown by my colleague Inka Busack. Diacylglycerol (DAG) is a second messenger that is responsible for neurotransmission by regulating synaptic vesicle release. $d g k$ 1 acts by phosphorylating DAG into phosphatidic acid, an action that reduces the amount of available DAG, inhibiting neurotransmitter release. unc-13 (a homolog of mUNC13), is the target of DAG that promotes neurotransmitter release. Both these genes act downstream of Gprotein coupled receptors to regulate quiescence ${ }^{[78]}$. Average activity of the RIS neuron as measured by GCaMP shows no differences in the $d g k-1$ or $u n c-13$ loss of function backgrounds, although the characteristic RIS peaks are absent (representative traces can be found at section 6.33). As expected the engineered de-polarized and hyper-polarized strains show, respectively, increased and decreased average RIS activity (Figure $33 \mathrm{~A}$ ). In all cases, the drop of speed that is observed by RIS activation in the wild-type is abolished. That, in turn, means that the inverse correlation of RIS activity and speed is lost (Figure 33 B). Finally, I investigated the signal complexity of RIS for each strain. Signal complexity of the electroencephalogram has been used as a predictive marker of brain health in neurodegenerative diseases, trauma, anesthesia and sleep-wakefulness studies ${ }^{[79]}$. Signal complexity, quantified here by fractal dimension, is higher in the $d g k-1$ loss of function background as well as the RIS de-polarized strain. On the other hand, unc-13 shows lower signal complexity and the RIS hyper-polarized strain shows no difference from the wild-type (Figure $33 \mathrm{C}$ ).

With this set of experiments, it is now evident that neurotransmission for sleep induction is not only dependent on RIS peaks, but also on the overall complexity of the neuronal signal. $d g k-1$ loss of function seems to ablate sleep in a way different than simply de- or hyper- polarizing RIS. 
A.

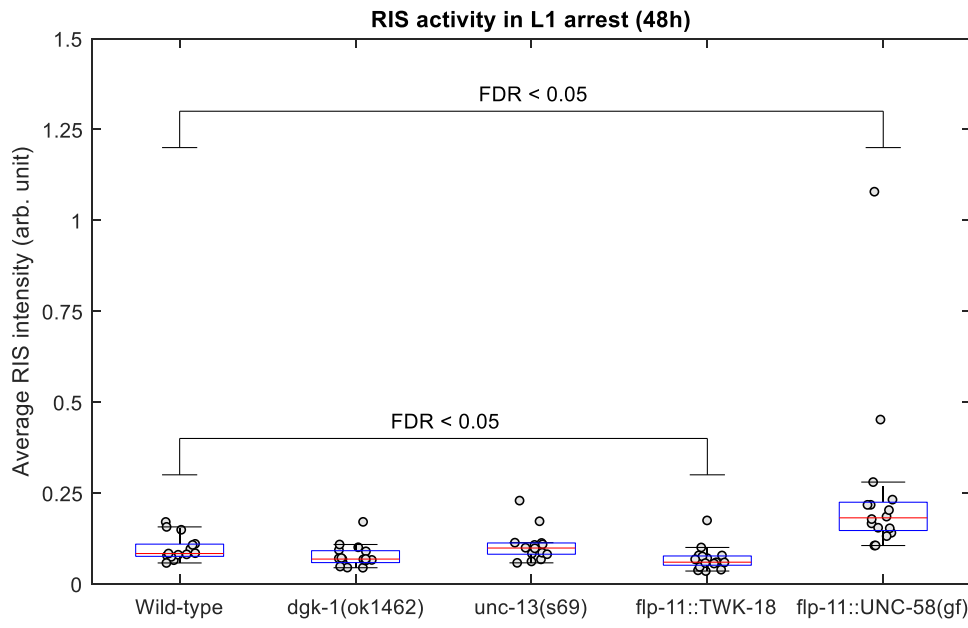

B.

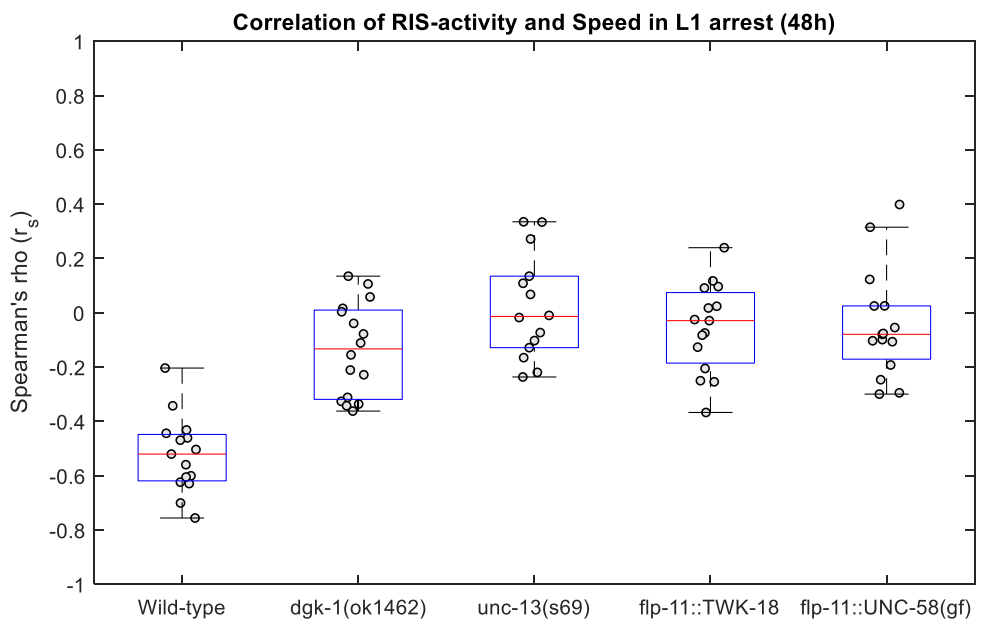

C.

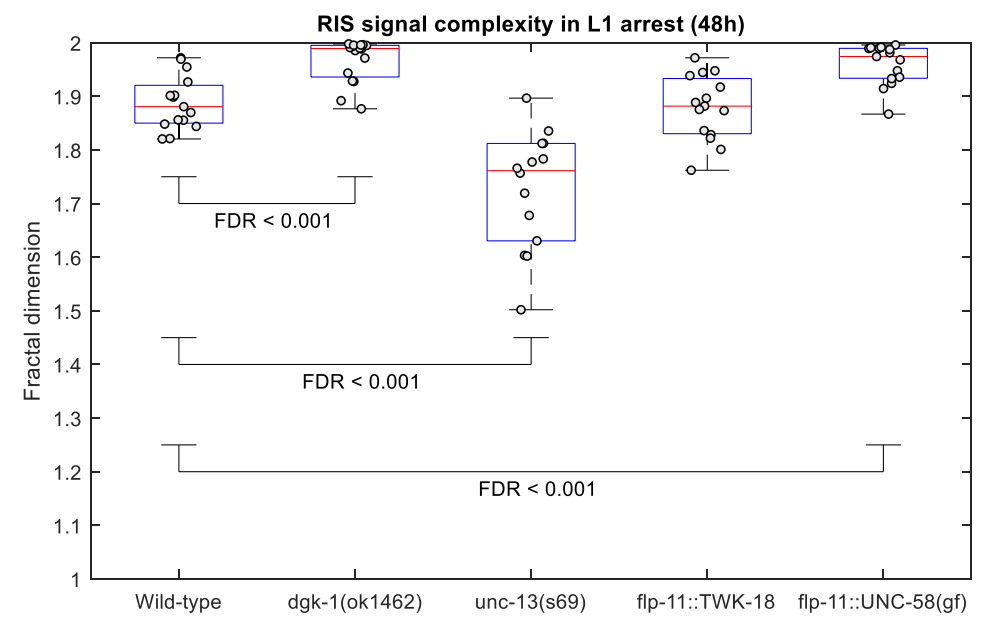

Figure 33. $d g k-1$ loss of function mutants abolish sleep by altering RIS function

(A) RIS average GCaMP activity levels are not changed by $d g k-1$ or $u n c-13$ loss of function. (B) All of the tested manipulations result in decoupling of RIS activity levels and speed (FDR $<0.05$ for all strains vs wild-type).

(C) RIS signal complexity is significantly higher in dgk-1(ok1462) and flp-11::UNC-58(gf) than the in the wild-type. Fractal dimension was calculated with Higuchi's algorithm $(\mathrm{k}=100)$. Wild-type: $\mathrm{n}=15$, dgk-1(ok1462): $\mathrm{n}=16$, unc-13(s69): $\mathrm{n}=14$, flp-11::TWK-18: $\mathrm{n}=15$, flp$11:: \mathrm{UNC}-58(\mathrm{gf}): \mathrm{n}=15$ 


\subsection{The transcription factor $\mathrm{XBP}-1$ regulates aspects of $L 1$ arrest quiescence}

In order to identify genes that could reverse the loss of developmental quiescence induced by the aptf-1 loss of function, an EMS mutagenesis assay was performed by Dr. Elisabeth Maluck (these results are documented in her Ph.D. thesis titled "Characterization of RIS presynaptic circuits for sleep regulation in Caenorhabditis elegans"). She was able to isolate 2 candidates, aptf-1 loss of function, strains that showed almost wild-type developmental quiescence (codenamed: Candidate 1 and Candidate 9). After she performed genetic sequencing on the 2 candidates, we collaborated for downstream analysis. I implemented an algorithm to find regions of high mutation density (hotspots) and eventually identify the bZIP transcription factor $x b p-1$ (Candidate 1) and the zinc-finger transcription factor $z t f-20$ (Candidate 9) as the genes with the highest probability of being responsible for the quiescence rescue (Figure $34 \mathrm{~A}, \mathrm{~B}$ ). The most cost-effective strategy was to obtain commercially available strains that carry mutations in these genes and screen them for quiescence. Dr. Maluck assessed quiescence in L1 lethargus and I assessed quiescence in L1 arrest.

Neither Dr. Maluck nor I could replicate the phenotype of Candidate 9 with the $z t f-20$ mutant in any condition. This might be due to the fact that the strain used did not exactly replicate the mutation identified in Candidate 9, but it was just a missense mutation (gk263563) with unknown effects on protein function.

However, $x b p-1$ loss of function was able to partially rescue the quiescence of the aptf- 1 loss of function mutant both in L1 lethargus (personal communication with Dr. Maluck) and in L1 arrest (Figure $34 \mathrm{C}$ ). It has to be noted here that the strain used did not exactly replicate the original mutation of Candidate 1 , but it contains a single nucleotide change that leads to a premature stop codon (zc12).

XBP-1 is the transcription factor responsible for the transcription of genes that take part in the unfolded protein response in the endoplasmic reticulum. Sleep deprivation is known to induce the unfolded protein response in young adult worms ${ }^{[80]}$. These findings taken together with my transcriptome analysis gene set enrichment analysis, show for the first time the interconnection of sleep loss in L1 arrest and the unfolded protein response in C. elegans. 
A.

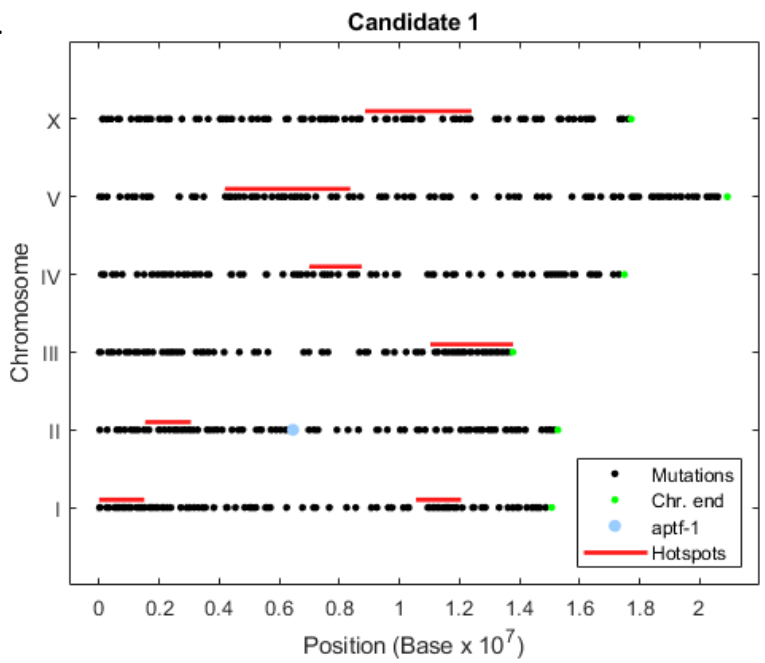

B.

Candidate 9

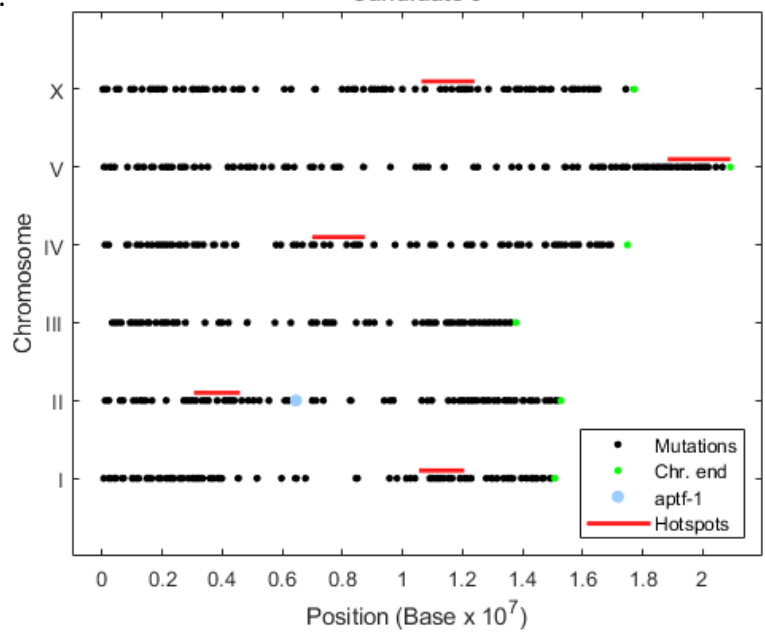

C.

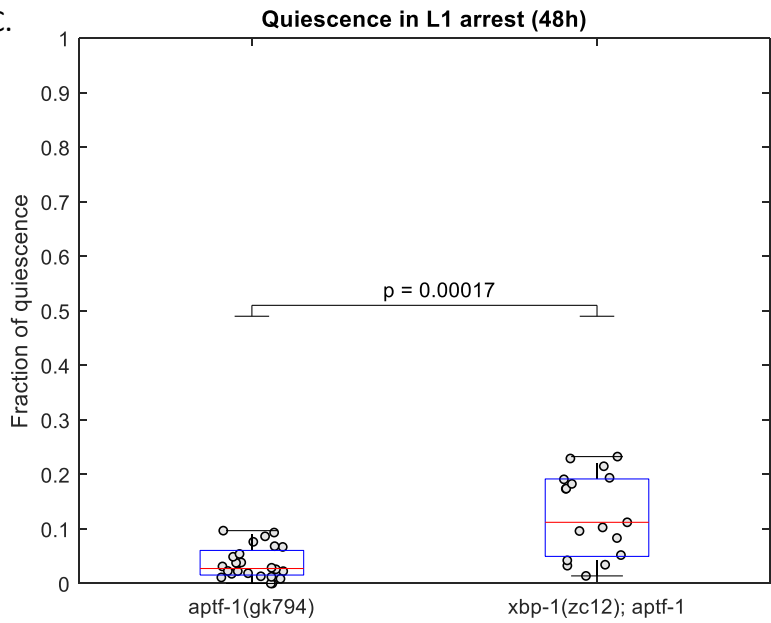

Figure 34. $x b p-1$ loss of function partially rescues the aptf-1(gk794) loss of quiescence (A-B) Hotspot regions as they were identified on the chromosomes of each candidate.

(C) $x b p-1$ loss of function results in a 2.1-times increase in quiescence of the aptf-1 loss of function mutant. aptf-1(gk794): $\mathrm{n}=24, x b p-1(\mathrm{zc} 12): \mathrm{n}=17$ 


\subsection{Sleep loss affects FOXO-dependent transcription in muscles}

As I have shown above, FOXO-dependent gene expression is greatly affected by sleep loss. I also presented the example of reduction in nuclear translocation of DAF-16 in intestinal cells. This is also the case for muscle cells in which decreased nuclear translocation of DAF-16 can be observed from day 2 of starvation (Figure $35 \mathrm{~A}$ ). For this reason, downstream class I targets of FOXO that get expressed in the muscle, such as $h s p-12.6$, are downregulated (Figure $35 \mathrm{C}$, D). The case of HSP-12.6 is a particular one, since it stands at the crossroads of starvation, sleep and aging, with its loss reducing the lifespan of L1 arrested worms (Figure 35 B).
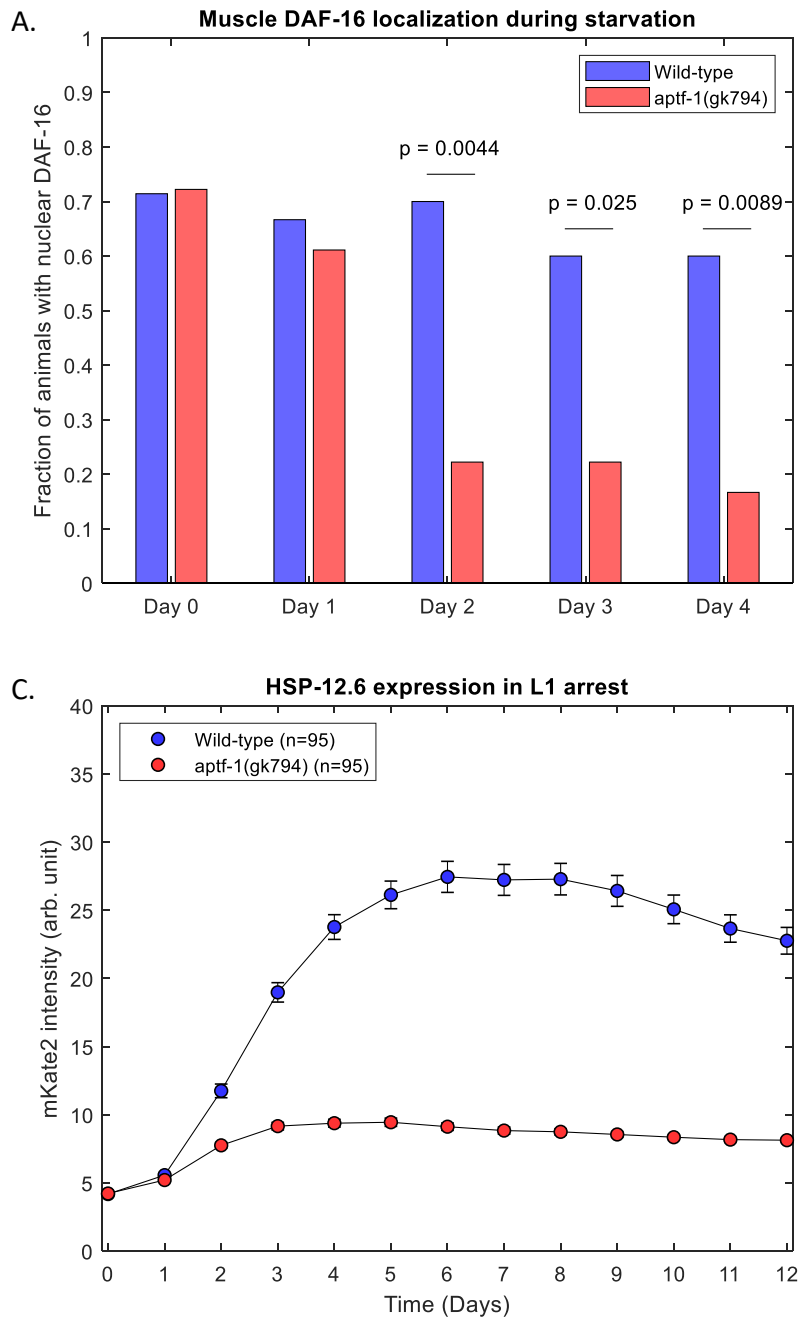

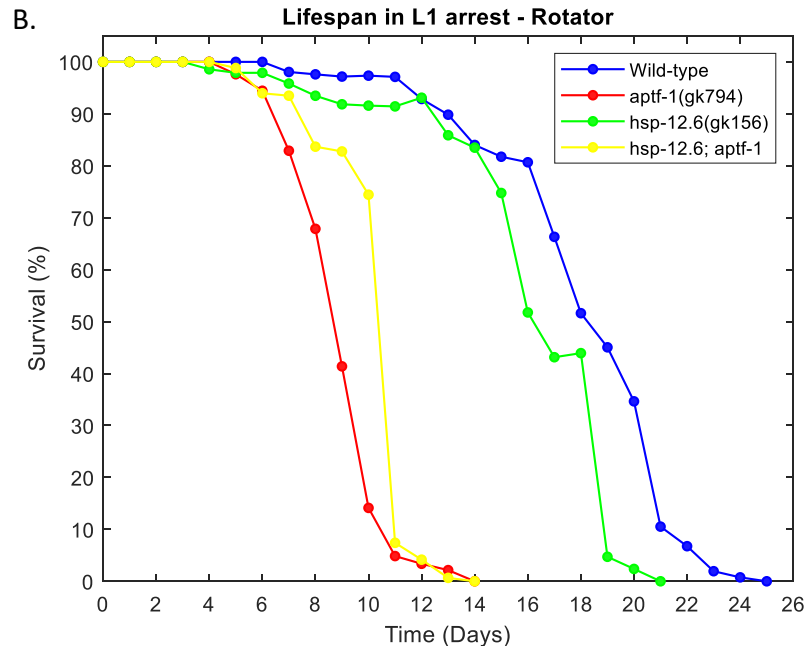

D.

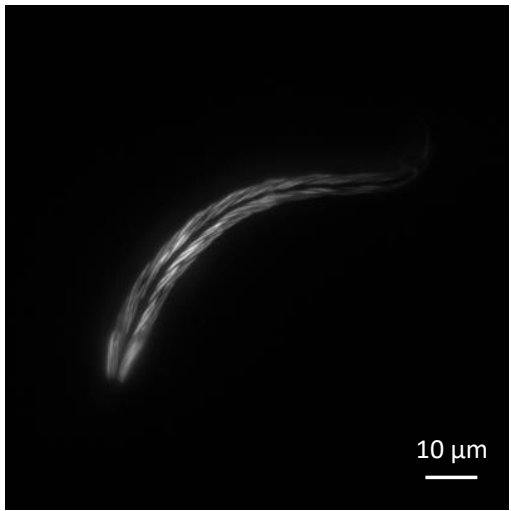

Figure 35. aptf-1 loss of function reduces FOXO nuclear localization and HSP-12.6

(A) Bar plot showing the fraction of animals with nuclear DAF-16 localization. Statistical significance was determined with Fisher's exact test. Wild-type: $\mathrm{n}=20$ and aptf-1(gk794): $\mathrm{n}=18$

(B) hsp-12.6 loss of function reduces the lifespan in the wild-type background (FDR $=3.7 \mathrm{x}$ $10^{-11}$ at day 19) while it does not affect the lifespan of aptf-1 (gk794) (FDR = 1 at day 12).

(C) HSP-12.6 expression is not induced as efficiently in the aptf-1(gk794) background during L1 starvation (FDR $<0.001$ for all time points, except day 0 and day 1 ).

(D) HSP-12.6 expression driven by the endogenous promoter is localized in body wall muscles. 


\subsection{RIS and the nervous system control HSP-12.6 expression in muscles}

In order to identify the rest of the pathway components that connect aptf-1 to hsp-12.6, I performed a screen of HSP-12.6 expression in 70 different conditions during the first 12 days of L1 arrest (Figure $36 \mathrm{~A}$ ). Although the FOXO transcription factor (daf-16) and the factors that control FOXO activity (daf-2, jnk-1,aak-1 and aak-2, rheb-1 and $f k h-9^{[63,81]}$ ) all affect the expression of HSP-12.6, they do it parallel to aptf-1 (Figure $36 \mathrm{~B}-\mathrm{E})$.

In order to address this issue, I hypothesized that HSP-12.6 expression is regulated by aptf-1 via a non-canonical pathway. For this reason, I tested the expression of HSP-12.6 in worms that were engineered to have de- or hyper- polarized RIS. RIS de-polarization leads to increased expression of HSP-12.6, while RIS hyper-polarization leads to decreased expression (Figure 37 A). Loss of function of other RIS-specific components, such as the neuropeptide FLP-11 or the serotonin receptor SER-4 ${ }^{[54]}$, increase or decrease the expression respectively (Figure $37 \mathrm{~B}$ ). After having established the role of RIS, I assessed the effect of neurotransmission. Increased neurotransmission resulting from $d g k-1$ loss of function, decreased the levels of HSP-12.6, while decreased neurotransmission resulting from unc-13 loss of function did not significantly alter the expression (Figure $37 \mathrm{C}$ ). With regard to cholinergic signaling, reducing acetylcholine by loss of function of choline O-acetyltransferase (cha-1) as well as increasing acetylcholine by adding $100 \mu \mathrm{M}$ of the acetylcholinesterase inhibitor Aldicarb, decreased HSP-12.6 levels (Figure $37 \mathrm{D}, \mathrm{E})$. However, addition of $100 \mu \mathrm{M}$ of the nicotinic receptor agonist Levamisole did not result in any change of the levels of HSP-12.6 (Figure 37 F).

By continuing the search for relevant neurotransmitters, I discovered that both supplementation of $10 \mu \mathrm{M}$ of octopamine and loss of function of tyramine $\beta$-hydroxylase ( $t b h-1)$ responsible for octopamine synthesis from tyramine, increase the expression of HSP-12.6 in the wild type while inducing only a marginal increase in aptf-1 loss of function background (Figure 38 A, B). Supplementation of $10 \mu \mathrm{M}$ of tyramine and loss of function of tyrosine decarboxylase $(t d c-1)$ responsible for tyramine synthesis, decrease the expression of HSP-12.6 (Figure $38 \mathrm{C}, \mathrm{D}$ ). Finally, while reduction of glutamate signaling does not change the levels of HSP-12.6 in the wild-type background, increase of inhibitory glutamate signaling by the addition of $1 \mu \mathrm{g} / \mathrm{ml}$ Ivermectin (glutamate-gated chloride channel agonist) decreases HSP-12.6 expression in the wild type but increases it in the aptf-1 loss of function background (Figure $38 \mathrm{E}, \mathrm{F}$ ). Plots for the rest of the screened conditions can be found in section 6.34 . 


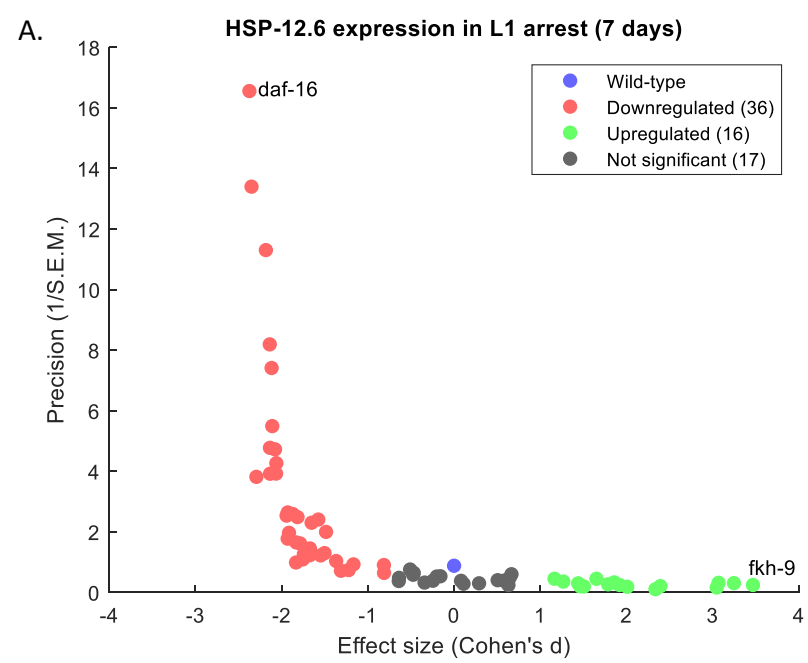

B.

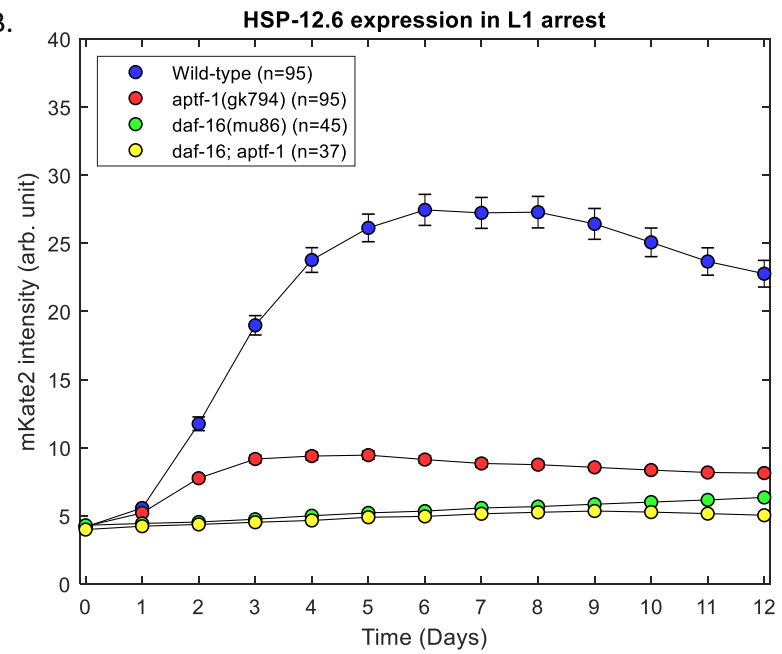

D.

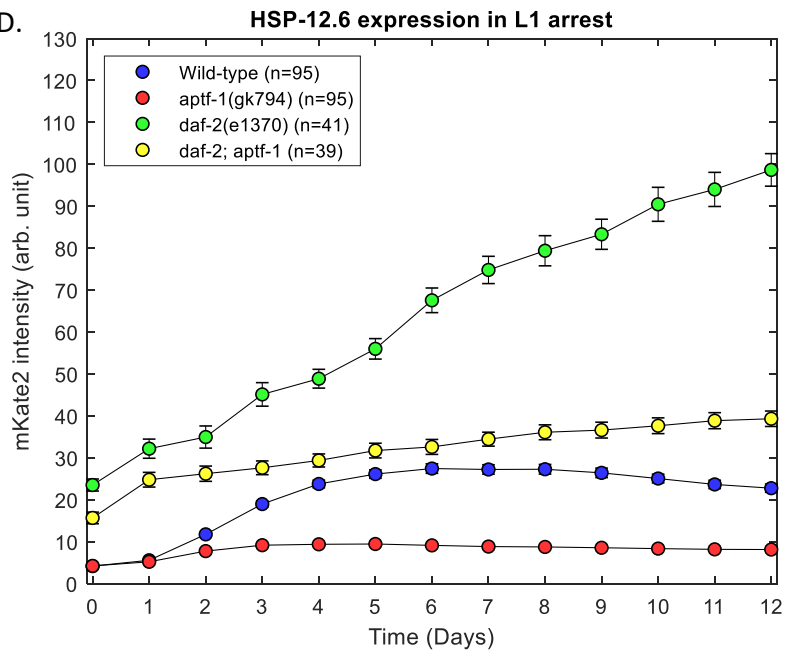

C.

C. HSP-12.6 expression in L1 arrest

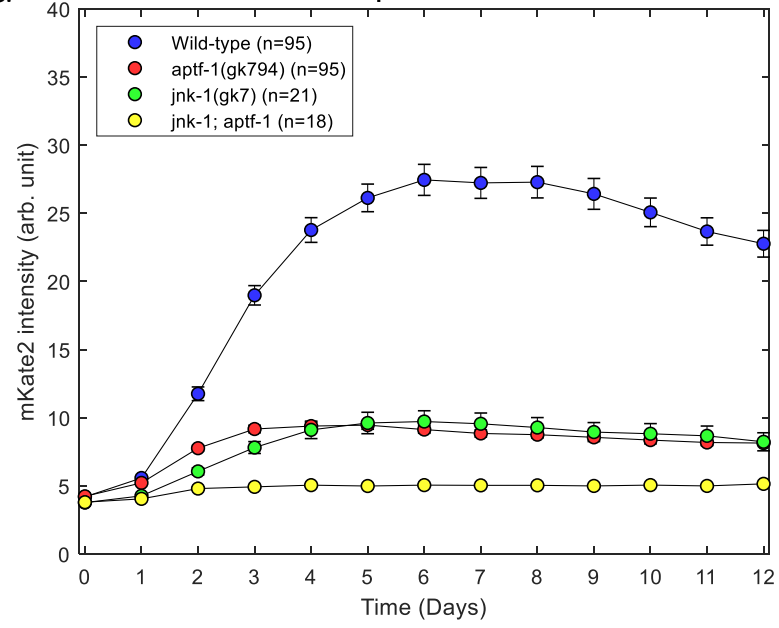

E.

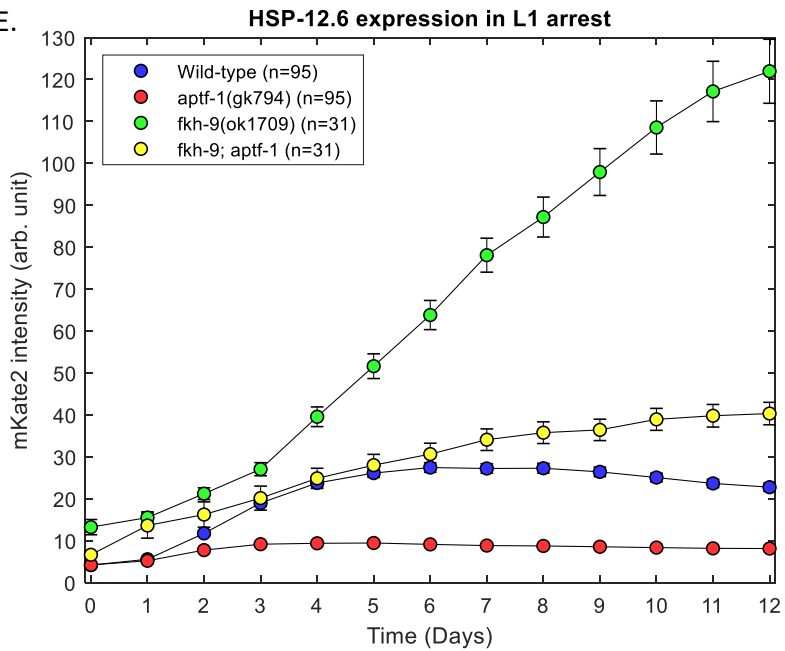

Figure 36. Regulation of HSP-12.6 by aptf-1 depends on FOXO homolog daf-16

(A) HSP-12.6 expression levels, when induced by starvation, plateau at the 7-day time point in the wild-type worm. This time point was selected as representative of the total expression.

(B-E) HSP-12.6 expression over time depends on daf-16 and the JNK pathway. It also depends on insulin signaling via $d a f-2$ as well as $f k h-9$, which both rescue the aptf-1 phenotype. In every case, FDR $<0.05$ for all time points except day 0 and 1. 
A.

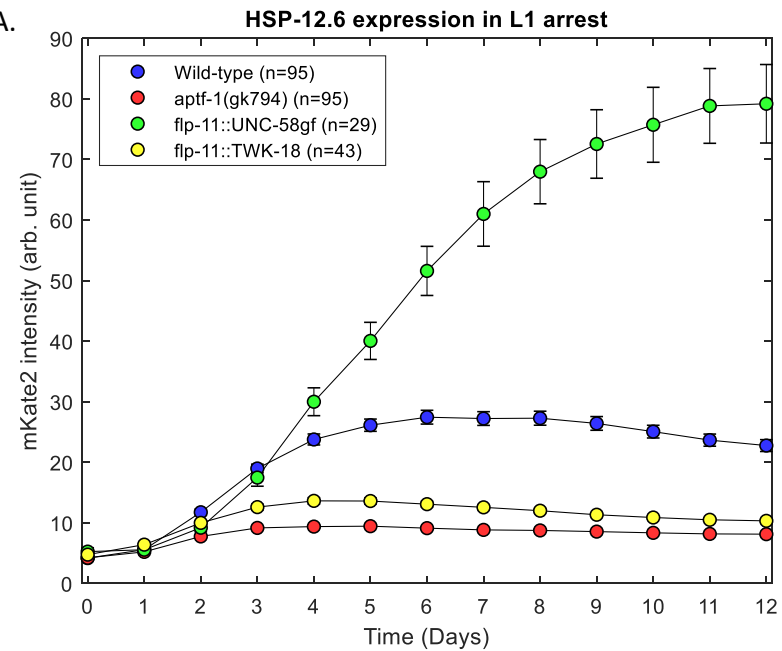

C.

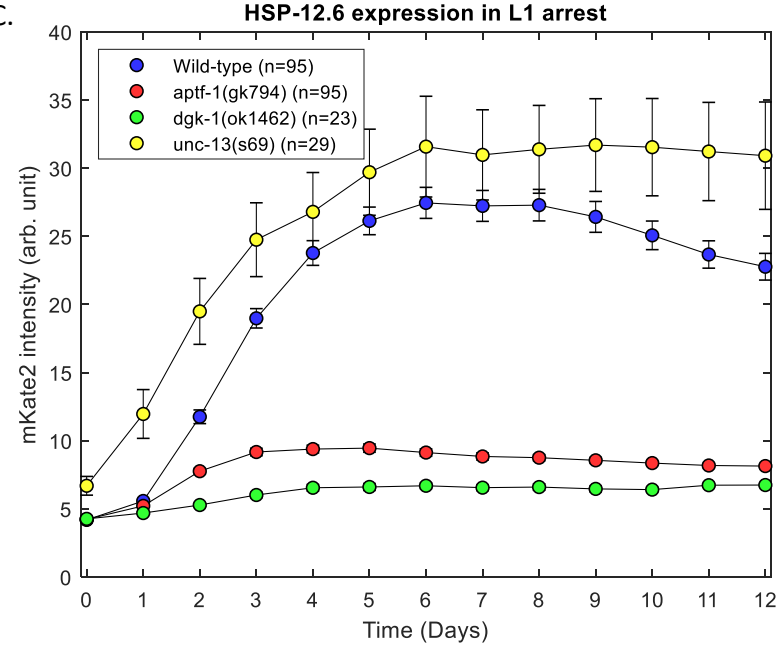

E.

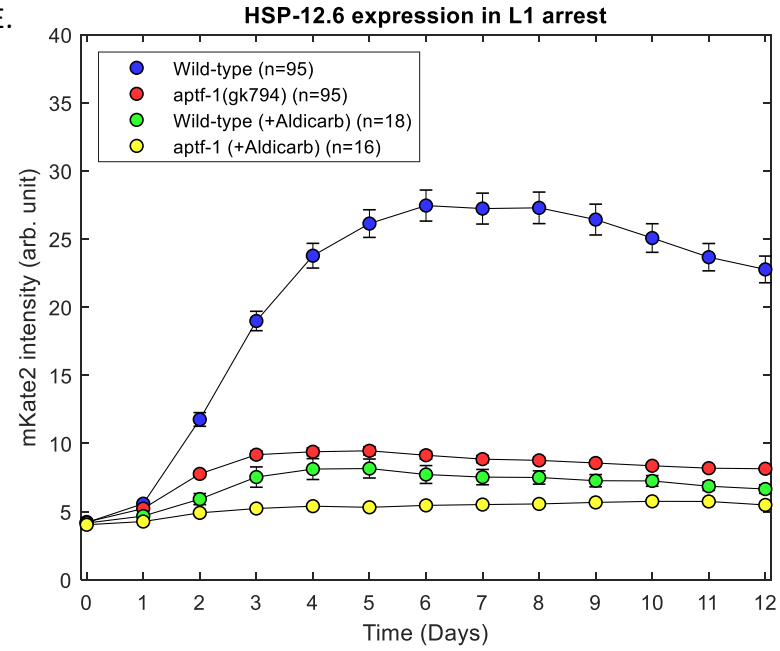

B.

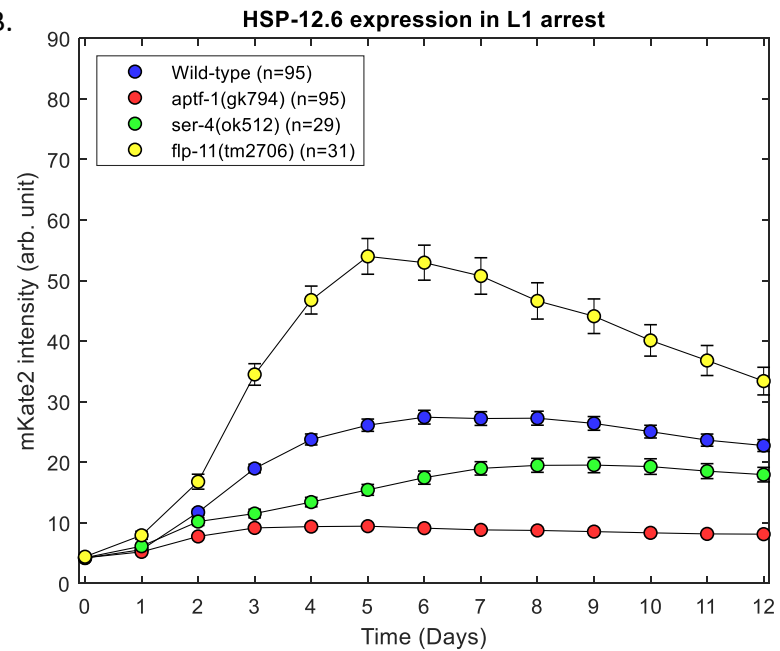

D.

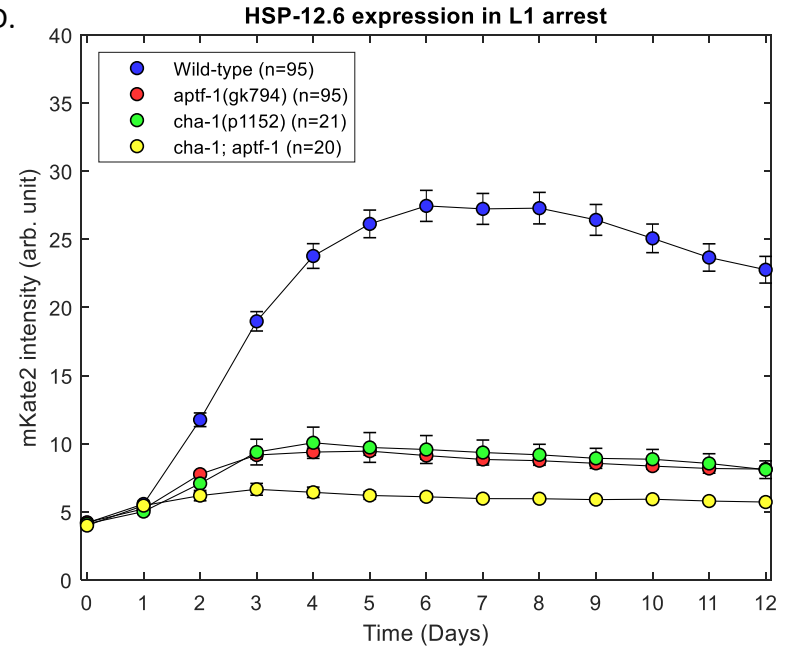

F.

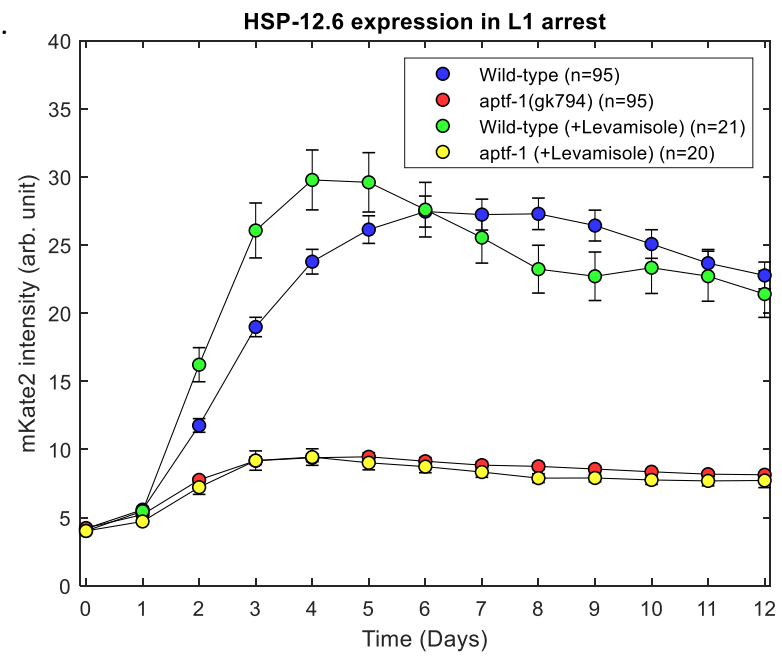

Figure 37. RIS and other neurons regulate HSP-12.6 expression in the muscle

(A) Constant RIS de- and hyper- polarization, increase and decrease HSP-12.6 levels respectively.

(B) Loss of function of $f l p-11$ increases HSP-12.6 expression, while loss of function of ser-4 leads to decrease.

(C) Increase in synaptic transmission via $d g k-1$ loss of function decreases HSP-12.6 expression.

(D-F) Decrease and increase in cholinergic signaling both lead to reduced HSP-12.6 levels.

Treatment with Levamisole does not change the expression. 
A.

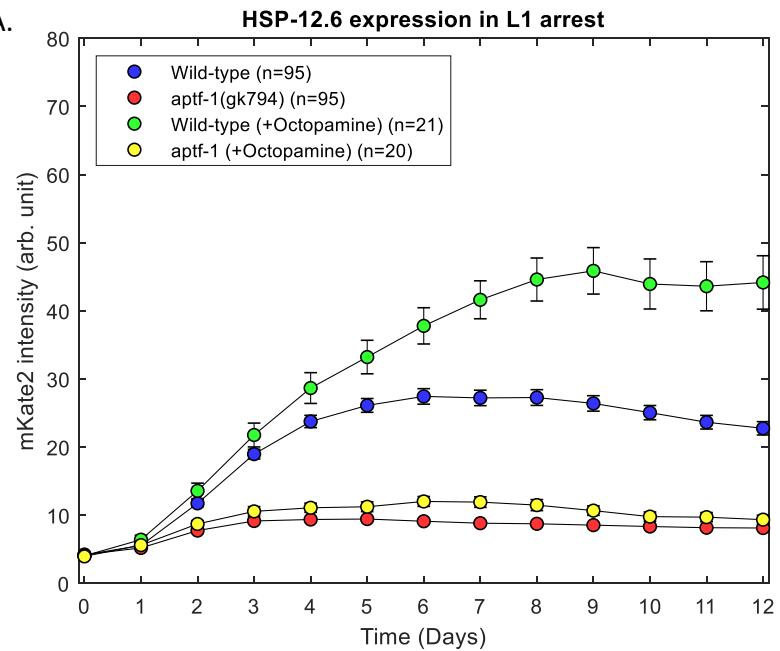

C.

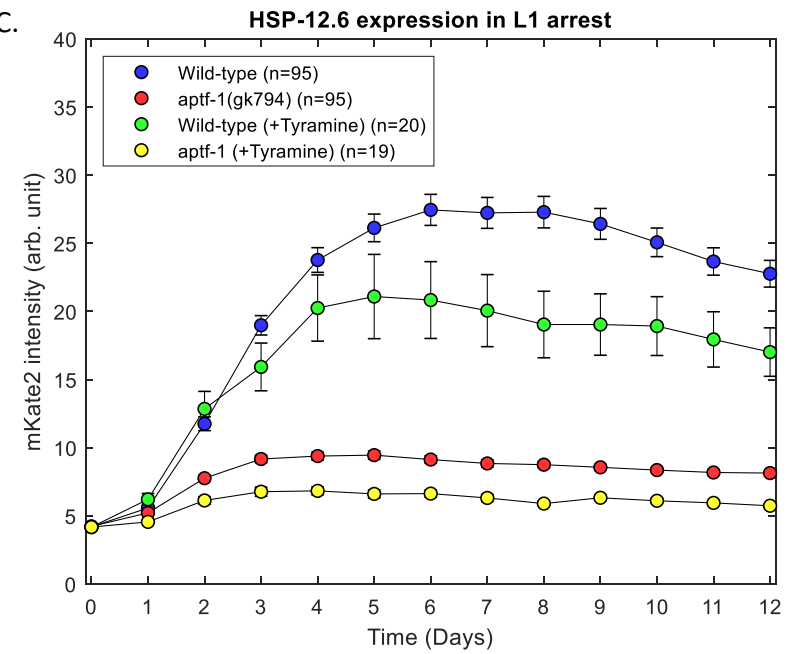

E.

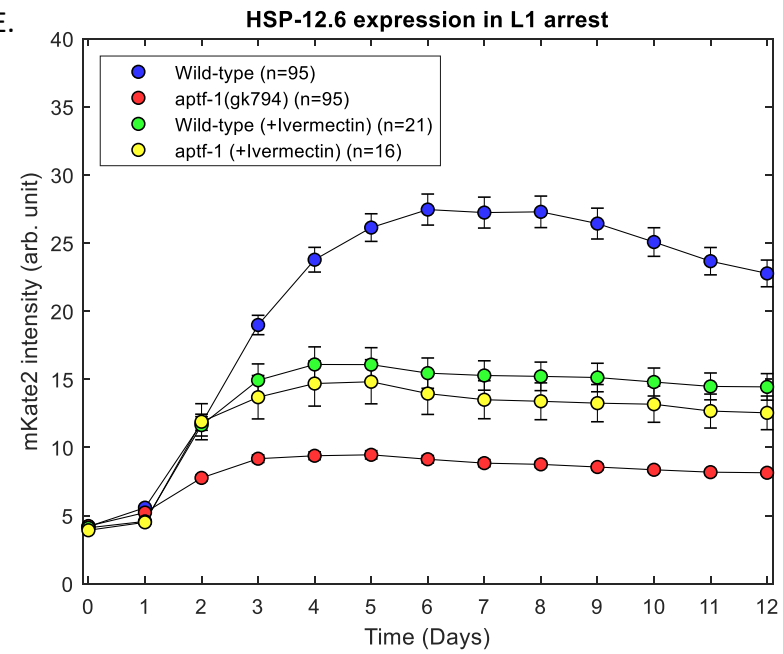

B.

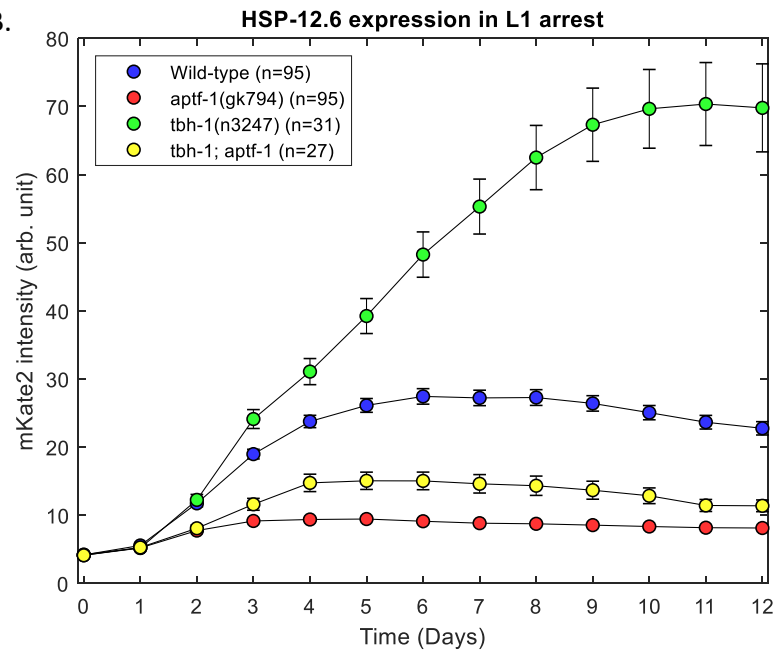

D.

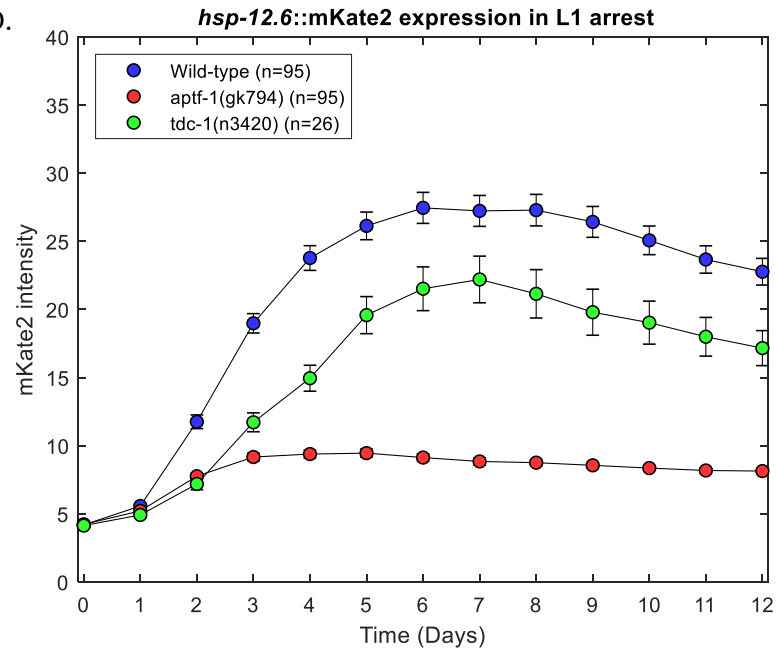

F.

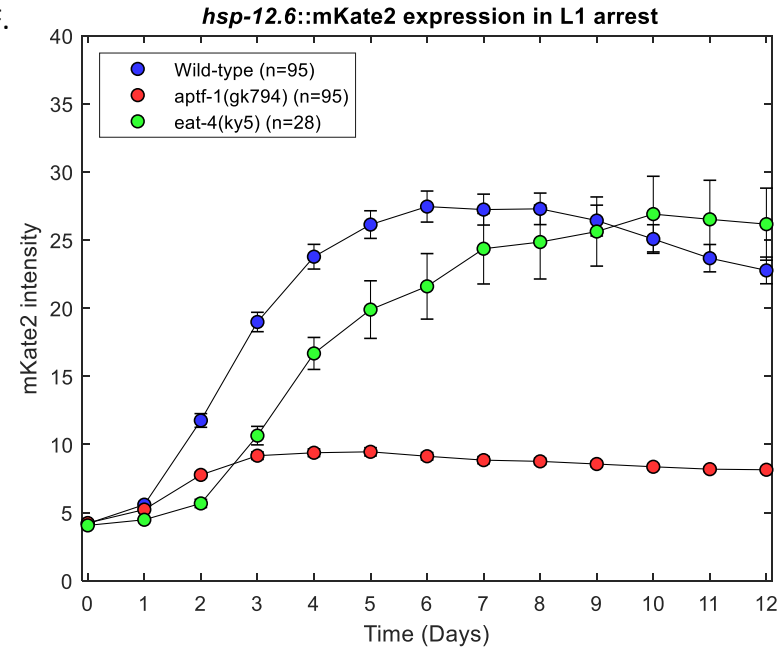

Figure 38. Octopamine, Tyramine and Glutamate affect HSP-12.6 expression

(A-B) Both loss and supplementation of Octopamine, increase the expression of HSP-12.6

(C-D) Both loss and supplementation of Tyramine decrease the expression of HSP-12.6

(E-F) Loss of glutamate vesicle transporter eat-4 does not change the expression of HSP-12.6 in the wild-type background. Glutamate receptor activation by Ivermectin results in reduced HSP-12.6 expression in the wild-type, but increased expression in aptf-1(gk794). 


\subsection{The endoplasmic reticulum unfolded protein response and the hypoxia response regulate HSP-12.6 expression in L1 arrest via aptf-1}

The connection of the transcription factor XBP-1 and sleep in L1 arrest that I established above, can be further extended by assessing HSP-12.6 expression in the $x b p-1$ loss of function condition. Indeed, the expression is decreased in the wild-type, while there is no difference in the aptf-1 loss of function background. This suggests that $x b p-1$ acts in the same pathway as aptf-1. However, there is no rescue of the aptf-1 phenotype, as was the case for the quiescence assessment (Figure 39 A). Loss of function of egl-9 which codes for a negative regulator of the hypoxia-inducible factor HIF-1 ${ }^{[82]}$, greatly increases HSP-12.6 expression in the wild-type but in the aptf- 1 loss of function mutant there is only a small increase of ambiguous biological relevance. This suggests that EGL-9 and APTF-1 act on the same pathway. egl-9 expression in enriched in RIS compared to all other neurons $\left(\log _{2}(\mathrm{FC})=1.28\right.$ and FDR $\left.=0.0061\right)$, as well as when compared to all other cells $\left(\log _{2}(\mathrm{FC})=2.66\right.$ and FDR $\left.=2.27 \times 10^{-12}\right)$ according to the dataset published by Cao et al. ${ }^{[54]}$. These results taken together with the low-quiescence phenotype of the egl-9 loss of function mutant that I discovered in the genetic screening discussed above, outline the interconnection of the hypoxia response and the starvation-induced quiescence in L1 arrest (Figure 39 B).

A.

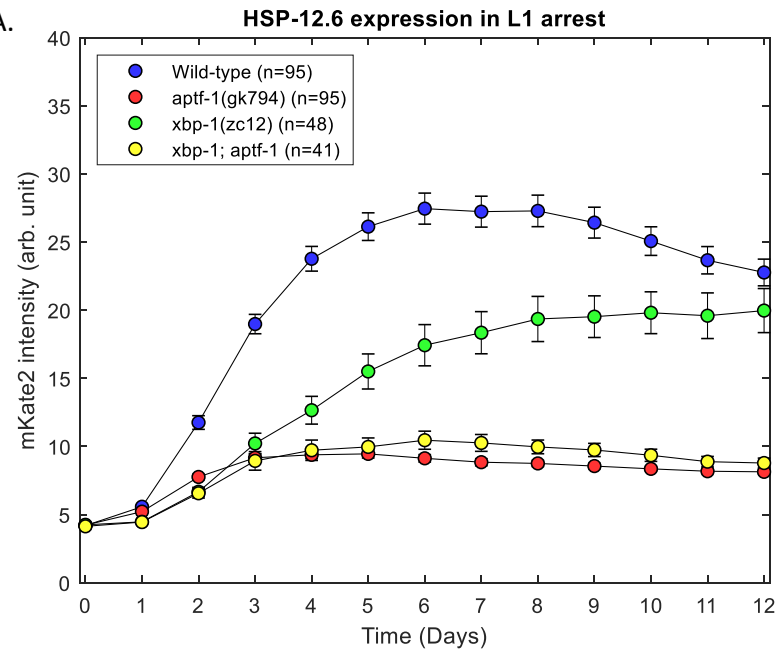

B.

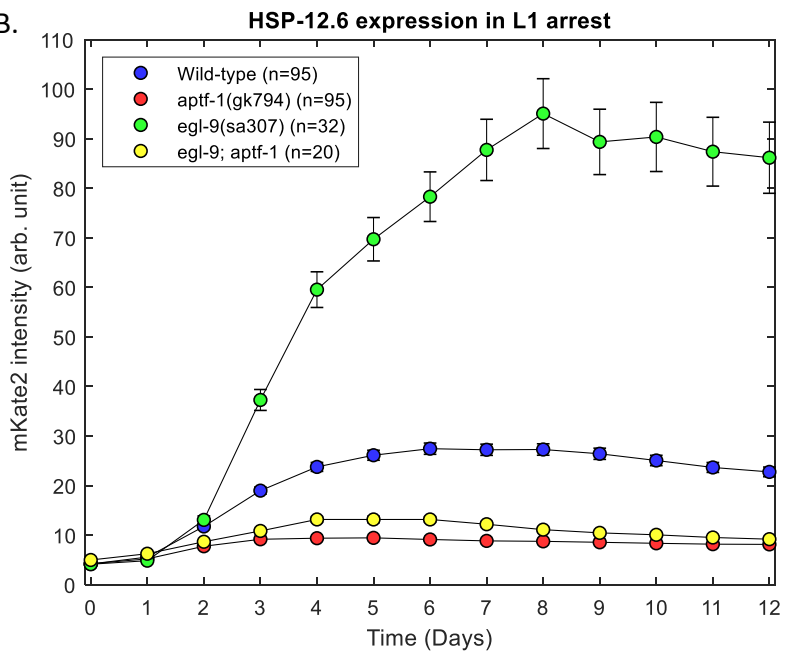

Figure 39. $x b p-1$ and egl-9 regulate HSP-12.6 expression via aptf-1

(A) $x b p-1$ (zc12) reduces the expression of HSP-12.6 in the wild-type (FDR $<0.001$ for all time points except days 0 and 1) but not in the aptf-1(gk794) (FDR $>0.05$ for all time points).

(B) egl-9(sa307) greatly increases HSP-12.6 expression in the wild type and to a much lesser extent in the aptf-1(gk794) (FDR $<0.05$ for all time points except days 0 and 1$)$. 


\subsection{Heat-shock induces the expression of HSP-12.6 regardless of aptf-1}

HSP-12.6 belongs to the family of small heat-shock proteins. Heat-shock has been shown to induce quiescence in a RIS-dependent manner, but it is the ALA neuron that conveys the protective - longevity-increasing effect. This "protective sleep" effect comes from the activation of EGF receptors that are present in both neurons ${ }^{[26]}$. Exposing L1 arrested worms to continuous heat-shock $\left(32{ }^{\circ} \mathrm{C}\right)$, I discovered that the expression does not depend on aptf- 1 . The fact that even in the aptf- 1 loss of function background, expression can be induced normally is indicative of the different nature of the heat-shock and the L1 starvation responses. However, ALA ablation by loss of function of ceh-14 does not affect the expression of HSP-12.6 during starvation in L1 arrest. This might indicate that the "protective role" that is played by ALA during heat stress of the adult worm, is played by RIS in the L1 arrest condition. Finally, gain of function of the EGF receptor homolog (let-23) increases HSP-12.6 expression only after 7 days of starvation (Figure 40).
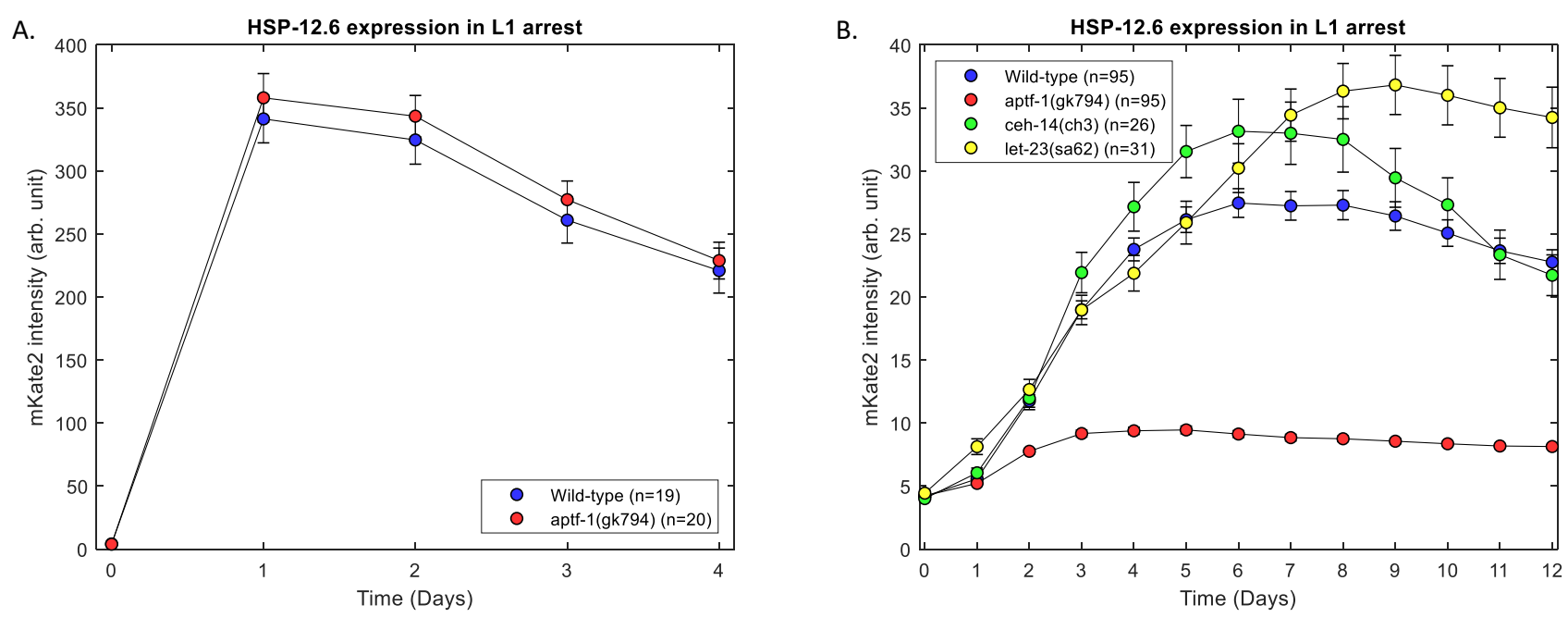

Figure 40. aptf-1 loss of function does not affect HSP-12.6 expression during heat-shock (A) Baseline measurements were taken right after hatching at $20{ }^{\circ} \mathrm{C}$. The worms were then incubated at $32{ }^{\circ} \mathrm{C}$ for 4 days. At day 3 animals started dying. By day 5 (not shown here) every animal was dead. There is no significant difference in expression between wild type and aptf1 (gk794) (FDR > 0.05 for every time point).

(B) Genetic ablation of ALA (ceh-14(ch3)), does not change HSP-12.6 expression in L1 arrest (FDR > 0.05 for all time points). Gain of function of EGF receptor (let-23(sa62)) induces a belated increase of HSP-12.6 after 7 days (FDR $<0.01$ from days 7 to 12 ). 


\subsection{0. $x b p-1$ partially rescues the survival of aptf-1 loss of function in L1 arrest}

In this study, I have used 3 different types of lifespan assays: agarose microchambers, 96-well plates with agarose and M9 and rotating Eppendorf tubes with M9. There is an assay-related variance in the results, where new phenotypes may emerge. This is the case for the aptf-1 loss of function mutant, which reproducibly shows no difference in longevity from the wild-type in the microchamber and 96-well plate assays, but shows a great reduction in the rotator assay. This is why I tried to find a condition, a genetic mutation, that rescues this phenotype. Plotting the time point of $50 \%$ survival in wild-type and aptf-1 backgrounds, has led to some potential candidates (Figure $41 \mathrm{~A})$.

The obvious candidate was the $d a f-2$ loss of function mutant, which has been shown to increase longevity ${ }^{[63]}$. Indeed, this mutation was able to rescue the aptf- 1 loss of function phenotype, but the difference between the wild-type and aptf-1(gk794) was the same in the control and the daf-2(e1370) background. In this case the rescue comes from a pathway parallel to aptf-1 (Figure $41 \mathrm{~B}$ ). With regard to $d a f-16$ and $j k k-1$ loss of function, there seems to be an epistatic relationship with aptf- 1 loss of function that leads to an even greater decrease in lifespan (Figure $41 \mathrm{C}, \mathrm{D})$. Other candidates tested such as $h s f-1, n p r l-3$, crh- 1 and rheb- 1 loss of function, did not significantly change the lifespan. However, jmjd-3.1 loss of function did show a small rescue of the aptf-1 loss of function phenotype (Figure $41 \mathrm{~F}$ ). jmjd-3.1 is a conserved histone demethylase that regulates lifespan by activating the mitochondrial unfolded protein response ${ }^{[83]}$. My colleague, Dr. Yin $\mathrm{Wu}$, found that loss of function in genes of the mitochondrial respiratory chain (isp-1 and nuo- 6 ) also rescue the aptf- 1 loss of function phenotype (these results are documented in her Ph.D. thesis titled "Mechanisms underlying the functions of sleep in aging during starvation in Caenorhabditis elegans"). These findings taken together show that disruption of important mitochondrial processes, such as the mitochondrial unfolded protein response and the respiratory chain, partially rescue the reduction in longevity of aptf-1 loss of function. Finally, $x b p-1$ loss of function is able to rescue the phenotype as well, although $x b p$ 1 in the wild-type background shows even more reduced lifespan than the aptf- 1 loss of function (Figure $41 \mathrm{E}$ ).

Survival plots for the rest of the conditions screened, can be found in section 6.35 . 
A.

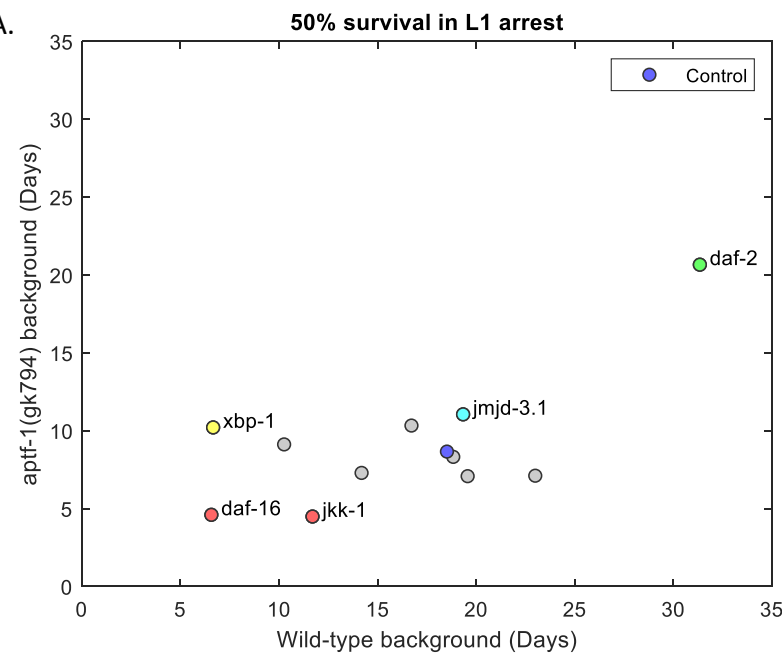

C.

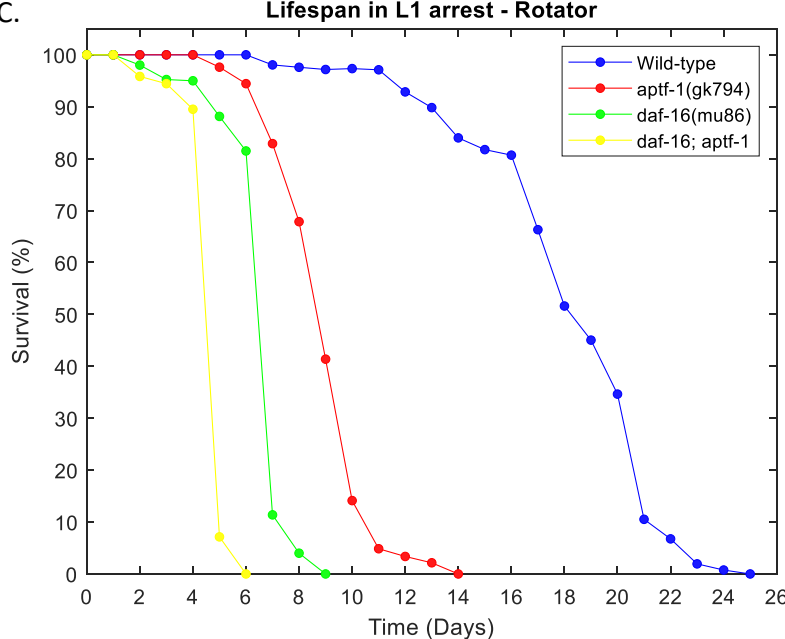

E.

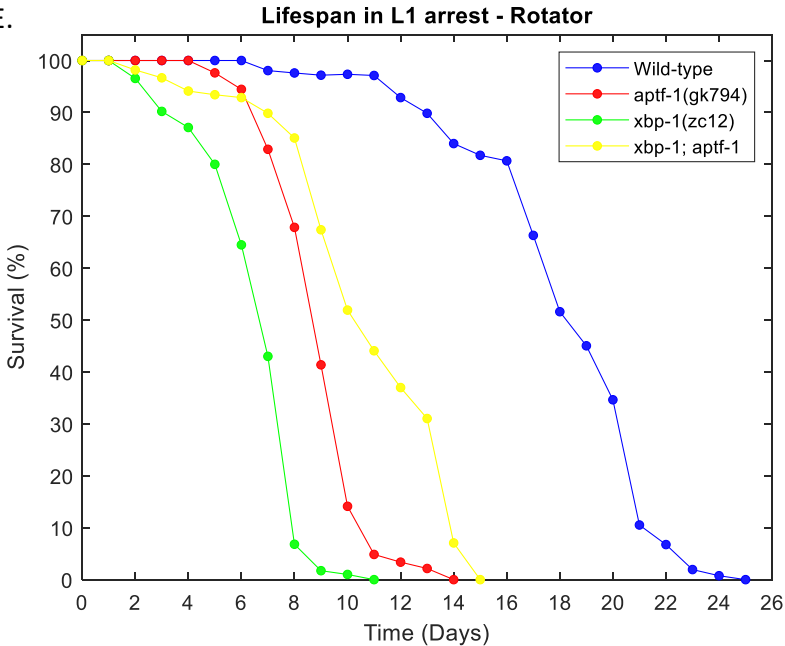

B.

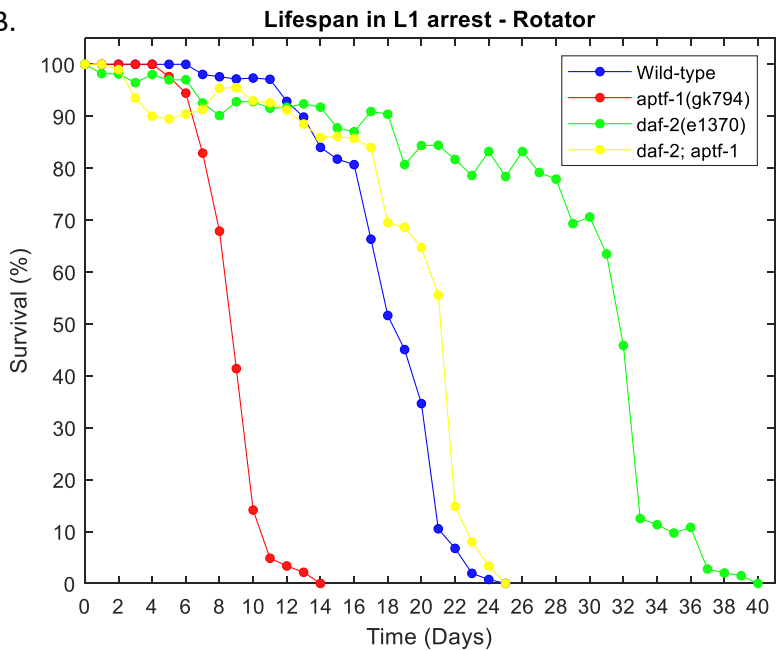

D.
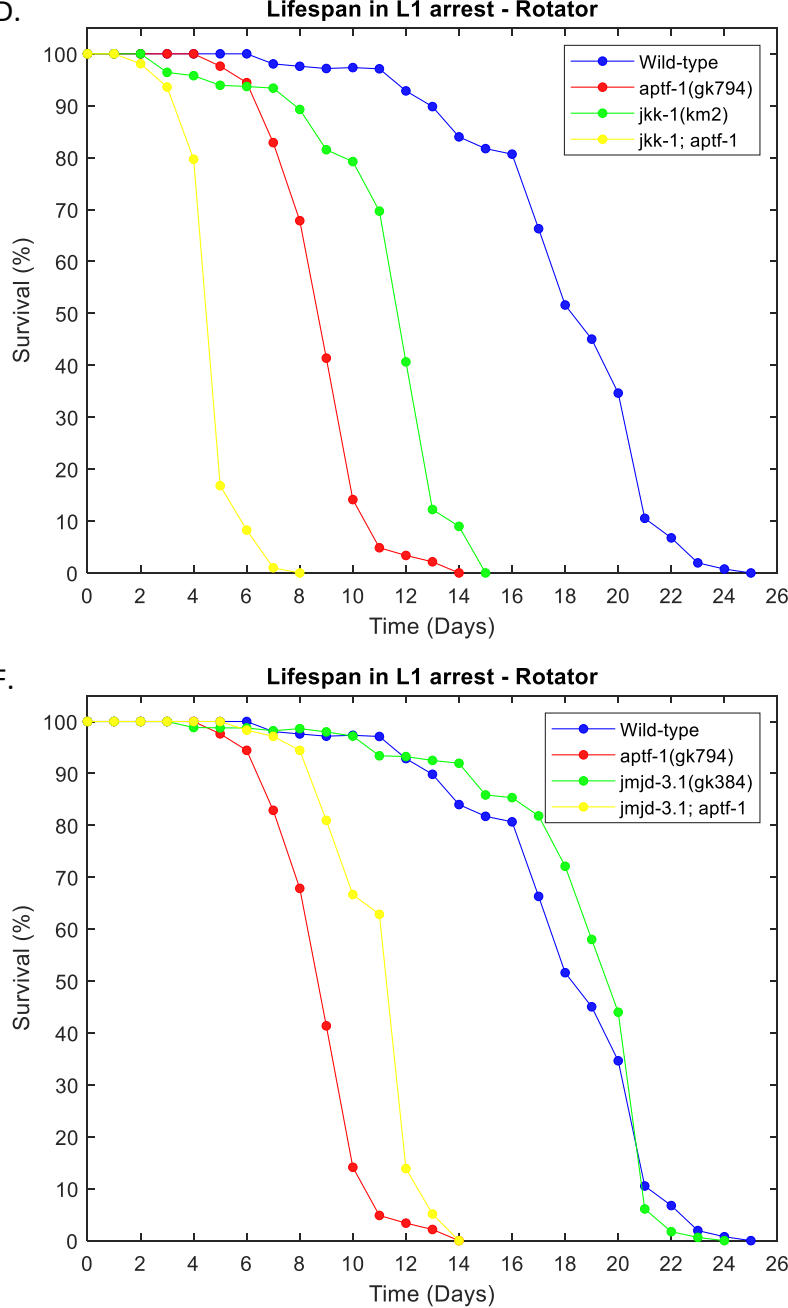

Figure 41. $x b p-1$ and $j m j d-3.1$ loss of function, partially rescue the reduced lifespan of aptf1 loss of function during $L 1$ arrest

(A) L1 arrest survival represented by the day that half the population has died. Conditions with at least $25 \%$ deviation from the control, were considered biologically significant.

(B-F) For the loss of function of $d a f-16, j k k-1, d a f-2$ and $x b p-1$, the differences in survival depicted are statistically significant, both in the wild-type and the aptf-1(gk794) mutant (FDR $<0.05$ for all days with Survival $<90 \%$ ). For jmjd-3.1 loss of function there is a significant difference only in the aptf-1(gk794) condition from day 7 to day 12 (FDR < 0.05). 


\subsection{RIS de-polarization via mechanical stimulation increases HSP-12.6 expression and lifespan, contrary to optogenetic hyper-polarization of RIS}

In order to strengthen my argument that RIS controls HSP-12.6 expression, I induced sleeploss by using the OptoGenBox for long-term optogenetic manipulation of RIS ${ }^{[49]}$. At first, I optogenetically inactivated RIS by expressing an Archaerhodopsin (ArchT) with the RISspecific $f l p-11$ promoter. This resulted in decreased HSP-12.6 expression on days 2 and 3 of L1 arrest (Figure 42 A). Then, I selectively activated the nociceptive sensory neuron ASH, by expressing a red-shifted Channelrhodopsin (ReaChR) with the sra-6 promoter, which has been shown to inactivate RIS via RIM ${ }^{[28]}$. In this case HSP-12.6 expression was also reduced on days 2 and 3 of L1 arrest (Figure 42 B). Finally, I activated the mechanosensory neurons by expressing ReaChR with the mec-4 promoter which is expressed in the body wall touch receptor neurons ALM, AVM, PLM and PVM, as well as the nose tip FLP neurons ${ }^{[84]}$. Their activation has been shown to cause RIS inactivation, probably via engagement of the PVC neuron ${ }^{[24]}$. However, this resulted in increase of HSP-12.6 expression on days 2, 3 and 4 of L1 arrest (Figure $42 \mathrm{C}$ ). Although ASH and the rest of the mechanosensory neurons are glutamatergic, the difference in the results may come from the fact that they engage different neurons presynaptic to RIS.

To complement these experiments, I sleep-deprived the worms by applying a noxious mechanical stimulus continuously over their lifetime. In this case, sleep-deprivation has been shown to be a result of RIS de-polarization via PVC activation ${ }^{[28]}$. This led to an increase in the expression of HSP-12.6 only in the wild-type background, while there was no difference in the aptf- 1 loss of function (Figure 43 A). Similarly, lifespan was extended only in the wild-type background after mechanical stimulation (Figure $43 \mathrm{~B}$ ). This is indicative of the importance of a functioning RIS neuron for protein expression and lifespan in L1 arrest. However, I was not able to get similar phenotypes from the unfolded protein response markers ( $h s p-4$ downstream of $x b p-1$ in the endoplasmic reticulum and $h s p-6$ downstream of jmjd-3.1 in the mitochondria). This might be because these 2 genes are heat-inducible genes that are not directly controlled by sleep (Figure 43 C, D). 
A.

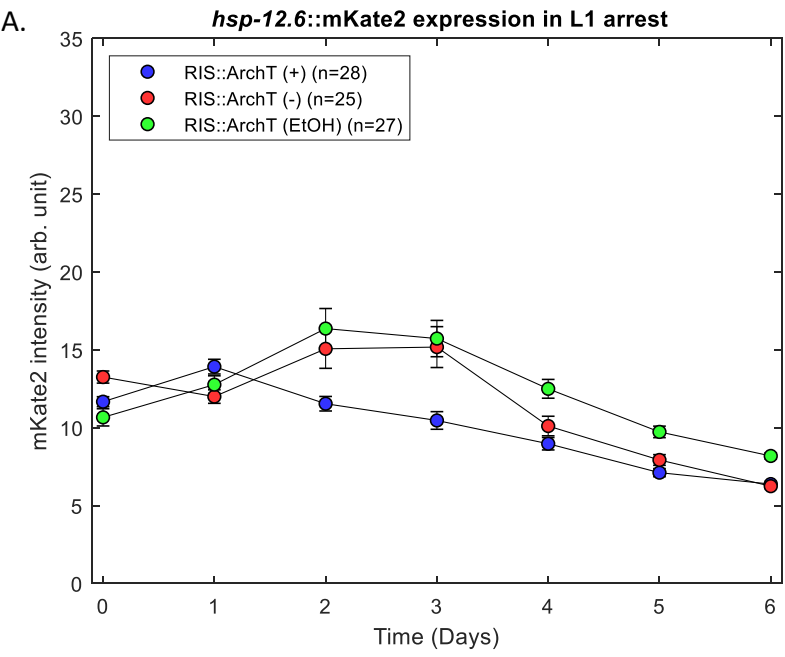

B.

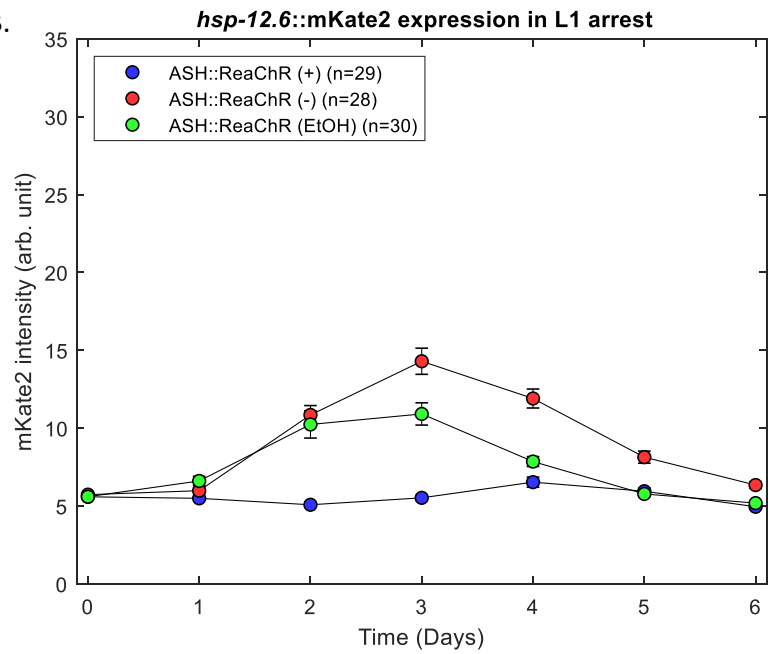

C.

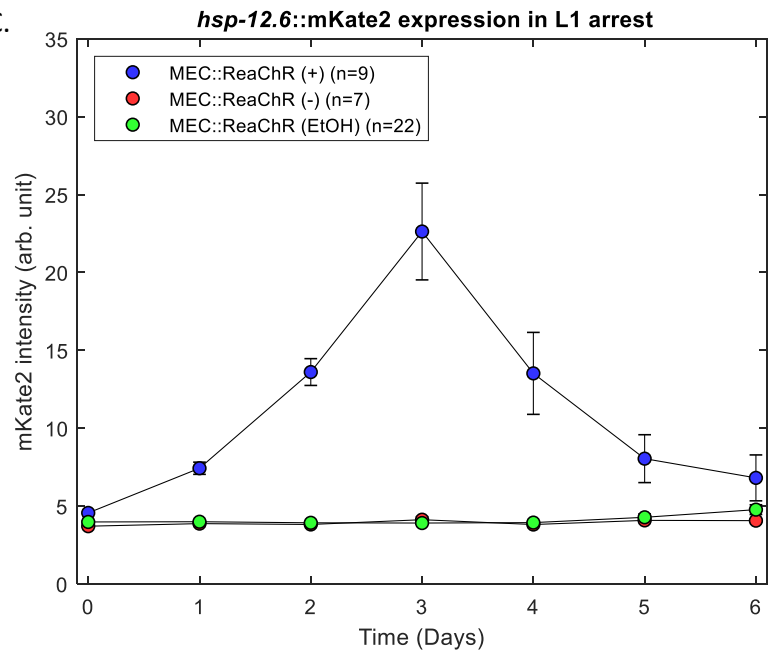

Figure 42. Optogenetic inactivation of RIS reduces HSP-12.6 expression

(A) Continuous RIS hyper-polarization of RIS results in reduced HSP-12.6 expression on days 2 and 3 (FDR < 0.05).

(B) Continuous ASH de-polarization results in reduced HSP-12.6 expression on days 2 and 3 (FDR $<0.05$ ).

(C) Continuous mechanosensory neuron de-polarization results in increased HSP-12.6 expression on days 2,3 and 4 (FDR < 0.05 ).

In all cases samples have been treated as follows: $(+)=$ Retinal, $(-)=$ Water, $($ EtOH $)=$ Ethanol 
A.

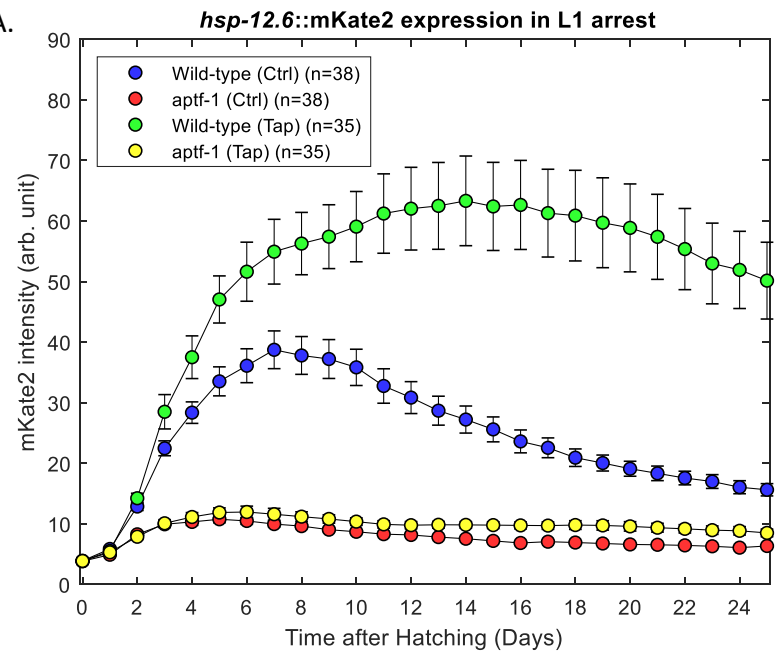

c

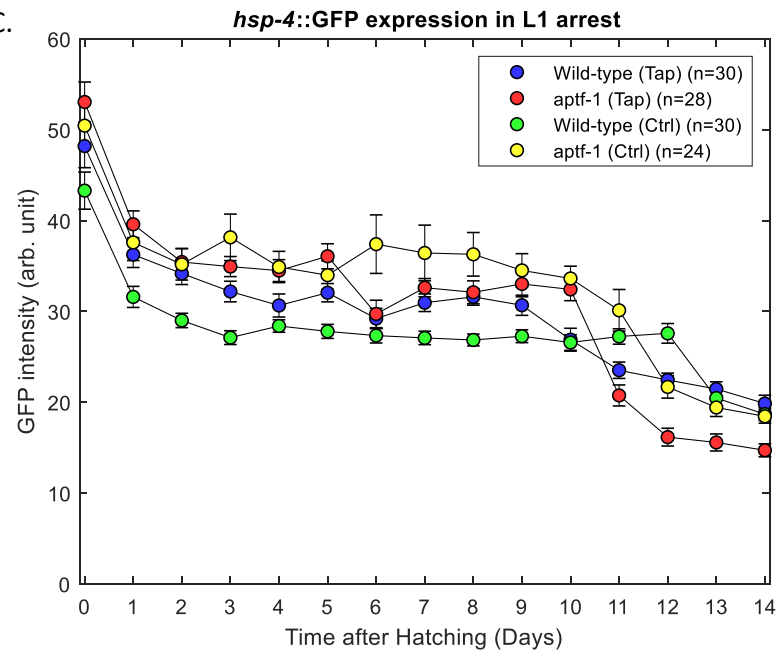

B.

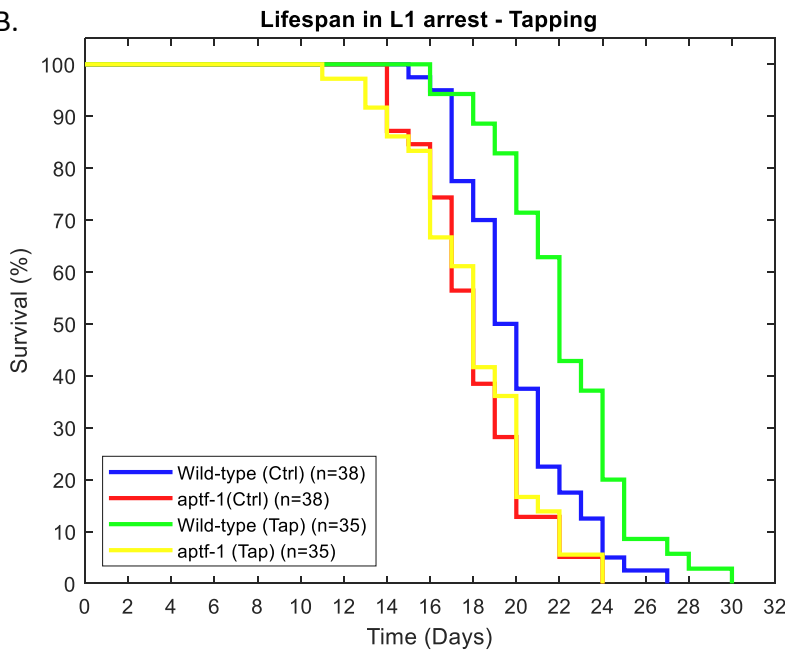

D.

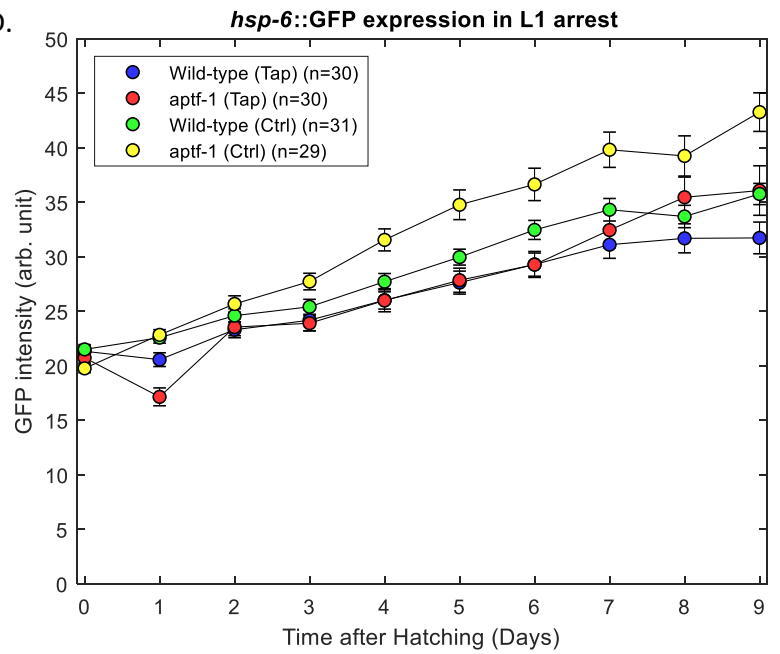

Figure 43. Mechanical stimulation increases HSP-12.6 expression and lifespan in an RISdependent way

(A) Sleep deprivation by mechanical stimulation increases HSP-12.6 expression in the wildtype (FDR < 0.01 from day 4 to day 24 ) but not in the aptf- 1 (gk794) background. The small increase in the expression of aptf-1(gk794) when it is mechanically stimulated does not correspond to HSP-12.6 expression, but rather comes from stress-induced intestinal vesicles.

(B) The lifespan of mechanically stimulated wild-type worms is significantly increased (FDR $=0.0028)$, in the aptf-1 (gk794) background it is unchanged $(\mathrm{FDR}=0.8151)$.

(C) $h s p-4$ expression is upregulated in aptf-1 (gk794) (FDR $<0.05$ from day 2 to day 10). Mechanical stimulation eliminates that difference by upregulating the wild-type condition.

(D) hsp-6 expression is upregulated in the aptf-1(gk794) (FDR $<0.05$ from day 4 to day 9). Mechanical stimulation does not affect the wild-type (FDR $>0.05$ for every time point) but rather decreases the expression of aptf-1(gk794) to wild-type levels. 


\section{Discussion and perspectives}

Sleep in C. elegans is traditionally connected to development ${ }^{[23]}$. Studying sleep in the L1 arrested state, uncouples it from worm development and provides the ground for discovery of those components that found their way to higher organisms through evolution. However, when this project started in late 2016, L1 arrest was only characterized as a state of developmental arrest in which quiescent behavior could be observed ${ }^{[31,85]}$. In 2018, Wu et al. showed that this quiescent behavior did in fact fulfill all the criteria to be characterized as sleep. This sleep state is controlled by the sleep-active neuron RIS, in a manner that involves the energy sensor AMPK and the FOXO homolog daf-16. Loss of sleep in L1 arrest results in shorter lifespan ${ }^{[24]}$. The work presented here aims to build on these observations and expand our understanding of sleep by utilizing omics approaches and other complementary assays.

\subsection{Sleep loss induces global metabolic changes in C. elegans}

\subsubsection{Dysregulation of the starvation response}

Sleep has been associated with metabolism in various instances. Changes in animal dietary habits, be it fasting or an eating disorder, impact the quality of sleep by affecting sleep architecture and melatonin levels ${ }^{[86,87,88,89]}$. When $C$. elegans hatches in the presence of food, L1 wild-type animals execute a developmental program that allows them to grow to adulthood, as long as there is an available nutrient source. If they hatch in a food-deprived environment, they enter L1 arrest and employ a series of metabolic changes to survive the fasting conditions. These manifest as changes (up- and down- regulation) in gene and protein expression and constitute the starvation response. In this thesis, I present evidence that lack of sleep hinders the proper execution of the starvation response. I have used the aptf- 1 loss of function and the RIS-ablated $f l p-11$ ::EGL-1 strains that show significantly reduced sleep fraction in L1 arrest to get a transcriptomic and proteomic profile of the starvation response. In both cases, the starvation response is compromised. In fact, a reversal of gene and protein expression takes place. Components that are normally upregulated in the wild type are downregulated in the sleepless animals and vice versa. This is the first piece of evidence towards my aim to understand how sleep affects metabolism. 


\subsubsection{Increase of the basal metabolic rate}

In any biological system, heat production is a measure of energy metabolism ${ }^{[90]}$. Sleep is characterized by an estimated $15 \%$ reduction in metabolic rate ${ }^{[91]}$. In order to discover differences in metabolism among the wild type and the 2 sleepless strains, I assessed their heat production over time by isothermal microcalorimetry. Indeed, sleeping worms produced less heat than the sleepless ones. After correcting for the amount of heat produced from movement during swimming (active metabolic rate) ${ }^{[92]}$, there is a $20 \%$ heat production that corresponds to the increased basal metabolic rate of sleepless animals. This calculation is consistent with the $15 \%$ reported in the literature.

\subsubsection{Increase in egg laying during adult starvation}

Sleep loss during pregnancy, has been shown to affect both the mother and the offspring, causing structural and metabolic changes ${ }^{[93,94,95]}$. Although in adult $C$. elegans the amount of sleep depends on food intake ${ }^{[24]}$, genetic ablation of sleep might interfere with offspring production. In well-fed animals the number of eggs laid, is invariant to sleep status. However, in starved adults the egg-laying capacity increases in sleep-deprived worms. The role of sleep loss during adult starvation, can be interpreted either as misallocation of continuously diminishing resources or as the last resort of a desperate animal to pass on its genes. Further experiments in this direction should assess egg viability as well as the percentage of hatched larvae that become fertile adults.

\subsubsection{Decrease in body size}

In the animal kingdom, poor sleep habits correlate negatively with development ${ }^{[96]}$. C. elegans size is regulated by the TGF- $\beta$ pathway in the hypodermis ${ }^{[58]}$. Considering the limited resources available to an L1-arrested worm, it was logical to ask whether there would be a developmental defect in sleepless animals. This was actually the case for the aptf- 1 loss of function strain, which shows a 5\% reduction in body size compared to the wild type, starting from the time point of hatching. Since APTF-1 expression is highest during late embryogenesis ${ }^{[27]}$, reduction of body size could be a developmental and not a sleep-related defect. Maternal defects in resource allocation in the aptf- 1 loss of function strain, could lead to the creation of egg yolks 
with lower nutritional value, that in turn impact size. Finally, it could also be the result of embryonic sleep ablation, although this would be very difficult to assess with mobility criteria.

\subsubsection{Increase in sleep-inducing metabolites}

In order to acquire an overview of the metabolic status of the sleepless worms, I performed a metabolome analysis at 48 hours and 6 days of starvation. In the aptf-1 loss of function strain, Tryptophan is upregulated at 48 hours, in accordance with the sleep deprivation literature for humans ${ }^{[36]}$. Tryptophan is an essential amino acid that can be metabolized to Serotonin, which in turn can be metabolized to Melatonin. All of these components have known roles in sleep induction and maintenance ${ }^{[97]}$. However, Serotonin was unchanged, and Melatonin was not detected in the metabolome analysis. Tryptophan can instead be converted into the neuroactive metabolite Kynurenic acid, which antagonizes the excitatory glutamatergic neurotransmission in the central nervous system. Sleep deprivation has been shown to increase the levels of Kynurenic acid in the hypothalamus and hippocampus of rat brains ${ }^{[98]}$. In C. elegans, Kynurenic acid is produced in the RIM neuron and is regulated by insulin signaling to control aspects of aging ${ }^{[76]}$. After 6 days of starvation, levels of Kynurenic acid increase. Taken together, these results show that the sleepless aptf-1 loss of function strain tries to induce sleep by upregulating somnogenic components and reducing excitatory neurotransmission. Ultimately this fails, probably because the sleep-inducing signal eventually has to go through RIS, which is not functional due to the aptf- 1 loss of function.

\subsubsection{Increase of oxidative stress and decrease of life-prolonging antioxidant factors}

Recent research has shown that sleep deprivation in rats and Drosophila, causes accumulation of ROS in the gut, which kills the organism prematurely and is reversed by administration of antioxidants ${ }^{[99]}$. Metabolomic analysis of the aptf- 1 loss of function strain, shows that there is upregulation of Allantoin and Glutathione (but not Oxidized glutathione which remains unchanged) at the 48-hour time point. Allantoin can only be produced as a result of oxidative stress in C. elegans ${ }^{[75]}$. Glutathione is a free radical scavenger and the ratio of Glutathione/Oxidized glutathione is under strict homeostatic control ${ }^{[100]}$. This suggests that sleepless worms are under severe oxidative stress. Also, AMP is upregulated, which increases the AMP/ATP ratio. This puts the whole organism in an energy deficit state and in turn activates the AMPK, which engages the catabolism of fatty acids via beta oxidation to produce more 
energy ${ }^{[101]}$. This process happens in the mitochondria and results in production of ROS ${ }^{[102]}$. However, transcription of antioxidant genes such as sod-3 is downregulated. This might explain the significantly reduced lifespan of the aptf- 1 loss of function strain in L1 arrest. Moreover, transcriptome analysis indicates that mitochondria are not functioning properly, because of extreme downregulation of the associated genes, especially those that are involved in energy production by oxidative phosphorylation. The link between fatty acid beta oxidation and oxidative phosphorylation is the TCA cycle, which is allosterically regulated by its metabolites. Upregulation of Succinic acid, which lies on the interface of the TCA cycle and complex III of the electron transport chain, may indicate the critical point of imbalance between energy and ROS production. Considering that AMPK activation can activate RIS ${ }^{[24]}$, we end up with a strong link of energy status, sleep and longevity.

\subsubsection{Increase of the unfolded protein response and dysregulation of proteostasis}

Sleep can be separated in two distinct components. The first one is the circadian component, in which light availability regulates the levels of melatonin to induce sleep and wakefulness ${ }^{[14,15]}$. In C. elegans, there is no circadian regulation of sleep in the same way it is for other animals, but it has been shown that lethargus intervals correlate with expression of PERIOD homolog lin-42 ${ }^{[103]}$. The second is the homeostatic component, in which cellular needs dictate molecule anabolism and catabolism. Protein metabolism is dynamically regulated at the levels of translation, folding, modification, localization and degradation, a process termed proteostasis. At the heart of proteostasis lies the unfolded protein response (UPR). UPR activation upregulates the expression of chaperone proteins e.g. BiP (homologous to C. elegans hsp-4), downregulates translation and increases the degradation of misfolded proteins. Sleep deprivation activates the UPR, while it has been shown that, in Drosophila, recovery sleep reduces BiP levels ${ }^{[104]}$.

My results indicate that in the aptf-1 loss of function strain, there is downregulation of ribosomal components at the transcript level, while in the meantime the essential amino acid and translation initiation component Methionine is also downregulated. Additionally, thiolation of Glutamine-tRNA wobble $\mathrm{U}_{34}$ is increased, decreasing the rate of translation of glutaminerich proteins ${ }^{[73]}$. These findings consent to protein regulation at the level of translation, consistent with downregulation due to activated UPR. 
Moreover, I observed increased expression of the BiP homolog $h s p-4$ at the transcript level in the RNA-seq and in the transgenic assay experiments in the aptf- 1 loss of function strain. Mechanical stimulation of the worm leads to activation of RIS ${ }^{[28]}$, which upregulates BiP in the wild type, but not in the aptf-1 loss of function. This result not only agrees with the Drosophila literature ${ }^{[104]}$, but indicates that RIS activity rather than sleep is responsible for the proper execution of the UPR. Furthermore, loss of function of the UPR-regulating transcription factor XBP1 (C. elegans homolog $x b p-1)$, partially rescues the behavioral quiescence and survival of the aptf-1 loss of function strain. Perhaps in this case, the downregulation of UPR offers temporary relief to the sleepless strain, mimicking the UPR downregulation of recovery sleep, which results in short-term survival rescue that cannot be maintained due to prolonged starvation.

In order to acquire a complete image of proteostasis, protein modifications, translocation and degradation need to be investigated further. Although glutathionylation is a well-known modification and the increased Glutathione observed in the aptf- 1 loss of function strain could affect protein homeostasis, it is not enough by itself to make safe conclusions.

\subsection{Changes in metabolism result in changes in sleep}

There is a two-way relationship between sleep and metabolism ${ }^{[86,87,88,89]}$ and I have already shown that impaired sleep alters the starvation response. In order to establish this connection in C. elegans, I performed an extensive genetic screening of strains with metabolic defects. Knock-down of components of the sphingolipid metabolism pathway lead to reduced sleep in L1 arrest. Sphingolipids are a class of metabolites that are massively downregulated in the aptf1 loss of function strain. Since sphingolipids can have both structural and signaling biological roles, further experiments need to be made in that direction. Ceramide, which is at the center of sphingolipid metabolism, plays a role apoptosis ${ }^{[105]}$. Loss of function of ced-3, a homolog of mammalian caspases, results in decreased sleep. Its role needs to be addressed in the context of RIS activation. Furthermore, I showed that loss of function of the C. elegans homolog of DKG $\theta$, $d g k-1$, results in almost complete loss of sleep and changes RIS physiology. DGK $\theta$ is a neuronal diacylglycerol kinase that is part of sphingolipid metabolism and synaptic vesicle trafficking by regulating DAG phosphorylation ${ }^{[78,106]}$. Finally, apart from the role of sphingolipids, the hypoxia response should be addressed in the context of sleep and RIS function since, loss of function of components in this pathway leads to reduced quiescence. 


\subsection{Sleep loss induces RIS-dependent tissue-wide changes}

The case of the sleepless $f l p-11$ ::EGL-1 strain is a peculiar one. This strain was originally created to induce apoptosis specifically in RIS. In the beginning it has been behaving as expected with decreased quiescence and lifespan. After it has been through some generations, the strain lost its survival phenotype. RIS was still visible and calcium imaging could not produce results. Since apoptosis is a process that involves calcium influx, we hypothesized that this strain is instead RIS-hyperactive and potentially upregulates the secretion of a neurotransmitter that is beneficial for survival, uncoupling it from sleep (personal communication with Prof. Henrik Bringmann). My datasets advocate for this hypothesis, since its body size is wild-type and its gene expression profile is very distinct both from the wild type and the aptf-1 loss of function strains. Our interest in studying the benefits of sleep separate from sleep behavior, led to the creation of the flp-11::UNC-58(gf) strain which was engineered to have a constantly active RIS neuron.

With regard to specific tissues both aptf-1 loss of function and $f l p-11::$ EGL-1 upregulate hypodermis-specific genes and downregulate intestine-specific genes. Muscle-specific genes are downregulated only in the RIS-hyperactive strain. Germline-specific genes and Notch signaling are downregulated in the aptf-1 loss of function strain, while they are upregulated in $f l p-11::$ EGL-1. Contrary to that, neuronal-specific gene expression is upregulated in the aptf-1 loss of function strain and downregulated in the RIS-hyperactive strain. While this is a very useful summary of RIS-dependent tissue-wide changes, research needs to focus on individual pathways. One such pathway involves the $\mathrm{GABA}_{\mathrm{A}}(\mathrm{UNC}-49)$ and $\mathrm{GABA}_{\mathrm{B}}$ (GBB-1, GBB-2) receptors which are upregulated in the aptf- 1 loss of function strain and downregulated in the RIS-hyperactive strain. This result taken together with the downregulation of glutamate receptors and Kynurenic acid in the RIS-hyperactive strain show an impact of RIS activity on excitatory glutamatergic signaling. One interesting perspective would be to research whether RIS activity controls the GABA shunt, which connects the GABAergic and glutamatergic signaling with the TCA cycle ${ }^{[107]}$. Finally, the relevance of the tyraminergic, peptidergic and glutamatergic neuron RIM ${ }^{[28]}$ should be further assessed since its activity levels are higher and tyrosine decarboxylase, $t d c-1$, loss of function enhances the reduction of the sleep phenotype in the aptf- 1 loss of function strain. Kynurenic acid production in RIM has been shown to affect lifespan ${ }^{[76]}$ and RIM can both inhibit and activate RIS ${ }^{[28]}$. 


\subsection{RIS controls the nuclear localization of FOXO homolog daf-16}

The most important finding of this thesis is the regulation of the FOXO transcription factor homolog daf-16 by RIS. Although RNA-seq data and reporter transgene expression experiments show that $d a f-16$ is upregulated in the aptf- 1 loss of function strain, this did not correlate well with the downregulation of the classic DAF-16 targets $h s p-12.6$ and sod-3. These genes are categorized as Class I because they were found to be downregulated in daf-16 loss of function conditions ${ }^{[62]}$. Therefore, since $d a f-16$ is upregulated in the aptf-1 loss of function background, the downstream targets should also be upregulated which is not the case. For this reason, I checked DAF-16 nuclear localization and found that it was decreased in the intestine and muscles of the aptf-1 loss of function strain. However, it is also decreased in the intestine of the RIS-hyperactive $f l p-11:: \mathrm{UNC}-58$ (gf) strain, which indicates that either the intestine is not the target tissue of RIS or that a normally functioning RIS is needed for proper nuclear DAF16 translocation. This needs to be addressed further as well as the localization of DAF-16 in neurons, also because of the fact that $d a f-16$ loss of function and insulin receptor homolog $d a f$ 2 loss of function produce strongly reduced and increased sleep phenotypes respectively.

Since the results of the DAF-16 localization assay are probabilistic in nature and can be subject to researcher bias, I used a fluorescent reporter fused to the endogenous locus of $h s p-12.6$, to track HSP-12.6 expression. This was used as an indirect, but more trustworthy measure of DAF16 activity. RIS ablation (aptf-1 loss of function and flp-11::TWK-18) downregulates the expression of HSP-12.6 while RIS hyperactivation upregulates its expression. Furthermore, mechanical stimulation, which was shown to activate RIS ${ }^{[28]}$, increased the expression of HSP12.6 and the lifespan in the wild type but not the aptf-1 background, proving that RIS hyperactivation can convey benefits to the animal regardless of sleep status. The regulation of HSP-12.6 is very complicated, since I showed that it is also regulated by cholinergic, tyraminergic, octopaminergic and glutamatergic stimuli. In my ordeal to find the regulatory pathway of RIS-controlled DAF-16 nuclear localization, I discovered that all the classical pathways described in the literature ${ }^{[63,81]}$, function upstream or in parallel to RIS. This means that there is a non-classical or not-identified pathway that controls DAF-16 localization downstream of RIS. As far as the current literature goes, there are only two promising candidate pathways: 1. GABA receptor signaling regulated by PKD ${ }^{[108]}$ and 2. Calmodulin/CAMKII signaling ${ }^{[109]}$. These pathways might eventually converge. 


\section{Appendix}

\subsection{Strain list}

\begin{tabular}{|c|c|c|c|}
\hline Strain & Description & Strain & Description \\
\hline $\mathrm{N} 2$ & Wild-type & DA472 & pha-2(ad472) X \\
\hline CB1189 & unc-51(e1189) V & DA521 & egl-4(ad450) IV \\
\hline CB189 & unc-32(e189) III & DA609 & npr-1(ad609) X \\
\hline CB369 & unc-51(e369) V & DG1867 & glp-1(tn777) III. \\
\hline EU552 & glp-1(or178) III & DG2389 & glp-1(bn18) III \\
\hline FX02586 & ptr-10(tm2586) & DR432 & ama-1(m118) IV \\
\hline FX04545 & tatn-1(tm4545) & EG9631 & unc-13(s69) I \\
\hline FX06853 & B0303.3(tm6853) & EW15 & bar-1(ga80) X \\
\hline FX07117 & rpia-1(tm7117) & FX00841 & cka-2(tm841) \\
\hline GG60 & glp-1(g60) III & FX00903 & dat-1(tm903) \\
\hline RB1950 & F38A6.3(ok2564) V & FX01035 & F47A4.5(tm1035) \\
\hline RB2071 & ced-3(ok2734) IV & FX01045 & acl-10(tm1045) \\
\hline RB2602 & F01G10.1(ok3624) IV & FX01097 & F20B4.6(tm1097) \\
\hline RB789 & tre-2(ok575) IV & FX01420 & C34B2.7(tm1420) \\
\hline VC2428 & sams-1(ok2946) X & FX01472 & asg-2(tm1472) \\
\hline VC2520 & aagr-2(ok3193) II & FX01584 & ipla-3(tm1584) \\
\hline VC410 & elo-5(gk208) IV & FX01655 & C54G7.2(tm1655) \\
\hline EC106 & $\begin{array}{l}\text { eeEx106[hil-1::GFP + } \\
\text { rol-6(su1006)] }\end{array}$ & FX01774 & C31C9.2(tm1774) \\
\hline AX1410 & flp-18(db99) X & FX01778 & acl-14(tm1778) \\
\hline BC119 & blmp-1(s71) I & FX01847 & acl-13(tm1847) \\
\hline BX106 & fat-6(tm331) IV & FX01929 & Y51H7C.9(tm1929) \\
\hline BX107 & fat-5(tm420) V & FX01936 & cdo-1(tm1936) \\
\hline BX14 & elo-1(wa7) IV & FX01989 & F18E3.7(tm1989) \\
\hline BX153 & fat-7(wa36) V & FX02028 & $\mathrm{F} 20 \mathrm{H} 11.5(\mathrm{tm} 2028)$ \\
\hline BX17 & fat-4(wa14) IV & FX02178 & T27F6.6(tm2178) \\
\hline $\mathrm{BX} 24$ & fat-1(wa9) IV & FX02311 & T01C8.4(tm2311) \\
\hline $\mathrm{BX} 26$ & fat-2(wa17) IV & FX02369 & $\mathrm{C} 46 \mathrm{C} 11.1(\mathrm{tm} 2369)$ \\
\hline $\mathrm{BX} 30$ & fat-3(wa22) IV & FX02390 & fce-1(tm2390) \\
\hline CB1111 & cat-1(e1111)X & FX02598 & fce-2(tm2598) \\
\hline CB1112 & cat-2(e1112)II & FX02680 & Y46G5A.24(tm2680) \\
\hline CB1370 & daf-2(e1370)III & FX02689 & acdh-2(tm2689) \\
\hline CB156 & unc-25(e156) III & FX02898 & prdx-6(tm2898) \\
\hline CB4876 & clk-1(e2519) III & FX03142 & $\operatorname{acl}-2(\operatorname{tm} 3142)$ \\
\hline CB5602 & vhl-1(ok161) X & FX03289 & acl-1(tm3289) \\
\hline CB6037 & bus-18(e2795) V & FX03314 & Y4C6B.6(tm3314) \\
\hline
\end{tabular}




\begin{tabular}{|c|c|c|c|}
\hline Strain & Description & Strain & Description \\
\hline CB6088 & egl-9(sa307) hif-1(ia4) V & FX03340 & $\operatorname{acl}-2(\operatorname{tm} 3340)$ \\
\hline CB6090 & $\begin{array}{c}\text { hif-1(ia4) V; vhl-1(ok161) } \\
\text { X }\end{array}$ & FX03349 & Y4C6B.6(tm3349) \\
\hline CE1255 & cep-1(ep347) I & FX03396 & acl-6(tm3396) \\
\hline CE541 & sbp-1(ep79) III & FX03424 & C14E2.2(tm3424) \\
\hline CE833 & sbp-1(ep176) III & FX03557 & mboa-4(tm3557) \\
\hline CF1038 & daf-16(mu86) I & FX03673 & Y69A2AR.5(tm3673) \\
\hline CF2218 & ncl-1(e1942) III & FX03788 & pyc-1(tm3788) \\
\hline CF376 & bar-1(mu63) X & FX03816 & $\mathrm{C} 33 \mathrm{C} 12.3(\mathrm{tm} 3816)$ \\
\hline CU2945 & scrm-1(tm805) I & FX04172 & $\mathrm{F} 14 \mathrm{~B} 4.2(\mathrm{tm} 4172)$ \\
\hline CW152 & gas-1(fc21) X & FX04174 & F14B4.2(tm4174) \\
\hline CZ3391 & vab-3(ju468) X & FX04284 & $\operatorname{prdx}-6(\operatorname{tm} 4284)$ \\
\hline FX04387 & $\mathrm{H} 12 \mathrm{D} 21.7(\mathrm{tm} 4387)$ & HBR02118 & F53H8.4(tm2613) \\
\hline FX04853 & acs-19(tm4853) & HBR02120 & maoc-1(tm5257) \\
\hline FX05123 & $\mathrm{B} 0365.1(\mathrm{tm} 5123)$ & HBR02122 & dgk-1(sy428) X \\
\hline FX05127 & W02H5.8(tm5127) & HBR02214 & metr-1(ok521) II \\
\hline FX05430 & R11F4.1(tm5430) & HBR02233 & $\begin{array}{l}\text { aptf-1(gk794); tut-1 } \\
\text { (tm1297) }\end{array}$ \\
\hline FX05491 & K07B1.4(tm5491) & HBR02121 & mes-2(tm5007) \\
\hline FX05954 & cpin-1(tm5954) & HBR02237 & vha-2(ok619) III \\
\hline FX06151 & dhs-3(tm6151) & HBR02242 & $\begin{array}{c}\text { elpc-1(tm2149); aptf- } \\
\text { 1(gk794) II }\end{array}$ \\
\hline FX06393 & C27A7.3(tm6393) & HBR02269 & $\begin{array}{l}\text { flp-11(tm2706) X; tdc- } \\
\text { 1(n3420) II }\end{array}$ \\
\hline FX06496 & $\operatorname{glna}-1(\operatorname{tm} 6496)$ & HBR00227 & aptf-1(gk794) II \\
\hline FX06644 & set-23(tm6644) & HBR02280 & sms-2 (gk608906) X \\
\hline FX06710 & pho-5(tm6710) & HBR02286 & $\begin{array}{l}\text { ser-2(pk1357) X; aptf-1 } \\
\text { (gk794) II }\end{array}$ \\
\hline FX06805 & set-23(tm6805) & HBR02298 & sms-2(tm2757) $X$ \\
\hline FX06830 & pho-5(tm6830) & HBR02312 & $\begin{array}{l}\text { glp-1(ar202); aptf-1 } \\
\text { (gk794) II }\end{array}$ \\
\hline FX06850 & rpia-1(tm6850) & HBR02315 & $\begin{array}{c}\text { aptf-1(gk794) II; tdc- } \\
\text { 1(n3420) II }\end{array}$ \\
\hline FX06913 & C01B4.6(tm6913) & HBR02317 & nlp-8(syb762) IV \\
\hline FX06977 & aldo-1(tm6977) & HBR02372 & $\begin{array}{c}\text { aptf-1(gk794) II; xbp- } \\
\text { 1(zc12) III }\end{array}$ \\
\hline FX06984 & C01G10.9(tm6984) & HBR02374 & $\begin{array}{c}\text { ztf-20(gk263563) V; aptf- } \\
\text { 1(gk794) II }\end{array}$ \\
\hline FX627 & $\operatorname{lgc}-11(\operatorname{tm} 627) X$ & SJ17 & $\begin{array}{c}\text { xbp-1(zc12) III; zcIs4[hsp- } \\
4:: \text { GFP] V }\end{array}$ \\
\hline GC833 & glp-1(ar202) III & HBR00507 & flp-11(tm2706) X \\
\hline GE68 & glp-1(e2144) III & HS184 & psa-4(os13) IV \\
\hline GG14 & emb-14(g14)I & HY520 & pod-2(ye60) II \\
\hline GG202 & ace-2(g72)I & IK589 & ttx-7(nj50) I \\
\hline GG43 & fasn-1(g43) I & JIN1375 & hlh-30(tm1978) IV \\
\hline
\end{tabular}




\begin{tabular}{|c|c|c|c|}
\hline Strain & Description & Strain & Description \\
\hline GR1307 & daf-16(mgDf50) I & JK289 & glp-1(e2142) III \\
\hline HBR01010 & nas-38(ok3407) X & JN1071 & snet-1(pe1063) X \\
\hline HBR00012 & goa-1(sa734) I & JT10800 & $\begin{array}{c}\text { ncr-2(nr2023) III; ncr- } \\
\text { 1(nr2022) X }\end{array}$ \\
\hline HBR01403 & $\begin{array}{c}\text { daf-2(e1370); aptf- } \\
\text { 1(gk794) II }\end{array}$ & JT307 & egl-9(sa307) V \\
\hline HBR01493 & flp-18(gk3063) X & KG2730 & clu-1(ok2) \\
\hline HBR01777 & $\begin{array}{l}\text { goeIs384 [pflp-11::egl- } \\
\text { 1::SL2-mkate2-flp-11- } \\
\text { 3'utr, unc-119(+)] }\end{array}$ & KJ550 & aco-1(jh131) X \\
\hline HBR2044 & C23H3.4(ok1693) II & KP1097 & dgk-1(nu62) $X$ \\
\hline HBR2045 & F13B12.4(ok3489) IV & KP2048 & ric-7(nu447) V \\
\hline HBR2046 & set-4(ok1481) II & KU12 & dlk-1(km12) I \\
\hline HBR2050 & dgk-1(ok1462) X & KU2 & jkk-1(km2) X \\
\hline HBR2051 & ckb-2(ok1922) III & LC143 & pcbd-1(tm5924) I \\
\hline HBR2057 & R09B5.6(ok2776) V & LC144 & agmo-1(e3016) III \\
\hline HBR2059 & ucr-2.3(ok3073) III. & LC33 & bas-1(tm351) III \\
\hline HBR2060 & Y46G5A.19(ok3421) II & LC73 & pah-1(tm520) II \\
\hline HBR2076 & set-4(n4600) II & $\mathrm{LC} 74$ & pah-1(ok687) II \\
\hline HBR02112 & hpd-1(ok1955) & LS505 & dyb-1(cx36) I \\
\hline HBR02116 & $\mathrm{D} 1005.1(\mathrm{tm} 5122)$ & MH1090 & $\operatorname{cog}-3(\mathrm{ku} 212) \mathrm{IV}$ \\
\hline HBR02117 & agxt-1(tm6307) & RB1003 & aco-1(ok924) X \\
\hline MH1955 & nhr-25(ku217) X & RB1005 & R02D3.1(ok926) IV \\
\hline MH5197 & nprl-3(ku540) IV & RB1025 & set-2(ok952) III \\
\hline MQ1333 & nuo-6(qm200) I & RB1029 & hdl-1(ok956) IV \\
\hline MQ1766 & $\begin{array}{c}\text { sod-2(ok1030) I; sod-5 } \\
\text { (tm1146) sod-1(tm783) II; } \\
\text { sod-4(gk101) III; } \\
\text { sod-3(tm760) X }\end{array}$ & RB1031 & fat-4(ok958) IV \\
\hline MQ770 & tpk-1(qm162) III & RB1036 & hyl-1(ok976) IV \\
\hline MQ989 & $\begin{array}{l}\text { isp-1(qm150) IV; ctb- } \\
\text { 1(qm189) }\end{array}$ & RB1046 & elo-9(ok993) II \\
\hline MT10549 & tdc-1(n3421) II & RB1053 & R05F9.10(ok1000) II \\
\hline MT10661 & tdc-1(n3420) II & RB1073 & dgk-4(ok1031) IV \\
\hline MT1083 & egl-8(n488) V & RB1075 & pes-9(ok1037) V \\
\hline MT1216 & egl-9(n586) V & RB1083 & F27C8.5(ok1050) IV \\
\hline MT13113 & tdc-1(n3419) II & RB1118 & ZK370.4(ok1107) III \\
\hline MT13293 & met-2(n4256) III & RB1123 & Y105E8A.10(ok1130) I \\
\hline MT14401 & set-12(n4442) X & RB1134 & Y105E8A.10(ok1157) I \\
\hline MT1443 & egl-10(n692) V & RB1160 & ckb-4(ok1195) V \\
\hline MT14480 & set-11(n4488) II & $\mathrm{RB} 1170$ & C04B4.2(ok1212) X \\
\hline MT14851 & set-2(n4589) III & RB1173 & plc-4(ok1215) IV \\
\hline MT1522 & ced-3(n717) IV & RB1188 & F23B12.6(ok1232) V \\
\hline MT16973 & met-1(n4337) I & RB1190 & amx-2(ok1235) I \\
\hline
\end{tabular}




\begin{tabular}{|c|c|c|c|}
\hline Strain & Description & Strain & Description \\
\hline MT2257 & lin-42(n1089) II & RB1203 & T10B11.2(ok1252) I \\
\hline MT7988 & bas-1(ad446) III & RB1206 & sks-1(ok1255) III \\
\hline MT8347 & ced-3(n2452) IV & RB1248 & ckb-1(ok1312) III \\
\hline MT8943 & $\begin{array}{c}\text { bas-1(ad446) III; cat- } \\
\text { 4(e1141) V }\end{array}$ & RB1264 & elo-4(ok1346) III \\
\hline MT9455 & tbh-1(n3247) X & RB1274 & F31C3.6(ok1365) I \\
\hline NL1832 & ucr-2.3(pk732) III & RB1304 & wdr-5.1(ok1417) III \\
\hline NM1568 & ehs-1(ok146) II & RB1326 & unc-129(ok1443) IV \\
\hline OH149 & ceh-23(ms 23) III & RB1370 & rabn-5(ok1555) III \\
\hline OH313 & ser-2(pk1357) X & RB1373 & gpdh-1(ok1558) I \\
\hline PD4588 & ceh-24(cc539) V & RB1400 & tre-3(ok394) V \\
\hline PD4605 & hlh-1(cc561) II & RB1404 & lec-1(ok1597) II \\
\hline PR1152 & cha-1(p1152) IV & RB1434 & mmcm-1(ok1637) III \\
\hline PS1839 & let-23(sa62) II & RB1482 & $\begin{array}{c}\text { gpdh-2 edit } \\
\text { K11H3.1(ok1733) III }\end{array}$ \\
\hline PS3551 & hsf-1(sy441) I & RB1483 & ifa-4(ok1734) X \\
\hline PS3653 & ipp-5(sy605) X & RB1484 & ath-1(ok1735) I \\
\hline QC122 & $\begin{array}{c}\text { paqr-2(tm3410) III; pcyt- } \\
\text { 1(et9) X }\end{array}$ & RB1487 & asm-3(ok1744) IV \\
\hline QC134 & nduf-7(et19) I & RB1498 & hyl-2(ok1766) X \\
\hline VC1625 & dylt-2(gk762) X & RB1525 & E02H9.5(ok1830) III \\
\hline VC1666 & met-1(ok2172) I & RB1527 & pnk-4(ok1832) X \\
\hline VC1667 & F55G7.2(ok2176) X & RB1548 & F53C3.13(ok1861) II \\
\hline VC1701 & mif-1(gk1027) III & RB1549 & klo-2(ok1862) III \\
\hline VC1737 & $\begin{array}{l}\text { F43C11.2(gk3131) II; } \\
\text { F13A2.3(gk3132) V; } \\
\text { W07E11.1(gk3133) }\end{array}$ & RB1579 & sptl-3(ok1927) V \\
\hline VC1876 & C39D10.3(ok2179) X & RB1588 & mxl-3(ok1947) X \\
\hline VC1896 & ckb-3(ok2310) III & RB1618 & hda-3(ok1991) I \\
\hline VC1897 & C32F10.8(tm2997) I & RB1633 & mif-1(ok2009) III \\
\hline VC199 & sir-2.1(ok434) IV & RB1688 & W05G11.6(ok2098) III \\
\hline VC20014 & gk246355 & RB1690 & ser-2(ok2103)X \\
\hline VC20058 & gk103693 & RB1704 & acp-2(ok2129) II \\
\hline VC20143 & gk115222 & RB1710 & Y39G10AR.18(ok2154) I \\
\hline VC20191 & gk237279 & $\mathrm{RB} 1715$ & aqp-2(ok2159) II \\
\hline VC20279 & $\mathrm{gk} 245731$ & RB1721 & Y71G12B.4(ok2189) I \\
\hline VC20300 & gk233890; gk947363 & RB1737 & pld-1(ok2222) II \\
\hline VC20317 & gk173266 & RB1764 & trxr-2(ok2267) III \\
\hline VC20339 & gk152785 & RB1789 & met-2(ok2307) III \\
\hline VC20346 & gk209511; gk246353 & RB1790 & D2096.3(ok2317) IV \\
\hline VC20392 & $\mathrm{gk} 322672$ & RB1793 & Y24D9A.2(ok2320) IV \\
\hline VC20400 & gk944084 & RB1794 & F53A2.7(ok2322) III \\
\hline VC20403 & gk324463 & RB1795 & fat-1(ok2323) IV \\
\hline
\end{tabular}




\begin{tabular}{|c|c|c|c|}
\hline Strain & Description & Strain & Description \\
\hline VC20453 & gk161469 & RB1796 & set-21(ok2327) IV \\
\hline VC20461 & gk284720 & RB1810 & cpr-5(ok2344) V \\
\hline VC20478 & gk169410 & RB1826 & T26A5.5(ok2364) III \\
\hline VC20488 & gk133451 & RB1828 & dgk-5(ok2366) II \\
\hline VC20504 & gk329791 & RB1843 & lin-42(ok2385) II \\
\hline VC20536 & gk255075 & RB1854 & sms-1(ok2399) IV \\
\hline VC20540 & gk173675 & RB1899 & acs-2(ok2457) V \\
\hline VC20545 & gk293929 & RB1919 & W07E6.3(ok2498) II \\
\hline VC20552 & gk278589 & RB1930 & spr-3(ok2525) X \\
\hline VC20560 & gk267976 & RB1932 & ZK418.8(ok2535) III \\
\hline VC20570 & gk109644 & RB1942 & ace-2(ok2545) I \\
\hline VC20602 & gk350360 & RB1954 & csp-1(ok2570) II \\
\hline VC20603 & gk350699 & RB1974 & smd-1(ok2602) I \\
\hline VC20624 & gk360120 & RB1997 & mtr-4(ok2642) IV \\
\hline VC20646 & gk368637 & RB2028 & set-12(ok2686) X \\
\hline VC20659 & gk941707 & RB2039 & set-13(ok2697) II \\
\hline VC20681 & gk371738 & RB2055 & ugt-1(ok2718) V \\
\hline VC20721 & gk382986 & RB2061 & ora-1(ok2724) IV \\
\hline VC20740 & gk963053 & RB2063 & gst-5(ok2726) II \\
\hline VC20773 & gk398679 & RB2067 & flp-9(ok2730) IV \\
\hline VC20777 & gk399608 & RB2070 & Y110A7A.6(ok2733) I \\
\hline VC20785 & gk963087 & RB2086 & C29E4.10(ok2752) III \\
\hline VC2142 & gpd-3(ok2870) X & RB2105 & T07D10.2(ok2780) I \\
\hline VC2156 & F59D12.1(gk1000) X & RB2114 & sodh-1(ok2799) V \\
\hline VC2171 & tkr-1(ok2886) III & RB2120 & Y6B3B.5(ok2805) I \\
\hline VC2175 & klo-1(ok2925) IV & RB2128 & idh-1(ok2832) IV \\
\hline VC218 & dgk-3(gk110) III & RB2135 & F33D4.4(ok2843) IV \\
\hline VC2210 & C12C8.2(ok2954) I & RB2165 & sams-3(ok2932) IV \\
\hline VC222 & raga-1(ok386) II & RB2206 & T25D3.3(ok2986) II \\
\hline VC225 & tps-1(ok373) X & RB2208 & H22K11.2(ok2990) X \\
\hline VC2285 & F59D12.1(gk1122) X & RB2240 & sams-1(ok3033) X \\
\hline VC2294 & W06D11.4(ok2831) X & RB2251 & $\begin{array}{c}\text { cpg-8 edit } \\
\text { K03B4.7(ok3045) V }\end{array}$ \\
\hline VC2309 & ZK669.4(ok3001) II & RB2263 & Y23B4A.2(ok3065) X \\
\hline VC2346 & hsp-12.3(ok3095) IV & RB2277 & ser-5 edit (ok3087) I \\
\hline VC2358 & sptl-2(ok2753) V & RB2285 & M02D8.4(ok3108) X \\
\hline VC239 & sams-5(gk147) IV & RB2301 & F32E10.2(ok3124) IV \\
\hline VC242 & tag-38(ok490) V & RB2347 & idh-2(ok3183) X \\
\hline VC2490 & $\begin{array}{c}\text { W07E11.1\&flp-2(gk1039) } \\
\text { X }\end{array}$ & RB2348 & idh-2(ok3184) X \\
\hline VC2524 & gpd-2(ok3243) X & RB2377 & gana-1(ok3230) V \\
\hline VC2569 & cth-1(ok3319) V & RB2420 & sams-4(ok3315) IV \\
\hline VC26 & pgp-12(gk19) X & RB2434 & asg-2(ok3344) X \\
\hline
\end{tabular}




\begin{tabular}{|c|c|c|c|}
\hline Strain & Description & Strain & Description \\
\hline VC2612 & cec-3(ok3432) III & RB2436 & $\begin{array}{c}\text { cysl-4 edit } \\
\text { F59A7.9(ok3359) V }\end{array}$ \\
\hline VC2683 & set-6(ok2195) X & RB2535 & K10H10.2(ok3516) II \\
\hline VC281 & hsp-12.6(gk156) IV & RB2549 & sms-3(ok3540) III \\
\hline VC30063 & gk407477 & RB2550 & ugt-23(ok3541) X \\
\hline VC30099 & gk949270 & RB2566 & T02G5.7(ok3574) II \\
\hline VC30165 & gk428189 & RB2588 & efk-1(ok3609) III \\
\hline VC30177 & gk960608 & RB2625 & F40F12.7(ok3684) III \\
\hline VC30220 & gk440213 & RB512 & mce-1(ok243) I \\
\hline VC30237 & gk963405 & RB608 & tag-96(ok336) IV \\
\hline VC40026 & gk146187 & RB681 & cat-1(ok411) X \\
\hline VC40041 & gk122498 & RB728 & tre-1(ok327) I \\
\hline VC40042 & gk153470 & RB737 & snt-4(ok503) I \\
\hline VC40057 & gk298765 & RB748 & gta-1(ok517) IV \\
\hline VC40071 & gk452553 & RB757 & nhr-111(ok519) V \\
\hline VC40107 & gk465651 & RB760 & tps-2(ok526) II \\
\hline VC40163 & gk492147 & RB766 & gei-7(ok531) V \\
\hline VC40209 & gk514674 & RB775 & T28D9.3(ok555) II \\
\hline VC40221 & gk520889 & RB782 & F27E5.1(ok564) II \\
\hline VC40235 & gk527451 & RB806 & tre-5(ok612) II \\
\hline VC40239 & gk529935 & RB810 & R07E5.3(ok622) III \\
\hline VC40249 & gk535232 & RB839 & F54A3.4(ok666) II \\
\hline VC40251 & gk536170 & RB865 & C05D2.3(ok703) III \\
\hline VC40283 & gk553614 & RB899 & C17G1.7(ok762) X \\
\hline VC40287 & gk555057 & RB910 & elo-3(ok777) IV \\
\hline VC40298 & gk560755 & RB927 & T11G6.8(ok801) IV \\
\hline VC40302 & gk563241 & RB938 & vha-12(ok821)X \\
\hline VC40328 & gk576881 & RB969 & fat-2(ok873) IV \\
\hline VC40336 & NA & RB976 & rhgf-1(ok880) X \\
\hline VC40341 & gk584646 & RB993 & tdc-1(ok914) II \\
\hline VC40365 & gk597083 & RM2702 & dat-1(ok157) III \\
\hline VC40390 & NA & SCL1 & mcp-1(tm2679) V \\
\hline VC40394 & gk616761 & SS579 & pgl-1(bn101) IV \\
\hline VC40396 & gk961143; gk961144 & SS580 & pgl-1(bn102) IV \\
\hline VC40402 & gk620458 & TG34 & gld-1(op236) I \\
\hline VC40428 & gk633662 & TJ1 & cep-1(gk138) I \\
\hline VC40434 & gk635549 & TK22 & mev-1(kn1) III \\
\hline VC40482 & gk658979 & TQ233 & trpa-1(ok999) IV \\
\hline VC40485 & gk660351 & TU228 & mec-18(u228) X \\
\hline VC40486 & gk660920 & TU55 & mec-14(u55) III \\
\hline VC40492 & gk962314 & VB1336 & nnt-1(tm358) X \\
\hline
\end{tabular}




\begin{tabular}{|c|c|c|c|}
\hline Strain & Description & Strain & Description \\
\hline VC40512 & gk672635 & VB1876 & tut-1(tm1297) \\
\hline VC40546 & gk687053 & VB1890 & elpc-1(tm2149) \\
\hline VC40549 & gk688401 & VB674 & nnt-1(sv34) X \\
\hline VC40551 & gk689405 & VC1072 & F29G6.2(gk455) X \\
\hline VC40585 & gk961365 & VC107 & tts-1(gk105) X \\
\hline VC40638 & $\mathrm{gk} 737178$ & VC1163 & T27A1.4(gk532) II \\
\hline VC40640 & gk738376 & VC1184 & T24H10.7(gk551) II \\
\hline VC40643 & gk961438 & VC1198 & Y39G8B.1(ok1682) II \\
\hline VC40650 & gk744178 & VC1307 & F52E4.1(ok1686) X \\
\hline VC40670 & gk942942 & VC1339 & Y49A10A.1(ok1856) X \\
\hline VC40679 & gk759089 & VC138 & elo-1(gk48) IV \\
\hline VC40790 & gk813443 & VC1383 & dgk-5 (gk631) II \\
\hline VC40807 & gk823783 & $\mathrm{VC} 142$ & dgk-2(gk124) X \\
\hline VC40823 & gk832037 & VC1432 & srh-159(ok1928) V \\
\hline VC40861 & gk852615 & VC1471 & nhr-186(gk732) V \\
\hline VC40884 & gk864611 & VC1555 & F13C5.2(gk716) X \\
\hline VC40887 & gk865337 & VC32 & haf-9(gk23) I \\
\hline VC40898 & gk872273 & VC333 & tap-1(gk202) X \\
\hline VC40899 & gk961751 & VC334 & hyl-1(gk203) IV \\
\hline VC40901 & $\mathrm{gk} 873395$ & VC335 & gly-2(gk204) I \\
\hline VC40905 & gk875298 & VC343 & glod-4(gk189) III \\
\hline VC40912 & gk961769 & VC345 & sgk-1(ok538) X \\
\hline VC40929 & gk886810 & VC377 & elo-5(gk182) IV \\
\hline VC40937 & gk889966 & VC393 & nduf-2.2(ok437) III \\
\hline VC40946 & gk948633; gk893848 & VC425 & elo-6(gk233) IV \\
\hline VC40964 & gk903134 & VC426 & hil-1(gk229) V \\
\hline VC40973 & gk907367 & VC430 & rhgf-1(gk217) X \\
\hline VC40979 & gk910077 & VC505 & ace-1(ok663) X \\
\hline VC40983 & gk912202 & VC545 & elo-3(gk236) IV \\
\hline VC41008 & gk925915 & VC549 & tdp-1(ok781) II \\
\hline VC41013 & gk927391 & VC566 & tag-174(gk274) III \\
\hline VC3028 & T03G6.3(ok3710) X & VC654 & lim-8(ok941) III \\
\hline VC3039 & col-184(ok3742) X & VC657 & gar-3(gk305) V \\
\hline VC3058 & thn-1(ok3735) IV & VC670 & gar-3(gk337) V \\
\hline VC3135 & gba-3(gk3287) IV & VC693 & cgt-1(ok1045) V \\
\hline VC788 & fat-3(ok1126) IV & VC736 & gfl-1(gk321) IV \\
\hline VC912 & tag-279(gk387) X & VC747 & lagr-1(gk327) I \\
\hline VC916 & sphk-1(ok1097) II & VC765 & lagr-1(gk331) I \\
\hline VC967 & set-32(ok1457) I & VC784 & ola-1(gk335) \\
\hline VF2 & pcs-1(tm1748) II & HBR02497 & $\begin{array}{c}\text { sqIs11 [lgg- } \\
1 \mathrm{p}:: \text { mCherry::GFP::lgg-1 + } \\
\text { rol-6] }\end{array}$ \\
\hline
\end{tabular}




\begin{tabular}{|c|c|c|c|}
\hline Strain & Description & Strain & Description \\
\hline VL1176 & alh-8(ww48) II & HBR02498 & $\begin{array}{c}\text { sqIs11 [lgg- } \\
\text { 1p::mCherry::GFP::lgg-1 + } \\
\text { rol-6]; aptf-1(gk794) II }\end{array}$ \\
\hline VM487 & nmr-1(ak4) II & HBR02332 & zIs356[daf-16::GFP] \\
\hline VS18 & maoc-1(hj13) II & HBR02482 & $\begin{array}{c}\text { flp-11(syb1445)X[flp-11- } \\
\text { SL2-unc-58(L428F)-linker- } \\
\text { mKate2]; zIs356[daf-16::GFP] } \\
\text { IV }\end{array}$ \\
\hline VS19 & maoc-1(hj14) II & HBR02333 & $\begin{array}{c}\text { aptf-1(gk794); zIs356 } \\
\text { [daf-16::GFP] }\end{array}$ \\
\hline VS24 & kat-1(tm1037) II & HBR02503 & $\begin{array}{l}\text { muIs126 [myo-3p::GFP::daf- } \\
16+\text { rol-6(su1006)] }\end{array}$ \\
\hline VT1343 & flh-1(bc374) IV & HBR02504 & $\begin{array}{c}\text { muIs126 [myo-3p::GFP::daf- } \\
16 \text { + rol-6(su1006)]; aptf- } \\
\text { 1(gk794) II }\end{array}$ \\
\hline WM99 & cdk-1(ne2257) III & SJ4100 & hsp-6::GFP(zcIs13) \\
\hline WU970 & haly-1(am132) X & HBR01810 & $\begin{array}{c}\text { aptf-1(gk794) II; zcIs 13[hsp- } \\
\text { 6::GFP]V. }\end{array}$ \\
\hline XA3101 & paf-1(tj11) I & HBR01370 & $\begin{array}{c}\text { goeIs309[pdat-1::SL1- } \\
\text { GCaMP3.35-SL2::mKate2- } \\
\text { unc-54-3'UTR, unc-119(+)]. }\end{array}$ \\
\hline XR3 & $\begin{array}{c}\operatorname{lagr-1(gk327)~I;~hyl-1(ok976)~} \\
\text { IV }\end{array}$ & HBR01417 & $\begin{array}{c}\text { aptf-1(gk794) II, } \\
\text { goeIs309[pdat-1::SL1- } \\
\text { GCaMP3.35-SL2::mKate2- } \\
\text { unc-54-3'UTR, unc-119(+)]. }\end{array}$ \\
\hline YH461 & ifta-2(tm1724) IV & HBR00560 & $\begin{array}{l}\text { goeIs120[ptdc-1::SL1- } \\
\text { GCaMP3.35-SL2::mKate2- } \\
\text { unc-54-3'utr,unc119(+)]. }\end{array}$ \\
\hline YM19 & cpb-3(bt17) I & HBR01118 & $\begin{array}{c}\text { aptf-1(gk794) II, } \\
\text { goeIs120[ptdc-1::SL1- } \\
\text { GCaMP3.35-SL2::mKate2- } \\
\text { unc-54-3'utr,unc119(+)]. }\end{array}$ \\
\hline YT17 & crh-1(tz2) III & HBR00205 & $\begin{array}{l}\text { goeIs22[pmec-4::SL1- } \\
\text { GCaMP3.35-SL2::mKate2- } \\
\text { unc-54-3'utr, unc-119(+)]. }\end{array}$ \\
\hline
\end{tabular}




\begin{tabular}{|c|c|c|c|}
\hline Strain & Description & Strain & Description \\
\hline ZG31 & hif-1(ia4) V & HBR00236 & $\begin{array}{c}\text { aptf-1(gk794) II; } \\
\text { goeIs22[pmec-4::SL1- } \\
\text { GCaMP3.35- } \\
\text { SL2::mKate2-unc-54-3'utr, } \\
\text { unc-119(+)]. }\end{array}$ \\
\hline ZR2 & jmjd-3.1 (gk384) X & HBR02201 & $\begin{array}{c}\text { goeIs304[pflp-11::SL1- } \\
\text { GCaMP3.35- } \\
\text { SL2::mKate2-unc-54- } \\
\text { 3'UTR, unc-119(+)], dgk- } \\
\text { 1(ok1462) X. }\end{array}$ \\
\hline ZZ12 & lev-11(x12) I & HBR02356 & $\begin{array}{c}\text { goeIs304[pflp-11::SL1- } \\
\text { GCaMP3.35- } \\
\text { SL2::mKate2-unc-54- } \\
\text { 3'UTR, unc-119(+)]; unc- } \\
13 \text { (s69) I }\end{array}$ \\
\hline BC12952 & $\begin{array}{c}\text { dpy-5(e907) I; sIs12952 } \\
{[\mathrm{rCes} \text { ZK112.1::GFP + }} \\
\text { pCeh361] }\end{array}$ & PHX2193 & $\begin{array}{c}\text { flp-11(syb2193) } \\
\text { [flp-11b-SL2(gpd-2)- } \\
\text { mKate2-linker-twk- } \\
\text { 18(e1913)] X. }\end{array}$ \\
\hline HBR02191 & $\begin{array}{c}\text { dpy-5(e907) I; sIs12952 } \\
\text { [rCes ZK112.1::GFP + } \\
\text { pCeh361], aptf-1(gk794) II }\end{array}$ & HBR02370 & $\begin{array}{c}\text { goeIs304[pflp-11::SL1- } \\
\text { GCaMP3.35- } \\
\text { SL2::mKate2-unc-54- } \\
\text { 3'UTR, unc-119(+)], flp- } \\
\text { 11(syb2193)[flp-11b- } \\
\text { SL2(gpd-2)-mKate2- } \\
\text { linker-twk-18(e1913)] }\end{array}$ \\
\hline HBR02212 & $\begin{array}{l}\text { eeEx106[hil-1::GFP + } \\
\text { rol-6(su1006)]; aptf- } \\
\text { 1(gk794) }\end{array}$ & HBR02340 & $\begin{array}{c}\text { flp-11(syb1445)X[flp-11- } \\
\text { SL2-unc-58(L428F)- } \\
\text { linker-mKate2] }\end{array}$ \\
\hline OP322 & $\begin{array}{c}\text { wgIs322 [ztf- } \\
\text { 4::TY1::EGFP:: } \\
\text { 3xFLAG(92C12); unc- } \\
\text { 119(+)] }\end{array}$ & HBR02371 & $\begin{array}{c}\text { goeIs304[pflp-11::SL1- } \\
\text { GCaMP3.35- } \\
\text { SL2::mKate2-unc-54- } \\
\text { 3'UTR, unc-119(+)], "flp- } \\
\text { 11(syb1445)X[flp-11- } \\
\text { SL2-unc-58(L428F)- } \\
\text { linker-mKate2]" }\end{array}$ \\
\hline SAL129 & $\begin{array}{c}\text { pha-1(e2123) III; denEx14. } \\
\text { [lys-1::GFP; pha-1(+)] }\end{array}$ & HBR02266 & $\begin{array}{l}\text { hsp-12.6 (gk196) IV; } \\
\text { aptf-1 (gk794) II }\end{array}$ \\
\hline HBR02213 & $\begin{array}{c}\text { pha-1(e2123) III; denEx14 } \\
\text { [lys-1::GFP + pha-1(+)]; } \\
\text { aptf-1(gk794) }\end{array}$ & HBR02270 & $\begin{array}{c}\text { hsp-12.6::mKate2 } \\
\text { (syb1364); aptf-1 (gk794) } \\
\text { II }\end{array}$ \\
\hline OP711 & $\begin{array}{c}\text { unc-119(tm4063) III; } \\
\text { wgIs711 } \\
\text { [fkh- } \\
\text { 7::TY1::EGFP::3xFLAG + } \\
\text { unc-119(+)] } \\
\end{array}$ & HBR02283 & $\begin{array}{c}\text { cutl-17(syb1652); hsp- } \\
\text { 12.6::mKate2 } \\
\text { (syb1364); aptf-1 (gk794) } \\
\text { II }\end{array}$ \\
\hline
\end{tabular}




\begin{tabular}{|c|c|c|c|}
\hline Strain & Description & Strain & Description \\
\hline HBR02240 & $\begin{array}{c}\text { wgIs711 [fkh- } \\
\text { 7::TY1::EGFP::3xFLAG + unc- } \\
\text { 119(+)]; aptf-1(gk794) }\end{array}$ & PHX1364 & $\begin{array}{l}\text { hsp-12.6::mKate2 } \\
\text { (syb1364) }\end{array}$ \\
\hline HBR02327 & $\begin{array}{c}\text { sEx15051 [rCes } \\
\text { C05D9.5::GFP + pCeh361] }\end{array}$ & PHX1652 & $\begin{array}{c}\text { cutl-17(syb1652); } \\
\text { hsp- } \\
\text { 12.6::mKate2 (syb1364) }\end{array}$ \\
\hline HBR02285 & $\begin{array}{l}\text { sEx15051[rCes C05D9.5::GFP + } \\
\text { pCeh361]; aptf-1(gk794) II }\end{array}$ & HBR02319 & $\begin{array}{c}\text { hsp-12.6::mKate2 } \\
\text { (syb1364); daf-16 (mu86) } \\
\text { I }\end{array}$ \\
\hline HBR02297 & $\begin{array}{l}\text { sEx10182[rCes R13A5.1a } \\
\quad:: G F P+\text { pCeh361] }\end{array}$ & HBR02320 & $\begin{array}{c}\text { hsp-12.6::mKate2 } \\
\text { (syb1364); aptf-1 } \\
\text { (gk794) II; daf-16 (mu86) } \\
\text { I }\end{array}$ \\
\hline HBR02241 & $\begin{array}{c}\text { sEx10182 } \\
{[\mathrm{rCes} \text { R13A5.1a::GFP + }} \\
\text { pCeh361]; aptf-1(gk794) }\end{array}$ & HBR02325 & $\begin{array}{c}\text { hsp-12.6::mKate2 } \\
\text { (syb1364); hsf-1(sy441) I }\end{array}$ \\
\hline NIS1012 & $\begin{array}{c}\text { kytEx1012[hsp-12.6p(1kb):: } \\
\text { hsp-12.6::GFP + rol-6(su1006)] }\end{array}$ & HBR02343 & $\begin{array}{c}\text { hsp-12.6::mKate2 } \\
\text { (syb1364); elt-3 (gk121) } \\
\text { X }\end{array}$ \\
\hline HBR02235 & $\begin{array}{c}\text { kytEx1012 [hsp-12.6p(1kb): } \\
\text { :hsp-12.6::GFP + rol-6(su1006)]; } \\
\text { aptf-1(gk794) }\end{array}$ & HBR02344 & $\begin{array}{c}\text { hsp-12.6::mKate2 } \\
\text { (syb1364); aptf-1 (gk794) } \\
\text { II; elt-3 (gk121) X }\end{array}$ \\
\hline NIS 1013 & $\begin{array}{c}\text { kytEx1013hsp-12.6p(5kb):: } \\
\text { hsp-12.6::GFP + rol-6(su1006)] }\end{array}$ & HBR02345 & $\begin{array}{c}\text { hsp-12.6::mKate2 } \\
\text { (syb1364); daf-2 (e1370) } \\
\text { III }\end{array}$ \\
\hline HBR02234 & $\begin{array}{c}\text { kytEx1013 [hsp-12.6p(5kb):: } \\
\text { hsp-12.6::GFP + rol-6(su1006)]; } \\
\text { aptf-1(gk794) }\end{array}$ & HBR02346 & $\begin{array}{c}\text { hsp-12.6::mKate2 } \\
\text { (syb1364); aptf-1 (gk794) } \\
\text { II; daf-2 (e1370) III }\end{array}$ \\
\hline CF1553 & $\begin{array}{c}\text { muIs84 }[(\mathrm{pAD} 76) \text { sod-3p::GFP + } \\
\text { rol-6(su1006)]; }\end{array}$ & HBR02346 & $\begin{array}{c}\text { hsp-12.6::mKate2 } \\
\text { (syb1364); aptf-1 (gk794) } \\
\text { II; daf-2 (e1370) III }\end{array}$ \\
\hline HBR02397 & $\begin{array}{l}\text { muIs84 [(pAD76) sod-3p::GFP + } \\
\text { rol-6(su1006)]; aptf-1(gk794) II. }\end{array}$ & HBR02347 & $\begin{array}{c}\text { hsp-12.6::mKate2 } \\
\text { (syb1364); blmp-1 (s71) I }\end{array}$ \\
\hline SS747 & $\begin{array}{c}\text { bnIs1[pie-1::GFP::pgl-1 + unc- } \\
119(+)]\end{array}$ & HBR02348 & $\begin{array}{c}\text { hsp-12.6::mKate2 } \\
\text { (syb1364); aptf-1 (gk794) } \\
\text { II; blmp-1 (s71) I }\end{array}$ \\
\hline HBR02102 & $\begin{array}{l}\text { aptf-1(gk794) II; bnIs1[pie- } \\
1:: \text { GFP::pgl-1 + unc-119(+)] }\end{array}$ & HBR02349 & $\begin{array}{c}\text { hsp-12.6::mKate2 } \\
\text { (syb1364); glp-1 (q224) } \\
\text { III } \\
\end{array}$ \\
\hline SJ4005 & zcIs4[hsp-4::GFP] V. & HBR02350 & $\begin{array}{c}\text { hsp-12.6::mKate2 } \\
\text { (syb1364); aptf-1 (gk794) } \\
\text { II; glp-1 (q224) III }\end{array}$ \\
\hline
\end{tabular}




\begin{tabular}{|c|c|c|c|}
\hline Strain & Description & Strain & Description \\
\hline HBR01809 & $\begin{array}{c}\text { aptf-1(gk794) II; zcIs4 [hsp- } \\
4:: G F P] ~ V .\end{array}$ & HBR02351 & $\begin{array}{c}\text { hsp-12.6::mKate2 } \\
\text { (syb1364); glp-1(tn777) } \\
\text { III }\end{array}$ \\
\hline HBR02384 & $\begin{array}{c}\text { unc-119(ed3) III;goeIs251[mec- } \\
\text { 4::ReaChr::mKate2-unc-54- } \\
\text { 3'utr,unc-119(+)]; hsp- } \\
\text { 12.6::mKate2 (syb1364) }\end{array}$ & HBR02352 & $\begin{array}{c}\text { hsp-12.6::mKate2 } \\
\text { (syb1364); aptf-1 (gk794) } \\
\text { II; glp-1(tn777) III }\end{array}$ \\
\hline HBR02410 & $\begin{array}{c}\text { hsp-12.6::mKate2 (syb1364); } \\
\text { jmjd-3.1 (gk384) X }\end{array}$ & HBR02353 & $\begin{array}{c}\text { hsp-12.6::mKate2 } \\
\text { (syb1364); glp-1(ar202) } \\
\text { III }\end{array}$ \\
\hline HBR02411 & $\begin{array}{l}\text { hsp-12.6::mKate2 (syb1364); aptf- } \\
1 \text { (gk794) II; jmjd-3.1 (gk384) X }\end{array}$ & HBR02354 & $\begin{array}{c}\text { hsp-12.6::mKate2 } \\
\text { (syb1364); aptf-1 (gk794) } \\
\text { II; glp-1(ar202) III }\end{array}$ \\
\hline HBR02412 & $\begin{array}{c}\text { hsp-12.6::mKate2 (syb1364); crh- } \\
1 \text { (tz2) III }\end{array}$ & HBR02326 & $\begin{array}{c}\text { hsp-12.6::mKate2 } \\
\text { (syb1364); aptf-1 } \\
\text { (gk794) II; hsf-1(sy441) I }\end{array}$ \\
\hline HBR02413 & $\begin{array}{l}\text { hsp-12.6::mKate2 (syb1364); aptf- } \\
1 \text { (gk794) II; crh-1 (tz2) III }\end{array}$ & HBR02378 & $\begin{array}{c}\text { flp-11(syb2193) } \\
\text { [flp-11b-SL2(gpd-2)- } \\
\text { mKate2-linker-twk- } \\
\text { 18(e1913)]; hsp- } \\
\text { 12.6::mKate2 (syb1364) }\end{array}$ \\
\hline HBR02414 & $\begin{array}{c}\text { hsp-12.6::mKate2 (syb1364); xbp- } \\
1 \text { (zc12) III }\end{array}$ & HBR02379 & $\begin{array}{l}\text { flp-11(syb1445)X[flp-11- } \\
\text { SL2-unc-58(L428F)- } \\
\text { linker-mKate2]; hsp- } \\
\text { 12.6::mKate2 (syb1364) }\end{array}$ \\
\hline HBR02415 & $\begin{array}{l}\text { hsp-12.6::mKate2 (syb1364); aptf- } \\
1 \text { (gk794) II; xbp-1 (zc12) III }\end{array}$ & HBR02380 & $\begin{array}{c}\text { goeIs307[flp-11 } \\
\text { prom::ArchT::SL2mKate- } \\
\text { 2-unc-54-3'utr,unc- } \\
\text { 119(+)]; hsp- } \\
\text { 12.6::mKate2 (syb1364) }\end{array}$ \\
\hline HBR02416 & $\begin{array}{c}\text { hsp-12.6::mKate2 (syb1364); efl-1 } \\
\text { (se1) V }\end{array}$ & HBR02381 & $\begin{array}{c}\text { goeIs232[psra- } \\
\text { 6::ReaChr::mKate2-unc- } \\
\text { 54-3'utr, unc-119(+)]; } \\
\text { hsp-12.6::mKate2 } \\
\text { (syb1364) }\end{array}$ \\
\hline HBR02417 & $\begin{array}{l}\text { hsp-12.6::mKate2 (syb1364); aptf- } \\
1 \text { (gk794) II; efl-1 (se1) V }\end{array}$ & HBR02382 & $\begin{array}{l}\text { eft-3p::TIR } 1:: \text { SEC; hsp- } \\
\text { 12.6::mKate2 (syb1364) }\end{array}$ \\
\hline HBR02418 & $\begin{array}{c}\text { hsp-12.6::mKate2 (syb1364); flp- } \\
11(\operatorname{tm} 2706) X\end{array}$ & HBR02383 & $\begin{array}{l}\text { eft-3p::TIR1::SEC; hsp- } \\
\text { 12.6::mKate2 (syb1364); } \\
\text { aptf-1(gk794) II }\end{array}$ \\
\hline HBR02419 & nprl-3 (ku540); aptf-1 (gk794) & HBR02456 & $\begin{array}{l}\text { hsp-12.6::mKate2 } \\
\text { (syb1364) IV; unc- } \\
\text { 13(s69) I }\end{array}$ \\
\hline
\end{tabular}




\begin{tabular}{|c|c|c|c|}
\hline Strain & Description & Strain & Description \\
\hline HBR02432 & $\begin{array}{l}\text { hsp-12.6::mKate2 (syb1364) IV; } \\
\text { tdc-1 (n3420) II }\end{array}$ & HBR02457 & $\begin{array}{l}\text { hsp-12.6::mKate2 } \\
\text { (syb1364) IV; ceh- } \\
\text { 14(ch3) X }\end{array}$ \\
\hline HBR02433 & $\begin{array}{l}\text { hsp-12.6::mKate2 (syb1364) IV; } \\
\text { tbh-1 (n3247) X }\end{array}$ & HBR02458 & $\begin{array}{l}\text { hsp-12.6::mKate2 } \\
\text { (syb1364) IV; cha- } \\
\text { 1(p1152) IV }\end{array}$ \\
\hline HBR02434 & $\begin{array}{l}\text { hsp-12.6::mKate2 (syb1364) IV; } \\
\text { cat-2 (n4547) II }\end{array}$ & HBR02459 & $\begin{array}{l}\text { hsp-12.6::mKate2 } \\
\text { (syb1364) IV; ceh- } \\
\text { 23(ms23) II }\end{array}$ \\
\hline HBR02435 & $\begin{array}{l}\text { hsp-12.6::mKate2 (syb1364) IV; } \\
\text { tph-1 (mg280) II }\end{array}$ & HBR02460 & $\begin{array}{l}\text { hsp-12.6::mKate2 } \\
\text { (syb1364) IV; ser- } \\
\text { 4(ok512)III }\end{array}$ \\
\hline HBR02436 & $\begin{array}{c}\text { hsp-12.6::mKate2 (syb1364) IV; } \\
\text { unc-25 (e156) III }\end{array}$ & HBR02461 & $\begin{array}{l}\text { hsp-12.6::mKate2 } \\
\text { (syb1364) IV; fkh- } \\
\text { 9(ok1709) X }\end{array}$ \\
\hline HBR02437 & $\begin{array}{c}\text { hsp-12.6::mKate2 (syb1364) IV; } \\
\text { dgk-1 (ok1462) X }\end{array}$ & HBR02462 & $\begin{array}{l}\text { hsp-12.6::mKate2 } \\
\text { (syb1364) IV; fkh- } \\
\text { 9(ok1709) X; aptf- } \\
\text { 1(gk794)II }\end{array}$ \\
\hline HBR02438 & $\begin{array}{c}\text { hsp-12.6::mKate2 (syb1364) IV; } \\
\text { eat-4 (ky5) III }\end{array}$ & HBR02463 & $\begin{array}{l}\text { hsp-12.6::mKate2 } \\
\text { (syb1364) IV; let- } \\
\text { 23(sa62) II }\end{array}$ \\
\hline HBR02439 & $\begin{array}{c}\text { hsp-12.6::mKate2 (syb1364) IV; } \\
\text { egl-3 (ok979) V }\end{array}$ & HBR02464 & $\begin{array}{c}\text { hsp-12.6::mKate2 } \\
\text { (syb1364) IV; aak-2 } \\
\text { (ok524) X; aak- } \\
\text { 1(tm1944) III }\end{array}$ \\
\hline HBR02454 & $\begin{array}{c}\text { hsp-12.6::mKate2 } \\
\text { (syb1364) IV; jkk-1(km2) X }\end{array}$ & HBR02473 & $\begin{array}{c}\text { hsp- } \\
\text { 12.6::mKate2(syb1364) } \\
\text { IV; aptf-1(gk794) II; } \\
\text { egl-9(sa307) V }\end{array}$ \\
\hline HBR02455 & $\begin{array}{l}\text { hsp-12.6::mKate2 (syb1364) IV; } \\
\text { tbh-1 (n3247) X; aptf-1 (gk794)II }\end{array}$ & HBR02474 & $\begin{array}{c}\text { hsp- } \\
\text { 12.6::mKate2(syb1364) } \\
\text { IV; aptf-1(gk794) II; } \\
\text { jkk-1(km2) X }\end{array}$ \\
\hline HBR02440 & $\begin{array}{c}\text { eft-3p::TIR1::SEC; hsp- } \\
\text { 12.6::mKate2 (syb1364) IV; rheb- } \\
\text { 1(re64 } \\
\text { re285[AID::mKate2::3xFlag::rheb- } \\
\text { 1]) III }\end{array}$ & HBR02465 & $\begin{array}{l}\text { hsp-12.6::mKate2 } \\
\text { (syb1364) IV; aptf-1 } \\
\text { (gk794) II; aak-2 } \\
\text { (ok524) X; aak- } \\
\text { 1(tm1944) III }\end{array}$ \\
\hline HBR02441 & $\begin{array}{c}\text { eft-3p::TIR1::SEC; hsp- } \\
\text { 12.6::mKate2 (syb1364) IV; aptf- } \\
\text { 1(gk794) II; rheb-1(re64 } \\
\text { re285[AID::mKate2::3xFlag::rheb- } \\
\text { 1]) III }\end{array}$ & HBR02466 & $\begin{array}{l}\text { hsp-12.6::mKate2 } \\
\text { (syb1364) IV; egl- } \\
\text { 9(sa307) V }\end{array}$ \\
\hline
\end{tabular}




\begin{tabular}{|c|c|c|c|}
\hline Strain & Description & Strain & Description \\
\hline HBR02475 & $\begin{array}{c}\text { hsp- } \\
\text { 12.6::mKate2(syb1364) } \\
\text { IV; asm-3(ok1744) IV }\end{array}$ & HBR02494 & $\begin{array}{l}\text { flp-11(syb2193)[flp-11b- } \\
\text { SL2(gpd-2)-mKate2- } \\
\text { linker-twk-18(e1913)] X; } \\
\text { hsp-12.6::mKate2 } \\
\text { (syb1364) IV; tph-1(tph-1 } \\
\text { (mg280) II) }\end{array}$ \\
\hline HBR02476 & $\begin{array}{c}\text { hsp- } \\
\text { 12.6::mKate2(syb1364) } \\
\text { IV; aptf-1(gk794) II; asm- } \\
\text { 3(ok1744) IV } \\
\end{array}$ & HBR02499 & $\begin{array}{c}\text { hsp- } \\
\text { 12.6::mKate2(syb1364) } \\
\text { IV; lpIs1 [jnk-1(+) + rol- } \\
\text { 6(su1006)] }\end{array}$ \\
\hline HBR02477 & $\begin{array}{c}\text { hsp- } \\
\text { 12.6::mKate2(syb1364) } \\
\text { IV; jnk-1(gk7) IV }\end{array}$ & HBR02500 & $\begin{array}{c}\text { hsp- } \\
\text { 12.6::mKate2(syb1364) } \\
\text { IV; lpIs1 [jnk-1(+) + rol- } \\
\text { 6(su1006)]; aptf-1(gk794) } \\
\text { II } \\
\end{array}$ \\
\hline HBR02478 & $\begin{array}{c}\text { hsp- } \\
\text { 12.6::mKate2(syb1364) } \\
\text { IV; aptf-1(gk794) II; jnk- } \\
\text { 1(gk7) IV }\end{array}$ & HBR02501 & $\begin{array}{c}\text { hsp- } \\
\text { 12.6::mKate2(syb1364) } \\
\text { IV; lpIs2 [jnk-1(+) + rol- } \\
\text { 6(su1006)] }\end{array}$ \\
\hline HBR02479 & $\begin{array}{c}\text { hsp- } \\
\text { 12.6::mKate2(syb1364) } \\
\text { IV; nkat-1(ok566) X }\end{array}$ & HBR02502 & $\begin{array}{c}\text { hsp- } \\
\text { 12.6::mKate2(syb1364) } \\
\text { IV; lpIs2 [jnk-1(+) + rol- } \\
\text { 6(su1006)]; aptf-1(gk794) } \\
\text { II } \\
\end{array}$ \\
\hline HBR02480 & $\begin{array}{c}\text { hsp- } \\
\text { 12.6::mKate2(syb1364) } \\
\text { IV; aptf-1(gk794) II; nkat- } \\
\text { 1(ok566) X }\end{array}$ & HBR02491 & $\begin{array}{c}\text { hsp- } \\
\text { 12.6::mKate2(syb1364) } \\
\text { IV; alg-1(gk214) X }\end{array}$ \\
\hline HBR02481 & $\begin{array}{c}\text { hsp- } \\
\text { 12.6::mKate2(syb1364) } \\
\text { IV; nsy-1(ag3) II }\end{array}$ & HBR02492 & $\begin{array}{c}\text { hsp-12.6::mKate2 } \\
\text { (syb1364) IV; cha- } \\
\text { 1(p1152) IV; aptf- } \\
\text { 1(gk794) II }\end{array}$ \\
\hline HBR02485 & $\begin{array}{c}\text { hsp- } \\
\text { 12.6::mKate2(syb1364) } \\
\text { IV; set-2(ok952) III. }\end{array}$ & HBR02493 & $\begin{array}{c}\text { hsp- } \\
\text { 12.6::mKate2(syb1364) } \\
\text { IV; jnk-1(gk7) IV; aptf- } \\
\text { 1(gk794) II }\end{array}$ \\
\hline HBR02486 & $\begin{array}{c}\text { aptf-1(gk794) II; set- } \\
\text { 2(ok952) III, hsp- } \\
\text { 12.6::mKate2(syb1364) IV }\end{array}$ & & \\
\hline
\end{tabular}




\subsection{Primer list}

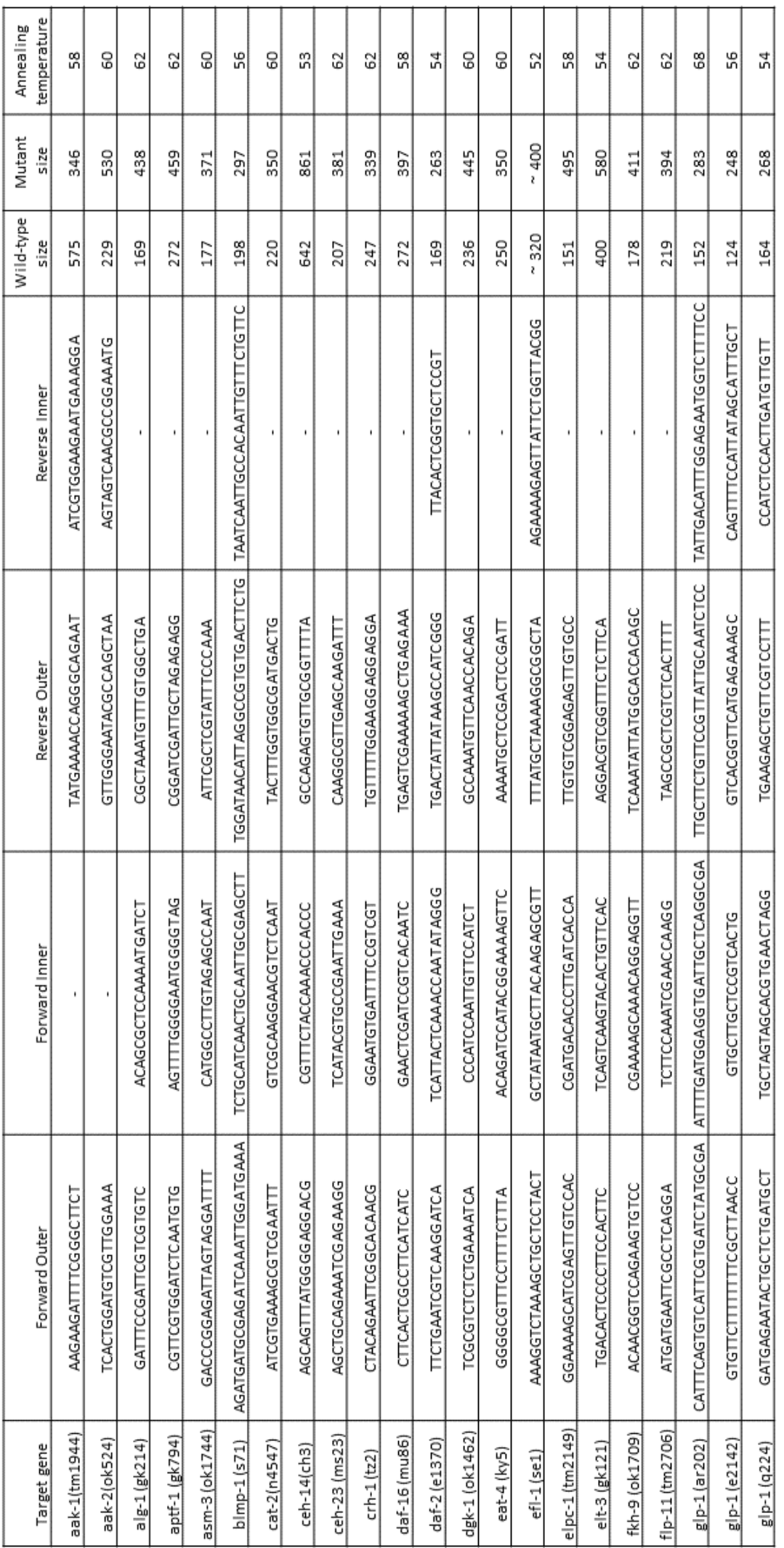




\begin{tabular}{|c|c|c|c|c|c|c|c|c|c|c|c|c|c|c|c|c|c|c|c|}
\hline 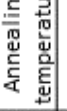 & เก & เి & 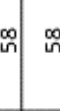 & S & $\widetilde{\sigma}$ & : & श & in 5 & $\begin{array}{l:l}0 \\
\end{array}$ & $|\tilde{\sigma}|$ & N & $:$ & $\begin{array}{lll}0 \\
\text { in }\end{array}$ & $p$ & 另 & : & 吕 & & \\
\hline 吝 & స్ & 㓏 & $\begin{array}{l}f \\
\text { fon }\end{array}$ & 诰 & \% & 郃 & ปี & 吕 & $\begin{array}{l}\infty \\
\end{array}$ & $\left|\begin{array}{c}0 \\
: \\
-1\end{array}\right|$ & 兽 & 骂 & $\underset{\sim}{2}$ & $\frac{b}{0}$ & $\vec{\wedge}$ & & 学 & & d. \\
\hline 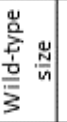 & की & & ปn] & $\underset{-1}{\infty}$ & 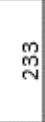 & i̊ & 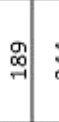 & 听 & 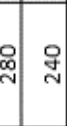 & 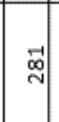 & 잉 & 원 & ปูป & 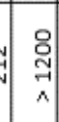 & ㅁ & & | & & of \\
\hline & . & 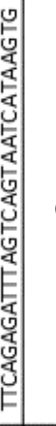 & & & & & $\frac{0}{0}$ & & $\begin{array}{ll}0 \\
0 \\
0 \\
0 \\
0 \\
0 \\
0 \\
0 \\
0 \\
0 \\
0 \\
0 \\
0 \\
0 \\
0 \\
0 \\
0 \\
0 \\
\quad \\
0\end{array}$ & 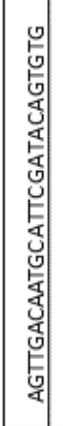 & & & 昜 & & & & & & 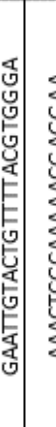 \\
\hline & 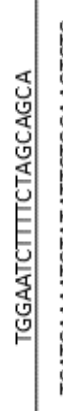 & 'ئ. & 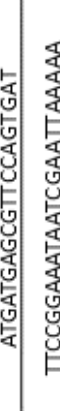 & 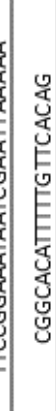 & 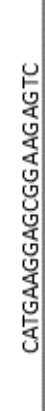 & $\begin{array}{l}0 \\
0 \\
\vdots \\
0 \\
0 \\
\hat{E} \\
0 \\
\vdots \\
0 \\
0 \\
0 \\
0 \\
0 \\
0 \\
0 \\
0\end{array}$ & 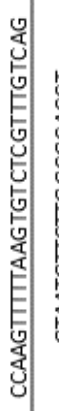 & 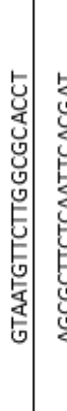 & 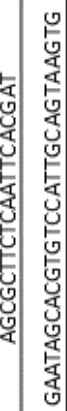 & 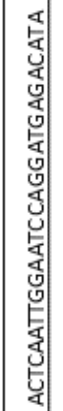 & $\begin{array}{l}0 \\
0 \\
0 \\
0 \\
0 \\
0 \\
0 \\
0 \\
0 \\
0 \\
0 \\
5 \\
0 \\
0\end{array}$ & 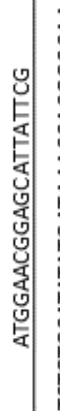 & 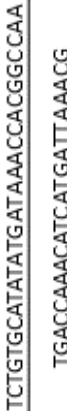 & 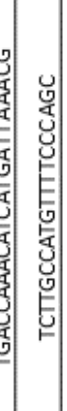 & $\begin{array}{l}\text { a } \\
0 \\
0 \\
0 \\
0 \\
0 \\
0 \\
0 \\
0 \\
0 \\
0 \\
0\end{array}$ & 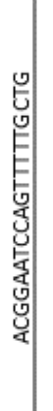 & 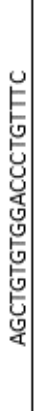 & 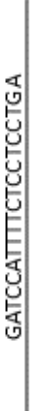 & |c| \\
\hline & 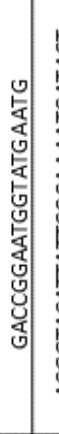 & لئ. & | & 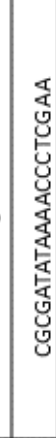 & 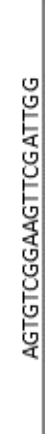 & $\begin{array}{l}0 \\
0 \\
0 \\
0 \\
0 \\
0 \\
\vdots \\
\vdots \\
\vdots \\
0 \\
0 \\
0 \\
0 \\
0\end{array}$ & $\begin{array}{l}0 \\
0 \\
0 \\
0 \\
0 \\
0 \\
0 \\
0 \\
0 \\
0 \\
0 \\
0\end{array}$ & 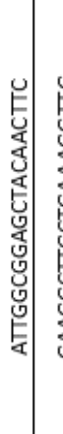 & 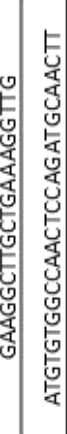 & 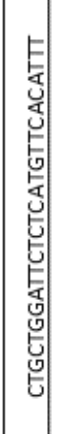 & 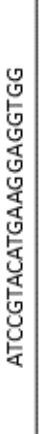 & 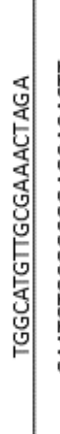 & 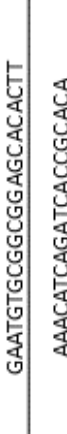 & & & & 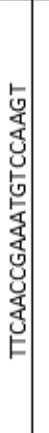 & 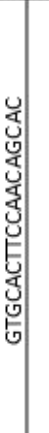 & 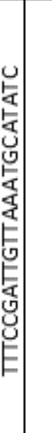 \\
\hline 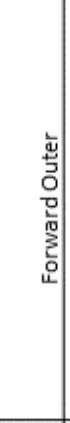 & $\begin{array}{l}0 \\
0 \\
0 \\
0 \\
0 \\
0 \\
0 \\
0 \\
0 \\
0 \\
0 \\
0 \\
0 \\
0\end{array}$ & |c. & 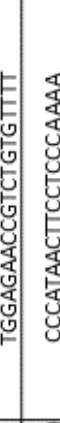 & 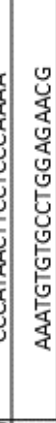 & 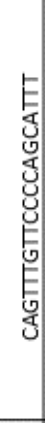 & 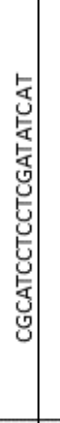 & 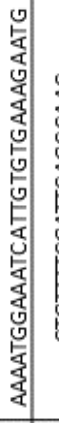 & 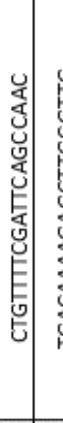 & 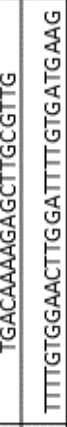 & 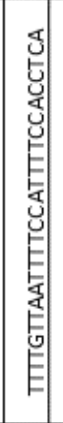 & \begin{tabular}{l|l}
0 \\
0 \\
0 \\
0 \\
0 \\
0 \\
0 \\
0 \\
0 \\
0 \\
0 \\
0 \\
0 \\
0 \\
0 \\
0
\end{tabular} & . & 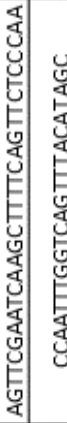 & 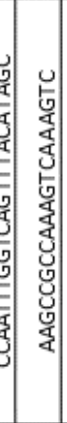 & $\begin{array}{l}\mathbf{4} \\
0 \\
0 \\
0 \\
0 \\
0 \\
0 \\
0 \\
0 \\
0 \\
0 \\
0\end{array}$ & 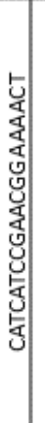 & 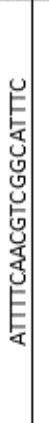 & & 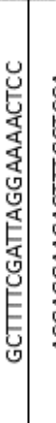 \\
\hline $\begin{array}{l}\text { 嚾 } \\
\text { 商 }\end{array}$ & 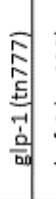 & 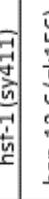 & $\begin{array}{l}0 \\
0 \\
0 \\
0\end{array}$ & 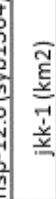 & 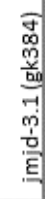 & 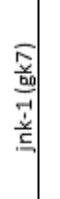 & $\frac{10}{m}$ & 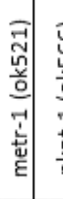 & 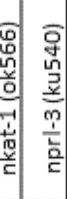 & $\begin{array}{l}\text { a } \\
\text { on } \\
7 \\
7\end{array}$ & 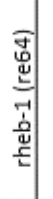 & 矛 & 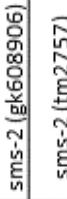 & 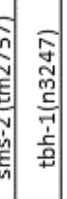 & ํํㅂ & 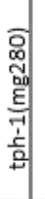 & 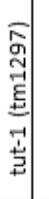 & & . \\
\hline
\end{tabular}




\subsection{Quiescence bout detection script}

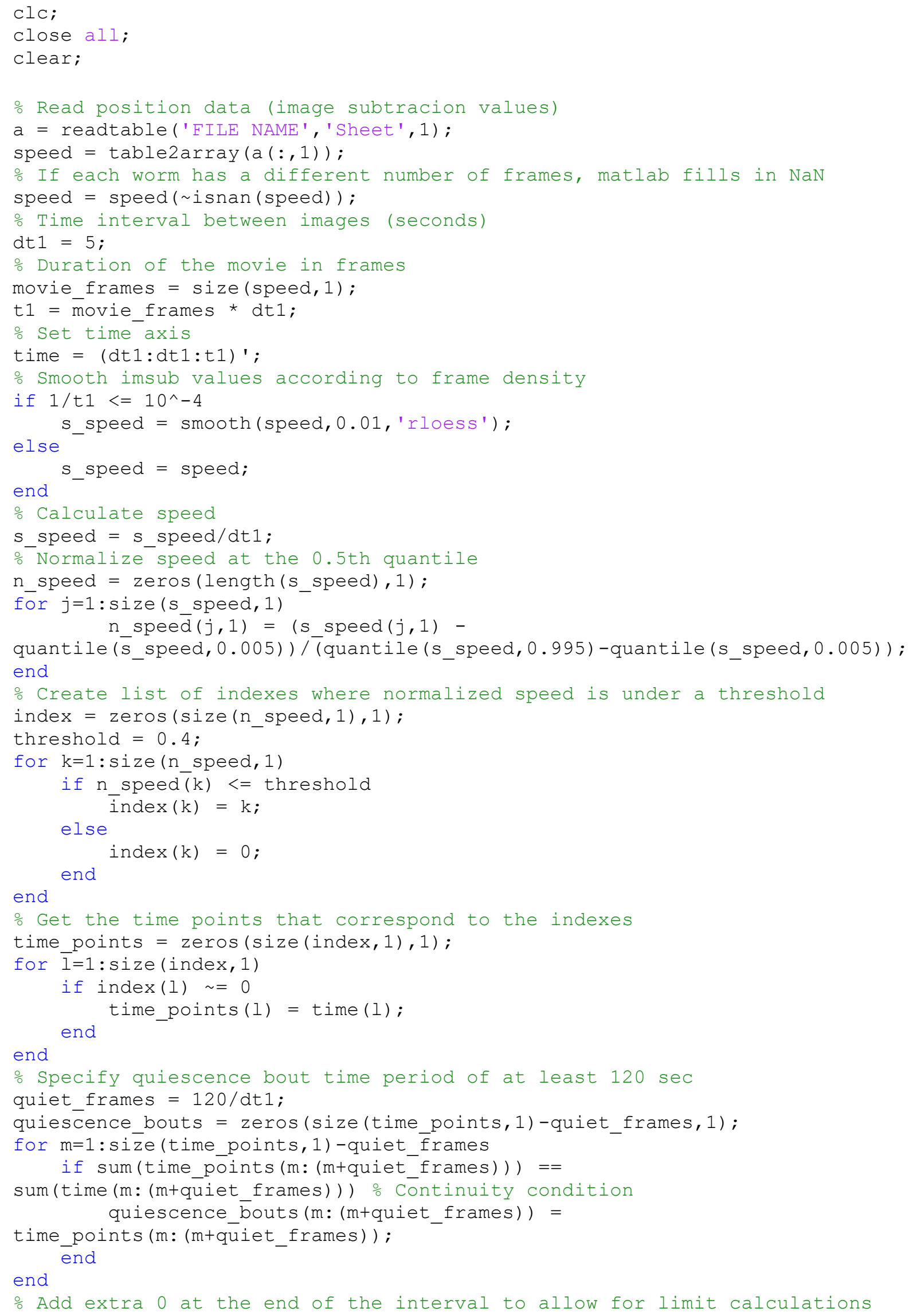




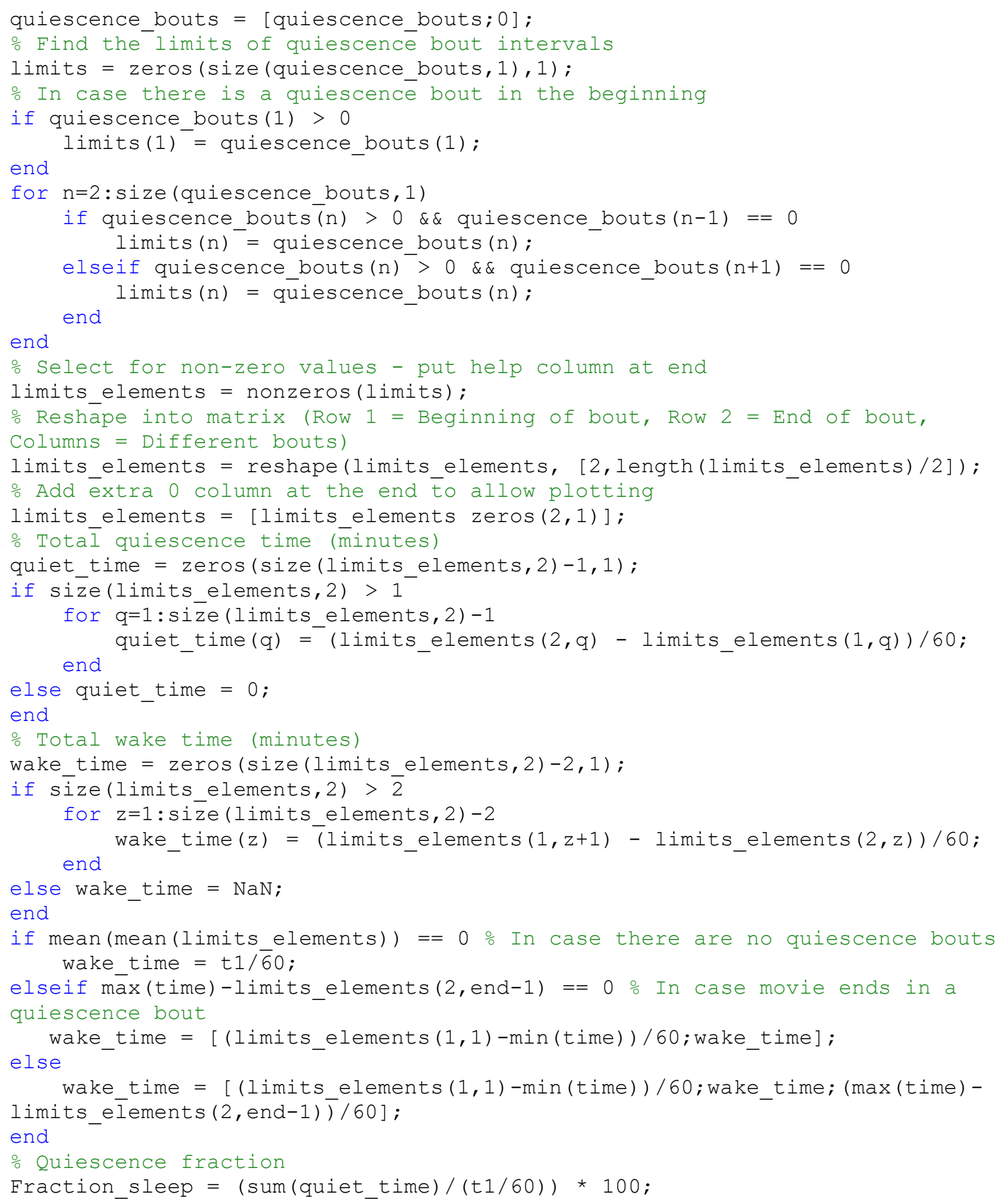




\subsection{RIS neuron automated detection script}

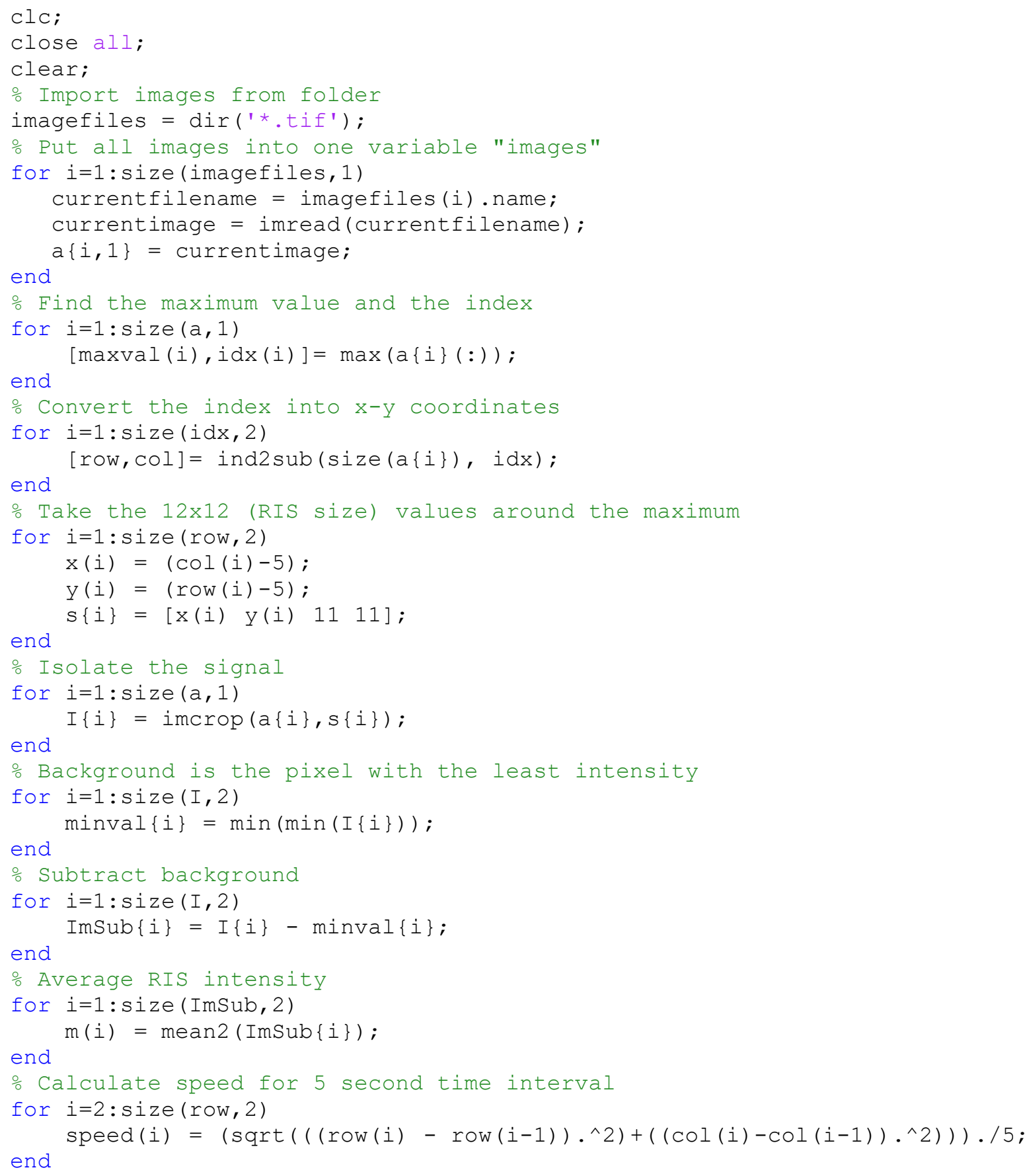




\subsection{RNA-seq differential expression analysis script}

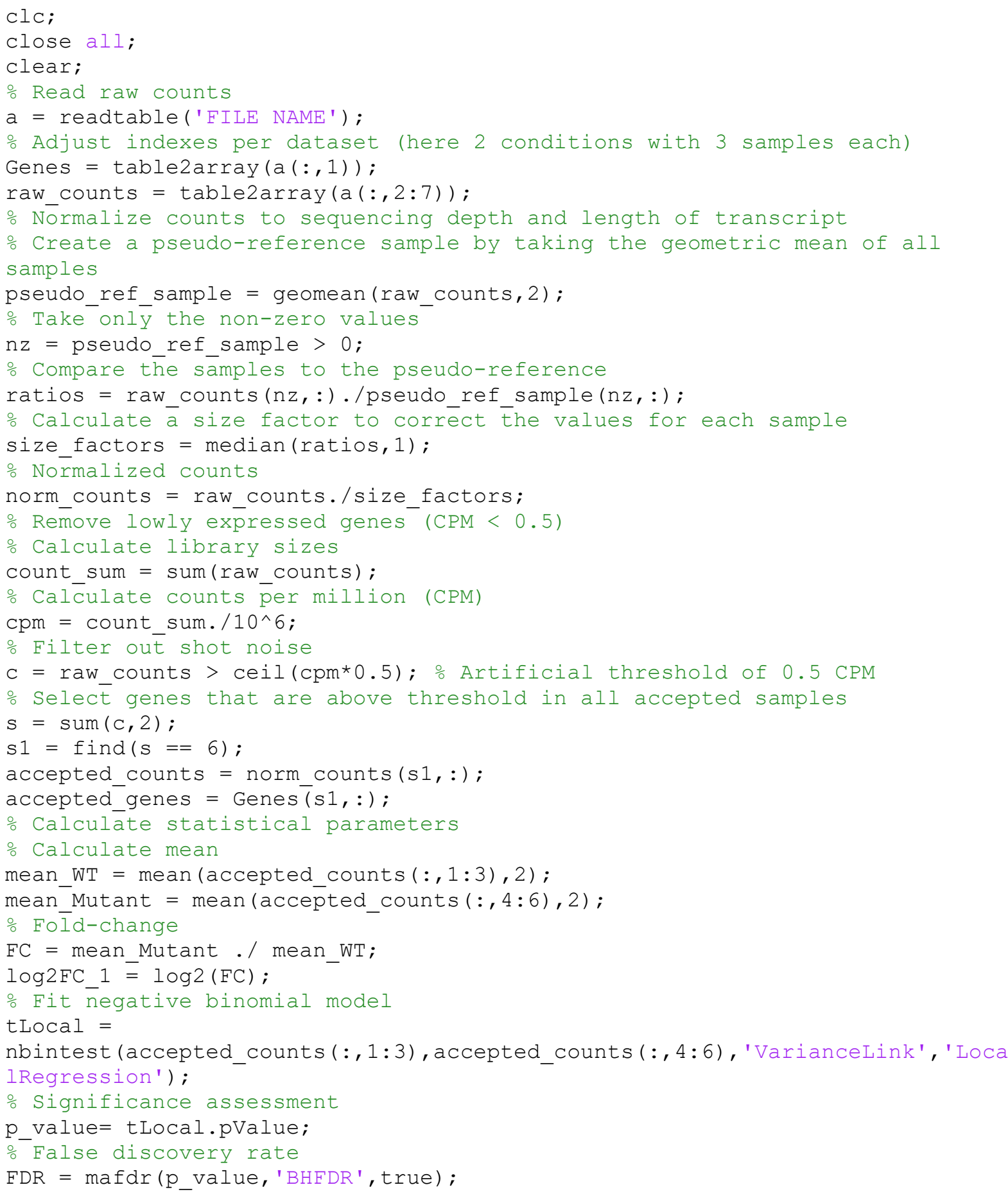




\subsection{Proteomics and metabolomics differential expression analysis script}

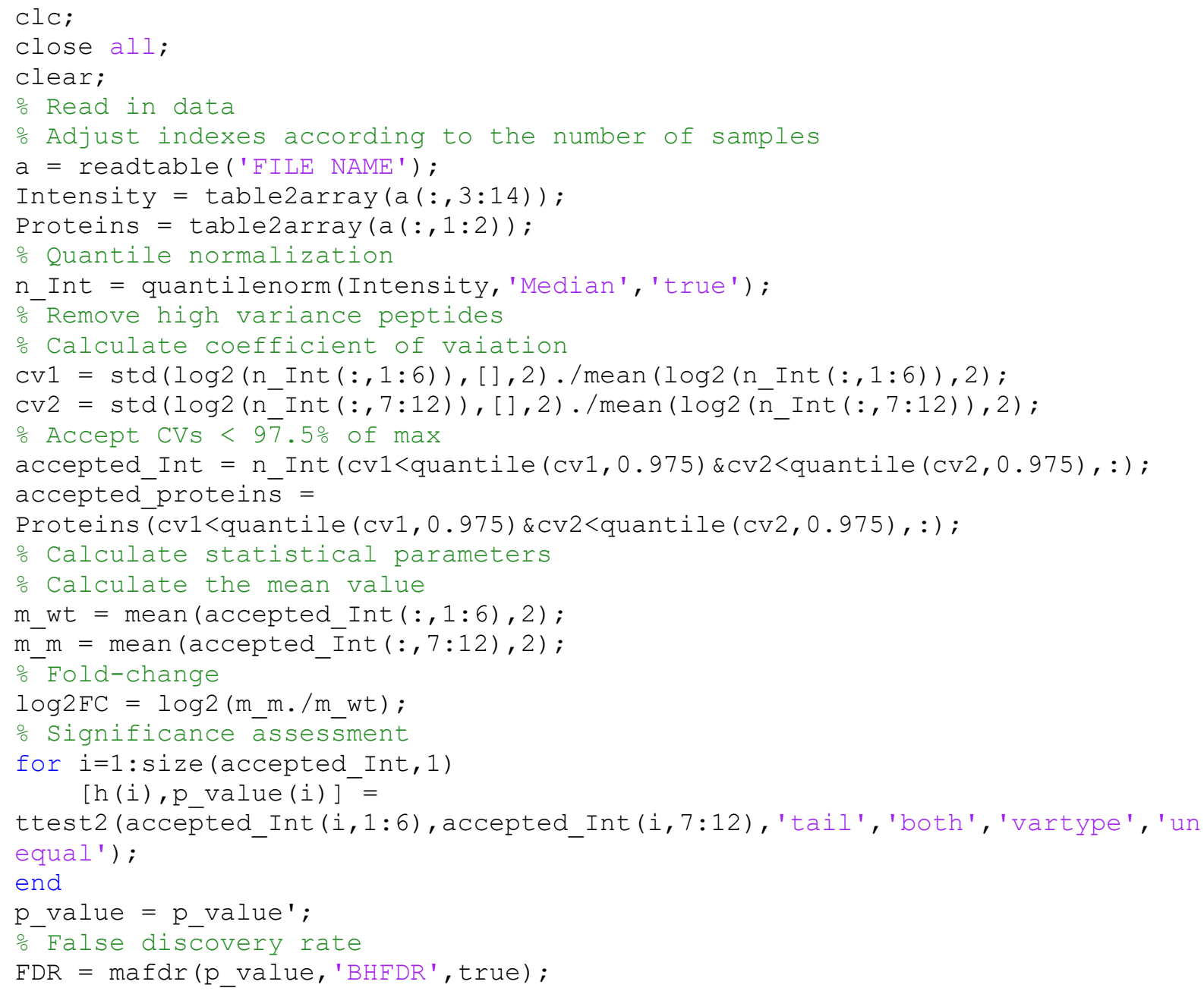




\subsection{RNA-seq (CeGaT) - N2 (wild-type) vs HBR227 (aptf-1) - 48h Starved}

\begin{tabular}{|c|c|c|c|c|c|c|c|}
\hline Gene & $\log 2 \mathrm{FC}$ & Gene & $\log 2 \mathrm{FC}$ & Gene & $\log 2 \mathrm{FC}$ & Gene & $\log 2 \mathrm{FC}$ \\
\hline gsto-1 & -3.31 & col-80 & -2 & W04E12.7 & -1.76 & $\mathrm{ctl}-2$ & -1.6 \\
\hline txt-4 & -3.14 & asm-2 & -1.99 & nnt-1 & -1.76 & Y73B6A.3 & -1.6 \\
\hline asp-13 & -3.01 & F45E1.5 & -1.98 & Y9С9A.8 & -1.75 & C08F11.3 & -1.59 \\
\hline dpy-5 & -2.78 & gst- 6 & -1.96 & arrd-11 & -1.74 & lys-7 & -1.59 \\
\hline dod-24 & -2.76 & F47B8.4 & -1.95 & sqst-2 & -1.74 & Y70C5A.3 & -1.59 \\
\hline sru-40 & -2.68 & pals-34 & -1.95 & pals-14 & -1.74 & hsp-16.41 & -1.58 \\
\hline col-50 & -2.66 & F22B7.9 & -1.95 & $\mathrm{C} 17 \mathrm{H} 12.8$ & -1.74 & C36B1.13 & -1.58 \\
\hline dhs-20 & -2.61 & hsp-12.3 & -1.94 & сур-29A2 & -1.73 & clec-52 & -1.58 \\
\hline asp-14 & -2.59 & grd-3 & -1.93 & F10A3.4 & -1.72 & F58B4.5 & -1.58 \\
\hline cest-9.1 & -2.58 & T05F1.11 & -1.92 & asp-1 & -1.71 & fbxa-185 & -1.58 \\
\hline F42C5.3 & -2.57 & fbxa-182 & -1.92 & pgap-3 & -1.71 & B0410.3 & -1.57 \\
\hline fbxa-172 & -2.49 & gldc-1 & -1.92 & col-159 & -1.71 & pck-2 & -1.57 \\
\hline asm-3 & -2.48 & $\mathrm{~F} 40 \mathrm{H} 3.2$ & -1.92 & C08F11.13 & -1.7 & W10G11.1 & -1.57 \\
\hline F55G11.8 & -2.48 & Imd-4 & -1.91 & gst-38 & -1.69 & F10G2.1 & -1.56 \\
\hline F12A10.1 & -2.45 & cest-13 & -1.9 & prmt- 6 & -1.69 & F37C4.6 & -1.56 \\
\hline asp-2 & -2.43 & sdz-24 & -1.9 & C33E10.8 & -1.69 & R09E10.13 & -1.56 \\
\hline linc-61 & -2.36 & T20D3.2 & -1.9 & fbxa-1 & -1.69 & $\mathrm{E} 02 \mathrm{H} 4.4$ & -1.55 \\
\hline C55A6.4 & -2.27 & B0403.6 & -1.89 & gst-41 & -1.69 & T05E7.1 & -1.55 \\
\hline ugt- 6 & -2.27 & col-93 & -1.89 & ZC196.2 & -1.68 & pho-11 & -1.55 \\
\hline scav-1 & -2.26 & F42C5.5 & -1.89 & $\mathrm{cdr}-2$ & -1.68 & pals-33 & -1.55 \\
\hline col-101 & -2.22 & C06G3.3 & -1.88 & F08H9.3 & -1.68 & clec- 55 & -1.55 \\
\hline $\mathrm{ctl}-1$ & -2.21 & F54H5.2 & -1.88 & afmd-1 & -1.66 & сур-32B1 & -1.53 \\
\hline dao-6 & -2.19 & nhr-17 & -1.88 & clec-60 & -1.66 & $\mathrm{C} 39 \mathrm{H} 7.4$ & -1.53 \\
\hline F18E3.11 & -2.18 & C24B5.4 & -1.88 & K08D12.6 & -1.66 & T25C12.3 & -1.52 \\
\hline haf-9 & -2.17 & col-160 & -1.87 & ddn-1 & -1.66 & pcp-1 & -1.52 \\
\hline T06C12.14 & -2.17 & $\mathrm{fbxa}-6$ & -1.86 & clec-17 & -1.65 & T08E11.1 & -1.52 \\
\hline C55A6.7 & -2.14 & C52E2.5 & -1.85 & klo-1 & -1.65 & Y47G6A.7 & -1.51 \\
\hline C24B9.3 & -2.14 & CC8.2 & -1.84 & hrg-1 & -1.64 & ZK593.3 & -1.51 \\
\hline ifc-1 & -2.12 & mul-1 & -1.83 & F19C7.1 & -1.64 & col-147 & -1.51 \\
\hline pmp-5 & -2.1 & gst-14 & -1.83 & Y34B4A.9 & -1.63 & zig-9 & -1.5 \\
\hline LLC1.2 & -2.07 & Y51H4A.24 & -1.82 & сур-34A10 & -1.63 & F40G12.5 & -1.5 \\
\hline far-5 & -2.06 & C31C9.7 & -1.82 & F13H8.11 & -1.63 & Y51B9A.9 & -1.5 \\
\hline gfi-1 & -2.05 & gpx-7 & -1.81 & hpo-8 & -1.61 & $\mathrm{mtl}-2$ & -1.5 \\
\hline vha- 6 & -2.05 & slc-17.9 & -1.8 & T28C6.8 & -1.61 & F56F10.1 & -1.5 \\
\hline col-98 & -2.04 & F35F10.1 & -1.8 & ZK1240.1 & -1.61 & F17B5.1 & -1.49 \\
\hline hpo-34 & -2.03 & R05F9.6 & -1.78 & Y37D8A.4 & -1.61 & Y105C5A.24 & -1.49 \\
\hline clec-53 & -2.03 & Y34B4A.6 & -1.78 & F57F4.4 & -1.61 & T05C3.6 & -1.49 \\
\hline ZK993.5 & -2.02 & $\mathrm{mtl}-1$ & -1.77 & $\mathrm{R} 13 \mathrm{H} 4.2$ & -1.6 & nep-17 & -1.48 \\
\hline swt-3 & -2.02 & Y51F10.7 & -1.77 & F44A6.5 & -1.6 & cpin-1 & -1.48 \\
\hline gpx-6 & -2 & haf-4 & -1.77 & F28A12.3 & -1.6 & paic-1 & -1.47 \\
\hline fmo-2 & -2 & col-176 & -1.77 & catp-3 & -1.6 & ZC250.4 & -1.47 \\
\hline
\end{tabular}




\begin{tabular}{|c|c|c|c|c|c|c|c|}
\hline Gene & $\log 2 \mathrm{FC}$ & Gene & $\log 2 \mathrm{FC}$ & Gene & $\log 2 \mathrm{FC}$ & Gene & $\log 2 \mathrm{FC}$ \\
\hline ugt-23 & -1.37 & F18E3.13 & -1.3 & T25B9.1 & -1.24 & tnt-2 & -1.19 \\
\hline R07E3.4 & -1.37 & $\mathrm{zmp}-4$ & -1.3 & vha- 8 & -1.24 & vha-13 & -1.19 \\
\hline aakg-4 & -1.37 & hlh-13 & -1.29 & asah-1 & -1.24 & R05A10.1 & -1.19 \\
\hline C54F6.6 & -1.36 & mdl-1 & -1.28 & F41E7.2 & -1.24 & clec-5 & -1.19 \\
\hline col-103 & -1.36 & hum-5 & -1.28 & F56C9.7 & -1.24 & C44E12.1 & -1.19 \\
\hline clec-72 & -1.36 & cpr-5 & -1.28 & C10A4.3 & -1.24 & F49C12.6 & -1.19 \\
\hline EGAP4.1 & -1.36 & Y7A5A.1 & -1.28 & dhs-21 & -1.24 & dhs-24 & -1.19 \\
\hline dhs-7 & -1.36 & сур-37B1 & -1.28 & F09C8.1 & -1.23 & K12C11.6 & -1.19 \\
\hline aat-1 & -1.36 & F21C10.11 & -1.27 & $x \operatorname{tr}-1$ & -1.23 & C46G7.2 & -1.18 \\
\hline mca-1 & -1.36 & F35E12.6 & -1.27 & clec-76 & -1.23 & T21H3.1 & -1.18 \\
\hline F14F3.5 & -1.36 & vha-5 & -1.27 & T10C6.6 & -1.23 & C06E4.3 & -1.18 \\
\hline cbl-1 & -1.35 & Y119D3B.14 & -1.27 & hpo-15 & -1.23 & sqd-1 & -1.18 \\
\hline F09B12.3 & -1.35 & B0334.3 & -1.27 & $n d x-3$ & -1.23 & irg-7 & -1.18 \\
\hline $\mathrm{fbxa}-54$ & -1.35 & F10E9.12 & -1.27 & M01E5.2 & -1.23 & C39D10.11 & -1.17 \\
\hline col-91 & -1.34 & gst-16 & -1.27 & irg-3 & -1.23 & T04A8.7 & -1.17 \\
\hline pcbd-1 & -1.34 & F46G10.1 & -1.27 & $\mathrm{~F} 47 \mathrm{H} 4.2$ & -1.23 & iars-2 & -1.17 \\
\hline gta-1 & -1.34 & ZC196.5 & -1.26 & gst-33 & -1.23 & T15B7.1 & -1.17 \\
\hline C49C3.4 & -1.34 & T19D12.1 & -1.26 & F29B9.1 & -1.23 & aakg-5 & -1.17 \\
\hline F21H7.12 & -1.33 & F48D6.4 & -1.26 & сур-25A3 & -1.22 & C49A9.3 & -1.17 \\
\hline C18B2.5 & -1.33 & $\mathrm{~F} 46 \mathrm{H} 5.7$ & -1.26 & gра-17 & -1.22 & $\mathrm{nft}-1$ & -1.16 \\
\hline ugt-2 & -1.33 & fat-4 & -1.26 & eol-1 & -1.22 & ucr-1 & -1.16 \\
\hline ech-7 & -1.33 & F10G8.9 & -1.26 & ctps-1 & -1.22 & Y65B4BL.7 & -1.16 \\
\hline Y67H2A.7 & -1.33 & idhg-2 & -1.26 & $\mathrm{C} 32 \mathrm{H} 11.4$ & -1.22 & F32D8.12 & -1.16 \\
\hline col-107 & -1.33 & T10C6.15 & -1.26 & F40A3.2 & -1.22 & pha-1 & -1.16 \\
\hline vha-15 & -1.33 & gcst-1 & -1.26 & flp-11 & -1.22 & T25C12.12 & -1.16 \\
\hline C50F4.1 & -1.33 & cars-1 & -1.26 & frm-4 & -1.22 & clec-84 & -1.16 \\
\hline lys-8 & -1.33 & E04F6.9 & -1.26 & asg-2 & -1.22 & clec-82 & -1.16 \\
\hline clec-85 & -1.32 & bath-46 & -1.25 & nstp-1 & -1.21 & Y43F8B.24 & -1.16 \\
\hline fbxa-162 & -1.32 & alh-1 & -1.25 & ZK550.6 & -1.21 & F41C3.2 & -1.16 \\
\hline $\operatorname{ttr}-24$ & -1.32 & VF13D12L.3 & -1.25 & B0393.9 & -1.21 & gba-4 & -1.16 \\
\hline Y34B4A.5 & -1.31 & F58F12.1 & -1.25 & Y43C5A.2 & -1.21 & $\operatorname{lgc}-25$ & -1.15 \\
\hline F52E1.5 & -1.31 & twk-18 & -1.25 & wars-1 & -1.21 & ctsa-3.1 & -1.15 \\
\hline$n c x-1$ & -1.31 & nmur-2 & -1.25 & spp-1 & -1.2 & gst-10 & -1.15 \\
\hline hda-5 & -1.31 & nhr-106 & -1.25 & prdx-6 & -1.2 & $\mathrm{C} 28 \mathrm{H} 8.14$ & -1.15 \\
\hline сур-34A9 & -1.31 & aagr-2 & -1.25 & cpg-9 & -1.2 & ceeh-2 & -1.15 \\
\hline exc-15 & -1.31 & mct-3 & -1.25 & drd-5 & -1.2 & pho-5 & -1.15 \\
\hline kcc-1 & -1.31 & Y45F3A.9 & -1.25 & T28F3.8 & -1.2 & ZC373.5 & -1.15 \\
\hline vgln-1 & -1.3 & maoc-1 & -1.24 & clu-1 & -1.19 & ucr-2.1 & -1.15 \\
\hline C01G10.9 & -1.3 & Y69A2AR.18 & -1.24 & chil-16 & -1.19 & C44E4.5 & -1.15 \\
\hline F52E1.14 & -1.3 & ril-2 & -1.24 & M28.10 & -1.19 & tkt-1 & -1.14 \\
\hline $\mathrm{T} 19 \mathrm{H} 12.3$ & -1.3 & vha-16 & -1.24 & dct-11 & -1.19 & F07H5.5 & -1.14 \\
\hline
\end{tabular}




\begin{tabular}{|c|c|c|c|c|c|c|c|}
\hline Gene & $\log 2 \mathrm{FC}$ & Gene & $\log 2 \mathrm{FC}$ & Gene & $\log 2 \mathrm{FC}$ & Gene & $\log 2 \mathrm{FC}$ \\
\hline ril-1 & -1.14 & asp-6 & -1.1 & $c d r-4$ & -1.06 & F32A5.8 & -1.02 \\
\hline T01D3.6 & -1.14 & C33A12.19 & -1.1 & D1022.4 & -1.06 & fig-1 & -1.02 \\
\hline gсp-2.1 & -1.14 & F26D11.12 & -1.1 & сур-33E1 & -1.06 & dig-1 & -1.02 \\
\hline C49G7.3 & -1.14 & trxr-2 & -1.1 & acp-6 & -1.06 & Y37E11B.5 & -1.02 \\
\hline col-142 & -1.14 & fbxa-105 & -1.1 & cpn-3 & -1.06 & adt-2 & -1.02 \\
\hline $\mathrm{gfm}-1$ & -1.14 & sek-1 & -1.1 & R10E8.6 & -1.06 & F25B5.5 & -1.01 \\
\hline B0035.13 & -1.14 & serp-1.2 & -1.1 & tsn-1 & -1.05 & sqrd-1 & -1.01 \\
\hline prx-6 & -1.14 & Imp-2 & -1.1 & C49A9.6 & -1.05 & let-611 & -1.01 \\
\hline сус-1 & -1.14 & vps-33.1 & -1.1 & cgr-1 & -1.05 & pals-24 & -1.01 \\
\hline T01G1.4 & -1.14 & F14F9.8 & -1.09 & $\operatorname{cox}-15$ & -1.05 & $\mathrm{R} 10 \mathrm{H} 10.3$ & -1.01 \\
\hline aex-5 & -1.13 & best-14 & -1.09 & $\mathrm{H} 18 \mathrm{~N} 23.2$ & -1.05 & mboa-7 & -1.01 \\
\hline sur-5 & -1.13 & M01F1.3 & -1.09 & gly-6 & -1.05 & nas-11 & -1.01 \\
\hline nuo-5 & -1.13 & gst-3 & -1.09 & dct-18 & -1.05 & F46C5.10 & -1.01 \\
\hline K02E11.7 & -1.13 & vars-1 & -1.09 & dhs-9 & -1.05 & tsp-10 & -1.01 \\
\hline F54D5.4 & -1.13 & gst-15 & -1.09 & vha-11 & -1.05 & pqn-25 & -1.01 \\
\hline coa-7 & -1.13 & pin-2 & -1.09 & skr-19 & -1.04 & prx-5 & -1.01 \\
\hline C14C6.5 & -1.13 & cal-5 & -1.09 & T08B1.1 & -1.04 & akt-2 & -1 \\
\hline F23B12.4 & -1.13 & tag-18 & -1.09 & clec-66 & -1.04 & myo-3 & -1 \\
\hline nstp-4 & -1.13 & acs-7 & -1.09 & hrpa-2 & -1.04 & ins-27 & -1 \\
\hline gst-27 & -1.13 & rom-2 & -1.09 & apm-1 & -1.04 & $\mathrm{mps}-2$ & -1 \\
\hline F31A3.3 & -1.13 & F43D2.7 & -1.09 & M01B2.13 & -1.04 & Y102A11A.3 & -1 \\
\hline egl-20 & -1.13 & ztf-17 & -1.09 & R07E3.1 & -1.04 & dsh-2 & 1 \\
\hline F08D12.3 & -1.13 & C01B10.6 & -1.09 & aqp-10 & -1.04 & col-121 & 1 \\
\hline C53A5.17 & -1.12 & T06A1.5 & -1.08 & abce-1 & -1.04 & spp-22 & 1.01 \\
\hline dct- 6 & -1.12 & cav-2 & -1.08 & dlc-3 & -1.04 & otub-3 & 1.01 \\
\hline clec-37 & -1.12 & des- 2 & -1.08 & R03H10.1 & -1.04 & $\mathrm{rnr}-1$ & 1.01 \\
\hline asb-2 & -1.12 & R05D3.12 & -1.08 & ctsa-1.1 & -1.03 & ZK973.9 & 1.01 \\
\hline faah-3 & -1.12 & pals-18 & -1.08 & C05D2.11 & -1.03 & nlp-64 & 1.01 \\
\hline gst-4 & -1.12 & mboa-2 & -1.08 & tric-1B.1 & -1.03 & F59A2.5 & 1.01 \\
\hline sek-4 & -1.11 & elo-1 & -1.08 & сур-32A1 & -1.03 & K05C4.7 & 1.01 \\
\hline ZK185.3 & -1.11 & gst-13 & -1.07 & ola-1 & -1.03 & Y37A1B.7 & 1.01 \\
\hline $\mathrm{T} 24 \mathrm{C} 12.3$ & -1.11 & $\mathrm{C} 38 \mathrm{H} 2.2$ & -1.07 & K12C11.1 & -1.03 & ceh-45 & 1.01 \\
\hline ZK1320.3 & -1.11 & Y6E2A.4 & -1.07 & F09E5.3 & -1.03 & dpy-3 & 1.01 \\
\hline Y54G11A.7 & -1.11 & dod-3 & -1.07 & pgrn-1 & -1.03 & F13A7.11 & 1.01 \\
\hline clp-1 & -1.11 & mus- 81 & -1.07 & zyg-11 & -1.03 & $w d r-48$ & 1.01 \\
\hline spp-3 & -1.11 & fat-1 & -1.07 & Y39A1A.14 & -1.03 & bus- 8 & 1.01 \\
\hline snet-1 & -1.11 & ttr-1 & -1.06 & T11B7.5 & -1.02 & nduo-3 & 1.01 \\
\hline C07D8.6 & -1.11 & F01D5.1 & -1.06 & gst-36 & -1.02 & igeg-1 & 1.02 \\
\hline pyr-1 & -1.11 & prmt-5 & -1.06 & mig-6 & -1.02 & rsp-4 & 1.02 \\
\hline alh-12 & -1.1 & gas-1 & -1.06 & T19D12.2 & -1.02 & suds-3 & 1.02 \\
\hline F58H1.3 & -1.1 & lipl-2 & -1.06 & spp-10 & -1.02 & R07E3.2 & 1.02 \\
\hline
\end{tabular}




\begin{tabular}{|c|c|c|c|c|c|c|c|}
\hline Gene & $\log 2 \mathrm{FC}$ & Gene & $\log 2 \mathrm{FC}$ & Gene & $\log 2 \mathrm{FC}$ & Gene & $\log 2 \mathrm{FC}$ \\
\hline dhs-27 & 1.02 & dscc-1 & 1.07 & Y7A9D.1 & 1.12 & grl-21 & 1.18 \\
\hline F58G11.3 & 1.02 & snrp-27 & 1.07 & F53B3.3 & 1.12 & Irr-1 & 1.18 \\
\hline noah-2 & 1.02 & T16G12.8 & 1.07 & cpr-4 & 1.12 & T11B7.2 & 1.19 \\
\hline tcab-1 & 1.02 & swp-1 & 1.07 & bath-41 & 1.12 & F25H9.6 & 1.19 \\
\hline ugt-28 & 1.02 & T22B7.3 & 1.07 & his-74 & 1.12 & abf-5 & 1.19 \\
\hline $\mathrm{knl}-2$ & 1.02 & cng-1 & 1.07 & K08E4.2 & 1.13 & K08B4.2 & 1.19 \\
\hline cdt-1 & 1.02 & F59A6.5 & 1.08 & F54D5.5 & 1.13 & pph-4.2 & 1.19 \\
\hline ugt-29 & 1.02 & nlp-16 & 1.08 & uri-1 & 1.13 & B0513.4 & 1.19 \\
\hline F16B4.4 & 1.03 & puf-8 & 1.08 & ins-28 & 1.13 & mei-1 & 1.19 \\
\hline Isd-1 & 1.03 & F14B4.1 & 1.08 & pgl-2 & 1.13 & bub-1 & 1.19 \\
\hline tre-5 & 1.03 & lem-3 & 1.08 & C47E8.11 & 1.14 & F52B5.2 & 1.19 \\
\hline thoc-7 & 1.03 & F59E12.15 & 1.08 & Y17D7C.3 & 1.14 & adal-1 & 1.19 \\
\hline blos-4 & 1.03 & mlt-11 & 1.09 & F22E5.9 & 1.14 & C02F5.13 & 1.19 \\
\hline R02F11.2 & 1.03 & C27B7. 5 & 1.09 & R08F11.7 & 1.14 & dnsn-1 & 1.2 \\
\hline rad-50 & 1.03 & fbxa-44 & 1.09 & Y46G5A.7 & 1.14 & pole-1 & 1.2 \\
\hline Y54G11A.3 & 1.03 & B0511.7 & 1.09 & rnh-2 & 1.14 & bath-10 & 1.2 \\
\hline W10C8.4 & 1.03 & C44C10.9 & 1.09 & F46E10.2 & 1.14 & ceh-86 & 1.2 \\
\hline rbmx-2 & 1.03 & let-49 & 1.09 & ego-1 & 1.14 & F28F5.4 & 1.2 \\
\hline marg-1 & 1.04 & aly-2 & 1.09 & T09F5.12 & 1.14 & ccch-3 & 1.2 \\
\hline C16C8.16 & 1.04 & Y87G2A.19 & 1.09 & K09F6.13 & 1.14 & hmg-4 & 1.2 \\
\hline $\mathrm{CO} \mathrm{H} 9.16$ & 1.04 & K07C10.3 & 1.09 & F56D2.2 & 1.15 & him-5 & 1.2 \\
\hline nasp-1 & 1.04 & nlp-79 & 1.09 & F15D4.5 & 1.15 & snpc-1.2 & 1.21 \\
\hline F42A9.6 & 1.05 & pri-1 & 1.09 & T09B9.1 & 1.15 & ung-1 & 1.21 \\
\hline$g \ln -5$ & 1.05 & ZK1053.4 & 1.1 & oma-2 & 1.15 & taf-8 & 1.21 \\
\hline pafo-1 & 1.05 & tim-1 & 1.1 & igeg-2 & 1.15 & pgp-8 & 1.21 \\
\hline F27E5.7 & 1.05 & K02B7.3 & 1.1 & $\mathrm{mcm}-3$ & 1.16 & apc-10 & 1.21 \\
\hline daf-18 & 1.05 & C49G7.12 & 1.1 & erh-2 & 1.16 & K09D9.1 & 1.21 \\
\hline F09G2.8 & 1.05 & Y65A5A.1 & 1.1 & Y68A4A.13 & 1.17 & C09B8.5 & 1.21 \\
\hline C07A9.9 & 1.05 & K08E4.3 & 1.1 & F44E2.10 & 1.17 & tnt-4 & 1.21 \\
\hline cup-15 & 1.06 & fbxc-36 & 1.1 & T14G8.4 & 1.17 & C14B1.12 & 1.22 \\
\hline Y69A2AR.21 & 1.06 & tsp-2 & 1.11 & M28.5 & 1.17 & Y54E10BR.2 & 1.22 \\
\hline F37H8.2 & 1.06 & T07F12.4 & 1.11 & ZK669.3 & 1.17 & F56F11.4 & 1.22 \\
\hline F45F2.11 & 1.06 & E01A2.2 & 1.11 & ceh-2 & 1.17 & gly-11 & 1.22 \\
\hline dsl-3 & 1.06 & T12G3.4 & 1.11 & ptr-13 & 1.18 & ech-1.1 & 1.22 \\
\hline cdk-4 & 1.06 & Y53F4B.9 & 1.11 & clec-196 & 1.18 & B0024.4 & 1.22 \\
\hline tni-4 & 1.06 & F54E2.1 & 1.11 & C44C1.6 & 1.18 & F43C1.7 & 1.22 \\
\hline $\mathrm{mcm}-5$ & 1.06 & pqn-75 & 1.11 & K09F6.6 & 1.18 & dnj-20 & 1.22 \\
\hline Ipr-7 & 1.06 & wrt-10 & 1.11 & lips-3 & 1.18 & D2005.4 & 1.22 \\
\hline C25G6.4 & 1.07 & F01D4.5 & 1.12 & F47B10.8 & 1.18 & W04E12.9 & 1.23 \\
\hline pgp-4 & 1.07 & $\mathrm{C} 06 \mathrm{H} 2.7$ & 1.12 & skpt-1 & 1.18 & alg-5 & 1.23 \\
\hline mre-11 & 1.07 & F55A11.4 & 1.12 & cht-1 & 1.18 & nlp-31 & 1.23 \\
\hline
\end{tabular}




\begin{tabular}{|c|c|c|c|c|c|c|c|}
\hline Gene & $\log 2 \mathrm{FC}$ & Gene & $\log 2 \mathrm{FC}$ & Gene & $\log 2 \mathrm{FC}$ & Gene & $\log 2 \mathrm{FC}$ \\
\hline Igc-11 & 1.23 & C30F2.4 & 1.28 & knl-3 & 1.32 & F55C5.2 & 1.4 \\
\hline pgph-2 & 1.23 & hсp-6 & 1.28 & capg-2 & 1.32 & F39F10.4 & 1.41 \\
\hline Y47D3A.1 & 1.23 & B0001.7 & 1.28 & mdf-2 & 1.32 & pri-2 & 1.41 \\
\hline him-8 & 1.23 & F31C3.6 & 1.28 & klp-19 & 1.33 & Y14H12B.2 & 1.41 \\
\hline F27C1.4 & 1.24 & F13A7.14 & 1.28 & skr-17 & 1.33 & Y75B8A.18 & 1.41 \\
\hline sas-5 & 1.24 & T04C12.7 & 1.28 & Y47H9C.1 & 1.33 & $\mathrm{cbd}-1$ & 1.41 \\
\hline $\mathrm{C} 31 \mathrm{H} 1.8$ & 1.24 & $\mathrm{~K} 08 \mathrm{H} 2.4$ & 1.29 & asns-2 & 1.33 & wago-1 & 1.41 \\
\hline T05B9.1 & 1.24 & dnj-11 & 1.29 & mesp-1 & 1.33 & cpg-1 & 1.41 \\
\hline Y46H3A.5 & 1.24 & K06H7.7 & 1.29 & pcn-1 & 1.33 & rmd-1 & 1.41 \\
\hline hсp-1 & 1.24 & B0348.2 & 1.29 & $\mathrm{fbxb}-104$ & 1.34 & C35E7.5 & 1.42 \\
\hline ZK1037.6 & 1.24 & C30G12.6 & 1.3 & irg-5 & 1.34 & W02B8.2 & 1.43 \\
\hline fbxa-206 & 1.25 & ndfl-4 & 1.3 & Y41D4B.26 & 1.34 & C40A11.2 & 1.43 \\
\hline cep-1 & 1.25 & C08F1.10 & 1.3 & pch-2 & 1.34 & F14F9.4 & 1.44 \\
\hline gla-3 & 1.25 & hecw-1 & 1.3 & tbb-6 & 1.34 & ndc -80 & 1.44 \\
\hline zig-4 & 1.25 & F25G6.1 & 1.3 & fbxb-95 & 1.35 & F26A1.9 & 1.44 \\
\hline nop-1 & 1.25 & gpr-1 & 1.3 & C33F10.4 & 1.35 & col-78 & 1.45 \\
\hline orc-4 & 1.25 & Y106G6H.6 & 1.3 & B0285.3 & 1.35 & fbxc-44 & 1.45 \\
\hline pemd-1 & 1.25 & col-111 & 1.3 & $\csc -1$ & 1.36 & K09F6.10 & 1.45 \\
\hline rab-11.2 & 1.26 & F52B5.7 & 1.3 & W01D2.6 & 1.36 & C03D6.9 & 1.45 \\
\hline in $x-2$ & 1.26 & Ү39АЗА.4 & 1.3 & T16G12.4 & 1.36 & klp-18 & 1.45 \\
\hline hasp-1 & 1.26 & tost-1 & 1.3 & Y17G9B.2 & 1.37 & $n x f-2$ & 1.46 \\
\hline sdz-28 & 1.26 & cutl-2 & 1.3 & Y49E10.4 & 1.37 & ttr-29 & 1.46 \\
\hline Y51F10.2 & 1.26 & ssna-1 & 1.3 & C25F9.2 & 1.37 & $\mathrm{~K} 10 \mathrm{H} 10.10$ & 1.46 \\
\hline hil-5 & 1.26 & pqn-60 & 1.31 & R13A5.7 & 1.37 & C14B1.9 & 1.46 \\
\hline C34C12.7 & 1.26 & pgl-3 & 1.31 & dlc-5 & 1.37 & Y58A7A.3 & 1.46 \\
\hline crn-4 & 1.26 & B0261.7 & 1.31 & clec-266 & 1.38 & C50F4.6 & 1.46 \\
\hline $\mathrm{C} 24 \mathrm{H} 12.1$ & 1.26 & $\mathrm{C} 42 \mathrm{C} 1.12$ & 1.31 & B0393.3 & 1.38 & C35E7.3 & 1.46 \\
\hline Ipr-3 & 1.27 & $\mathrm{C} 25 \mathrm{H} 3.1$ & 1.31 & chaf-2 & 1.38 & exo-3 & 1.46 \\
\hline F17C11.11 & 1.27 & $\mathrm{C} 18 \mathrm{H} 7.11$ & 1.31 & ify-1 & 1.38 & T10B11.8 & 1.46 \\
\hline $\mathrm{rsp}-5$ & 1.27 & hrde-1 & 1.31 & flh-3 & 1.39 & T01C3.2 & 1.46 \\
\hline $\mathrm{cmt}-1$ & 1.27 & $a b f-6$ & 1.31 & ima-2 & 1.39 & T11B7.1 & 1.46 \\
\hline rde-4 & 1.27 & zeel-1 & 1.31 & cdc-7 & 1.39 & oef-1 & 1.47 \\
\hline icp-1 & 1.27 & ncr-2 & 1.31 & ptr-22 & 1.39 & C41D11.9 & 1.47 \\
\hline $9 / 1 / 2020$ & 1.27 & fbxb-75 & 1.31 & col-169 & 1.39 & iff-1 & 1.47 \\
\hline F10D2.10 & 1.27 & $\mathrm{~F} 36 \mathrm{~A} 2.10$ & 1.32 & cec-4 & 1.39 & kbp-3 & 1.47 \\
\hline acox -1.2 & 1.27 & dnj-22 & 1.32 & F41E6.7 & 1.39 & gena-1 & 1.47 \\
\hline pitr-3 & 1.27 & pid-3 & 1.32 & Y73F4A.3 & 1.39 & F56A12.2 & 1.48 \\
\hline T02C1.1 & 1.28 & M01D1.12 & 1.32 & W04A8.1 & 1.39 & aptf-2 & 1.48 \\
\hline T22F3.11 & 1.28 & K09F6.9 & 1.32 & scc-1 & 1.39 & T25B6.5 & 1.48 \\
\hline сур-33С6 & 1.28 & hch-1 & 1.32 & gpdh-1 & 1.4 & fbxc-18 & 1.48 \\
\hline F48E3.6 & 1.28 & C47D2.1 & 1.32 & Y4C6B.1 & 1.4 & sym-1 & 1.49 \\
\hline
\end{tabular}




\begin{tabular}{|c|c|c|c|c|c|c|c|}
\hline Gene & $\log 2 \mathrm{FC}$ & Gene & $\log 2 \mathrm{FC}$ & Gene & $\log 2 \mathrm{FC}$ & Gene & $\log 2 \mathrm{FC}$ \\
\hline T04C12.32 & 1.49 & R144.10 & 1.57 & air-2 & 1.65 & $\mathrm{rbm}-3.1$ & 1.76 \\
\hline cdk-1 & 1.49 & msh-2 & 1.57 & syp-1 & 1.66 & fbxb-54 & 1.76 \\
\hline C05D9.4 & 1.49 & col-181 & 1.57 & R05H5.3 & 1.66 & C38D4.4 & 1.76 \\
\hline $\mathrm{K} 10 \mathrm{H} 10.4$ & 1.49 & Y45F10D.2 & 1.57 & T05D4.2 & 1.66 & F40F11.3 & 1.77 \\
\hline nhr-2 & 1.5 & ttr-27 & 1.57 & Ү39АЗА. 3 & 1.66 & $\mathrm{kbp}-2$ & 1.78 \\
\hline polh-1 & 1.5 & $\mathrm{mcm}-4$ & 1.57 & cec- 8 & 1.67 & Y39G10AL.1 & 1.78 \\
\hline C08E3.1 & 1.5 & W04A8.5 & 1.58 & Y37H2A.14 & 1.67 & T04D3.1 & 1.78 \\
\hline egg-3 & 1.5 & ctf-4 & 1.58 & pole-4 & 1.67 & kbp-4 & 1.78 \\
\hline him-10 & 1.5 & clx-1 & 1.58 & fbxa-50 & 1.67 & pash-1 & 1.78 \\
\hline C06E2.5 & 1.5 & D2005.7 & 1.58 & K04G2.10 & 1.67 & spp-20 & 1.8 \\
\hline htp-1 & 1.5 & F13A7.12 & 1.58 & rsa-1 & 1.67 & Y45G5AM.5 & 1.81 \\
\hline klc-1 & 1.5 & F22B5.4 & 1.58 & ZK829.9 & 1.68 & T05A7.1 & 1.82 \\
\hline K07D4.4 & 1.51 & Y17D7B.3 & 1.59 & F59C12.4 & 1.69 & T03G11.3 & 1.82 \\
\hline Iron-1 & 1.52 & Y52E8A.2 & 1.59 & $\mathrm{fbxb}-3$ & 1.69 & vet-2 & 1.82 \\
\hline kbp-1 & 1.52 & C23G10.11 & 1.59 & san-1 & 1.69 & F08F8.4 & 1.82 \\
\hline mei-2 & 1.52 & chaf-1 & 1.59 & ttr-28 & 1.69 & F15A4.2 & 1.83 \\
\hline puf-3 & 1.52 & C55B7.11 & 1.6 & W03C9.2 & 1.7 & clec- 172 & 1.83 \\
\hline F32A7.8 & 1.52 & Y38E10A.14 & 1.6 & such-1 & 1.7 & cah-4 & 1.83 \\
\hline set-31 & 1.52 & ZK1127.13 & 1.6 & kbp-5 & 1.7 & psf-1 & 1.83 \\
\hline Y106G6D.2 & 1.53 & $\mathrm{fbxc}-25$ & 1.6 & F08F3.4 & 1.71 & hil-4 & 1.83 \\
\hline fbxb-15 & 1.53 & set-9 & 1.6 & F13E9.14 & 1.72 & vet-1 & 1.83 \\
\hline C09B8.4 & 1.53 & syp-6 & 1.6 & pot-2 & 1.72 & mbtr-1 & 1.84 \\
\hline ins-11 & 1.53 & nhr-161 & 1.6 & C48B4.9 & 1.72 & T05H10.4 & 1.84 \\
\hline ZK622.4 & 1.53 & ttr-38 & 1.6 & F11E6.7 & 1.72 & Y58A7A.4 & 1.85 \\
\hline nlp-67 & 1.53 & $\mathrm{~K} 03 \mathrm{H} 1.7$ & 1.61 & gna-2 & 1.73 & skr-10 & 1.85 \\
\hline ttr-50 & 1.54 & C50F7.5 & 1.61 & hpr-17 & 1.73 & dut-1 & 1.86 \\
\hline C13A10.2 & 1.54 & ced-8 & 1.62 & $\mathrm{mcm}-7$ & 1.73 & hсp-2 & 1.86 \\
\hline crn-1 & 1.54 & cey-3 & 1.62 & $s d z-4$ & 1.73 & Y106G6D.1 & 1.86 \\
\hline col-145 & 1.54 & valv-1 & 1.62 & hal-2 & 1.73 & skr-13 & 1.86 \\
\hline hal-3 & 1.54 & immt-2 & 1.62 & F54F7.6 & 1.73 & R11.4 & 1.86 \\
\hline $\mathrm{fbxb}-84$ & 1.54 & memi-2 & 1.62 & $\mathrm{C} 24 \mathrm{H} 10.1$ & 1.73 & T28A8.3 & 1.87 \\
\hline $\mathrm{fbxc}-40$ & 1.54 & psf-2 & 1.63 & K08E5.1 & 1.74 & spo-11 & 1.87 \\
\hline C08F1.6 & 1.55 & aptf-4 & 1.63 & Y59A8B.12 & 1.74 & mis-12 & 1.87 \\
\hline mom-2 & 1.55 & neg-1 & 1.63 & C37C3.9 & 1.74 & Y43B11AL.1 & 1.87 \\
\hline F09C6.3 & 1.55 & psf-3 & 1.63 & F23A7.4 & 1.74 & $\mathrm{~K} 04 \mathrm{H} 4.5$ & 1.88 \\
\hline axl-1 & 1.55 & T25E12.6 & 1.64 & ogr-2 & 1.74 & $\mathrm{mcm}-10$ & 1.89 \\
\hline F15B9.6 & 1.55 & C05D12.4 & 1.64 & fbxa-155 & 1.74 & F14B6.3 & 1.9 \\
\hline rnr-2 & 1.55 & toca-2 & 1.65 & btb-11 & 1.75 & oig-3 & 1.9 \\
\hline ptr-2 & 1.55 & atz-1 & 1.65 & gld-1 & 1.75 & skr-7 & 1.92 \\
\hline fzy-1 & 1.56 & ndnf-1 & 1.65 & $\mathrm{fbxc}-32$ & 1.76 & C29A12.1 & 1.92 \\
\hline hcp-4 & 1.56 & C01B12.8 & 1.65 & cyb-1 & 1.76 & T28A8.4 & 1.92 \\
\hline
\end{tabular}




\begin{tabular}{|c|c|c|c|c|c|}
\hline Gene & $\log 2 \mathrm{FC}$ & Gene & $\log 2 \mathrm{FC}$ & Gene & $\log 2 \mathrm{FC}$ \\
\hline ZC434.8 & 1.92 & gyg-2 & 2.17 & ceh-83 & 2.49 \\
\hline fbxb-72 & 1.93 & orc-3 & 2.17 & ZC308.4 & 2.5 \\
\hline vet -6 & 1.94 & F09C6.1 & 2.19 & cdc-25.3 & 2.5 \\
\hline K02B12.2 & 1.94 & ins-20 & 2.2 & F09E10.15 & 2.51 \\
\hline R09F10.8 & 1.94 & pie-1 & 2.2 & cyb-3 & 2.52 \\
\hline $\mathrm{F} 22 \mathrm{H} 10.2$ & 1.94 & mct-5 & 2.21 & F32D1.7 & 2.55 \\
\hline gpd-4 & 1.94 & him-3 & 2.21 & C08E8.3 & 2.59 \\
\hline F19B10.10 & 1.95 & sun-1 & 2.22 & cyb-2.2 & 2.6 \\
\hline$g \ln -6$ & 1.95 & mans-4 & 2.22 & gpd-1 & 2.64 \\
\hline T07D3.9 & 1.95 & tipn-1 & 2.22 & mex-1 & 2.67 \\
\hline sld-2 & 1.96 & puf-5 & 2.23 & Y39B6A.10 & 2.72 \\
\hline skr-9 & 1.97 & F56F4.8 & 2.24 & EEED8.3 & 2.72 \\
\hline flh-1 & 1.97 & puf-11 & 2.25 & $3 / 3 / 2020$ & 2.79 \\
\hline linc- 6 & 1.97 & $\mathrm{~T} 19 \mathrm{H} 12.2$ & 2.25 & nasp-2 & 2.81 \\
\hline gei-14 & 1.97 & syp-5 & 2.25 & Isy-27 & 2.85 \\
\hline Y42H9B.3 & 1.98 & set-22 & 2.26 & F08F3.6 & 2.85 \\
\hline skr-8 & 1.98 & htp-2 & 2.26 & pos-1 & 2.88 \\
\hline F53F8.3 & 1.99 & T05F1.2 & 2.26 & ceh-49 & 2.93 \\
\hline mut-2 & 2.01 & szy-4 & 2.27 & C42C1.8 & 2.96 \\
\hline C17E4.2 & 2.01 & mex-5 & 2.29 & clec-91 & 2.97 \\
\hline Y111B2A.2 & 2.02 & C09F12.2 & 2.29 & nspe-1 & 2.98 \\
\hline skr-14 & 2.03 & T21C9.13 & 2.3 & C05C10.5 & 3.02 \\
\hline btb- 6 & 2.04 & W06B4.1 & 2.31 & F23A7.8 & 3.05 \\
\hline sdz-30 & 2.05 & Y54G9A.5 & 2.32 & T12G3.6 & 3.07 \\
\hline DY3.8 & 2.05 & glp-1 & 2.32 & clec- 88 & 3.09 \\
\hline ZC155.4 & 2.06 & C08F8.3 & 2.33 & F14D7.2 & 3.1 \\
\hline sip-1 & 2.06 & R04D3.2 & 2.35 & ins-3 & 3.28 \\
\hline $\mathrm{F} 54 \mathrm{H} 12.5$ & 2.06 & skr-15 & 2.36 & era-1 & 3.31 \\
\hline Y60C6A.2 & 2.07 & sdz-14 & 2.36 & $\mathrm{~F} 14 \mathrm{H} 3.6$ & 3.39 \\
\hline gld-3 & 2.07 & Y71A12B.11 & 2.39 & clec- 87 & 3.49 \\
\hline in $x-14$ & 2.07 & str-112 & 2.42 & & \\
\hline trt-1 & 2.08 & cey-2 & 2.42 & & \\
\hline C01G6.3 & 2.08 & F56C9.6 & 2.42 & & \\
\hline B0281.5 & 2.1 & ctf-8 & 2.42 & & \\
\hline hmg-3 & 2.1 & taf-11.2 & 2.43 & & \\
\hline htp-3 & 2.1 & W05F2.3 & 2.44 & & \\
\hline C44B9.3 & 2.11 & fbxa-215 & 2.44 & & \\
\hline C04A2.15 & 2.11 & Y110A2AR.1 & 2.46 & & \\
\hline dsl-2 & 2.12 & $\operatorname{trcs}-1$ & 2.46 & & \\
\hline nspe-7 & 2.13 & F18A1.7 & 2.47 & & \\
\hline orc-1 & 2.16 & spn-4 & 2.47 & & \\
\hline
\end{tabular}


6.8. RNA-seq (CeGaT) - N2 (wild-type) vs HBR227 (aptf-1) - 4h Fed

\begin{tabular}{|c|c|c|c|c|c|c|c|}
\hline Gene & $\log 2 \mathrm{FC}$ & Gene & $\log 2 \mathrm{FC}$ & Gene & $\log 2 \mathrm{FC}$ & Gene & $\log 2 \mathrm{FC}$ \\
\hline gst-33 & -2.25 & clec- 230 & -1.48 & phat-3 & -1.3 & F09F7.6 & -1.17 \\
\hline nspc-16 & -2.2 & F15E6.10 & -1.47 & irg-3 & -1.3 & clec-166 & -1.16 \\
\hline F11C7.7 & -2.15 & asm-3 & -1.47 & C07H4.1 & -1.29 & T02B11.4 & -1.16 \\
\hline cyp-13A6 & -2.09 & C06E4.3 & -1.47 & clec-199 & -1.29 & ins-29 & -1.16 \\
\hline сур-33С2 & -1.98 & $\mathrm{~T} 10 \mathrm{H} 4.13$ & -1.46 & grd-6 & -1.29 & F45E10.2 & -1.16 \\
\hline gba-2 & -1.97 & W03D2.6 & -1.46 & gst-19 & -1.28 & F25E5.8 & -1.16 \\
\hline cpr-2 & -1.97 & F19C6.4 & -1.46 & gst-12 & -1.27 & $\mathrm{~F} 55 \mathrm{H} 12.2$ & -1.16 \\
\hline flp-11 & -1.94 & fbxa-203 & -1.45 & F42A8.1 & -1.27 & ZC395.5 & -1.15 \\
\hline F16B4.5 & -1.91 & F14F3.5 & -1.45 & scl-5 & -1.27 & R08F11.4 & -1.14 \\
\hline Y65B4BR.1 & -1.89 & F40E3.5 & -1.45 & dct-16 & -1.26 & F32H5.3 & -1.14 \\
\hline fipr-7 & -1.83 & T05A10.6 & -1.45 & K02E11.6 & -1.26 & papl-1 & -1.14 \\
\hline сyp-33D1 & -1.81 & T05E12.6 & -1.44 & T05E7.1 & -1.26 & nduo-3 & -1.14 \\
\hline clec-55 & -1.8 & F09C8.1 & -1.43 & Y73F4A.1 & -1.25 & D2023.1 & -1.14 \\
\hline F57H12.6 & -1.75 & B0478.3 & -1.43 & Т19H12.3 & -1.25 & ftn-1 & -1.14 \\
\hline rpr-1 & -1.75 & gst-8 & -1.42 & F22B7.9 & -1.24 & glt-5 & -1.13 \\
\hline gst-38 & -1.74 & M01B2.13 & -1.42 & C06B8.2 & -1.24 & grd-14 & -1.13 \\
\hline C08F11.1 & -1.73 & ent-4 & -1.41 & F17B5.1 & -1.24 & H11E01.2 & -1.13 \\
\hline srb-16 & -1.72 & pud-4 & -1.41 & cdr-2 & -1.24 & ugt-61 & -1.13 \\
\hline gst-14 & -1.71 & lips-17 & -1.41 & col-153 & -1.24 & rncs-1 & -1.13 \\
\hline $\mathrm{C} 40 \mathrm{H} 1.7$ & -1.69 & fbxa-38 & -1.4 & pho-6 & -1.23 & F53F4.13 & -1.12 \\
\hline Y34F4.2 & -1.69 & B0334.13 & -1.4 & ZK593.2 & -1.23 & msrp-7 & -1.12 \\
\hline clec-61 & -1.68 & C35A11.4 & -1.4 & F18E3.13 & -1.23 & ZK822.6 & -1.11 \\
\hline ora-1 & -1.68 & $\mathrm{mtl}-2$ & -1.39 & T11G6.3 & -1.22 & ndfl-4 & -1.11 \\
\hline F46C5.10 & -1.67 & F44G4.6 & -1.39 & fipr-26 & -1.22 & C50F4.8 & -1.11 \\
\hline pgp-12 & -1.67 & W02D7.11 & -1.38 & Y46G5A.36 & -1.22 & F41G3.10 & -1.1 \\
\hline srg-34 & -1.66 & C27B7.9 & -1.38 & spp-18 & -1.21 & W10C8.6 & -1.1 \\
\hline C24A11.1 & -1.64 & Y73B6A.3 & -1.37 & C06B3.6 & -1.21 & ZK593.3 & -1.1 \\
\hline cyp-25A2 & -1.64 & F22D6.15 & -1.37 & R06F6.14 & -1.21 & nlp-18 & -1.1 \\
\hline nspc-14 & -1.64 & W07G4.8 & -1.37 & F08H9.3 & -1.2 & сур-13A10 & -1.1 \\
\hline F18E3.11 & -1.62 & nspc-18 & -1.36 & bca-1 & -1.2 & ZK353.10 & -1.09 \\
\hline Y55F3BR.10 & -1.61 & srsx-34 & -1.35 & vap-1 & -1.2 & folt-2 & -1.09 \\
\hline snet-1 & -1.61 & B0410.3 & -1.35 & H32K16.2 & -1.2 & sulp-5 & -1.09 \\
\hline col-184 & -1.6 & сур-13A7 & -1.35 & nspc-20 & -1.19 & $\mathrm{cdr}-4$ & -1.09 \\
\hline ZK384.7 & -1.57 & T11B7.5 & -1.34 & F11C7.2 & -1.19 & K07A1.3 & -1.09 \\
\hline pudl-2 & -1.56 & F57A8.4 & -1.34 & F25D1.5 & -1.19 & fip-2 & -1.09 \\
\hline F12E12.11 & -1.56 & R13D7.2 & -1.32 & sodh-1 & -1.19 & C06G3.3 & -1.09 \\
\hline C06E8.5 & -1.54 & Y39B6A.29 & -1.32 & ilys-5 & -1.18 & txt-4 & -1.09 \\
\hline K01D12.9 & -1.53 & F32D8.11 & -1.32 & gpx-7 & -1.18 & R05D8.7 & -1.08 \\
\hline T25G12.6 & -1.52 & txt-3 & -1.31 & Y69A2AR.22 & -1.18 & F56D6.17 & -1.08 \\
\hline T07G12.5 & -1.51 & Y54G2A.41 & -1.31 & col-58 & -1.18 & T12B3.3 & -1.08 \\
\hline С03АЗ.3 & -1.51 & fipr-6 & -1.31 & F21C10.10 & -1.17 & fmo-2 & -1.08 \\
\hline
\end{tabular}




\begin{tabular}{|c|c|c|c|c|c|c|c|}
\hline Gene & $\log 2 \mathrm{FC}$ & Gene & $\log 2 \mathrm{FC}$ & Gene & $\log 2 \mathrm{FC}$ & Gene & $\log 2 \mathrm{FC}$ \\
\hline ZK1320.9 & -1.07 & apc-11 & 1.01 & cdk-4 & 1.04 & spd-5 & 1.1 \\
\hline $\mathrm{CC} 8.2$ & -1.07 & ergo-1 & 1.01 & him-6 & 1.04 & R17.3 & 1.1 \\
\hline ZK822.4 & -1.07 & clec-266 & 1.01 & klp-19 & 1.04 & Ipr-4 & 1.1 \\
\hline C10C5.7 & -1.06 & F22E5.9 & 1.01 & ZK688.9 & 1.04 & hyl-1 & 1.1 \\
\hline T22C8.6 & -1.06 & mett-10 & 1.01 & pafo-1 & 1.05 & dpy-2 & 1.1 \\
\hline Y51F10.7 & -1.06 & dpy-7 & 1.01 & ent-1 & 1.05 & atf-8 & 1.1 \\
\hline ddo-2 & -1.06 & R11H6.4 & 1.01 & cdc- 25.2 & 1.05 & T22C1.5 & 1.11 \\
\hline asp-14 & -1.06 & com-1 & 1.02 & F33E2.5 & 1.05 & ZC449.1 & 1.11 \\
\hline spp-2 & -1.06 & Y82E9BR.17 & 1.02 & Y105C5B.19 & 1.05 & drsh-1 & 1.11 \\
\hline cest- 26 & -1.06 & C50D2.8 & 1.02 & tbp-1 & 1.05 & $\mathrm{C} 16 \mathrm{C} 8.16$ & 1.11 \\
\hline F23F12.12 & -1.05 & $\mathrm{~F} 54 \mathrm{H} 12.5$ & 1.02 & unc-59 & 1.06 & ska-3 & 1.11 \\
\hline acs-2 & -1.05 & suds-3 & 1.02 & ntp-1 & 1.06 & B0205.9 & 1.11 \\
\hline Y49E10.18 & -1.05 & fbxa-101 & 1.02 & C27H6.9 & 1.06 & C48B4.11 & 1.11 \\
\hline T16G1.4 & -1.04 & arle-14 & 1.02 & B0511.7 & 1.06 & R07B7.10 & 1.12 \\
\hline F29C6.1 & -1.04 & snpc-3.4 & 1.02 & F35D2.3 & 1.06 & F36F12.1 & 1.12 \\
\hline T10C6.15 & -1.04 & F48E3.6 & 1.02 & $\mathrm{fkb}-7$ & 1.06 & phf-10 & 1.12 \\
\hline C38C3.10 & -1.04 & $\mathrm{rnp}-5$ & 1.03 & drh-3 & 1.06 & tag-342 & 1.12 \\
\hline gstk-1 & -1.04 & Y65A5A.1 & 1.03 & fnci-1 & 1.06 & trpl-2 & 1.12 \\
\hline F59A7.5 & -1.03 & $\mathrm{~F} 32 \mathrm{H} 2.10$ & 1.03 & mdt-6 & 1.07 & cec- 6 & 1.12 \\
\hline frpr-19 & -1.03 & mom-5 & 1.03 & K02B12.2 & 1.07 & hcf-1 & 1.12 \\
\hline F19F10.3 & -1.03 & F33H1.3 & 1.03 & ZK1127.13 & 1.07 & dsl-6 & 1.12 \\
\hline Y41C4A.17 & -1.03 & lin-42 & 1.03 & T20F5.4 & 1.07 & F52D2.7 & 1.12 \\
\hline nlp-20 & -1.02 & F41G3.6 & 1.03 & F13A7.14 & 1.07 & slx-1 & 1.12 \\
\hline ZK1307.1 & -1.02 & wrt-1 & 1.03 & F01F1.11 & 1.07 & vang-1 & 1.13 \\
\hline col-77 & -1.02 & Y69A2AR.28 & 1.03 & ego-1 & 1.08 & chl-1 & 1.13 \\
\hline fig-1 & -1.02 & C15A11.4 & 1.03 & hrpk-2 & 1.08 & nspe-7 & 1.13 \\
\hline fip-6 & -1.02 & C25F6.7 & 1.03 & egl-46 & 1.08 & isw-1 & 1.13 \\
\hline nlp-69 & -1.01 & mes- 6 & 1.03 & ZK973.1 & 1.08 & T24D1.2 & 1.13 \\
\hline fipr-1 & -1.01 & C09F9.2 & 1.03 & rsp-4 & 1.08 & cep-1 & 1.13 \\
\hline C31C9.7 & -1.01 & hhat-1 & 1.03 & $\operatorname{lin}-37$ & 1.09 & EEED8.14 & 1.13 \\
\hline nduo-6 & -1.01 & F09E10.6 & 1.03 & T25B6.5 & 1.09 & $\mathrm{Y} 69 \mathrm{H} 2.7$ & 1.13 \\
\hline C49A9.3 & -1.01 & Y48B6A.13 & 1.03 & F39F10.4 & 1.09 & algn-6 & 1.13 \\
\hline dod-3 & -1 & H03E18.1 & 1.04 & Y105E8A.14 & 1.09 & dpy-3 & 1.13 \\
\hline C33G8.4 & -1 & dnj-11 & 1.04 & pes- 5 & 1.09 & F52C6.2 & 1.13 \\
\hline tag-209 & -1 & Y46G5A.7 & 1.04 & ari-1.4 & 1.09 & C27F2.10 & 1.14 \\
\hline F10E9.3 & 1 & K10D3.4 & 1.04 & in $x-3$ & 1.09 & F44B9.8 & 1.14 \\
\hline C56C10.9 & 1 & $\mathrm{rsp}-6$ & 1.04 & spdl-1 & 1.1 & Ipr-5 & 1.14 \\
\hline ssna-1 & 1 & Y41D4B.14 & 1.04 & Y37A1B.7 & 1.1 & D1081.6 & 1.14 \\
\hline C08F1.10 & 1.01 & T09B9.1 & 1.04 & F01G4.4 & 1.1 & ppm-1.G & 1.14 \\
\hline R05D11.9 & 1.01 & B0432.8 & 1.04 & erh-1 & 1.1 & $\mathrm{fbxc}-18$ & 1.14 \\
\hline D1007.8 & 1.01 & nfyc-1 & 1.04 & mrt-1 & 1.1 & R05H10.3 & 1.14 \\
\hline
\end{tabular}




\begin{tabular}{|c|c|c|c|c|c|c|c|}
\hline Gene & $\log 2 \mathrm{FC}$ & Gene & $\log 2 \mathrm{FC}$ & Gene & $\log 2 \mathrm{FC}$ & Gene & $\log 2 \mathrm{FC}$ \\
\hline T26C12.3 & 1.15 & rfc-2 & 1.2 & Y52B11A.8 & 1.25 & cdc- 6 & 1.31 \\
\hline Y73F8A.13 & 1.15 & unc-85 & 1.2 & tyms-1 & 1.26 & trt-1 & 1.31 \\
\hline zipt-1 & 1.15 & pig-1 & 1.2 & pssy-2 & 1.26 & C48B4.10 & 1.31 \\
\hline C45G3.3 & 1.15 & hcp-3 & 1.2 & $\operatorname{ttr}-50$ & 1.26 & evl-18 & 1.31 \\
\hline ced-4 & 1.15 & wrt-10 & 1.2 & sdz-38 & 1.26 & lin-54 & 1.31 \\
\hline rsp-5 & 1.15 & ZK829.9 & 1.2 & zyg-1 & 1.26 & $\mathrm{fbxc}-25$ & 1.31 \\
\hline ztf-1 & 1.15 & F42A9.6 & 1.21 & cec-4 & 1.26 & C40A11.2 & 1.31 \\
\hline C14F11.6 & 1.15 & swd-2.1 & 1.21 & dna-2 & 1.27 & Y39G10AL.1 & 1.32 \\
\hline Y38A10A.7 & 1.15 & pigk-1 & 1.21 & Ist-1 & 1.27 & F23A7.4 & 1.32 \\
\hline npp-18 & 1.15 & sdha-2 & 1.21 & him-8 & 1.27 & C16C8.11 & 1.32 \\
\hline$|s|-1$ & 1.15 & C02F5.13 & 1.21 & let-49 & 1.27 & CzW-1 & 1.32 \\
\hline W04A8.6 & 1.15 & Y46G5A.8 & 1.21 & hmbx-1 & 1.27 & F09G2.8 & 1.32 \\
\hline K05C4.7 & 1.15 & nasp-1 & 1.21 & Ipr-6 & 1.27 & top-2 & 1.33 \\
\hline C06E7.88 & 1.16 & btb-11 & 1.21 & Y53G8AR.6 & 1.27 & eme-1 & 1.33 \\
\hline such-1 & 1.16 & htz-1 & 1.21 & B0001.2 & 1.28 & brc-1 & 1.33 \\
\hline ndc- 80 & 1.16 & T03F6.3 & 1.22 & E_BE45912.2 & 1.28 & Y106G6D.2 & 1.33 \\
\hline F26F4.5 & 1.16 & F59A6.5 & 1.22 & K08E4.2 & 1.28 & noah-2 & 1.33 \\
\hline ostb-1 & 1.16 & hmg-20 & 1.22 & $\mathrm{fbxb}-104$ & 1.28 & Y49E10.4 & 1.33 \\
\hline aos-1 & 1.16 & npp-2 & 1.22 & F55A11.8 & 1.28 & F27C1.4 & 1.33 \\
\hline cpf-1 & 1.16 & tim-1 & 1.22 & tdc-1 & 1.28 & dsh-2 & 1.33 \\
\hline parp-2 & 1.16 & C13F10.7 & 1.22 & C48B6.9 & 1.28 & C03D6.9 & 1.33 \\
\hline gras-1 & 1.16 & C49F5.6 & 1.22 & C04A11.2 & 1.28 & cec-3 & 1.34 \\
\hline hcp-4 & 1.16 & Y34D9A.7 & 1.22 & kbp-3 & 1.28 & D1046.2 & 1.34 \\
\hline C46A5.6 & 1.17 & damt-1 & 1.22 & sid-1 & 1.28 & dnsn-1 & 1.34 \\
\hline ect-2 & 1.17 & spr-3 & 1.23 & F54D5.9 & 1.29 & ZK616.5 & 1.34 \\
\hline rad-54.B & 1.17 & ZK813.6 & 1.23 & ptr-2 & 1.29 & $\mathrm{pgl}-2$ & 1.35 \\
\hline skr-9 & 1.17 & bub-3 & 1.23 & nduf-2.2 & 1.29 & bmk-1 & 1.35 \\
\hline $\mathrm{dsl}-3$ & 1.17 & mrg-1 & 1.23 & imp-1 & 1.29 & mut-7 & 1.35 \\
\hline F46E10.2 & 1.18 & in $x-2$ & 1.23 & ugt-63 & 1.29 & eaf-1 & 1.35 \\
\hline T04D3.5 & 1.18 & gbas-1 & 1.23 & C13G5.2 & 1.29 & R13A5.7 & 1.35 \\
\hline ZK1053.4 & 1.18 & ceh-86 & 1.23 & wago-4 & 1.29 & R07E4.5 & 1.36 \\
\hline ceh-40 & 1.19 & wrt-2 & 1.23 & cpar-1 & 1.3 & C35E7.5 & 1.36 \\
\hline $\operatorname{div}-1$ & 1.19 & sna-2 & 1.23 & apc-2 & 1.3 & T26A5.8 & 1.36 \\
\hline mig-32 & 1.19 & kca-1 & 1.24 & mlt-11 & 1.3 & metl-9 & 1.36 \\
\hline F44F1.6 & 1.19 & cye-1 & 1.24 & coh-4 & 1.3 & $\mathrm{knl}-3$ & 1.37 \\
\hline Y48G10A.2 & 1.19 & C17G10.1 & 1.25 & ssup-72 & 1.3 & Y53F4B.9 & 1.37 \\
\hline lin-53 & 1.19 & set-9 & 1.25 & skr-8 & 1.3 & ani-1 & 1.37 \\
\hline simr-1 & 1.19 & B0035.18 & 1.25 & T08G5.3 & 1.3 & asb-1 & 1.38 \\
\hline ZK643.2 & 1.19 & sex-1 & 1.25 & $\mathrm{JC} 8.7$ & 1.3 & R57.2 & 1.38 \\
\hline $\mathrm{nhr}-23$ & 1.2 & pid-2 & 1.25 & npp-23 & 1.31 & F59G1.8 & 1.39 \\
\hline unc-71 & 1.2 & fbxb-66 & 1.25 & noah-1 & 1.31 & Y75B8A.16 & 1.39 \\
\hline
\end{tabular}




\begin{tabular}{|c|c|c|c|c|c|c|c|}
\hline Gene & $\log 2 \mathrm{FC}$ & Gene & $\log 2 \mathrm{FC}$ & Gene & $\log 2 \mathrm{FC}$ & Gene & $\log 2 \mathrm{FC}$ \\
\hline inft-2 & 1.4 & F44E2.10 & 1.47 & psf-3 & 1.56 & chaf-2 & 1.68 \\
\hline icp-1 & 1.4 & $\mathrm{mcm}-10$ & 1.47 & fubl-4 & 1.56 & F01D4.5 & 1.68 \\
\hline tofu-1 & 1.4 & rod-1 & 1.47 & chaf-1 & 1.56 & chk-1 & 1.68 \\
\hline Y51H7C.3 & 1.4 & mis-12 & 1.48 & Y66D12A.16 & 1.57 & daf-18 & 1.68 \\
\hline Y41D4B.26 & 1.4 & sas-1 & 1.48 & fncm-1 & 1.57 & hcp-1 & 1.7 \\
\hline lem-3 & 1.41 & F35C11.5 & 1.48 & erh-2 & 1.57 & $f b x b-53$ & 1.7 \\
\hline C47F8.1 & 1.41 & C44F1.1 & 1.48 & mre-11 & 1.57 & F15B10.3 & 1.7 \\
\hline orc-4 & 1.41 & fbxb-35 & 1.48 & $\mathrm{mlh}-1$ & 1.57 & hrde-2 & 1.7 \\
\hline Y95D11A.3 & 1.41 & rpa-1 & 1.49 & msh-6 & 1.57 & ctf-8 & 1.7 \\
\hline tipn-1 & 1.42 & fbxb-15 & 1.49 & C41D11.9 & 1.58 & C40A11.4 & 1.7 \\
\hline W02B8.2 & 1.42 & pola-1 & 1.49 & skr-10 & 1.58 & sas- 6 & 1.71 \\
\hline T13F2.6 & 1.42 & aspm-1 & 1.49 & hlh-3 & 1.58 & Y106G6H.6 & 1.71 \\
\hline crn-1 & 1.42 & W04A8.5 & 1.49 & hcp-6 & 1.58 & mans-4 & 1.71 \\
\hline F54D10.5 & 1.43 & unc-61 & 1.5 & ekl-5 & 1.58 & dvc-1 & 1.71 \\
\hline cdt-1 & 1.43 & mei-1 & 1.5 & pal-1 & 1.58 & Y49F6B.9 & 1.72 \\
\hline F56D2.2 & 1.43 & F08F8.4 & 1.51 & wrt-4 & 1.59 & W05F2.6 & 1.72 \\
\hline F48A11.4 & 1.43 & T05D4.2 & 1.51 & F15A4.2 & 1.59 & pid-3 & 1.72 \\
\hline D2030.8 & 1.43 & npp-22 & 1.51 & C36B1.11 & 1.6 & Y71F9AL.6 & 1.72 \\
\hline C39E9.12 & 1.44 & C56A3.8 & 1.52 & cec-7 & 1.6 & hcp-2 & 1.73 \\
\hline tag-63 & 1.44 & cyk-4 & 1.52 & bub-1 & 1.6 & sygl-1 & 1.73 \\
\hline hmg-12 & 1.44 & hal-3 & 1.52 & fbxb-54 & 1.61 & skr-2 & 1.73 \\
\hline him-10 & 1.44 & Ipr-3 & 1.52 & F07H5.10 & 1.61 & K04G2.10 & 1.74 \\
\hline tcab-1 & 1.44 & fbn-1 & 1.53 & $\mathrm{dpl}-1$ & 1.61 & neg-1 & 1.74 \\
\hline thk-1 & 1.44 & xpo-2 & 1.53 & C48B4.9 & 1.61 & snrp-40.2 & 1.75 \\
\hline Y71F9AL.7 & 1.44 & aly-2 & 1.53 & B0041.8 & 1.61 & glh-3 & 1.75 \\
\hline fbxa-107 & 1.44 & pph-4.2 & 1.53 & Y43B11AL.1 & 1.62 & zipt-7.1 & 1.75 \\
\hline$w d r-48$ & 1.44 & cutl-16 & 1.53 & Y37E11AL.3 & 1.62 & Y48G1A.2 & 1.76 \\
\hline pri-1 & 1.44 & brd-1 & 1.53 & cutl-2 & 1.62 & dsl-2 & 1.76 \\
\hline ifa-3 & 1.45 & Y39B6A.42 & 1.54 & pole-1 & 1.63 & ced-8 & 1.76 \\
\hline cdc-25.1 & 1.45 & T07C4.3 & 1.54 & prg-1 & 1.63 & F12F6.7 & 1.76 \\
\hline adal-1 & 1.45 & iff-1 & 1.54 & efl-1 & 1.64 & sld-2 & 1.77 \\
\hline $\mathrm{mcm}-6$ & 1.46 & gpr-2 & 1.54 & F52B5.2 & 1.65 & $\mathrm{scc}-1$ & 1.77 \\
\hline got-2.1 & 1.46 & T16G12.4 & 1.54 & F21D5.1 & 1.65 & Isd-1 & 1.78 \\
\hline Y17G9B.2 & 1.46 & zhp-3 & 1.54 & fbxa-83 & 1.65 & klp-18 & 1.78 \\
\hline rmh-1 & 1.46 & R11.4 & 1.54 & $\mathrm{C} 28 \mathrm{C} 12.12$ & 1.66 & taf-11.2 & 1.78 \\
\hline bath-15 & 1.46 & $z w l-1$ & 1.55 & rnp-8 & 1.66 & plk-1 & 1.78 \\
\hline Y75B8A.18 & 1.47 & rfc-4 & 1.55 & mes-4 & 1.67 & F54F7.6 & 1.79 \\
\hline rnh-2 & 1.47 & F45F2.11 & 1.55 & T26A5.2 & 1.67 & irg-5 & 1.79 \\
\hline cup-15 & 1.47 & rfc-3 & 1.55 & fbxc-29 & 1.67 & K07A1.1 & 1.79 \\
\hline otub-3 & 1.47 & sgo-1 & 1.56 & gpaa-1 & 1.67 & syp-1 & 1.8 \\
\hline cks-1 & 1.47 & vet-2 & 1.56 & cdl-1 & 1.67 & sdz-30 & 1.8 \\
\hline
\end{tabular}




\begin{tabular}{|c|c|c|c|c|c|c|c|}
\hline Gene & $\log 2 \mathrm{FC}$ & Gene & $\log 2 \mathrm{FC}$ & Gene & $\log 2 \mathrm{FC}$ & Gene & $\log 2 \mathrm{FC}$ \\
\hline fbxc-44 & 1.8 & gcna-1 & 1.91 & rnr-1 & 2.05 & plk-2 & 2.21 \\
\hline B0281.5 & 1.8 & D1081.7 & 1.91 & sep-1 & 2.05 & apc-10 & 2.21 \\
\hline F31B9.3 & 1.81 & pif-1 & 1.92 & C01G6.3 & 2.06 & ogr-2 & 2.21 \\
\hline C17E4.2 & 1.81 & Y4C6B.1 & 1.92 & $\mathrm{mcm}-4$ & 2.06 & syp-2 & 2.21 \\
\hline tdpt-1 & 1.81 & deps-1 & 1.92 & tofu- 6 & 2.07 & $\mathrm{mcm}-2$ & 2.21 \\
\hline knl-1 & 1.82 & T04D3.1 & 1.92 & ung-1 & 2.07 & сур-31А3 & 2.22 \\
\hline let-99 & 1.82 & T05H10.4 & 1.92 & kbp-5 & 2.07 & him-3 & 2.23 \\
\hline dhs- 11 & 1.82 & T28A8.4 & 1.92 & exo-1 & 2.08 & C06E2.5 & 2.23 \\
\hline skr-17 & 1.83 & F58G11.3 & 1.92 & orc-3 & 2.08 & F14B6.3 & 2.23 \\
\hline fbxc-32 & 1.83 & hal-2 & 1.92 & fbxa-206 & 2.08 & skr-15 & 2.24 \\
\hline F40F11.3 & 1.83 & dscc-1 & 1.92 & tpxl-1 & 2.08 & Y51F10.2 & 2.25 \\
\hline T25E12.6 & 1.83 & gln-5 & 1.92 & edg-1 & 2.08 & set-31 & 2.25 \\
\hline $\mathrm{kbp}-4$ & 1.83 & F11E6.7 & 1.93 & gmn-1 & 2.08 & C27B7.5 & 2.25 \\
\hline gpr-1 & 1.83 & K07A1.17 & 1.93 & pemd-1 & 2.1 & set-32 & 2.25 \\
\hline M03B6.4 & 1.83 & ceh-83 & 1.94 & aptf-2 & 2.1 & fog-2 & 2.25 \\
\hline K03H1.7 & 1.84 & fem-3 & 1.94 & prom-1 & 2.11 & pcn-1 & 2.25 \\
\hline Y97E10AR.4 & 1.84 & gei-14 & 1.94 & F56F4.8 & 2.12 & F59A3.12 & 2.25 \\
\hline ced-3 & 1.84 & meg-3 & 1.96 & C01B12.8 & 2.12 & alg-5 & 2.26 \\
\hline $\mathrm{kbp}-1$ & 1.84 & F19B10.10 & 1.96 & btb-19 & 2.13 & $n x f-2$ & 2.26 \\
\hline dsb-2 & 1.85 & $\mathrm{cmt}-1$ & 1.97 & mdf-2 & 2.13 & ima-2 & 2.26 \\
\hline pash-1 & 1.85 & rmd-1 & 1.97 & W04A8.1 & 2.14 & hrde-1 & 2.27 \\
\hline flh-1 & 1.85 & fbxb-95 & 1.97 & C50C3.1 & 2.14 & htp-2 & 2.27 \\
\hline nemp-1 & 1.85 & H04M03.3 & 1.98 & air-1 & 2.14 & rbm-3.1 & 2.28 \\
\hline aptf-4 & 1.85 & skr-13 & 1.99 & sdz-28 & 2.15 & fbxa-210 & 2.28 \\
\hline cdk-1 & 1.85 & sas-5 & 1.99 & T16G12.8 & 2.15 & cyk-7 & 2.29 \\
\hline polh-1 & 1.86 & kbp-2 & 2 & spo-11 & 2.15 & syp-5 & 2.29 \\
\hline F10E9.7 & 1.86 & B0001.7 & 2 & bath-41 & 2.16 & $\mathrm{mcm}-3$ & 2.3 \\
\hline skr-7 & 1.86 & C37C3.9 & 2 & egg-6 & 2.17 & pch-2 & 2.3 \\
\hline zim-3 & 1.86 & gla-3 & 2.01 & nop-1 & 2.18 & eri-5 & 2.3 \\
\hline Y111B2A.3 & 1.86 & rad-51 & 2.01 & ctf-4 & 2.18 & hpo-40 & 2.32 \\
\hline W06D11.1 & 1.86 & D2030.7 & 2.01 & F13D12.5 & 2.18 & F54D5.2 & 2.32 \\
\hline vet-1 & 1.86 & pri-2 & 2.02 & ucr-2.3 & 2.18 & klc-1 & 2.33 \\
\hline$w d r-5.3$ & 1.87 & rsa-2 & 2.03 & tost-1 & 2.18 & D2005.4 & 2.33 \\
\hline air-2 & 1.87 & btb-6 & 2.03 & zen-4 & 2.19 & T10B11.8 & 2.33 \\
\hline $\mathrm{mcm}-5$ & 1.89 & F54D5.5 & 2.03 & W03C9.2 & 2.19 & Irr-1 & 2.34 \\
\hline capg-2 & 1.89 & csc-1 & 2.03 & $\mathrm{rnr}-2$ & 2.19 & nhr-2 & 2.34 \\
\hline msh-2 & 1.89 & skr-14 & 2.03 & toe-2 & 2.19 & C48B4.7 & 2.34 \\
\hline tofu-7 & 1.9 & cpg-4 & 2.04 & orc-5 & 2.19 & ani-2 & 2.35 \\
\hline Y73B3A.1 & 1.9 & san-1 & 2.04 & $\mathrm{mcm}-7$ & 2.2 & mbtr-1 & 2.35 \\
\hline bir-2 & 1.9 & F30F8.1 & 2.05 & psf-1 & 2.2 & pigv-1 & 2.36 \\
\hline flh-3 & 1.9 & cdc-7 & 2.05 & gpd-4 & 2.21 & htp-3 & 2.37 \\
\hline
\end{tabular}




\begin{tabular}{|c|c|c|c|c|c|c|c|}
\hline Gene & $\log 2 \mathrm{FC}$ & Gene & $\log 2 \mathrm{FC}$ & Gene & $\log 2 \mathrm{FC}$ & Gene & $\log 2 \mathrm{FC}$ \\
\hline cdc-25.3 & 2.37 & orc-1 & 2.56 & sip-1 & 2.89 & DY3.8 & 3.36 \\
\hline fzy-1 & 2.37 & F23A7.8 & 2.57 & Isy-27 & 2.89 & memi-2 & 3.37 \\
\hline mut-2 & 2.37 & cyn-9 & 2.58 & gld-1 & 2.9 & spn-4 & 3.37 \\
\hline C14B1.9 & 2.38 & mom-2 & 2.59 & Y110A7A.15 & 2.91 & szy-4 & 3.39 \\
\hline psf-2 & 2.38 & T28A8.3 & 2.59 & R04D3.2 & 2.92 & cpg-1 & 3.45 \\
\hline set-22 & 2.38 & wee-1.3 & 2.59 & zif-1 & 2.94 & nasp-2 & 3.45 \\
\hline T05B9.1 & 2.39 & pid-4 & 2.61 & inx-14 & 2.94 & $\mathrm{cbd}-1$ & 3.45 \\
\hline cyb-3 & 2.39 & F33E11.2 & 2.62 & C01G8.1 & 2.95 & mei-2 & 3.48 \\
\hline B0304.2 & 2.4 & T22D1.5 & 2.62 & gln-6 & 2.95 & cey-2 & 3.48 \\
\hline vet- 6 & 2.41 & C29A12.1 & 2.64 & mex-5 & 2.96 & puf-3 & 3.5 \\
\hline exo-3 & 2.41 & syx-4 & 2.65 & ify-1 & 2.96 & clec-87 & 3.57 \\
\hline Y71A12B.11 & 2.41 & T28A8.5 & 2.65 & gyg-2 & 2.98 & F14D7.2 & 3.6 \\
\hline C38D4.4 & 2.42 & F18A1.7 & 2.65 & gpd-1 & 2.99 & $\operatorname{trcs}-1$ & 3.62 \\
\hline snpc-1.2 & 2.42 & hpr-17 & 2.66 & wago-1 & 3.02 & puf-11 & 3.67 \\
\hline cyb-1 & 2.43 & sdz-14 & 2.67 & C08F8.3 & 3.02 & cey-3 & 3.7 \\
\hline rsa-1 & 2.44 & egg-3 & 2.67 & T05F1.2 & 3.03 & oma-2 & 3.76 \\
\hline immt-2 & 2.45 & B0393.3 & 2.68 & daz-1 & 3.04 & $\mathrm{C} 31 \mathrm{H} 1.8$ & 4.31 \\
\hline K07F5.12 & 2.45 & pgl-1 & 2.69 & glp-1 & 3.05 & puf-5 & 4.57 \\
\hline oef-1 & 2.46 & ZC308.4 & 2.7 & pie-1 & 3.05 & & \\
\hline cpg-3 & 2.46 & Y54G11A.3 & 2.71 & F53F8.3 & 3.06 & & \\
\hline Y42Н9B.3 & 2.47 & dut-1 & 2.71 & mar-3 & 3.06 & & \\
\hline F56F11.4 & 2.48 & $\mathrm{C} 42 \mathrm{C} 1.8$ & 2.71 & fbxa-215 & 3.07 & & \\
\hline his-74 & 2.48 & R09F10.8 & 2.74 & era-1 & 3.08 & & \\
\hline nos-1 & 2.48 & Y14H12B.2 & 2.74 & W06B4.1 & 3.09 & & \\
\hline ZC434.8 & 2.48 & mct-5 & 2.78 & T12G3.6 & 3.09 & & \\
\hline Y111B2A.2 & 2.48 & Y39B6A.10 & 2.78 & EEED8.3 & 3.13 & & \\
\hline R05H5.3 & 2.48 & T21C9.13 & 2.79 & cyb-2.2 & 3.14 & & \\
\hline $\mathrm{pgl}-3$ & 2.48 & ceh-49 & 2.8 & F14H3.6 & 3.15 & & \\
\hline mesp-1 & 2.48 & zim-1 & 2.81 & Y110A2AR.1 & 3.15 & & \\
\hline htp-1 & 2.49 & Y54G9A.5 & 2.81 & D2005.7 & 3.17 & & \\
\hline R144.10 & 2.5 & hil-5 & 2.81 & Y73B6BL.23 & 3.18 & & \\
\hline F08F3.6 & 2.51 & hil-4 & 2.82 & sun-1 & 3.18 & & \\
\hline F56C9.3 & 2.51 & gna-2 & 2.82 & pos-1 & 3.22 & & \\
\hline Y59A8B.12 & 2.51 & hmg-3 & 2.84 & W05F2.3 & 3.24 & & \\
\hline skpt-1 & 2.51 & B0261.7 & 2.85 & clec- 88 & 3.29 & & \\
\hline C55B7.11 & 2.52 & toca-2 & 2.86 & F56C9.6 & 3.29 & & \\
\hline puf-8 & 2.53 & Т19H12.2 & 2.86 & Y52E8A.2 & 3.3 & & \\
\hline syp-6 & 2.53 & pot-2 & 2.87 & clec-91 & 3.32 & & \\
\hline H04D03.2 & 2.55 & F32D1.7 & 2.88 & mex-1 & 3.35 & & \\
\hline spsb-2 & 2.56 & xnd-1 & 2.88 & $\mathrm{C} 05 \mathrm{C} 10.5$ & 3.35 & & \\
\hline C44B9.3 & 2.56 & ZC155.4 & 2.88 & gld-3 & 3.35 & & \\
\hline
\end{tabular}


6.9. Proteome (TMT) - N2 (wild-type) vs HBR227 (aptf-1) - 48h Starved

\begin{tabular}{|c|c|c|c|c|c|c|c|}
\hline Protein & $\log 2 \mathrm{FC}$ & Protein & $\log 2 \mathrm{FC}$ & Protein & $\log 2 \mathrm{FC}$ & Protein & $\log 2 \mathrm{FC}$ \\
\hline perm-4 & -4.57 & asp-5 & -1.94 & vit-2 & -1.49 & dao-2 & -1.22 \\
\hline ilys-5 & -4.29 & vit-3 & -1.94 & F35C8.5 & -1.49 & set-18 & -1.22 \\
\hline Y59E9AR.1 & -3.84 & dct-16 & -1.93 & dod-19 & -1.48 & $\operatorname{gln}-6$ & -1.21 \\
\hline madd-3 & -3.61 & fmo-5 & -1.9 & pmp-5 & -1.48 & pid-3 & -1.21 \\
\hline folt-2 & -3.55 & F56C9.7 & -1.9 & glh-2 & -1.48 & F55G1.9 & -1.21 \\
\hline $\mathrm{K} 12 \mathrm{H} 4.7$ & -3.55 & ctsa-1.2 & -1.89 & vit-1 & -1.48 & ifp-1 & -1.21 \\
\hline dpy-10 & -3.54 & hpo-40 & -1.87 & gfat-1 & -1.47 & asp-14 & -1.19 \\
\hline ule- 5 & -3.46 & nuc-1 & -1.85 & pcp-4 & -1.45 & gldc-1 & -1.19 \\
\hline glh-1 & -3.36 & nrf-6 & -1.83 & ifc-1 & -1.45 & Y7A5A.1 & -1.19 \\
\hline Y62H9A.5 & -3.27 & asp-13 & -1.83 & lec-8 & -1.43 & ugt-62 & -1.18 \\
\hline D1054.10 & -3.14 & ost-1 & -1.82 & LLC1.2 & -1.43 & nhl-1 & -1.17 \\
\hline ZK813.3 & -3.14 & W05F2.3 & -1.81 & pgp-3 & -1.42 & hpo-34 & -1.16 \\
\hline $\mathrm{cbd}-1$ & -2.98 & T21H3.1 & -1.8 & asp-2 & -1.41 & R74.8 & -1.16 \\
\hline Y51F10.7 & -2.92 & T05H10.3 & -1.78 & $\mathrm{C} 23 \mathrm{H} 5.8$ & -1.39 & him-17 & -1.16 \\
\hline pgp-1 & -2.76 & hach-1 & -1.77 & $\mathrm{rnr}-1$ & -1.39 & T16G12.1 & -1.15 \\
\hline csr-1 & -2.73 & spp-5 & -1.75 & M02H5.8 & -1.39 & R10F2.4 & -1.15 \\
\hline T28F3.8 & -2.64 & chat-1 & -1.75 & gfi-1 & -1.38 & Y54E2A.4 & -1.15 \\
\hline C17G1.2 & -2.64 & vit-6 & -1.72 & far-2 & -1.35 & asp-3 & -1.15 \\
\hline cey-2 & -2.63 & $\mathrm{R} 10 \mathrm{H} 10.3$ & -1.72 & tba-4 & -1.35 & clec-87 & -1.15 \\
\hline hrg-7 & -2.61 & F19C7.1 & -1.7 & dhs- 25 & -1.34 & aagr-2 & -1.14 \\
\hline lys-4 & -2.6 & anp-1 & -1.69 & skpo-3 & -1.34 & timm-23 & -1.13 \\
\hline asp-1 & -2.51 & ima-2 & -1.69 & dnc-3 & -1.34 & afd-1 & -1.13 \\
\hline F54E2.1 & -2.5 & Iron-8 & -1.67 & W04B5.3 & -1.33 & rars-2 & -1.13 \\
\hline perm-2 & -2.49 & clec-63 & -1.67 & Iron-7 & -1.33 & mec-1 & -1.12 \\
\hline ule-1 & -2.48 & egg-1 & -1.66 & B0379.6 & -1.32 & sptl-1 & -1.11 \\
\hline F17E9.5 & -2.46 & vit-4 & -1.64 & grl-7 & -1.32 & bigr-1 & -1.11 \\
\hline acdh-1 & -2.41 & $\mathrm{C} 34 \mathrm{H} 4.2$ & -1.63 & R193.2 & -1.31 & sur-5 & -1.11 \\
\hline asp-6 & -2.37 & F52E1.14 & -1.63 & F58B4.5 & -1.31 & sago-2 & -1.11 \\
\hline F17E9.4 & -2.34 & F28B4.3 & -1.63 & F55G11.8 & -1.3 & Y69E1A.5 & -1.1 \\
\hline col-92 & -2.29 & H20J04.1 & -1.61 & irg-7 & -1.3 & C35A5.11 & -1.1 \\
\hline asns-2 & -2.29 & dot-1.1 & -1.6 & Y34B4A.6 & -1.29 & hcp-1 & -1.09 \\
\hline spp-3 & -2.29 & spp-2 & -1.59 & $\mathrm{~T} 25 \mathrm{C} 12.3$ & -1.29 & Y41E3.1 & -1.09 \\
\hline rme-2 & -2.25 & ugt-26 & -1.59 & cdr-4 & -1.28 & mct-2 & -1.09 \\
\hline F21C10.9 & -2.12 & glh-4 & -1.59 & rnp-1 & -1.27 & Czw-1 & -1.08 \\
\hline F09C8.1 & -2.1 & hphd-1 & -1.58 & ech-6 & -1.27 & aagr-1 & -1.08 \\
\hline ZK6.11 & -2.1 & dnj-11 & -1.58 & argk-1 & -1.27 & sip-1 & -1.07 \\
\hline F57F5.1 & -2.06 & dut-1 & -1.56 & oxy-4 & -1.27 & $d d x-17$ & -1.06 \\
\hline сур-13A5 & -2.03 & leo-1 & -1.55 & C29F3.7 & -1.26 & gst-35 & -1.05 \\
\hline clec-50 & -1.99 & pod-2 & -1.55 & gcst-1 & -1.26 & cdk-1 & -1.05 \\
\hline cpr-4 & -1.97 & vit-5 & -1.55 & C05C10.3 & -1.25 & hsp-6 & -1.04 \\
\hline C14F11.4 & -1.97 & F53F8.4 & -1.53 & spp-14 & -1.24 & pgp-6 & -1.04 \\
\hline cey-3 & -1.94 & Y34B4A.9 & -1.51 & spin-1 & -1.24 & K08D8.6 & -1.04 \\
\hline
\end{tabular}




\begin{tabular}{|c|c|c|c|c|c|}
\hline Protein & $\log 2 \mathrm{FC}$ & Protein & $\log 2 \mathrm{FC}$ & Protein & $\log 2 \mathrm{FC}$ \\
\hline R102.4 & -1.04 & hsp-16.2 & 1.09 & Ifi-1 & 1.26 \\
\hline Y48A6B.7 & -1.04 & F59F3.6 & 1.09 & tni-3 & 1.27 \\
\hline ints-13 & -1.03 & gpx-5 & 1.09 & $\operatorname{ttr}-23$ & 1.28 \\
\hline M18.3 & -1.03 & Y53F4B.23 & 1.1 & hmg-3 & 1.31 \\
\hline repo-1 & -1.03 & C02B10.3 & 1.1 & ZK863.8 & 1.31 \\
\hline ipla-1 & -1.02 & C32D5.8 & 1.12 & T10B10.3 & 1.35 \\
\hline wago-1 & -1.02 & ZC434.4 & 1.12 & T03F1.11 & 1.37 \\
\hline mctp-1 & -1.02 & pdl-1 & 1.12 & sod-3 & 1.37 \\
\hline vbh-1 & -1.01 & F47B8.2 & 1.13 & nlp-24 & 1.38 \\
\hline $\mathrm{mcm}-4$ & -1.01 & sgn-1 & 1.13 & pqn-94 & 1.4 \\
\hline Y65B4A.2 & 1 & srap-1 & 1.13 & rpi-2 & 1.42 \\
\hline viro-2 & 1 & pghm-1 & 1.14 & F26G1.2 & 1.42 \\
\hline exp-2 & 1.01 & nol-16 & 1.14 & F07C3.2 & 1.43 \\
\hline F54E4.3 & 1.01 & $\mathrm{scl}-3$ & 1.14 & col-39 & 1.43 \\
\hline sod-4 & 1.02 & E02C12.9 & 1.14 & tnt-2 & 1.43 \\
\hline F59B10.3 & 1.02 & exc-13 & 1.14 & cnnm-1 & 1.44 \\
\hline unc-27 & 1.02 & hil-3 & 1.14 & droe-4 & 1.44 \\
\hline ttr-16 & 1.02 & F55F8.3 & 1.14 & F58H1.7 & 1.46 \\
\hline his-24 & 1.02 & F17H10.2 & 1.14 & pud-1.1 & 1.47 \\
\hline gstk-2 & 1.02 & W03G11.3 & 1.15 & ttr-5 & 1.53 \\
\hline F39H12.3 & 1.02 & $\mathrm{ptl}-1$ & 1.15 & best-14 & 1.54 \\
\hline C55A6.6 & 1.02 & C24A3.2 & 1.15 & ensa-1 & 1.57 \\
\hline $\mathrm{H} 14 \mathrm{~N} 18.4$ & 1.03 & T03D8.6 & 1.15 & F53B3.6 & 1.64 \\
\hline F45D11.15 & 1.04 & F52E4.5 & 1.16 & F43C9.2 & 1.71 \\
\hline dig-1 & 1.05 & che-10 & 1.16 & K02F3.9 & 1.72 \\
\hline F40A3.2 & 1.05 & W05H12.1 & 1.17 & ftn-1 & 1.76 \\
\hline rnp-5 & 1.05 & T27C4.1 & 1.17 & sod-5 & 1.76 \\
\hline gana-1 & 1.05 & spp-13 & 1.17 & grd-3 & 1.77 \\
\hline pud-2.1 & 1.05 & $\mathrm{R} 10 \mathrm{H} 10.4$ & 1.17 & E04F6.9 & 1.83 \\
\hline ric-4 & 1.05 & $\mathrm{zmp}-3$ & 1.18 & $\mathrm{C} 18 \mathrm{H} 7.1$ & 1.83 \\
\hline C15C7.7 & 1.06 & C53D6.7 & 1.18 & msa-1 & 1.92 \\
\hline T28B11.1 & 1.06 & slc-36.5 & 1.18 & M01H9.3 & 1.93 \\
\hline F45E1.1 & 1.06 & Y52E8A.3 & 1.18 & C53B7.3 & 2.21 \\
\hline cat-1 & 1.06 & rab-3 & 1.19 & hil-1 & 2.22 \\
\hline tni-4 & 1.06 & R07B1.9 & 1.2 & F36H9.7 & 2.22 \\
\hline fkh-7 & 1.07 & F41D9.2 & 1.21 & Y53C10A.6 & 2.39 \\
\hline gst-20 & 1.07 & Y39B6A.5 & 1.22 & Y54G2A.57 & 2.57 \\
\hline ttr-46 & 1.07 & plpr-1 & 1.22 & cut-1 & 2.74 \\
\hline Iron-2 & 1.07 & mec-5 & 1.24 & asp-9 & 3.01 \\
\hline sng-1 & 1.07 & Y44A6D.2 & 1.25 & H39E23.3 & 3.29 \\
\hline F54C8.6 & 1.08 & sup-1 & 1.25 & scl-11 & 3.33 \\
\hline hsp-16.48 & 1.08 & dpf-1 & 1.25 & scl-12 & 3.78 \\
\hline
\end{tabular}


6.10. Proteome (TMT) - N2 (wild-type) vs HBR227 (aptf-1) - 4h Fed

\begin{tabular}{|c|c|c|c|c|c|}
\hline Protein & $\log 2 \mathrm{FC}$ & Protein & $\log 2 \mathrm{FC}$ & Protein & $\log 2 \mathrm{FC}$ \\
\hline $\mathrm{K} 12 \mathrm{H} 4.7$ & -3.3 & let-805 & -1.15 & vps-18 & 1.27 \\
\hline col-158 & -3.26 & F13D12.5 & -1.13 & lact-3 & 1.28 \\
\hline perm-4 & -3.15 & T21B6.3 & -1.13 & F25B4.7 & 1.28 \\
\hline cey-3 & -3.13 & unc-7 & -1.12 & F53F4.16 & 1.29 \\
\hline folt-2 & -2.92 & $\mathrm{~F} 23 \mathrm{H} 11.5$ & -1.11 & $\mathrm{~F} 39 \mathrm{H} 11.1$ & 1.3 \\
\hline Y62H9A.5 & -2.74 & endu-1 & -1.1 & eif-2Balpha & 1.3 \\
\hline ilys-5 & -2.53 & F17E9.5 & -1.07 & F41D9.2 & 1.34 \\
\hline scl-11 & -2.53 & kdp-1 & -1.07 & $\mathrm{C} 49 \mathrm{H} 3.6$ & 1.35 \\
\hline glh-1 & -2.49 & srp-1 & -1.06 & $\mathrm{M} 02 \mathrm{H} 5.8$ & 1.41 \\
\hline pqn-94 & -2.46 & сур-34A9 & -1.06 & B0432.7 & 1.41 \\
\hline grl-23 & -2.46 & grl-16 & -1.06 & comt-4 & 1.45 \\
\hline C14F11.4 & -2.4 & gst-19 & -1.06 & unc- 85 & 1.46 \\
\hline hex-2 & -2.17 & unc-52 & -1.06 & sft-1 & 1.48 \\
\hline perm-2 & -2.14 & hpo-32 & -1.06 & nol-10 & 1.5 \\
\hline cut-1 & -2.01 & $\mathrm{R} 02 \mathrm{C} 2.7$ & -1.04 & vit-1 & 1.5 \\
\hline tftc-3 & -1.89 & ZK185.5 & -1.01 & cka-2 & 1.51 \\
\hline lec- 8 & -1.89 & spr-4 & -1.01 & trpp-8 & 1.52 \\
\hline pud-2.1 & -1.87 & Y51F10.7 & -1.01 & vit-4 & 1.54 \\
\hline pud-1.1 & -1.8 & syd-9 & 1 & $\operatorname{csn}-5$ & 1.55 \\
\hline col-39 & -1.72 & $\mathrm{fdps}-1$ & 1.01 & trpa-2 & 1.58 \\
\hline D1054.10 & -1.6 & Y54E5A.5 & 1.02 & T10G3.3 & 1.6 \\
\hline cey-2 & -1.57 & egg-1 & 1.03 & rbm-3.2 & 1.66 \\
\hline H39E23.3 & -1.53 & dpy-30 & 1.03 & Y69E1A.5 & 1.68 \\
\hline scl-12 & -1.53 & F09F7.6 & 1.04 & ger-1 & 1.69 \\
\hline ZK6.11 & -1.52 & C45G9.5 & 1.05 & T26G10.1 & 1.74 \\
\hline cbd-1 & -1.51 & Y104H12D.2 & 1.05 & C09G9.1 & 1.78 \\
\hline chat-1 & -1.47 & rps-10 & 1.07 & vit-5 & 1.82 \\
\hline imp-1 & -1.44 & F09G2.8 & 1.08 & C39D10.8 & 1.88 \\
\hline sec-11 & -1.44 & Y59A8B.12 & 1.11 & ceh-20 & 1.89 \\
\hline dpy-10 & -1.42 & cdf-1 & 1.12 & $\mathrm{ikb}-1$ & 1.91 \\
\hline ZK813.3 & -1.4 & mrpl-13 & 1.12 & Y53C10A.6 & 2.02 \\
\hline T12D8.5 & -1.34 & cdk-5 & 1.13 & fip-5 & 2.19 \\
\hline asp-9 & -1.29 & grl-21 & 1.14 & vit-3 & 2.31 \\
\hline pfs-2 & -1.29 & ZK970.7 & 1.14 & vps-36 & 2.32 \\
\hline F58B4.5 & -1.29 & mbk-1 & 1.14 & asps-1 & 2.41 \\
\hline F10D11.6 & -1.25 & vti-1 & 1.18 & rpc-25 & 3.01 \\
\hline $\mathrm{hmt}-1$ & -1.24 & atg-16.2 & 1.23 & Y39H10A.6 & 3.69 \\
\hline dpy-14 & -1.22 & nola-3 & 1.24 & $\mathrm{C} 01 \mathrm{H} 6.8$ & 3.92 \\
\hline ule-5 & -1.19 & mig-2 & 1.24 & & \\
\hline dpy-17 & -1.18 & nemp-1 & 1.24 & & \\
\hline C17G1.2 & -1.18 & aps-2 & 1.25 & & \\
\hline R05H10.1 & -1.17 & $\operatorname{ttr}-35$ & 1.26 & & \\
\hline
\end{tabular}


6.11. RNA-seq (CeGaT) - N2 (wild-type) vs HBR1777 (flp-11::EGL-1) - 48h Starved

\begin{tabular}{|c|c|c|c|c|c|c|c|}
\hline Gene & $\log 2 \mathrm{FC}$ & Gene & $\log 2 \mathrm{FC}$ & Gene & $\log 2 \mathrm{FC}$ & Gene & $\log 2 \mathrm{FC}$ \\
\hline$c d r-4$ & -2.46 & F23D12.11 & -1.5 & C45B11.7 & -1.21 & dhs-20 & -1.07 \\
\hline gst-37 & -2.43 & F18E3.12 & -1.48 & C12D12.1 & -1.2 & mdl-1 & -1.07 \\
\hline F18E3.11 & -2.42 & F55G11.8 & -1.48 & F41C3.2 & -1.2 & T05F1.11 & -1.06 \\
\hline linc-72 & -2.38 & C36B1.13 & -1.47 & gst-41 & -1.19 & pals-37 & -1.06 \\
\hline gba-2 & -2.29 & F22B7.9 & -1.46 & R13A1.10 & -1.19 & $\mathrm{C} 40 \mathrm{H} 1.7$ & -1.06 \\
\hline sru-40 & -2.14 & F12E12.11 & -1.46 & hsp-12.3 & -1.18 & col-98 & -1.05 \\
\hline Y2H9A.6 & -2.13 & clec-1 & -1.45 & F47B8.4 & -1.18 & сур-29A2 & -1.05 \\
\hline gsto-2 & -2.13 & F14F3.5 & -1.43 & C55A6.7 & -1.17 & gba-4 & -1.04 \\
\hline col-101 & -2.05 & B0507.8 & -1.43 & linc-96 & -1.17 & F31E3.6 & -1.04 \\
\hline gst-33 & -2.03 & eol-1 & -1.43 & Y71H2AM.14 & -1.17 & $\mathrm{R} 09 \mathrm{H} 10.5$ & -1.04 \\
\hline catp-3 & -1.93 & C06E4.3 & -1.42 & gst-14 & -1.17 & gldc-1 & -1.04 \\
\hline gsto-1 & -1.93 & B0507.6 & -1.41 & R05D8.7 & -1.17 & $c d r-2$ & -1.04 \\
\hline gst-12 & -1.86 & R08F11.4 & -1.4 & irg-7 & -1.17 & F08H9.3 & -1.03 \\
\hline gst- 8 & -1.84 & сур-13A10 & -1.38 & $\mathrm{C} 17 \mathrm{H} 12.8$ & -1.16 & cpn-3 & -1.03 \\
\hline swt-3 & -1.82 & F09C8.1 & -1.38 & asm-3 & -1.16 & twk-18 & -1.03 \\
\hline ZK742.4 & -1.82 & C46G7.2 & -1.37 & R07E3.4 & -1.16 & C24B5.4 & -1.03 \\
\hline pals-2 & -1.81 & sod-3 & -1.37 & nhr-17 & -1.15 & hpo-34 & -1.03 \\
\hline cest-9.1 & -1.81 & aat-1 & -1.35 & R13H4.2 & -1.15 & Y42G9A.3 & -1.02 \\
\hline ddn-1 & -1.75 & Y9C9A.8 & -1.34 & ctl-1 & -1.14 & fbxa-54 & -1.02 \\
\hline fbxa-182 & -1.74 & C53D6.11 & -1.33 & Y34B4A.6 & -1.14 & gst-16 & -1.01 \\
\hline C55A6.4 & -1.74 & fat-3 & -1.33 & T08E11.1 & -1.13 & B0310.1 & -1.01 \\
\hline gst-39 & -1.71 & gst-5 & -1.33 & ctsa-3.1 & -1.13 & T16G1.9 & -1.01 \\
\hline F42C5.3 & -1.7 & gpx-6 & -1.32 & pmp-5 & -1.13 & C24B9.3 & -1 \\
\hline F54H5.2 & -1.67 & haf-9 & -1.32 & C17C3.15 & -1.13 & сур-33E2 & -1 \\
\hline scav-1 & -1.66 & sdz-24 & -1.3 & sdz-8 & -1.12 & csc-1 & 1 \\
\hline cdr-1 & -1.62 & oac-14 & -1.29 & ugt-16 & -1.11 & Y46H3A.5 & 1 \\
\hline clec-143 & -1.61 & C33E10.8 & -1.29 & T05E7.1 & -1.11 & $\mathrm{kbp}-2$ & 1 \\
\hline txt-4 & -1.6 & gst-30 & -1.28 & T07E3.4 & -1.11 & Y38E10A.14 & 1 \\
\hline gfi-1 & -1.6 & F13H8.11 & -1.28 & D2023.1 & -1.11 & phat-3 & 1.01 \\
\hline Y37A1A.2 & -1.6 & pgap-3 & -1.28 & col-176 & -1.11 & cpg-8 & 1.01 \\
\hline F18E3.13 & -1.57 & col-93 & -1.27 & asp-13 & -1.11 & nduo-6 & 1.01 \\
\hline slc-17.9 & -1.56 & F20G2.1 & -1.27 & gpx-7 & -1.1 & mlt-9 & 1.01 \\
\hline dpy-5 & -1.56 & hsp-16.2 & -1.26 & F19C7.1 & -1.1 & his-74 & 1.01 \\
\hline hsp-16.41 & -1.55 & ugt-31 & -1.26 & F44A6.5 & -1.1 & skr-8 & 1.01 \\
\hline T19D12.1 & -1.55 & pals-39 & -1.26 & fbxa-59 & -1.1 & mdf-2 & 1.03 \\
\hline сур-25A3 & -1.55 & D1022.3 & -1.26 & $\mathrm{~F} 40 \mathrm{H} 3.2$ & -1.1 & col-74 & 1.03 \\
\hline gst-31 & -1.54 & K08D12.6 & -1.24 & F38E11.9 & -1.09 & K09F6.6 & 1.03 \\
\hline T25C12.3 & -1.54 & Y46G5A.20 & -1.23 & fbxa-61 & -1.08 & T05H10.3 & 1.03 \\
\hline gst-7 & -1.5 & T20D3.2 & -1.23 & K02E11.7 & -1.08 & K02B12.2 & 1.04 \\
\hline col-159 & -1.5 & pals-14 & -1.23 & zyg-11 & -1.07 & aptf-2 & 1.04 \\
\hline
\end{tabular}




\begin{tabular}{|c|c|c|c|c|c|c|c|}
\hline Gene & $\log 2 \mathrm{FC}$ & Gene & $\log 2 \mathrm{FC}$ & Gene & $\log 2 \mathrm{FC}$ & Gene & $\log 2 \mathrm{FC}$ \\
\hline bus- 8 & 1.04 & $\mathrm{C} 31 \mathrm{H} 2.3$ & 1.11 & hal-3 & 1.2 & Y14H12B.2 & 1.29 \\
\hline pri-2 & 1.04 & dpy-7 & 1.11 & valv-1 & 1.2 & unc-119 & 1.29 \\
\hline col-48 & 1.04 & T26G10.5 & 1.11 & $\mathrm{~F} 22 \mathrm{H} 10.2$ & 1.2 & htp-3 & 1.29 \\
\hline C25G6.4 & 1.04 & clec-196 & 1.12 & gld-1 & 1.2 & Y111B2A.2 & 1.29 \\
\hline mlt-8 & 1.04 & hcp-2 & 1.12 & K03B4.4 & 1.2 & T25E12.6 & 1.31 \\
\hline Y69A2AR.28 & 1.05 & ptr-22 & 1.12 & Y65B4BL. 6 & 1.2 & taf-11.2 & 1.32 \\
\hline F41F3.3 & 1.05 & C08F1.10 & 1.12 & T21C9.9 & 1.2 & sym-1 & 1.32 \\
\hline dpy-17 & 1.05 & kbp-5 & 1.12 & F32H5.1 & 1.21 & F56C9.6 & 1.32 \\
\hline him-10 & 1.05 & col-169 & 1.12 & Т20В3.14 & 1.21 & ins-34 & 1.32 \\
\hline cah-4 & 1.05 & klp-18 & 1.13 & C44B9.3 & 1.21 & btb-6 & 1.32 \\
\hline clec- 266 & 1.05 & fzy-1 & 1.13 & hecw-1 & 1.21 & B0563.5 & 1.32 \\
\hline F41B4.2 & 1.05 & cec-4 & 1.13 & C44C1.6 & 1.21 & Ipr-3 & 1.32 \\
\hline $\mathrm{R} 02 \mathrm{C} 2.7$ & 1.06 & $\mathrm{kbp}-4$ & 1.14 & skr-10 & 1.21 & C50C3.1 & 1.32 \\
\hline nspc-19 & 1.06 & sas-5 & 1.14 & F53F8.3 & 1.22 & $\mathrm{hmg}-3$ & 1.32 \\
\hline T11B7.2 & 1.06 & nlp-67 & 1.14 & ZK669.3 & 1.22 & nspe-7 & 1.34 \\
\hline C26B9.7 & 1.07 & cec-8 & 1.14 & cyb-1 & 1.22 & $\mathrm{H} 42 \mathrm{~K} 12.3$ & 1.34 \\
\hline hcp-4 & 1.07 & F15E6.3 & 1.14 & ced-8 & 1.23 & wrt-10 & 1.36 \\
\hline grl-5 & 1.07 & oma-2 & 1.15 & F46E10.2 & 1.23 & cutl-2 & 1.36 \\
\hline zig-4 & 1.07 & ZC308.4 & 1.15 & Y41D4B.26 & 1.23 & ndfl-4 & 1.36 \\
\hline K02B7.3 & 1.07 & B0261.7 & 1.15 & W06B4.1 & 1.23 & nspc-20 & 1.36 \\
\hline ZK1053.4 & 1.08 & flh-1 & 1.15 & ZK813.6 & 1.23 & R144.10 & 1.37 \\
\hline wrt-2 & 1.08 & psf-1 & 1.15 & $\mathrm{mcm}-7$ & 1.24 & fbxc- 25 & 1.38 \\
\hline F39F10.4 & 1.08 & $\mathrm{scl}-3$ & 1.16 & F23A7.4 & 1.24 & F18A1.7 & 1.38 \\
\hline C02E7.6 & 1.08 & lips-15 & 1.16 & ZK829.9 & 1.25 & F08F3.4 & 1.38 \\
\hline ptr-16 & 1.08 & F48G7.4 & 1.16 & F36A2.10 & 1.25 & DY3.8 & 1.39 \\
\hline $\mathrm{K} 10 \mathrm{H} 10.4$ & 1.08 & hil-5 & 1.16 & Ipr-7 & 1.25 & F23A7.8 & 1.39 \\
\hline col-121 & 1.08 & immt-2 & 1.17 & col-181 & 1.25 & ceh-83 & 1.39 \\
\hline tost-1 & 1.09 & R05H5.3 & 1.17 & nlp-64 & 1.25 & air-2 & 1.39 \\
\hline F14B6.3 & 1.09 & dlc-5 & 1.17 & skr-15 & 1.26 & skr-7 & 1.4 \\
\hline gnrr-3 & 1.09 & ung-1 & 1.18 & syp-1 & 1.26 & gpd-4 & 1.4 \\
\hline lips-3 & 1.09 & K08E7.5 & 1.18 & hch-1 & 1.26 & B0281.5 & 1.4 \\
\hline mis-12 & 1.1 & rbm-3.1 & 1.18 & C17E4.2 & 1.26 & skr-14 & 1.4 \\
\hline F01D5.6 & 1.1 & T05D4.2 & 1.18 & ctf-8 & 1.27 & R11.4 & 1.41 \\
\hline col-111 & 1.1 & skr-13 & 1.18 & cpr-4 & 1.27 & mex-5 & 1.42 \\
\hline F35C11.5 & 1.1 & T05F1.2 & 1.18 & cut-2 & 1.27 & puf-5 & 1.43 \\
\hline rmd-1 & 1.1 & C05D9.9 & 1.18 & orc-1 & 1.28 & T05H10.4 & 1.44 \\
\hline col-145 & 1.1 & C04A2.15 & 1.19 & cdc-25.3 & 1.28 & gld-3 & 1.44 \\
\hline grl-7 & 1.1 & rsa-1 & 1.19 & Y65A5A.1 & 1.28 & C09F12.2 & 1.44 \\
\hline $\mathrm{dsl}-2$ & 1.1 & ndnf-1 & 1.19 & C30F2.4 & 1.28 & cey-3 & 1.46 \\
\hline $\mathrm{F} 22 \mathrm{H} 10.10$ & 1.11 & daf-28 & 1.19 & C13A10.2 & 1.29 & scl-24 & 1.46 \\
\hline
\end{tabular}




\begin{tabular}{|c|c|c|c|}
\hline Gene & $\log 2 \mathrm{FC}$ & Gene & $\log 2 \mathrm{FC}$ \\
\hline F32D1.7 & 1.46 & pie-1 & 1.78 \\
\hline szy-4 & 1.48 & C08F8.3 & 1.79 \\
\hline F09C6.1 & 1.48 & linc-6 & 1.8 \\
\hline gyg-2 & 1.49 & T05A7.1 & 1.8 \\
\hline ins-28 & 1.5 & capa-1 & 1.82 \\
\hline orc-3 & 1.5 & nspc-16 & 1.83 \\
\hline htp-2 & 1.5 & T22F3.11 & 1.83 \\
\hline him-3 & 1.5 & spn-4 & 1.89 \\
\hline nspc-17 & 1.51 & C05C10.5 & 1.91 \\
\hline $3 / 3 / 2020$ & 1.51 & F09E10.15 & 1.91 \\
\hline gln-6 & 1.51 & cey-2 & 1.91 \\
\hline inx-14 & 1.51 & W05F2.3 & 1.91 \\
\hline pud-4 & 1.51 & nasp-2 & 1.93 \\
\hline T21C9.13 & 1.53 & nspc- 18 & 1.96 \\
\hline $\mathrm{T} 19 \mathrm{H} 12.2$ & 1.54 & pos-1 & 2.06 \\
\hline $\operatorname{trcs}-1$ & 1.54 & C08E8.3 & 2.06 \\
\hline R09F10.8 & 1.55 & nspc-14 & 2.07 \\
\hline C42C1.8 & 1.56 & clec-91 & 2.08 \\
\hline ceh-49 & 1.57 & F14H3.6 & 2.12 \\
\hline tipn-1 & 1.58 & T07D3.9 & 2.15 \\
\hline F15D4.5 & 1.58 & clec- 88 & 2.16 \\
\hline F08F3.6 & 1.59 & w09C3.7 & 2.17 \\
\hline sun-1 & 1.6 & ins-20 & 2.21 \\
\hline puf-11 & 1.6 & mex-1 & 2.26 \\
\hline $\mathrm{fbxa}-215$ & 1.61 & era-1 & 2.29 \\
\hline Y39B6A.10 & 1.61 & clec-87 & 2.31 \\
\hline Y54G9A.5 & 1.62 & Isy-27 & 2.44 \\
\hline nspe-1 & 1.63 & egl-1 & 3.86 \\
\hline EEED8.3 & 1.67 & & \\
\hline sip-1 & 1.69 & & \\
\hline F14D7.2 & 1.71 & & \\
\hline gpd-1 & 1.71 & & \\
\hline cyb-2.2 & 1.73 & & \\
\hline flp-11 & 1.73 & & \\
\hline hil-4 & 1.73 & & \\
\hline cyb-3 & 1.74 & & \\
\hline Y110A2AR.1 & 1.74 & & \\
\hline Ү39В6А.1 & 1.75 & & \\
\hline C01G6.3 & 1.76 & & \\
\hline T12G3.6 & 1.77 & & \\
\hline
\end{tabular}


6.12. RNA-seq (CeGaT) - N2 (wild-type) vs HBR1777 (flp-11::EGL-1) - 4h Fed

\begin{tabular}{|c|c|c|c|c|c|c|c|}
\hline Gene & $\log 2 \mathrm{FC}$ & Gene & $\log 2 \mathrm{FC}$ & Gene & $\log 2 \mathrm{FC}$ & Gene & $\log 2 \mathrm{FC}$ \\
\hline fmo-2 & -2.4 & Y51H7BR.4 & -1.54 & F14H8.4 & -1.38 & C49A9.3 & -1.28 \\
\hline Y40B10B.1 & -2.31 & C07G1.15 & -1.54 & $\mathrm{C} 18 \mathrm{H} 9.5$ & -1.37 & gst-33 & -1.28 \\
\hline ugt-37 & -2.2 & $\mathrm{C} 28 \mathrm{H} 8.2$ & -1.51 & C09D4.2 & -1.37 & lipl-2 & -1.28 \\
\hline clec-143 & -1.94 & drh-2 & -1.51 & gcy-15 & -1.37 & W03D2.6 & -1.28 \\
\hline txt-3 & -1.85 & $\mathrm{ckr}-1$ & -1.49 & B0432.14 & -1.37 & T19D12.9 & -1.27 \\
\hline сур-35B1 & -1.85 & egl-47 & -1.49 & nduo-2 & -1.36 & ace-2 & -1.27 \\
\hline F53A2.9 & -1.85 & T16G1.6 & -1.49 & ugt-18 & -1.36 & nduo- 6 & -1.27 \\
\hline gst-2 & -1.84 & F47B8.4 & -1.49 & $\mathrm{~T} 10 \mathrm{H} 4.13$ & -1.35 & Y20C6A.1 & -1.27 \\
\hline сур-34A9 & -1.82 & F25E5.4 & -1.48 & Y51B9A.9 & -1.35 & T16G1.7 & -1.27 \\
\hline hsp-12.6 & -1.81 & amt-2 & -1.48 & dlhd-1 & -1.35 & W04E12.7 & -1.27 \\
\hline Y65B4BR.1 & -1.77 & ntr-2 & -1.48 & aagr-1 & -1.35 & Y70G10A.3 & -1.27 \\
\hline F11C7.7 & -1.75 & dop-2 & -1.48 & nca-2 & -1.35 & tyra-3 & -1.26 \\
\hline cpr-2 & -1.74 & gst-8 & -1.47 & pgp-1 & -1.34 & ugt-34 & -1.26 \\
\hline C15B12.2 & -1.73 & frpr-15 & -1.46 & F32H5.3 & -1.34 & K09C8.8 & -1.26 \\
\hline $\mathrm{mgl}-2$ & -1.73 & сур-13A6 & -1.46 & C07G3.15 & -1.34 & asm-2 & -1.26 \\
\hline F16B4.5 & -1.71 & C08F11.1 & -1.45 & H37A05.2 & -1.33 & kvs-1 & -1.26 \\
\hline T04C12.7 & -1.69 & mod-1 & -1.45 & F44A2.3 & -1.33 & сур-35A2 & -1.26 \\
\hline C50B6.7 & -1.68 & mltn-1 & -1.44 & gst-14 & -1.33 & C13C4.6 & -1.26 \\
\hline lgc-49 & -1.67 & exc-13 & -1.44 & rncs-1 & -1.33 & C47E12.9 & -1.26 \\
\hline F23F12.12 & -1.66 & D1086.2 & -1.44 & F40A3.7 & -1.32 & $\mathrm{fbxa}-80$ & -1.25 \\
\hline str-112 & -1.66 & clec-186 & -1.44 & сур-37B1 & -1.32 & unc-31 & -1.25 \\
\hline $\mathrm{nmr}-2$ & -1.65 & F46C5.10 & -1.43 & clec-198 & -1.32 & clec-166 & -1.25 \\
\hline col-142 & -1.64 & ndfl-4 & -1.43 & $\mathrm{mtl}-2$ & -1.32 & C13A10.1 & -1.25 \\
\hline gba-2 & -1.64 & glt-4 & -1.43 & F08A7.1 & -1.32 & T22F3.7 & -1.25 \\
\hline C45E5.4 & -1.63 & $\mathrm{~F} 28 \mathrm{H} 7.2$ & -1.42 & gap-1 & -1.31 & cest-9.1 & -1.25 \\
\hline nlp-20 & -1.63 & lurp-4 & -1.42 & F56A12.2 & -1.31 & F55G11.2 & -1.25 \\
\hline C35E7.6 & -1.63 & clec-41 & -1.41 & $\mathrm{mps}-1$ & -1.31 & $\mathrm{C} 40 \mathrm{H} 1.7$ & -1.25 \\
\hline F43C11.7 & -1.63 & $\mathrm{H} 23 \mathrm{~N} 18.6$ & -1.41 & nduo-3 & -1.31 & Y42G9A.3 & -1.25 \\
\hline clec-76 & -1.62 & F38C2.4 & -1.41 & Y110A2AL.9 & -1.3 & F58B4.5 & -1.25 \\
\hline сур-25A2 & -1.62 & $\mathrm{C} 32 \mathrm{H} 11.3$ & -1.41 & Y41D4B.24 & -1.3 & F17B5.8 & -1.25 \\
\hline hizr-1 & -1.62 & B0379.7 & -1.41 & clh-2 & -1.3 & scl-14 & -1.25 \\
\hline del-9 & -1.61 & F25B3.2 & -1.41 & anr-27 & -1.3 & F41E7.20 & -1.25 \\
\hline T16G1.4 & -1.6 & sprr-3 & -1.41 & asm-3 & -1.3 & glb-20 & -1.24 \\
\hline F56H9.9 & -1.59 & C08F11.3 & -1.4 & T23B3.6 & -1.29 & tsp-10 & -1.24 \\
\hline nhr-144 & -1.58 & frpr-4 & -1.4 & twk-5 & -1.29 & str-176 & -1.24 \\
\hline acr-18 & -1.57 & fil-1 & -1.39 & T05A10.6 & -1.29 & npr-27 & -1.24 \\
\hline cyp-32B1 & -1.57 & gab-1 & -1.38 & ZK1307.7 & -1.29 & C30B5.7 & -1.24 \\
\hline spp-8 & -1.55 & F23D12.11 & -1.38 & dhs-20 & -1.29 & T10C6.15 & -1.24 \\
\hline chil-22 & -1.55 & ttll-11 & -1.38 & best- 5 & -1.29 & clec-57 & -1.24 \\
\hline sulp-5 & -1.54 & fbxa-190 & -1.38 & fbxc-7 & -1.29 & irld-34 & -1.24 \\
\hline
\end{tabular}




\begin{tabular}{|c|c|c|c|c|c|c|c|}
\hline Gene & $\log 2 \mathrm{FC}$ & Gene & $\log 2 \mathrm{FC}$ & Gene & $\log 2 \mathrm{FC}$ & Gene & $\log 2 \mathrm{FC}$ \\
\hline Y40H7A.10 & -1.24 & gst-6 & -1.18 & Y42A5A.1 & -1.14 & sad-1 & -1.11 \\
\hline $\mathrm{C} 18 \mathrm{H} 7.6$ & -1.24 & dod-24 & -1.18 & Y62H9A.15 & -1.14 & K06A9.1 & -1.1 \\
\hline ugt-55 & -1.23 & glt-5 & -1.18 & C03B1.5 & -1.14 & scl-5 & -1.1 \\
\hline clec-55 & -1.23 & asah-2 & -1.18 & asah-1 & -1.14 & Y110A2AL.3 & -1.1 \\
\hline C17C3.15 & -1.23 & F59E12.6 & -1.18 & $x b x-5$ & -1.14 & pcp-4 & -1.1 \\
\hline flp-20 & -1.23 & $\mathrm{mxl}-3$ & -1.18 & F25E5.8 & -1.14 & ZK930.2 & -1.1 \\
\hline ptr-3 & -1.23 & C39D10.11 & -1.18 & H04M03.12 & -1.14 & R05A10.4 & -1.1 \\
\hline slc-36.3 & -1.23 & F14D2.19 & -1.18 & F18E3.11 & -1.14 & pbo-5 & -1.1 \\
\hline C05D12.2 & -1.23 & F31F7.1 & -1.18 & F42A10.7 & -1.14 & klp-13 & -1.1 \\
\hline F20G2.5 & -1.22 & F22B7.9 & -1.18 & F47G3.1 & -1.14 & T19D12.1 & -1.1 \\
\hline srv-5 & -1.22 & linc-72 & -1.17 & $\mathrm{nlp}-80$ & -1.14 & Y6G8.2 & -1.1 \\
\hline C31C9.6 & -1.22 & col-181 & -1.17 & ceh-79 & -1.13 & sulp-2 & -1.1 \\
\hline K05F6.10 & -1.22 & npr-35 & -1.17 & spp-18 & -1.13 & C16D9.9 & -1.1 \\
\hline ent-4 & -1.22 & F42A10.6 & -1.17 & F27D4.6 & -1.13 & F15E6.6 & -1.1 \\
\hline T03F6.9 & -1.22 & pgp-11 & -1.17 & acr-5 & -1.13 & B0035.13 & -1.1 \\
\hline C34D1.4 & -1.22 & F40E3.5 & -1.17 & lact-3 & -1.13 & F18E3.13 & -1.1 \\
\hline $\operatorname{lgc}-37$ & -1.22 & ttr-39 & -1.17 & F37C12.10 & -1.13 & $\mathrm{mfb}-1$ & -1.1 \\
\hline acr-17 & -1.22 & endu-2 & -1.17 & nhr-36 & -1.13 & rbf-1 & -1.1 \\
\hline des-2 & -1.22 & irg-3 & -1.17 & glc-3 & -1.13 & bgal-2 & -1.09 \\
\hline F18E3.12 & -1.21 & poml-3 & -1.16 & acr-16 & -1.13 & C01B9.1 & -1.09 \\
\hline ggr-3 & -1.21 & ptr-13 & -1.16 & T15B7.1 & -1.13 & tsp-6 & -1.09 \\
\hline C08F11.13 & -1.21 & W02H5.2 & -1.16 & parg-2 & -1.12 & T02B11.4 & -1.09 \\
\hline gst-41 & -1.21 & C32D5.6 & -1.16 & mec-14 & -1.12 & cnnm-2 & -1.09 \\
\hline far -6 & -1.21 & Igc-1 & -1.16 & ZC395.5 & -1.12 & osm-9 & -1.09 \\
\hline twk-25 & -1.21 & C54D10.3 & -1.16 & nspc-18 & -1.12 & lite-1 & -1.09 \\
\hline nhr-107 & -1.21 & pho-4 & -1.16 & tts- 1 & -1.12 & $\mathrm{C} 32 \mathrm{H} 11.4$ & -1.09 \\
\hline mdl-1 & -1.21 & slc-17.3 & -1.16 & Y55F3BR.10 & -1.12 & cest-13 & -1.09 \\
\hline daf-37 & -1.21 & W07A12.4 & -1.16 & ZK250.13 & -1.12 & sqst-2 & -1.09 \\
\hline T09F5.12 & -1.2 & glna-1 & -1.16 & asp-14 & -1.12 & Y49E10.18 & -1.09 \\
\hline ndnf-1 & -1.2 & W02D7.11 & -1.16 & nlp-59 & -1.11 & F14H3.12 & -1.09 \\
\hline ZC239.17 & -1.2 & pho-6 & -1.16 & F22F1.3 & -1.11 & Y8A9A.2 & -1.08 \\
\hline D1044.7 & -1.2 & dgk-5 & -1.15 & $\mathrm{H} 06 \mathrm{H} 21.8$ & -1.11 & aex-2 & -1.08 \\
\hline math-35 & -1.2 & col-151 & -1.15 & C47E8.3 & -1.11 & C54D10.5 & -1.08 \\
\hline F09C8.1 & -1.2 & gba-1 & -1.15 & chil-13 & -1.11 & daf-2 & -1.08 \\
\hline C50F4.8 & -1.19 & smp-2 & -1.15 & F59B2.13 & -1.11 & T03D8.7 & -1.08 \\
\hline nhr-19 & -1.19 & F22D6.15 & -1.15 & del-5 & -1.11 & egl-20 & -1.08 \\
\hline F23F12.3 & -1.19 & mec-1 & -1.15 & $\mathrm{~F} 52 \mathrm{H} 2.3$ & -1.11 & F25B3.4 & -1.08 \\
\hline Y82E9BR.5 & -1.19 & lys-4 & -1.15 & T05E7.1 & -1.11 & nhr-21 & -1.08 \\
\hline W06H8.6 & -1.18 & fozi-1 & -1.15 & kvs-4 & -1.11 & K04D7.6 & -1.08 \\
\hline ZK1240.2 & -1.18 & T05C12.11 & -1.14 & hpo-11 & -1.11 & Y43F8A.1 & -1.08 \\
\hline
\end{tabular}




\begin{tabular}{|c|c|c|c|c|c|c|c|}
\hline Gene & $\log 2 \mathrm{FC}$ & Gene & $\log 2 \mathrm{FC}$ & Gene & $\log 2 \mathrm{FC}$ & Gene & $\log 2 \mathrm{FC}$ \\
\hline T23F2.2 & -1.08 & clec-65 & -1.04 & $\mathrm{C} 05 \mathrm{C} 12.6$ & -1.01 & cpar-1 & 1.02 \\
\hline $\mathrm{C} 17 \mathrm{H} 12.8$ & -1.08 & B0410.3 & -1.04 & ceeh-2 & -1.01 & ceh-93 & 1.02 \\
\hline К09H9.5 & -1.08 & spp-6 & -1.04 & pсp-2 & -1.01 & bcl-7 & 1.02 \\
\hline ZK593.2 & -1.08 & daao-1 & -1.04 & Y69A2AR.22 & -1.01 & ulp-2 & 1.02 \\
\hline gtl-2 & -1.08 & smg-2 & -1.04 & ugt-16 & -1.01 & C25A11.2 & 1.02 \\
\hline nlp-40 & -1.08 & cng-1 & -1.04 & dop-5 & -1.01 & K02E10.4 & 1.02 \\
\hline ZK353.10 & -1.07 & Ibp-2 & -1.04 & unc-13 & -1.01 & dnj-22 & 1.03 \\
\hline egl-2 & -1.07 & lin-14 & -1.04 & F45E10.2 & -1.01 & T19C3.2 & 1.03 \\
\hline ggr-2 & -1.07 & C09E7.6 & -1.04 & txt-8 & -1.01 & R07E3.6 & 1.03 \\
\hline fbxa-173 & -1.07 & gst-19 & -1.04 & $\mathrm{R} 10 \mathrm{H} 10.3$ & -1.01 & F26F4.5 & 1.03 \\
\hline $\mathrm{cdr}-1$ & -1.07 & F17C11.13 & -1.04 & clec-61 & -1.01 & hst-1 & 1.03 \\
\hline clec-52 & -1.07 & unc-2 & -1.04 & clec-54 & -1.01 & B0035.3 & 1.03 \\
\hline Y105C5A.17 & -1.07 & F35B12.10 & -1.04 & nhr-5 & -1.01 & che-14 & 1.03 \\
\hline acox-1.2 & -1.07 & ZK822.6 & -1.04 & twk-1 & -1.01 & unc-37 & 1.03 \\
\hline daf-12 & -1.07 & H11E01.2 & -1.04 & F13E9.8 & -1.01 & ent-1 & 1.03 \\
\hline F43H9.4 & -1.07 & mec-9 & -1.03 & egl-10 & -1.01 & C05C10.2 & 1.03 \\
\hline dop-1 & -1.06 & ges-1 & -1.03 & nhr-88 & -1.01 & aipl-1 & 1.03 \\
\hline C13C4.7 & -1.06 & srsx-34 & -1.03 & $\mathrm{~F} 23 \mathrm{C} 8.14$ & -1.01 & grl-7 & 1.03 \\
\hline F17B5.1 & -1.06 & C15C7.4 & -1.03 & C29F7.2 & -1.01 & pign-1 & 1.03 \\
\hline ZK822.4 & -1.06 & rhgf-1 & -1.03 & fbxa-33 & -1 & C44C1.1 & 1.03 \\
\hline сур-37A1 & -1.06 & glb-24 & -1.03 & ZK673.1 & -1 & ZK1127.3 & 1.03 \\
\hline tom-1 & -1.06 & npr-8 & -1.03 & spp-14 & -1 & sys-1 & 1.03 \\
\hline H20E11.1 & -1.06 & R10E8.3 & -1.03 & clec-74 & -1 & tbp-1 & 1.03 \\
\hline Y38C1AA.6 & -1.06 & del- 6 & -1.03 & sodh-1 & -1 & spcs-2 & 1.03 \\
\hline C36A4.11 & -1.06 & faah-3 & -1.03 & $\mathrm{~T} 16 \mathrm{H} 12.9$ & -1 & hot- 6 & 1.03 \\
\hline clec-84 & -1.06 & $\mathrm{~F} 55 \mathrm{H} 12.2$ & -1.03 & F23F1.10 & 1 & Y94H6A.3 & 1.04 \\
\hline C05E11.6 & -1.06 & B0361.9 & -1.03 & gfi-3 & 1 & spt-4 & 1.04 \\
\hline frpr-16 & -1.06 & Y45F10D.2 & -1.03 & eri-1 & 1 & K06H7.7 & 1.04 \\
\hline F56H9.2 & -1.06 & nspc-16 & -1.02 & C14B1.8 & 1.01 & $\mathrm{~F} 25 \mathrm{H} 2.4$ & 1.04 \\
\hline C09B9.1 & -1.05 & ist-1 & -1.02 & wht-2 & 1.01 & rde-8 & 1.04 \\
\hline F32D8.11 & -1.05 & math-34 & -1.02 & spd-2 & 1.01 & mdf-1 & 1.04 \\
\hline B0524.6 & -1.05 & F45D3.4 & -1.02 & Y71G12B.25 & 1.01 & C56E6.2 & 1.04 \\
\hline tag-89 & -1.05 & npr-29 & -1.02 & C47E8.9 & 1.01 & $\mathrm{mls}-2$ & 1.04 \\
\hline C50A2.3 & -1.05 & clec-53 & -1.02 & B0310.6 & 1.01 & isw-1 & 1.04 \\
\hline T09B4.6 & -1.05 & cnnm-3 & -1.02 & B0336.13 & 1.02 & sptf-1 & 1.04 \\
\hline nhr-83 & -1.05 & Y69H2.10 & -1.02 & spp-7 & 1.02 & taf-6.1 & 1.04 \\
\hline npr-4 & -1.05 & C36B1.13 & -1.02 & $\mathrm{fbxb}-85$ & 1.02 & B0205.9 & 1.04 \\
\hline T07E3.4 & -1.05 & R07C12.2 & -1.02 & $\mathrm{C} 06 \mathrm{H} 2.7$ & 1.02 & aly-3 & 1.04 \\
\hline asp-3 & -1.04 & cest-19 & -1.02 & hst-2 & 1.02 & pigk-1 & 1.04 \\
\hline gсy-28 & -1.04 & flp-16 & -1.02 & R11H6.5 & 1.02 & tab-1 & 1.05 \\
\hline
\end{tabular}




\begin{tabular}{|c|c|c|c|c|c|c|c|}
\hline Gene & $\log 2 \mathrm{FC}$ & Gene & $\log 2 \mathrm{FC}$ & Gene & $\log 2 \mathrm{FC}$ & Gene & $\log 2 \mathrm{FC}$ \\
\hline snr-2 & 1.05 & F36D3.1 & 1.08 & npp-15 & 1.11 & F52B5.7 & 1.14 \\
\hline C55A6.1 & 1.05 & C53A5.2 & 1.08 & F33D4.6 & 1.11 & mat-2 & 1.14 \\
\hline col-131 & 1.05 & pcaf-1 & 1.08 & $|s|-1$ & 1.11 & C27C12.1 & 1.14 \\
\hline lido-12 & 1.05 & rad-54.B & 1.08 & sdha-2 & 1.11 & col-105 & 1.14 \\
\hline cec- 6 & 1.05 & fkh-2 & 1.08 & dnj-11 & 1.11 & Y44E3A.1 & 1.14 \\
\hline B0432.8 & 1.05 & lig-1 & 1.08 & E_BE45912.2 & 1.11 & Y105С5B.19 & 1.14 \\
\hline eri-7 & 1.05 & K05C4.7 & 1.08 & T27A1.2 & 1.11 & fbxa-137 & 1.14 \\
\hline C29A12.6 & 1.05 & T13F2.6 & 1.08 & kle-2 & 1.11 & сур-23A1 & 1.14 \\
\hline hrpf-1 & 1.05 & dhhc-3 & 1.08 & lido-1 & 1.11 & ostb-1 & 1.15 \\
\hline erg-28 & 1.05 & F33A8.7 & 1.08 & zipt-1 & 1.11 & chmp-7 & 1.15 \\
\hline gras-1 & 1.06 & F13A7.12 & 1.08 & osm-8 & 1.11 & wrt-6 & 1.15 \\
\hline T05H10.3 & 1.06 & dpy-7 & 1.08 & C38C6.3 & 1.11 & F55G1.6 & 1.15 \\
\hline Y17D7C.4 & 1.06 & $\mathrm{mbd}-2$ & 1.08 & Y37E11B.6 & 1.11 & ZK675.4 & 1.15 \\
\hline H03E18.1 & 1.06 & F37B4.10 & 1.09 & $\operatorname{csn}-1$ & 1.12 & phf-30 & 1.15 \\
\hline hut-1 & 1.06 & pot-3 & 1.09 & C30G12.4 & 1.12 & cya-1 & 1.16 \\
\hline bkip-1 & 1.06 & F35C11.5 & 1.09 & ent- 6 & 1.12 & gip-2 & 1.16 \\
\hline C48B4.6 & 1.06 & F25H9.6 & 1.09 & alh-13 & 1.12 & $\mathrm{Y} 69 \mathrm{H} 2.7$ & 1.16 \\
\hline B0205.1 & 1.06 & K10D2.5 & 1.09 & hcf-1 & 1.12 & K02E7.6 & 1.16 \\
\hline tag-96 & 1.06 & gtf-2H3 & 1.09 & F44E7.5 & 1.12 & pigu-1 & 1.16 \\
\hline C17E4.20 & 1.06 & prp-38 & 1.09 & fbxa-101 & 1.12 & swsn-6 & 1.16 \\
\hline ooc-5 & 1.06 & C16C8.14 & 1.09 & ifa-3 & 1.12 & T03F1.12 & 1.16 \\
\hline F22E5.9 & 1.07 & mans-1 & 1.1 & $\mathrm{~F} 32 \mathrm{H} 2.10$ & 1.12 & set-1 & 1.16 \\
\hline $\operatorname{lin}-35$ & 1.07 & rde-2 & 1.1 & apc-11 & 1.12 & mek-5 & 1.16 \\
\hline nspb-11 & 1.07 & $\mathrm{fkb}-3$ & 1.1 & F32E10.5 & 1.12 & bath-5 & 1.16 \\
\hline C09G4.4 & 1.07 & smut-1 & 1.1 & W09D6.1 & 1.13 & snpc-3.4 & 1.16 \\
\hline txdc-12.2 & 1.07 & K08E4.2 & 1.1 & mdt-11 & 1.13 & $\mathrm{pgl}-2$ & 1.16 \\
\hline T13H5.8 & 1.07 & R03E9.2 & 1.1 & E01A2.2 & 1.13 & dpy-14 & 1.17 \\
\hline cnc- 6 & 1.07 & B0285.3 & 1.1 & chl-1 & 1.13 & unc-59 & 1.17 \\
\hline T14B4.3 & 1.07 & dmd-5 & 1.1 & $\mathrm{~F} 33 \mathrm{H} 2.3$ & 1.13 & F57B1.6 & 1.17 \\
\hline pst-2 & 1.07 & $\mathrm{R} 10 \mathrm{H} 10.6$ & 1.1 & ufd-3 & 1.13 & ccch-3 & 1.17 \\
\hline pup-3 & 1.07 & K08B12.1 & 1.1 & arp-6 & 1.13 & cbn-1 & 1.17 \\
\hline tag-273 & 1.07 & his-3 & 1.1 & cutl-18 & 1.13 & F55A11.4 & 1.17 \\
\hline C16C10.4 & 1.07 & let-268 & 1.1 & K02E7.5 & 1.13 & hira-1 & 1.17 \\
\hline npp-5 & 1.07 & swt-4 & 1.1 & T01D1.8 & 1.13 & hrpk-2 & 1.17 \\
\hline mdt-18 & 1.07 & T12G3.4 & 1.1 & dhfr-1 & 1.13 & atg-10 & 1.17 \\
\hline slx-1 & 1.07 & T04A8.8 & 1.1 & set-5 & 1.13 & T10E9.2 & 1.17 \\
\hline dhs-27 & 1.07 & suds-3 & 1.11 & cdc- 6 & 1.13 & Y54F10AM.6 & 1.18 \\
\hline emb-27 & 1.07 & spd-5 & 1.11 & C27F2.7 & 1.13 & hhat-2 & 1.18 \\
\hline C25A1.1 & 1.08 & pqn-98 & 1.11 & C08B6.4 & 1.14 & mett-10 & 1.18 \\
\hline rba-1 & 1.08 & F26A1.1 & 1.11 & parp-2 & 1.14 & D1007.8 & 1.18 \\
\hline
\end{tabular}




\begin{tabular}{|c|c|c|c|c|c|c|c|}
\hline Gene & $\log 2 \mathrm{FC}$ & Gene & $\log 2 \mathrm{FC}$ & Gene & $\log 2 \mathrm{FC}$ & Gene & $\log 2 \mathrm{FC}$ \\
\hline mlt-11 & 1.18 & F49E12.12 & 1.21 & pssy-2 & 1.25 & ZC434.3 & 1.32 \\
\hline hex-4 & 1.18 & mdt- 6 & 1.21 & such-1 & 1.25 & tpk-1 & 1.32 \\
\hline Y69A2AR.28 & 1.18 & cyn-6 & 1.22 & taf-8 & 1.25 & F21D9.6 & 1.32 \\
\hline rnp-5 & 1.18 & Y51H7C.7 & 1.22 & $\mathrm{JC} 8.7$ & 1.25 & gana-1 & 1.32 \\
\hline R07B1.13 & 1.18 & K07B1.4 & 1.22 & $\mathrm{rnp}-2$ & 1.25 & C48B4.11 & 1.32 \\
\hline cpt-6 & 1.18 & ZK1248.13 & 1.22 & blos-4 & 1.25 & npp-23 & 1.32 \\
\hline strm-1 & 1.18 & nduf-2.2 & 1.22 & Y105E8B.9 & 1.25 & swd-2.1 & 1.32 \\
\hline ZK829.9 & 1.18 & drsh-1 & 1.22 & Y51H7C.3 & 1.25 & R13A5.7 & 1.32 \\
\hline F13B9. 2 & 1.19 & unc-119 & 1.22 & $z w l-1$ & 1.25 & spr-3 & 1.33 \\
\hline C05D9.7 & 1.19 & C16C8.5 & 1.22 & pid-2 & 1.26 & Y47G6A.14 & 1.33 \\
\hline F41G3.6 & 1.19 & emb-1 & 1.22 & M01D1.12 & 1.26 & F52B5.3 & 1.33 \\
\hline Y43F4B.10 & 1.19 & C49F5.6 & 1.22 & npp-1 & 1.26 & unc- 85 & 1.33 \\
\hline polk-1 & 1.19 & pcp-5 & 1.22 & com-1 & 1.26 & acp-2 & 1.33 \\
\hline F33H1.3 & 1.19 & C02E7.6 & 1.22 & C17G10.1 & 1.26 & rsp-5 & 1.33 \\
\hline ZK973.1 & 1.19 & Y55F3C.9 & 1.22 & B0393.5 & 1.26 & phf-10 & 1.33 \\
\hline K08F4.3 & 1.19 & $\mathrm{rsp}-4$ & 1.22 & C10C5.4 & 1.26 & C54G4.9 & 1.33 \\
\hline $\mathrm{mrg}-1$ & 1.19 & cec-3 & 1.22 & oac-51 & 1.26 & F52C6.2 & 1.34 \\
\hline F56A8.4 & 1.2 & F28B3.5 & 1.22 & C33F10.4 & 1.27 & $\mathrm{R} 05 \mathrm{H} 10.3$ & 1.34 \\
\hline swip-10 & 1.2 & smo-1 & 1.23 & drh-3 & 1.27 & ppt-1 & 1.34 \\
\hline par-2 & 1.2 & sucl-2 & 1.23 & gadr-5 & 1.27 & grl-15 & 1.34 \\
\hline hse-5 & 1.2 & rpb-4 & 1.23 & cpf-1 & 1.27 & C01H6.8 & 1.34 \\
\hline B0304.4 & 1.2 & nasp-1 & 1.23 & сур-31A3 & 1.28 & K08E7.5 & 1.34 \\
\hline col-121 & 1.2 & ppm-1.G & 1.23 & pafo-1 & 1.28 & smc-5 & 1.34 \\
\hline zip-8 & 1.2 & ari-1.4 & 1.23 & B0310.2 & 1.29 & in $x-3$ & 1.34 \\
\hline henn-1 & 1.2 & mom-5 & 1.23 & Ist-1 & 1.29 & sna-2 & 1.34 \\
\hline Y66D12A.16 & 1.2 & T01C3.2 & 1.23 & hlh-32 & 1.29 & $\mathrm{C} 11 \mathrm{H} 1.7$ & 1.34 \\
\hline cec- 8 & 1.2 & npp-2 & 1.23 & aman-1 & 1.29 & dna-2 & 1.34 \\
\hline ptr-2 & 1.2 & R08B4.3 & 1.23 & cutl-16 & 1.3 & F43C11.6 & 1.34 \\
\hline abu-13 & 1.21 & Y11D7A.9 & 1.23 & mut-7 & 1.3 & $\mathrm{C} 27 \mathrm{~F} 2.10$ & 1.35 \\
\hline evl-18 & 1.21 & isy-1 & 1.23 & cht-2 & 1.3 & C16C8.4 & 1.35 \\
\hline rsp-6 & 1.21 & cyn-10 & 1.24 & R05D11.9 & 1.31 & C56А3.8 & 1.35 \\
\hline srv-7 & 1.21 & R12E2.8 & 1.24 & R07E4.5 & 1.31 & F59B2.9 & 1.35 \\
\hline C06E1.1 & 1.21 & gbas-1 & 1.24 & ZK688.9 & 1.31 & K01D12.8 & 1.35 \\
\hline hlh-2 & 1.21 & unc-39 & 1.24 & B0511.7 & 1.31 & galt-1 & 1.35 \\
\hline Y75B8A.31 & 1.21 & row-1 & 1.24 & EEED8.14 & 1.31 & F58A4.2 & 1.36 \\
\hline Y34D9A.7 & 1.21 & him-8 & 1.24 & cutl-28 & 1.31 & wrt-4 & 1.36 \\
\hline acs-3 & 1.21 & lin-42 & 1.24 & F09E10.6 & 1.31 & tofu-5 & 1.36 \\
\hline cdk-4 & 1.21 & ztf-11 & 1.24 & ZC449.2 & 1.31 & hil-2 & 1.36 \\
\hline nfyc-1 & 1.21 & unc-71 & 1.24 & ptr-16 & 1.31 & F44B9.8 & 1.36 \\
\hline cep-1 & 1.21 & his-60 & 1.25 & $\mathrm{C} 27 \mathrm{H} 6.9$ & 1.32 & F09F9.2 & 1.36 \\
\hline
\end{tabular}




\begin{tabular}{|c|c|c|c|c|c|c|c|}
\hline Gene & $\log 2 \mathrm{FC}$ & Gene & $\log 2 \mathrm{FC}$ & Gene & $\log 2 \mathrm{FC}$ & Gene & $\log 2 \mathrm{FC}$ \\
\hline B0001.2 & 1.36 & ego-1 & 1.4 & F21H7.3 & 1.45 & W05F2.6 & 1.51 \\
\hline fbxb-72 & 1.36 & mlh-1 & 1.4 & wrt-10 & 1.46 & tyms-1 & 1.52 \\
\hline fbxc-51 & 1.36 & pes-5 & 1.4 & rnh-2 & 1.46 & npp-22 & 1.52 \\
\hline $\operatorname{div}-1$ & 1.37 & vang-1 & 1.41 & mre-11 & 1.46 & C45G3.3 & 1.52 \\
\hline noah-1 & 1.37 & Y41D4A.3 & 1.41 & C10B5.1 & 1.46 & Y52B11A.8 & 1.52 \\
\hline F33E2.5 & 1.37 & lem-3 & 1.41 & mam-5 & 1.46 & C50F4.6 & 1.52 \\
\hline tag-63 & 1.37 & mom-1 & 1.41 & F21D5.1 & 1.47 & pph-4.2 & 1.52 \\
\hline his-61 & 1.37 & rfc-2 & 1.41 & osr-1 & 1.47 & cdc-25.1 & 1.52 \\
\hline F11E6.4 & 1.37 & Y69A2AR.21 & 1.41 & B0511.11 & 1.47 & pole-4 & 1.52 \\
\hline F54D5.9 & 1.37 & T28C6.10 & 1.41 & C09B8.5 & 1.47 & Y65A5A.1 & 1.53 \\
\hline mrt-1 & 1.37 & cye-1 & 1.41 & М03B6.3 & 1.47 & Y39B6A.42 & 1.53 \\
\hline bub-3 & 1.37 & pen-2 & 1.41 & pig-1 & 1.48 & $\mathrm{knl}-2$ & 1.53 \\
\hline $\mathrm{fbxb}-7$ & 1.37 & W04A8.6 & 1.41 & C33E10.3 & 1.48 & T09F5.1 & 1.54 \\
\hline bath-1 & 1.37 & Y73E7A.8 & 1.42 & ZK1127.13 & 1.48 & F09G2.8 & 1.54 \\
\hline lin-53 & 1.38 & C50F4.12 & 1.42 & $\mathrm{C} 28 \mathrm{C} 12.12$ & 1.48 & C44H9.7 & 1.54 \\
\hline mltn-9 & 1.38 & CzW-1 & 1.42 & damt-1 & 1.48 & F54D10.5 & 1.55 \\
\hline npp-18 & 1.38 & asb-1 & 1.43 & F55A11.8 & 1.49 & Y53F4B.9 & 1.55 \\
\hline C16C8.16 & 1.38 & R07B7.10 & 1.43 & C13G5.2 & 1.49 & C16C8.11 & 1.55 \\
\hline ani-1 & 1.38 & ceh-36 & 1.43 & set-10 & 1.49 & T03F6.3 & 1.55 \\
\hline $\mathrm{C} 02 \mathrm{~F} 4.4$ & 1.38 & zyg-1 & 1.43 & C36B1.14 & 1.49 & hasp-1 & 1.55 \\
\hline xpo-2 & 1.39 & C56G2.15 & 1.43 & kca-1 & 1.49 & cuti-1 & 1.55 \\
\hline R04B3.2 & 1.39 & T26A5.8 & 1.43 & hmg-12 & 1.49 & F47B8.5 & 1.55 \\
\hline inft-2 & 1.39 & dpy-10 & 1.43 & B0035.18 & 1.49 & cyk-4 & 1.55 \\
\hline eme-1 & 1.39 & coh-4 & 1.43 & $\mathrm{mcm}-6$ & 1.49 & $f b x b-88$ & 1.55 \\
\hline mig-32 & 1.39 & let-49 & 1.43 & prg-1 & 1.49 & Y38A10A.7 & 1.56 \\
\hline mam-3 & 1.39 & Y106G6D.1 & 1.43 & hcp-4 & 1.49 & hcp-3 & 1.56 \\
\hline hmg-20 & 1.39 & C13F10.7 & 1.44 & zipt-7.1 & 1.49 & T22C8.4 & 1.56 \\
\hline $\mathrm{fkb}-7$ & 1.39 & dtmk-1 & 1.44 & Y38A10A.2 & 1.49 & gei-14 & 1.56 \\
\hline C02F5.13 & 1.39 & ssna-1 & 1.44 & nstp-3 & 1.5 & F12F6.7 & 1.56 \\
\hline grl-5 & 1.39 & F25E5.2 & 1.44 & lin-54 & 1.5 & C32D5.12 & 1.57 \\
\hline W04H10.2 & 1.39 & Y41D4B.6 & 1.44 & rbbp-5 & 1.5 & ZK616.5 & 1.57 \\
\hline F46F11.7 & 1.39 & ska-3 & 1.44 & metl-9 & 1.5 & pola-1 & 1.57 \\
\hline C48B6.9 & 1.39 & clec-266 & 1.44 & ekl-5 & 1.5 & F54H12.5 & 1.57 \\
\hline set-9 & 1.39 & ect-2 & 1.44 & tmem-17 & 1.5 & msh-6 & 1.57 \\
\hline tofu-1 & 1.39 & $\mathrm{~K} 11 \mathrm{H} 12.5$ & 1.45 & erh-1 & 1.5 & tim-1 & 1.57 \\
\hline wago-4 & 1.4 & zeel-1 & 1.45 & apc-2 & 1.5 & F36H9.5 & 1.58 \\
\hline C14A4.9 & 1.4 & imp-1 & 1.45 & got-2.1 & 1.51 & bmk-1 & 1.58 \\
\hline htz-1 & 1.4 & ZK1098.3 & 1.45 & F39F10.4 & 1.51 & Y95D11A.3 & 1.58 \\
\hline B0212.3 & 1.4 & dpy-2 & 1.45 & D1046.2 & 1.51 & F39G3.2 & 1.58 \\
\hline F59B2.8 & 1.4 & spdl-1 & 1.45 & T07C4.3 & 1.51 & Y106G6H.16 & 1.58 \\
\hline
\end{tabular}




\begin{tabular}{|c|c|c|c|c|c|c|c|}
\hline Gene & $\log 2 \mathrm{FC}$ & Gene & $\log 2 \mathrm{FC}$ & Gene & $\log 2 \mathrm{FC}$ & Gene & $\log 2 \mathrm{FC}$ \\
\hline hrde-2 & 1.58 & grl-11 & 1.64 & Y111B2A.3 & 1.71 & unc-61 & 1.79 \\
\hline tag-342 & 1.58 & $\mathrm{~K} 11 \mathrm{H} 12.11$ & 1.64 & ZK829.3 & 1.71 & tipn-1 & 1.79 \\
\hline fbxa-107 & 1.58 & cpg-3 & 1.64 & dao-2 & 1.71 & C17E7.13 & 1.8 \\
\hline D2030.8 & 1.59 & C39E9.12 & 1.64 & F07H5.10 & 1.71 & $\mathrm{knl}-3$ & 1.8 \\
\hline C36B1.11 & 1.59 & Y37E11AL.3 & 1.64 & cut-6 & 1.71 & deps-1 & 1.8 \\
\hline C42D4.13 & 1.59 & top-2 & 1.65 & W01C9.1 & 1.71 & gla-3 & 1.8 \\
\hline rnp-8 & 1.59 & Y54G2A.76 & 1.66 & wrt-1 & 1.72 & bath-15 & 1.8 \\
\hline cutl-9 & 1.59 & F48A11.4 & 1.66 & rrn-3.1 & 1.72 & rod-1 & 1.8 \\
\hline F59G1.8 & 1.59 & glh-3 & 1.66 & C35A5.11 & 1.72 & flp-11 & 1.81 \\
\hline rpa-1 & 1.59 & Y49E10.4 & 1.66 & thk-1 & 1.72 & $\mathrm{~T} 22 \mathrm{H} 2.4$ & 1.81 \\
\hline F18C5.9 & 1.59 & cec-7 & 1.66 & K07A1.1 & 1.72 & pri-1 & 1.81 \\
\hline cec- 4 & 1.59 & rmh-1 & 1.66 & sgo-1 & 1.72 & ceh-40 & 1.81 \\
\hline fubl-4 & 1.6 & C07E3.10 & 1.66 & ced-3 & 1.72 & zim-3 & 1.81 \\
\hline lam-3 & 1.6 & F52G3.5 & 1.66 & C40A11.3 & 1.72 & cks-1 & 1.81 \\
\hline cav-1 & 1.6 & $\mathrm{~F} 15 \mathrm{H} 10.8$ & 1.67 & brc-1 & 1.72 & $9 / 1 / 2020$ & 1.82 \\
\hline F01F1.11 & 1.6 & crn-1 & 1.67 & $\mathrm{fbxb}-78$ & 1.73 & Y49F6B.9 & 1.82 \\
\hline T04D3.5 & 1.6 & otub-3 & 1.67 & hrde-1 & 1.73 & bub-1 & 1.82 \\
\hline his-59 & 1.61 & cutl-17 & 1.67 & ZK287.4 & 1.73 & B0365.9 & 1.82 \\
\hline mei-1 & 1.61 & adal-1 & 1.67 & erh-2 & 1.73 & lips-15 & 1.82 \\
\hline $\mathrm{dpl}-1$ & 1.61 & $w d r-48$ & 1.67 & B0041.8 & 1.73 & orc-4 & 1.82 \\
\hline his-32 & 1.61 & sygl-1 & 1.68 & R11G1.7 & 1.74 & sex-1 & 1.82 \\
\hline pal-1 & 1.61 & tdpt-1 & 1.68 & ntp-1 & 1.74 & ces- 2 & 1.83 \\
\hline dpy-3 & 1.61 & gln-5 & 1.68 & Y48G1A.2 & 1.74 & brd-1 & 1.83 \\
\hline cpg-4 & 1.61 & F13A7.14 & 1.68 & hal-3 & 1.74 & ugt-57 & 1.83 \\
\hline B0205.4 & 1.61 & atf-8 & 1.68 & Y73F8A.13 & 1.75 & cdc-25.2 & 1.84 \\
\hline Y53G8AR.6 & 1.62 & aly-2 & 1.68 & Y75B8A.16 & 1.75 & nemp-1 & 1.84 \\
\hline cdt-1 & 1.62 & iсp-1 & 1.69 & plk-2 & 1.75 & R57.2 & 1.84 \\
\hline tcab-1 & 1.62 & rfc-3 & 1.69 & C04A11.2 & 1.75 & wdr-5.3 & 1.84 \\
\hline C48B4.9 & 1.62 & ndc- 80 & 1.69 & F30A10.2 & 1.76 & Y45G5AM.5 & 1.84 \\
\hline D1044.1 & 1.62 & $\mathrm{fbxb}-77$ & 1.69 & taf-11.2 & 1.76 & dnsn-1 & 1.85 \\
\hline bus- 12 & 1.62 & daf-18 & 1.69 & fkb-5 & 1.76 & skr-17 & 1.85 \\
\hline ssup-72 & 1.63 & ZK813.6 & 1.69 & Y37A1B.7 & 1.77 & plk-1 & 1.85 \\
\hline $\mathrm{kbp}-3$ & 1.63 & pid-3 & 1.7 & pgph-3 & 1.77 & chk-1 & 1.85 \\
\hline dvc-1 & 1.63 & mes-4 & 1.7 & F44E2.10 & 1.77 & T28A8.4 & 1.85 \\
\hline rrn-3.56 & 1.63 & T03G6.1 & 1.7 & gmn-1 & 1.78 & hcp-1 & 1.86 \\
\hline C39B5.2 & 1.63 & Y58A7A.2 & 1.7 & nos-1 & 1.78 & gpaa-1 & 1.86 \\
\hline trt-1 & 1.63 & gpr-2 & 1.7 & clec-196 & 1.78 & C05D12.4 & 1.86 \\
\hline eaf-1 & 1.63 & F23A7.4 & 1.7 & dhs- 11 & 1.78 & F59A6.5 & 1.86 \\
\hline F36F12.1 & 1.63 & T26C12.3 & 1.7 & neg-1 & 1.79 & hsp-12.1 & 1.86 \\
\hline F45F2.11 & 1.64 & D1081.6 & 1.7 & fncm-1 & 1.79 & sas-6 & 1.87 \\
\hline
\end{tabular}




\begin{tabular}{|c|c|c|c|c|c|c|c|}
\hline Gene & $\log 2 \mathrm{FC}$ & Gene & $\log 2 \mathrm{FC}$ & Gene & $\log 2 \mathrm{FC}$ & Gene & $\log 2 \mathrm{FC}$ \\
\hline dsh-2 & 1.87 & aspm-1 & 1.94 & hal-2 & 2.04 & Y23H5B.8 & 2.15 \\
\hline K01D12.1 & 1.87 & msh-2 & 1.94 & cdk-1 & 2.04 & let-99 & 2.15 \\
\hline T28C6.3 & 1.87 & scc-1 & 1.94 & atz-1 & 2.04 & fbxa-206 & 2.15 \\
\hline C40A11.4 & 1.88 & prom-1 & 1.94 & F46F3.3 & 2.04 & fbxb-45 & 2.15 \\
\hline inx-2 & 1.88 & C26B9.7 & 1.94 & Y17G9B.2 & 2.05 & T05C1.3 & 2.15 \\
\hline chaf-2 & 1.88 & Isd-1 & 1.94 & Y68A4B.3 & 2.05 & elo-4 & 2.15 \\
\hline Y97E10AR.4 & 1.88 & iff-1 & 1.94 & him-3 & 2.05 & Y11D7A.5 & 2.16 \\
\hline D1014.5 & 1.88 & F13D12.5 & 1.95 & Y64H9A.2 & 2.05 & hhat-1 & 2.16 \\
\hline txdc-12.1 & 1.89 & T04D3.1 & 1.95 & Y106G6H.6 & 2.06 & sld-2 & 2.16 \\
\hline mis-12 & 1.89 & edg-1 & 1.95 & F58G11.3 & 2.06 & F07H5.13 & 2.17 \\
\hline EEED8.15 & 1.89 & ZC449.1 & 1.95 & syp-1 & 2.06 & kbp-5 & 2.17 \\
\hline F52B5.2 & 1.89 & Y41D4B.14 & 1.96 & dsl-3 & 2.07 & cyk-7 & 2.17 \\
\hline C14F11.6 & 1.89 & chaf-1 & 1.96 & $n \times f-2$ & 2.07 & meg-3 & 2.17 \\
\hline F54D5.2 & 1.89 & cdl-1 & 1.96 & $\mathrm{mcm}-5$ & 2.08 & $\mathrm{rnr}-1$ & 2.17 \\
\hline F37A4.3 & 1.89 & psf-3 & 1.96 & B0034.7 & 2.08 & К03H1.7 & 2.17 \\
\hline T02C1.1 & 1.89 & rmd-1 & 1.97 & set-31 & 2.08 & tpxl-1 & 2.17 \\
\hline pole-1 & 1.9 & K05F6.4 & 1.97 & W02B8.2 & 2.08 & D2030.7 & 2.17 \\
\hline F27C1.4 & 1.9 & $\mathrm{mcm}-10$ & 1.97 & W03C9.2 & 2.08 & skr-9 & 2.18 \\
\hline jmjd-3.2 & 1.9 & $\mathrm{R} 12 \mathrm{C} 12.10$ & 1.97 & F40F11.3 & 2.09 & exo-1 & 2.18 \\
\hline $\mathrm{F} 58 \mathrm{H} 1.2$ & 1.9 & bgnt-1.2 & 1.98 & ceh-86 & 2.09 & tofu-7 & 2.18 \\
\hline D1081.7 & 1.9 & him-10 & 1.98 & egg-6 & 2.1 & srpr-2.2 & 2.18 \\
\hline T26A5.2 & 1.91 & fbxb-24 & 1.98 & $\mathrm{fbxb}-75$ & 2.1 & T26C5.2 & 2.18 \\
\hline F01D4.5 & 1.91 & K07A1.17 & 1.99 & knl-1 & 2.1 & Y4C6B.1 & 2.18 \\
\hline zhp-3 & 1.91 & sul-1 & 1.99 & F46E10.2 & 2.1 & T11B7.1 & 2.18 \\
\hline F44F1.6 & 1.91 & air-2 & 1.99 & K08B4.2 & 2.1 & nhr-127 & 2.18 \\
\hline btb-19 & 1.91 & F30F8.1 & 1.99 & klc-1 & 2.1 & $f b x b-53$ & 2.18 \\
\hline glf-1 & 1.91 & pash-1 & 2 & cup-15 & 2.11 & rad-51 & 2.19 \\
\hline pgl-3 & 1.91 & mans-4 & 2 & snrp-40.2 & 2.11 & C01G6.9 & 2.19 \\
\hline hcp-6 & 1.92 & F08F8.4 & 2.01 & C41D11.9 & 2.11 & fbxc-44 & 2.19 \\
\hline F59A3.12 & 1.92 & $\mathrm{fbxb}-84$ & 2.01 & fem-3 & 2.12 & rrn-2.1 & 2.19 \\
\hline efl-1 & 1.92 & Ipr-1 & 2.01 & $\mathrm{C} 24 \mathrm{H} 12.1$ & 2.12 & set-32 & 2.19 \\
\hline Y46G5A.8 & 1.92 & ZK643.2 & 2.02 & C35E7.3 & 2.12 & dscc-1 & 2.19 \\
\hline fbn-1 & 1.92 & polh-1 & 2.02 & B0001.7 & 2.13 & C06E7.88 & 2.19 \\
\hline syp-2 & 1.92 & ins-34 & 2.03 & ctf-8 & 2.13 & $\mathrm{fbxc}-32$ & 2.2 \\
\hline ima-2 & 1.92 & hcp-2 & 2.03 & klp-18 & 2.13 & F36H5.4 & 2.2 \\
\hline Y73В3А.1 & 1.92 & hmbx-1 & 2.03 & C08F1.10 & 2.13 & egl-1 & 2.2 \\
\hline sdz-38 & 1.92 & C35E7.5 & 2.03 & bath-10 & 2.13 & pemd-1 & 2.21 \\
\hline Y47G6A.31 & 1.93 & rsa-2 & 2.03 & C50C3.1 & 2.13 & T10E9.8 & 2.21 \\
\hline $\mathrm{K} 08 \mathrm{H} 2.4$ & 1.93 & dsb-2 & 2.04 & rfc-4 & 2.13 & Y47D3A.1 & 2.21 \\
\hline pif-1 & 1.93 & skr-2 & 2.04 & F49D11.6 & 2.13 & Y71F9AL.7 & 2.21 \\
\hline
\end{tabular}




\begin{tabular}{|c|c|c|c|c|c|c|c|}
\hline Gene & $\log 2 \mathrm{FC}$ & Gene & $\log 2 \mathrm{FC}$ & Gene & $\log 2 \mathrm{FC}$ & Gene & $\log 2 \mathrm{FC}$ \\
\hline ani-2 & 2.22 & swah-1 & 2.3 & tost-1 & 2.43 & spsb-2 & 2.56 \\
\hline cmt-1 & 2.22 & spp-13 & 2.31 & T08G5.3 & 2.43 & $\mathrm{mcm}-7$ & 2.57 \\
\hline sas-5 & 2.22 & T06A1.1 & 2.31 & ctf-4 & 2.44 & $\mathrm{fbxa}-210$ & 2.57 \\
\hline Y82E9BR.17 & 2.22 & hpo-40 & 2.31 & Y75B8A.18 & 2.44 & sdz-30 & 2.58 \\
\hline W07G4.1 & 2.22 & F19B10.10 & 2.31 & flh-1 & 2.44 & Y102A11A.5 & 2.58 \\
\hline T16G12.4 & 2.23 & Y106G6D.2 & 2.32 & $\mathrm{kbp}-1$ & 2.44 & wee-1.3 & 2.58 \\
\hline gcna-1 & 2.23 & fbxa-83 & 2.33 & zen-4 & 2.45 & mct-5 & 2.59 \\
\hline bir-2 & 2.23 & F11E6.7 & 2.33 & T28A8.5 & 2.45 & btb-11 & 2.59 \\
\hline psf-1 & 2.24 & dsl-6 & 2.33 & K07F5.12 & 2.45 & cutl-2 & 2.59 \\
\hline T05B9.1 & 2.24 & C55B7.11 & 2.34 & oef-1 & 2.45 & cyn-9 & 2.59 \\
\hline cdc-7 & 2.24 & air-1 & 2.34 & fbxc-18 & 2.46 & his-74 & 2.59 \\
\hline Y46G5A.7 & 2.25 & H04M03.3 & 2.34 & fbxb-65 & 2.47 & orc-1 & 2.6 \\
\hline gpd-4 & 2.25 & $\mathrm{mcm}-3$ & 2.35 & C27B7.5 & 2.47 & F56F4.8 & 2.61 \\
\hline Y41D4B.26 & 2.25 & tofu-6 & 2.35 & san-1 & 2.48 & orc-3 & 2.62 \\
\hline alg-5 & 2.25 & $\csc -1$ & 2.36 & F54F7.6 & 2.48 & F08F3.6 & 2.62 \\
\hline $\mathrm{pgl}-1$ & 2.25 & F10E9.7 & 2.36 & orc-5 & 2.48 & Y54G11A.3 & 2.63 \\
\hline C14B1.9 & 2.25 & snpc-1.2 & 2.37 & sdz-4 & 2.48 & C29A12.1 & 2.63 \\
\hline $\mathrm{H} 23 \mathrm{~N} 18.5$ & 2.26 & bath-41 & 2.37 & pcn-1 & 2.48 & ZC434.8 & 2.63 \\
\hline K02B12.2 & 2.26 & fbxc- 25 & 2.37 & F54D5.5 & 2.49 & rsa-1 & 2.64 \\
\hline puf- 8 & 2.26 & exo-3 & 2.38 & syx-4 & 2.49 & aptf-4 & 2.64 \\
\hline $\mathrm{mcm}-4$ & 2.26 & F56C9.3 & 2.38 & F48E3.6 & 2.49 & srpr-2.1 & 2.64 \\
\hline C37C3.9 & 2.27 & C38D4.4 & 2.38 & H04D03.2 & 2.5 & C17E4.2 & 2.64 \\
\hline F33E11.2 & 2.27 & ucr-2.3 & 2.39 & Y39G10AL.1 & 2.5 & C48B4.7 & 2.65 \\
\hline Irr-1 & 2.27 & htp-1 & 2.39 & pigv-1 & 2.51 & F15B10.3 & 2.65 \\
\hline htp-2 & 2.27 & $\mathrm{fbxb}-100$ & 2.39 & R144.10 & 2.51 & egg-3 & 2.66 \\
\hline W04A8.1 & 2.27 & W06D11.1 & 2.4 & R05H5.3 & 2.51 & fbxc- 29 & 2.66 \\
\hline T10B11.8 & 2.27 & skr-8 & 2.4 & rbm-3.1 & 2.51 & pch-2 & 2.66 \\
\hline spo-11 & 2.27 & mut-2 & 2.4 & $m d f-2$ & 2.51 & T16G12.8 & 2.66 \\
\hline pri-2 & 2.28 & flh-3 & 2.4 & T22D1.5 & 2.52 & M03B6.4 & 2.67 \\
\hline capg-2 & 2.28 & C47F8.1 & 2.4 & ogr-2 & 2.52 & dut-1 & 2.67 \\
\hline F56D2.2 & 2.28 & $f b x b-3$ & 2.4 & R11.4 & 2.53 & eri-5 & 2.68 \\
\hline rnr-2 & 2.28 & vet-2 & 2.4 & ung-1 & 2.53 & fog-2 & 2.68 \\
\hline linc-13 & 2.28 & nop-1 & 2.4 & T05H10.4 & 2.53 & F56F11.4 & 2.68 \\
\hline kbp-4 & 2.28 & $\mathrm{C} 24 \mathrm{H} 10.3$ & 2.41 & F46G11.6 & 2.53 & ceh-83 & 2.68 \\
\hline gpr-1 & 2.29 & apc-10 & 2.41 & skpt-1 & 2.53 & aptf-2 & 2.69 \\
\hline Y51F10.2 & 2.29 & kbp-2 & 2.41 & syp-5 & 2.54 & Y14H12B.2 & 2.69 \\
\hline $\mathrm{mcm}-2$ & 2.29 & D2005.4 & 2.42 & C01G8.1 & 2.54 & cyb-3 & 2.7 \\
\hline htp-3 & 2.29 & ttr-50 & 2.42 & dsl-2 & 2.56 & fbxb-54 & 2.7 \\
\hline hlh-3 & 2.29 & toe- 2 & 2.43 & mom-2 & 2.56 & fbxb-104 & 2.71 \\
\hline ZK1053.4 & 2.3 & B0304.2 & 2.43 & mbtr-1 & 2.56 & mesp-1 & 2.71 \\
\hline
\end{tabular}




\begin{tabular}{|c|c|c|c|c|c|c|c|}
\hline Gene & $\log 2 \mathrm{FC}$ & Gene & $\log 2 \mathrm{FC}$ & Gene & $\log 2 \mathrm{FC}$ & Gene & $\log 2 \mathrm{FC}$ \\
\hline immt-2 & 2.72 & C44B9. 3 & 2.88 & F14B6.3 & 3.11 & oma-2 & 3.61 \\
\hline C01G6.3 & 2.73 & T25E12.6 & 2.89 & cyb-2.2 & 3.12 & F14D7.2 & 3.63 \\
\hline skr-10 & 2.74 & K04G2.10 & 2.89 & sdz-28 & 3.12 & trcs-1 & 3.77 \\
\hline C08F1.6 & 2.74 & T05D4.2 & 2.89 & era-1 & 3.14 & clec-87 & 3.78 \\
\hline T28A8.3 & 2.74 & fbxb-35 & 2.89 & nhr-2 & 3.14 & $\mathrm{C} 31 \mathrm{H} 1.8$ & 4.19 \\
\hline Y42H9B.3 & 2.75 & Y71F9AL.6 & 2.91 & C08F8.3 & 3.16 & puf-5 & 4.23 \\
\hline cdc-25.3 & 2.75 & hpr-17 & 2.92 & Y110A2AR.1 & 3.17 & & \\
\hline W04A8.5 & 2.75 & syp-6 & 2.92 & fbxa-215 & 3.17 & & \\
\hline pid-4 & 2.77 & hil-5 & 2.93 & pie-1 & 3.18 & & \\
\hline cyb-1 & 2.77 & B0393.3 & 2.94 & glp-1 & 3.18 & & \\
\hline zim-1 & 2.77 & cpg-1 & 2.94 & $\mathrm{~T} 19 \mathrm{H} 12.2$ & 3.19 & & \\
\hline F23A7.8 & 2.77 & gpd-1 & 2.94 & R04D3.2 & 3.2 & & \\
\hline C06E2.5 & 2.78 & B0281.5 & 2.95 & F14H3.6 & 3.2 & & \\
\hline xnd-1 & 2.78 & ify-1 & 2.96 & pot-2 & 3.21 & & \\
\hline $\mathrm{fbxb}-15$ & 2.78 & F32D1.7 & 2.96 & gld-3 & 3.22 & & \\
\hline $\mathrm{C} 42 \mathrm{C} 1.8$ & 2.78 & sip-1 & 2.97 & F56C9.6 & 3.23 & & \\
\hline fzy-1 & 2.78 & $3 / 3 / 2020$ & 2.98 & szy-4 & 3.25 & & \\
\hline Y43B11AL.1 & 2.79 & ZC308.4 & 2.98 & W05F2.3 & 3.25 & & \\
\hline F15A4.2 & 2.79 & $g \ln -6$ & 2.99 & Y39B6A.10 & 3.27 & & \\
\hline C01B12.8 & 2.8 & inx-14 & 3 & sdz-14 & 3.27 & & \\
\hline hil-4 & 2.8 & daz-1 & 3.01 & F53F8.3 & 3.28 & & \\
\hline skr-7 & 2.8 & $\mathrm{cbd}-1$ & 3.02 & D2005.7 & 3.29 & & \\
\hline gna-2 & 2.81 & cey-2 & 3.02 & sun-1 & 3.29 & & \\
\hline$f b x b-66$ & 2.81 & ced-8 & 3.03 & puf-11 & 3.3 & & \\
\hline vet-1 & 2.82 & skr-15 & 3.03 & EEED8.3 & 3.31 & & \\
\hline Y110A7A.15 & 2.82 & btb- 6 & 3.03 & vet- 6 & 3.32 & & \\
\hline Y54G9A.5 & 2.82 & Y73B6BL.23 & 3.04 & nasp-2 & 3.33 & & \\
\hline psf-2 & 2.82 & T12G3.6 & 3.04 & cey-3 & 3.34 & & \\
\hline gyg-2 & 2.83 & C40A11.2 & 3.05 & mei-2 & 3.36 & & \\
\hline C03D6.9 & 2.83 & T05F1.2 & 3.05 & clec-88 & 3.36 & & \\
\hline hmg-3 & 2.84 & puf-3 & 3.05 & ceh-49 & 3.39 & & \\
\hline Y111B2A.2 & 2.84 & mex-5 & 3.06 & fbxb-95 & 3.4 & & \\
\hline toca-2 & 2.84 & R09F10.8 & 3.06 & mex-1 & 3.41 & & \\
\hline set-22 & 2.86 & T21C9.13 & 3.07 & pos-1 & 3.41 & & \\
\hline gld-1 & 2.86 & zif-1 & 3.07 & spn-4 & 3.46 & & \\
\hline skr-13 & 2.86 & memi-2 & 3.07 & C05C10.5 & 3.48 & & \\
\hline wago-1 & 2.86 & skr-14 & 3.09 & DY3.8 & 3.48 & & \\
\hline F18A1.7 & 2.86 & W06B4.1 & 3.09 & Isy-27 & 3.52 & & \\
\hline Y59A8B.12 & 2.87 & ZC155.4 & 3.1 & Y52E8A.2 & 3.54 & & \\
\hline B0261.7 & 2.88 & Y71A12B.11 & 3.1 & clec-91 & 3.59 & & \\
\hline
\end{tabular}


6.13. Proteome (TMT) - N2 (wild-type) vs HBR1777 (flp-11::EGL-1) - 48h Starved

\begin{tabular}{|c|c|c|c|c|c|c|c|}
\hline Protein & $\log 2 \mathrm{FC}$ & Protein & $\log 2 \mathrm{FC}$ & Protein & $\log 2 \mathrm{FC}$ & Protein & $\log 2 \mathrm{FC}$ \\
\hline ilys-5 & -4.79 & tba-4 & -2.07 & F28B4.3 & -1.53 & spin-1 & -1.27 \\
\hline Y62H9A.5 & -4.79 & F09C8.1 & -2.06 & mct-2 & -1.52 & F13D12.5 & -1.27 \\
\hline Y59E9AR.1 & -4.66 & anp-1 & -2.03 & ifc- 1 & -1.52 & C39D10.8 & -1.26 \\
\hline ule-5 & -4.66 & F57F5.1 & -2 & pud-2.1 & -1.51 & W10C8.5 & -1.26 \\
\hline perm-4 & -4.44 & bigr-1 & -1.98 & T01D1.4 & -1.5 & $\mathrm{C} 23 \mathrm{H} 3.2$ & -1.26 \\
\hline $\mathrm{cbd}-1$ & -4.01 & nrf-6 & -1.96 & dod-19 & -1.5 & rnp-1 & -1.24 \\
\hline folt-2 & -3.92 & R193.2 & -1.94 & Y37H2A.14 & -1.49 & phy-2 & -1.23 \\
\hline ZK813.3 & -3.75 & $\mathrm{~F} 21 \mathrm{C} 10.9$ & -1.92 & $\mathrm{~T} 25 \mathrm{C} 12.3$ & -1.49 & AC3.5 & -1.23 \\
\hline D1054.10 & -3.72 & F58B4.5 & -1.89 & Idp-1 & -1.48 & F25B4.7 & -1.21 \\
\hline $\mathrm{R} 10 \mathrm{H} 10.3$ & -3.52 & D1086.3 & -1.89 & sptl-3 & -1.48 & txdc-12.2 & -1.21 \\
\hline perm-2 & -3.5 & argk-1 & -1.89 & gst-27 & -1.46 & fpn-1.1 & -1.19 \\
\hline cey-2 & -3.39 & elo-1 & -1.88 & hро-40 & -1.46 & T10G3.3 & -1.19 \\
\hline glh-1 & -3.37 & fmo-5 & -1.88 & gfi-1 & -1.46 & $\mathrm{C} 23 \mathrm{H} 5.8$ & -1.18 \\
\hline $\mathrm{K} 12 \mathrm{H} 4.7$ & -3.36 & spp-3 & -1.85 & ugt-16 & -1.45 & pgp-6 & -1.17 \\
\hline hrg-7 & -3.3 & F35C8.5 & -1.84 & Y67D2.7 & -1.45 & ZK185.5 & -1.17 \\
\hline T28F3.8 & -3.27 & poml-3 & -1.83 & Y58A7A.1 & -1.44 & ost-1 & -1.16 \\
\hline ule-1 & -3.23 & rhr-1 & -1.79 & ugt-22 & -1.44 & F47B8.2 & -1.15 \\
\hline pgp-1 & -3.03 & Y7A5A.1 & -1.79 & Y54F10AM.8 & -1.44 & imp-1 & -1.15 \\
\hline F17E9.5 & -3.03 & asp-13 & -1.76 & hach-1 & -1.43 & acp-6 & -1.15 \\
\hline C14F11.4 & -2.96 & aagr-2 & -1.76 & gldc-1 & -1.41 & Y16B4A.2 & -1.14 \\
\hline lec-8 & -2.94 & pgp-3 & -1.75 & F55G11.8 & -1.39 & mlt-7 & -1.14 \\
\hline dct-16 & -2.9 & sur-5 & -1.75 & pod-2 & -1.39 & gfat-1 & -1.14 \\
\hline grl-23 & -2.67 & asp-1 & -1.74 & asns-2 & -1.38 & ifp-1 & -1.14 \\
\hline col-92 & -2.66 & C17G1.2 & -1.72 & Iron-7 & -1.36 & ech-6 & -1.13 \\
\hline F54E2.1 & -2.66 & ugt-62 & -1.72 & coq-1 & -1.35 & K12B6.9 & -1.13 \\
\hline Y51F10.7 & -2.59 & F19C7.1 & -1.72 & irg-7 & -1.35 & $\mathrm{R} 09 \mathrm{H} 10.5$ & -1.13 \\
\hline far-2 & -2.58 & lec-10 & -1.66 & spp-2 & -1.34 & hpo-34 & -1.13 \\
\hline madd-3 & -2.52 & Y75B8A.14 & -1.65 & pud-1.1 & -1.33 & $\mathrm{~T} 21 \mathrm{H} 3.1$ & -1.12 \\
\hline cey-3 & -2.5 & hphd-1 & -1.64 & $c d r-4$ & -1.33 & pept-1 & -1.11 \\
\hline lys-4 & -2.48 & W05F2.3 & -1.64 & R10F2.4 & -1.33 & T07D3.9 & -1.1 \\
\hline ZK6.11 & -2.39 & immp-1 & -1.63 & Y34B4A.6 & -1.32 & Y23H5B.6 & -1.1 \\
\hline acdh-1 & -2.38 & Y34B4A.9 & -1.62 & Y14H12B.1 & -1.29 & sago-2 & -1.1 \\
\hline ctsa-1.2 & -2.31 & $\mathrm{C} 34 \mathrm{H} 4.2$ & -1.6 & oxy-4 & -1.29 & pmt-2 & -1.1 \\
\hline ugt-26 & -2.28 & pcp-4 & -1.58 & C53D5.5 & -1.29 & acdh-9 & -1.09 \\
\hline csr-1 & -2.24 & LLC1.2 & -1.57 & aqp-7 & -1.29 & wago-1 & -1.09 \\
\hline cpr-4 & -2.17 & asp-6 & -1.56 & T16G12.1 & -1.29 & mpc-1 & -1.09 \\
\hline сур-13A5 & -2.14 & nuc-1 & -1.55 & spp-5 & -1.29 & zipt-15 & -1.08 \\
\hline clec-50 & -2.1 & let-767 & -1.55 & glh-2 & -1.29 & timm-23 & -1.08 \\
\hline F17E9.4 & -2.08 & W04B5.3 & -1.54 & clec-63 & -1.28 & C05C10.3 & -1.08 \\
\hline F56C9.7 & -2.08 & dhs-25 & -1.54 & pmp-5 & -1.27 & sptl-1 & -1.07 \\
\hline
\end{tabular}




\begin{tabular}{|c|c|c|c|c|c|}
\hline Protein & $\log 2 \mathrm{FC}$ & Protein & $\log 2 \mathrm{FC}$ & Protein & $\log 2 F C$ \\
\hline Y54E2A.4 & -1.07 & col-121 & 1.1 & ZK863.8 & 1.43 \\
\hline asp-5 & -1.06 & Y55D9A.2 & 1.1 & F13D11.3 & 1.45 \\
\hline leo-1 & -1.06 & dpy-30 & 1.12 & nlp-24 & 1.47 \\
\hline cpn-1 & -1.06 & Y52E8A.3 & 1.12 & fbn-1 & 1.47 \\
\hline ifc- 2 & -1.06 & hil-2 & 1.13 & F36H9.7 & 1.48 \\
\hline rop-1 & -1.05 & Iron-2 & 1.13 & K02F3.9 & 1.54 \\
\hline fmo-3 & -1.05 & hsp-16.11 & 1.13 & cht-1 & 1.61 \\
\hline let-716 & -1.05 & grd-13 & 1.14 & Y53F4B.23 & 1.64 \\
\hline iff-2 & -1.04 & vps-60 & 1.15 & F43C9.2 & 1.67 \\
\hline soap-1 & -1.03 & E04F6.9 & 1.15 & hsp-16.2 & 1.67 \\
\hline nog-1 & -1.02 & F10D11.6 & 1.15 & $\mathrm{scl}-3$ & 1.67 \\
\hline C15A11.7 & -1.02 & F17H10.2 & 1.16 & Y44A6D.2 & 1.68 \\
\hline col-159 & -1.02 & msa-1 & 1.16 & slc-36.5 & 1.7 \\
\hline dpy-18 & -1.02 & rgs-3 & 1.16 & cut-3 & 1.71 \\
\hline F09B12.3 & -1.01 & C33A12.4 & 1.17 & $\mathrm{C} 18 \mathrm{H} 7.1$ & 1.73 \\
\hline C35A5.11 & -1.01 & nlp-31 & 1.18 & T03F1.11 & 1.79 \\
\hline alh-8 & -1.01 & his-35 & 1.21 & hmg-3 & 1.81 \\
\hline nas-11 & -1 & clec-266 & 1.21 & ttr-5 & 1.83 \\
\hline F56C9.8 & 1.02 & nep-1 & 1.22 & hsp-16.48 & 1.93 \\
\hline rpi-2 & 1.02 & hil-3 & 1.23 & E02C12.9 & 1.93 \\
\hline pdl-1 & 1.02 & F55F8.3 & 1.24 & ensa-1 & 1.97 \\
\hline C25F9.11 & 1.02 & Ipr-3 & 1.24 & C01G6.3 & 1.99 \\
\hline F53E10.1 & 1.03 & ipla-2 & 1.25 & hil-1 & 2.01 \\
\hline clec-13 & 1.04 & plpr-1 & 1.25 & dpy-10 & 2.04 \\
\hline F59B10.3 & 1.04 & sod-4 & 1.27 & T10B10.3 & 2.04 \\
\hline exc-13 & 1.04 & sod-5 & 1.27 & F14B4.1 & 2.2 \\
\hline$r n r-2$ & 1.04 & C15C7.7 & 1.28 & grl-16 & 2.31 \\
\hline C06A5.3 & 1.04 & $\mathrm{~F} 58 \mathrm{H} 1.7$ & 1.28 & M01H9.3 & 2.36 \\
\hline tni-4 & 1.05 & $\mathrm{dsl}-3$ & 1.29 & Y53C10A.6 & 2.6 \\
\hline oig-3 & 1.05 & ttr-23 & 1.31 & C53B7.3 & 2.61 \\
\hline hmg-1.1 & 1.05 & Т10H9.8 & 1.32 & Y39B6A.5 & 2.91 \\
\hline nlt-1 & 1.05 & cwc-15 & 1.33 & dpy-14 & 2.95 \\
\hline C03G6.17 & 1.05 & nhr-48 & 1.35 & dpy-17 & 3.06 \\
\hline W10C8.4 & 1.06 & cnnm-1 & 1.38 & col-158 & 3.25 \\
\hline fkh-7 & 1.07 & T11B7.5 & 1.38 & col-39 & 3.42 \\
\hline $\operatorname{ttr}-33$ & 1.08 & F07C3.2 & 1.38 & fipr-22 & 4.23 \\
\hline F26G1.2 & 1.08 & T04C9.1 & 1.39 & H39E23.3 & 7.15 \\
\hline snu-23 & 1.1 & D2063.1 & 1.39 & & \\
\hline F29B9.5 & 1.1 & far-7 & 1.4 & & \\
\hline $\operatorname{ttr}-16$ & 1.1 & gst-34 & 1.41 & & \\
\hline
\end{tabular}


6.14. Proteome (TMT) - N2 (wild-type) vs HBR1777 (flp-11::EGL-1) - 4h Fed

\begin{tabular}{|c|c|c|c|c|c|}
\hline Protein & $\log 2 \mathrm{FC}$ & Protein & $\log 2 \mathrm{FC}$ & Protein & $\log 2 \mathrm{FC}$ \\
\hline scl-11 & -2.88 & col-92 & 1.07 & far-3 & 2.03 \\
\hline $\mathrm{scl}-12$ & -2.05 & B0001.8 & 1.07 & vps-36 & 2.16 \\
\hline smr-1 & -1.85 & F53F4.16 & 1.08 & asps-1 & 2.3 \\
\hline mec-9 & -1.68 & $\mathrm{fkb}-5$ & 1.09 & Y67D2.7 & 2.34 \\
\hline asp-9 & -1.65 & Y104H12D.2 & 1.12 & rpc-25 & 2.46 \\
\hline exp-2 & -1.64 & nhl-1 & 1.12 & fip-5 & 2.74 \\
\hline madd-3 & -1.61 & syd-9 & 1.14 & Y39H10A.6 & 3.49 \\
\hline sec-11 & -1.51 & vps-18 & 1.15 & C01H6.8 & 4.3 \\
\hline hmg-3 & -1.48 & Y45F10B.13 & 1.16 & & \\
\hline T04F8.7 & -1.48 & grd-13 & 1.16 & & \\
\hline tag-65 & -1.47 & Y48C 3 A. 20 & 1.17 & & \\
\hline gst-19 & -1.37 & F53B1.8 & 1.17 & & \\
\hline $\mathrm{R} 02 \mathrm{C} 2.7$ & -1.37 & comt-4 & 1.17 & & \\
\hline lars-2 & -1.36 & bigr-1 & 1.18 & & \\
\hline pqbp-1.1 & -1.34 & cdk-5 & 1.19 & & \\
\hline rpi-2 & -1.32 & $\mathrm{~F} 39 \mathrm{H} 11.1$ & 1.19 & & \\
\hline clh-2 & -1.29 & nemp-1 & 1.2 & & \\
\hline pqn-94 & -1.21 & C39D10.8 & 1.21 & & \\
\hline sir-2.4 & -1.21 & ceh-20 & 1.22 & & \\
\hline F55G1.9 & -1.18 & F20G2.1 & 1.25 & & \\
\hline rfc-2 & -1.17 & F44G4.1 & 1.25 & & \\
\hline bli-4 & -1.16 & mbk-1 & 1.26 & & \\
\hline F13D12.5 & -1.12 & Y10G11A.1 & 1.27 & & \\
\hline wrb-1 & -1.1 & lact-3 & 1.29 & & \\
\hline zipt-15 & -1.1 & ger-1 & 1.3 & & \\
\hline cubn-1 & -1.09 & Y48A6C.4 & 1.32 & & \\
\hline ipla-2 & -1.08 & nol-10 & 1.33 & & \\
\hline T04C9.1 & -1.08 & F41D9.2 & 1.36 & & \\
\hline $\mathrm{F} 52 \mathrm{H} 2.1$ & -1.07 & atg-16.2 & 1.36 & & \\
\hline R151.6 & -1.07 & rbm-3.2 & 1.38 & & \\
\hline H39E23.3 & -1.03 & $\operatorname{csn}-5$ & 1.41 & & \\
\hline ZK863.8 & -1.02 & eif-2Balpha & 1.42 & & \\
\hline bcs-1 & -1.02 & F09F7.6 & 1.46 & & \\
\hline B0379.6 & -1.02 & T10B10.3 & 1.49 & & \\
\hline С49H3.6 & 1.01 & aps-2 & 1.58 & & \\
\hline chd-3 & 1.02 & trpp-8 & 1.59 & & \\
\hline W01B11.6 & 1.03 & C09G9.1 & 1.6 & & \\
\hline nola-3 & 1.04 & Y53C10A.6 & 1.62 & & \\
\hline Y15E3A.4 & 1.04 & T26G10.1 & 1.63 & & \\
\hline $\mathrm{C} 23 \mathrm{H} 3.2$ & 1.05 & $\mathrm{ikb}-1$ & 1.63 & & \\
\hline M02H5.8 & 1.06 & trpa-2 & 1.92 & & \\
\hline $\operatorname{cog} c-2$ & 1.07 & B0432.7 & 1.93 & & \\
\hline
\end{tabular}


6.15. RNA-seq (CeGaT) - 4h Fed vs 48h Starved - N2 (wild-type)

\begin{tabular}{|c|c|c|c|c|c|c|c|}
\hline Gene & $\log 2 \mathrm{FC}$ & Gene & $\log 2 \mathrm{FC}$ & Gene & $\log 2 \mathrm{FC}$ & Gene & $\log 2 \mathrm{FC}$ \\
\hline T22F3.11 & -6.5 & К02B7.3 & -2.88 & col-14 & -2.61 & clec- 80 & -2.42 \\
\hline Y39B6A.1 & -6.14 & E01G6.1 & -2.87 & idh-1 & -2.6 & misc-1 & -2.41 \\
\hline T07D3.9 & -5.54 & ugt-41 & -2.86 & clec-180 & -2.6 & clx-1 & -2.41 \\
\hline $\mathrm{R} 02 \mathrm{C} 2.7$ & -4.99 & bca-1 & -2.85 & pgp-14 & -2.59 & sodh-1 & -2.41 \\
\hline $\mathrm{cpr}-4$ & -4.62 & mua-6 & -2.84 & Y60C6A.2 & -2.59 & B0403.5 & -2.41 \\
\hline C23G10.11 & -4.48 & col-33 & -2.84 & T04F8.8 & -2.58 & daao-1 & -2.4 \\
\hline W10C8.5 & -4.08 & col-145 & -2.83 & $\mathrm{C} 49 \mathrm{H} 3.16$ & -2.58 & Y105C5B.5 & -2.4 \\
\hline F46F2.3 & -3.97 & col-109 & -2.82 & phg-1 & -2.57 & alh-5 & -2.4 \\
\hline nlp-30 & -3.79 & zig-4 & -2.81 & F57F4.4 & -2.55 & tyr-2 & -2.39 \\
\hline gst-26 & -3.68 & Y37H2A.14 & -2.81 & $\mathrm{~F} 55 \mathrm{H} 12.4$ & -2.54 & ZK829.9 & -2.39 \\
\hline F46C5.1 & -3.64 & Y47H9C.1 & -2.81 & pqn-74 & -2.54 & abu-12 & -2.39 \\
\hline mdh-2 & -3.47 & K02G10.15 & -2.8 & Y37D8A.3 & -2.53 & $\mathrm{C} 18 \mathrm{H} 7.11$ & -2.38 \\
\hline irg-4 & -3.43 & nas-38 & -2.79 & cut-2 & -2.53 & R03C1.1 & -2.37 \\
\hline F35B3.4 & -3.41 & acn-1 & -2.79 & F09E10.15 & -2.53 & F13D12.9 & -2.37 \\
\hline F22B5.4 & -3.39 & asns-2 & -2.77 & aqp-7 & -2.53 & F09E10.1 & -2.36 \\
\hline acs-2 & -3.37 & $\mathrm{fbp}-1$ & -2.77 & Y22D7AL.10 & -2.52 & F07H5.8 & -2.35 \\
\hline C35C5.8 & -3.35 & nlp-34 & -2.77 & M02G9.1 & -2.51 & $\mathrm{C} 31 \mathrm{H} 2.4$ & -2.35 \\
\hline hphd-1 & -3.34 & C23H5.8 & -2.76 & F53F1.4 & -2.51 & cnc-4 & -2.35 \\
\hline T19B10.2 & -3.31 & ugt-34 & -2.75 & math-45 & -2.5 & tag-10 & -2.35 \\
\hline cah-4 & -3.31 & icl-1 & -2.75 & gst-28 & -2.48 & kat-1 & -2.34 \\
\hline folt-2 & -3.24 & clec- 48 & -2.75 & M03F8.1 & -2.48 & mec-7 & -2.34 \\
\hline Ion-1 & -3.22 & col-97 & -2.73 & F20B10.3 & -2.48 & W08E12.3 & -2.34 \\
\hline F17A9.5 & -3.19 & col-166 & -2.73 & lec-8 & -2.48 & T06A4.1 & -2.34 \\
\hline ilys-5 & -3.13 & F41E6.5 & -2.73 & F21C10.9 & -2.47 & ptr-2 & -2.33 \\
\hline $\mathrm{F} 22 \mathrm{H} 10.2$ & -3.09 & nspe-1 & -2.72 & Idh-1 & -2.47 & F38B2.4 & -2.33 \\
\hline ZK1320.9 & -3.07 & C09B8.4 & -2.72 & ttr-2 & -2.47 & Y106G6D.1 & -2.33 \\
\hline col-113 & -3.06 & kreg-1 & -2.71 & R09E12.9 & -2.47 & T10E10.4 & -2.33 \\
\hline C45B2.2 & -3.05 & pho-11 & -2.71 & grl-21 & -2.47 & skpo-2 & -2.33 \\
\hline C42D4.3 & -3.05 & nlp-77 & -2.71 & C01G10.15 & -2.46 & K01A6.4 & -2.33 \\
\hline best-1 & -3.04 & fmo-3 & -2.7 & T06D8.10 & -2.46 & col-144 & -2.32 \\
\hline pqn-26 & -3.04 & nspe-7 & -2.68 & F15D3.6 & -2.46 & ddo-2 & -2.32 \\
\hline nlp-31 & -3.02 & ZK1037.6 & -2.68 & T20B6.3 & -2.45 & acdh-1 & -2.32 \\
\hline col-48 & -3.02 & hacd-1 & -2.68 & ech-9 & -2.44 & F56D2.3 & -2.32 \\
\hline F32D8.11 & -3.02 & F15B9.6 & -2.68 & srg-31 & -2.44 & pqn-22 & -2.3 \\
\hline F40E10.5 & -3.01 & dod-19 & -2.67 & ptr-22 & -2.44 & F16H11.1 & -2.3 \\
\hline $\mathrm{F} 49 \mathrm{C} 12.7$ & -3.01 & $\mathrm{~K} 10 \mathrm{H} 10.4$ & -2.66 & F10D11.6 & -2.44 & col-111 & -2.3 \\
\hline valv-1 & -3 & F59A7.2 & -2.66 & daf-36 & -2.43 & C54D2.1 & -2.29 \\
\hline spp-20 & -2.98 & E04F6.6 & -2.66 & ttr-31 & -2.42 & ndnf-1 & -2.29 \\
\hline dct-16 & -2.93 & ZK6.11 & -2.65 & pqn-63 & -2.42 & K08D8.3 & -2.29 \\
\hline B0024.4 & -2.91 & Ibp-6 & -2.61 & D1054.9 & -2.42 & F44E5.4 & -2.28 \\
\hline C50F7.5 & -2.89 & F13E9.14 & -2.61 & act-5 & -2.42 & C08F11.1 & -2.28 \\
\hline
\end{tabular}




\begin{tabular}{|c|c|c|c|c|c|c|c|}
\hline Gene & $\log 2 \mathrm{FC}$ & Gene & $\log 2 \mathrm{FC}$ & Gene & $\log 2 \mathrm{FC}$ & Gene & $\log 2 \mathrm{FC}$ \\
\hline F13D12.3 & -2.28 & T22F3.10 & -2.16 & dex-1 & -2.09 & $\lg x-1$ & -2 \\
\hline lys-3 & -2.28 & nhr-137 & -2.16 & F20G2.2 & -2.09 & T02G5.7 & -2 \\
\hline pqn-60 & -2.28 & cpr-3 & -2.16 & Y65B4BL.1 & -2.09 & nas-30 & -2 \\
\hline hsp-17 & -2.28 & ptr-1 & -2.16 & ugt-19 & -2.08 & Igc-33 & -2 \\
\hline lec-6 & -2.27 & cyn-17 & -2.16 & $\operatorname{ttr}-15$ & -2.08 & rde-4 & -2 \\
\hline $\mathrm{T} 24 \mathrm{C} 12.4$ & -2.26 & ugt-33 & -2.16 & srh-2 & -2.08 & nspe-5 & -2 \\
\hline anmt-3 & -2.26 & hро-6 & -2.15 & cdh-5 & -2.08 & irg-5 & -1.99 \\
\hline grl-4 & -2.26 & nduo- 6 & -2.15 & col-182 & -2.07 & C55A6.12 & -1.99 \\
\hline F41E6.11 & -2.26 & ifd-2 & -2.15 & dpy-1 & -2.07 & grl-6 & -1.99 \\
\hline T26C5.4 & -2.26 & Y57G11C.42 & -2.15 & grd-14 & -2.07 & smd-1 & -1.99 \\
\hline Ibp-5 & -2.25 & pqn-32 & -2.15 & Iron-2 & -2.06 & ttr-38 & -1.98 \\
\hline col-169 & -2.25 & let-754 & -2.14 & era-1 & -2.06 & col-54 & -1.98 \\
\hline R193.2 & -2.25 & hsp-60 & -2.14 & K01A6.7 & -2.06 & ugt-40 & -1.98 \\
\hline bus- 8 & -2.25 & ugt-45 & -2.13 & haao-1 & -2.06 & B0035.15 & -1.98 \\
\hline hch-1 & -2.24 & Y49F6B.12 & -2.13 & F38A6.4 & -2.06 & F15D4.5 & -1.98 \\
\hline rab-11.2 & -2.23 & nas-10 & -2.13 & tag-297 & -2.05 & col-3 & -1.97 \\
\hline abu-14 & -2.23 & acer-1 & -2.13 & Y16B4A.2 & -2.05 & Iron-1 & -1.97 \\
\hline F13H8.5 & -2.23 & col-167 & -2.13 & T06G6.12 & -2.05 & acox -1.2 & -1.97 \\
\hline C46A5.4 & -2.23 & F42A8.1 & -2.13 & ZC513.7 & -2.05 & cutl-23 & -1.97 \\
\hline col-115 & -2.23 & C35A5.3 & -2.13 & F54E2.1 & -2.05 & mtp-18 & -1.97 \\
\hline T06A4.3 & -2.23 & nspb-12 & -2.13 & cest-33 & -2.05 & Y49E10.18 & -1.97 \\
\hline ZK662.2 & -2.22 & aqp-1 & -2.13 & T27E9.2 & -2.05 & F45E4.5 & -1.96 \\
\hline R09E10.5 & -2.21 & Y7A9A.79 & -2.12 & C01B10.11 & -2.04 & R04B5.5 & -1.96 \\
\hline ZK180.5 & -2.21 & plpr-1 & -2.12 & T11B7.2 & -2.04 & cutl-8 & -1.96 \\
\hline cpr-1 & -2.21 & F28B4.3 & -2.12 & lips-10 & -2.04 & C34C12.8 & -1.96 \\
\hline T17H7.7 & -2.21 & spp-2 & -2.12 & $\mathrm{mtd}-1$ & -2.03 & ZK622.4 & -1.96 \\
\hline C35B1.5 & -2.21 & apy-1 & -2.12 & act-3 & -2.03 & ram-5 & -1.95 \\
\hline C35A5.10 & -2.2 & F46C3.6 & -2.11 & F46C8.8 & -2.03 & cnc-10 & -1.95 \\
\hline Y43F8B.3 & -2.2 & acdh-6 & -2.11 & T05H4.7 & -2.03 & K09D9.1 & -1.95 \\
\hline R02F11.1 & -2.2 & Y39A1A.21 & -2.11 & cysl-2 & -2.02 & nduo-3 & -1.95 \\
\hline pho-14 & -2.2 & ttr-18 & -2.11 & F26A1.9 & -2.02 & Y37A1B.5 & -1.95 \\
\hline M03E7.4 & -2.2 & pqn-71 & -2.1 & col-146 & -2.02 & EGAP4.1 & -1.95 \\
\hline twk-11 & -2.18 & mlt-9 & -2.1 & $\operatorname{ttr}-45$ & -2.02 & gsnl-1 & -1.95 \\
\hline F01F1.3 & -2.18 & F59A6.10 & -2.1 & R12A1.3 & -2.02 & M153.1 & -1.94 \\
\hline F44E5.5 & -2.18 & col-104 & -2.1 & F27D9.2 & -2.02 & Ibp-3 & -1.94 \\
\hline C54G4.4 & -2.18 & Ipr-3 & -2.1 & acs-11 & -2.02 & gst-21 & -1.94 \\
\hline nstp-2 & -2.18 & ndfl-4 & -2.09 & axl-1 & -2.02 & F59D6.1 & -1.93 \\
\hline R07E5.4 & -2.18 & F08B12.4 & -2.09 & K08D12.4 & -2.02 & ins-26 & -1.93 \\
\hline F59B10.3 & -2.17 & pqn-29 & -2.09 & cnc- 8 & -2.02 & Y57G11A.4 & -1.93 \\
\hline F32D8.7 & -2.17 & C50D2.1 & -2.09 & $\operatorname{ttr}-32$ & -2.01 & ZK1290.10 & -1.93 \\
\hline C18E9.7 & -2.17 & K06A9.3 & -2.09 & sym-1 & -2.01 & hpo-36 & -1.92 \\
\hline
\end{tabular}




\begin{tabular}{|c|c|c|c|c|c|c|c|}
\hline Gene & $\log 2 \mathrm{FC}$ & Gene & $\log 2 \mathrm{FC}$ & Gene & $\log 2 \mathrm{FC}$ & Gene & $\log 2 \mathrm{FC}$ \\
\hline K11G9.5 & -1.92 & C06G1.1 & -1.85 & K04H4.2 & -1.79 & C34E7.4 & -1.74 \\
\hline abu-8 & -1.91 & Ipr-6 & -1.85 & pho-1 & -1.79 & C03B1.14 & -1.74 \\
\hline F11A5.9 & -1.91 & F27E5.9 & -1.85 & R07E3.2 & -1.79 & C49F8.3 & -1.74 \\
\hline C34C12.7 & -1.91 & K08D12.8 & -1.85 & sdz-24 & -1.79 & M03F4.6 & -1.74 \\
\hline mdh-1 & -1.91 & F53H4.3 & -1.84 & F09C8.1 & -1.79 & idhg-2 & -1.74 \\
\hline papl-1 & -1.91 & ttr-7 & -1.84 & F13E9.13 & -1.79 & C52G5.2 & -1.74 \\
\hline col-155 & -1.91 & H02F09.3 & -1.84 & pfn-1 & -1.79 & ugt-13 & -1.74 \\
\hline $\operatorname{lgc}-27$ & -1.9 & dpy-18 & -1.84 & col-65 & -1.79 & ZK180.6 & -1.74 \\
\hline col-61 & -1.9 & Ipr-7 & -1.84 & chs- 2 & -1.79 & pges-2 & -1.74 \\
\hline ZC513.14 & -1.9 & bca-2 & -1.84 & col-78 & -1.78 & Y47H10A.5 & -1.74 \\
\hline T20B3.1 & -1.9 & ugt-9 & -1.84 & ztf-26 & -1.78 & M04C3.2 & -1.73 \\
\hline T14G8.4 & -1.9 & gst-19 & -1.84 & $\operatorname{ctg}-2$ & -1.78 & metr-1 & -1.73 \\
\hline Y57G7A.1 & -1.9 & nas-13 & -1.84 & asns- 1 & -1.78 & pho-8 & -1.73 \\
\hline pqn-67 & -1.9 & col-177 & -1.84 & col-130 & -1.78 & ech-4 & -1.73 \\
\hline ZK84.1 & -1.89 & grl-16 & -1.84 & ech-6 & -1.78 & grd-16 & -1.73 \\
\hline $\operatorname{ttr}-30$ & -1.89 & ugt-63 & -1.83 & tsp-1 & -1.77 & tts-2 & -1.73 \\
\hline R07B1.8 & -1.89 & abu-10 & -1.83 & Y41G9A.5 & -1.77 & $\mathrm{R} 74.2$ & -1.73 \\
\hline aagr-4 & -1.89 & R186.8 & -1.83 & $\mathrm{D} 1005.2$ & -1.77 & C30B5.6 & -1.73 \\
\hline M7.12 & -1.89 & linc- 6 & -1.83 & W05F2.3 & -1.77 & F29B9.5 & -1.72 \\
\hline $\mathrm{fbxc}-40$ & -1.89 & mlt-8 & -1.83 & сyp-33D1 & -1.77 & cht-1 & -1.72 \\
\hline clec-78 & -1.89 & pgk-1 & -1.83 & Y38E10A.28 & -1.77 & clec-172 & -1.72 \\
\hline slc-36.4 & -1.89 & cnc-3 & -1.82 & Igc-21 & -1.77 & lips-17 & -1.72 \\
\hline ZC123.1 & -1.89 & cut-5 & -1.82 & T16G1.2 & -1.77 & C30G12.6 & -1.72 \\
\hline C05C10.3 & -1.88 & ZK809.8 & -1.82 & K09B3.1 & -1.77 & ora-1 & -1.72 \\
\hline T16G12.1 & -1.88 & nol-56 & -1.82 & npax-2 & -1.77 & dhs- 8 & -1.72 \\
\hline cutl-10 & -1.88 & T06E4.14 & -1.81 & F41F3.3 & -1.77 & hog-1 & -1.72 \\
\hline F01D5.6 & -1.87 & F44E2.4 & -1.81 & T24F1.7 & -1.77 & pqn-36 & -1.72 \\
\hline fipr-7 & -1.87 & linc-17 & -1.81 & twk-22 & -1.76 & K08D9.4 & -1.72 \\
\hline linc-1 & -1.87 & D2092.8 & -1.81 & lagr-1 & -1.76 & Y47D3B.6 & -1.72 \\
\hline ZK970.7 & -1.87 & F13C5.5 & -1.81 & C25F9.4 & -1.76 & Y68A4A.13 & -1.72 \\
\hline hil-3 & -1.87 & сур-35C1 & -1.81 & C25E10.5 & -1.76 & jud-4 & -1.71 \\
\hline T19C4.1 & -1.87 & dod-3 & -1.81 & Y32G9B.1 & -1.76 & D2096.6 & -1.71 \\
\hline Y57G11C.39 & -1.86 & T05A7.1 & -1.8 & F09F7.6 & -1.76 & mpst-3 & -1.7 \\
\hline wrt-9 & -1.86 & $\mathrm{H} 42 \mathrm{~K} 12.3$ & -1.8 & F15E6.3 & -1.76 & B0205.13 & -1.7 \\
\hline abu-11 & -1.86 & nex-1 & -1.8 & C18D11.3 & -1.76 & F55A11.7 & -1.7 \\
\hline ptr-18 & -1.86 & $\operatorname{ttr}-28$ & -1.8 & W02F12.8 & -1.76 & grd-13 & -1.7 \\
\hline F53B3.3 & -1.85 & C08E8.3 & -1.8 & slc-25A10 & -1.75 & nhr-202 & -1.7 \\
\hline Y48G1BR.1 & -1.85 & C35D10.5 & -1.8 & argk-1 & -1.75 & hsp-12.2 & -1.7 \\
\hline C31C9.2 & -1.85 & tth-1 & -1.79 & F35A5.4 & -1.75 & Y43F8C.13 & -1.7 \\
\hline pqn-73 & -1.85 & C34E11.2 & -1.79 & $\mathrm{~F} 52 \mathrm{H} 2.3$ & -1.75 & $\operatorname{ttr}-33$ & -1.7 \\
\hline col-74 & -1.85 & noah-2 & -1.79 & dlc-2 & -1.74 & Y37A1A.4 & -1.7 \\
\hline
\end{tabular}




\begin{tabular}{|c|c|c|c|c|c|c|c|}
\hline Gene & $\log 2 \mathrm{FC}$ & Gene & $\log 2 \mathrm{FC}$ & Gene & $\log 2 \mathrm{FC}$ & Gene & $\log 2 \mathrm{FC}$ \\
\hline Y59H11AR.4 & -1.69 & cec- 8 & -1.64 & C24D10.6 & -1.6 & cblc-1 & -1.55 \\
\hline R12E2.7 & -1.69 & F10C1.9 & -1.64 & R04B3.3 & -1.59 & C34B4.2 & -1.55 \\
\hline K02D3.2 & -1.69 & C15F1.1 & -1.64 & H10E21.4 & -1.59 & F15G9.6 & -1.55 \\
\hline lips-3 & -1.69 & Irx-1 & -1.64 & clec-42 & -1.59 & clec- 88 & -1.55 \\
\hline col-125 & -1.69 & col-165 & -1.64 & Y62E10A.19 & -1.59 & $\mathrm{C} 08 \mathrm{H} 9.15$ & -1.55 \\
\hline T10G3.3 & -1.69 & elo-6 & -1.64 & $n d x-2$ & -1.59 & W08E12.2 & -1.54 \\
\hline arrd-16 & -1.69 & C48B4.13 & -1.64 & nep-22 & -1.59 & C05E7.2 & -1.54 \\
\hline R11A5.3 & -1.69 & C06C3.4 & -1.64 & T05H10.3 & -1.59 & Y87G2A.19 & -1.54 \\
\hline F30H5.3 & -1.69 & daf- 6 & -1.63 & ztf-30 & -1.59 & pcs-1 & -1.54 \\
\hline T25E4.1 & -1.68 & ptr-4 & -1.63 & $\mathrm{C} 46 \mathrm{C} 2.7$ & -1.58 & ctf-8 & -1.54 \\
\hline fip-5 & -1.68 & Y53F4B.27 & -1.63 & $\mathrm{~F} 57 \mathrm{H} 12.6$ & -1.58 & fbxb-72 & -1.54 \\
\hline clec-266 & -1.68 & asp-8 & -1.63 & pсp-2 & -1.58 & hil-7 & -1.54 \\
\hline F43D9.1 & -1.68 & F56B3.2 & -1.63 & C04G6.10 & -1.58 & ZK822.1 & -1.54 \\
\hline C45E5.1 & -1.68 & atf-2 & -1.63 & Y82E9BR.6 & -1.58 & T01B7.8 & -1.54 \\
\hline tipn-1 & -1.68 & gst-9 & -1.63 & ins-11 & -1.58 & phat-2 & -1.53 \\
\hline C16D9.1 & -1.68 & lys-1 & -1.63 & Y17D7C.4 & -1.58 & nhr-12 & -1.53 \\
\hline T07F10.6 & -1.68 & H37A05.2 & -1.63 & mec-12 & -1.58 & dhrs-4 & -1.53 \\
\hline R13D11.4 & -1.68 & F02C12.1 & -1.63 & such-1 & -1.58 & F27D9.7 & -1.53 \\
\hline F46G11.2 & -1.67 & $\mathrm{R} 03 \mathrm{H} 10.2$ & -1.62 & $\mathrm{~F} 36 \mathrm{H} 1.11$ & -1.58 & Y62E10A.13 & -1.53 \\
\hline col-168 & -1.67 & lips-7 & -1.62 & F54E7.6 & -1.57 & taf-11.2 & -1.53 \\
\hline F17C11.11 & -1.67 & Y65B4BL. 6 & -1.62 & C48B4.9 & -1.57 & col-58 & -1.53 \\
\hline gcsh-1 & -1.67 & R08E5.3 & -1.62 & T23F2.3 & -1.56 & T19D12.4 & -1.53 \\
\hline W03D2.9 & -1.67 & fip-6 & -1.62 & $m t x-1$ & -1.56 & R03G8.3 & -1.53 \\
\hline nlp-16 & -1.67 & C29F3.7 & -1.62 & F48G7.5 & -1.56 & D2005.6 & -1.53 \\
\hline Isy-27 & -1.67 & tsp-2 & -1.62 & ZK596.1 & -1.56 & F47D12.3 & -1.53 \\
\hline C06C3.10 & -1.67 & col-186 & -1.62 & clec-173 & -1.56 & acs-19 & -1.53 \\
\hline ugt-47 & -1.67 & cpt-4 & -1.62 & $\mathrm{~F} 10 \mathrm{C} 1.8$ & -1.56 & $\mathrm{~F} 14 \mathrm{H} 12.3$ & -1.53 \\
\hline H01G02.1 & -1.67 & B0034.1 & -1.62 & cyb-2.2 & -1.56 & D1053.4 & -1.53 \\
\hline F32A7.8 & -1.66 & cdc-50.B & -1.62 & F55F8.8 & -1.56 & hsp-25 & -1.53 \\
\hline ZC416.2 & -1.66 & $\mathrm{~F} 21 \mathrm{C} 10.10$ & -1.61 & C15B12.8 & -1.56 & abhd-11.2 & -1.52 \\
\hline grl-1 & -1.66 & ifp-1 & -1.61 & C07A4.2 & -1.56 & dhcr-24 & -1.52 \\
\hline Y69A2AR.28 & -1.66 & ttr-6 & -1.61 & phat-4 & -1.56 & F13B6.1 & -1.52 \\
\hline $\mathrm{M} 02 \mathrm{H} 5.8$ & -1.66 & R12E2.14 & -1.61 & ttr-26 & -1.56 & Y58A7A.3 & -1.52 \\
\hline ZK154.1 & -1.66 & prx-19 & -1.61 & F53F4.2 & -1.56 & F52E4.5 & -1.52 \\
\hline far-3 & -1.65 & K02D3.1 & -1.61 & cyb-3 & -1.56 & gpd-4 & -1.52 \\
\hline mex-1 & -1.65 & T23F2.5 & -1.61 & Т19С3.3 & -1.56 & fib-1 & -1.52 \\
\hline C05D12.1 & -1.65 & nlp-29 & -1.61 & F45D3.3 & -1.56 & K08D8.6 & -1.52 \\
\hline C18D11.6 & -1.65 & vglu-2 & -1.6 & set-20 & -1.55 & acaa-2 & -1.52 \\
\hline mlc-6 & -1.65 & hxk-1 & -1.6 & F13E9.15 & -1.55 & lgc-34 & -1.52 \\
\hline anmt-2 & -1.65 & C06E7.4 & -1.6 & ptps-1 & -1.55 & W04A8.4 & -1.51 \\
\hline cpr-5 & -1.65 & B0294.1 & -1.6 & Y80D3A.9 & -1.55 & ppgn-1 & -1.51 \\
\hline
\end{tabular}




\begin{tabular}{|c|c|c|c|c|c|c|c|}
\hline Gene & $\log 2 \mathrm{FC}$ & Gene & $\log 2 \mathrm{FC}$ & Gene & $\log 2 \mathrm{FC}$ & Gene & $\log 2 \mathrm{FC}$ \\
\hline R13D7.2 & -1.51 & daf-22 & -1.47 & T21D12.14 & -1.44 & nlp-27 & -1.42 \\
\hline agmo-1 & -1.51 & str-112 & -1.47 & K07D4.4 & -1.44 & С03АЗ.3 & -1.42 \\
\hline Y68A4B.3 & -1.51 & C13A10.2 & -1.47 & T08B1.1 & -1.44 & Y41G9A.10 & -1.42 \\
\hline F56C11.3 & -1.51 & T09B9.1 & -1.47 & K03A1.4 & -1.44 & сур-13В1 & -1.41 \\
\hline F14D7.12 & -1.51 & C15A11.4 & -1.47 & F57F5.1 & -1.44 & T14E8.4 & -1.41 \\
\hline Y38E10A.14 & -1.51 & T23F6.1 & -1.47 & ZK742.6 & -1.44 & C17C3.1 & -1.41 \\
\hline sqt-3 & -1.51 & C01G6.3 & -1.47 & $\mathrm{~F} 40 \mathrm{H} 3.3$ & -1.44 & F32D8.12 & -1.41 \\
\hline $\mathrm{T} 10 \mathrm{H} 10.2$ & -1.5 & dnj-11 & -1.47 & R05D3.9 & -1.44 & col-174 & -1.41 \\
\hline F15B9.8 & -1.5 & lact- 4 & -1.47 & F26G1.5 & -1.44 & moc-1 & -1.41 \\
\hline sptl-2 & -1.5 & cpg-7 & -1.47 & B0334.13 & -1.44 & rocf-1 & -1.41 \\
\hline ugt-29 & -1.5 & $\mathrm{~K} 10 \mathrm{H} 10.12$ & -1.47 & F08F1.3 & -1.44 & T05C1.1 & -1.41 \\
\hline F53B1.4 & -1.5 & linc-4 & -1.47 & T20B3.14 & -1.44 & ZK1010.4 & -1.4 \\
\hline F01D5.10 & -1.5 & F13A7.11 & -1.47 & R07B1.9 & -1.44 & gly-11 & -1.4 \\
\hline BE0003N10.1 & -1.5 & F18E9.4 & -1.47 & C16B8.2 & -1.44 & F44E7.5 & -1.4 \\
\hline sdhd-1 & -1.5 & F39D8.3 & -1.46 & T12B3.2 & -1.44 & air-2 & -1.4 \\
\hline $\mathrm{C} 28 \mathrm{C} 12.4$ & -1.5 & $r d y-2$ & -1.46 & K06G5.1 & -1.44 & sod-5 & -1.4 \\
\hline mis-12 & -1.5 & T23F2.4 & -1.46 & T01H8.2 & -1.44 & F44G4.3 & -1.4 \\
\hline K01D12.5 & -1.5 & C03F11.2 & -1.46 & mppa-1 & -1.44 & Y40B10B.1 & -1.4 \\
\hline T05E12.6 & -1.49 & R11H6.4 & -1.46 & F55G1.1 & -1.43 & W03F9.4 & -1.4 \\
\hline F56A12.2 & -1.49 & cpn-4 & -1.46 & tni-3 & -1.43 & C15H9.11 & -1.39 \\
\hline T07A9.14 & -1.49 & pqn-37 & -1.46 & F22F7.7 & -1.43 & T05A7.7 & -1.39 \\
\hline src-2 & -1.49 & clec- 41 & -1.46 & Y105C5A.1268 & -1.43 & cisd-3.1 & -1.39 \\
\hline VF13D12L.3 & -1.49 & ttr-51 & -1.46 & best-13 & -1.43 & H2OE11.1 & -1.39 \\
\hline F10D7.2 & -1.49 & act-1 & -1.46 & T20G5.8 & -1.43 & dhs-23 & -1.39 \\
\hline F29G6.1 & -1.49 & C06E1.1 & -1.46 & phy-2 & -1.43 & сpi-1 & -1.39 \\
\hline F48E3.2 & -1.49 & Y39A3CL.3 & -1.45 & cdk-5 & -1.43 & Y63D3A.7 & -1.39 \\
\hline D2023.4 & -1.48 & Y17D7B.3 & -1.45 & ubc-12 & -1.43 & ric-7 & -1.39 \\
\hline C55A1.6 & -1.48 & $\mathrm{C} 34 \mathrm{H} 4.2$ & -1.45 & nhr-161 & -1.43 & cysl-1 & -1.39 \\
\hline mrps-28 & -1.48 & clec-187 & -1.45 & fipr-6 & -1.43 & F26E4.3 & -1.38 \\
\hline T26C5.5 & -1.48 & grl-7 & -1.45 & $\mathrm{R} 13 \mathrm{H} 4.8$ & -1.43 & hach-1 & -1.38 \\
\hline Y46G5A.36 & -1.48 & hic-1 & -1.45 & Y47D7A.13 & -1.42 & pgph-2 & -1.38 \\
\hline Y56АЗА.19 & -1.48 & cest-35.2 & -1.45 & Y55D5A.4 & -1.42 & Y45G12B.3 & -1.38 \\
\hline wrt-2 & -1.48 & pos-1 & -1.45 & spn-4 & -1.42 & F58G1.7 & -1.38 \\
\hline col-180 & -1.48 & R05G9R.1 & -1.45 & lact-7 & -1.42 & C05D2.8 & -1.38 \\
\hline Y53F4B.23 & -1.48 & T12G3.6 & -1.45 & C25E10.10 & -1.42 & gpd-1 & -1.38 \\
\hline pqn-13 & -1.48 & nhr-65 & -1.45 & eppl-1 & -1.42 & zipt-7.1 & -1.38 \\
\hline col-117 & -1.48 & dpy-17 & -1.45 & T14B4.5 & -1.42 & F35C12.3 & -1.38 \\
\hline pgp-8 & -1.48 & C36B7.5 & -1.45 & $\operatorname{tr} x-3$ & -1.42 & C14C6.5 & -1.38 \\
\hline Y40A1A.3 & -1.48 & vab-2 & -1.45 & hpr-9 & -1.42 & srr-1 & -1.38 \\
\hline ifb-1 & -1.47 & R12E2.15 & -1.44 & nep-16 & -1.42 & C05C10.5 & -1.38 \\
\hline cts-1 & -1.47 & F58E6.13 & -1.44 & acs-1 & -1.42 & F13D11.3 & -1.38 \\
\hline
\end{tabular}




\begin{tabular}{|c|c|c|c|c|c|c|c|}
\hline Gene & $\log 2 \mathrm{FC}$ & Gene & $\log 2 \mathrm{FC}$ & Gene & $\log 2 \mathrm{FC}$ & Gene & $\log 2 \mathrm{FC}$ \\
\hline F20G2.5 & -1.38 & tomm-40 & -1.34 & unc-59 & -1.32 & сур-23A1 & -1.29 \\
\hline doxa-1 & -1.38 & C54E4.4 & -1.34 & W06F12.2 & -1.32 & gmeb-2 & -1.29 \\
\hline cest-12 & -1.38 & ZK909.3 & -1.34 & klp-19 & -1.32 & T12B3.3 & -1.29 \\
\hline F16B4.4 & -1.38 & mex-5 & -1.34 & K03B4.4 & -1.31 & lgc-37 & -1.29 \\
\hline C06G1.2 & -1.38 & ect-2 & -1.34 & ugt-48 & -1.31 & F49E12.8 & -1.28 \\
\hline F14H3.6 & -1.38 & F49B2.6 & -1.34 & grl-10 & -1.31 & aco-2 & -1.28 \\
\hline Y39F10A.1 & -1.38 & F21A3.3 & -1.34 & tyr-6 & -1.31 & nhr-21 & -1.28 \\
\hline pash-1 & -1.37 & M28.5 & -1.34 & dhs- 19 & -1.31 & M05D6.5 & -1.28 \\
\hline ZK822.5 & -1.37 & $\mathrm{C} 23 \mathrm{H} 4.8$ & -1.34 & dhs-30 & -1.31 & F45D3.4 & -1.28 \\
\hline F25B3.2 & -1.37 & $\mathrm{C} 13 \mathrm{C} 12.2$ & -1.34 & vha-12 & -1.31 & lec-1 & -1.28 \\
\hline scrm-4 & -1.37 & nhr-105 & -1.34 & C07E3.3 & -1.31 & C01G10.7 & -1.28 \\
\hline ptr-17 & -1.37 & col-172 & -1.34 & gst-36 & -1.31 & cdh-8 & -1.28 \\
\hline pfn-3 & -1.37 & got-2.2 & -1.34 & C29F9.6 & -1.31 & mlp-1 & -1.28 \\
\hline $\mathrm{rcs}-1$ & -1.37 & grd-10 & -1.33 & Y47D3A.20 & -1.31 & W03C9.2 & -1.28 \\
\hline F54H12.5 & -1.37 & cpn-2 & -1.33 & col-75 & -1.31 & tag-293 & -1.28 \\
\hline inx-3 & -1.37 & F01G10.6 & -1.33 & K02E10.4 & -1.31 & R102.2 & -1.27 \\
\hline Y71H2AM.6 & -1.37 & nlp-33 & -1.33 & F31D4.5 & -1.3 & C18B10.6 & -1.27 \\
\hline fmo-2 & -1.37 & M04C9.3 & -1.33 & pck-1 & -1.3 & srr-6 & -1.27 \\
\hline acox-1.1 & -1.37 & dnj-27 & -1.33 & E04D5.4 & -1.3 & $\mathrm{C} 42 \mathrm{C} 1.8$ & -1.27 \\
\hline $\mathrm{mcm}-7$ & -1.37 & C45B2.8 & -1.33 & F13B6.2 & -1.3 & T01C8.2 & -1.27 \\
\hline Igc- 22 & -1.36 & tnt-4 & -1.33 & Т02H6.11 & -1.3 & F56D3.1 & -1.27 \\
\hline T13C2.2 & -1.36 & cpg-9 & -1.33 & T02C12.5 & -1.3 & F44D12.2 & -1.27 \\
\hline col-121 & -1.36 & dhs-27 & -1.33 & his-24 & -1.3 & oig-5 & -1.27 \\
\hline ttr-59 & -1.36 & lir-3 & -1.33 & C09F9.2 & -1.3 & F53A9.3 & -1.27 \\
\hline C08E3.1 & -1.36 & cnc- 6 & -1.33 & set-19 & -1.3 & mlt-11 & -1.27 \\
\hline pie-1 & -1.36 & rsp-5 & -1.33 & F59E11.5 & -1.3 & cnp-2 & -1.27 \\
\hline Y18H1A.9 & -1.36 & inf-1 & -1.33 & $\mathrm{~T} 24 \mathrm{H} 10.4$ & -1.3 & F59E12.15 & -1.27 \\
\hline F53A9.7 & -1.36 & M60.6 & -1.33 & lec-7 & -1.3 & ztf-9 & -1.27 \\
\hline natc-2 & -1.36 & F47E1.1 & -1.33 & $\mathrm{~F} 23 \mathrm{H} 12.5$ & -1.3 & gln-3 & -1.27 \\
\hline $3 / 3 / 2020$ & -1.36 & Y51H4A.5 & -1.33 & $\mathrm{~T} 28 \mathrm{C} 12.6$ & -1.3 & R13A5.10 & -1.27 \\
\hline romo-1 & -1.36 & ZK742.3 & -1.33 & trh-1 & -1.3 & pgp-15 & -1.26 \\
\hline K11D2.1 & -1.36 & $\mathrm{C} 14 \mathrm{C} 11.7$ & -1.33 & hсp-2 & -1.29 & cut-3 & -1.26 \\
\hline R02E4.3 & -1.36 & H11E01.2 & -1.32 & mup-4 & -1.29 & F41E6.12 & -1.26 \\
\hline mev-1 & -1.35 & Y58A7A.4 & -1.32 & spp-17 & -1.29 & amt-4 & -1.26 \\
\hline cutl-12 & -1.35 & hcp-4 & -1.32 & C34C12.6 & -1.29 & R02F2.8 & -1.26 \\
\hline acly-2 & -1.35 & ttc-36 & -1.32 & C29A12.6 & -1.29 & ceh-74 & -1.26 \\
\hline phat-1 & -1.35 & flh-1 & -1.32 & C56C10.4 & -1.29 & exc- 6 & -1.26 \\
\hline ifa-3 & -1.35 & F59C12.4 & -1.32 & piit-1 & -1.29 & nhr-22 & -1.26 \\
\hline Y71F9B.1 & -1.35 & dod-18 & -1.32 & him-3 & -1.29 & glrx-10 & -1.26 \\
\hline dpy-7 & -1.35 & F36G3.1 & -1.32 & Ipr-4 & -1.29 & slc-17.8 & -1.26 \\
\hline F02E9.1 & -1.35 & zig-12 & -1.32 & M02D8.6 & -1.29 & K08E3.5 & -1.26 \\
\hline
\end{tabular}




\begin{tabular}{|c|c|c|c|c|c|c|c|}
\hline Gene & $\log 2 \mathrm{FC}$ & Gene & $\log 2 \mathrm{FC}$ & Gene & $\log 2 \mathrm{FC}$ & Gene & $\log 2 \mathrm{FC}$ \\
\hline $\mathrm{mxl}-3$ & -1.26 & W02B12.13 & -1.23 & $\mathrm{~F} 28 \mathrm{H} 7.3$ & -1.21 & W01C8.5 & -1.18 \\
\hline EGAP5.1 & -1.26 & T03F6.10 & -1.23 & T03D8.6 & -1.21 & Y57G11C.44 & -1.18 \\
\hline C02B8.3 & -1.25 & nhr-237 & -1.23 & eat-5 & -1.21 & T06E4.9 & -1.18 \\
\hline F35E12.6 & -1.25 & F38B6.4 & -1.23 & cec-4 & -1.21 & T06E4.8 & -1.18 \\
\hline C15C7.5 & -1.25 & ZC443.1 & -1.23 & Y73B3A.1 & -1.21 & orai-1 & -1.18 \\
\hline T09A5.15 & -1.25 & W08E12.6 & -1.23 & enol-1 & -1.2 & M163.8 & -1.18 \\
\hline dif-1 & -1.25 & K08E4.3 & -1.23 & col-68 & -1.2 & cri-3 & -1.18 \\
\hline mai-2 & -1.25 & T21G5.2 & -1.23 & F26F12.4 & -1.2 & gpn-1 & -1.17 \\
\hline Y73F4A.1 & -1.25 & F59B1.10 & -1.23 & oig-2 & -1.2 & T19B10.5 & -1.17 \\
\hline best-24 & -1.25 & atad-3 & -1.23 & F21D5.3 & -1.2 & lec-10 & -1.17 \\
\hline F31E9.6 & -1.25 & C06B8.2 & -1.23 & cdh-9 & -1.2 & F33G12.7 & -1.17 \\
\hline C09F12.2 & -1.25 & Y46H3A.4 & -1.23 & mth-1 & -1.2 & Y71G12B.10 & -1.17 \\
\hline R11.4 & -1.25 & F01G10.10 & -1.23 & dpy-14 & -1.2 & mrps-6 & -1.17 \\
\hline F46C5.10 & -1.25 & gba-1 & -1.23 & R09F10.5 & -1.2 & sdha-2 & -1.17 \\
\hline F28H7.8 & -1.25 & F33H2.3 & -1.22 & pcyt-1 & -1.2 & $\mathrm{~F} 35 \mathrm{H} 10.2$ & -1.17 \\
\hline lys-4 & -1.25 & wrt-10 & -1.22 & acbp-3 & -1.2 & F10D2.10 & -1.17 \\
\hline bra-2 & -1.25 & F21E9.2 & -1.22 & W09G12.10 & -1.2 & C15H9.9 & -1.17 \\
\hline F32D8.5 & -1.25 & Y39A3CL.4 & -1.22 & ugt-22 & -1.2 & $m x t-1$ & -1.17 \\
\hline bag-1 & -1.25 & $\mathrm{C} 38 \mathrm{H} 2.3$ & -1.22 & ZC455.1 & -1.2 & cdr-6 & -1.17 \\
\hline poml-2 & -1.25 & gtr-1 & -1.22 & col-89 & -1.2 & cutl-14 & -1.17 \\
\hline ppw-1 & -1.25 & T19A5.3 & -1.22 & lev-8 & -1.19 & irg-2 & -1.17 \\
\hline ZK512.1 & -1.24 & cpn-1 & -1.22 & C16D9.4 & -1.19 & Y71G12B.17 & -1.17 \\
\hline pyp-1 & -1.24 & puf-11 & -1.22 & K02E7.5 & -1.19 & Y52E8A.3 & -1.17 \\
\hline bcmo-1 & -1.24 & elf-1 & -1.22 & ZK1307.8 & -1.19 & F56D6.17 & -1.17 \\
\hline C30F2.4 & -1.24 & F14B4.1 & -1.22 & C08F8.9 & -1.19 & rpb-11 & -1.17 \\
\hline Iron-10 & -1.24 & clec-166 & -1.22 & Y92H12BL.5 & -1.19 & rad-8 & -1.17 \\
\hline Y119D3B.12 & -1.24 & ech-1.1 & -1.22 & F25A2.1 & -1.19 & dnj-9 & -1.17 \\
\hline rsp-4 & -1.24 & nmy-1 & -1.22 & Y60A3A.21 & -1.19 & slc-25A42 & -1.16 \\
\hline cpt-5 & -1.24 & $\operatorname{dim}-1$ & -1.22 & ugt-17 & -1.18 & K01A2.5 & -1.16 \\
\hline сур-14A5 & -1.24 & Y69A2AR.3 & -1.21 & $\mathrm{crn}-1$ & -1.18 & F14F9.4 & -1.16 \\
\hline col-154 & -1.24 & C26B2.7 & -1.21 & sod-2 & -1.18 & T19C3.2 & -1.16 \\
\hline Y73C8B.1 & -1.24 & sod-1 & -1.21 & Y73C8B.2 & -1.18 & Y38C1AA.9 & -1.16 \\
\hline set-9 & -1.24 & Y71H2AM.11 & -1.21 & Y87G2A.16 & -1.18 & $n d x-8$ & -1.16 \\
\hline R03A10.5 & -1.24 & $\mathrm{~F} 33 \mathrm{H} 2.6$ & -1.21 & zip-10 & -1.18 & T05H10.4 & -1.16 \\
\hline bigr-1 & -1.23 & M199.2 & -1.21 & efn-4 & -1.18 & C36C9.5 & -1.16 \\
\hline enu-3.5 & -1.23 & Y73C8B.3 & -1.21 & $\mathrm{crn}-6$ & -1.18 & W02F12.2 & -1.16 \\
\hline Y75B7AR.1 & -1.23 & W04B5.3 & -1.21 & Y54G2A.45 & -1.18 & Y113G7A.14 & -1.16 \\
\hline ttr-20 & -1.23 & $\mathrm{fkb}-4$ & -1.21 & parg-2 & -1.18 & pole-4 & -1.15 \\
\hline T09B4.5 & -1.23 & K02E10.6 & -1.21 & cat-1 & -1.18 & $a m x-2$ & -1.15 \\
\hline Y54G11A.4 & -1.23 & C49F5.6 & -1.21 & unc-52 & -1.18 & tba- 6 & -1.15 \\
\hline del-10 & -1.23 & col-131 & -1.21 & C07G3.15 & -1.18 & $\mathrm{~F} 22 \mathrm{H} 10.3$ & -1.15 \\
\hline
\end{tabular}




\begin{tabular}{|c|c|c|c|c|c|c|c|}
\hline Gene & $\log 2 \mathrm{FC}$ & Gene & $\log 2 \mathrm{FC}$ & Gene & $\log 2 \mathrm{FC}$ & Gene & $\log 2 \mathrm{FC}$ \\
\hline F21D5.4 & -1.15 & col-10 & -1.14 & chw-1 & -1.12 & spg-7 & -1.1 \\
\hline ugt-53 & -1.15 & $\mathrm{C} 46 \mathrm{H} 11.7$ & -1.14 & dlhd-1 & -1.12 & T01D1.4 & -1.1 \\
\hline C05D12.3 & -1.15 & uri-1 & -1.13 & mom-2 & -1.12 & F41G3.21 & -1.1 \\
\hline ZK682.7 & -1.15 & Y6D1A. 2 & -1.13 & clec-83 & -1.12 & alx-1 & -1.1 \\
\hline Y56A3A.7 & -1.15 & Y97E10C.1 & -1.13 & far-8 & -1.11 & ZC21.3 & -1.1 \\
\hline mpst-1 & -1.15 & ZC449.5 & -1.13 & C45G9.2 & -1.11 & tdc-1 & -1.09 \\
\hline F19C6.4 & -1.15 & ost-1 & -1.13 & tag-307 & -1.11 & ech-8 & -1.09 \\
\hline cng-1 & -1.15 & $\mathrm{C} 40 \mathrm{H} 1.7$ & -1.13 & gly-8 & -1.11 & T22E5.1 & -1.09 \\
\hline F38B2.2 & -1.15 & F14F9.3 & -1.13 & glt-1 & -1.11 & nas-33 & -1.09 \\
\hline glb-13 & -1.15 & vdac-1 & -1.13 & K07C5.9 & -1.11 & ceh-49 & -1.09 \\
\hline T05E7.1 & -1.15 & gei-14 & -1.13 & clec-87 & -1.11 & acs-7 & -1.09 \\
\hline kdp-1 & -1.15 & F18C5.5 & -1.13 & cfim-1 & -1.11 & clik-1 & -1.09 \\
\hline $\operatorname{arx}-5$ & -1.15 & acds-10 & -1.13 & Y39A3А.4 & -1.11 & nas-7 & -1.09 \\
\hline C26B2.8 & -1.15 & nduf- 6 & -1.13 & T05F1.2 & -1.11 & F13D12.8 & -1.09 \\
\hline nex-3 & -1.15 & skat-1 & -1.13 & K12G11.6 & -1.11 & Ipr-5 & -1.09 \\
\hline nadk-2 & -1.15 & Y113G7B.11 & -1.13 & mrpl-19 & -1.11 & $\operatorname{tfg}-1$ & -1.09 \\
\hline dmd-9 & -1.15 & eat-20 & -1.13 & vem-1 & -1.11 & hrg-7 & -1.09 \\
\hline $\mathrm{F} 33 \mathrm{H} 12.7$ & -1.14 & Y48A6B.7 & -1.13 & pat-12 & -1.11 & T11F8.12 & -1.09 \\
\hline rig-5 & -1.14 & F10A3.4 & -1.13 & taco-1 & -1.11 & $\mathrm{R} 10 \mathrm{H} 10.4$ & -1.09 \\
\hline Y67A10A.9 & -1.14 & swip-10 & -1.13 & tbc-19 & -1.11 & C54E4.12 & -1.09 \\
\hline gpx-5 & -1.14 & igcm-1 & -1.13 & ucr-11 & -1.11 & F25D1.5 & -1.09 \\
\hline T12G3.4 & -1.14 & C13B4.1 & -1.13 & C08B6.4 & -1.11 & lin-24 & -1.09 \\
\hline sco-1 & -1.14 & C25D7.5 & -1.13 & F09C3.2 & -1.11 & his-72 & -1.09 \\
\hline sun-1 & -1.14 & Y58A7A.5 & -1.13 & glt-5 & -1.1 & Ipd-5 & -1.09 \\
\hline $\mathrm{fkb}-1$ & -1.14 & gst-35 & -1.12 & M04B2.4 & -1.1 & kca-1 & -1.09 \\
\hline K08B5.2 & -1.14 & nspe-4 & -1.12 & pyc-1 & -1.1 & ZK675.4 & -1.09 \\
\hline hal-3 & -1.14 & Y10G11A.1 & -1.12 & C47E8.4 & -1.1 & rig-3 & -1.09 \\
\hline T03G11.9 & -1.14 & ZC477.3 & -1.12 & mam-8 & -1.1 & inx-2 & -1.09 \\
\hline ckc-1 & -1.14 & K02E10.1 & -1.12 & Y105E8A.36 & -1.1 & $\mathrm{R} 05 \mathrm{H} 10.1$ & -1.09 \\
\hline B0205.12 & -1.14 & tir-1 & -1.12 & htz-1 & -1.1 & B0205.14 & -1.09 \\
\hline T05G5.1 & -1.14 & nhr-28 & -1.12 & clec-56 & -1.1 & inx-13 & -1.09 \\
\hline let-383 & -1.14 & coq-5 & -1.12 & nlp-66 & -1.1 & bus- 2 & -1.09 \\
\hline F53A9.9 & -1.14 & Y71G12B.6 & -1.12 & phb-1 & -1.1 & T28A8.4 & -1.09 \\
\hline F18E9.1 & -1.14 & C44B7.7 & -1.12 & ugt-21 & -1.1 & Y48G10A.1 & -1.09 \\
\hline Y92H12BR.2 & -1.14 & K08E7.6 & -1.12 & eat-2 & -1.1 & ifa-1 & -1.09 \\
\hline ZK1307.7 & -1.14 & igeg-2 & -1.12 & cdd-2 & -1.1 & rnp-8 & -1.08 \\
\hline C33B4.4 & -1.14 & E01A2.2 & -1.12 & F18A11.2 & -1.1 & coq-8 & -1.08 \\
\hline mif-1 & -1.14 & pfkb-1.2 & -1.12 & fat-5 & -1.1 & Y4C6B.1 & -1.08 \\
\hline C44C1.1 & -1.14 & tpi-1 & -1.12 & lips-9 & -1.1 & F37H8.2 & -1.08 \\
\hline C07G3.10 & -1.14 & C05D12.4 & -1.12 & abu-13 & -1.1 & daf-28 & -1.08 \\
\hline C25F9.9 & -1.14 & gst-27 & -1.12 & Y102A11A.7 & -1.1 & T20F5.4 & -1.08 \\
\hline
\end{tabular}




\begin{tabular}{|c|c|c|c|c|c|c|c|}
\hline Gene & $\log 2 \mathrm{FC}$ & Gene & $\log 2 \mathrm{FC}$ & Gene & $\log 2 \mathrm{FC}$ & Gene & $\log 2 \mathrm{FC}$ \\
\hline Ion-8 & -1.08 & ZK1053.4 & -1.06 & bus-4 & -1.04 & F35D11.4 & -1.02 \\
\hline Y105C5B.19 & -1.08 & ptr-16 & -1.06 & nhr-158 & -1.04 & ugt-16 & -1.02 \\
\hline ZC262.2 & -1.08 & T02D1.8 & -1.06 & pfk-1.1 & -1.04 & nlp-79 & -1.02 \\
\hline W02B8.1 & -1.08 & pole-1 & -1.06 & nasp-2 & -1.04 & W05B10.4 & -1.02 \\
\hline T08G2.2 & -1.08 & oat-1 & -1.06 & ceh-2 & -1.04 & col-12 & -1.02 \\
\hline haf-1 & -1.08 & ina-1 & -1.06 & T21C9.9 & -1.04 & C04F12.5 & -1.02 \\
\hline acp-6 & -1.08 & F35H10.10 & -1.05 & C56G2.4 & -1.03 & B0507.1 & -1.02 \\
\hline dhs-3 & -1.08 & arrd-25 & -1.05 & dhs- 25 & -1.03 & F09E10.7 & -1.02 \\
\hline $\mathrm{F} 23 \mathrm{C} 8.14$ & -1.08 & сур-33С5 & -1.05 & F08F3.6 & -1.03 & dma-1 & -1.02 \\
\hline enu-3.3 & -1.08 & snr-2 & -1.05 & $\mathrm{~F} 56 \mathrm{H} 11.2$ & -1.03 & ztf-4 & -1.01 \\
\hline cisd-3.2 & -1.08 & dhs-29 & -1.05 & T13H5.6 & -1.03 & F36H9.2 & -1.01 \\
\hline alh-10 & -1.08 & C53A5.2 & -1.05 & F33C8.4 & -1.03 & hsp-4 & -1.01 \\
\hline ucp-4 & -1.08 & T26E3.10 & -1.05 & F31F7.1 & -1.03 & Y55F3C.17 & -1.01 \\
\hline sups-1 & -1.08 & adt-1 & -1.05 & lat-2 & -1.03 & C49G7.10 & -1.01 \\
\hline $\mathrm{C} 25 \mathrm{H} 3.14$ & -1.08 & C01A2.4 & -1.05 & clec-4 & -1.03 & W01A11.1 & -1.01 \\
\hline ttr-8 & -1.08 & C38C6.3 & -1.05 & aqp-11 & -1.03 & B0284.3 & -1.01 \\
\hline T27F6.8 & -1.07 & F53F1.6 & -1.05 & F58A6.1 & -1.03 & grl-5 & -1.01 \\
\hline dog-1 & -1.07 & C53H9.3 & -1.05 & nmgp-1 & -1.03 & $\operatorname{cox}-6 \mathrm{C}$ & -1.01 \\
\hline dhs-5 & -1.07 & R04A9.9 & -1.05 & elo-9 & -1.03 & B0238.12 & -1.01 \\
\hline F58H1.5 & -1.07 & upp-1 & -1.05 & ZK1055.6 & -1.03 & plst-1 & -1.01 \\
\hline nlp-24 & -1.07 & Y38H6C.20 & -1.05 & ceh-91 & -1.03 & zipt-13 & -1.01 \\
\hline tbb-6 & -1.07 & F20D1.3 & -1.05 & C45G7.4 & -1.03 & F15G9.1 & -1.01 \\
\hline cah-5 & -1.07 & brd-1 & -1.05 & gfl-1 & -1.03 & R06C1.4 & -1.01 \\
\hline ZK973.9 & -1.07 & C44B9. 3 & -1.05 & clec-91 & -1.02 & dhhc-1 & -1.01 \\
\hline sel-7 & -1.07 & $\mathrm{~K} 11 \mathrm{H} 12.7$ & -1.05 & Y48G1A.1 & -1.02 & sdz-4 & -1.01 \\
\hline nduo-2 & -1.07 & phat-5 & -1.05 & ZK228.4 & -1.02 & $\mathrm{dsl}-2$ & -1.01 \\
\hline swt-5 & -1.07 & pqn-31 & -1.05 & pgp-6 & -1.02 & F18A1.7 & -1.01 \\
\hline С18H9.6 & -1.07 & Y53G8B.2 & -1.05 & DC2.5 & -1.02 & K10C9.1 & -1.01 \\
\hline F13A7.12 & -1.07 & cdc- 25.3 & -1.05 & Y7A9A.1 & -1.02 & F01D5.5 & -1.01 \\
\hline cht-2 & -1.07 & Y25C1A.8 & -1.04 & zig-8 & -1.02 & asic-2 & -1 \\
\hline cutl-25 & -1.07 & sdha-1 & -1.04 & $\mathrm{mcm}-3$ & -1.02 & F57C2.5 & -1 \\
\hline F23A7.4 & -1.07 & $\operatorname{cox}-17$ & -1.04 & F36H9.7 & -1.02 & gbh-1 & -1 \\
\hline T11G6.3 & -1.06 & $\mathrm{cpd}-2$ & -1.04 & Y94H6A.16 & -1.02 & tyr-4 & -1 \\
\hline ddl-3 & -1.06 & dpy-31 & -1.04 & Y87G2A.2 & -1.02 & math-22 & -1 \\
\hline tps-2 & -1.06 & pqm-1 & -1.04 & swt-6 & -1.02 & B0495.8 & -1 \\
\hline tyms-1 & -1.06 & suds-3 & -1.04 & F59F4.3 & -1.02 & F10D7.1 & -1 \\
\hline $\mathrm{C} 02 \mathrm{~F} 4.4$ & -1.06 & B0272.4 & -1.04 & ero-1 & -1.02 & calu-1 & -1 \\
\hline lin-42 & -1.06 & Iron-8 & -1.04 & thoc-5 & -1.02 & W01B11.6 & -1 \\
\hline R07E3.6 & -1.06 & hex-1 & -1.04 & Y39G10AR.32 & -1.02 & mrps-34 & -1 \\
\hline iсp-1 & -1.06 & B0272.3 & -1.04 & $\mathrm{~K} 12 \mathrm{H} 4.5$ & -1.02 & Y42H9AR.5 & -1 \\
\hline C02E7.7 & -1.06 & F13A7.14 & -1.04 & svh-1 & -1.02 & mlt-4 & -1 \\
\hline
\end{tabular}




\begin{tabular}{|c|c|c|c|c|c|c|c|}
\hline Gene & $\log 2 \mathrm{FC}$ & Gene & $\log 2 \mathrm{FC}$ & Gene & $\log 2 \mathrm{FC}$ & Gene & $\log 2 \mathrm{FC}$ \\
\hline C01B10.3 & -1 & T22D1.3 & 1.02 & Y104H12D.2 & 1.03 & srpa-72 & 1.05 \\
\hline ddp-1 & -1 & cyl-1 & 1.02 & F02A9.7 & 1.03 & F55A3.2 & 1.05 \\
\hline gpc-2 & -1 & twk-8 & 1.02 & ZK1240.3 & 1.03 & glct- 6 & 1.06 \\
\hline syp-1 & -1 & F21D9.4 & 1.02 & npr-5 & 1.03 & smg-1 & 1.06 \\
\hline mecr-1 & 1 & catp-5 & 1.02 & tads-1 & 1.03 & nspc-20 & 1.06 \\
\hline mrpl-2 & 1 & Y55D9A.2 & 1.02 & Y71H2AM.3 & 1.04 & C01C4.3 & 1.06 \\
\hline C49C3.10 & 1 & K08D10.14 & 1.02 & R07E3.4 & 1.04 & F52E10.4 & 1.06 \\
\hline F52B11.5 & 1 & fbxc-23 & 1.02 & $x b x-5$ & 1.04 & C17H11.6 & 1.06 \\
\hline pes-4 & 1 & trpl-1 & 1.02 & ttll-4 & 1.04 & nhr-190 & 1.06 \\
\hline gtsf-1 & 1 & tdpo-1 & 1.02 & gtf-2A2 & 1.04 & smu-2 & 1.06 \\
\hline arrd-13 & 1 & cyk-3 & 1.02 & T09F3.2 & 1.04 & far-7 & 1.06 \\
\hline cdc-14 & 1 & dhhc-9 & 1.02 & $n \times f-1$ & 1.04 & gck-3 & 1.06 \\
\hline ubc-3 & 1 & dgk-4 & 1.02 & rom-2 & 1.04 & nhr-5 & 1.06 \\
\hline egl-20 & 1 & Y39G10AR.9 & 1.02 & C25A11.2 & 1.04 & C03B1.13 & 1.06 \\
\hline srw-85 & 1 & let-526 & 1.02 & F13B12.1 & 1.04 & col-129 & 1.06 \\
\hline daf-15 & 1.01 & bgal-2 & 1.02 & spp-1 & 1.04 & R12E2.13 & 1.06 \\
\hline ced-2 & 1.01 & F54C8.4 & 1.02 & ints-2 & 1.04 & set-6 & 1.06 \\
\hline H05C05.1 & 1.01 & hpk-1 & 1.02 & nas-39 & 1.04 & ZK1307.1 & 1.06 \\
\hline F26F4.12 & 1.01 & F53A2.9 & 1.02 & prmt-6 & 1.04 & Y102A11A.5 & 1.06 \\
\hline figo-1 & 1.01 & M04B2.2 & 1.03 & Y20C6A.1 & 1.04 & W09D10.5 & 1.06 \\
\hline C05D11.8 & 1.01 & oaz-1 & 1.03 & $\operatorname{atg}-13$ & 1.04 & mbk-2 & 1.06 \\
\hline rle-1 & 1.01 & CD4.1 & 1.03 & egl-5 & 1.04 & hda- 6 & 1.07 \\
\hline epn-1 & 1.01 & $\mathrm{~T} 16 \mathrm{H} 12.3$ & 1.03 & kel-1 & 1.04 & F52H2.1 & 1.07 \\
\hline taf-12 & 1.01 & tag-30 & 1.03 & asp-12 & 1.04 & trip-4 & 1.07 \\
\hline T12D8.10 & 1.01 & ZK1127.12 & 1.03 & nhr-274 & 1.04 & srpa-68 & 1.07 \\
\hline daf-8 & 1.01 & Y48G10A.3 & 1.03 & tbc-9 & 1.04 & ZK1128.7 & 1.07 \\
\hline F49E2.2 & 1.01 & T21B4.15 & 1.03 & aakg-1 & 1.05 & nhr-184 & 1.07 \\
\hline pgph-3 & 1.01 & ceh-38 & 1.03 & unc-2 & 1.05 & gcy-11 & 1.07 \\
\hline zif-1 & 1.01 & dolk-1 & 1.03 & mul-1 & 1.05 & F40G9.5 & 1.07 \\
\hline $\mathrm{kcc}-1$ & 1.01 & Y55F3BR.2 & 1.03 & nhr-126 & 1.05 & W02D7.6 & 1.07 \\
\hline R06F6.12 & 1.01 & F40G9.17 & 1.03 & cdk-2 & 1.05 & sur- 6 & 1.07 \\
\hline tat-4 & 1.01 & F58G11.4 & 1.03 & mrp-5 & 1.05 & D1022.3 & 1.07 \\
\hline rpl-9 & 1.01 & tbg-1 & 1.03 & Y75B8A.14 & 1.05 & dsh-1 & 1.08 \\
\hline C16D9.9 & 1.01 & T01B10.5 & 1.03 & sop-2 & 1.05 & crtc-1 & 1.08 \\
\hline tre-2 & 1.01 & rpl-3 & 1.03 & ZC196.5 & 1.05 & F15D4.2 & 1.08 \\
\hline dhs-21 & 1.01 & gap-1 & 1.03 & D2023.1 & 1.05 & $|s|-1$ & 1.08 \\
\hline edc-4 & 1.01 & ubr-5 & 1.03 & $\mathrm{~F} 47 \mathrm{H} 4.2$ & 1.05 & T09B4.8 & 1.08 \\
\hline Y41D4B.1 & 1.02 & ant-1.1 & 1.03 & M116.2 & 1.05 & nipa-1 & 1.08 \\
\hline$r c q-5$ & 1.02 & linc-91 & 1.03 & B0507.6 & 1.05 & F45F2.9 & 1.08 \\
\hline rrn-2.1 & 1.02 & pals-24 & 1.03 & R02F2.7 & 1.05 & $\mathrm{ncl}-1$ & 1.08 \\
\hline $\mathrm{rbm}-28$ & 1.02 & Y53F4B.21 & 1.03 & ccdc- 55 & 1.05 & mei-2 & 1.08 \\
\hline
\end{tabular}




\begin{tabular}{|c|c|c|c|c|c|c|c|}
\hline Gene & $\log 2 \mathrm{FC}$ & Gene & $\log 2 \mathrm{FC}$ & Gene & $\log 2 \mathrm{FC}$ & Gene & $\log 2 \mathrm{FC}$ \\
\hline $\mathrm{cbp}-3$ & 1.08 & gbas-1 & 1.1 & pab-2 & 1.11 & cdc-25.1 & 1.13 \\
\hline $\operatorname{try}-10$ & 1.08 & Y48E1C.1 & 1.1 & nrps-1 & 1.11 & pqn-85 & 1.13 \\
\hline cil-1 & 1.08 & stau-1 & 1.1 & rpl-4 & 1.11 & T06A1.5 & 1.13 \\
\hline$x r n-1$ & 1.08 & F45E10.2 & 1.1 & athp-2 & 1.11 & sma-9 & 1.13 \\
\hline R11G1.2 & 1.08 & fut-1 & 1.1 & snf-6 & 1.11 & ncx-7 & 1.14 \\
\hline rfth-1 & 1.08 & $\mathrm{ikb}-1$ & 1.1 & acox-1.6 & 1.11 & ZK131.11 & 1.14 \\
\hline mrps-5 & 1.08 & cat-4 & 1.1 & $\operatorname{tr} x-2$ & 1.12 & $\mathrm{zmp}-4$ & 1.14 \\
\hline F35H8.2 & 1.08 & rbpl-1 & 1.1 & Y95D11A.3 & 1.12 & pro-2 & 1.14 \\
\hline T25C12.11 & 1.08 & rskn-2 & 1.1 & gpd-3 & 1.12 & pir-1 & 1.14 \\
\hline F14B6.6 & 1.08 & tam-1 & 1.1 & F49E12.12 & 1.12 & efsc-1 & 1.14 \\
\hline F57C12.6 & 1.08 & par-4 & 1.1 & snx-13 & 1.12 & rde-2 & 1.14 \\
\hline cec- 2 & 1.08 & nlp-7 & 1.1 & nuaf-1 & 1.12 & $\mathrm{rrn}-3.1$ & 1.14 \\
\hline rab-14 & 1.08 & ptc-1 & 1.1 & prdx-6 & 1.12 & npr-28 & 1.14 \\
\hline nol-2 & 1.09 & Y51F10.3 & 1.1 & C34C12.9 & 1.12 & lipl-7 & 1.14 \\
\hline F01D5.1 & 1.09 & mca-2 & 1.1 & kynu-1 & 1.12 & rnf-1 & 1.14 \\
\hline C18B2.5 & 1.09 & T03E6.8 & 1.1 & H23N18.6 & 1.12 & eif-3.D & 1.14 \\
\hline syp-2 & 1.09 & F46B6.12 & 1.1 & F58G6.7 & 1.12 & cdap-2 & 1.14 \\
\hline B0035.13 & 1.09 & T19C3.6 & 1.1 & uev-1 & 1.12 & $c d r-4$ & 1.14 \\
\hline M03A1.8 & 1.09 & B0495.9 & 1.11 & fbxa-101 & 1.12 & C18F10.7 & 1.14 \\
\hline F35C11.4 & 1.09 & kqt-2 & 1.11 & let-70 & 1.12 & dct- 6 & 1.14 \\
\hline rnh-1.0 & 1.09 & sri-30 & 1.11 & imp-2 & 1.12 & gld-2 & 1.15 \\
\hline W01G7.4 & 1.09 & acs-22 & 1.11 & Y39B6A.34 & 1.13 & $\mathrm{~F} 27 \mathrm{C} 1.2$ & 1.15 \\
\hline C04G6.4 & 1.09 & fbxa-59 & 1.11 & $\mathrm{ftn}-1$ & 1.13 & clec-178 & 1.15 \\
\hline Y56A3А.16 & 1.09 & spk-1 & 1.11 & ctsa-4.1 & 1.13 & $\mathrm{~F} 36 \mathrm{H} 2.3$ & 1.15 \\
\hline T05F1.11 & 1.09 & T26C12.1 & 1.11 & Y55F3BL.4 & 1.13 & rpc-11 & 1.15 \\
\hline saeg-1 & 1.09 & F33D4.4 & 1.11 & mtm-5 & 1.13 & $r s r-2$ & 1.15 \\
\hline F21D5.5 & 1.09 & Y22D7AL.4 & 1.11 & rpi-2 & 1.13 & F58B4.5 & 1.15 \\
\hline W08A12.2 & 1.09 & C35D10.6 & 1.11 & elt-7 & 1.13 & Imtr-5 & 1.15 \\
\hline C06G3.6 & 1.09 & egl-10 & 1.11 & eri-12 & 1.13 & T19D7.6 & 1.15 \\
\hline $\mathrm{C} 31 \mathrm{H} 1.8$ & 1.09 & C49A9.9 & 1.11 & M01H9.3 & 1.13 & fahd-1 & 1.15 \\
\hline nmur-2 & 1.09 & bcl-11 & 1.11 & spp-5 & 1.13 & cdh-3 & 1.15 \\
\hline C08G9.1 & 1.09 & T26A8.1 & 1.11 & Y39H10В.2 & 1.13 & zer-1 & 1.15 \\
\hline Y43F4B.9 & 1.09 & wht-2 & 1.11 & E01A2.5 & 1.13 & eef-1G & 1.15 \\
\hline T07D1.3 & 1.1 & pstk-1 & 1.11 & dop-6 & 1.13 & Irp-2 & 1.15 \\
\hline apb-3 & 1.1 & C32E8.6 & 1.11 & R12B2.2 & 1.13 & clec-146 & 1.15 \\
\hline$d d x-52$ & 1.1 & Y110A7A.21 & 1.11 & pqn-48 & 1.13 & ZC250.4 & 1.15 \\
\hline taf-5 & 1.1 & fic-1 & 1.11 & $\operatorname{ttr}-1$ & 1.13 & $\mathrm{crm}-1$ & 1.15 \\
\hline dhs-13 & 1.1 & tag-294 & 1.11 & ZK993.5 & 1.13 & srpr-2.1 & 1.15 \\
\hline aatf-1 & 1.1 & K09F5.6 & 1.11 & sars-1 & 1.13 & M176.4 & 1.15 \\
\hline Y46G5A.15 & 1.1 & C45G7.13 & 1.11 & F58G6.8 & 1.13 & F10E9.12 & 1.15 \\
\hline ets- 6 & 1.1 & ZK1058.3 & 1.11 & egl-43 & 1.13 & T20D3.5 & 1.15 \\
\hline
\end{tabular}




\begin{tabular}{|c|c|c|c|c|c|c|c|}
\hline Gene & $\log 2 \mathrm{FC}$ & Gene & $\log 2 \mathrm{FC}$ & Gene & $\log 2 \mathrm{FC}$ & Gene & $\log 2 \mathrm{FC}$ \\
\hline Y2H9A.4 & 1.15 & pxmp-4 & 1.17 & Y105E8A.1 & 1.19 & ZK1127.5 & 1.21 \\
\hline Y75B8A.7 & 1.15 & frpr-8 & 1.17 & F58D5.5 & 1.19 & M01F1.9 & 1.21 \\
\hline nhr-110 & 1.15 & del-9 & 1.17 & F09G2.1 & 1.19 & clp-1 & 1.21 \\
\hline $\mathrm{C} 03 \mathrm{H} 12.1$ & 1.15 & chd-7 & 1.17 & F13C5.1 & 1.19 & zipt-9 & 1.21 \\
\hline C56A3.4 & 1.15 & $\mathrm{mrp}-7$ & 1.17 & Y50D7A.1 & 1.19 & Y59A8B.21 & 1.21 \\
\hline F46H5.7 & 1.16 & vps-22 & 1.17 & txt-18 & 1.19 & Y40H7A.11 & 1.21 \\
\hline Y47A7.2 & 1.16 & Y71F9AL.8 & 1.17 & Ipd-8 & 1.19 & Y92H12A.2 & 1.21 \\
\hline F17B5.1 & 1.16 & slcf-1 & 1.17 & Y49A3A.3 & 1.19 & C25G4.2 & 1.21 \\
\hline hecd-1 & 1.16 & Y73В3А. 3 & 1.17 & gstk-2 & 1.19 & F55G11.8 & 1.21 \\
\hline rps-16 & 1.16 & C37H5.5 & 1.17 & $\mathrm{mps}-1$ & 1.19 & K02D10.4 & 1.21 \\
\hline C24G6.8 & 1.16 & cest-9.1 & 1.17 & frm-7 & 1.19 & fpn-1.1 & 1.21 \\
\hline pus-1 & 1.16 & clec-76 & 1.18 & vps-26 & 1.19 & F46A8.13 & 1.21 \\
\hline aexr-1 & 1.16 & T22C1.6 & 1.18 & hda-4 & 1.19 & $\mathrm{~T} 25 \mathrm{C} 12.12$ & 1.21 \\
\hline rps-23 & 1.16 & mdt-15 & 1.18 & C09E7.6 & 1.2 & eas-1 & 1.21 \\
\hline tsp-11 & 1.16 & W03D8.8 & 1.18 & F11E6.6 & 1.2 & F13H8.1 & 1.21 \\
\hline W05H9.3 & 1.16 & Y34B4A.5 & 1.18 & F07H5.5 & 1.2 & $\mathrm{mfn}-1$ & 1.21 \\
\hline gei-4 & 1.16 & srh-210 & 1.18 & linc-35 & 1.2 & C29G2.6 & 1.21 \\
\hline gly-6 & 1.16 & rpoa-49 & 1.18 & tct-1 & 1.2 & ain-2 & 1.21 \\
\hline $\mathrm{C} 27 \mathrm{H} 5.2$ & 1.16 & F57B10.8 & 1.18 & gur-3 & 1.2 & Y6B3B.9 & 1.21 \\
\hline pals-31 & 1.16 & Y39B6A.3 & 1.18 & ubc-21 & 1.2 & pinn-4 & 1.21 \\
\hline R13A1.10 & 1.16 & egl-8 & 1.18 & dct-1 & 1.2 & mes- 6 & 1.21 \\
\hline gfi-3 & 1.16 & nhr-227 & 1.18 & gap-3 & 1.2 & $\mathrm{C} 15 \mathrm{C} 8.7$ & 1.22 \\
\hline Y40B1A.3 & 1.16 & drd-1 & 1.18 & R06A10.1 & 1.2 & piki-1 & 1.22 \\
\hline M03F8.3 & 1.16 & C13F10.5 & 1.18 & F59C6.11 & 1.2 & pqn-21 & 1.22 \\
\hline Y22D7AR.2 & 1.16 & ttll-5 & 1.18 & R02E4.2 & 1.2 & mys-4 & 1.22 \\
\hline faah-4 & 1.16 & C10B5.3 & 1.18 & C48B6.3 & 1.2 & aka-1 & 1.22 \\
\hline snap-29 & 1.16 & F56C4.4 & 1.18 & C30F12.3 & 1.2 & daf-16 & 1.22 \\
\hline F33D11.16 & 1.17 & prcc-1 & 1.18 & fog-2 & 1.2 & mct-3 & 1.22 \\
\hline F35E12.4 & 1.17 & M176.11 & 1.18 & rps-2 & 1.2 & M02E1.3 & 1.22 \\
\hline hpo-31 & 1.17 & Y57G11C.1135 & 1.18 & $\operatorname{lin}-15 A$ & 1.2 & atg-9 & 1.22 \\
\hline tftc-1 & 1.17 & W02B12.1 & 1.18 & zipt-7.2 & 1.2 & ncs-3 & 1.22 \\
\hline ima-3 & 1.17 & unc-31 & 1.19 & dct-11 & 1.2 & Y50D7A.8 & 1.22 \\
\hline $\mathrm{rpl}-2$ & 1.17 & col-181 & 1.19 & spat-2 & 1.2 & $\mathrm{~T} 10 \mathrm{H} 4.13$ & 1.22 \\
\hline $\mathrm{C} 05 \mathrm{C} 8.5$ & 1.17 & nhr-101 & 1.19 & Irp-1 & 1.2 & mig-18 & 1.22 \\
\hline ptl-1 & 1.17 & col-95 & 1.19 & kgb-2 & 1.2 & K12D12.4 & 1.22 \\
\hline B0244.10 & 1.17 & gsto-2 & 1.19 & acp-2 & 1.2 & tat- 6 & 1.23 \\
\hline Y45G5AL.1 & 1.17 & klo-1 & 1.19 & memo-1 & 1.2 & $\mathrm{acl}-2$ & 1.23 \\
\hline C14C10.2 & 1.17 & B0462.5 & 1.19 & Y9C2UA.1 & 1.2 & ubc- 8 & 1.23 \\
\hline pdcd-2 & 1.17 & clh-6 & 1.19 & $\mathrm{~K} 12 \mathrm{H} 4.7$ & 1.2 & H35N09.2 & 1.23 \\
\hline anr-36 & 1.17 & F01G4.6 & 1.19 & nrde-4 & 1.21 & tub-2 & 1.23 \\
\hline fbxa-53 & 1.17 & T08E11.1 & 1.19 & F26H9.5 & 1.21 & ZK757.1 & 1.23 \\
\hline
\end{tabular}




\begin{tabular}{|c|c|c|c|c|c|c|c|}
\hline Gene & $\log 2 \mathrm{FC}$ & Gene & $\log 2 \mathrm{FC}$ & Gene & $\log 2 \mathrm{FC}$ & Gene & $\log 2 \mathrm{FC}$ \\
\hline supr-1 & 1.23 & Y73B6BL.31 & 1.26 & qars-1 & 1.29 & gta-1 & 1.31 \\
\hline $\mathrm{C} 28 \mathrm{H} 8.2$ & 1.23 & Y38C1AA.12 & 1.26 & Y54F10BM.12 & 1.29 & $\mathrm{~F} 47 \mathrm{H} 4.12$ & 1.31 \\
\hline rpl-16 & 1.23 & plk-2 & 1.26 & ugt-23 & 1.29 & atg-16.2 & 1.31 \\
\hline C07B5.3 & 1.23 & ubh-3 & 1.26 & rpia-1 & 1.29 & cpna-1 & 1.31 \\
\hline mrp-3 & 1.23 & asp-1 & 1.26 & smk-1 & 1.29 & glb-30 & 1.32 \\
\hline puf-9 & 1.23 & K10B3.5 & 1.26 & Y52B11A.12 & 1.29 & fbxb-53 & 1.32 \\
\hline let-711 & 1.23 & K05C4.2 & 1.26 & cyn-5 & 1.29 & F10G2.1 & 1.32 \\
\hline fbxa-189 & 1.23 & $\mathrm{C} 43 \mathrm{H} 6.4$ & 1.26 & rev-1 & 1.29 & gcs-1 & 1.32 \\
\hline btb-4 & 1.24 & hrg-1 & 1.26 & sms-1 & 1.29 & K04F10.7 & 1.32 \\
\hline pgp-11 & 1.24 & adpr-1 & 1.27 & nhr-90 & 1.29 & W09D6.1 & 1.32 \\
\hline Y43C5A.2 & 1.24 & rabx-5 & 1.27 & Y49F6B.2 & 1.29 & M142.8 & 1.32 \\
\hline Y18H1A.11 & 1.24 & nuc-1 & 1.27 & gtbp-1 & 1.29 & lis-1 & 1.32 \\
\hline C27D8.4 & 1.24 & nlp-17 & 1.27 & T24B8.7 & 1.29 & C01G6.4 & 1.32 \\
\hline $\operatorname{lgg}-2$ & 1.24 & faah- 6 & 1.27 & W09C5.1 & 1.3 & Y55F3C.9 & 1.32 \\
\hline kin-4 & 1.24 & $\mathrm{nlp}-2$ & 1.27 & F42C5.4 & 1.3 & Y73E7A.1 & 1.32 \\
\hline rps-20 & 1.24 & slc-25A26 & 1.27 & F22B7.1 & 1.3 & C28D4.10 & 1.32 \\
\hline catp-7 & 1.24 & nhr-10 & 1.27 & $\operatorname{trxr}-1$ & 1.3 & elt-4 & 1.32 \\
\hline $\mathrm{scl}-2$ & 1.24 & F49H6.5 & 1.27 & pde-4 & 1.3 & Y53F4B.13 & 1.32 \\
\hline Y53G8AM.7 & 1.24 & glb-26 & 1.27 & T20F7.5 & 1.3 & clec-37 & 1.32 \\
\hline ZK250.13 & 1.24 & mdt-26 & 1.27 & ZK971.1 & 1.3 & pef-1 & 1.32 \\
\hline zhit-2 & 1.24 & Y38F1A.1 & 1.27 & $\mathrm{R} 03 \mathrm{H} 10.1$ & 1.3 & moe-3 & 1.32 \\
\hline F11C7.2 & 1.24 & Y41E3.22 & 1.27 & spr-3 & 1.3 & cpd-1 & 1.33 \\
\hline alg-1 & 1.24 & T25D10.1 & 1.27 & hgo-1 & 1.3 & F21H7.2 & 1.33 \\
\hline Y39A1A.14 & 1.24 & B0507.2 & 1.27 & Ipr-2 & 1.3 & ife-1 & 1.33 \\
\hline igdb-2 & 1.24 & dhps-1 & 1.27 & snx -6 & 1.3 & ace-2 & 1.33 \\
\hline $\operatorname{lin}-49$ & 1.25 & Y42A5A.1 & 1.28 & swan-2 & 1.3 & pals-38 & 1.33 \\
\hline W02D3.4 & 1.25 & dhs-15 & 1.28 & F59C6.5 & 1.31 & Y56A3A.18 & 1.33 \\
\hline F42A9.9 & 1.25 & Y71F9AL.10 & 1.28 & C45E5.3 & 1.31 & brp-1 & 1.33 \\
\hline deg-3 & 1.25 & ahr-1 & 1.28 & Y67H2A.7 & 1.31 & F35B12.10 & 1.33 \\
\hline ppfr-1 & 1.25 & wdfy-3 & 1.28 & ZK1320.3 & 1.31 & cest-31 & 1.33 \\
\hline C01C10.2 & 1.25 & К09Н9.8 & 1.28 & sdc-3 & 1.31 & C25F9.5 & 1.33 \\
\hline ubc-7 & 1.25 & $\mathrm{mtl}-1$ & 1.28 & D1014.2 & 1.31 & R102.4 & 1.33 \\
\hline sax-1 & 1.25 & R144.3 & 1.28 & cif-1 & 1.31 & C17G1.5 & 1.33 \\
\hline F22D6.14 & 1.25 & T26H5.8 & 1.28 & $\mathrm{C} 32 \mathrm{H} 11.3$ & 1.31 & tfbm-1 & 1.33 \\
\hline F09E5.10 & 1.25 & prx-5 & 1.28 & atg-16.1 & 1.31 & $z z z-1$ & 1.33 \\
\hline B0563.5 & 1.26 & F22D6.2 & 1.28 & C32D5.11 & 1.31 & larp-5 & 1.33 \\
\hline M04D8.7 & 1.26 & btb-9 & 1.28 & сур-34A9 & 1.31 & flp-20 & 1.33 \\
\hline dhp-1 & 1.26 & Y53F4B.1 & 1.28 & C06E1.9 & 1.31 & Y55F3AM.13 & 1.34 \\
\hline henn-1 & 1.26 & twk-12 & 1.28 & cest-35.1 & 1.31 & Y51H4A.7 & 1.34 \\
\hline ZK993.2 & 1.26 & mrpl-9 & 1.28 & F13D11.4 & 1.31 & rрac-40 & 1.34 \\
\hline C36B1.13 & 1.26 & $f b x c-6$ & 1.28 & K07E3.1 & 1.31 & cept-2 & 1.34 \\
\hline
\end{tabular}




\begin{tabular}{|c|c|c|c|c|c|c|c|}
\hline Gene & $\log 2 \mathrm{FC}$ & Gene & $\log 2 \mathrm{FC}$ & Gene & $\log 2 \mathrm{FC}$ & Gene & $\log 2 \mathrm{FC}$ \\
\hline ugt-56 & 1.34 & twk-10 & 1.36 & fbxb-65 & 1.38 & F59C6.16 & 1.41 \\
\hline сур-34A10 & 1.34 & C45G3.3 & 1.36 & eme-1 & 1.39 & T26H5.14 & 1.41 \\
\hline gра-16 & 1.34 & cof-2 & 1.36 & pry-1 & 1.39 & F12A10.1 & 1.41 \\
\hline$a m x-3$ & 1.34 & irg-3 & 1.36 & F40F12.9 & 1.39 & сур-13A3 & 1.41 \\
\hline klp-4 & 1.34 & T21H3.1 & 1.37 & ZK858.5 & 1.39 & F53C11.9 & 1.41 \\
\hline T05G5.5 & 1.34 & endu-2 & 1.37 & K02E11.9 & 1.39 & pdpr-1 & 1.41 \\
\hline drap-1 & 1.34 & cct-2 & 1.37 & ctl-1 & 1.39 & $\mathrm{~T} 24 \mathrm{C} 4.8$ & 1.41 \\
\hline rpb-9 & 1.34 & lite-1 & 1.37 & $\mathrm{R} 01 \mathrm{H} 2.7$ & 1.39 & nubp-1 & 1.42 \\
\hline ptr-24 & 1.34 & pgp-9 & 1.37 & ZK822.4 & 1.39 & arrd-20 & 1.42 \\
\hline scav-1 & 1.34 & tgt-2 & 1.37 & trpp-9 & 1.39 & $\mathrm{C} 28 \mathrm{H} 8.14$ & 1.42 \\
\hline txt-3 & 1.34 & Y71H2B.2 & 1.37 & sgk-1 & 1.39 & pha-1 & 1.42 \\
\hline C18A11.1 & 1.34 & C15H9.2 & 1.37 & ears-1 & 1.39 & $\mathrm{mtl}-2$ & 1.42 \\
\hline yars-2 & 1.34 & ZK632.14 & 1.37 & F17C11.4 & 1.4 & pfd-5 & 1.42 \\
\hline $\mathrm{C} 38 \mathrm{H} 2.2$ & 1.34 & iars-2 & 1.37 & afmd-1 & 1.4 & dcp-66 & 1.43 \\
\hline F40F4.7 & 1.34 & Y66H1A.5 & 1.37 & tag-280 & 1.4 & C45B11.7 & 1.43 \\
\hline T02G5.14 & 1.34 & pgap-1 & 1.37 & Y92H12BL.1 & 1.4 & spp-10 & 1.43 \\
\hline eol-1 & 1.35 & K11E4.1 & 1.37 & sinh-1 & 1.4 & F16B4.5 & 1.43 \\
\hline emc-3 & 1.35 & $\operatorname{lgc}-23$ & 1.37 & scav- 6 & 1.4 & F46G10.1 & 1.43 \\
\hline mrpl-1 & 1.35 & Y43F8C.6 & 1.37 & nhr-119 & 1.4 & des-2 & 1.43 \\
\hline Y95B8A.6 & 1.35 & pals-19 & 1.37 & C28C12.11 & 1.4 & K07F5.15 & 1.43 \\
\hline T08B1.4 & 1.35 & E04F6.8 & 1.37 & hsp-6 & 1.4 & pap-1 & 1.43 \\
\hline F52E1.5 & 1.35 & $\mathrm{R} 10 \mathrm{H} 1.1$ & 1.37 & Y71H2B.11 & 1.4 & Y48C 3 A.3 & 1.43 \\
\hline byn-1 & 1.35 & ctf-18 & 1.37 & pup-2 & 1.4 & W08F4.5 & 1.43 \\
\hline haf-4 & 1.35 & $\mathrm{AH} 9.4$ & 1.37 & C17G10.7 & 1.4 & asm-3 & 1.43 \\
\hline pqbp-1.1 & 1.35 & $\mathrm{~F} 49 \mathrm{C} 12.10$ & 1.38 & icd-2 & 1.4 & T26G10.1 & 1.43 \\
\hline F20B6.4 & 1.35 & akt-2 & 1.38 & $\mathrm{rbm}-34$ & 1.4 & pab-1 & 1.43 \\
\hline pigs-1 & 1.35 & akap-1 & 1.38 & zyg-11 & 1.4 & rrp-1 & 1.44 \\
\hline C05E11.6 & 1.35 & pcf-11 & 1.38 & C54D10.10 & 1.4 & Y22D7AR.6 & 1.44 \\
\hline E04F6.9 & 1.35 & ctns- 1 & 1.38 & eif-2alpha & 1.41 & $\mathrm{R} 13 \mathrm{H} 4.2$ & 1.44 \\
\hline gba-3 & 1.35 & $\mathrm{mxl}-1$ & 1.38 & $n c x-2$ & 1.41 & $\operatorname{atg}-10$ & 1.44 \\
\hline eif-3.F & 1.35 & T19D12.3 & 1.38 & plpp-1.1 & 1.41 & Y71H2AM.2 & 1.44 \\
\hline nhr-219 & 1.36 & cap-2 & 1.38 & K10G6.9 & 1.41 & cest- 25 & 1.44 \\
\hline $\mathrm{C} 17 \mathrm{H} 12.8$ & 1.36 & mig-1 & 1.38 & rmh-2 & 1.41 & pmt-2 & 1.44 \\
\hline Y48C3A.12 & 1.36 & $\mathrm{E} 02 \mathrm{H} 4.4$ & 1.38 & pals-16 & 1.41 & T16G1.9 & 1.44 \\
\hline enpl-1 & 1.36 & ZK813.4 & 1.38 & Y50D4A.1 & 1.41 & C39D10.8 & 1.44 \\
\hline eif-2gamma & 1.36 & lips-1 & 1.38 & C34F6.5 & 1.41 & F44A6.5 & 1.44 \\
\hline $\mathrm{F} 13 \mathrm{H} 8.11$ & 1.36 & asp-4 & 1.38 & ekl-5 & 1.41 & pho-4 & 1.44 \\
\hline fnip-2 & 1.36 & abhd-14 & 1.38 & cct-1 & 1.41 & B0228.7 & 1.44 \\
\hline col-107 & 1.36 & rpap-2 & 1.38 & noca-1 & 1.41 & mrps-11 & 1.44 \\
\hline nhr-64 & 1.36 & gcst-1 & 1.38 & C29F5.3 & 1.41 & Y18D10A.23 & 1.44 \\
\hline T05A12.3 & 1.36 & swsn-9 & 1.38 & sir-2.4 & 1.41 & epg-4 & 1.44 \\
\hline
\end{tabular}




\begin{tabular}{|c|c|c|c|c|c|c|c|}
\hline Gene & $\log 2 \mathrm{FC}$ & Gene & $\log 2 \mathrm{FC}$ & Gene & $\log 2 \mathrm{FC}$ & Gene & $\log 2 \mathrm{FC}$ \\
\hline F08F8.9 & 1.44 & egl-45 & 1.48 & lars-1 & 1.52 & lin-28 & 1.55 \\
\hline fkh-7 & 1.44 & Y94H6A.5 & 1.48 & C49F5.7 & 1.52 & Y71G12B.31 & 1.56 \\
\hline $\mathrm{C} 44 \mathrm{H} 9.5$ & 1.45 & hex-3 & 1.48 & nlp-59 & 1.52 & ascc-1 & 1.56 \\
\hline C25E10.16 & 1.45 & dao-5 & 1.48 & ZC443.2 & 1.53 & ins-35 & 1.56 \\
\hline dhs-20 & 1.45 & H04M03.3 & 1.48 & alkb-8 & 1.53 & F36H5.10 & 1.56 \\
\hline T07D4.2 & 1.45 & F42F12.3 & 1.49 & epg-6 & 1.53 & nac-2 & 1.56 \\
\hline aex-5 & 1.45 & F29C6.1 & 1.49 & abce-1 & 1.53 & $c n x-1$ & 1.56 \\
\hline rsd-6 & 1.45 & exc-14 & 1.49 & pigw-1 & 1.53 & $w d r-5.2$ & 1.56 \\
\hline sma-2 & 1.45 & coa-7 & 1.49 & Y59C2A.1 & 1.53 & mca-1 & 1.56 \\
\hline C33D9.3 & 1.45 & pno-1 & 1.49 & K04F10.1 & 1.53 & ercc-1 & 1.56 \\
\hline Y54G2A.41 & 1.46 & unk-1 & 1.49 & Y92H12A.5 & 1.53 & Y43F8C.3 & 1.56 \\
\hline C45E5.4 & 1.46 & C04F5.8 & 1.49 & adr-1 & 1.53 & F58H1.3 & 1.56 \\
\hline Y41D4A.6 & 1.46 & Y71H2AM.12 & 1.49 & $d d b-1$ & 1.53 & adss-1 & 1.56 \\
\hline ZK285.2 & 1.46 & mboa-2 & 1.49 & F08G12.11 & 1.53 & T10H9.8 & 1.56 \\
\hline pinn-1 & 1.46 & nmad-1 & 1.49 & mtcu-1 & 1.53 & eif-3.I & 1.57 \\
\hline btb-19 & 1.46 & his-60 & 1.5 & nca-2 & 1.54 & hil-1 & 1.57 \\
\hline F14H3.12 & 1.46 & C14A11.2 & 1.5 & tag-89 & 1.54 & clec-63 & 1.57 \\
\hline R02F2.1 & 1.46 & $\mathrm{~F} 53 \mathrm{H} 4.4$ & 1.5 & M01E5.2 & 1.54 & lido-13 & 1.57 \\
\hline nhl-1 & 1.46 & fat-2 & 1.5 & asp-14 & 1.54 & C09D4.4 & 1.57 \\
\hline $\mathrm{mfb}-1$ & 1.46 & math-27 & 1.5 & slcr-46.1 & 1.54 & T05H4.10 & 1.57 \\
\hline R05A10.1 & 1.46 & Y106G6G.2 & 1.5 & abcf-3 & 1.54 & fbxa-182 & 1.57 \\
\hline $\operatorname{ttr}-24$ & 1.46 & R06C1.6 & 1.5 & F58A3.4 & 1.54 & Y6E2A.4 & 1.57 \\
\hline ZK180.8 & 1.46 & ints-1 & 1.5 & Y54E5A.8 & 1.54 & C47B2.2 & 1.57 \\
\hline R186.1 & 1.47 & tpra-1 & 1.5 & pals-5 & 1.54 & plc-3 & 1.57 \\
\hline mdt-27 & 1.47 & nhr-48 & 1.51 & ifb-2 & 1.54 & F54E7.9 & 1.57 \\
\hline unc-54 & 1.47 & Y32F6A.4 & 1.51 & $\operatorname{trm}-2 \mathrm{~A}$ & 1.54 & C10B5.1 & 1.57 \\
\hline egl-27 & 1.47 & F19B6.1 & 1.51 & C07D8.6 & 1.54 & B0454.5 & 1.57 \\
\hline Y18D10A.9 & 1.47 & Y41C4A.8 & 1.51 & atf-6 & 1.54 & ampd-1 & 1.57 \\
\hline car-1 & 1.47 & F42C5.6 & 1.51 & $\mathrm{ncr}-2$ & 1.54 & D2045.8 & 1.57 \\
\hline rog-1 & 1.47 & asna-1 & 1.51 & frm-5.1 & 1.55 & F08A7.1 & 1.58 \\
\hline clh-4 & 1.47 & fbxa-74 & 1.51 & rla-0 & 1.55 & acl-7 & 1.58 \\
\hline C54C8.8 & 1.47 & $F 36 F 2.2$ & 1.51 & mff-2 & 1.55 & F59A7.8 & 1.58 \\
\hline ZK1098.11 & 1.47 & сур-13A1 & 1.51 & fbxa-48 & 1.55 & zgpa-1 & 1.58 \\
\hline sru-7 & 1.47 & mop-25.1 & 1.51 & ZC443.4 & 1.55 & T06E6.1 & 1.58 \\
\hline K03H1.11 & 1.47 & C44E12.1 & 1.52 & K02E7.11 & 1.55 & pals-18 & 1.58 \\
\hline $\operatorname{trxr}-2$ & 1.47 & zip-12 & 1.52 & cnnm-2 & 1.55 & C06G3.3 & 1.58 \\
\hline T27A10.6 & 1.47 & M03C11.6 & 1.52 & smp-2 & 1.55 & pigk-1 & 1.58 \\
\hline C36B1.6 & 1.47 & F55A4.7 & 1.52 & hecw-1 & 1.55 & nhr-152 & 1.58 \\
\hline irld-2 & 1.47 & mrpl-37 & 1.52 & D1054.18 & 1.55 & F07E5.5 & 1.58 \\
\hline$x d h-1$ & 1.48 & $z n f x-1$ & 1.52 & pcbd-1 & 1.55 & B0563.7 & 1.58 \\
\hline nrde-1 & 1.48 & F32E10.5 & 1.52 & him-4 & 1.55 & $\mathrm{cpz}-2$ & 1.58 \\
\hline
\end{tabular}




\begin{tabular}{|c|c|c|c|c|c|c|c|}
\hline Gene & $\log 2 \mathrm{FC}$ & Gene & $\log 2 \mathrm{FC}$ & Gene & $\log 2 \mathrm{FC}$ & Gene & $\log 2 \mathrm{FC}$ \\
\hline F54F2.7 & 1.58 & clec-186 & 1.63 & R03D7.5 & 1.67 & ril-2 & 1.72 \\
\hline Y71F9AL.12 & 1.58 & fkh-9 & 1.63 & F26A3.1 & 1.67 & sorf-2 & 1.72 \\
\hline R05D3.12 & 1.59 & adt-3 & 1.63 & srap-1 & 1.67 & F16C3.1 & 1.72 \\
\hline Y61A9LA.10 & 1.59 & $\mathrm{CO} 3 \mathrm{H} 5.3$ & 1.63 & nhl-2 & 1.68 & sqst-2 & 1.72 \\
\hline ZC443.3 & 1.59 & K02D10.8 & 1.63 & adk-1 & 1.68 & timm-23 & 1.72 \\
\hline $\mathrm{C} 33 \mathrm{H} 5.13$ & 1.59 & Y43C5A.3 & 1.63 & dhs-11 & 1.68 & fbxa-10 & 1.72 \\
\hline mak-2 & 1.59 & ccdc-47 & 1.64 & B0403.6 & 1.68 & exos-8 & 1.72 \\
\hline F53F4.14 & 1.59 & F37A8.5 & 1.64 & nhr-106 & 1.68 & ensa-1 & 1.73 \\
\hline col-42 & 1.6 & coel-1 & 1.64 & F55F8.2 & 1.68 & T23D8.3 & 1.73 \\
\hline T01D3.6 & 1.6 & F28B12.6 & 1.64 & gldc-1 & 1.68 & fbxa-138 & 1.73 \\
\hline cct-7 & 1.6 & F28B4.4 & 1.64 & F56D5.6 & 1.68 & nst-1 & 1.73 \\
\hline F26F12.3 & 1.6 & cpg-3 & 1.64 & srh-48 & 1.68 & apm-1 & 1.73 \\
\hline F46B6.5 & 1.6 & gsa-1 & 1.64 & Y51H4A.24 & 1.68 & fbxa-163 & 1.73 \\
\hline F29B9.1 & 1.6 & Y82E9BR.22 & 1.64 & hpo-11 & 1.69 & K12C11.6 & 1.73 \\
\hline slc-28.1 & 1.6 & ric-19 & 1.64 & nhr-210 & 1.69 & F31A3.3 & 1.73 \\
\hline C56A3.5 & 1.6 & hars-1 & 1.64 & B0303.4 & 1.69 & T10B11.5 & 1.74 \\
\hline ptp-1 & 1.6 & nsy-1 & 1.64 & pals-2 & 1.69 & flp-10 & 1.74 \\
\hline efk-1 & 1.6 & T21F4.1 & 1.65 & daz-1 & 1.69 & R151.1 & 1.74 \\
\hline eif-3.G & 1.6 & sca-1 & 1.65 & fbxa-175 & 1.69 & lipl-5 & 1.74 \\
\hline pfas-1 & 1.6 & nhr-8 & 1.65 & tric-1B.1 & 1.7 & M01H9.4 & 1.74 \\
\hline lin-41 & 1.6 & cps- 6 & 1.65 & mom-1 & 1.7 & linc-39 & 1.74 \\
\hline F54D11.4 & 1.6 & F09B12.3 & 1.65 & plc-2 & 1.7 & R12E2.8 & 1.74 \\
\hline pudl-2 & 1.61 & hрo-34 & 1.65 & C13B9.2 & 1.7 & C42D8.1 & 1.74 \\
\hline sydn-1 & 1.61 & ddn-1 & 1.65 & R07C12.2 & 1.7 & chil-23 & 1.75 \\
\hline F57C12.2 & 1.61 & pro-3 & 1.65 & odr-10 & 1.7 & $\mathrm{C} 25 \mathrm{H} 3.18$ & 1.75 \\
\hline C34F6.11 & 1.61 & cct-5 & 1.65 & rab-21 & 1.7 & T28C6.8 & 1.75 \\
\hline eef-1A.1 & 1.61 & T10G3.1 & 1.65 & nhr-80 & 1.7 & Y49E10.16 & 1.75 \\
\hline F37B12.3 & 1.61 & K03B4.1 & 1.66 & F32D8.13 & 1.7 & aakb-2 & 1.75 \\
\hline gst- 6 & 1.61 & mtq-2 & 1.66 & B0513.4 & 1.7 & rpac-19 & 1.75 \\
\hline W05F2.6 & 1.61 & mekk-3 & 1.66 & B0393.9 & 1.71 & C35B8.3 & 1.75 \\
\hline slc-17.9 & 1.61 & M163.1 & 1.66 & $\operatorname{ttr}-40$ & 1.71 & ufc-1 & 1.75 \\
\hline kin-10 & 1.61 & Y6B3B.4 & 1.66 & C06C3.11 & 1.71 & F46B6.4 & 1.75 \\
\hline hsp-90 & 1.61 & uev-3 & 1.66 & eri-5 & 1.71 & Y56A3А.31 & 1.75 \\
\hline F18C5.10 & 1.61 & xpg-1 & 1.66 & dph-3 & 1.71 & M18.3 & 1.75 \\
\hline K10G4.3 & 1.61 & nstp-4 & 1.66 & pot-3 & 1.71 & $\mathrm{crn}-3$ & 1.75 \\
\hline Y39B6A.37 & 1.62 & D2030.3 & 1.66 & col-147 & 1.71 & polg-1 & 1.75 \\
\hline nstp-1 & 1.62 & C16A3.6 & 1.67 & csr-1 & 1.71 & C27A7.5 & 1.76 \\
\hline F41G3.18 & 1.62 & cfim-2 & 1.67 & C08F11.3 & 1.71 & F31B12.3 & 1.76 \\
\hline fbxa-32 & 1.62 & C05D11.1 & 1.67 & lin-14 & 1.71 & C47E12.12 & 1.76 \\
\hline pde-6 & 1.62 & frm-4 & 1.67 & yap-1 & 1.71 & aldo-2 & 1.76 \\
\hline Y92H12BL.4 & 1.63 & ccdc-149 & 1.67 & noa-1 & 1.71 & C16C10.2 & 1.76 \\
\hline
\end{tabular}




\begin{tabular}{|c|c|c|c|c|c|c|c|}
\hline Gene & $\log 2 \mathrm{FC}$ & Gene & $\log 2 \mathrm{FC}$ & Gene & $\log 2 \mathrm{FC}$ & Gene & $\log 2 \mathrm{FC}$ \\
\hline sul-3 & 1.76 & acs-16 & 1.81 & crt-1 & 1.88 & K02E11.7 & 1.93 \\
\hline got-2.1 & 1.76 & M01F1.3 & 1.81 & Y41C4A.9 & 1.88 & F37C12.21 & 1.93 \\
\hline rrbs-1 & 1.77 & col-93 & 1.81 & vbh-1 & 1.88 & frk-1 & 1.93 \\
\hline col-91 & 1.77 & ugt-6 & 1.82 & T10B5.3 & 1.88 & T12D8.5 & 1.93 \\
\hline F20D6.5 & 1.77 & gtl-1 & 1.82 & F13G3.6 & 1.88 & cgr-1 & 1.93 \\
\hline mus- 81 & 1.77 & $\mathrm{nft}-1$ & 1.83 & Y48C3A.20 & 1.88 & M4.1 & 1.93 \\
\hline C44E4.5 & 1.77 & F10E7.5 & 1.83 & ugt-1 & 1.89 & nhr-170 & 1.93 \\
\hline fshr-1 & 1.77 & К09H9.5 & 1.83 & ztf-7 & 1.89 & Y70С5A.3 & 1.94 \\
\hline T01C8.3 & 1.78 & hlh-11 & 1.83 & eef-2 & 1.89 & dohh-1 & 1.94 \\
\hline nape-2 & 1.78 & abcf-2 & 1.83 & ctn-1 & 1.89 & $\mathrm{C} 40 \mathrm{H} 1.9$ & 1.94 \\
\hline F35F10.1 & 1.78 & moc-2 & 1.83 & Y51B9A.9 & 1.89 & T28F3.8 & 1.94 \\
\hline clec-75 & 1.78 & ubc-26 & 1.83 & swt-3 & 1.89 & glb-17 & 1.95 \\
\hline klp-15 & 1.78 & C47D12.5 & 1.83 & gfat-1 & 1.9 & smi-1 & 1.95 \\
\hline klp-16 & 1.78 & F26G1.10 & 1.84 & gра-8 & 1.9 & F21A3.11 & 1.95 \\
\hline K07G5.5 & 1.78 & T20D3.6 & 1.84 & T10C6.6 & 1.9 & lact-2 & 1.95 \\
\hline pkn-1 & 1.78 & let-611 & 1.84 & fat -6 & 1.9 & nape-1 & 1.95 \\
\hline T24H7.2 & 1.79 & asah-1 & 1.84 & tag-243 & 1.9 & pals-34 & 1.95 \\
\hline siah-1 & 1.79 & W08G11.3 & 1.85 & K02A11.4 & 1.9 & ztf-1 & 1.96 \\
\hline F58A3.3 & 1.79 & mrps-17 & 1.85 & fbxa-115 & 1.9 & xpo-1 & 1.96 \\
\hline C11D2.3 & 1.79 & $\mathrm{C} 04 \mathrm{H} 5.1$ & 1.85 & nspc-14 & 1.9 & F23B2.10 & 1.96 \\
\hline $\mathrm{cpz}-1$ & 1.79 & C27A7.3 & 1.85 & pud-4 & 1.9 & prmt-1 & 1.96 \\
\hline nlp-69 & 1.79 & B0564.2 & 1.85 & F35D11.3 & 1.9 & fars-3 & 1.96 \\
\hline alh-8 & 1.79 & hlh-13 & 1.85 & tag-151 & 1.91 & clec-17 & 1.96 \\
\hline fem-2 & 1.79 & $\mathrm{R} 01 \mathrm{H} 10.4$ & 1.85 & nhr-133 & 1.91 & scrm-2 & 1.97 \\
\hline duxl-1 & 1.79 & fbxa-185 & 1.86 & H20E11.3 & 1.91 & gра-17 & 1.97 \\
\hline Y54G2A.10 & 1.79 & ZC239.16 & 1.86 & F41G4.7 & 1.91 & ipla-7 & 1.97 \\
\hline dhs-7 & 1.79 & H35B03.1 & 1.86 & clec-82 & 1.91 & C04F12.16 & 1.97 \\
\hline col-101 & 1.8 & Y51H4A.15 & 1.86 & $\mathrm{H} 06 \mathrm{H} 21.8$ & 1.91 & $\operatorname{csp}-2$ & 1.98 \\
\hline C10E2.2 & 1.8 & pyr-1 & 1.87 & ZK792.5 & 1.91 & H34I24.2 & 1.98 \\
\hline eng-1 & 1.8 & nhr-35 & 1.87 & cest-13 & 1.91 & ZK550.2 & 1.98 \\
\hline nhr-55 & 1.8 & peb-1 & 1.87 & $\mathrm{C} 23 \mathrm{H} 3.5$ & 1.92 & dhs-1 & 1.98 \\
\hline$m t x-2$ & 1.8 & copz-1 & 1.87 & $\operatorname{tax}-6$ & 1.92 & T28A11.2 & 1.98 \\
\hline ugt-2 & 1.8 & exos-9 & 1.87 & F41E7.2 & 1.92 & Y106G6E.4 & 1.98 \\
\hline T22C8.4 & 1.8 & asp-3 & 1.87 & tag-229 & 1.92 & C02F5.3 & 1.99 \\
\hline F53H4.6 & 1.8 & F16B3.2 & 1.87 & T28C6.10 & 1.92 & skr-19 & 1.99 \\
\hline Y54F10AR.2 & 1.81 & nhr-108 & 1.87 & C15B12.4 & 1.92 & aakg-5 & 1.99 \\
\hline iff-2 & 1.81 & nhr-221 & 1.87 & M01E5.3 & 1.92 & fubl-1 & 1.99 \\
\hline drd-5 & 1.81 & Y45G5AM.7 & 1.88 & F13B12.2 & 1.92 & cct-4 & 1.99 \\
\hline M60.7 & 1.81 & E02H9.2 & 1.88 & paqr-1 & 1.92 & clec-60 & 2 \\
\hline R09B5.11 & 1.81 & C16C10.8 & 1.88 & lep-5 & 1.93 & bath-43 & 2 \\
\hline F31E3.6 & 1.81 & mlst-8 & 1.88 & Y32H12A.8 & 1.93 & cdc-48.3 & 2 \\
\hline
\end{tabular}




\begin{tabular}{|c|c|c|c|c|c|c|c|}
\hline Gene & $\log 2 \mathrm{FC}$ & Gene & $\log 2 \mathrm{FC}$ & Gene & $\log 2 \mathrm{FC}$ & Gene & $\log 2 \mathrm{FC}$ \\
\hline btb-17 & 2 & C17G10.6 & 2.08 & W07E6.2 & 2.18 & wars-1 & 2.28 \\
\hline F44G4.1 & 2 & F40F8.5 & 2.08 & W06E11.1 & 2.18 & cct-3 & 2.28 \\
\hline $\mathrm{E} 02 \mathrm{H} 1.1$ & 2 & Y32B12C.5 & 2.08 & cars-1 & 2.19 & Y46G5A.34 & 2.28 \\
\hline C05D9.9 & 2.01 & $\mathrm{C} 28 \mathrm{H} 8.3$ & 2.08 & bath-47 & 2.19 & unc-83 & 2.29 \\
\hline R11D1.13 & 2.01 & tpk-1 & 2.08 & AH9.7 & 2.2 & R09F10.1 & 2.29 \\
\hline nhr-103 & 2.01 & F35H8.1 & 2.09 & ZK512.2 & 2.2 & C53H9.2 & 2.29 \\
\hline nog-1 & 2.01 & ptr-21 & 2.09 & Y6G8.2 & 2.21 & gst-3 & 2.29 \\
\hline F09G2.2 & 2.01 & riok-2 & 2.09 & obr-2 & 2.21 & fbxa-173 & 2.29 \\
\hline ads-1 & 2.01 & D2096.7 & 2.09 & pgap-3 & 2.21 & sek-4 & 2.29 \\
\hline ugt-54 & 2.01 & twk-7 & 2.1 & gpx-6 & 2.22 & riok-3 & 2.3 \\
\hline T25B9.1 & 2.01 & dhs-24 & 2.1 & aakg-4 & 2.22 & C09D4.2 & 2.3 \\
\hline hsp-12.3 & 2.02 & $d d x-17$ & 2.11 & nhr-129 & 2.22 & R119.2 & 2.3 \\
\hline F41G4.8 & 2.02 & JC8.2 & 2.11 & rab-8 & 2.22 & exos-7 & 2.3 \\
\hline smg-3 & 2.02 & rev-3 & 2.11 & K09C8.7 & 2.22 & kin-14 & 2.3 \\
\hline rars-1 & 2.02 & Y113G7A.13 & 2.12 & lec-2 & 2.23 & F45E1.5 & 2.3 \\
\hline ubc-1 & 2.02 & psa-3 & 2.12 & nhr-88 & 2.23 & W06H8.6 & 2.31 \\
\hline asp-5 & 2.02 & Y53C12B.7 & 2.12 & D1054.3 & 2.23 & C27F2.4 & 2.31 \\
\hline aat-8 & 2.02 & alh-12 & 2.13 & T09B4.6 & 2.23 & Y18D10A.3 & 2.31 \\
\hline C54F6.6 & 2.02 & C15C7.4 & 2.13 & memb-2 & 2.23 & Y97E10AL.1 & 2.31 \\
\hline F10G8.9 & 2.02 & C03G6.17 & 2.13 & Y37H9A.5 & 2.24 & $\mathrm{fbxc}-7$ & 2.32 \\
\hline clec-61 & 2.03 & Ipd-7 & 2.14 & nhr-92 & 2.24 & C45E1.4 & 2.32 \\
\hline mrpl-55 & 2.03 & linc-43 & 2.14 & W07G4.5 & 2.24 & C52E2.4 & 2.32 \\
\hline Y67D2.5 & 2.03 & Y119D3B.14 & 2.14 & T22F3.2 & 2.24 & far-5 & 2.32 \\
\hline daf-2 & 2.03 & gst-37 & 2.14 & clec-62 & 2.24 & fil-1 & 2.32 \\
\hline ZC8.6 & 2.03 & smf-3 & 2.14 & K08F9.1 & 2.24 & Y71H2AM.1 & 2.32 \\
\hline aak-1 & 2.03 & ulp-3 & 2.14 & D1022.4 & 2.25 & wrb-1 & 2.32 \\
\hline elpc-4 & 2.03 & imb-3 & 2.14 & eif-3.E & 2.25 & srp-2 & 2.33 \\
\hline sel-8 & 2.03 & clec-8 & 2.15 & twk-5 & 2.25 & ZC239.17 & 2.33 \\
\hline linc-96 & 2.04 & kin-3 & 2.15 & F30A10.9 & 2.25 & ZK1290.13 & 2.33 \\
\hline $\mathrm{ccm}-2$ & 2.05 & F08H9.3 & 2.15 & Y71G10AR.4 & 2.25 & B0379.7 & 2.33 \\
\hline Y34F4.5 & 2.06 & ZK430.7 & 2.16 & Imd-4 & 2.26 & B0524.6 & 2.34 \\
\hline upb-1 & 2.06 & pho-6 & 2.16 & B0511.6 & 2.26 & pho-5 & 2.34 \\
\hline Y54F10AM.8 & 2.06 & C50F4.1 & 2.17 & clec-65 & 2.26 & nhr-146 & 2.34 \\
\hline F53F10.1 & 2.07 & F53F4.11 & 2.17 & Y61A9LA.1 & 2.26 & F32A5.8 & 2.34 \\
\hline nhr-85 & 2.07 & Y57G11B.2 & 2.17 & K11D12.12 & 2.26 & ubc-15 & 2.34 \\
\hline gst-23 & 2.07 & $\mathrm{rsd}-3$ & 2.17 & C36E8.1 & 2.26 & $\mathrm{~F} 49 \mathrm{C} 12.6$ & 2.34 \\
\hline $\mathrm{C} 24 \mathrm{H} 12.4$ & 2.07 & nsun-2 & 2.17 & F23F12.3 & 2.26 & cpr-6 & 2.35 \\
\hline C53C11.2 & 2.07 & afmd-2 & 2.17 & Y45F3A.9 & 2.27 & nlp-52 & 2.35 \\
\hline T19D12.2 & 2.07 & rpom-1 & 2.18 & T01B7.5 & 2.27 & Igc- 25 & 2.35 \\
\hline rars-2 & 2.07 & W02D7.11 & 2.18 & nap-1 & 2.28 & $\mathrm{C} 39 \mathrm{H} 7.4$ & 2.35 \\
\hline T08G11.4 & 2.08 & $\mathrm{C} 25 \mathrm{H} 3.11$ & 2.18 & F26D11.12 & 2.28 & spp-6 & 2.35 \\
\hline
\end{tabular}




\begin{tabular}{|c|c|c|c|c|c|c|c|}
\hline Gene & $\log 2 \mathrm{FC}$ & Gene & $\log 2 \mathrm{FC}$ & Gene & $\log 2 \mathrm{FC}$ & Gene & $\log 2 \mathrm{FC}$ \\
\hline ZK355.8 & 2.36 & F31E9.3 & 2.54 & W04E12.7 & 2.66 & F40G12.5 & 2.98 \\
\hline epg-5 & 2.36 & C33E10.3 & 2.54 & Y7A5A.6 & 2.67 & F36A2.2 & 2.99 \\
\hline T26H5.4 & 2.37 & Y9C9A.8 & 2.54 & asah-2 & 2.67 & hpo-15 & 2.99 \\
\hline gst-22 & 2.38 & lido-10 & 2.54 & asp-6 & 2.67 & ZK593.2 & 3.01 \\
\hline math-37 & 2.39 & zig-9 & 2.54 & F54F12.1 & 2.67 & cest-1.2 & 3.02 \\
\hline T26C11.9 & 2.39 & Y47G6A.7 & 2.55 & Y43B11AR.3 & 2.67 & T01G1.4 & 3.02 \\
\hline B0507.3 & 2.4 & mboa-7 & 2.56 & tts-1 & 2.68 & F56H9.2 & 3.04 \\
\hline arch-1 & 2.4 & nhr-18 & 2.56 & R10E8.6 & 2.68 & metl-18 & 3.05 \\
\hline C49C8.8 & 2.41 & col-160 & 2.56 & C28G1.6 & 2.69 & $\mathrm{fbxa}-6$ & 3.06 \\
\hline eif-3.B & 2.41 & linc-103 & 2.56 & pin-2 & 2.69 & F56A8.5 & 3.07 \\
\hline metl-2 & 2.42 & F18G5.6 & 2.57 & clec-85 & 2.71 & acp-5 & 3.07 \\
\hline T10B5.7 & 2.42 & col-92 & 2.57 & ech-7 & 2.72 & col-39 & 3.07 \\
\hline btb-16 & 2.42 & R09E10.13 & 2.57 & C39D10.11 & 2.72 & fbxa-162 & 3.07 \\
\hline vps-33.1 & 2.42 & F08F8.7 & 2.57 & chil-13 & 2.73 & F10G7.5 & 3.12 \\
\hline Igc-28 & 2.42 & T25G3.3 & 2.57 & Y54H5A.1 & 2.73 & cup-16 & 3.13 \\
\hline F42C5.3 & 2.43 & rrp-8 & 2.57 & Y52B11A.10 & 2.73 & faah-3 & 3.13 \\
\hline ttr-34 & 2.44 & nhr-94 & 2.58 & Y47G6A.5 & 2.75 & C08F11.13 & 3.14 \\
\hline K02C4.5 & 2.45 & Y39B6A.33 & 2.58 & nas-9 & 2.76 & clec-84 & 3.15 \\
\hline acl-9 & 2.45 & T08A9.13 & 2.58 & T08B6.2 & 2.76 & сур-37B1 & 3.15 \\
\hline pals-14 & 2.46 & F48E8.3 & 2.58 & C52E2.5 & 2.76 & $\mathrm{~T} 28 \mathrm{H} 10.1$ & 3.16 \\
\hline fbxa-80 & 2.46 & C29G2.2 & 2.59 & ZK688.11 & 2.77 & ZK470.14 & 3.16 \\
\hline F16A11.5 & 2.46 & Y47H10A.3 & 2.59 & $\mathrm{C} 18 \mathrm{H} 9.5$ & 2.79 & C53A5.17 & 3.18 \\
\hline nhr-232 & 2.47 & F42A9.18 & 2.6 & R07E3.1 & 2.8 & C25F9.12 & 3.18 \\
\hline nhr-109 & 2.47 & mnk-1 & 2.6 & W03D8.2 & 2.8 & ctps-1 & 3.2 \\
\hline col-118 & 2.47 & R10E8.1 & 2.6 & Y42G9A.1 & 2.81 & B0546.3 & 3.21 \\
\hline Y56A3A.33 & 2.47 & gsp-2 & 2.6 & pqn-62 & 2.82 & hrg-2 & 3.22 \\
\hline Inp-1 & 2.48 & Y54H5A.2 & 2.61 & cpr-2 & 2.82 & F52E1.14 & 3.23 \\
\hline Y37E11B.5 & 2.48 & fbxa-69 & 2.62 & T13F2.6 & 2.82 & chil-16 & 3.25 \\
\hline Y47D3A.32 & 2.49 & nnt-1 & 2.62 & riok-1 & 2.82 & F47B8.8 & 3.26 \\
\hline Y54E10BR.4 & 2.5 & pmt-1 & 2.63 & sek-1 & 2.82 & F47B8.4 & 3.27 \\
\hline gdh-1 & 2.5 & F53F1.14 & 2.63 & pqn-59 & 2.83 & F35C8.5 & 3.28 \\
\hline M01B2.13 & 2.5 & puf-12 & 2.63 & pbo-6 & 2.86 & srv-4 & 3.32 \\
\hline elpc-3 & 2.51 & $\mathrm{C} 32 \mathrm{H} 11.4$ & 2.63 & dao-6 & 2.86 & T06C12.14 & 3.32 \\
\hline F40E3.5 & 2.51 & Y22D7AL.15 & 2.63 & clec-264 & 2.87 & exc-13 & 3.35 \\
\hline T22H9.1 & 2.51 & T18D3.6 & 2.64 & Y48G8AL.13 & 2.88 & F43D2.7 & 3.39 \\
\hline C16A3.4 & 2.51 & ulp-4 & 2.64 & D1086.2 & 2.89 & fard-1 & 3.39 \\
\hline ngp-1 & 2.52 & gst-20 & 2.64 & C34E11.4 & 2.89 & C32F10.4 & 3.42 \\
\hline F54D5.3 & 2.52 & asm-2 & 2.65 & Y15E3A.4 & 2.94 & T10C6.15 & 3.49 \\
\hline ZK1240.2 & 2.52 & adah-1 & 2.65 & Y40C5A.4 & 2.95 & K05F6.10 & 3.52 \\
\hline C23F12.4 & 2.52 & dph-1 & 2.65 & W03D2.6 & 2.95 & Y65B4BL.7 & 3.52 \\
\hline C49A9.6 & 2.53 & C33E10.8 & 2.65 & tut-1 & 2.96 & T26E3.4 & 3.56 \\
\hline
\end{tabular}




\begin{tabular}{|c|c|}
\hline Gene & log2FC \\
\hline ttr-49 & 3.59 \\
\hline W08E12.8 & 3.6 \\
\hline F43C11.7 & 3.6 \\
\hline spp-8 & 3.64 \\
\hline col-50 & 3.64 \\
\hline hda-5 & 3.71 \\
\hline cyp-32B1 & 3.79 \\
\hline F21C10.11 & 3.93 \\
\hline ZK185.5 & 3.93 \\
\hline F56F11.1 & 3.97 \\
\hline C10A4.3 & 4.01 \\
\hline T28A11.16 & 4.04 \\
\hline arrd-11 & 4.07 \\
\hline W10G11.1 & 4.14 \\
\hline F21H7.12 & 4.27 \\
\hline hsp-12.6 & 5.24 \\
\hline dpy-5 & 6.22 \\
\hline
\end{tabular}


6.16. RNA-seq (CeGaT) - 4h Fed vs 48h Starved - HBR227 (aptf-1)

\begin{tabular}{|c|c|c|c|c|c|c|c|}
\hline Gene & $\log 2 \mathrm{FC}$ & Gene & $\log 2 \mathrm{FC}$ & Gene & $\log 2 \mathrm{FC}$ & Gene & $\log 2 \mathrm{FC}$ \\
\hline F46F2.3 & -5.06 & R12A1.3 & -2.65 & dod-19 & -2.41 & F30H5.3 & -2.26 \\
\hline T22F3.11 & -4.98 & R193.2 & -2.65 & asp-13 & -2.41 & clec-78 & -2.26 \\
\hline Y39B6A.1 & -4.93 & C42D4.3 & -2.64 & clec-180 & -2.41 & pqn-71 & -2.26 \\
\hline $\mathrm{R} 02 \mathrm{C} 2.7$ & -4.52 & puf-11 & -2.64 & acdh-1 & -2.4 & ZK662.2 & -2.25 \\
\hline irg-4 & -3.83 & col-109 & -2.62 & nlp-77 & -2.39 & T10E10.4 & -2.25 \\
\hline gst-26 & -3.83 & kreg-1 & -2.62 & Y53F4B.23 & -2.38 & nlp-34 & -2.24 \\
\hline W10C8.5 & -3.8 & col-14 & -2.62 & tag-10 & -2.38 & cut-2 & -2.23 \\
\hline F57F4.4 & -3.7 & cpr-5 & -2.61 & tyr-2 & -2.37 & grl-21 & -2.22 \\
\hline gsto-1 & -3.53 & grl-4 & -2.58 & F46C8.8 & -2.37 & Ipr-6 & -2.21 \\
\hline pho-11 & -3.38 & abu-10 & -2.58 & gfi-1 & -2.37 & Y16B4A.2 & -2.21 \\
\hline F49C12.7 & -3.36 & W05F2.3 & -2.57 & F56D2.3 & -2.37 & C35C5.8 & -2.21 \\
\hline T07D3.9 & -3.17 & EGAP4.1 & -2.57 & F44E2.4 & -2.36 & fmo-3 & -2.21 \\
\hline col-166 & -3.16 & R02F11.1 & -2.56 & Ipr-3 & -2.35 & pie-1 & -2.21 \\
\hline C45B2.2 & -3.02 & cey-3 & -2.56 & F28B4.3 & -2.34 & F53F1.4 & -2.2 \\
\hline dod-24 & -2.97 & $\mathrm{cbd}-1$ & -2.56 & mex-1 & -2.33 & grd-3 & -2.2 \\
\hline col-48 & -2.96 & ZK180.6 & -2.55 & act-3 & -2.32 & phat-1 & -2.2 \\
\hline col-97 & -2.95 & idh-1 & -2.55 & spn-4 & -2.32 & ZK6.11 & -2.2 \\
\hline C15A11.4 & -2.94 & act-5 & -2.55 & ttr-31 & -2.32 & F56C9.3 & -2.19 \\
\hline mdh-2 & -2.91 & F35B3.4 & -2.54 & C09F9.2 & -2.32 & cutl-8 & -2.19 \\
\hline $\mathrm{C} 23 \mathrm{H} 5.8$ & -2.9 & K08D8.3 & -2.52 & gst-28 & -2.32 & F38B6.4 & -2.18 \\
\hline alh-5 & -2.9 & hrg-7 & -2.52 & К02B7.3 & -2.31 & Idh-1 & -2.18 \\
\hline puf-3 & -2.89 & M02G9.1 & -2.51 & smd-1 & -2.31 & pqn-22 & -2.17 \\
\hline ugt-63 & -2.82 & F10D11.6 & -2.49 & ech-9 & -2.31 & acox-1.5 & -2.17 \\
\hline col-113 & -2.81 & Y57G11C.42 & -2.48 & col-33 & -2.3 & E01G6.1 & -2.17 \\
\hline C23G10.11 & -2.81 & F40E10.5 & -2.48 & R11H6.4 & -2.3 & ZK180.5 & -2.17 \\
\hline lbp-6 & -2.81 & abu-12 & -2.46 & B0403.5 & -2.3 & nas-13 & -2.17 \\
\hline hphd-1 & -2.79 & T08B1.1 & -2.46 & acn-1 & -2.3 & Y14H12B.2 & -2.16 \\
\hline clec-48 & -2.78 & F46C5.1 & -2.46 & gsnl-1 & -2.29 & $\lg x-1$ & -2.16 \\
\hline Y65B4BL.1 & -2.77 & oma-2 & -2.46 & fmo-2 & -2.29 & Ion-1 & -2.16 \\
\hline Т19B10.2 & -2.77 & F13D12.3 & -2.45 & grd-10 & -2.29 & $\mathrm{mcm}-3$ & -2.16 \\
\hline C01G10.15 & -2.77 & F35E12.6 & -2.45 & hpo-6 & -2.29 & ifa-3 & -2.16 \\
\hline R09E10.5 & -2.75 & irg-5 & -2.44 & nas-30 & -2.28 & abu-8 & -2.16 \\
\hline sdz-24 & -2.74 & dct-16 & -2.44 & Ipr-5 & -2.27 & F09E10.1 & -2.16 \\
\hline ilys-5 & -2.73 & ugt-33 & -2.43 & Y22D7AL.10 & -2.27 & mom-2 & -2.16 \\
\hline cpr-4 & -2.72 & nas-38 & -2.43 & idhg-2 & -2.27 & skpo-2 & -2.16 \\
\hline txt-4 & -2.71 & mua-6 & -2.42 & C01G8.1 & -2.27 & nas-10 & -2.15 \\
\hline folt-2 & -2.7 & T24C12.4 & -2.42 & C06C3.10 & -2.27 & plpr-1 & -2.14 \\
\hline pqn-26 & -2.69 & F59A6.10 & -2.42 & F38B2.4 & -2.27 & pqn-32 & -2.14 \\
\hline nspb-12 & -2.68 & cnc-4 & -2.42 & gld-3 & -2.26 & nlp-30 & -2.14 \\
\hline Y7A9A.79 & -2.68 & abu-14 & -2.41 & F20B10.3 & -2.26 & C25F6.7 & -2.14 \\
\hline C48B4.13 & -2.67 & F41E6.5 & -2.41 & F13H8.5 & -2.26 & phg-1 & -2.13 \\
\hline
\end{tabular}




\begin{tabular}{|c|c|c|c|c|c|c|c|}
\hline Gene & $\log 2 \mathrm{FC}$ & Gene & $\log 2 \mathrm{FC}$ & Gene & $\log 2 \mathrm{FC}$ & Gene & $\log 2 \mathrm{FC}$ \\
\hline slc-17.8 & -2.13 & Ibp-5 & -2.04 & F41E6.11 & -1.97 & kat-1 & -1.88 \\
\hline Y73В3А.1 & -2.13 & C54G4.4 & -2.04 & C50F7.5 & -1.96 & K02G10.15 & -1.88 \\
\hline C06G1.1 & -2.13 & ZK970.7 & -2.03 & $\mathrm{C} 49 \mathrm{H} 3.16$ & -1.96 & T05F1.2 & -1.88 \\
\hline grd-13 & -2.13 & Y34B4A.6 & -2.02 & zipt-7.1 & -1.96 & E04F6.6 & -1.88 \\
\hline pgp-14 & -2.13 & cyn-17 & -2.02 & F39D8.3 & -1.96 & D1054.9 & -1.88 \\
\hline F48G7.5 & -2.13 & phat-4 & -2.02 & cey-2 & -1.96 & vha-12 & -1.88 \\
\hline F10A3.4 & -2.12 & hacd-1 & -2.02 & C29F3.7 & -1.96 & daao-1 & -1.87 \\
\hline egg-6 & -2.12 & tts-2 & -2.02 & air-1 & -1.96 & dod-3 & -1.87 \\
\hline B0024.4 & -2.11 & asns-2 & -2.02 & gst-21 & -1.95 & Y43F8B.3 & -1.87 \\
\hline C35A5.10 & -2.11 & hsp-60 & -2.02 & $\operatorname{ttr}-15$ & -1.95 & T06A4.1 & -1.87 \\
\hline srg-31 & -2.11 & pqn-73 & -2.02 & T20B6.3 & -1.95 & K08D12.4 & -1.87 \\
\hline pqn-74 & -2.11 & ptr-18 & -2.01 & mec-7 & -1.95 & gld-1 & -1.86 \\
\hline C30B5.6 & -2.11 & $\mathrm{~K} 04 \mathrm{H} 4.2$ & -2.01 & wrt-2 & -1.94 & F16H11.1 & -1.86 \\
\hline sun-1 & -2.11 & ZK84.1 & -2.01 & C50D2.1 & -1.94 & col-182 & -1.85 \\
\hline T06A4.3 & -2.11 & Y73B6BL.23 & -2.01 & C52G5.2 & -1.94 & cpg-9 & -1.85 \\
\hline cyb-2.2 & -2.1 & W03D2.9 & -2.01 & lys-1 & -1.93 & sod-1 & -1.85 \\
\hline asp-8 & -2.1 & dex-1 & -2.01 & Y47H9C.1 & -1.93 & grl-16 & -1.84 \\
\hline acp-6 & -2.1 & mex-5 & -2.01 & cpg-1 & -1.93 & R09E12.9 & -1.84 \\
\hline noah-2 & -2.1 & F55A11.7 & -2 & valv-1 & -1.93 & abu-11 & -1.84 \\
\hline cut-5 & -2.1 & F54D5.2 & -2 & F17A9.5 & -1.93 & ttr-45 & -1.84 \\
\hline gcsh-1 & -2.09 & $\mathrm{C} 34 \mathrm{H} 4.2$ & -2 & col-167 & -1.92 & T05B9.1 & -1.84 \\
\hline cutl-23 & -2.09 & T19A5.3 & -2 & pqn-13 & -1.92 & puf-5 & -1.84 \\
\hline ptr-1 & -2.09 & col-144 & -2 & tth-1 & -1.92 & F56D3.1 & -1.84 \\
\hline Y110A7A.15 & -2.09 & twk-11 & -2 & dpy-1 & -1.92 & ugt-22 & -1.83 \\
\hline VF13D12L.3 & -2.08 & F01D5.6 & -1.99 & Y47D3B.6 & -1.92 & $\mathrm{mcm}-7$ & -1.83 \\
\hline nos-1 & -2.07 & act-1 & -1.99 & Ipr-4 & -1.91 & $r n r-1$ & -1.83 \\
\hline tpxl-1 & -2.07 & $\mathrm{mcm}-2$ & -1.99 & bus- 8 & -1.91 & zig-4 & -1.83 \\
\hline F32D8.7 & -2.07 & pcp-2 & -1.99 & ttr-2 & -1.91 & wee-1.3 & -1.83 \\
\hline ptr-2 & -2.07 & C18E9.7 & -1.98 & C55A6.4 & -1.91 & jud-4 & -1.83 \\
\hline phat-2 & -2.07 & clec-51 & -1.98 & W08E12.3 & -1.91 & haf-9 & -1.83 \\
\hline lec- 8 & -2.07 & $\mathrm{C} 31 \mathrm{H} 1.8$ & -1.98 & C14C6.5 & -1.91 & col-80 & -1.83 \\
\hline C46A5.4 & -2.06 & ugt-41 & -1.98 & ZK829.9 & -1.91 & F21C10.9 & -1.83 \\
\hline col-98 & -2.06 & ugt-40 & -1.98 & F32D8.3 & -1.91 & era-1 & -1.83 \\
\hline sqt-3 & -2.06 & ptr-4 & -1.98 & cpr-1 & -1.91 & szy-4 & -1.83 \\
\hline lec-6 & -2.06 & pqn-63 & -1.98 & Y51F10.2 & -1.91 & ech-6 & -1.83 \\
\hline mlt-8 & -2.06 & otpl-8 & -1.97 & rad-51 & -1.91 & ZC513.14 & -1.83 \\
\hline hpo-36 & -2.05 & R05F9.6 & -1.97 & col-159 & -1.9 & nstp-2 & -1.82 \\
\hline $\mathrm{F} 23 \mathrm{H} 12.5$ & -2.05 & cdh-5 & -1.97 & col-3 & -1.9 & W04A8.4 & -1.82 \\
\hline mlt-9 & -2.05 & ani-2 & -1.97 & hch-1 & -1.89 & col-145 & -1.82 \\
\hline set-19 & -2.04 & lys-3 & -1.97 & $\mathrm{~F} 14 \mathrm{H} 12.3$ & -1.89 & gst-41 & -1.82 \\
\hline F20G2.2 & -2.04 & $f b p-1$ & -1.97 & F43D9.1 & -1.89 & H42K12.3 & -1.82 \\
\hline
\end{tabular}




\begin{tabular}{|c|c|c|c|c|c|c|c|}
\hline Gene & $\log 2 \mathrm{FC}$ & Gene & $\log 2 \mathrm{FC}$ & Gene & $\log 2 \mathrm{FC}$ & Gene & $\log 2 \mathrm{FC}$ \\
\hline let-754 & -1.81 & F07H5.8 & -1.75 & clec-41 & -1.69 & atp-1 & -1.65 \\
\hline D2005.6 & -1.81 & pho-14 & -1.75 & fip-5 & -1.69 & cutl-10 & -1.65 \\
\hline D2096.6 & -1.81 & ugt-34 & -1.75 & H01G02.1 & -1.69 & C01B10.11 & -1.65 \\
\hline F59F5.7 & -1.8 & T04F8.8 & -1.75 & M03F8.1 & -1.69 & sdhd-1 & -1.65 \\
\hline clec-49 & -1.8 & $\operatorname{lgc}-22$ & -1.75 & nspe-7 & -1.69 & Y111B2A.3 & -1.65 \\
\hline cest-33 & -1.8 & clec- 88 & -1.75 & pcn-1 & -1.68 & C12D12.1 & -1.65 \\
\hline hil-4 & -1.79 & C50C3.1 & -1.74 & his-74 & -1.68 & T17H7.7 & -1.65 \\
\hline $\mathrm{F} 35 \mathrm{H} 10.10$ & -1.79 & T20F5.4 & -1.74 & nasp-2 & -1.68 & Iron-2 & -1.64 \\
\hline apy-1 & -1.79 & col-117 & -1.74 & snpc-1.2 & -1.68 & tag-297 & -1.64 \\
\hline Y75B7AR.1 & -1.79 & chs-2 & -1.74 & ZK1320.9 & -1.68 & R09F10.8 & -1.64 \\
\hline pos-1 & -1.79 & cdl-1 & -1.74 & mdh-1 & -1.68 & gst-19 & -1.64 \\
\hline K11G9.5 & -1.79 & chtl-1 & -1.73 & puf-8 & -1.68 & toca-2 & -1.63 \\
\hline nspe-5 & -1.79 & F30F8.1 & -1.73 & $\operatorname{ttr}-7$ & -1.68 & dhcr-24 & -1.63 \\
\hline gpd-4 & -1.79 & daf- 6 & -1.73 & F08F1.3 & -1.68 & K10D3.4 & -1.63 \\
\hline col-115 & -1.79 & gpd-1 & -1.73 & T06D8.10 & -1.68 & pals-26 & -1.63 \\
\hline clik-1 & -1.79 & K01A6.4 & -1.73 & zwl-1 & -1.68 & brd-1 & -1.63 \\
\hline $\operatorname{trcs}-1$ & -1.78 & tost-1 & -1.73 & F58G1.7 & -1.68 & wrt-9 & -1.63 \\
\hline grl-6 & -1.78 & R144.10 & -1.72 & F13D12.9 & -1.68 & ify-1 & -1.63 \\
\hline hil-5 & -1.78 & Y48G1BR.1 & -1.72 & C14B1.9 & -1.68 & ZK154.1 & -1.63 \\
\hline Y48G1A.2 & -1.78 & T16G1.2 & -1.72 & Igc-34 & -1.67 & eat-20 & -1.62 \\
\hline ifd-2 & -1.78 & C04G6.10 & -1.72 & $\mathrm{~F} 40 \mathrm{H} 3.3$ & -1.67 & $3 / 3 / 2020$ & -1.62 \\
\hline T23F6.1 & -1.78 & ttr-18 & -1.72 & С14B9.3 & -1.67 & R04B5.5 & -1.62 \\
\hline Y69A2AR.28 & -1.78 & F01D5.10 & -1.72 & R12E2.7 & -1.67 & acly-2 & -1.62 \\
\hline clec-53 & -1.78 & C49F5.6 & -1.72 & cnc- 8 & -1.67 & air-2 & -1.62 \\
\hline col-10 & -1.78 & Isy-27 & -1.72 & ced-3 & -1.67 & R07E5.4 & -1.62 \\
\hline pgk-1 & -1.78 & mes-4 & -1.72 & col-104 & -1.67 & clec-72 & -1.61 \\
\hline F12A10.1 & -1.78 & T27E9.2 & -1.72 & col-155 & -1.67 & egl-46 & -1.61 \\
\hline $\mathrm{R} 13 \mathrm{H} 4.8$ & -1.77 & F54E7.6 & -1.71 & Y41G9A.10 & -1.66 & C29A12.6 & -1.61 \\
\hline M03E7.2 & -1.77 & W06B4.1 & -1.71 & aagr-4 & -1.66 & B0304.2 & -1.61 \\
\hline lec-10 & -1.77 & M03F4.6 & -1.7 & C05D12.1 & -1.66 & tag-18 & -1.61 \\
\hline srp-1 & -1.77 & F54D5.4 & -1.7 & zif-1 & -1.66 & F08D12.2 & -1.61 \\
\hline deps-1 & -1.77 & Y37A1B.5 & -1.7 & $\operatorname{lgc}-27$ & -1.66 & sas- 6 & -1.61 \\
\hline W03C9.2 & -1.77 & C05C10.5 & -1.7 & prom-1 & -1.66 & zen-4 & -1.61 \\
\hline gyg-2 & -1.77 & atf-2 & -1.7 & ifp-1 & -1.66 & Y4C6B.1 & -1.6 \\
\hline clec-10 & -1.76 & F08B12.4 & -1.7 & pfn-1 & -1.66 & K01A6.7 & -1.6 \\
\hline $\mathrm{pgl}-3$ & -1.76 & F18E9.4 & -1.7 & spp-17 & -1.66 & nlp-31 & -1.6 \\
\hline R03C1.1 & -1.76 & F20G2.5 & -1.7 & T09B4.5 & -1.66 & F27E5.9 & -1.6 \\
\hline wrt-4 & -1.76 & R05H5.3 & -1.69 & lys-8 & -1.66 & haao-1 & -1.6 \\
\hline T28C12.6 & -1.76 & F13B6.2 & -1.69 & T19D12.1 & -1.65 & Y53F4B.39 & -1.6 \\
\hline F45E1.4 & -1.76 & D2092.8 & -1.69 & F59A7.2 & -1.65 & dpy-17 & -1.6 \\
\hline lys-4 & -1.76 & pmp-5 & -1.69 & F10C1.9 & -1.65 & F32D8.12 & -1.59 \\
\hline
\end{tabular}




\begin{tabular}{|c|c|c|c|c|c|c|c|}
\hline Gene & $\log 2 \mathrm{FC}$ & Gene & $\log 2 \mathrm{FC}$ & Gene & $\log 2 \mathrm{FC}$ & Gene & $\log 2 \mathrm{FC}$ \\
\hline T25E4.1 & -1.59 & C35B1.5 & -1.55 & cld-9 & -1.51 & T06E4.14 & -1.47 \\
\hline ect-2 & -1.59 & T02G5.7 & -1.55 & aqp-2 & -1.51 & sym-1 & -1.47 \\
\hline F09C8.1 & -1.59 & anmt-2 & -1.55 & R07B1.9 & -1.51 & col-177 & -1.47 \\
\hline hsp-25 & -1.59 & alg-5 & -1.55 & acs-11 & -1.51 & T12G3.6 & -1.47 \\
\hline ZK105.13 & -1.59 & $\mathrm{R} 03 \mathrm{H} 10.2$ & -1.54 & gst-27 & -1.51 & Y32G9B.1 & -1.47 \\
\hline acaa-2 & -1.59 & ugt-13 & -1.54 & W09G12.10 & -1.51 & ctf-4 & -1.46 \\
\hline C56G2.4 & -1.59 & Igc-21 & -1.54 & C29G2.3 & -1.5 & kdp-1 & -1.46 \\
\hline ZK1290.10 & -1.59 & col-34 & -1.54 & $\mathrm{M02H} 5.8$ & -1.5 & syp-6 & -1.46 \\
\hline ugt-45 & -1.59 & B0001.7 & -1.54 & C44B9.3 & -1.5 & tyms-1 & -1.45 \\
\hline cyb-1 & -1.59 & $\operatorname{dim}-1$ & -1.54 & col-154 & -1.5 & plk-1 & -1.45 \\
\hline $\mathrm{knl}-1$ & -1.59 & F57F5.1 & -1.54 & nol-56 & -1.5 & T22D1.5 & -1.45 \\
\hline unc-59 & -1.58 & toe- 2 & -1.54 & lec-1 & -1.5 & dhs- 8 & -1.45 \\
\hline rnp-8 & -1.58 & K08D8.6 & -1.53 & Igc-33 & -1.5 & div-1 & -1.45 \\
\hline F53B1.4 & -1.58 & icl-1 & -1.53 & spsb-2 & -1.5 & daf-22 & -1.45 \\
\hline F20G2.1 & -1.58 & M02D8.6 & -1.53 & B0261.7 & -1.5 & tdpt-1 & -1.45 \\
\hline C08F8.3 & -1.58 & $\mathrm{~T} 10 \mathrm{H} 10.2$ & -1.53 & gst-4 & -1.49 & C01G6.3 & -1.45 \\
\hline cdh-8 & -1.58 & fbxa-215 & -1.53 & hsp-17 & -1.49 & Y54G11A.3 & -1.44 \\
\hline in $x-3$ & -1.58 & Y58A7A.1 & -1.53 & K08D12.6 & -1.49 & T02H6.11 & -1.44 \\
\hline C06C3.4 & -1.58 & dpy-18 & -1.53 & F56C9.7 & -1.49 & R74.2 & -1.44 \\
\hline mam-1 & -1.58 & $g \ln -6$ & -1.53 & grl-1 & -1.49 & R02E4.3 & -1.44 \\
\hline col-186 & -1.58 & pfk-1.1 & -1.53 & pole-1 & -1.49 & C34C12.8 & -1.44 \\
\hline cyk-7 & -1.58 & F42A10.9 & -1.53 & skpt-1 & -1.49 & mpst-1 & -1.44 \\
\hline C15C6.1 & -1.58 & ech-4 & -1.53 & C15F1.1 & -1.49 & T03F6.3 & -1.44 \\
\hline Y110A2AR.1 & -1.58 & col-169 & -1.53 & W01A11.1 & -1.49 & rocf-1 & -1.44 \\
\hline F38A6.4 & -1.58 & noah-1 & -1.53 & $\mathrm{~K} 10 \mathrm{H} 10.4$ & -1.49 & hcp-1 & -1.44 \\
\hline DY3.8 & -1.58 & C55A6.12 & -1.53 & R08E5.3 & -1.49 & ost-1 & -1.43 \\
\hline ttr-59 & -1.57 & sptl-2 & -1.53 & clec-42 & -1.49 & C45B2.8 & -1.43 \\
\hline best-1 & -1.57 & tnt-2 & -1.53 & C54D2.1 & -1.49 & C49F8.3 & -1.43 \\
\hline unc-52 & -1.57 & $\mathrm{dpl}-1$ & -1.52 & $\mathrm{ctl}-2$ & -1.49 & oac-14 & -1.43 \\
\hline F22B5.4 & -1.57 & zim-1 & -1.52 & ZK1037.6 & -1.49 & F26F12.4 & -1.43 \\
\hline tdc-1 & -1.57 & F35D2.3 & -1.52 & Y51F10.7 & -1.48 & K08E3.5 & -1.43 \\
\hline$n m y-1$ & -1.57 & sip-1 & -1.52 & bath-26 & -1.48 & ung-1 & -1.43 \\
\hline pho-1 & -1.56 & F19C7.1 & -1.52 & mlt-11 & -1.48 & cyb-3 & -1.43 \\
\hline сур-35C1 & -1.56 & F56C9.6 & -1.52 & F41G3.21 & -1.48 & F33D4.6 & -1.43 \\
\hline argk-1 & -1.56 & F15B9.8 & -1.52 & papl-1 & -1.48 & cyk-4 & -1.42 \\
\hline dpy-7 & -1.56 & T19C4.1 & -1.52 & ZC443.1 & -1.48 & ZK675.4 & -1.42 \\
\hline zig-12 & -1.55 & phat-5 & -1.52 & Y37H2A.14 & -1.48 & lec-9 & -1.42 \\
\hline ZC123.1 & -1.55 & sdha-2 & -1.52 & slc- $25 A 10$ & -1.48 & edg-1 & -1.42 \\
\hline M04C9.1 & -1.55 & pqn-60 & -1.51 & $\mathrm{mcm}-6$ & -1.48 & F15D3.6 & -1.42 \\
\hline ttr-33 & -1.55 & inx-14 & -1.51 & ifc-1 & -1.47 & spp-2 & -1.41 \\
\hline mup-4 & -1.55 & $s d z-28$ & -1.51 & T21C9.13 & -1.47 & T23F2.5 & -1.41 \\
\hline
\end{tabular}




\begin{tabular}{|c|c|c|c|c|c|c|c|}
\hline Gene & $\log 2 \mathrm{FC}$ & Gene & $\log 2 \mathrm{FC}$ & Gene & $\log 2 \mathrm{FC}$ & Gene & $\log 2 \mathrm{FC}$ \\
\hline fzy-1 & -1.41 & acs-7 & -1.35 & F54D5.5 & -1.3 & mthf-1 & -1.24 \\
\hline pqn-36 & -1.41 & gna-2 & -1.35 & dpy-14 & -1.29 & chaf-2 & -1.24 \\
\hline $\mathrm{cpr}-3$ & -1.41 & asns-1 & -1.35 & F31D4.5 & -1.29 & $\operatorname{ttr}-30$ & -1.24 \\
\hline hrde-1 & -1.41 & F11E6.3 & -1.35 & T21B6.3 & -1.29 & T05H10.4 & -1.24 \\
\hline dhs-30 & -1.41 & Y41G9A.5 & -1.35 & rsa-1 & -1.29 & F39B2.3 & -1.24 \\
\hline F22B7.9 & -1.41 & hmg-3 & -1.34 & col-111 & -1.29 & capg-2 & -1.24 \\
\hline pri-2 & -1.4 & F33H2.3 & -1.34 & misc-1 & -1.29 & сур-13В1 & -1.24 \\
\hline C36B1.11 & -1.4 & cks-1 & -1.34 & hsp-16.41 & -1.29 & tsp-1 & -1.24 \\
\hline aqp-7 & -1.4 & fat-1 & -1.34 & F21D5.1 & -1.29 & aly-2 & -1.24 \\
\hline pdp-1 & -1.4 & lys-7 & -1.34 & mai-2 & -1.29 & cts-1 & -1.23 \\
\hline F26G1.5 & -1.4 & ril-1 & -1.34 & ifo-1 & -1.29 & bca-2 & -1.22 \\
\hline mec-12 & -1.4 & htz-1 & -1.34 & bca-1 & -1.29 & ttr-20 & -1.22 \\
\hline agmo-1 & -1.4 & F44B9. 2 & -1.34 & prg-1 & -1.29 & cdk-1 & -1.22 \\
\hline sodh-1 & -1.39 & T25B9.9 & -1.34 & pigv-1 & -1.28 & acbp-3 & -1.22 \\
\hline ucr-2.2 & -1.39 & pch-2 & -1.34 & F40A3.6 & -1.28 & pfn-3 & -1.22 \\
\hline gst-36 & -1.39 & Ist-1 & -1.34 & T19D12.4 & -1.28 & mab-20 & -1.22 \\
\hline dut-1 & -1.39 & enol-1 & -1.33 & metr-1 & -1.28 & dnj-11 & -1.22 \\
\hline ppm-1.G & -1.39 & pam-1 & -1.33 & F11A5.9 & -1.28 & gsy-1 & -1.22 \\
\hline nlp-29 & -1.38 & T04F3.1 & -1.33 & orc-1 & -1.28 & lagr-1 & -1.21 \\
\hline LLC1.2 & -1.38 & $\operatorname{tfg}-1$ & -1.32 & aco-2 & -1.28 & $\mathrm{rsp}-5$ & -1.21 \\
\hline rmd-1 & -1.38 & dpy-2 & -1.32 & tag-307 & -1.27 & tsfm-1 & -1.21 \\
\hline Y60C6A.2 & -1.38 & pges-2 & -1.32 & F20D6.11 & -1.27 & flh-1 & -1.21 \\
\hline ZK809.8 & -1.38 & wago-1 & -1.32 & H10E21.4 & -1.27 & col-130 & -1.2 \\
\hline acs-2 & -1.37 & F42A8.1 & -1.32 & F59B10.3 & -1.27 & smc-3 & -1.2 \\
\hline far-2 & -1.37 & mev-1 & -1.32 & sdha-1 & -1.27 & let-2 & -1.2 \\
\hline swt-6 & -1.37 & bub-1 & -1.32 & B0272.3 & -1.27 & F58E6.13 & -1.19 \\
\hline$n \times f-2$ & -1.37 & Y71G12B.6 & -1.32 & cysl-1 & -1.27 & atp-5 & -1.19 \\
\hline C15C7.5 & -1.37 & clec-266 & -1.32 & rsa-2 & -1.27 & ech-8 & -1.19 \\
\hline clec-91 & -1.37 & grl-7 & -1.32 & Y67A10A.9 & -1.27 & F58A6.1 & -1.19 \\
\hline top-2 & -1.37 & vha-11 & -1.32 & C17C3.1 & -1.27 & dnj-27 & -1.19 \\
\hline cri-3 & -1.36 & K06G5.1 & -1.31 & vha-8 & -1.27 & perm-5 & -1.17 \\
\hline cnc-10 & -1.36 & tbb-1 & -1.31 & ZC513.7 & -1.27 & Y63D3A.7 & -1.17 \\
\hline vha-13 & -1.36 & clec-5 & -1.31 & T19H12.2 & -1.26 & pck-1 & -1.12 \\
\hline $\mathrm{mdf}-2$ & -1.36 & $\mathrm{mcm}-4$ & -1.31 & pqn-52 & -1.26 & vdac-1 & -1.12 \\
\hline cpn-1 & -1.36 & wrt-10 & -1.31 & col-125 & -1.25 & acer-1 & -1.11 \\
\hline $\operatorname{ttr}-6$ & -1.36 & taco-1 & -1.31 & ZK1058.9 & -1.25 & R04F11.2 & -1.1 \\
\hline Y57G11A.4 & -1.36 & tni-3 & -1.31 & Y52B11A.8 & -1.25 & act-4 & -1.01 \\
\hline nex-1 & -1.36 & xpo-2 & -1.3 & W02F12.2 & -1.25 & rpl-16 & 1 \\
\hline kbp-5 & -1.35 & rsp-4 & -1.3 & Y71G12B.10 & -1.25 & tatn-1 & 1.01 \\
\hline col-74 & -1.35 & unc-95 & -1.3 & cee-1 & -1.25 & egl-21 & 1.01 \\
\hline pgl-1 & -1.35 & Y56АЗА.19 & -1.3 & T20D3.2 & -1.24 & eri-12 & 1.01 \\
\hline
\end{tabular}




\begin{tabular}{|c|c|c|c|c|c|c|c|}
\hline Gene & $\log 2 \mathrm{FC}$ & Gene & $\log 2 \mathrm{FC}$ & Gene & $\log 2 \mathrm{FC}$ & Gene & $\log 2 \mathrm{FC}$ \\
\hline F27C1.2 & 1.02 & pho-4 & 1.13 & F08F8.9 & 1.22 & akap-1 & 1.27 \\
\hline osm-11 & 1.03 & lact-2 & 1.14 & Y47G6A.7 & 1.22 & C16C10.2 & 1.27 \\
\hline pdcd-2 & 1.03 & pah-1 & 1.14 & R06F6.14 & 1.22 & Y59C2A.1 & 1.28 \\
\hline xpo-1 & 1.04 & $\mathrm{R} 03 \mathrm{H} 10.6$ & 1.14 & ZC395.5 & 1.22 & ZK1127.5 & 1.28 \\
\hline aldo-2 & 1.04 & flp-16 & 1.14 & nlp-11 & 1.22 & cct-3 & 1.28 \\
\hline pqn-48 & 1.05 & nlp-8 & 1.14 & klf-1 & 1.23 & msrp-7 & 1.28 \\
\hline F58D5.5 & 1.05 & F52G3.6 & 1.15 & pfd-5 & 1.23 & Y39E4B.6 & 1.28 \\
\hline hsp-90 & 1.07 & tag-209 & 1.15 & $\mathrm{~F} 26 \mathrm{H} 9.5$ & 1.23 & mrps-11 & 1.28 \\
\hline cct-7 & 1.07 & exc-14 & 1.15 & T07F12.1 & 1.23 & M01E5.3 & 1.29 \\
\hline cct-5 & 1.07 & $\operatorname{lgg}-2$ & 1.15 & $m t x-2$ & 1.23 & C01C10.2 & 1.29 \\
\hline byn-1 & 1.08 & nhr-10 & 1.16 & bath-43 & 1.23 & F13G3.6 & 1.29 \\
\hline K12B6.9 & 1.08 & hlh-11 & 1.16 & Y66D12A.8 & 1.23 & F56F10.2 & 1.29 \\
\hline egl-3 & 1.08 & nhr-87 & 1.16 & K07D4.9 & 1.23 & Y54F10AR.2 & 1.29 \\
\hline flp-15 & 1.08 & abhd-14 & 1.17 & F41E7.7 & 1.23 & F15D4.2 & 1.29 \\
\hline flp-14 & 1.08 & smg-3 & 1.17 & dct-1 & 1.24 & Y22D7AR.6 & 1.29 \\
\hline hgo-1 & 1.08 & cdr-4 & 1.17 & ace-2 & 1.24 & F22D6.2 & 1.29 \\
\hline $\mathrm{T} 05 \mathrm{H} 4.10$ & 1.08 & nxf-1 & 1.17 & T28F3.8 & 1.24 & gstk-2 & 1.29 \\
\hline ascc-1 & 1.09 & rpia-1 & 1.17 & fat-2 & 1.24 & $\mathrm{C} 03 \mathrm{H} 5.3$ & 1.29 \\
\hline kin-10 & 1.09 & skr-19 & 1.18 & unc- 80 & 1.24 & $\mathrm{~F} 17 \mathrm{H} 10.2$ & 1.29 \\
\hline Y54E5A.5 & 1.09 & nlp-17 & 1.18 & efk-1 & 1.24 & rpl-24.2 & 1.29 \\
\hline Y45G5AM.7 & 1.1 & F22F4.4 & 1.18 & spp-18 & 1.25 & C38C3.4 & 1.3 \\
\hline hars-1 & 1.1 & nhr-138 & 1.18 & $\mathrm{fbxl}-1$ & 1.25 & B0432.14 & 1.3 \\
\hline adk-1 & 1.1 & T21F4.1 & 1.18 & Y49F6B.2 & 1.25 & cct-4 & 1.3 \\
\hline eef-2 & 1.1 & gpx-3 & 1.19 & Y37E11B.5 & 1.25 & К03B4.1 & 1.3 \\
\hline ubc-3 & 1.1 & ptr-24 & 1.19 & nhr-55 & 1.25 & sca-1 & 1.3 \\
\hline T28B11.1 & 1.1 & F02E11.2 & 1.19 & C35C5.9 & 1.26 & dao-6 & 1.3 \\
\hline $\mathrm{rpb}-9$ & 1.11 & rab-21 & 1.19 & sma-10 & 1.26 & bath-38 & 1.3 \\
\hline Ipin-1 & 1.11 & nnt-1 & 1.19 & W07E6.2 & 1.26 & Y73E7A.1 & 1.31 \\
\hline rla-0 & 1.11 & nhr-90 & 1.19 & F18C5.10 & 1.26 & Y105E8B.7 & 1.31 \\
\hline$n c x-2$ & 1.12 & T26H5.9 & 1.19 & M03D4.3 & 1.26 & E02A10.3 & 1.31 \\
\hline ptl-1 & 1.12 & F54D11.4 & 1.19 & ist-1 & 1.26 & $\mathrm{nhl}-2$ & 1.31 \\
\hline asna-1 & 1.12 & R119.5 & 1.19 & ads-1 & 1.26 & $\mathrm{ikb}-1$ & 1.31 \\
\hline eef-1A.1 & 1.13 & gst-12 & 1.2 & Y50D4A.1 & 1.26 & W02D3.12 & 1.31 \\
\hline ptp-1 & 1.13 & nhr-101 & 1.2 & ZK792.5 & 1.26 & $\mathrm{mtl}-2$ & 1.32 \\
\hline ril-2 & 1.13 & T23G7.3 & 1.2 & Y43F8C.3 & 1.27 & ZK1098.11 & 1.32 \\
\hline F59C6.5 & 1.13 & lite-1 & 1.21 & Y47A7.2 & 1.27 & Y39B6A.37 & 1.32 \\
\hline $\mathrm{mfn}-1$ & 1.13 & ipla-7 & 1.21 & F13D11.4 & 1.27 & C01C4.3 & 1.32 \\
\hline F43G6.8 & 1.13 & slc-25A29 & 1.21 & $\mathrm{nlp}-53$ & 1.27 & Y39B6A.3 & 1.32 \\
\hline Y54F10AM.8 & 1.13 & Y42A5A.1 & 1.21 & nlp-43 & 1.27 & drh-2 & 1.32 \\
\hline sma-9 & 1.13 & icd-2 & 1.21 & nhr-100 & 1.27 & F54E7.9 & 1.32 \\
\hline ida-1 & 1.13 & $d d x-52$ & 1.22 & ZC8.6 & 1.27 & mlc-2 & 1.32 \\
\hline
\end{tabular}




\begin{tabular}{|c|c|c|c|c|c|c|c|}
\hline Gene & $\log 2 \mathrm{FC}$ & Gene & $\log 2 \mathrm{FC}$ & Gene & $\log 2 \mathrm{FC}$ & Gene & $\log 2 \mathrm{FC}$ \\
\hline C50F4.1 & 1.32 & K02E11.5 & 1.38 & iff-2 & 1.43 & ZK1240.9 & 1.48 \\
\hline F43C9.2 & 1.33 & frm-7 & 1.38 & cyn-2 & 1.43 & T09E11.11 & 1.48 \\
\hline mboa-1 & 1.33 & kin-14 & 1.38 & R02E4.2 & 1.43 & H34I24.2 & 1.48 \\
\hline F17C11.4 & 1.33 & C35B8.3 & 1.38 & alh-12 & 1.43 & R11G10.3 & 1.49 \\
\hline M176.11 & 1.34 & ccdc-149 & 1.38 & $\mathrm{C} 28 \mathrm{H} 8.2$ & 1.43 & del-5 & 1.49 \\
\hline F58H1.7 & 1.34 & K07G5.5 & 1.38 & F59B2.13 & 1.43 & asm-2 & 1.49 \\
\hline Y40B1B.7 & 1.34 & $d d x-17$ & 1.39 & irg-3 & 1.44 & K01A2.10 & 1.49 \\
\hline fkh-9 & 1.34 & duxl-1 & 1.39 & T08B1.4 & 1.44 & T26H5.8 & 1.49 \\
\hline ttr-27 & 1.34 & lipl-5 & 1.39 & F35B12.10 & 1.44 & F23A7.3 & 1.5 \\
\hline ZK858.5 & 1.34 & F13H8.1 & 1.39 & endu-2 & 1.44 & his-60 & 1.5 \\
\hline vps-33.1 & 1.34 & F36H5.10 & 1.4 & cgr-1 & 1.44 & nrf-5 & 1.5 \\
\hline droe-4 & 1.34 & F35D11.3 & 1.4 & btb-4 & 1.44 & $\mathrm{C} 33 \mathrm{H} 5.13$ & 1.5 \\
\hline pde-4 & 1.34 & C08G9.1 & 1.4 & pin-2 & 1.44 & F35B12.3 & 1.5 \\
\hline rrp-1 & 1.35 & C49F5.7 & 1.4 & F09C6.3 & 1.44 & marg-1 & 1.5 \\
\hline Y66H1A.5 & 1.35 & mop-25.1 & 1.4 & eif-3.E & 1.44 & clec- 8 & 1.5 \\
\hline zig- 6 & 1.35 & MTCE.33 & 1.4 & VC5.2 & 1.44 & F41E7.2 & 1.5 \\
\hline ubc- 8 & 1.35 & Y71H2B.11 & 1.41 & C17G1.5 & 1.45 & C25G4.2 & 1.5 \\
\hline D2023.1 & 1.35 & F44A2.3 & 1.41 & nhr-210 & 1.45 & B0507.3 & 1.5 \\
\hline lin-28 & 1.35 & Y67D2.5 & 1.41 & ZK131.11 & 1.45 & M01F1.3 & 1.51 \\
\hline twk-8 & 1.35 & CD4.1 & 1.41 & asah-1 & 1.45 & nhr-110 & 1.51 \\
\hline asp-5 & 1.35 & ZK1128.7 & 1.41 & daf-2 & 1.46 & cdd-1 & 1.51 \\
\hline T08G11.4 & 1.35 & Y41E3.22 & 1.41 & flp-9 & 1.46 & Y49E10.16 & 1.51 \\
\hline M176.4 & 1.35 & Y34F4.5 & 1.41 & C07H4.1 & 1.46 & imb-3 & 1.51 \\
\hline F43C1.7 & 1.35 & haly-1 & 1.41 & crt-1 & 1.46 & ZC47.8 & 1.51 \\
\hline R10H1.1 & 1.36 & C30G4.4 & 1.42 & zig-9 & 1.46 & fbxa-138 & 1.51 \\
\hline T25B9.1 & 1.36 & K04F10.1 & 1.42 & hil-1 & 1.46 & Y51H4A.7 & 1.51 \\
\hline T07D4.2 & 1.36 & lido-13 & 1.42 & prmt-1 & 1.46 & nhr-85 & 1.51 \\
\hline nlp-15 & 1.36 & lin-14 & 1.42 & nuaf-1 & 1.46 & F26F12.3 & 1.52 \\
\hline eif-3.G & 1.36 & gst-22 & 1.42 & ctn-1 & 1.46 & riok-3 & 1.52 \\
\hline $\mathrm{C} 06 \mathrm{H} 5.8$ & 1.36 & far- 6 & 1.42 & fubl-1 & 1.47 & C54F6.6 & 1.52 \\
\hline gei-3 & 1.36 & mig-18 & 1.42 & F09G2.2 & 1.47 & C02F5.3 & 1.52 \\
\hline far-7 & 1.37 & H10D18.1 & 1.42 & C10E2.2 & 1.47 & F07E5.5 & 1.53 \\
\hline $\operatorname{tax}-6$ & 1.37 & clec-75 & 1.42 & mak-2 & 1.47 & him-4 & 1.53 \\
\hline K09E2.2 & 1.37 & T13F2.6 & 1.42 & pho-5 & 1.47 & T26H5.14 & 1.53 \\
\hline Y61A9LA.10 & 1.37 & Y73F4A.3 & 1.42 & timm-23 & 1.47 & pgp-9 & 1.53 \\
\hline cdc-48.3 & 1.37 & eif-3.I & 1.43 & W09C5.1 & 1.47 & elpc-4 & 1.53 \\
\hline pno-1 & 1.37 & aatf-1 & 1.43 & $\mathrm{C} 28 \mathrm{H} 8.3$ & 1.47 & tag-151 & 1.53 \\
\hline srw-85 & 1.37 & C25G6.4 & 1.43 & Y73В3А.18 & 1.48 & ZC443.3 & 1.53 \\
\hline M01H9.4 & 1.37 & uev-3 & 1.43 & C33G8.4 & 1.48 & Y106G6E.4 & 1.54 \\
\hline $\mathrm{cpz}-2$ & 1.37 & ztf-7 & 1.43 & psa-3 & 1.48 & adt-3 & 1.54 \\
\hline K11E4.1 & 1.37 & nhr-35 & 1.43 & exos-9 & 1.48 & ins-20 & 1.54 \\
\hline
\end{tabular}




\begin{tabular}{|c|c|c|c|c|c|c|c|}
\hline Gene & $\log 2 \mathrm{FC}$ & Gene & $\log 2 \mathrm{FC}$ & Gene & $\log 2 \mathrm{FC}$ & Gene & $\log 2 \mathrm{FC}$ \\
\hline $\mathrm{CO3H} 5.7$ & 1.54 & $n c x-7$ & 1.58 & M163.1 & 1.63 & nhl-1 & 1.68 \\
\hline nhr-146 & 1.54 & R06C1.6 & 1.58 & moc-2 & 1.63 & Y71H2AM.1 & 1.69 \\
\hline K07F5.15 & 1.54 & $\mathrm{fbxa}-44$ & 1.58 & scl-24 & 1.63 & F32H5.1 & 1.69 \\
\hline oig-3 & 1.54 & ZC404.1 & 1.58 & R07C12.2 & 1.63 & nhr-219 & 1.69 \\
\hline $\mathrm{scl}-2$ & 1.54 & W06E11.1 & 1.58 & pals-38 & 1.63 & chil-23 & 1.69 \\
\hline F53F4.14 & 1.54 & C49A9.6 & 1.58 & Y71G12B.31 & 1.64 & dohh-1 & 1.69 \\
\hline F33D11.16 & 1.54 & M116.2 & 1.59 & col-184 & 1.64 & D1022.4 & 1.69 \\
\hline nape-1 & 1.55 & gpa-6 & 1.59 & pbo-6 & 1.64 & C33D9.3 & 1.69 \\
\hline gtl-1 & 1.55 & T23D8.3 & 1.59 & gсу-11 & 1.64 & gdh-1 & 1.69 \\
\hline F22D6.14 & 1.55 & F14D2.19 & 1.59 & Y57G11B.2 & 1.64 & tpra-1 & 1.69 \\
\hline F45E10.2 & 1.55 & F26D11.12 & 1.59 & brp-1 & 1.64 & sek-1 & 1.69 \\
\hline bath-47 & 1.55 & F26A3.1 & 1.59 & del-9 & 1.65 & dph-1 & 1.7 \\
\hline coel-1 & 1.55 & rsd-3 & 1.59 & fnip-2 & 1.65 & tos- -1 & 1.7 \\
\hline fbxc-57 & 1.55 & nhr-119 & 1.59 & $\mathrm{C} 05 \mathrm{C} 10.7$ & 1.65 & srb-16 & 1.7 \\
\hline C10B5.3 & 1.56 & nhr-232 & 1.59 & abf-5 & 1.65 & F40F12.9 & 1.7 \\
\hline col-156 & 1.56 & gst-3 & 1.59 & fbxa-140 & 1.65 & F31E3.6 & 1.7 \\
\hline C54D10.10 & 1.56 & $\ln p-1$ & 1.6 & $\mathrm{fbxa}-115$ & 1.65 & R11D1.13 & 1.7 \\
\hline F56C4.4 & 1.56 & riok-2 & 1.6 & rars-1 & 1.65 & Y32H12A.8 & 1.7 \\
\hline adss-1 & 1.56 & сур-33С3 & 1.6 & zip-12 & 1.65 & Y39B6А.29 & 1.7 \\
\hline mtq-2 & 1.56 & nhr-5 & 1.6 & K10G4.3 & 1.65 & F40G12.5 & 1.7 \\
\hline F19C6.12 & 1.56 & D2096.7 & 1.6 & F20D6.5 & 1.66 & $\operatorname{ttr}-40$ & 1.7 \\
\hline Y6G8.2 & 1.56 & F58G6.7 & 1.6 & dhp-1 & 1.66 & fbxa-50 & 1.7 \\
\hline kin-3 & 1.56 & K02A11.4 & 1.6 & cat-4 & 1.66 & nmad-1 & 1.7 \\
\hline scl-5 & 1.56 & F59A7.5 & 1.61 & slcf-2 & 1.66 & M04D8.7 & 1.71 \\
\hline B0310.3 & 1.56 & sir-2.4 & 1.61 & Y43C5A.3 & 1.66 & T20F7.5 & 1.71 \\
\hline $\mathrm{fbxa}-203$ & 1.56 & C04A2.15 & 1.61 & mrps-17 & 1.66 & T01C8.3 & 1.71 \\
\hline ttr-34 & 1.57 & R09F10.1 & 1.61 & fbxa-10 & 1.66 & F55H12.7 & 1.72 \\
\hline math-27 & 1.57 & hpo-11 & 1.61 & asp-3 & 1.67 & Y56A3A.18 & 1.72 \\
\hline $\mathrm{ccm}-2$ & 1.57 & F36F2.2 & 1.61 & Y95B8A.6 & 1.67 & T04C12.7 & 1.72 \\
\hline F27D4.3 & 1.57 & rrp-8 & 1.61 & rrbs-1 & 1.67 & ins-35 & 1.73 \\
\hline T06E6.1 & 1.57 & Y51H4A.15 & 1.61 & Ipr-2 & 1.67 & ZK512.2 & 1.73 \\
\hline Y116A8A.10 & 1.57 & R02F2.1 & 1.61 & T09F5.12 & 1.68 & $\mathrm{C} 18 \mathrm{H} 7.1$ & 1.73 \\
\hline nst-1 & 1.57 & fbxc-41 & 1.61 & alh-8 & 1.68 & F10E7.5 & 1.73 \\
\hline C18A11.1 & 1.57 & srbc-20 & 1.61 & unc-83 & 1.68 & mltn-1 & 1.73 \\
\hline F46A8.13 & 1.57 & H35В03.1 & 1.61 & C36E8.1 & 1.68 & catp-7 & 1.74 \\
\hline Y7A9C.1 & 1.57 & aakb-2 & 1.61 & nhr-133 & 1.68 & T20D3.6 & 1.74 \\
\hline D1054.3 & 1.57 & Y113G7A.13 & 1.62 & C47E8.11 & 1.68 & T10G3.1 & 1.74 \\
\hline ZK180.8 & 1.57 & D2045.8 & 1.62 & Y92H12BL.1 & 1.68 & K02D10.8 & 1.74 \\
\hline capa-1 & 1.58 & btb-17 & 1.63 & сур-33C2 & 1.68 & F53A2.9 & 1.74 \\
\hline clec- 62 & 1.58 & F11E6.11 & 1.63 & $\mathrm{C} 25 \mathrm{H} 3.11$ & 1.68 & rnt-1 & 1.74 \\
\hline R12E2.13 & 1.58 & F02A9.7 & 1.63 & F08H9.3 & 1.68 & nspc-18 & 1.74 \\
\hline
\end{tabular}




\begin{tabular}{|c|c|c|c|c|c|c|c|}
\hline Gene & $\log 2 \mathrm{FC}$ & Gene & $\log 2 \mathrm{FC}$ & Gene & $\log 2 \mathrm{FC}$ & Gene & $\log 2 \mathrm{FC}$ \\
\hline K09C8.7 & 1.75 & nlp-47 & 1.82 & gsp-2 & 1.94 & C16D9.9 & 2.04 \\
\hline T01G1.4 & 1.75 & aak-1 & 1.83 & fbxa-6 & 1.94 & Y82E9BR.22 & 2.04 \\
\hline C52E2.4 & 1.75 & F55A4.4 & 1.83 & F49C12.6 & 1.94 & T01B7.5 & 2.05 \\
\hline ubc-1 & 1.75 & R09E10.13 & 1.83 & gst-23 & 1.94 & C27F2.4 & 2.05 \\
\hline nspc-19 & 1.75 & $\mathrm{nlp}-7$ & 1.84 & ZK1307.1 & 1.94 & T22H9.1 & 2.05 \\
\hline pstk-1 & 1.75 & Y105C5A.17 & 1.85 & frk-1 & 1.94 & nhr-109 & 2.06 \\
\hline smf-3 & 1.75 & cps- 6 & 1.85 & C29F5.3 & 1.94 & nspc-20 & 2.06 \\
\hline F41G4.8 & 1.76 & C47D12.5 & 1.85 & srh-210 & 1.94 & C53C11.2 & 2.06 \\
\hline $\mathrm{cpz}-1$ & 1.76 & F48B9.1 & 1.85 & paqr-1 & 1.95 & ZC443.4 & 2.06 \\
\hline C49C8.8 & 1.76 & ech-7 & 1.86 & F49H6.5 & 1.95 & W07G4.5 & 2.06 \\
\hline Y42G9A.1 & 1.77 & C16A3.6 & 1.86 & ZK430.7 & 1.95 & C14A11.2 & 2.06 \\
\hline Y75B7AL.2 & 1.77 & asp-6 & 1.86 & nlp-59 & 1.96 & lep-5 & 2.06 \\
\hline ptr-13 & 1.77 & twk-7 & 1.86 & lec-2 & 1.96 & ZK1240.2 & 2.07 \\
\hline W03D8.8 & 1.78 & gрср-1 & 1.86 & gpa-8 & 1.97 & mrpl-55 & 2.07 \\
\hline T10B5.3 & 1.78 & nhr-221 & 1.87 & rpr-1 & 1.97 & fbxa-163 & 2.07 \\
\hline memb-2 & 1.78 & ZC239.16 & 1.87 & E02H9.2 & 1.98 & ins-29 & 2.07 \\
\hline B0478.3 & 1.79 & C47D2.1 & 1.87 & $\mathrm{R} 01 \mathrm{H} 10.4$ & 1.98 & nhr-103 & 2.07 \\
\hline exos-7 & 1.79 & F53F4.11 & 1.88 & F44G4.1 & 1.99 & tre-5 & 2.08 \\
\hline srg-34 & 1.79 & flp-20 & 1.88 & pals-31 & 1.99 & Y53C12B.7 & 2.08 \\
\hline nap-1 & 1.79 & F21H7.2 & 1.88 & C08F11.13 & 1.99 & T12D8.5 & 2.08 \\
\hline wrb-1 & 1.79 & C15B12.4 & 1.88 & clec-65 & 1.99 & ulp-4 & 2.09 \\
\hline yap-1 & 1.79 & F02C12.3 & 1.88 & btb-16 & 1.99 & lgc-28 & 2.09 \\
\hline rncs-1 & 1.8 & K02E7.11 & 1.89 & F43D2.7 & 2 & math-37 & 2.09 \\
\hline C38D4.7 & 1.8 & nog-1 & 1.89 & B0511.6 & 2 & riok-1 & 2.09 \\
\hline K09E4.4 & 1.8 & ulp-3 & 1.9 & upb-1 & 2 & obr-2 & 2.1 \\
\hline $\mathrm{C} 25 \mathrm{H} 3.18$ & 1.8 & T28A11.2 & 1.9 & $\mathrm{JC} 8.2$ & 2 & C28G1.6 & 2.1 \\
\hline nape-2 & 1.8 & eif-3.B & 1.9 & F30A10.9 & 2 & $\mathrm{C} 40 \mathrm{H} 1.9$ & 2.1 \\
\hline F11C7.2 & 1.81 & nhr-94 & 1.91 & ZK355.8 & 2 & adah-1 & 2.11 \\
\hline gcy-15 & 1.81 & C23F12.4 & 1.91 & ZK971.1 & 2 & W02D7.11 & 2.11 \\
\hline C27B7.9 & 1.81 & moe-3 & 1.91 & C03G6.17 & 2.01 & puf-12 & 2.11 \\
\hline peb-1 & 1.81 & R10E8.6 & 1.91 & fbxa-162 & 2.02 & К09H9.5 & 2.11 \\
\hline elpc-3 & 1.81 & F37A8.5 & 1.91 & F55C5.2 & 2.02 & B0563.5 & 2.11 \\
\hline fbxa-3 & 1.81 & acl-9 & 1.91 & $\mathrm{C} 24 \mathrm{H} 12.4$ & 2.02 & Y54H5A.1 & 2.11 \\
\hline T19С3.6 & 1.81 & gst-20 & 1.92 & nhr-80 & 2.02 & E02H1.1 & 2.11 \\
\hline F40F8.5 & 1.81 & K02C4.5 & 1.92 & siah-1 & 2.02 & $\mathrm{mfb}-1$ & 2.12 \\
\hline K09F6.10 & 1.81 & K02A2.5 & 1.92 & ZK1290.13 & 2.02 & odr-10 & 2.13 \\
\hline ZK822.4 & 1.82 & clec-186 & 1.92 & hpo-15 & 2.02 & С53H9.2 & 2.14 \\
\hline Y47G6A.5 & 1.82 & B0564.2 & 1.93 & nas-9 & 2.03 & $\mathrm{H} 06 \mathrm{H} 21.8$ & 2.14 \\
\hline R02F11.2 & 1.82 & rab-8 & 1.93 & cpr-6 & 2.03 & C34E11.4 & 2.14 \\
\hline fbxa-155 & 1.82 & clec-146 & 1.93 & Ipd-7 & 2.03 & F21A3.11 & 2.14 \\
\hline C39D10.11 & 1.82 & linc-39 & 1.94 & sma-2 & 2.04 & linc-96 & 2.14 \\
\hline
\end{tabular}




\begin{tabular}{|c|c|c|c|c|c|c|c|}
\hline Gene & $\log 2 \mathrm{FC}$ & Gene & $\log 2 \mathrm{FC}$ & Gene & $\log 2 \mathrm{FC}$ & Gene & $\log 2 \mathrm{FC}$ \\
\hline C10A4.3 & 2.15 & Y34F4.2 & 2.27 & B0524.6 & 2.4 & ZC239.17 & 2.61 \\
\hline R09B5.11 & 2.15 & W06H8.6 & 2.27 & F54D5.3 & 2.4 & AH9.7 & 2.61 \\
\hline T22F3.2 & 2.16 & F10G7.5 & 2.28 & nhr-129 & 2.4 & Y32B12C.5 & 2.61 \\
\hline F57C12.6 & 2.16 & lido-10 & 2.28 & tag-243 & 2.4 & chil-16 & 2.65 \\
\hline clec- 85 & 2.16 & Y54G2A.41 & 2.28 & Y65B4BL.7 & 2.4 & Y37H9A.5 & 2.65 \\
\hline ZK550.2 & 2.16 & T25G3.3 & 2.29 & $\mathrm{~T} 28 \mathrm{H} 10.1$ & 2.41 & Y52B11A.10 & 2.66 \\
\hline T18D3.6 & 2.17 & ZK688.11 & 2.29 & srp-2 & 2.41 & F47H4.12 & 2.66 \\
\hline fbxa-69 & 2.17 & D1054.18 & 2.29 & gst-37 & 2.42 & flp-10 & 2.67 \\
\hline F36A2.2 & 2.17 & $\mathrm{ftn}-1$ & 2.29 & $\operatorname{ttr}-49$ & 2.42 & Y56A3A.33 & 2.68 \\
\hline arch-1 & 2.17 & dph-3 & 2.29 & C05E11.6 & 2.42 & cup-16 & 2.69 \\
\hline acp-5 & 2.17 & F08F3.4 & 2.29 & Y39B6A.33 & 2.44 & lips-1 & 2.72 \\
\hline$f b x a-80$ & 2.17 & F09C6.1 & 2.29 & Y54G2A.10 & 2.45 & F41G4.7 & 2.73 \\
\hline pudl-1 & 2.18 & T02G5.14 & 2.29 & ttr-29 & 2.45 & pud-4 & 2.75 \\
\hline F48E8.3 & 2.18 & C16C10.8 & 2.3 & clec-84 & 2.45 & T04C12.32 & 2.76 \\
\hline Y57G11C.1135 & 2.19 & Y7A5A.6 & 2.3 & F08F8.7 & 2.46 & tut-1 & 2.79 \\
\hline nhr-18 & 2.19 & K11D12.12 & 2.3 & ctps-1 & 2.46 & C25F9.12 & 2.81 \\
\hline Y40C5A.4 & 2.2 & F47B8.4 & 2.3 & nhr-88 & 2.47 & col-95 & 2.82 \\
\hline C15C7.4 & 2.2 & C45E5.4 & 2.31 & $\mathrm{~F} 26 \mathrm{G} 1.10$ & 2.47 & Y15E3A.4 & 2.82 \\
\hline T08B6.2 & 2.21 & W03D8.2 & 2.31 & M60.7 & 2.47 & pudl-2 & 2.83 \\
\hline Y71G10AR.4 & 2.21 & linc-43 & 2.32 & metl-18 & 2.47 & pqn-59 & 2.86 \\
\hline F29C6.1 & 2.22 & B0546.3 & 2.33 & F52E1.14 & 2.48 & F18G5.6 & 2.87 \\
\hline $\mathrm{C} 32 \mathrm{H} 11.4$ & 2.22 & mnk-1 & 2.34 & Y97E10AL.1 & 2.48 & сур-13A3 & 2.87 \\
\hline Y54H5A.2 & 2.22 & C25F9.2 & 2.34 & T26C11.9 & 2.49 & chil-13 & 2.87 \\
\hline F08G12.11 & 2.23 & T06C12.14 & 2.34 & col-118 & 2.5 & M01B2.13 & 2.88 \\
\hline Y47D3A.32 & 2.23 & srap-1 & 2.35 & faah-3 & 2.5 & twk-5 & 2.88 \\
\hline C53A5.17 & 2.23 & C11D2.3 & 2.35 & linc-103 & 2.5 & spp-8 & 2.9 \\
\hline metl-2 & 2.23 & F58A3.3 & 2.35 & tag-89 & 2.51 & spp-6 & 2.91 \\
\hline fil-1 & 2.24 & col-39 & 2.35 & Y22D7AL.15 & 2.51 & F54F12.1 & 2.91 \\
\hline R03D7.5 & 2.24 & asah-2 & 2.36 & F56A8.5 & 2.52 & nlp-69 & 2.93 \\
\hline fbxa-173 & 2.24 & tts-1 & 2.36 & col-50 & 2.53 & clec-61 & 2.94 \\
\hline F53F1.14 & 2.24 & ngp-1 & 2.36 & C09D4.2 & 2.54 & Y48G8AL.13 & 2.96 \\
\hline T10H9.8 & 2.25 & сур-33С6 & 2.36 & T03G11.3 & 2.54 & nspc-16 & 2.96 \\
\hline txt-3 & 2.25 & fat-6 & 2.37 & Y54E10BR.4 & 2.54 & arrd-11 & 2.97 \\
\hline F53F10.1 & 2.25 & F31E9.3 & 2.37 & $\mathrm{nlp}-2$ & 2.54 & ZK470.14 & 2.98 \\
\hline Y47H10A.3 & 2.25 & C16A3.4 & 2.38 & fard-1 & 2.55 & nlp-52 & 3 \\
\hline сур-37B1 & 2.26 & pqn-62 & 2.38 & T09B4.6 & 2.56 & сур-32B1 & 3 \\
\hline T22B7.3 & 2.26 & $\mathrm{~T} 10 \mathrm{H} 4.13$ & 2.38 & C29G2.2 & 2.58 & W03D2.6 & 3.02 \\
\hline F21C10.11 & 2.26 & F55A4.7 & 2.38 & C24A11.1 & 2.58 & T26H5.4 & 3.03 \\
\hline Y43B11AR.3 & 2.27 & R10E8.1 & 2.39 & Y46G5A.34 & 2.59 & C17G10.6 & 3.06 \\
\hline R07E3.1 & 2.27 & F59A7.8 & 2.39 & exc-13 & 2.6 & F35H8.1 & 3.07 \\
\hline F31B12.3 & 2.27 & pho-6 & 2.4 & T10B5.7 & 2.61 & hda-5 & 3.08 \\
\hline
\end{tabular}




\begin{tabular}{|c|c|}
\hline Gene & log2FC \\
\hline T26E3.4 & 3.1 \\
\hline D1086.2 & 3.11 \\
\hline pmt-1 & 3.15 \\
\hline cest-1.2 & 3.16 \\
\hline F16A11.5 & 3.17 \\
\hline F35C8.5 & 3.17 \\
\hline F36A2.10 & 3.17 \\
\hline F16B4.5 & 3.19 \\
\hline F23F12.3 & 3.19 \\
\hline K05F6.10 & 3.19 \\
\hline hecW-1 & 3.23 \\
\hline nspc-14 & 3.24 \\
\hline C05D9.9 & 3.26 \\
\hline T10C6.15 & 3.28 \\
\hline hrg-2 & 3.31 \\
\hline F43C11.7 & 3.32 \\
\hline C32F10.4 & 3.37 \\
\hline clec-264 & 3.39 \\
\hline C45E1.4 & 3.41 \\
\hline C18H9.5 & 3.42 \\
\hline W10G11.1 & 3.42 \\
\hline T08A9.13 & 3.43 \\
\hline col-181 & 3.47 \\
\hline ncr-2 & 3.48 \\
\hline ZK593.2 & 3.48 \\
\hline F42A9.18 & 3.5 \\
\hline F40E3.5 & 3.59 \\
\hline ZK185.5 & 3.6 \\
\hline B0379.7 & 3.61 \\
\hline F21H7.12 & 3.62 \\
\hline F56F11.1 & 3.68 \\
\hline B0513.4 & 3.79 \\
\hline F47B8.8 & 3.79 \\
\hline F56H9.2 & 3.81 \\
\hline cpr-2 & 3.84 \\
\hline W08E12.8 & 3.89 \\
\hline ins-3 & 4.01 \\
\hline dpy-5 & 4.82 \\
\hline T28A11.16 & 4.86 \\
\hline srv-4 & 5.01 \\
\hline hsp-12.6 & 5.35 \\
\hline
\end{tabular}


6.17. RNA-seq (CeGaT) - 4h Fed vs 48h Starved - HBR1777 (flp-11::EGL-1)

\begin{tabular}{|c|c|c|c|c|c|c|c|}
\hline Gene & $\log 2 \mathrm{FC}$ & Gene & $\log 2 \mathrm{FC}$ & Gene & $\log 2 \mathrm{FC}$ & Gene & $\log 2 \mathrm{FC}$ \\
\hline T22F3.11 & -4.54 & skr-15 & -2.76 & set-22 & -2.56 & mbtr-1 & -2.41 \\
\hline C23G10.11 & -4.08 & gld-3 & -2.75 & sdz-30 & -2.56 & act-5 & -2.41 \\
\hline Ү39B6A.1 & -3.89 & grl-21 & -2.75 & hacd-1 & -2.56 & col-145 & -2.41 \\
\hline W10C8.5 & -3.69 & kreg-1 & -2.74 & nspb-12 & -2.55 & F44E7.5 & -2.41 \\
\hline $\mathrm{R} 02 \mathrm{C} 2.7$ & -3.59 & Isy-27 & -2.74 & asns-1 & -2.55 & R04B5.5 & -2.4 \\
\hline F46F2.3 & -3.59 & Y7A9A.79 & -2.74 & ora-1 & -2.54 & ctf-8 & -2.4 \\
\hline T07D3.9 & -3.5 & F57F4.4 & -2.74 & clec-91 & -2.54 & pcn-1 & -2.4 \\
\hline gst-26 & -3.25 & clec- 88 & -2.74 & Y47D3B.6 & -2.53 & W03C9. 2 & -2.39 \\
\hline W05F2.3 & -3.11 & Ibp-6 & -2.74 & idh-1 & -2.53 & Y73В3А.1 & -2.39 \\
\hline C35C5.8 & -3.08 & T12G3.6 & -2.72 & Ion-1 & -2.53 & tyr-2 & -2.39 \\
\hline C09B8.4 & -3.04 & vet-2 & -2.72 & T21C9.13 & -2.52 & Y73F8A.13 & -2.39 \\
\hline mdh-2 & -3.01 & С44B9.3 & -2.71 & nhr-2 & -2.52 & C14B1.9 & -2.39 \\
\hline spn-4 & -3 & Y71A12B.11 & -2.71 & cyb-3 & -2.51 & best-1 & -2.38 \\
\hline mex-5 & -2.99 & pqn-22 & -2.7 & cdc- 25.3 & -2.51 & $\mathrm{cbd}-1$ & -2.38 \\
\hline aptf-4 & -2.98 & $\mathrm{mcm}-7$ & -2.69 & Y68A4B.3 & -2.5 & ZK1290.10 & -2.38 \\
\hline T05F1.2 & -2.98 & Y39G10AL.1 & -2.69 & $\mathrm{fbxb}-104$ & -2.5 & ZC155.4 & -2.38 \\
\hline C45B2.2 & -2.98 & srh-237 & -2.69 & F35B3.4 & -2.49 & Y42H9B.3 & -2.38 \\
\hline cyb-2.2 & -2.95 & mom-2 & -2.68 & F18A1.7 & -2.49 & abu-8 & -2.38 \\
\hline $\mathrm{C} 05 \mathrm{C} 10.5$ & -2.95 & F22B5.4 & -2.68 & $\mathrm{C} 42 \mathrm{C} 1.8$ & -2.49 & B0393.3 & -2.38 \\
\hline $\mathrm{sdz}-28$ & -2.95 & abu-10 & -2.67 & T04D3.1 & -2.48 & gld-1 & -2.37 \\
\hline R09E12.9 & -2.93 & glp-1 & -2.67 & szy-4 & -2.48 & $\mathrm{mcm}-2$ & -2.37 \\
\hline puf-11 & -2.92 & Y43B11AL.1 & -2.66 & dct-16 & -2.48 & T06E4.14 & -2.37 \\
\hline ceh-49 & -2.92 & ZC308.4 & -2.65 & cnc- 6 & -2.48 & jmjd-3.2 & -2.37 \\
\hline era-1 & -2.91 & C42D4.3 & -2.65 & F54F7.6 & -2.48 & gena-1 & -2.37 \\
\hline vet- 6 & -2.86 & $\mathrm{~F} 14 \mathrm{H} 12.3$ & -2.65 & gna-2 & -2.48 & R04D3.2 & -2.37 \\
\hline trcs-1 & -2.85 & $\mathrm{~F} 54 \mathrm{H} 12.5$ & -2.65 & $\mathrm{fbxa}-215$ & -2.47 & gpd-4 & -2.37 \\
\hline hphd-1 & -2.85 & $s d z-4$ & -2.65 & pho-11 & -2.47 & ctf-4 & -2.36 \\
\hline F17A9.5 & -2.85 & col-166 & -2.64 & cyb-1 & -2.47 & tpxl-1 & -2.36 \\
\hline sun-1 & -2.84 & C35E7.3 & -2.64 & pqn-26 & -2.47 & cey-3 & -2.36 \\
\hline puf-3 & -2.83 & col-113 & -2.62 & $\mathrm{C} 31 \mathrm{H} 1.8$ & -2.46 & cah-4 & -2.36 \\
\hline folt-2 & -2.82 & flh-1 & -2.62 & Y106G6D.2 & -2.45 & F38B2.4 & -2.36 \\
\hline mar-3 & -2.82 & F09E10.1 & -2.61 & dsl-2 & -2.45 & EEED8.3 & -2.36 \\
\hline$f b x b-35$ & -2.8 & gpd-1 & -2.61 & phat-4 & -2.45 & gsnl-1 & -2.36 \\
\hline pos-1 & -2.8 & $\mathrm{mcm}-3$ & -2.61 & T19B10.2 & -2.45 & R11.4 & -2.36 \\
\hline mex-1 & -2.8 & acdh-1 & -2.6 & F14H3.6 & -2.45 & Y110A7A.15 & -2.36 \\
\hline F46C5.1 & -2.79 & clec-87 & -2.58 & col-33 & -2.45 & F28B4.3 & -2.36 \\
\hline W06B4.1 & -2.79 & Y106G6D.1 & -2.58 & nasp-2 & -2.44 & DY3.8 & -2.36 \\
\hline vet-1 & -2.79 & ZK1037.6 & -2.58 & syp- 6 & -2.44 & $\mathrm{C} 23 \mathrm{H} 5.8$ & -2.35 \\
\hline pqn-63 & -2.78 & mct-5 & -2.57 & C01G6.3 & -2.43 & M195.2 & -2.35 \\
\hline F41E6.11 & -2.78 & pqn-74 & -2.56 & C29A12.1 & -2.42 & Y4C6B.1 & -2.35 \\
\hline pie-1 & -2.76 & ptr-2 & -2.56 & flh-3 & -2.42 & fmo-3 & -2.35 \\
\hline cpr-4 & -2.76 & F56C9.6 & -2.56 & W04A8.5 & -2.41 & K11G9.5 & -2.35 \\
\hline
\end{tabular}




\begin{tabular}{|c|c|c|c|c|c|c|c|}
\hline Gene & $\log 2 \mathrm{FC}$ & Gene & $\log 2 \mathrm{FC}$ & Gene & $\log 2 \mathrm{FC}$ & Gene & $\log 2 \mathrm{FC}$ \\
\hline R09F10.8 & -2.35 & grl-4 & -2.26 & rnr-1 & -2.2 & scc-1 & -2.14 \\
\hline pqn-71 & -2.35 & pch-2 & -2.26 & $\mathrm{knl}-1$ & -2.2 & T05B9.1 & -2.14 \\
\hline ZK662.2 & -2.34 & F13E9.14 & -2.26 & hcp-2 & -2.2 & in $x-14$ & -2.14 \\
\hline F23A7.8 & -2.34 & $f b x b-3$ & -2.26 & col-48 & -2.2 & C06E2.5 & -2.14 \\
\hline asns- 2 & -2.34 & $\mathrm{fbxb}-95$ & -2.26 & mut-2 & -2.2 & Y54G9A.5 & -2.13 \\
\hline T11B7.1 & -2.33 & nlp-30 & -2.26 & orc-1 & -2.2 & sld-2 & -2.13 \\
\hline T05D4.2 & -2.33 & Y22D7AL.10 & -2.25 & pole-1 & -2.2 & T14B4.5 & -2.13 \\
\hline ZK829.9 & -2.33 & col-97 & -2.25 & zen-4 & -2.19 & D2096.6 & -2.13 \\
\hline pash-1 & -2.33 & Y38E10A.28 & -2.25 & ify-1 & -2.19 & C55B7.11 & -2.13 \\
\hline $\mathrm{F} 22 \mathrm{H} 10.2$ & -2.33 & K01A6.4 & -2.25 & tost-1 & -2.19 & W08E12.3 & -2.13 \\
\hline toca-2 & -2.32 & T04F8.8 & -2.25 & K07A1.17 & -2.19 & W08E12.6 & -2.13 \\
\hline C50F7.5 & -2.32 & sdz-14 & -2.25 & sdz-24 & -2.19 & T25E12.6 & -2.12 \\
\hline F14D7.2 & -2.32 & T05H10.4 & -2.25 & Y47H9C.1 & -2.19 & btb-11 & -2.12 \\
\hline nlp-34 & -2.32 & fzy-1 & -2.25 & chaf-2 & -2.19 & Y47D3A.1 & -2.12 \\
\hline egg-3 & -2.32 & K03H1.7 & -2.24 & F11E6.7 & -2.19 & abu-14 & -2.12 \\
\hline Y39B6A.10 & -2.32 & dod-19 & -2.24 & R11A5.3 & -2.19 & acs-2 & -2.11 \\
\hline K04G2.10 & -2.32 & Y14H12B.2 & -2.24 & lec- 6 & -2.18 & F56F4.8 & -2.11 \\
\hline egg-6 & -2.32 & $\mathrm{C} 23 \mathrm{H} 4.8$ & -2.24 & C01G8.1 & -2.18 & $\mathrm{hmg}-3$ & -2.11 \\
\hline oma-2 & -2.31 & hlh-3 & -2.23 & skr-7 & -2.17 & F32D1.7 & -2.11 \\
\hline puf-5 & -2.31 & T20B6.3 & -2.23 & fbp-1 & -2.17 & $\mathrm{mcm}-4$ & -2.11 \\
\hline sas- 6 & -2.31 & skr-14 & -2.23 & act-3 & -2.17 & C27A2.5 & -2.11 \\
\hline Т19H12.2 & -2.3 & W03F9.4 & -2.23 & F08F8.4 & -2.17 & pqn-60 & -2.1 \\
\hline Y110A2AR.1 & -2.3 & F19B10.10 & -2.23 & cnc-4 & -2.17 & ZK1320.9 & -2.1 \\
\hline F59A7.2 & -2.3 & fbxc-18 & -2.23 & T16G12.8 & -2.17 & $\mathrm{~F} 16 \mathrm{H} 11.1$ & -2.1 \\
\hline cdc-7 & -2.3 & tofu-7 & -2.23 & $\mathrm{C} 31 \mathrm{H} 2.4$ & -2.17 & hpo-6 & -2.1 \\
\hline capg-2 & -2.3 & ect-2 & -2.22 & F13A7.14 & -2.16 & Y49F6B.12 & -2.09 \\
\hline wee-1.3 & -2.3 & F56C9.3 & -2.22 & F58G6.3 & -2.16 & col-109 & -2.09 \\
\hline nlp-31 & -2.29 & $\mathrm{fbxb}-72$ & -2.22 & EGAP4.1 & -2.16 & F54D5.2 & -2.09 \\
\hline gyg-2 & -2.29 & Y51F10.2 & -2.22 & air-1 & -2.15 & pgph-2 & -2.09 \\
\hline grd-13 & -2.29 & brd-1 & -2.22 & C48B4.9 & -2.15 & cdk-1 & -2.09 \\
\hline T03F6.3 & -2.29 & pid-4 & -2.22 & ZK896.4 & -2.15 & in $x-2$ & -2.09 \\
\hline mis-12 & -2.28 & ttr-31 & -2.22 & ttr-7 & -2.15 & irg-4 & -2.09 \\
\hline R12A1.3 & -2.28 & ZK6.11 & -2.22 & T10B11.8 & -2.15 & T09F5.1 & -2.08 \\
\hline F53F8.3 & -2.28 & R05H5.3 & -2.21 & nlp-77 & -2.15 & F21C10.9 & -2.08 \\
\hline B0024.4 & -2.28 & F20B10.3 & -2.21 & C08F1.6 & -2.15 & col-14 & -2.08 \\
\hline btb-6 & -2.28 & rad-51 & -2.21 & M03E7.4 & -2.15 & clec-266 & -2.07 \\
\hline ZK1053.4 & -2.27 & T28A8.3 & -2.21 & F48E3.6 & -2.15 & zipt-7.1 & -2.07 \\
\hline W04A8.1 & -2.27 & valv-1 & -2.21 & Y43F8B.3 & -2.14 & grl-6 & -2.07 \\
\hline alg-5 & -2.27 & C38D4.4 & -2.21 & ceh-83 & -2.14 & bus- 17 & -2.07 \\
\hline C08F8.3 & -2.27 & B0281.5 & -2.21 & idhg-2 & -2.14 & D2005.7 & -2.07 \\
\hline T24C12.4 & -2.27 & dut-1 & -2.21 & T01B7.8 & -2.14 & F08F3.6 & -2.06 \\
\hline ugt-19 & -2.27 & ZK970.7 & -2.2 & Idh-1 & -2.14 & mltn-9 & -2.06 \\
\hline
\end{tabular}




\begin{tabular}{|c|c|c|c|c|c|c|c|}
\hline Gene & $\log 2 \mathrm{FC}$ & Gene & $\log 2 \mathrm{FC}$ & Gene & $\log 2 \mathrm{FC}$ & Gene & $\log 2 \mathrm{FC}$ \\
\hline Y37H2A.14 & -2.06 & tyms-1 & -2.02 & cdl-1 & -1.98 & $c l x-1$ & -1.94 \\
\hline pig-1 & -2.06 & ttr-2 & -2.02 & col-146 & -1.98 & cks-1 & -1.94 \\
\hline dhcr-24 & -2.06 & R03C1.1 & -2.02 & nas-38 & -1.97 & adal-1 & -1.94 \\
\hline syp-5 & -2.06 & skr-17 & -2.01 & D1054.9 & -1.97 & Y60C6A. 2 & -1.93 \\
\hline col-144 & -2.06 & smd-1 & -2.01 & plk-1 & -1.97 & anmt-2 & -1.93 \\
\hline B0001.7 & -2.06 & cey-2 & -2.01 & C49F5.6 & -1.97 & grd-10 & -1.93 \\
\hline hsp-16.41 & -2.06 & pole-4 & -2.01 & clec-78 & -1.97 & hmbx-1 & -1.93 \\
\hline mua-6 & -2.06 & Y38H6A.3 & -2.01 & B0403.5 & -1.97 & cpt-4 & -1.93 \\
\hline K05F6.4 & -2.06 & F53F1.4 & -2.01 & orc-3 & -1.97 & noah-2 & -1.93 \\
\hline skr-10 & -2.06 & phg-1 & -2 & F09C8.1 & -1.97 & R144.10 & -1.93 \\
\hline C54D2.1 & -2.05 & dod-18 & -2 & psf-2 & -1.97 & fip-5 & -1.93 \\
\hline К02B7.3 & -2.05 & $g \ln -6$ & -2 & kbp-3 & -1.97 & pqn-29 & -1.93 \\
\hline $\mathrm{mcm}-6$ & -2.05 & fbxb-66 & -2 & ptr-1 & -1.97 & cmt-1 & -1.93 \\
\hline F13H8.5 & -2.05 & ZC434.8 & -2 & $\mathrm{mcm}-5$ & -1.96 & Y45G5AM.5 & -1.93 \\
\hline toe-2 & -2.05 & F56F11.4 & -2 & such-1 & -1.96 & zim-1 & -1.93 \\
\hline abu-11 & -2.05 & hil-5 & -2 & Ibp-5 & -1.96 & C01B12.8 & -1.93 \\
\hline T16G12.4 & -2.05 & air-2 & -2 & $\mathrm{~F} 55 \mathrm{H} 12.4$ & -1.96 & F58G11.3 & -1.93 \\
\hline ZK742.3 & -2.05 & acdh-6 & -2 & B0034.7 & -1.96 & W02B8.2 & -1.92 \\
\hline hpo-36 & -2.05 & T10E10.4 & -2 & mre-11 & -1.96 & dhs-27 & -1.92 \\
\hline clec-48 & -2.04 & phat-1 & -2 & sip-1 & -1.96 & cnc-8 & -1.92 \\
\hline W03D2.9 & -2.04 & snpc-1.2 & -1.99 & aqp-7 & -1.96 & mlc-7 & -1.92 \\
\hline T06D8.10 & -2.04 & inx-3 & -1.99 & $\mathrm{R} 12 \mathrm{C} 12.10$ & -1.96 & cup-15 & -1.92 \\
\hline mdf-2 & -2.04 & lys-3 & -1.99 & nhr-12 & -1.96 & pri-1 & -1.92 \\
\hline C36B7.5 & -2.04 & $\mathrm{fbxc}-32$ & -1.99 & acox-1.4 & -1.96 & Y41D4B.15 & -1.92 \\
\hline$f b x b-54$ & -2.04 & F44E2.4 & -1.99 & M03B6.4 & -1.96 & cpg-1 & -1.92 \\
\hline F54D10.5 & -2.04 & C05D12.4 & -1.99 & C35E7.5 & -1.96 & C03D6.9 & -1.92 \\
\hline T25E4.1 & -2.03 & R74.2 & -1.99 & acn-1 & -1.96 & F13D12.3 & -1.92 \\
\hline clec-180 & -2.03 & T27A1.2 & -1.99 & F41E6.5 & -1.96 & dpl-1 & -1.92 \\
\hline T05E12.6 & -2.03 & ilys-5 & -1.99 & EEED8.15 & -1.96 & C40A11.2 & -1.92 \\
\hline abu-12 & -2.03 & nspe-5 & -1.99 & crn-1 & -1.95 & brc-1 & -1.92 \\
\hline rod-1 & -2.03 & cdt-1 & -1.98 & hpo-40 & -1.95 & ung-1 & -1.92 \\
\hline skr-13 & -2.03 & spp-20 & -1.98 & K08D8.3 & -1.95 & skr-8 & -1.92 \\
\hline M153.3 & -2.03 & taf-11.2 & -1.98 & prom-1 & -1.95 & wago-1 & -1.91 \\
\hline Y59A8B.12 & -2.03 & F20G2.2 & -1.98 & $f b x b-15$ & -1.95 & bub-1 & -1.91 \\
\hline cest-33 & -2.03 & F07H5.13 & -1.98 & Y82E9BR.17 & -1.95 & noah-1 & -1.91 \\
\hline pri-2 & -2.03 & ZK84.1 & -1.98 & $\mathrm{C} 49 \mathrm{H} 3.16$ & -1.95 & bath-10 & -1.91 \\
\hline skr-9 & -2.03 & srg-31 & -1.98 & Y46G5A.7 & -1.95 & his-74 & -1.91 \\
\hline kbp-5 & -2.02 & rsa-1 & -1.98 & D2030.7 & -1.94 & icl-1 & -1.91 \\
\hline$f b x b-24$ & -2.02 & srpr-2.1 & -1.98 & F54D5.5 & -1.94 & $\mathrm{mcm}-10$ & -1.91 \\
\hline $\mathrm{fbxb}-75$ & -2.02 & kbp-2 & -1.98 & cht-2 & -1.94 & C06G1.1 & -1.91 \\
\hline zif-1 & -2.02 & lec-8 & -1.98 & F49C12.7 & -1.94 & inft-2 & -1.91 \\
\hline nspe-7 & -2.02 & F07H5.8 & -1.98 & F15A4.2 & -1.94 & C08F1.10 & -1.91 \\
\hline
\end{tabular}




\begin{tabular}{|c|c|c|c|c|c|c|c|}
\hline Gene & $\log 2 \mathrm{FC}$ & Gene & $\log 2 \mathrm{FC}$ & Gene & $\log 2 \mathrm{FC}$ & Gene & $\log 2 \mathrm{FC}$ \\
\hline T28A8.4 & -1.91 & F54E7.6 & -1.87 & $\mathrm{~K} 10 \mathrm{H} 10.4$ & -1.83 & Y111B2A.2 & -1.79 \\
\hline cyn-9 & -1.9 & Y52E8A.2 & -1.87 & aptf-2 & -1.83 & C35B1.5 & -1.79 \\
\hline aly-2 & -1.9 & F15G9.6 & -1.87 & dpy-1 & -1.83 & Y69A2AR.28 & -1.79 \\
\hline $\mathrm{R} 05 \mathrm{H} 10.3$ & -1.9 & elo-6 & -1.87 & lem-3 & -1.83 & C36B1.11 & -1.79 \\
\hline $\mathrm{pgl}-3$ & -1.9 & C26B2.8 & -1.86 & cutl-23 & -1.83 & Y58A7A.2 & -1.79 \\
\hline T06E4.8 & -1.9 & $\mathrm{C} 24 \mathrm{H} 12.1$ & -1.86 & Y73B6BL.23 & -1.83 & $\mathrm{msh}-2$ & -1.79 \\
\hline F55A11.7 & -1.9 & сур-23A1 & -1.86 & T23F2.5 & -1.83 & $\mathrm{sdz}-38$ & -1.79 \\
\hline Y46G5A.8 & -1.9 & acs-11 & -1.86 & mesp-1 & -1.83 & C55A1.6 & -1.78 \\
\hline F11E6.4 & -1.9 & neg-1 & -1.86 & grl-1 & -1.83 & D1081.6 & -1.78 \\
\hline immt-2 & -1.89 & T09B9.1 & -1.86 & skpt-1 & -1.83 & T12G3.4 & -1.78 \\
\hline F49D11.6 & -1.89 & C55A6.12 & -1.86 & htp-3 & -1.83 & F59B10.3 & -1.78 \\
\hline hcp-1 & -1.89 & san-1 & -1.86 & memi-2 & -1.82 & fncm-1 & -1.78 \\
\hline hcp-6 & -1.89 & сур-35С1 & -1.86 & elo-4 & -1.82 & htp-1 & -1.78 \\
\hline tipn-1 & -1.89 & dscc-1 & -1.86 & dnsn-1 & -1.82 & klp-18 & -1.78 \\
\hline skpo-2 & -1.89 & rsp-5 & -1.86 & misc-1 & -1.82 & F58G1.7 & -1.78 \\
\hline orc-5 & -1.89 & klc-1 & -1.86 & nas-13 & -1.82 & dnj-11 & -1.78 \\
\hline nstp-2 & -1.89 & Y57G11C.42 & -1.86 & nos-1 & -1.81 & vang-1 & -1.78 \\
\hline F39D8.3 & -1.89 & C28C12.4 & -1.85 & lam-3 & -1.81 & gla-3 & -1.78 \\
\hline K02B12.2 & -1.89 & T05H4.7 & -1.85 & ttr-50 & -1.81 & C45B2.8 & -1.78 \\
\hline hsp-60 & -1.89 & ndc- -80 & -1.85 & R07B1.9 & -1.81 & Y111B2A.3 & -1.78 \\
\hline hsp-16.2 & -1.89 & C56C10.4 & -1.85 & trt-1 & -1.81 & pemd-1 & -1.78 \\
\hline $\mathrm{rrn}-2.1$ & -1.89 & T06A4.3 & -1.85 & ced-3 & -1.81 & fem-3 & -1.78 \\
\hline top-2 & -1.89 & Y52B11A.8 & -1.85 & K08D12.4 & -1.81 & ech-6 & -1.78 \\
\hline gst-28 & -1.88 & cnc-3 & -1.85 & fbxc-44 & -1.81 & Y71F9AL.6 & -1.77 \\
\hline math-45 & -1.88 & glh-3 & -1.85 & $\operatorname{ttr}-45$ & -1.81 & kbp-1 & -1.77 \\
\hline him-10 & -1.88 & tag-63 & -1.85 & ifa-3 & -1.8 & cdd-2 & -1.77 \\
\hline aagr-4 & -1.88 & M03F8.1 & -1.85 & syp-1 & -1.8 & Y37A1B.5 & -1.77 \\
\hline ttr-15 & -1.88 & bca-1 & -1.85 & Ibp-3 & -1.8 & F32D8.11 & -1.77 \\
\hline $\mathrm{F} 53 \mathrm{H} 4.3$ & -1.88 & hpr-17 & -1.84 & col-182 & -1.8 & grl-16 & -1.77 \\
\hline xnd-1 & -1.88 & C37C3.9 & -1.84 & F32D8.7 & -1.8 & oac-31 & -1.77 \\
\hline T08B1.1 & -1.88 & act-1 & -1.84 & fib-1 & -1.8 & dsh-2 & -1.77 \\
\hline F40E10.5 & -1.88 & F10D11.6 & -1.84 & F54E2.1 & -1.8 & $\mathrm{C} 24 \mathrm{H} 10.3$ & -1.77 \\
\hline mes-4 & -1.87 & Y75B8A.18 & -1.84 & $\mathrm{C} 46 \mathrm{C} 2.7$ & -1.8 & C06E1.1 & -1.77 \\
\hline B0310.6 & -1.87 & him-3 & -1.84 & lys-1 & -1.8 & K07F5.12 & -1.77 \\
\hline spo-11 & -1.87 & atz-1 & -1.84 & mdh-1 & -1.8 & ech-4 & -1.77 \\
\hline linc-13 & -1.87 & Iron-2 & -1.84 & lips-7 & -1.8 & C35A5.10 & -1.77 \\
\hline іср-1 & -1.87 & hsp-17 & -1.84 & $\csc -1$ & -1.8 & Y105C5B.19 & -1.77 \\
\hline K10D11.7 & -1.87 & F10D2.8 & -1.84 & col-186 & -1.8 & $\mathrm{fkb}-7$ & -1.77 \\
\hline $\mathrm{fbxb}-78$ & -1.87 & let-754 & -1.84 & $\mathrm{rnr}-2$ & -1.8 & cpg-9 & -1.77 \\
\hline pqn-32 & -1.87 & C17E4.2 & -1.84 & R09E10.5 & -1.8 & C06C3.4 & -1.77 \\
\hline hil-4 & -1.87 & $\mathrm{rbm}-3.1$ & -1.84 & ZK180.5 & -1.8 & unc-59 & -1.77 \\
\hline F31D4.8 & -1.87 & fbxa-83 & -1.83 & hasp-1 & -1.79 & F44E2.10 & -1.76 \\
\hline
\end{tabular}




\begin{tabular}{|c|c|c|c|c|c|c|c|}
\hline Gene & $\log 2 \mathrm{FC}$ & Gene & $\log 2 \mathrm{FC}$ & Gene & $\log 2 \mathrm{FC}$ & Gene & $\log 2 \mathrm{FC}$ \\
\hline C09B8.5 & -1.76 & Y42A5A.5 & -1.73 & F30F8.1 & -1.7 & hal-3 & -1.69 \\
\hline D2005.4 & -1.76 & F01D4.5 & -1.73 & K08B4.2 & -1.7 & hch-1 & -1.68 \\
\hline ceh-86 & -1.76 & Y32G9B.1 & -1.73 & Y106G6H.6 & -1.7 & M199.2 & -1.68 \\
\hline fbxc-29 & -1.76 & cutl-10 & -1.73 & cutl-9 & -1.7 & ins-26 & -1.68 \\
\hline$n x f-2$ & -1.76 & C48B4.13 & -1.73 & C27B7.5 & -1.7 & apy-1 & -1.68 \\
\hline aspm-1 & -1.76 & mlt-11 & -1.72 & $\operatorname{div}-1$ & -1.7 & ZK829.3 & -1.68 \\
\hline $\mathrm{H} 01 \mathrm{G} 02.1$ & -1.76 & pqn-36 & -1.72 & Ipr-1 & -1.7 & Y57G11C.39 & -1.68 \\
\hline oef-1 & -1.76 & mam-1 & -1.72 & R186.8 & -1.7 & dhs- 8 & -1.68 \\
\hline $\mathrm{F} 33 \mathrm{H} 2.3$ & -1.76 & Y97E10AR.4 & -1.72 & C56E6.2 & -1.7 & dhs-23 & -1.68 \\
\hline K04C2.5 & -1.76 & K01D12.8 & -1.72 & F55F8.8 & -1.7 & che-14 & -1.68 \\
\hline cut-2 & -1.76 & K08D12.8 & -1.72 & col-115 & -1.7 & erh-2 & -1.68 \\
\hline T04D3.5 & -1.76 & hal-2 & -1.72 & col-174 & -1.7 & kin-15 & -1.68 \\
\hline E01G6.1 & -1.75 & tofu- 6 & -1.72 & ram-5 & -1.7 & cyk-4 & -1.68 \\
\hline par-2 & -1.75 & R04B3.3 & -1.72 & rmd-1 & -1.7 & nop-1 & -1.68 \\
\hline C53A5.2 & -1.75 & gsto-1 & -1.72 & F53A2.3 & -1.7 & fbxb-84 & -1.68 \\
\hline C15C6.1 & -1.75 & nhr-137 & -1.72 & F46G11.6 & -1.7 & htz-1 & -1.68 \\
\hline C56G2.15 & -1.75 & T26C12.3 & -1.72 & bmk-1 & -1.7 & mans-4 & -1.68 \\
\hline Y53F4B.27 & -1.75 & F32D8.12 & -1.72 & F58E6.13 & -1.7 & col-104 & -1.68 \\
\hline argk-1 & -1.75 & T16G12.1 & -1.72 & Y71G12B.6 & -1.7 & cutl-17 & -1.68 \\
\hline D2030.8 & -1.75 & set-31 & -1.72 & lgc-34 & -1.69 & F30H5.3 & -1.68 \\
\hline F42A8.1 & -1.75 & $\mathrm{fbxb}-85$ & -1.72 & F02C12.1 & -1.69 & C48B6.9 & -1.68 \\
\hline F14B6.3 & -1.75 & Y11D7A.9 & -1.72 & Y68A4A.13 & -1.69 & fbn-1 & -1.68 \\
\hline T19C4.1 & -1.75 & F59A6.5 & -1.72 & ZK512.1 & -1.69 & B0261.7 & -1.68 \\
\hline $\mathrm{K} 08 \mathrm{H} 2.4$ & -1.75 & pgp-14 & -1.72 & W08E12.2 & -1.69 & nol-56 & -1.67 \\
\hline gbh-1 & -1.75 & T02G5.7 & -1.72 & chtl-1 & -1.69 & R02F11.1 & -1.67 \\
\hline $\mathrm{nhr}-127$ & -1.75 & $\mathrm{R} 11 \mathrm{H} 6.4$ & -1.72 & glf-1 & -1.69 & С39B5.2 & -1.67 \\
\hline papl-1 & -1.75 & pho-14 & -1.71 & F14D7.12 & -1.69 & K01D12.1 & -1.67 \\
\hline cdk-4 & -1.75 & Y48G1A.2 & -1.71 & col-131 & -1.69 & prg-1 & -1.67 \\
\hline hcp-4 & -1.75 & F13E9.13 & -1.71 & F46C8.8 & -1.69 & B0205.13 & -1.67 \\
\hline M153.1 & -1.74 & W04H10.2 & -1.71 & F46C3.6 & -1.69 & cec-4 & -1.67 \\
\hline $\mathrm{kbp}-4$ & -1.74 & D2092.8 & -1.71 & C44C1.1 & -1.69 & ZK596.3 & -1.67 \\
\hline pfn-1 & -1.74 & C46A5.4 & -1.71 & C34C12.7 & -1.69 & eat-5 & -1.67 \\
\hline psf-3 & -1.74 & grl-11 & -1.71 & sodh-1 & -1.69 & C54G4.4 & -1.67 \\
\hline txdc-12.1 & -1.74 & srpr-2.2 & -1.71 & C34C12.8 & -1.69 & F10E9.7 & -1.67 \\
\hline htp-2 & -1.74 & sptl-2 & -1.71 & tsp-1 & -1.69 & sdha-2 & -1.67 \\
\hline VF13D12L.3 & -1.74 & slc-25A10 & -1.71 & C17C3.1 & -1.69 & C18E9.7 & -1.67 \\
\hline let-99 & -1.74 & bir-2 & -1.71 & Y39D8A.1 & -1.69 & fkb-4 & -1.67 \\
\hline gst-39 & -1.73 & C09F9.2 & -1.71 & F44F1.6 & -1.69 & mlt-9 & -1.66 \\
\hline hrde-1 & -1.73 & cht-1 & -1.71 & rmh-1 & -1.69 & ZK643.2 & -1.66 \\
\hline psf-1 & -1.73 & cec- 8 & -1.71 & rnp-8 & -1.69 & fbxc- 40 & -1.66 \\
\hline E04F6.6 & -1.73 & alh-5 & -1.7 & atf-8 & -1.69 & cuti-1 & -1.66 \\
\hline C16D9.1 & -1.73 & dex-1 & -1.7 & rfc-4 & -1.69 & lin-53 & -1.66 \\
\hline
\end{tabular}




\begin{tabular}{|c|c|c|c|c|c|c|c|}
\hline Gene & $\log 2 \mathrm{FC}$ & Gene & $\log 2 \mathrm{FC}$ & Gene & $\log 2 \mathrm{FC}$ & Gene & $\log 2 \mathrm{FC}$ \\
\hline acaa-2 & -1.66 & hsp-12.1 & -1.63 & ifd-2 & -1.6 & F36H5.4 & -1.58 \\
\hline ptr-18 & -1.66 & C40A11.3 & -1.63 & orc-4 & -1.6 & col-58 & -1.58 \\
\hline smc-5 & -1.66 & C32D5.12 & -1.63 & cutl-8 & -1.6 & nstp-3 & -1.58 \\
\hline kat-1 & -1.66 & F46F3.3 & -1.63 & $\mathrm{C} 02 \mathrm{~F} 4.4$ & -1.6 & F53A9.3 & -1.58 \\
\hline fip-6 & -1.66 & mei-1 & -1.63 & gcsh-1 & -1.6 & $\mathrm{fbxb}-45$ & -1.58 \\
\hline chs-2 & -1.66 & set-9 & -1.63 & rde-4 & -1.6 & F32A7.8 & -1.58 \\
\hline$w d r-5.3$ & -1.65 & ska-3 & -1.63 & F46G11.2 & -1.6 & $\mathrm{knl}-3$ & -1.58 \\
\hline F31D4.5 & -1.65 & pid-3 & -1.63 & F27C1.4 & -1.6 & slc- 17.8 & -1.58 \\
\hline bath-41 & -1.65 & oac-14 & -1.63 & K08B4.7 & -1.6 & C08B6.4 & -1.58 \\
\hline hog-1 & -1.65 & bus- 12 & -1.62 & ima-2 & -1.59 & T16G1.2 & -1.58 \\
\hline R12E2.7 & -1.65 & ugt-29 & -1.62 & C29F3.7 & -1.59 & paqr-3 & -1.58 \\
\hline nhr-205 & -1.65 & wrt-4 & -1.62 & F41C3.11 & -1.59 & Y47G6A.31 & -1.58 \\
\hline col-54 & -1.65 & F59A6.10 & -1.62 & C49G7.7 & -1.59 & sdhd-1 & -1.58 \\
\hline C48B4.11 & -1.65 & cpr-5 & -1.62 & $\mathrm{R} 13 \mathrm{H} 4.8$ & -1.59 & $\mathrm{~T} 28 \mathrm{C} 12.6$ & -1.58 \\
\hline zeel-1 & -1.65 & Ist-1 & -1.62 & nex-1 & -1.59 & F44E5.4 & -1.58 \\
\hline tag-307 & -1.65 & agmo-1 & -1.62 & $\mathrm{fbxc}-51$ & -1.59 & $\mathrm{~F} 33 \mathrm{H} 2.6$ & -1.58 \\
\hline chw-1 & -1.65 & C26B9.7 & -1.62 & cutl-28 & -1.59 & ugt-57 & -1.58 \\
\hline Y65B4BL.1 & -1.64 & C14A4.9 & -1.62 & F40F11.3 & -1.59 & F07H5.10 & -1.57 \\
\hline Irr-1 & -1.64 & algn-6 & -1.62 & smo-1 & -1.59 & gei-14 & -1.57 \\
\hline F20G2.1 & -1.64 & cutl-18 & -1.62 & T26C5.2 & -1.59 & spd-5 & -1.57 \\
\hline F59B2.8 & -1.64 & T27E9.2 & -1.62 & dpy-18 & -1.59 & pola-1 & -1.57 \\
\hline cpr-1 & -1.64 & col-154 & -1.62 & F26A1.9 & -1.59 & F43D9.1 & -1.57 \\
\hline ztf-11 & -1.64 & ttr-30 & -1.62 & Y49E10.4 & -1.59 & K06A9.3 & -1.57 \\
\hline Y16B4A.2 & -1.64 & bgnt-1.2 & -1.62 & fbxc-25 & -1.59 & klp-19 & -1.57 \\
\hline fog-2 & -1.64 & fbxa-210 & -1.62 & ogr-2 & -1.59 & R08A2.5 & -1.57 \\
\hline metr-1 & -1.64 & mec-7 & -1.62 & F56D2.3 & -1.59 & R08F11.4 & -1.57 \\
\hline cut-3 & -1.64 & F36F12.1 & -1.61 & B0393.5 & -1.59 & E01A2.2 & -1.57 \\
\hline phat-2 & -1.64 & F44E5.5 & -1.61 & ZK675.4 & -1.59 & tts-2 & -1.57 \\
\hline $\mathrm{rsp}-4$ & -1.64 & pal-1 & -1.61 & ced-8 & -1.59 & Ipr-5 & -1.57 \\
\hline daf- 6 & -1.64 & F30A10.2 & -1.61 & cest-10 & -1.59 & pck-1 & -1.57 \\
\hline nas-30 & -1.64 & W06D11.1 & -1.61 & F15B10.3 & -1.59 & gfi-1 & -1.57 \\
\hline Y39A1A.21 & -1.64 & ptr-15 & -1.61 & pgk-1 & -1.59 & nspe-1 & -1.57 \\
\hline C41D11.9 & -1.64 & M03F4.6 & -1.61 & ckb-2 & -1.59 & F08B12.4 & -1.57 \\
\hline C18D11.6 & -1.64 & $\mathrm{C} 46 \mathrm{H} 11.7$ & -1.61 & T06G6.12 & -1.59 & ZK180.6 & -1.56 \\
\hline $\mathrm{F} 15 \mathrm{H} 10.8$ & -1.64 & ech-1.1 & -1.61 & mtp-18 & -1.59 & ZK742.4 & -1.56 \\
\hline Y23H5B.8 & -1.64 & spdl-1 & -1.61 & Y71F9AL.7 & -1.58 & tag-297 & -1.56 \\
\hline exo-1 & -1.64 & T05H10.3 & -1.61 & M04C9.3 & -1.58 & cpna-3 & -1.56 \\
\hline T17H7.7 & -1.64 & cyn-10 & -1.61 & lips-3 & -1.58 & sys-1 & -1.56 \\
\hline nep-16 & -1.63 & fipr-7 & -1.61 & Y75B7AR.1 & -1.58 & chaf-1 & -1.56 \\
\hline swip-10 & -1.63 & F29G6.1 & -1.6 & plpr-1 & -1.58 & clec-196 & -1.56 \\
\hline K02E10.4 & -1.63 & snr-2 & -1.6 & C30B5.6 & -1.58 & ani-2 & -1.56 \\
\hline $\mathrm{F} 35 \mathrm{H} 10.10$ & -1.63 & pho-1 & -1.6 & hmg-20 & -1.58 & cnc-11 & -1.56 \\
\hline
\end{tabular}




\begin{tabular}{|c|c|c|c|c|c|c|c|}
\hline Gene & $\log 2 \mathrm{FC}$ & Gene & $\log 2 \mathrm{FC}$ & Gene & $\log 2 \mathrm{FC}$ & Gene & $\log 2 \mathrm{FC}$ \\
\hline pigv-1 & -1.56 & F52B5.2 & -1.54 & F18E3.12 & -1.52 & daf-18 & -1.49 \\
\hline nas-10 & -1.56 & ceh-30 & -1.54 & edg-1 & -1.52 & H10E21.4 & -1.49 \\
\hline dod-3 & -1.56 & T07F10.6 & -1.54 & dhs-30 & -1.52 & Y63D3A.7 & -1.49 \\
\hline T02C1.1 & -1.56 & ins-34 & -1.54 & eppl-1 & -1.52 & gpdh-2 & -1.49 \\
\hline ugt-63 & -1.56 & tth-1 & -1.54 & D1081.7 & -1.51 & Y57G11A.4 & -1.49 \\
\hline F26G1.5 & -1.56 & $n d x-2$ & -1.54 & Iron-8 & -1.51 & ZK1098.3 & -1.49 \\
\hline C50C3.1 & -1.55 & $\mathrm{C} 01 \mathrm{H} 6.8$ & -1.53 & rocf-1 & -1.51 & C45G9.2 & -1.49 \\
\hline swah-1 & -1.55 & F18A11.2 & -1.53 & Ipr-6 & -1.51 & rfc-2 & -1.49 \\
\hline tcab-1 & -1.55 & $\operatorname{ttr}-18$ & -1.53 & sqt-3 & -1.51 & lin-54 & -1.49 \\
\hline F56D2.2 & -1.55 & ZC449.1 & -1.53 & sid-1 & -1.51 & jud-4 & -1.49 \\
\hline dnj-27 & -1.55 & Y39B6A.42 & -1.53 & M28.5 & -1.51 & VH15N14R.1 & -1.49 \\
\hline ces-2 & -1.55 & coq-5 & -1.53 & F54D5.9 & -1.51 & $\mathrm{knl}-2$ & -1.49 \\
\hline rsa-2 & -1.55 & alh-13 & -1.53 & F08G12.5 & -1.51 & dsl-6 & -1.49 \\
\hline H03E18.1 & -1.55 & C45E5.1 & -1.53 & dhs-19 & -1.51 & F35A5.4 & -1.49 \\
\hline cutl-2 & -1.55 & tag-10 & -1.53 & T26A5.2 & -1.51 & Y37A1B.7 & -1.49 \\
\hline D1005.2 & -1.55 & pсp-2 & -1.53 & C50D2.1 & -1.51 & col-74 & -1.48 \\
\hline T14G8.4 & -1.55 & vap-2 & -1.53 & T19C3.2 & -1.5 & F09F9.2 & -1.48 \\
\hline ugt-34 & -1.55 & linc-1 & -1.53 & npp-22 & -1.5 & let-268 & -1.48 \\
\hline clik-1 & -1.55 & syx-4 & -1.53 & gpaa-1 & -1.5 & hira-1 & -1.48 \\
\hline fan-1 & -1.55 & dnj-2 & -1.53 & cutl-16 & -1.5 & fbxa-206 & -1.48 \\
\hline inf-1 & -1.55 & $\operatorname{ctg}-2$ & -1.53 & $\mathrm{C} 05 \mathrm{C} 10.3$ & -1.5 & daf-36 & -1.48 \\
\hline ssna-1 & -1.55 & suds-3 & -1.53 & Ipr-3 & -1.5 & aman-1 & -1.48 \\
\hline dif-1 & -1.55 & F23A7.4 & -1.53 & F58H1.2 & -1.5 & F18C5.9 & -1.48 \\
\hline $\mathrm{F} 33 \mathrm{H} 12.7$ & -1.55 & tdpt-1 & -1.53 & Iron-1 & -1.5 & Y73F8A.26 & -1.48 \\
\hline C05E7.2 & -1.55 & $z w \mid-1$ & -1.53 & irg-5 & -1.5 & pot-2 & -1.48 \\
\hline ZC123.1 & -1.55 & ttr-33 & -1.53 & unc-61 & -1.5 & grd-12 & -1.48 \\
\hline ppm-1.G & -1.54 & gpr-1 & -1.53 & T06E4.9 & -1.5 & F41G3.21 & -1.48 \\
\hline F15D3.6 & -1.54 & exo-3 & -1.53 & Y17D7C.4 & -1.5 & C31C9.2 & -1.48 \\
\hline col-75 & -1.54 & twk-11 & -1.53 & lact-4 & -1.5 & C44B7.7 & -1.48 \\
\hline F21H7.3 & -1.54 & M02G9.1 & -1.52 & C05D11.5 & -1.5 & C04A11.2 & -1.48 \\
\hline W04A8.6 & -1.54 & cutl-5 & -1.52 & F38A6.4 & -1.5 & fat-5 & -1.48 \\
\hline Y73F4A.1 & -1.54 & col-111 & -1.52 & col-130 & -1.5 & C35A5.11 & -1.48 \\
\hline pqn-73 & -1.54 & F01D5.6 & -1.52 & rba-1 & -1.5 & K01D12.5 & -1.48 \\
\hline scl-6 & -1.54 & C01B10.11 & -1.52 & cutl-25 & -1.49 & dpy-2 & -1.48 \\
\hline W01C9.1 & -1.54 & cut-5 & -1.52 & T10E9.8 & -1.49 & Y47D7A.13 & -1.48 \\
\hline bus- 8 & -1.54 & dna-2 & -1.52 & vha-12 & -1.49 & $\mathrm{C} 27 \mathrm{H} 6.9$ & -1.48 \\
\hline $\mathrm{dsl}-3$ & -1.54 & cpg-4 & -1.52 & sas-5 & -1.49 & abu-13 & -1.48 \\
\hline ifp-1 & -1.54 & spsb-2 & -1.52 & col-169 & -1.49 & sgo-1 & -1.48 \\
\hline Y11D7A.5 & -1.54 & $\operatorname{ttr}-26$ & -1.52 & $\operatorname{lgc}-27$ & -1.49 & Y41G9A.5 & -1.48 \\
\hline col-167 & -1.54 & cnc-10 & -1.52 & dyf-7 & -1.49 & F29B9.5 & -1.48 \\
\hline ugt-33 & -1.54 & C06C3.10 & -1.52 & C25F9.4 & -1.49 & anmt-3 & -1.48 \\
\hline C15A11.4 & -1.54 & F15B9.8 & -1.52 & B0432.8 & -1.49 & col-121 & -1.48 \\
\hline
\end{tabular}




\begin{tabular}{|c|c|c|c|c|c|c|c|}
\hline Gene & $\log 2 \mathrm{FC}$ & Gene & $\log 2 \mathrm{FC}$ & Gene & $\log 2 \mathrm{FC}$ & Gene & $\log 2 \mathrm{FC}$ \\
\hline tim-1 & -1.48 & mlt-8 & -1.45 & col-177 & -1.42 & ntp-1 & -1.4 \\
\hline Y37A1A.4 & -1.47 & B0272.3 & -1.45 & dtmk-1 & -1.42 & ttr-20 & -1.4 \\
\hline Igc-21 & -1.47 & F52G3.5 & -1.45 & F44G4.3 & -1.42 & F56C11.5 & -1.4 \\
\hline T03G6.1 & -1.47 & tdc-1 & -1.45 & Y53F4B.23 & -1.42 & ZK154.1 & -1.4 \\
\hline F53B1.4 & -1.47 & $\mathrm{fkb}-5$ & -1.45 & ptps-1 & -1.42 & Y54G11A.3 & -1.4 \\
\hline Igc-33 & -1.47 & msh-6 & -1.45 & F31E3.12 & -1.42 & pges-2 & -1.4 \\
\hline F59A3.12 & -1.47 & B0205.4 & -1.45 & cblc-1 & -1.42 & F10C1.9 & -1.4 \\
\hline clec-4 & -1.47 & R08D7.7 & -1.45 & npp-15 & -1.42 & ZK228.4 & -1.4 \\
\hline C39E9.12 & -1.47 & cee-1 & -1.45 & deps-1 & -1.42 & B0334.13 & -1.4 \\
\hline dhhc-1 & -1.47 & $\mathrm{~K} 11 \mathrm{H} 12.5$ & -1.45 & $w d r-48$ & -1.42 & cpt- 6 & -1.39 \\
\hline grl-15 & -1.47 & $\mathrm{R} 05 \mathrm{H} 11.2$ & -1.45 & C01G6.9 & -1.42 & ttr-32 & -1.39 \\
\hline puf-8 & -1.47 & cest-35.2 & -1.44 & hsp-25 & -1.42 & unc-71 & -1.39 \\
\hline tag-342 & -1.47 & bub-3 & -1.44 & acdh-12 & -1.42 & erd-2.2 & -1.39 \\
\hline T22D1.5 & -1.47 & rbbp-5 & -1.44 & $\mathrm{C} 34 \mathrm{H} 4.2$ & -1.42 & ZK1307.8 & -1.39 \\
\hline pqn-67 & -1.47 & B0041.8 & -1.44 & ZK287.4 & -1.42 & F14F9.3 & -1.39 \\
\hline efl-1 & -1.47 & fcd-2 & -1.44 & $\mathrm{rfc}-3$ & -1.41 & K02D3.2 & -1.39 \\
\hline emb-27 & -1.47 & cutl-12 & -1.44 & T09B4.5 & -1.41 & F44B9.2 & -1.39 \\
\hline grd-15 & -1.47 & npax-2 & -1.44 & sex-1 & -1.41 & meg-3 & -1.39 \\
\hline Y62E10A.13 & -1.46 & bca-2 & -1.44 & swt-5 & -1.41 & set-32 & -1.39 \\
\hline C52G5.2 & -1.46 & F55G1.6 & -1.44 & col-105 & -1.41 & pph-4.2 & -1.39 \\
\hline dpy-10 & -1.46 & R02E4.3 & -1.44 & $\mathrm{R} 10 \mathrm{H} 10.6$ & -1.41 & ZK596.1 & -1.39 \\
\hline ugt-22 & -1.46 & bath-15 & -1.44 & dpy-14 & -1.41 & pot-1 & -1.39 \\
\hline F12F6.7 & -1.46 & acer-1 & -1.44 & $\mathrm{C} 28 \mathrm{C} 12.12$ & -1.41 & dhfr-1 & -1.39 \\
\hline polh-1 & -1.46 & nas-7 & -1.44 & ttr-53 & -1.41 & lir-3 & -1.39 \\
\hline swt-6 & -1.46 & hhat-2 & -1.44 & ucr-2.3 & -1.41 & F46E10.2 & -1.39 \\
\hline T11G6.4 & -1.46 & F56D3.1 & -1.44 & ugt-45 & -1.41 & lec-10 & -1.39 \\
\hline K09D9.1 & -1.46 & $\mathrm{M} 02 \mathrm{H} 5.8$ & -1.44 & $\lg x-1$ & -1.41 & cyn-17 & -1.39 \\
\hline col-155 & -1.46 & F33D4.6 & -1.44 & pqn-13 & -1.41 & F42A10.7 & -1.39 \\
\hline $\mathrm{T} 24 \mathrm{H} 10.4$ & -1.46 & cpr-3 & -1.44 & otub-3 & -1.4 & smc-3 & -1.39 \\
\hline C15F1.1 & -1.46 & $\mathrm{Y} 69 \mathrm{H} 2.7$ & -1.43 & sod-1 & -1.4 & pam-1 & -1.39 \\
\hline Y53G8AR.6 & -1.46 & M01D1.12 & -1.43 & iff-1 & -1.4 & dpy-17 & -1.39 \\
\hline txt-4 & -1.46 & T06E4.10 & -1.43 & haao-1 & -1.4 & zim-3 & -1.39 \\
\hline pfkb-1.2 & -1.46 & R07E5.4 & -1.43 & ceh-39 & -1.4 & F52B5.7 & -1.39 \\
\hline dsb-2 & -1.46 & ZK816.4 & -1.43 & cutl-11 & -1.4 & blos-4 & -1.38 \\
\hline Y66C5A.1 & -1.46 & B0035.15 & -1.43 & gsto-2 & -1.4 & F43C11.6 & -1.38 \\
\hline oac-51 & -1.45 & F26F12.4 & -1.43 & T23F6.1 & -1.4 & W02F12.8 & -1.38 \\
\hline Y62E10A.19 & -1.45 & atf-2 & -1.43 & ZK1127.13 & -1.4 & C14C6.5 & -1.38 \\
\hline $\mathrm{fbxb}-77$ & -1.45 & Y55F3C.17 & -1.43 & F45F2.11 & -1.4 & ZK973.1 & -1.38 \\
\hline wrt-1 & -1.45 & cri-3 & -1.43 & chk-1 & -1.4 & set-10 & -1.38 \\
\hline phy-2 & -1.45 & lec-7 & -1.42 & sym-1 & -1.4 & C50F4.6 & -1.38 \\
\hline C48B4.7 & -1.45 & Y69A2AR.21 & -1.42 & spd-1 & -1.4 & ugt-9 & -1.38 \\
\hline hhat-1 & -1.45 & Y18H1A.9 & -1.42 & ZC449.2 & -1.4 & F40A3.6 & -1.38 \\
\hline
\end{tabular}




\begin{tabular}{|c|c|c|c|c|c|c|c|}
\hline Gene & $\log 2 \mathrm{FC}$ & Gene & $\log 2 \mathrm{FC}$ & Gene & $\log 2 \mathrm{FC}$ & Gene & $\log 2 \mathrm{FC}$ \\
\hline C35A5.3 & -1.38 & dao-2 & -1.35 & $t x \mid-1$ & -1.33 & gst-31 & -1.31 \\
\hline $\operatorname{dim}-1$ & -1.38 & nlp-33 & -1.35 & eri-7 & -1.33 & C35D10.5 & -1.31 \\
\hline $\mathrm{hmg}-4$ & -1.38 & hcp-3 & -1.35 & C49F8.3 & -1.33 & Ism-1 & -1.31 \\
\hline ppw-1 & -1.38 & $\mathrm{C} 18 \mathrm{H} 7.11$ & -1.35 & dpy-3 & -1.33 & efl-3 & -1.31 \\
\hline K02G10.15 & -1.38 & $\mathrm{~F} 40 \mathrm{H} 3.3$ & -1.35 & Y41D4B.26 & -1.33 & swt-4 & -1.31 \\
\hline C24D10.6 & -1.38 & col-180 & -1.35 & cye-1 & -1.33 & Ibp-4 & -1.31 \\
\hline grl-7 & -1.38 & Y38E10A.14 & -1.35 & mth-1 & -1.33 & F59B1.8 & -1.31 \\
\hline C34E11.2 & -1.38 & ZK1010.4 & -1.35 & pgp-15 & -1.33 & slc-25A46 & -1.31 \\
\hline hrpk-2 & -1.38 & F35D2.3 & -1.35 & tofu-1 & -1.33 & rad-8 & -1.31 \\
\hline T06A1.1 & -1.37 & K06H7.7 & -1.35 & osr-1 & -1.33 & aly-3 & -1.31 \\
\hline B0511.7 & -1.37 & cdc-25.2 & -1.35 & unc-85 & -1.33 & ssup-72 & -1.31 \\
\hline swt-7 & -1.37 & B0464.6 & -1.35 & perm-5 & -1.33 & F25E5.2 & -1.31 \\
\hline grd-14 & -1.37 & acbp-3 & -1.35 & K01A6.7 & -1.33 & cav-1 & -1.31 \\
\hline crn-6 & -1.37 & pcs-1 & -1.35 & C47E8.4 & -1.33 & mlc-6 & -1.31 \\
\hline ttr-6 & -1.37 & uri-1 & -1.35 & nemp-1 & -1.33 & kca-1 & -1.31 \\
\hline bcmo-1 & -1.37 & col-125 & -1.34 & R08E5.3 & -1.33 & F13B9.2 & -1.31 \\
\hline Y69A2AR.3 & -1.37 & ugt-41 & -1.34 & phat-5 & -1.33 & H04D03.2 & -1.31 \\
\hline F01G10.10 & -1.37 & C15C7.5 & -1.34 & nasp-1 & -1.33 & $\mathrm{imp}-1$ & -1.31 \\
\hline npp-1 & -1.37 & mup-4 & -1.34 & F01F1.11 & -1.33 & nhr-237 & -1.31 \\
\hline osm-8 & -1.37 & T24F1.7 & -1.34 & zip-8 & -1.33 & cup-5 & -1.31 \\
\hline taco-1 & -1.37 & ttr-59 & -1.34 & B0310.1 & -1.33 & K08B12.1 & -1.31 \\
\hline rsp-6 & -1.37 & C11D2.4 & -1.34 & grl-5 & -1.33 & C26B2.7 & -1.31 \\
\hline T26C5.4 & -1.37 & C09G4.4 & -1.34 & ego-1 & -1.33 & F10C1.8 & -1.31 \\
\hline sep-1 & -1.36 & $f b x b-100$ & -1.34 & ubc-12 & -1.32 & far-3 & -1.3 \\
\hline rnp-5 & -1.36 & thk-1 & -1.34 & ppgn-1 & -1.32 & T01D1.8 & -1.3 \\
\hline cut-6 & -1.36 & C07E3.10 & -1.34 & mev-1 & -1.32 & F09E10.6 & -1.3 \\
\hline col-165 & -1.36 & col-68 & -1.34 & wrt-10 & -1.32 & dhs-4 & -1.3 \\
\hline F25A2.1 & -1.36 & sups-1 & -1.34 & gly-8 & -1.32 & pif-1 & -1.3 \\
\hline$m t x-1$ & -1.36 & M03E7.2 & -1.34 & $\mathrm{R} 09 \mathrm{H} 10.5$ & -1.32 & K08D8.4 & -1.3 \\
\hline zig-12 & -1.36 & slc-30A5 & -1.34 & gst-12 & -1.32 & F45E4.5 & -1.3 \\
\hline gst-21 & -1.36 & bus- 4 & -1.34 & dpy-7 & -1.32 & Y6D1A.2 & -1.3 \\
\hline glrx-10 & -1.36 & hsp-70 & -1.34 & C15B12.8 & -1.32 & mppa-1 & -1.3 \\
\hline gst-9 & -1.36 & eat-20 & -1.34 & $\mathrm{~F} 36 \mathrm{H} 1.11$ & -1.32 & gad-1 & -1.3 \\
\hline D1014.5 & -1.36 & C34E7.4 & -1.34 & $\mathrm{col}-3$ & -1.32 & F25D1.5 & -1.3 \\
\hline ztf-30 & -1.36 & B0205.1 & -1.34 & ZK822.1 & -1.32 & dog-1 & -1.3 \\
\hline Y17G9B.2 & -1.36 & txdc-12.2 & -1.34 & cfim-1 & -1.32 & trul-1 & -1.3 \\
\hline F46F11.7 & -1.36 & tbb-1 & -1.33 & F41F3.3 & -1.32 & $\mathrm{fbxb}-88$ & -1.3 \\
\hline elo-9 & -1.36 & lips-10 & -1.33 & $\mathrm{mrg}-1$ & -1.32 & R05D3.9 & -1.3 \\
\hline mut-7 & -1.35 & sul-1 & -1.33 & Igc-22 & -1.32 & memb-1 & -1.3 \\
\hline $\mathrm{C} 11 \mathrm{H} 1.7$ & -1.35 & BE0003N10.1 & -1.33 & F17C11.13 & -1.31 & H21P03.2 & -1.3 \\
\hline $\mathrm{ttr}-38$ & -1.35 & C35D10.10 & -1.33 & gst-36 & -1.31 & H04M03.3 & -1.3 \\
\hline R04A9.9 & -1.35 & F44B9.8 & -1.33 & gpr-2 & -1.31 & C48B6.10 & -1.3 \\
\hline
\end{tabular}




\begin{tabular}{|c|c|c|c|c|c|c|c|}
\hline Gene & $\log 2 \mathrm{FC}$ & Gene & $\log 2 \mathrm{FC}$ & Gene & $\log 2 \mathrm{FC}$ & Gene & $\log 2 \mathrm{FC}$ \\
\hline $\mathrm{R} 03 \mathrm{H} 10.2$ & -1.3 & T28A8.5 & -1.28 & C29A12.6 & -1.26 & let-49 & -1.24 \\
\hline xpo-2 & -1.29 & ZC434.3 & -1.28 & hsp-4 & -1.26 & pmp-5 & -1.24 \\
\hline сур-13B1 & -1.29 & exc- 6 & -1.28 & F01D5.10 & -1.26 & Y43F4B.10 & -1.24 \\
\hline F39B2.3 & -1.29 & pnn-1 & -1.28 & Y56A3A.19 & -1.26 & aff-1 & -1.24 \\
\hline his-3 & -1.29 & Y55D5A.4 & -1.28 & F16B4.4 & -1.26 & F57C2.5 & -1.24 \\
\hline K11D2.1 & -1.29 & nlp-29 & -1.27 & сур-13A7 & -1.26 & K05C4.7 & -1.24 \\
\hline E01G4.5 & -1.29 & bra-2 & -1.27 & C05D12.1 & -1.26 & M88.4 & -1.24 \\
\hline T19D12.4 & -1.29 & F57B1.6 & -1.27 & Y44E3A.1 & -1.26 & ceh-74 & -1.24 \\
\hline spp-7 & -1.29 & spl-2 & -1.27 & col-117 & -1.25 & C42C1.12 & -1.24 \\
\hline Y75B8A.16 & -1.29 & F21D5.3 & -1.27 & srr-6 & -1.25 & Y106G6D.8 & -1.24 \\
\hline B0024.13 & -1.29 & lido-1 & -1.27 & Ipr-7 & -1.25 & hach-1 & -1.24 \\
\hline Y62F5A.9 & -1.29 & strm-1 & -1.27 & ostb-1 & -1.25 & rnh-2 & -1.23 \\
\hline col-65 & -1.29 & apc-2 & -1.27 & pcyt-1 & -1.25 & lagr-1 & -1.23 \\
\hline sdha-1 & -1.29 & $\mathrm{C} 06 \mathrm{H} 2.7$ & -1.27 & C29G2.3 & -1.25 & eif-2D & -1.23 \\
\hline F10A3.4 & -1.29 & R02D5.8 & -1.27 & fipr-6 & -1.25 & F38B6.4 & -1.23 \\
\hline ani-1 & -1.29 & F55A11.4 & -1.27 & C25E10.5 & -1.25 & F53F4.2 & -1.23 \\
\hline mei-2 & -1.29 & zipt-17 & -1.27 & gtr-1 & -1.25 & rpn-12 & -1.23 \\
\hline F25H9.6 & -1.29 & $\mathrm{H} 42 \mathrm{~K} 12.3$ & -1.27 & pup-3 & -1.25 & F13D12.5 & -1.23 \\
\hline K02G10.5 & -1.29 & hxk-1 & -1.27 & F21C10.10 & -1.25 & usp-39 & -1.23 \\
\hline erh-1 & -1.29 & F13C5.5 & -1.27 & T11B7.2 & -1.25 & ccch-3 & -1.23 \\
\hline T20G5.8 & -1.29 & H23N18.5 & -1.27 & F01G10.9 & -1.25 & tfg-1 & -1.23 \\
\hline ptr-16 & -1.29 & C34C12.6 & -1.27 & ZC513.7 & -1.25 & row-1 & -1.23 \\
\hline pdp-1 & -1.28 & ran-4 & -1.27 & C18D11.3 & -1.25 & C14F11.6 & -1.23 \\
\hline C08H9.15 & -1.28 & lec-1 & -1.27 & W02F12.2 & -1.25 & K08F8.7 & -1.23 \\
\hline F26E4.3 & -1.28 & C05E7.1 & -1.27 & rsp-1 & -1.25 & Y82E9BR.6 & -1.23 \\
\hline grl-12 & -1.28 & his-24 & -1.27 & spp-13 & -1.25 & M02D8.6 & -1.23 \\
\hline Y57G7A.1 & -1.28 & $\operatorname{csn}-1$ & -1.27 & ZK809.8 & -1.25 & mdt-6 & -1.23 \\
\hline C16C10.4 & -1.28 & JC8.7 & -1.27 & F27E5.9 & -1.25 & enol-1 & -1.23 \\
\hline T06A4.1 & -1.28 & acly-2 & -1.27 & grl-10 & -1.25 & ger-1 & -1.23 \\
\hline Y105C5B.5 & -1.28 & сур-14A5 & -1.27 & K12G11.6 & -1.25 & eya-1 & -1.23 \\
\hline D1007.10 & -1.28 & tab-1 & -1.27 & dad-1 & -1.25 & Y71H2AM.11 & -1.23 \\
\hline F55G1.1 & -1.28 & D1046.2 & -1.27 & C02E7.7 & -1.25 & $\mathrm{~F} 25 \mathrm{H} 2.4$ & -1.23 \\
\hline $\mathrm{CO} 8 \mathrm{H} 9.16$ & -1.28 & sod-2 & -1.27 & ddo-2 & -1.25 & M106.8 & -1.23 \\
\hline ugt-53 & -1.28 & thoc-5 & -1.27 & R12E2.14 & -1.25 & got-2.2 & -1.23 \\
\hline ooc-5 & -1.28 & gale-1 & -1.27 & C52D10.3 & -1.25 & mrps-28 & -1.23 \\
\hline tag-18 & -1.28 & pqn-37 & -1.26 & T10G3.3 & -1.25 & F52A8.1 & -1.23 \\
\hline hmg-12 & -1.28 & tag-72 & -1.26 & dnj-23 & -1.24 & mpst-1 & -1.23 \\
\hline Y38A10A.7 & -1.28 & Ipr-4 & -1.26 & kdp-1 & -1.24 & F13A7.11 & -1.22 \\
\hline grd-6 & -1.28 & F33E11.2 & -1.26 & pfk-1.1 & -1.24 & C38C6.3 & -1.22 \\
\hline nfyc-1 & -1.28 & $\mathrm{~T} 13 \mathrm{C} 2.2$ & -1.26 & T02H6.11 & -1.24 & K09A11.1 & -1.22 \\
\hline pgl-1 & -1.28 & W04A8.4 & -1.26 & D2063.1 & -1.24 & $\operatorname{lin}-42$ & -1.22 \\
\hline B0304.2 & -1.28 & сpi-1 & -1.26 & E02H9.3 & -1.24 & nlp-27 & -1.22 \\
\hline
\end{tabular}




\begin{tabular}{|c|c|c|c|c|c|c|c|}
\hline Gene & $\log 2 \mathrm{FC}$ & Gene & $\log 2 \mathrm{FC}$ & Gene & $\log 2 \mathrm{FC}$ & Gene & $\log 2 \mathrm{FC}$ \\
\hline svh-1 & -1.22 & Y106G6H.16 & -1.2 & T03D8.6 & -1.18 & Ism-4 & -1.16 \\
\hline C55A6.1 & -1.22 & ubl-5 & -1.2 & F36G3.1 & -1.18 & E04D5.4 & -1.16 \\
\hline grd-16 & -1.22 & C54D10.13 & -1.2 & ZK1058.9 & -1.18 & M28.8 & -1.16 \\
\hline skr-2 & -1.22 & nprl-2 & -1.2 & C06G1.2 & -1.18 & Y54G2A.45 & -1.16 \\
\hline set-20 & -1.22 & nlp-24 & -1.2 & gna-1 & -1.18 & daao-1 & -1.16 \\
\hline F33A8.7 & -1.22 & $\operatorname{tag}-275$ & -1.2 & C10F3.7 & -1.18 & $\mathrm{~F} 25 \mathrm{H} 5.10$ & -1.16 \\
\hline ZK353.2 & -1.22 & nsun-4 & -1.2 & dhs-29 & -1.18 & B0035.3 & -1.16 \\
\hline mig-17 & -1.22 & cld-9 & -1.2 & Y108G3AL.2 & -1.18 & C03F11.2 & -1.16 \\
\hline F59F5.7 & -1.22 & Y34B4A.6 & -1.2 & plpp-1.3 & -1.18 & B0205.9 & -1.16 \\
\hline cysl-2 & -1.22 & gst-7 & -1.2 & T01C3.2 & -1.18 & ubc-23 & -1.16 \\
\hline $\mathrm{rsp}-2$ & -1.22 & Т25B9.9 & -1.2 & T01H8.2 & -1.18 & $\mathrm{fkb}-3$ & -1.16 \\
\hline F44D12.2 & -1.22 & ost-1 & -1.2 & C27F2.10 & -1.18 & F18E3.11 & -1.15 \\
\hline mek-5 & -1.22 & K07B1.4 & -1.2 & F09G2.8 & -1.18 & Y40A1A.3 & -1.15 \\
\hline W09G12.10 & -1.22 & doxa-1 & -1.2 & gras-1 & -1.18 & ZK813.6 & -1.15 \\
\hline acs-19 & -1.22 & T21C12.8 & -1.2 & zip-10 & -1.18 & Y65B4BL. 6 & -1.15 \\
\hline hil-3 & -1.21 & F25B4.4 & -1.2 & T26A5.8 & -1.18 & lips-9 & -1.15 \\
\hline pyp-1 & -1.21 & R07E3.2 & -1.2 & F10E9.3 & -1.17 & H14E04.2 & -1.15 \\
\hline H02F09.3 & -1.21 & $\mathrm{~F} 23 \mathrm{H} 12.5$ & -1.2 & R08D7.1 & -1.17 & rsp-3 & -1.15 \\
\hline Y73E7A.8 & -1.21 & pals-37 & -1.19 & R11H6.5 & -1.17 & mib-1 & -1.15 \\
\hline $\mathrm{C} 44 \mathrm{H} 9.7$ & -1.21 & C01G10.7 & -1.19 & hil-2 & -1.17 & wapl-1 & -1.15 \\
\hline unc-52 & -1.21 & tba- 6 & -1.19 & sgcb-1 & -1.17 & F10D2.10 & -1.15 \\
\hline cpn-4 & -1.21 & Y92H12BR.2 & -1.19 & let-721 & -1.17 & aco-2 & -1.15 \\
\hline mus-101 & -1.21 & wago-4 & -1.19 & C14B1.8 & -1.17 & T19D12.1 & -1.15 \\
\hline Y46H3A.4 & -1.21 & linc-17 & -1.19 & K02E7.5 & -1.17 & rpb-11 & -1.15 \\
\hline $\operatorname{lin}-35$ & -1.21 & T01D1.4 & -1.19 & T05B11.1 & -1.17 & $\operatorname{ttr}-8$ & -1.15 \\
\hline F37A4.3 & -1.21 & Irx-1 & -1.19 & B0507.1 & -1.17 & exc-12 & -1.15 \\
\hline jmjc-1 & -1.21 & R57.2 & -1.19 & tnt-2 & -1.17 & R07G3.7 & -1.15 \\
\hline $\mathrm{mlp}-1$ & -1.21 & K07C5.6 & -1.19 & hrde-2 & -1.17 & Y119D3B.12 & -1.15 \\
\hline $\operatorname{lin}-32$ & -1.21 & mam-3 & -1.19 & atad-3 & -1.17 & aipl-1 & -1.15 \\
\hline ZK930.6 & -1.21 & K08D8.6 & -1.19 & srh-2 & -1.17 & set-19 & -1.15 \\
\hline $\mathrm{C} 13 \mathrm{C} 12.2$ & -1.21 & F55A11.8 & -1.19 & sec-61.B & -1.17 & hsp-12.2 & -1.15 \\
\hline tyr-4 & -1.21 & Y51H7C.3 & -1.19 & mttu-1 & -1.16 & T05E7.1 & -1.15 \\
\hline T23F2.4 & -1.21 & F10D7.2 & -1.19 & arv-1 & -1.16 & $\mathrm{~F} 23 \mathrm{C} 8.5$ & -1.15 \\
\hline C06E7.88 & -1.21 & D1053.4 & -1.19 & alkb-7 & -1.16 & anmt-1 & -1.15 \\
\hline Y54G2A.76 & -1.2 & Y41D4B.6 & -1.19 & ptr-4 & -1.16 & pgp-6 & -1.15 \\
\hline F52E4.5 & -1.2 & fbxa-137 & -1.19 & hst-1 & -1.16 & rрa-1 & -1.15 \\
\hline T07C4.3 & -1.2 & C14C11.7 & -1.19 & ttr-51 & -1.16 & C07D10.5 & -1.15 \\
\hline tag-293 & -1.2 & T21D12.7 & -1.19 & C34B4. 2 & -1.16 & unc-95 & -1.15 \\
\hline srr-1 & -1.2 & npp-5 & -1.19 & $\mathrm{~F} 28 \mathrm{H} 7.8$ & -1.16 & ubc-18 & -1.15 \\
\hline K06G5.1 & -1.2 & Т19B10.5 & -1.18 & lin-9 & -1.16 & hut-1 & -1.15 \\
\hline smut-1 & -1.2 & zig-4 & -1.18 & Y53F4B.9 & -1.16 & rrn-3.56 & -1.15 \\
\hline daf-22 & -1.2 & tpi-1 & -1.18 & F08D12.2 & -1.16 & gst-19 & -1.15 \\
\hline
\end{tabular}




\begin{tabular}{|c|c|c|c|c|c|c|c|}
\hline Gene & $\log 2 \mathrm{FC}$ & Gene & $\log 2 \mathrm{FC}$ & Gene & $\log 2 \mathrm{FC}$ & Gene & $\log 2 \mathrm{FC}$ \\
\hline T05G5.1 & -1.15 & nhr-123 & -1.13 & F37C12.3 & -1.12 & F58G6.9 & -1.1 \\
\hline igcm-1 & -1.15 & D2005.6 & -1.13 & Y39G10AR.32 & -1.12 & mbf-1 & -1.1 \\
\hline Y73C8B.1 & -1.14 & R07E3.6 & -1.13 & linc-42 & -1.12 & $\operatorname{csn}-3$ & -1.1 \\
\hline F45E1.4 & -1.14 & pde-12 & -1.13 & hcf-1 & -1.12 & F56B3.2 & -1.09 \\
\hline let-653 & -1.14 & dlat-1 & -1.13 & C46G7.2 & -1.12 & Y39A1A.20 & -1.09 \\
\hline poml-2 & -1.14 & best-13 & -1.13 & C07D10.1 & -1.12 & F01G4.4 & -1.09 \\
\hline atn-1 & -1.14 & ZK688.9 & -1.13 & nud-1 & -1.12 & ubc-9 & -1.09 \\
\hline T28A8.6 & -1.14 & F20D1.3 & -1.13 & F21C10.7 & -1.12 & dvc-1 & -1.09 \\
\hline drsh-1 & -1.14 & Y38H6C.16 & -1.13 & nep-22 & -1.12 & K08E7.5 & -1.09 \\
\hline $\mathrm{nmy-1}$ & -1.14 & pafo-1 & -1.13 & rnp-2 & -1.12 & ZC416.2 & -1.09 \\
\hline wrt-6 & -1.14 & R02F2.8 & -1.13 & W06H8.2 & -1.11 & T20F5.4 & -1.09 \\
\hline Y57E12AL.6 & -1.14 & pgp-8 & -1.13 & zhp-3 & -1.11 & R07B1.8 & -1.09 \\
\hline T08G5.3 & -1.14 & taf-8 & -1.13 & F48E3.2 & -1.11 & frg-1 & -1.09 \\
\hline hic-1 & -1.14 & rpt-2 & -1.13 & clec- 80 & -1.11 & gst-4 & -1.09 \\
\hline him-8 & -1.14 & F42A10.9 & -1.13 & npp-2 & -1.11 & szy-5 & -1.09 \\
\hline snrp-27 & -1.14 & eaf-1 & -1.13 & $\mathrm{mls}-2$ & -1.11 & K08E4.2 & -1.09 \\
\hline sna-2 & -1.14 & pho-8 & -1.13 & F33E2.5 & -1.11 & fkh-2 & -1.09 \\
\hline $\mathrm{F} 39 \mathrm{H} 2.3$ & -1.14 & col-12 & -1.13 & linc- 4 & -1.11 & mat-2 & -1.09 \\
\hline cpn-1 & -1.14 & his-32 & -1.13 & npp-3 & -1.11 & set-5 & -1.09 \\
\hline coh-4 & -1.14 & ZC374.2 & -1.13 & W01A11.1 & -1.11 & F37C4.5 & -1.09 \\
\hline Isd-1 & -1.14 & gip-2 & -1.13 & B0001.2 & -1.11 & C36A4.4 & -1.09 \\
\hline F36H9.5 & -1.14 & F58E6.11 & -1.12 & ubxn-4 & -1.11 & T04F3.1 & -1.09 \\
\hline chmp-7 & -1.14 & cysl-1 & -1.12 & $\mathrm{~F} 45 \mathrm{H} 11.8$ & -1.11 & ugt-21 & -1.09 \\
\hline Ion-8 & -1.14 & sucl-2 & -1.12 & F21D9.6 & -1.11 & F21D5.1 & -1.09 \\
\hline dlc-2 & -1.14 & T07D4.5 & -1.12 & drh-1 & -1.11 & F09F7.6 & -1.09 \\
\hline B0495.8 & -1.14 & hse-5 & -1.12 & F14B4.1 & -1.11 & col-89 & -1.09 \\
\hline F26A1.1 & -1.14 & prx-19 & -1.12 & cpsf-4 & -1.11 & ugt-13 & -1.09 \\
\hline Y48G10A.2 & -1.14 & tomm-40 & -1.12 & hrpf-1 & -1.11 & eif-2Bbeta & -1.09 \\
\hline B0280.7 & -1.14 & rap-3 & -1.12 & nhr-145 & -1.11 & ina-1 & -1.09 \\
\hline F56C9.7 & -1.14 & pfn-2 & -1.12 & cpt-5 & -1.1 & snr-5 & -1.09 \\
\hline dgat-2 & -1.13 & Y37E3.30 & -1.12 & F39H11.1 & -1.1 & dhs-9 & -1.09 \\
\hline rpn-9 & -1.13 & wrt-9 & -1.12 & Y69E1A.5 & -1.1 & C15H9.11 & -1.09 \\
\hline B0365.9 & -1.13 & F54D5.12 & -1.12 & Y54G11A.4 & -1.1 & bag-1 & -1.09 \\
\hline sod-5 & -1.13 & Y73C8B.2 & -1.12 & fust-1 & -1.1 & col-172 & -1.08 \\
\hline ent-1 & -1.13 & usp-14 & -1.12 & nstp-10 & -1.1 & B0212.3 & -1.08 \\
\hline C02E7.6 & -1.13 & cutl-13 & -1.12 & nhr-23 & -1.1 & F45D3.3 & -1.08 \\
\hline algn-8 & -1.13 & W01C8.5 & -1.12 & F47B8. 5 & -1.1 & C33D9.13 & -1.08 \\
\hline C05D2.8 & -1.13 & ugt-16 & -1.12 & his-59 & -1.1 & F15B9.6 & -1.08 \\
\hline F56D6.16 & -1.13 & isw-1 & -1.12 & K08E4.3 & -1.1 & nduf-6 & -1.08 \\
\hline acox-1.1 & -1.13 & cts-1 & -1.12 & F53B3.3 & -1.1 & F42A9.6 & -1.08 \\
\hline K08E3.5 & -1.13 & ZK1055.6 & -1.12 & atp-1 & -1.1 & M01F1.4 & -1.08 \\
\hline F18C5.5 & -1.13 & viln-1 & -1.12 & sqv-3 & -1.1 & algn-10 & -1.08 \\
\hline
\end{tabular}




\begin{tabular}{|c|c|c|c|c|c|c|c|}
\hline Gene & $\log 2 \mathrm{FC}$ & Gene & $\log 2 \mathrm{FC}$ & Gene & $\log 2 \mathrm{FC}$ & Gene & $\log 2 \mathrm{FC}$ \\
\hline wdr-5.1 & -1.08 & Y66D12A.16 & -1.07 & mpdu-1 & -1.06 & clc-1 & -1.04 \\
\hline mam-5 & -1.08 & psr-1 & -1.07 & oac-7 & -1.06 & $\mathrm{~T} 13 \mathrm{C} 2.3$ & -1.04 \\
\hline T05C1.3 & -1.08 & C06E4.3 & -1.07 & pen-2 & -1.06 & eif-2Bgamma & -1.04 \\
\hline exos-2 & -1.08 & tmed-13 & -1.07 & cdh-8 & -1.06 & stl-1 & -1.04 \\
\hline efn-4 & -1.08 & mdt-10 & -1.07 & $\mathrm{rpb}-4$ & -1.06 & Y97E10AR.2 & -1.04 \\
\hline T09A5.15 & -1.08 & F48A11.4 & -1.07 & Y53C10A.6 & -1.05 & drh-3 & -1.04 \\
\hline C06E7.4 & -1.08 & F13B12.4 & -1.07 & R05D11.9 & -1.05 & ceh-91 & -1.04 \\
\hline T28C6.3 & -1.08 & gpx-5 & -1.07 & tag-296 & -1.05 & arx-4 & -1.04 \\
\hline Y54G2A.18 & -1.08 & ced-11 & -1.07 & cyk-7 & -1.05 & F58F9.4 & -1.04 \\
\hline pcp-5 & -1.08 & VW02B12L.2 & -1.07 & lec-9 & -1.05 & C47E12.2 & -1.04 \\
\hline tmem-17 & -1.08 & Y66D12A.7 & -1.07 & rcs-1 & -1.05 & cutl-14 & -1.04 \\
\hline F01F1.3 & -1.08 & acp-6 & -1.07 & rrn-3.1 & -1.05 & $\operatorname{cox}-18$ & -1.04 \\
\hline pfn-3 & -1.08 & Y34D9A.7 & -1.07 & col-61 & -1.05 & B0272.4 & -1.04 \\
\hline vps-28 & -1.08 & F13E9.15 & -1.07 & inx-13 & -1.05 & $\mathrm{~F} 13 \mathrm{H} 10.5$ & -1.04 \\
\hline enu-3.2 & -1.08 & zipt-1 & -1.07 & phf-30 & -1.05 & W02D7.5 & -1.04 \\
\hline damt-1 & -1.08 & tsp-7 & -1.07 & F22E12.3 & -1.05 & W04B5.3 & -1.04 \\
\hline rec-1 & -1.08 & D1007.5 & -1.07 & W04D2.4 & -1.05 & rig-4 & -1.04 \\
\hline F13D12.9 & -1.08 & Y46G5A.20 & -1.07 & F58A6.1 & -1.05 & Y92H12BL.5 & -1.04 \\
\hline F53F8.7 & -1.08 & spe-10 & -1.06 & $\mathrm{rbm}-7$ & -1.05 & F13A7.12 & -1.04 \\
\hline ztf-4 & -1.08 & cpn-2 & -1.06 & C30F12.2 & -1.05 & Y25C1A.13 & -1.04 \\
\hline F11A5.9 & -1.07 & $\mathrm{~T} 10 \mathrm{H} 10.2$ & -1.06 & C33F10.4 & -1.05 & F18E3.13 & -1.04 \\
\hline spcs-2 & -1.07 & cisd-3.2 & -1.06 & K08D12.6 & -1.05 & acox-1.5 & -1.04 \\
\hline C47F8.1 & -1.07 & Y64H9A.2 & -1.06 & C42D4.13 & -1.05 & snrp-40.2 & -1.04 \\
\hline acds-10 & -1.07 & B0303.3 & -1.06 & wip-1 & -1.05 & $\mathrm{ccm}-3$ & -1.03 \\
\hline gsy-1 & -1.07 & bed-3 & -1.06 & B0238.12 & -1.05 & cep-1 & -1.03 \\
\hline gasr-8 & -1.07 & hpo-26 & -1.06 & M7.12 & -1.05 & npp-13 & -1.03 \\
\hline bigr-1 & -1.07 & F32D8.5 & -1.06 & CzW-1 & -1.05 & cpg-7 & -1.03 \\
\hline C01A2.2 & -1.07 & nlp-63 & -1.06 & F01F1.15 & -1.05 & act-2 & -1.03 \\
\hline dnj-22 & -1.07 & ska-1 & -1.06 & clec-194 & -1.05 & alx-1 & -1.03 \\
\hline ran-1 & -1.07 & eri-5 & -1.06 & rab-11.2 & -1.05 & F49C12.9 & -1.03 \\
\hline nspe-4 & -1.07 & dlc-1 & -1.06 & Y75B8A.31 & -1.05 & gln-5 & -1.03 \\
\hline popl-7 & -1.07 & romo-1 & -1.06 & nbet-1 & -1.05 & vdac-1 & -1.03 \\
\hline bud-31 & -1.07 & C16C8.16 & -1.06 & T10E9.2 & -1.05 & $\mathrm{smr}-1$ & -1.03 \\
\hline fbxa-50 & -1.07 & sdz-27 & -1.06 & F53C3.3 & -1.05 & gst-37 & -1.03 \\
\hline F39H12.1 & -1.07 & srf-3 & -1.06 & Y37E11AL.3 & -1.05 & dnj-18 & -1.03 \\
\hline C18B2.3 & -1.07 & hpo-18 & -1.06 & Y48G1BR.1 & -1.05 & hpr-9 & -1.03 \\
\hline T07F10.3 & -1.07 & cuc-1 & -1.06 & nhr-63 & -1.04 & bcl-7 & -1.03 \\
\hline $\mathrm{mbd}-2$ & -1.07 & Ism-8 & -1.06 & hsb-1 & -1.04 & epi-1 & -1.03 \\
\hline ZK177.8 & -1.07 & col-10 & -1.06 & $r d y-2$ & -1.04 & zyg-1 & -1.03 \\
\hline egl-46 & -1.07 & cec-7 & -1.06 & mrps-15 & -1.04 & K01D12.9 & -1.03 \\
\hline T19C9.8 & -1.07 & F20D6.11 & -1.06 & trpl-2 & -1.04 & mig-32 & -1.03 \\
\hline $\mathrm{F} 32 \mathrm{H} 2.10$ & -1.07 & nep-12 & -1.06 & C02F5.13 & -1.04 & cdh-5 & -1.03 \\
\hline
\end{tabular}




\begin{tabular}{|c|c|c|c|c|c|c|c|}
\hline Gene & $\log 2 \mathrm{FC}$ & Gene & $\log 2 \mathrm{FC}$ & Gene & $\log 2 \mathrm{FC}$ & Gene & $\log 2 \mathrm{FC}$ \\
\hline ctsa-3.1 & -1.03 & B0035.18 & -1.01 & epn-1 & 1 & car-1 & 1.02 \\
\hline F58D5.2 & -1.03 & trpa-1 & -1.01 & eif-3.D & 1 & Y6B3В.9 & 1.02 \\
\hline emc-5 & -1.03 & spr-3 & -1.01 & gck-3 & 1 & C18F10.7 & 1.02 \\
\hline madf-8 & -1.03 & mrt-2 & -1.01 & pus-1 & 1 & pqn-41 & 1.02 \\
\hline mtd-1 & -1.03 & algn-13 & -1.01 & btb-14 & 1.01 & pll-1 & 1.02 \\
\hline $\operatorname{lin}-3$ & -1.03 & vha-11 & -1.01 & K10G6.9 & 1.01 & clec-7 & 1.02 \\
\hline letm-1 & -1.03 & Y62F5A.12 & -1.01 & Igc-50 & 1.01 & Y18H1A.11 & 1.02 \\
\hline $\mathrm{K} 03 \mathrm{H} 1.13$ & -1.03 & wrt-2 & -1.01 & $\mathrm{C} 07 \mathrm{H} 4.1$ & 1.01 & dhs-31 & 1.02 \\
\hline ins-11 & -1.02 & mrpl-19 & -1.01 & gopc-1 & 1.01 & magi-1 & 1.02 \\
\hline linc-72 & -1.02 & T12B3.2 & -1.01 & Y37A1A.3 & 1.01 & T07D1.2 & 1.02 \\
\hline tsfm-1 & -1.02 & nol-58 & -1.01 & M04B2.7 & 1.01 & $\mathrm{~F} 53 \mathrm{H} 2.1$ & 1.02 \\
\hline M110.10 & -1.02 & emc-1 & -1.01 & glc-1 & 1.01 & abf-5 & 1.02 \\
\hline R12E2.15 & -1.02 & nex-3 & -1.01 & fbxa-55 & 1.01 & kvs-5 & 1.03 \\
\hline gtf-2H3 & -1.02 & ZK1248.19 & -1.01 & rpl-6 & 1.01 & pcrg-1 & 1.03 \\
\hline Y39A3CL.4 & -1.02 & F22F7.7 & -1.01 & F25B3.4 & 1.01 & arrd-22 & 1.03 \\
\hline nas-33 & -1.02 & C18H9.6 & -1.01 & nhr-54 & 1.01 & fpn-1.1 & 1.03 \\
\hline $\mathrm{K} 11 \mathrm{H} 12.11$ & -1.02 & mlc-1 & -1.01 & cla-1 & 1.01 & B0222.11 & 1.03 \\
\hline gst-27 & -1.02 & fecl-1 & -1.01 & Y34D9A.8 & 1.01 & ubq-2 & 1.03 \\
\hline trap-3 & -1.02 & F15G9.1 & -1.01 & gos-28 & 1.01 & reps-1 & 1.03 \\
\hline Y48G10A.1 & -1.02 & nars-1 & -1.01 & gur-4 & 1.01 & kvs-4 & 1.03 \\
\hline F22E5.6 & -1.02 & B0310.2 & -1.01 & K06A9.2 & 1.01 & trpp-12 & 1.03 \\
\hline M04C9.1 & -1.02 & T05C1.1 & -1 & ilcr-1 & 1.01 & pek-1 & 1.03 \\
\hline eri-1 & -1.02 & polk-1 & -1 & unc-16 & 1.01 & F57B9.3 & 1.03 \\
\hline ZK909.3 & -1.02 & Y38A10A.2 & -1 & T25B2.2 & 1.01 & D2005.3 & 1.03 \\
\hline pccb-1 & -1.02 & Y43F8C.13 & -1 & clh-6 & 1.01 & T05A8.5 & 1.03 \\
\hline mex-3 & -1.02 & metl-9 & -1 & tag-180 & 1.01 & Y71H2B.4 & 1.03 \\
\hline F59B2.9 & -1.02 & pes-5 & -1 & iars-2 & 1.01 & $\mathrm{~F} 52 \mathrm{H} 2.1$ & 1.03 \\
\hline grd-5 & -1.02 & cpar-1 & -1 & kynu-1 & 1.01 & srd-35 & 1.03 \\
\hline mdt-11 & -1.02 & nhx-9 & -1 & Y39E4B.10 & 1.01 & Y41E3.6 & 1.03 \\
\hline C16C8.4 & -1.02 & Y37A1A.2 & -1 & C16A11.3 & 1.01 & rpm-1 & 1.03 \\
\hline Isy-13 & -1.02 & T23F2.3 & -1 & F46F11.1 & 1.02 & fbxa-146 & 1.03 \\
\hline hsp-110 & -1.02 & rps-8 & 1 & cept-1 & 1.02 & rps-23 & 1.03 \\
\hline K08F4.3 & -1.02 & qars-1 & 1 & spat-2 & 1.02 & F21D5.5 & 1.03 \\
\hline K08D9.4 & -1.02 & trpp-9 & 1 & pigw-1 & 1.02 & F23B12.4 & 1.03 \\
\hline slc-17.2 & -1.02 & unc-96 & 1 & nlp-40 & 1.02 & K12C11.3 & 1.03 \\
\hline $\mathrm{T} 20 \mathrm{H} 4.5$ & -1.02 & supr-1 & 1 & C25G6.3 & 1.02 & ZC116.1 & 1.03 \\
\hline ufd-3 & -1.02 & linc-91 & 1 & unc-10 & 1.02 & F19C7.2 & 1.03 \\
\hline nra-2 & -1.02 & col-153 & 1 & Y56АЗА.2 & 1.02 & fln-2 & 1.03 \\
\hline natc-2 & -1.02 & fbxa-156 & 1 & Y43C5A.7 & 1.02 & unc-75 & 1.03 \\
\hline acr-8 & -1.02 & snt-7 & 1 & gld-4 & 1.02 & F15G9.5 & 1.03 \\
\hline F49E11.2 & -1.02 & clec-205 & 1 & aat- 6 & 1.02 & Y56A3A.30 & 1.03 \\
\hline blos-1 & -1.02 & fbxa-157 & 1 & Y71H10B.1 & 1.02 & cct-1 & 1.03 \\
\hline
\end{tabular}




\begin{tabular}{|c|c|c|c|c|c|c|c|}
\hline Gene & $\log 2 \mathrm{FC}$ & Gene & $\log 2 \mathrm{FC}$ & Gene & $\log 2 \mathrm{FC}$ & Gene & $\log 2 \mathrm{FC}$ \\
\hline ssl-1 & 1.03 & $\mathrm{~F} 49 \mathrm{C} 12.10$ & 1.05 & linc-109 & 1.07 & obr-1 & 1.08 \\
\hline dop-2 & 1.03 & cdo-1 & 1.05 & F28H6.6 & 1.07 & acs-22 & 1.08 \\
\hline pik-1 & 1.03 & T07D3.4 & 1.05 & T05G5.5 & 1.07 & aka-1 & 1.08 \\
\hline ZK287.9 & 1.04 & Y50D4C.5 & 1.05 & R10F2.6 & 1.07 & dlk-1 & 1.08 \\
\hline vps-26 & 1.04 & R08F11.7 & 1.05 & $\mathrm{ckr}-2$ & 1.07 & Y67D8A.2 & 1.08 \\
\hline klp-16 & 1.04 & sulp-4 & 1.05 & F10D7.5 & 1.07 & abts-3 & 1.08 \\
\hline Y71A12B.18 & 1.04 & math-28 & 1.05 & itr-1 & 1.07 & C01B10.6 & 1.08 \\
\hline sre-23 & 1.04 & T22D1.3 & 1.05 & rme-4 & 1.07 & unc-104 & 1.08 \\
\hline nhr-79 & 1.04 & elt-3 & 1.05 & wnk-1 & 1.07 & C28D4.10 & 1.08 \\
\hline K10D3.10 & 1.04 & sulp-7 & 1.05 & F13H8.11 & 1.07 & R10E9.3 & 1.08 \\
\hline slc-25A26 & 1.04 & $\mathrm{C} 44 \mathrm{H} 4.6$ & 1.05 & Y40B10A.5 & 1.07 & lev-9 & 1.08 \\
\hline C05D12.7 & 1.04 & col-107 & 1.05 & ppfr-1 & 1.07 & stau-1 & 1.08 \\
\hline C47E12.12 & 1.04 & ZK1058.3 & 1.05 & T26G10.5 & 1.07 & apt-9 & 1.08 \\
\hline flp-7 & 1.04 & tmbi-4 & 1.05 & F40F9.10 & 1.07 & $\mathrm{rpl}-3$ & 1.08 \\
\hline F53A9.8 & 1.04 & Irp-1 & 1.05 & crh-2 & 1.07 & akt-2 & 1.08 \\
\hline Y41D4A.6 & 1.04 & K02E2.6 & 1.05 & K01A11.1 & 1.07 & flp-28 & 1.08 \\
\hline Y110A7A.21 & 1.04 & F43H9.4 & 1.05 & nlp-1 & 1.07 & F45F2.9 & 1.08 \\
\hline spe-48 & 1.04 & ctsa-4.2 & 1.05 & fbxa-199 & 1.07 & nrps-1 & 1.09 \\
\hline B0310.3 & 1.04 & best- 5 & 1.05 & T28D6.7 & 1.07 & seb-3 & 1.09 \\
\hline bet-1 & 1.04 & lin-7 & 1.05 & F13B12.3 & 1.07 & btbd-10 & 1.09 \\
\hline ins-18 & 1.04 & kcc-1 & 1.05 & F53B2.8 & 1.07 & fem-2 & 1.09 \\
\hline pha-1 & 1.04 & $\mathrm{rpl}-5$ & 1.05 & K11D12.8 & 1.07 & cdk-2 & 1.09 \\
\hline F32B4.4 & 1.04 & dhcr-7 & 1.06 & $\mathrm{mdl}-1$ & 1.07 & srw-85 & 1.09 \\
\hline rpl-4 & 1.04 & rrf-2 & 1.06 & F28B12.6 & 1.07 & ZK836.3 & 1.09 \\
\hline H06I04.6 & 1.04 & Y105E8A.25 & 1.06 & vamp-7 & 1.07 & K10D11.6 & 1.09 \\
\hline nhr-41 & 1.04 & clec-178 & 1.06 & K07C11.7 & 1.08 & T19D7.6 & 1.09 \\
\hline scav- 6 & 1.04 & rimb-1 & 1.06 & $\mathrm{dbl}-1$ & 1.08 & arrd-21 & 1.09 \\
\hline mecr-1 & 1.04 & $\mathrm{~F} 26 \mathrm{H} 9.5$ & 1.06 & $\mathrm{mlc}-3$ & 1.08 & F49D11.2 & 1.09 \\
\hline tdo-2 & 1.04 & kin-19 & 1.06 & glc-3 & 1.08 & Y119D3B.13 & 1.09 \\
\hline K07E1.1 & 1.05 & pde-2 & 1.06 & srg-34 & 1.08 & C39F7.5 & 1.09 \\
\hline drn-1 & 1.05 & C55A6.10 & 1.06 & F01E11.18 & 1.08 & F57A8.1 & 1.09 \\
\hline$a b f-2$ & 1.05 & $\mathrm{C} 30 \mathrm{H} 6.12$ & 1.06 & mig-18 & 1.08 & cdc-14 & 1.09 \\
\hline glr-4 & 1.05 & Y67H2A.7 & 1.06 & larp-5 & 1.08 & F07F6.7 & 1.09 \\
\hline nhr-14 & 1.05 & nhr-36 & 1.06 & Y53H1B.2 & 1.08 & del-6 & 1.09 \\
\hline Itah-1.2 & 1.05 & somi-1 & 1.06 & sta-1 & 1.08 & F57C7.4 & 1.09 \\
\hline fip-2 & 1.05 & unc-7 & 1.06 & nmur-1 & 1.08 & ссрр-6 & 1.09 \\
\hline F20G2.7 & 1.05 & F58D5.5 & 1.06 & sprr-3 & 1.08 & svh-2 & 1.09 \\
\hline dpf-6 & 1.05 & nfki-1 & 1.06 & fig-1 & 1.08 & F19G12.3 & 1.09 \\
\hline pup-2 & 1.05 & F31E3.2 & 1.06 & B0546.4 & 1.08 & Irp-2 & 1.09 \\
\hline $\mathrm{ncl}-1$ & 1.05 & rpl-7 & 1.06 & F27C1.11 & 1.08 & ncs-2 & 1.09 \\
\hline F11C7.7 & 1.05 & F41B4.2 & 1.06 & T12B3.1 & 1.08 & snx-6 & 1.09 \\
\hline gnrr-5 & 1.05 & ptc-1 & 1.07 & pkc-2 & 1.08 & odr-3 & 1.09 \\
\hline
\end{tabular}




\begin{tabular}{|c|c|c|c|c|c|c|c|}
\hline Gene & $\log 2 \mathrm{FC}$ & Gene & $\log 2 \mathrm{FC}$ & Gene & $\log 2 \mathrm{FC}$ & Gene & $\log 2 \mathrm{FC}$ \\
\hline lgc-26 & 1.09 & cfi-1 & 1.11 & glna-1 & 1.12 & ttr-23 & 1.14 \\
\hline tub-1 & 1.09 & F52C6.12 & 1.11 & $\mathrm{C} 38 \mathrm{H} 2.2$ & 1.12 & B0462.5 & 1.14 \\
\hline C49A1.10 & 1.09 & C49A9.9 & 1.11 & clip-1 & 1.12 & R11G1.6 & 1.14 \\
\hline mab-31 & 1.09 & F25E5.4 & 1.11 & F08D12.7 & 1.13 & F58H1.3 & 1.14 \\
\hline sfxn-1.5 & 1.09 & C04F12.8 & 1.11 & Y92H12BR.7 & 1.13 & Y23H5B.6 & 1.14 \\
\hline C18B2.5 & 1.09 & ercc-1 & 1.11 & cgt-2 & 1.13 & hlh-13 & 1.14 \\
\hline Y37H2C.1 & 1.09 & nhr-32 & 1.11 & zer-1 & 1.13 & ZC204.12 & 1.14 \\
\hline F52D1.2 & 1.1 & C25F9.5 & 1.11 & hpk-1 & 1.13 & rpac-19 & 1.15 \\
\hline pals- 25 & 1.1 & Y40H7A.10 & 1.11 & ahr-1 & 1.13 & $\mathrm{C} 05 \mathrm{C} 12.6$ & 1.15 \\
\hline crh-1 & 1.1 & pab-2 & 1.11 & flp-21 & 1.13 & T01E8.1 & 1.15 \\
\hline ocr-2 & 1.1 & F28F9.2 & 1.11 & C47B2.2 & 1.13 & R04E5.8 & 1.15 \\
\hline T19C3.7 & 1.1 & T28B8.3 & 1.11 & asp-1 & 1.13 & sbp-1 & 1.15 \\
\hline pmk-2 & 1.1 & faah-1 & 1.11 & T28C6.8 & 1.13 & marg-1 & 1.15 \\
\hline $\mathrm{F} 47 \mathrm{H} 4.2$ & 1.1 & flp-34 & 1.11 & ogdh-1 & 1.13 & R05A10.4 & 1.15 \\
\hline rps- 6 & 1.1 & Y23B4A.1 & 1.11 & M01G12.9 & 1.13 & efsc-1 & 1.15 \\
\hline ZK1127.5 & 1.1 & T12A7.6 & 1.11 & twk-46 & 1.13 & tcer-1 & 1.15 \\
\hline col-156 & 1.1 & rpl-9 & 1.11 & Igc-46 & 1.13 & Y41E3.22 & 1.15 \\
\hline ttIl-5 & 1.1 & spp-3 & 1.12 & ZC196.5 & 1.13 & C09D4.1 & 1.15 \\
\hline mctp-1 & 1.1 & fbxa-219 & 1.12 & nas-32 & 1.14 & tatn-1 & 1.15 \\
\hline F07H5.5 & 1.1 & T09B9.3 & 1.12 & eef-1G & 1.14 & T26C12.1 & 1.15 \\
\hline ZC123.4 & 1.1 & nhr-110 & 1.12 & F38C2.4 & 1.14 & F40F4.7 & 1.15 \\
\hline F42C5.6 & 1.1 & C09G5.13 & 1.12 & $\mathrm{nmr}-2$ & 1.14 & pes-4 & 1.15 \\
\hline B0302.5 & 1.1 & $\mathrm{rpb}-9$ & 1.12 & K11D2.4 & 1.14 & nlp-13 & 1.15 \\
\hline C04G6.4 & 1.1 & T02H6.1 & 1.12 & spk-1 & 1.14 & mrpl-41 & 1.15 \\
\hline npr-26 & 1.1 & gmeb-1 & 1.12 & pqn-18 & 1.14 & aex-4 & 1.15 \\
\hline Y55D5A.1 & 1.1 & frpr-19 & 1.12 & R06F6.14 & 1.14 & olrn-1 & 1.15 \\
\hline C34F6.5 & 1.1 & txt-18 & 1.12 & T02D1.4 & 1.14 & pdf-1 & 1.15 \\
\hline frpr-16 & 1.1 & wdr-5.2 & 1.12 & Y43F8C.11 & 1.14 & сур-33E1 & 1.16 \\
\hline mlk-1 & 1.1 & madf-10 & 1.12 & nhr-42 & 1.14 & daf-15 & 1.16 \\
\hline tsct-1 & 1.1 & C08F1.8 & 1.12 & C30B5.7 & 1.14 & nhl-3 & 1.16 \\
\hline cyk-3 & 1.1 & nhr-95 & 1.12 & nhr-195 & 1.14 & daf-7 & 1.16 \\
\hline lin-1 & 1.1 & nlt-1 & 1.12 & pde-3 & 1.14 & slc-36.3 & 1.16 \\
\hline scpl-1 & 1.1 & linc-84 & 1.12 & C50A2.3 & 1.14 & suco-1 & 1.16 \\
\hline $\mathrm{F} 42 \mathrm{C} 5.4$ & 1.1 & fahd-1 & 1.12 & clec- 224 & 1.14 & snt-2 & 1.16 \\
\hline tba-7 & 1.11 & nipa-1 & 1.12 & ent-2 & 1.14 & Y44A6D.3 & 1.16 \\
\hline pdf-2 & 1.11 & T01B4.3 & 1.12 & ncr-1 & 1.14 & dgk-3 & 1.16 \\
\hline H10E21.1 & 1.11 & C17E4.10 & 1.12 & Y55F3BR.10 & 1.14 & sut-2 & 1.16 \\
\hline C01G5.25 & 1.11 & C11G10.1 & 1.12 & $\mathrm{H} 05 \mathrm{C} 05.1$ & 1.14 & mboa-2 & 1.16 \\
\hline mef-2 & 1.11 & slc-25A29 & 1.12 & serp-1.1 & 1.14 & Y43F8B.11 & 1.16 \\
\hline F23F12.13 & 1.11 & ccdc- 55 & 1.12 & C01G6.4 & 1.14 & Y71H2B.8 & 1.16 \\
\hline R11G10.3 & 1.11 & sro-1 & 1.12 & col-147 & 1.14 & osm-7 & 1.16 \\
\hline F13D12.10 & 1.11 & $\mathrm{rcq}-5$ & 1.12 & F25B3.5 & 1.14 & cyl-1 & 1.16 \\
\hline
\end{tabular}




\begin{tabular}{|c|c|c|c|c|c|c|c|}
\hline Gene & $\log 2 \mathrm{FC}$ & Gene & $\log 2 \mathrm{FC}$ & Gene & $\log 2 \mathrm{FC}$ & Gene & $\log 2 \mathrm{FC}$ \\
\hline F54F7.2 & 1.16 & $\mathrm{mbl}-1$ & 1.18 & unc-77 & 1.2 & Y77E11A.14 & 1.22 \\
\hline C07G1.15 & 1.16 & $\mathrm{~F} 23 \mathrm{H} 11.7$ & 1.18 & C44C1.6 & 1.2 & nhr-206 & 1.22 \\
\hline eif-3.F & 1.16 & rskn-1 & 1.18 & C44E4.5 & 1.2 & nlp-21 & 1.22 \\
\hline clec-55 & 1.16 & osm-11 & 1.18 & twk-31 & 1.2 & cca-1 & 1.22 \\
\hline Y38C1AA.6 & 1.16 & F35E12.4 & 1.18 & ZC443.2 & 1.2 & rskn-2 & 1.22 \\
\hline C25E10.13 & 1.16 & elt-7 & 1.18 & Y39A1A.22 & 1.2 & pxmp-4 & 1.22 \\
\hline Y39G8B.9 & 1.17 & W04C9.8 & 1.18 & fbxa-4 & 1.2 & C14C11.4 & 1.22 \\
\hline cof-2 & 1.17 & grk-2 & 1.18 & C25F9.14 & 1.2 & cdap-2 & 1.22 \\
\hline tct-1 & 1.17 & dop-3 & 1.18 & F54G2.1 & 1.2 & tftc-1 & 1.22 \\
\hline C30F12.3 & 1.17 & emc-3 & 1.18 & rog-1 & 1.2 & B0222.1 & 1.22 \\
\hline MTCE.33 & 1.17 & ZK809.9 & 1.18 & rps-16 & 1.2 & M01E5.2 & 1.22 \\
\hline hex-3 & 1.17 & hrg-1 & 1.18 & srh-271 & 1.2 & serp-1.2 & 1.22 \\
\hline glb-11 & 1.17 & Y71F9AL.10 & 1.18 & twk-42 & 1.2 & Y11D7A.8 & 1.22 \\
\hline ghi-1 & 1.17 & F21D12.3 & 1.18 & sup-9 & 1.2 & H04M03.12 & 1.22 \\
\hline Y62H9A.13 & 1.17 & C49A9.4 & 1.18 & pro-2 & 1.2 & ZK822.6 & 1.22 \\
\hline lgc-49 & 1.17 & F25B5.3 & 1.18 & nhr-177 & 1.2 & Y40B1A.3 & 1.22 \\
\hline kcnl-2 & 1.17 & clp-1 & 1.18 & jkk-1 & 1.2 & uev-1 & 1.22 \\
\hline rrc-1 & 1.17 & smk-1 & 1.19 & M01F1.9 & 1.2 & C51E3.10 & 1.22 \\
\hline islo-1 & 1.17 & nlp-35 & 1.19 & ZK1127.12 & 1.2 & R04E5.9 & 1.22 \\
\hline sam-10 & 1.17 & F36H5.10 & 1.19 & cest-9.2 & 1.2 & M116.2 & 1.22 \\
\hline ccch-1 & 1.17 & Y39A1A.3 & 1.19 & F20C5.5 & 1.2 & pfas-1 & 1.22 \\
\hline F09F7.5 & 1.17 & sms-1 & 1.19 & F26A3.4 & 1.2 & pgrn-1 & 1.22 \\
\hline mask-1 & 1.17 & svh-5 & 1.19 & sop-2 & 1.2 & fbxa-95 & 1.22 \\
\hline D2030.3 & 1.17 & npr-31 & 1.19 & M03C11.3 & 1.21 & F41E7.6 & 1.22 \\
\hline T19D2.3 & 1.17 & sad-1 & 1.19 & aakg-3 & 1.21 & F37B12.3 & 1.22 \\
\hline$x b x-3$ & 1.17 & pcp-3 & 1.19 & catp-5 & 1.21 & Y105C5A.15 & 1.22 \\
\hline rgs-4 & 1.17 & gta-1 & 1.19 & ags-3 & 1.21 & twk-23 & 1.22 \\
\hline F52C12.6 & 1.17 & nep-21 & 1.19 & Y17D7C.2 & 1.21 & Y105C5A.24 & 1.22 \\
\hline C26E1.2 & 1.17 & pitp-1 & 1.19 & frpr-11 & 1.21 & rbm-34 & 1.22 \\
\hline Y71H2B.1 & 1.17 & col-129 & 1.19 & K06A4.7 & 1.21 & Y9C12A.1 & 1.23 \\
\hline C27A7.5 & 1.17 & C35D10.17 & 1.19 & K07E3.1 & 1.21 & pdfr-1 & 1.23 \\
\hline din-1 & 1.17 & C25F9.11 & 1.19 & $\mathrm{~T} 16 \mathrm{H} 12.3$ & 1.21 & icmt-1 & 1.23 \\
\hline K09C4.1 & 1.17 & gра-9 & 1.19 & flp-26 & 1.21 & $\mathrm{Y} 69 \mathrm{H} 2.10$ & 1.23 \\
\hline C31H5.1 & 1.17 & F10G2.1 & 1.19 & atg-16.2 & 1.21 & srbc-20 & 1.23 \\
\hline T26C5.3 & 1.18 & C49A9.3 & 1.19 & aexr-1 & 1.21 & rpac-40 & 1.23 \\
\hline ubc-3 & 1.18 & rab-14 & 1.19 & $\operatorname{lin}-45$ & 1.21 & sbt-1 & 1.23 \\
\hline $\mathrm{fbl}-1$ & 1.18 & old-1 & 1.19 & clec-92 & 1.21 & hlh-10 & 1.23 \\
\hline $\mathrm{F} 34 \mathrm{H} 10.3$ & 1.18 & scrm-2 & 1.2 & C06A5.3 & 1.21 & F37C12.21 & 1.23 \\
\hline $\mathrm{T} 25 \mathrm{C} 12.12$ & 1.18 & Y47H10A.4 & 1.2 & cdd-1 & 1.21 & clec-143 & 1.23 \\
\hline acy-2 & 1.18 & R05D3.12 & 1.2 & Imtr-5 & 1.21 & F28C1.3 & 1.23 \\
\hline $\mathrm{F} 22 \mathrm{H} 10.6$ & 1.18 & F10G2.7 & 1.2 & C09B9.1 & 1.21 & gldc-1 & 1.23 \\
\hline M02E1.1 & 1.18 & sprr-2 & 1.2 & zig-5 & 1.22 & ugt-58 & 1.23 \\
\hline
\end{tabular}




\begin{tabular}{|c|c|c|c|c|c|c|c|}
\hline Gene & $\log 2 \mathrm{FC}$ & Gene & $\log 2 \mathrm{FC}$ & Gene & $\log 2 \mathrm{FC}$ & Gene & $\log 2 \mathrm{FC}$ \\
\hline F58B3.4 & 1.23 & mrpl-47 & 1.25 & F59C6.16 & 1.27 & klp-15 & 1.29 \\
\hline T05C12.8 & 1.23 & slcf-2 & 1.25 & nlp-53 & 1.27 & F17C8.3 & 1.29 \\
\hline cbp-1 & 1.23 & K09F6.6 & 1.25 & Y67A10A.7 & 1.27 & dao-5 & 1.29 \\
\hline $\mathrm{mgl}-3$ & 1.23 & F35F10.1 & 1.25 & Y4С6B. 2 & 1.27 & $\mathrm{C} 17 \mathrm{H} 11.6$ & 1.3 \\
\hline glb-19 & 1.24 & ugt-49 & 1.26 & bcl-11 & 1.28 & fbxa-222 & 1.3 \\
\hline gcst-1 & 1.24 & T05A12.3 & 1.26 & $\mathrm{mlc}-2$ & 1.28 & ife-1 & 1.3 \\
\hline T28B11.1 & 1.24 & tbc-14 & 1.26 & F29B9.12 & 1.28 & K02E11.5 & 1.3 \\
\hline M03D4.3 & 1.24 & R102.4 & 1.26 & gld-2 & 1.28 & math-35 & 1.3 \\
\hline T09F3.2 & 1.24 & C07C7.1 & 1.26 & F08D12.3 & 1.28 & F58A6.2 & 1.3 \\
\hline Y67A10A.10 & 1.24 & ctns-1 & 1.26 & mrpl-9 & 1.28 & tag-280 & 1.3 \\
\hline kcc-2 & 1.24 & R151.6 & 1.26 & nlp-18 & 1.28 & srw-86 & 1.3 \\
\hline sri-30 & 1.24 & pgp-1 & 1.26 & W05F2.4 & 1.28 & nhr-10 & 1.3 \\
\hline frpr-13 & 1.24 & flp-32 & 1.26 & K03A11.5 & 1.28 & F47B8.3 & 1.3 \\
\hline amt-2 & 1.24 & glo-4 & 1.26 & snt-4 & 1.28 & F54E7.9 & 1.3 \\
\hline slcf-1 & 1.24 & sir-2.4 & 1.26 & yars-2 & 1.28 & T06A1.5 & 1.3 \\
\hline C04E6.13 & 1.24 & dhs- 1 & 1.26 & сур-33С6 & 1.28 & rpl-16 & 1.3 \\
\hline $\mathrm{C} 05 \mathrm{C} 12.4$ & 1.24 & lin-41 & 1.26 & cil-1 & 1.28 & glb-3 & 1.3 \\
\hline F32D1.8 & 1.24 & asna-2 & 1.26 & kcnl-1 & 1.28 & B0228.7 & 1.3 \\
\hline amt-3 & 1.24 & $\mathrm{R} 12 \mathrm{E} 2.13$ & 1.26 & nlp-68 & 1.28 & K04F10.7 & 1.3 \\
\hline hecd-1 & 1.24 & atg-2 & 1.26 & ins-17 & 1.29 & rab-39 & 1.3 \\
\hline flp-5 & 1.24 & T09B4.8 & 1.26 & rlbp-1 & 1.29 & let-70 & 1.3 \\
\hline C31C9.6 & 1.24 & alkb-8 & 1.26 & F46B6.5 & 1.29 & $\mathrm{crn}-2$ & 1.3 \\
\hline algn-7 & 1.24 & Y51H7BR.4 & 1.26 & ddl-2 & 1.29 & F18H3.1 & 1.3 \\
\hline egl-15 & 1.24 & math-34 & 1.26 & clec-36 & 1.29 & rga-6 & 1.3 \\
\hline ZK593.3 & 1.25 & enpl-1 & 1.26 & Y43F8A.1 & 1.29 & ZK1320.2 & 1.3 \\
\hline clh-2 & 1.25 & obr-4 & 1.26 & Y38C1AA.12 & 1.29 & R05G6.10 & 1.3 \\
\hline Y73ВЗА.3 & 1.25 & T25D10.1 & 1.26 & fbxa-172 & 1.29 & H40L08.3 & 1.3 \\
\hline F35H8.2 & 1.25 & W05F2.7 & 1.26 & clec-34 & 1.29 & $\operatorname{lgc}-12$ & 1.3 \\
\hline nhr-122 & 1.25 & piki-1 & 1.27 & mdt-27 & 1.29 & F42A10.6 & 1.3 \\
\hline Y39E4B.6 & 1.25 & let-526 & 1.27 & egl-45 & 1.29 & acl-2 & 1.31 \\
\hline C26B9.6 & 1.25 & acd-5 & 1.27 & pinn-4 & 1.29 & F46F2.5 & 1.31 \\
\hline let-19 & 1.25 & Ipd-3 & 1.27 & noca-1 & 1.29 & clec-60 & 1.31 \\
\hline Y51F10.3 & 1.25 & nlp-64 & 1.27 & Y55F3AM.13 & 1.29 & Y39G10AR.11 & 1.31 \\
\hline fbxa-3 & 1.25 & hpo-34 & 1.27 & ZC190.6 & 1.29 & Y59A8B.21 & 1.31 \\
\hline dsh-1 & 1.25 & F10E9.12 & 1.27 & $\mathrm{mpz}-1$ & 1.29 & $\operatorname{lgc}-38$ & 1.31 \\
\hline nhr-85 & 1.25 & osta-1 & 1.27 & nlp-42 & 1.29 & T04A6.1 & 1.31 \\
\hline cpna-5 & 1.25 & rps-2 & 1.27 & pals-38 & 1.29 & F58E2.3 & 1.31 \\
\hline fbxc-41 & 1.25 & $\mathrm{fbxc}-45$ & 1.27 & klu-2 & 1.29 & nhr-227 & 1.31 \\
\hline T04F8.3 & 1.25 & wdfy-3 & 1.27 & Y75B8A.7 & 1.29 & C47E8.3 & 1.31 \\
\hline rpl-2 & 1.25 & B0303.4 & 1.27 & arrd-14 & 1.29 & pyr-1 & 1.31 \\
\hline T13H5.1 & 1.25 & lin-49 & 1.27 & kqt-2 & 1.29 & asic-1 & 1.31 \\
\hline sre- 6 & 1.25 & $\mathrm{C} 17 \mathrm{H} 12.8$ & 1.27 & R12B2.2 & 1.29 & phat-3 & 1.31 \\
\hline
\end{tabular}




\begin{tabular}{|c|c|c|c|c|c|c|c|}
\hline Gene & $\log 2 \mathrm{FC}$ & Gene & $\log 2 \mathrm{FC}$ & Gene & $\log 2 \mathrm{FC}$ & Gene & $\log 2 \mathrm{FC}$ \\
\hline ZC47.11 & 1.31 & $\mathrm{fbxc}-6$ & 1.34 & cif-1 & 1.35 & gnrr-3 & 1.38 \\
\hline daf-12 & 1.31 & dpy-22 & 1.34 & F57C9.6 & 1.36 & F52G2.3 & 1.38 \\
\hline Y73F8A.32 & 1.31 & F47B10.8 & 1.34 & nhr-8 & 1.36 & ZK285.2 & 1.38 \\
\hline gcy-28 & 1.31 & T27C4.1 & 1.34 & zhit-2 & 1.36 & pqn-15 & 1.38 \\
\hline $\mathrm{C} 31 \mathrm{H} 2.3$ & 1.31 & nhr-87 & 1.34 & msi-1 & 1.36 & F23A7.3 & 1.38 \\
\hline F53C3.6 & 1.32 & cyld-1 & 1.34 & nep-2 & 1.36 & inos-1 & 1.38 \\
\hline W02H5.2 & 1.32 & miz-1 & 1.34 & F56F10.2 & 1.36 & twk-2 & 1.38 \\
\hline F57B10.8 & 1.32 & F15D4.4 & 1.34 & rgef-1 & 1.36 & $\mathrm{cnx}-1$ & 1.38 \\
\hline kin-29 & 1.32 & F16C3.2 & 1.34 & F22D6.2 & 1.36 & flr-4 & 1.38 \\
\hline C24G6.8 & 1.32 & C15H9.2 & 1.34 & memo-1 & 1.36 & B0393.9 & 1.38 \\
\hline sars-1 & 1.32 & djr-1.2 & 1.34 & fbxa-124 & 1.36 & plpp-1.1 & 1.38 \\
\hline C25G6.4 & 1.32 & npr-23 & 1.34 & linc-11 & 1.36 & ZC449.7 & 1.39 \\
\hline gсу-12 & 1.32 & nhr-101 & 1.34 & unc- 82 & 1.36 & fut-1 & 1.39 \\
\hline gei-4 & 1.32 & cpna-1 & 1.34 & asp-12 & 1.36 & nhr-138 & 1.39 \\
\hline Y34F4.1 & 1.32 & Ife-2 & 1.34 & F43D2.6 & 1.36 & nubp-1 & 1.39 \\
\hline F22D6.14 & 1.32 & gyf-1 & 1.34 & rpn-6.2 & 1.36 & mrps-5 & 1.39 \\
\hline C49F5.11 & 1.32 & str-112 & 1.34 & icap-1 & 1.36 & R02F2.7 & 1.39 \\
\hline ZK792.5 & 1.32 & C44E12.1 & 1.34 & M18.3 & 1.36 & flp-33 & 1.39 \\
\hline ctsa-4.1 & 1.32 & copz-1 & 1.34 & Y39B6A.3 & 1.36 & eif-2gamma & 1.39 \\
\hline ceeh-2 & 1.32 & F54F2.7 & 1.34 & F35G2.1 & 1.36 & flp-18 & 1.39 \\
\hline unc-14 & 1.32 & gap-3 & 1.34 & C08B6.10 & 1.36 & K09F5.6 & 1.39 \\
\hline tag-196 & 1.32 & nep-11 & 1.35 & irk-3 & 1.36 & W08G11.3 & 1.39 \\
\hline cyn-5 & 1.33 & C13B9.2 & 1.35 & F14B6.6 & 1.37 & $g \mid b-5$ & 1.39 \\
\hline C04F12.16 & 1.33 & T13F3.7 & 1.35 & rnh-1.0 & 1.37 & F40F8.4 & 1.39 \\
\hline Y45G5AM.7 & 1.33 & linc-96 & 1.35 & H09F14.1 & 1.37 & mig-1 & 1.4 \\
\hline gpx-3 & 1.33 & unc-132 & 1.35 & C37C3.2 & 1.37 & Y54E5A.5 & 1.4 \\
\hline $\mathrm{mxl}-1$ & 1.33 & T19С3.4 & 1.35 & Y71F9B.13 & 1.37 & oaz-1 & 1.4 \\
\hline osm-3 & 1.33 & M01H9.3 & 1.35 & R01B10.3 & 1.37 & pab-1 & 1.4 \\
\hline T27D12.6 & 1.33 & molo-1 & 1.35 & dmd-7 & 1.37 & F13B12.2 & 1.4 \\
\hline gсy-23 & 1.33 & ima-3 & 1.35 & C13C4.6 & 1.37 & flp-15 & 1.4 \\
\hline pqn-20 & 1.33 & F43G6.8 & 1.35 & nlp-82 & 1.37 & F46H5.7 & 1.4 \\
\hline F41E6.17 & 1.33 & sdc-3 & 1.35 & nlp-55 & 1.37 & C27D6.12 & 1.4 \\
\hline K02E11.9 & 1.33 & $3 / 5 / 2020$ & 1.35 & C33E10.8 & 1.37 & gab-1 & 1.4 \\
\hline ZK858.5 & 1.33 & Y39B6A.34 & 1.35 & W03A5.1 & 1.37 & K02F2.5 & 1.4 \\
\hline T24B8.7 & 1.33 & vps-22 & 1.35 & dlc-3 & 1.37 & Y111B2A.12 & 1.4 \\
\hline dab-1 & 1.33 & F46G10.2 & 1.35 & B0564.2 & 1.37 & daf-8 & 1.4 \\
\hline Y119D3B.14 & 1.33 & Ipin-1 & 1.35 & cap-2 & 1.37 & cal-2 & 1.4 \\
\hline F46B6.4 & 1.33 & $\mathrm{~F} 55 \mathrm{C} 12.5$ & 1.35 & T26H5.14 & 1.37 & T05C1.2 & 1.4 \\
\hline $\mathrm{T} 16 \mathrm{H} 12.9$ & 1.33 & $\mathrm{~F} 27 \mathrm{C} 1.2$ & 1.35 & egl-47 & 1.37 & B0250.4 & 1.4 \\
\hline drh-2 & 1.33 & VC5.2 & 1.35 & ins-1 & 1.37 & C56A3.5 & 1.4 \\
\hline R10F2.4 & 1.33 & Y92H12BM.1 & 1.35 & pef-1 & 1.37 & tyra-3 & 1.4 \\
\hline W05E10.5 & 1.34 & pmt-2 & 1.35 & Y57G11C.5 & 1.37 & epg-6 & 1.41 \\
\hline
\end{tabular}




\begin{tabular}{|c|c|c|c|c|c|c|c|}
\hline Gene & $\log 2 \mathrm{FC}$ & Gene & $\log 2 \mathrm{FC}$ & Gene & $\log 2 \mathrm{FC}$ & Gene & $\log 2 \mathrm{FC}$ \\
\hline T04C12.7 & 1.41 & K05C4.2 & 1.43 & npr-4 & 1.45 & kgb-2 & 1.47 \\
\hline M04G7.1 & 1.41 & fat-2 & 1.43 & F46B6.12 & 1.46 & $\mathrm{~T} 25 \mathrm{C} 12.11$ & 1.47 \\
\hline nhr-274 & 1.41 & C39D10.8 & 1.43 & $\mathrm{~T} 05 \mathrm{H} 4.10$ & 1.46 & npr-18 & 1.47 \\
\hline tre-5 & 1.41 & C26D10.4 & 1.43 & Y110A2AL.3 & 1.46 & $\mathrm{~K} 03 \mathrm{H} 1.11$ & 1.47 \\
\hline frm-4 & 1.41 & unc-54 & 1.43 & F36A2.14 & 1.46 & $\mathrm{C} 16 \mathrm{C} 10.2$ & 1.47 \\
\hline gei-3 & 1.41 & ntr-2 & 1.43 & C13C4.7 & 1.46 & C34D1.4 & 1.48 \\
\hline K11H12.9 & 1.41 & T22B7.8 & 1.43 & Y69A2AR.22 & 1.46 & C04F5.8 & 1.48 \\
\hline D1054.19 & 1.41 & $\mathrm{rbm}-28$ & 1.43 & nlp-15 & 1.46 & Y45G5AL.1 & 1.48 \\
\hline F44D12.6 & 1.41 & klp-13 & 1.43 & txt-5 & 1.46 & cytb-5.2 & 1.48 \\
\hline flp-4 & 1.41 & M03B6.5 & 1.43 & lgg-2 & 1.46 & rbf-1 & 1.48 \\
\hline F01G4.6 & 1.41 & pqn-92 & 1.43 & F19G12.9 & 1.46 & aakg-5 & 1.48 \\
\hline ttr-1 & 1.41 & nlp-43 & 1.43 & eif-2alpha & 1.46 & M176.11 & 1.48 \\
\hline rps-20 & 1.41 & mrpl-1 & 1.43 & $d d x-52$ & 1.46 & glb-21 & 1.48 \\
\hline C13F10.5 & 1.41 & E04F6.8 & 1.44 & egl-20 & 1.46 & C47E12.9 & 1.48 \\
\hline nuaf-1 & 1.41 & F36G3.3 & 1.44 & T23F2.2 & 1.46 & pcf-11 & 1.48 \\
\hline flp-2 & 1.41 & kin-16 & 1.44 & mltn-1 & 1.46 & C30G12.2 & 1.49 \\
\hline vps-20 & 1.41 & Y105E8A.1 & 1.44 & F32D8.13 & 1.46 & Y45F3A.9 & 1.49 \\
\hline F57B1.5 & 1.41 & smg-1 & 1.44 & alg-1 & 1.46 & mekk-3 & 1.49 \\
\hline hsp-12.3 & 1.41 & K12H4.7 & 1.44 & K12C11.6 & 1.46 & saeg-1 & 1.49 \\
\hline mca-2 & 1.42 & ttr-46 & 1.44 & pghm-1 & 1.46 & smg-2 & 1.49 \\
\hline twk-10 & 1.42 & dhs-17 & 1.44 & Y53F4B.1 & 1.46 & F29B9.1 & 1.49 \\
\hline pah-1 & 1.42 & Y47G6A.13 & 1.44 & gtf-2A2 & 1.46 & sul-2 & 1.49 \\
\hline aagr-1 & 1.42 & pqn-48 & 1.44 & $\mathrm{~F} 36 \mathrm{H} 2.3$ & 1.46 & gpx-6 & 1.49 \\
\hline $\mathrm{scl}-2$ & 1.42 & Y71H2B.11 & 1.44 & W08A12.2 & 1.47 & rla-0 & 1.49 \\
\hline сур-13A1 & 1.42 & C54C8.8 & 1.44 & T26G10.1 & 1.47 & pdcd-2 & 1.49 \\
\hline K10B3.5 & 1.42 & smi-1 & 1.44 & F41G3.18 & 1.47 & daf-16 & 1.5 \\
\hline T05A8.3 & 1.42 & F58B4.5 & 1.44 & hsp-6 & 1.47 & tos-1 & 1.5 \\
\hline nhr-152 & 1.42 & C45G7.13 & 1.44 & Y42G9A.2 & 1.47 & Y75B8A.14 & 1.5 \\
\hline Y49F6B.2 & 1.42 & $\mathrm{C} 04 \mathrm{H} 5.1$ & 1.44 & Y38F1A.1 & 1.47 & pfk-1.2 & 1.5 \\
\hline Y105C5A.8 & 1.42 & Iron-4 & 1.45 & F18C5.10 & 1.47 & E02H4.5 & 1.5 \\
\hline clec-86 & 1.42 & Y116A8C.40 & 1.45 & srsx-19 & 1.47 & F48E8.4 & 1.5 \\
\hline lis-1 & 1.42 & spp-10 & 1.45 & F55F8.2 & 1.47 & C32E12.4 & 1.5 \\
\hline ensa-1 & 1.42 & spin-4 & 1.45 & C54D10.3 & 1.47 & rpc-11 & 1.5 \\
\hline nft-1 & 1.42 & B0403.6 & 1.45 & F53H4.4 & 1.47 & Y50D4A.1 & 1.5 \\
\hline nstp-1 & 1.42 & F16B3.2 & 1.45 & C52B11.5 & 1.47 & pals-14 & 1.5 \\
\hline mlst-8 & 1.42 & Y38H6C.23 & 1.45 & $\operatorname{tr} x-2$ & 1.47 & B0035.13 & 1.5 \\
\hline Y41D4A.7 & 1.42 & ain-2 & 1.45 & W02G9.4 & 1.47 & lin-48 & 1.5 \\
\hline C06E1.9 & 1.42 & Y9С9A.8 & 1.45 & fipr-10 & 1.47 & rgs-2 & 1.5 \\
\hline kel-1 & 1.42 & Y39H10A.6 & 1.45 & trxr-1 & 1.47 & cbp-3 & 1.5 \\
\hline egl-5 & 1.43 & mlck-1 & 1.45 & F59B2.13 & 1.47 & ceh-14 & 1.5 \\
\hline arrd-18 & 1.43 & exos-8 & 1.45 & unc-13 & 1.47 & rpi-2 & 1.5 \\
\hline F35B3.7 & 1.43 & rev-3 & 1.45 & B0507.2 & 1.47 & F33D4.4 & 1.5 \\
\hline
\end{tabular}




\begin{tabular}{|c|c|c|c|c|c|c|c|}
\hline Gene & $\log 2 \mathrm{FC}$ & Gene & $\log 2 \mathrm{FC}$ & Gene & $\log 2 \mathrm{FC}$ & Gene & $\log 2 \mathrm{FC}$ \\
\hline plc-1 & 1.5 & nhr-190 & 1.53 & $\mathrm{ctl}-1$ & 1.56 & C32D5.11 & 1.58 \\
\hline Y41C4A.8 & 1.5 & npr-24 & 1.53 & $\mathrm{zmp}-4$ & 1.56 & gcy-21 & 1.59 \\
\hline flp-14 & 1.5 & ZK673.1 & 1.53 & abcf-3 & 1.56 & ears-1 & 1.59 \\
\hline flp-6 & 1.5 & F59E12.6 & 1.53 & $\mathrm{~F} 14 \mathrm{H} 12.8$ & 1.56 & kin-14 & 1.59 \\
\hline Y41D4B.1 & 1.51 & col-91 & 1.53 & cwp-5 & 1.56 & tub-2 & 1.59 \\
\hline F42F12.3 & 1.51 & $\mathrm{C} 05 \mathrm{C} 10.7$ & 1.53 & C05D11.1 & 1.56 & Y92H12A.2 & 1.59 \\
\hline ttr-24 & 1.51 & nceh-1 & 1.53 & F52E10.4 & 1.56 & Y54E5A.8 & 1.59 \\
\hline fozi-1 & 1.51 & C13A10.1 & 1.53 & F32A5.8 & 1.56 & nuc-1 & 1.59 \\
\hline rbpl-1 & 1.51 & nhr-100 & 1.53 & B0454.5 & 1.56 & F41E7.7 & 1.59 \\
\hline Y34B4A.5 & 1.51 & C10B5.3 & 1.53 & T03F6.9 & 1.56 & klo-1 & 1.59 \\
\hline klf-1 & 1.51 & spp-1 & 1.53 & F3OH5.4 & 1.56 & $\mathrm{~T} 21 \mathrm{H} 3.1$ & 1.59 \\
\hline rnt-1 & 1.51 & fbxa-99 & 1.53 & rpia-1 & 1.56 & zig-6 & 1.6 \\
\hline noa-1 & 1.51 & $\mathrm{~F} 22 \mathrm{H} 10.10$ & 1.53 & far- 7 & 1.56 & abhd-14 & 1.6 \\
\hline ceh-79 & 1.51 & trk-1 & 1.53 & pals-34 & 1.56 & dop-5 & 1.6 \\
\hline T10C6.6 & 1.51 & mdt-15 & 1.53 & glb-24 & 1.56 & sulp-5 & 1.6 \\
\hline pdzd-8 & 1.51 & T05A10.6 & 1.54 & Y8A9A.2 & 1.56 & haly-1 & 1.6 \\
\hline atg-9 & 1.51 & F49F1.14 & 1.54 & $\mathrm{C} 03 \mathrm{H} 5.7$ & 1.56 & Y18D10A.23 & 1.6 \\
\hline F52E1.5 & 1.51 & Y75B12B.3 & 1.54 & $\mathrm{C} 27 \mathrm{H} 5.4$ & 1.56 & igdb-2 & 1.6 \\
\hline K08D8.7 & 1.51 & chil-12 & 1.54 & F59F5.3 & 1.56 & F54D1.1 & 1.6 \\
\hline сур-13АЗ & 1.51 & F40G9.17 & 1.54 & nhr-144 & 1.57 & T02B11.3 & 1.6 \\
\hline scd-2 & 1.51 & flp-22 & 1.54 & eef-1A.1 & 1.57 & nhr-126 & 1.6 \\
\hline F49E2.2 & 1.52 & F54D5.7 & 1.54 & asm-3 & 1.57 & ZK180.7 & 1.6 \\
\hline $\mathrm{ikb}-1$ & 1.52 & C36A4.11 & 1.54 & $\mathrm{C} 18 \mathrm{H} 7.6$ & 1.57 & Y79H2A.12 & 1.6 \\
\hline fkh-7 & 1.52 & mod-1 & 1.54 & F02E11.7 & 1.57 & nlp-5 & 1.6 \\
\hline T10B11.5 & 1.52 & F09C6.1 & 1.54 & unc- -80 & 1.57 & C17G1.5 & 1.6 \\
\hline cct-2 & 1.52 & pfd-5 & 1.54 & egl-43 & 1.57 & F53B1.8 & 1.61 \\
\hline nstp-4 & 1.52 & glb-27 & 1.54 & byn-1 & 1.57 & $\mathrm{C} 44 \mathrm{H} 9.5$ & 1.61 \\
\hline spv-1 & 1.52 & C47D2.1 & 1.54 & rars-2 & 1.57 & Y61A9LA.1 & 1.61 \\
\hline C18A11.2 & 1.52 & F11C7.6 & 1.55 & mus-81 & 1.57 & lact-3 & 1.61 \\
\hline dgk-1 & 1.52 & T02B11.4 & 1.55 & bath-38 & 1.57 & rab-21 & 1.61 \\
\hline $\mathrm{C} 46 \mathrm{H} 11.10$ & 1.52 & nhr-96 & 1.55 & linc-35 & 1.57 & ggr-3 & 1.61 \\
\hline $\mathrm{fbxl}-1$ & 1.52 & ptr-13 & 1.55 & T03E6.8 & 1.58 & $\mathrm{~F} 17 \mathrm{H} 10.2$ & 1.61 \\
\hline rrp-1 & 1.52 & $\operatorname{trm}-2 \mathrm{~A}$ & 1.55 & ttr-39 & 1.58 & $\mathrm{C} 32 \mathrm{H} 11.3$ & 1.61 \\
\hline Y18D10A.9 & 1.52 & ceh-57 & 1.55 & Y20C6A.1 & 1.58 & Y92H12A.5 & 1.61 \\
\hline F32B5.6 & 1.52 & R119.5 & 1.55 & Y48G10A.3 & 1.58 & F58H1.7 & 1.61 \\
\hline ugt-46 & 1.52 & K08E5.1 & 1.55 & spp-18 & 1.58 & ufc-1 & 1.61 \\
\hline aakg-1 & 1.52 & C06G3.3 & 1.55 & Y70C5A.3 & 1.58 & CD4.1 & 1.61 \\
\hline R10E8.8 & 1.52 & pqn-25 & 1.55 & K07G5.5 & 1.58 & T07F12.1 & 1.61 \\
\hline F01D5.1 & 1.53 & T27A10.6 & 1.55 & deg-3 & 1.58 & F20B6.4 & 1.61 \\
\hline adr-1 & 1.53 & gcy-7 & 1.55 & W02D3.12 & 1.58 & fbxa-21 & 1.61 \\
\hline Y39H10B.2 & 1.53 & Y61A9LA.10 & 1.56 & ugt-18 & 1.58 & Y51H4A.8 & 1.61 \\
\hline R10E8.7 & 1.53 & egl-2 & 1.56 & F57C12.2 & 1.58 & afmd-1 & 1.61 \\
\hline
\end{tabular}




\begin{tabular}{|c|c|c|c|c|c|c|c|}
\hline Gene & $\log 2 \mathrm{FC}$ & Gene & $\log 2 \mathrm{FC}$ & Gene & $\log 2 \mathrm{FC}$ & Gene & $\log 2 \mathrm{FC}$ \\
\hline $\mathrm{C} 27 \mathrm{H} 5.2$ & 1.61 & Y105E8B.7 & 1.65 & dop-1 & 1.68 & ubc- 8 & 1.71 \\
\hline pro-3 & 1.61 & T02C5.1 & 1.65 & pdpr-1 & 1.68 & flp-12 & 1.72 \\
\hline T24C2.5 & 1.61 & ZK1240.9 & 1.66 & pkn-1 & 1.68 & spp-5 & 1.72 \\
\hline mlt-7 & 1.62 & gur-3 & 1.66 & C29F7.2 & 1.68 & F45E10.2 & 1.72 \\
\hline nhr-83 & 1.62 & hda-4 & 1.66 & nas-39 & 1.68 & C30G4.4 & 1.72 \\
\hline ZK1320.3 & 1.62 & sptf-2 & 1.66 & K02D10.4 & 1.68 & F46G10.1 & 1.72 \\
\hline vbh-1 & 1.62 & C05D11.8 & 1.66 & scl-14 & 1.69 & cest-31 & 1.72 \\
\hline F55A4.4 & 1.62 & mod-5 & 1.66 & sgk-1 & 1.69 & nhr-48 & 1.72 \\
\hline R08B4.4 & 1.62 & Y73E7A.1 & 1.66 & nhr-99 & 1.69 & M4.1 & 1.72 \\
\hline F36F2.7 & 1.62 & F15D4.2 & 1.66 & $\mathrm{mtl}-1$ & 1.69 & icd-2 & 1.72 \\
\hline $\operatorname{ttr}-29$ & 1.62 & cest- 25 & 1.66 & F11E6.6 & 1.69 & sul-3 & 1.72 \\
\hline ZC404.1 & 1.63 & ugt-52 & 1.66 & duxl-1 & 1.69 & egl-27 & 1.72 \\
\hline efk-1 & 1.63 & fbxa-185 & 1.66 & Y73В3А.18 & 1.69 & E04F6.9 & 1.72 \\
\hline tric-1B.1 & 1.63 & lido-13 & 1.66 & snap-29 & 1.69 & abcf-2 & 1.72 \\
\hline crtc-1 & 1.63 & Y53F4B.21 & 1.66 & hars-1 & 1.69 & fbxa-189 & 1.72 \\
\hline rncs-1 & 1.63 & ccdc-149 & 1.67 & let-23 & 1.69 & ifb-2 & 1.73 \\
\hline sel-8 & 1.63 & D1025.10 & 1.67 & ascc-1 & 1.69 & nhr-5 & 1.73 \\
\hline let-611 & 1.63 & C27B7.7 & 1.67 & egl-21 & 1.69 & klp-4 & 1.73 \\
\hline sma-9 & 1.64 & F35B12.3 & 1.67 & nlp-11 & 1.7 & dct-11 & 1.73 \\
\hline ZK1098.11 & 1.64 & F43C9.2 & 1.67 & sax-1 & 1.7 & K07D4.9 & 1.73 \\
\hline$m f n-1$ & 1.64 & T28C12.2 & 1.67 & R04E5.7 & 1.7 & K12B6.9 & 1.73 \\
\hline F40A3.7 & 1.64 & F17C11.4 & 1.67 & mrpl-37 & 1.7 & eif-3.I & 1.73 \\
\hline sma-10 & 1.64 & C33D9.3 & 1.67 & B0286.1 & 1.7 & uev-3 & 1.73 \\
\hline $\mathrm{fbxa}-140$ & 1.64 & sorf-2 & 1.67 & Y40B1B.7 & 1.7 & Y37F4.8 & 1.74 \\
\hline nlp-61 & 1.64 & Y71H2AM.2 & 1.67 & flp-3 & 1.7 & glo-3 & 1.74 \\
\hline F02E11.2 & 1.64 & atf-6 & 1.67 & tag-209 & 1.7 & nlp-80 & 1.74 \\
\hline F59C6.5 & 1.64 & dhs-20 & 1.67 & C38D4.7 & 1.7 & T09E11.11 & 1.74 \\
\hline ttr-10 & 1.64 & frpr-18 & 1.67 & Y73В3А.9 & 1.7 & daf-37 & 1.74 \\
\hline lipl-2 & 1.64 & Y54G2A.10 & 1.67 & cct-5 & 1.7 & cyn-2 & 1.74 \\
\hline egl-10 & 1.65 & nape-1 & 1.67 & egl-8 & 1.71 & hsp-90 & 1.74 \\
\hline mca-1 & 1.65 & ugt-55 & 1.67 & znfx-1 & 1.71 & glct-6 & 1.74 \\
\hline cct-7 & 1.65 & F40F12.9 & 1.67 & C08G9.1 & 1.71 & F02A9.1 & 1.74 \\
\hline F58G6.7 & 1.65 & gcy-35 & 1.67 & $\mathrm{C} 25 \mathrm{H} 3.11$ & 1.71 & nhr-232 & 1.75 \\
\hline fezf-1 & 1.65 & $\mathrm{C} 44 \mathrm{H} 4.8$ & 1.68 & kin-10 & 1.71 & F16B4.2 & 1.75 \\
\hline haf-4 & 1.65 & T03G11.3 & 1.68 & R10F2.5 & 1.71 & lgc- 25 & 1.75 \\
\hline$x d h-1$ & 1.65 & F13C5.1 & 1.68 & cest-35.1 & 1.71 & ser-4 & 1.75 \\
\hline cpx-2 & 1.65 & K07F5.15 & 1.68 & Y94H6A.5 & 1.71 & adpr-1 & 1.75 \\
\hline fbxa-74 & 1.65 & msrp-7 & 1.68 & lars-1 & 1.71 & F08H9.3 & 1.75 \\
\hline mboa-7 & 1.65 & dct-1 & 1.68 & Y75B7AL.2 & 1.71 & K04F10.1 & 1.75 \\
\hline $\operatorname{ttr}-40$ & 1.65 & Y39B6A.37 & 1.68 & irld-34 & 1.71 & C07F11.2 & 1.75 \\
\hline T24H7.2 & 1.65 & drd-5 & 1.68 & F10G8.9 & 1.71 & far-5 & 1.75 \\
\hline dcp-66 & 1.65 & T23B3.6 & 1.68 & ampd-1 & 1.71 & tom-1 & 1.75 \\
\hline
\end{tabular}




\begin{tabular}{|c|c|c|c|c|c|c|c|}
\hline Gene & $\log 2 \mathrm{FC}$ & Gene & $\log 2 \mathrm{FC}$ & Gene & $\log 2 \mathrm{FC}$ & Gene & $\log 2 \mathrm{FC}$ \\
\hline$n \times f-1$ & 1.75 & $\mathrm{ptl}-1$ & 1.78 & T13F2.6 & 1.82 & F20A1.6 & 1.87 \\
\hline C54F6.6 & 1.75 & adk-1 & 1.78 & pin-2 & 1.83 & zip-12 & 1.87 \\
\hline frm-7 & 1.75 & ugt-56 & 1.78 & Y71H2AM.12 & 1.83 & F09B12.3 & 1.87 \\
\hline clh-4 & 1.75 & nlp-9 & 1.79 & pde- 6 & 1.83 & aatf-1 & 1.87 \\
\hline F58G6.8 & 1.75 & F17B5.1 & 1.79 & glt-4 & 1.83 & dgk-5 & 1.87 \\
\hline $\mathrm{ccm}-2$ & 1.75 & linc-56 & 1.79 & pudl-2 & 1.83 & tsp-6 & 1.87 \\
\hline T26H5.8 & 1.76 & dhs-15 & 1.79 & Y32F6A.4 & 1.83 & Y110A2AL.9 & 1.87 \\
\hline C33G8.4 & 1.76 & C08F11.116 & 1.79 & nhr-128 & 1.84 & F53F4.14 & 1.87 \\
\hline сур-34A2 & 1.76 & ugt-54 & 1.79 & hizr-1 & 1.84 & C52E2.5 & 1.87 \\
\hline glt-7 & 1.76 & Y9C2UA.1 & 1.79 & lin-28 & 1.84 & osm-9 & 1.87 \\
\hline Y43C5A.2 & 1.76 & C29G2.6 & 1.79 & fars -3 & 1.84 & Y46G5A.15 & 1.88 \\
\hline F16C3.1 & 1.76 & Y73B6BL.31 & 1.79 & ZK355.8 & 1.84 & far-6 & 1.88 \\
\hline gрср-1 & 1.76 & twk-25 & 1.79 & Y92H12BL.4 & 1.84 & asna-1 & 1.88 \\
\hline T23G7.3 & 1.76 & C04E7.3 & 1.79 & F08F8.9 & 1.84 & cct-4 & 1.88 \\
\hline$z z z-1$ & 1.76 & aat-8 & 1.79 & ccdc- 47 & 1.84 & $\mathrm{C} 40 \mathrm{H} 1.9$ & 1.88 \\
\hline B0478.3 & 1.76 & ric-19 & 1.79 & flp-27 & 1.84 & ubc-15 & 1.88 \\
\hline plc-3 & 1.76 & nsy-1 & 1.79 & dop-6 & 1.84 & frk-1 & 1.88 \\
\hline pvf-1 & 1.76 & rpl-24.2 & 1.8 & moe-3 & 1.84 & rabx-5 & 1.88 \\
\hline nhr-90 & 1.76 & spp-14 & 1.8 & gfat-1 & 1.84 & C36B1.6 & 1.88 \\
\hline K03B4.1 & 1.76 & pals-19 & 1.8 & egl-3 & 1.84 & nhr-106 & 1.88 \\
\hline nlp-17 & 1.76 & nhr-64 & 1.8 & F13H8.1 & 1.85 & C29F5.3 & 1.88 \\
\hline dhp-1 & 1.76 & gba-3 & 1.8 & ZK131.11 & 1.85 & $\mathrm{crn}-3$ & 1.88 \\
\hline C34F6.11 & 1.77 & eri-12 & 1.8 & unk-1 & 1.85 & flp-16 & 1.88 \\
\hline K01A2.10 & 1.77 & slcr-46.1 & 1.8 & btb-9 & 1.85 & R151.1 & 1.88 \\
\hline npr-28 & 1.77 & T16G1.7 & 1.8 & gst- 6 & 1.85 & W06E11.1 & 1.88 \\
\hline bgal-2 & 1.77 & nlp-8 & 1.8 & shpk-1 & 1.85 & cab-1 & 1.88 \\
\hline W08F4.5 & 1.77 & Y39B6A.29 & 1.8 & Y41C4A.9 & 1.85 & $x b x-5$ & 1.89 \\
\hline clec-37 & 1.77 & ZK993.2 & 1.8 & F14D2.19 & 1.85 & R06C1.6 & 1.89 \\
\hline pgp-11 & 1.78 & acl-7 & 1.8 & eef-2 & 1.85 & T08G11.4 & 1.89 \\
\hline B0563.7 & 1.78 & psa-3 & 1.8 & Y67D2.5 & 1.85 & F55H12.7 & 1.89 \\
\hline Y57G11B.2 & 1.78 & K02A2.5 & 1.81 & Y32H12A.8 & 1.85 & T21F4.1 & 1.89 \\
\hline Igc-19 & 1.78 & H10D18.1 & 1.81 & hlh-11 & 1.85 & sek-4 & 1.89 \\
\hline K08D10.14 & 1.78 & F49H6.5 & 1.81 & unc-2 & 1.85 & сур-37A1 & 1.89 \\
\hline ech-7 & 1.78 & F47G3.1 & 1.81 & K11E4.1 & 1.86 & C42D8.1 & 1.89 \\
\hline npr-5 & 1.78 & T21B4.15 & 1.81 & col-184 & 1.86 & glb-26 & 1.9 \\
\hline ncs-3 & 1.78 & D2096.7 & 1.81 & $\mathrm{ftn}-1$ & 1.86 & $\mathrm{~F} 26 \mathrm{D} 11.12$ & 1.9 \\
\hline nlp-38 & 1.78 & Y116A8C.25 & 1.82 & gstk-2 & 1.86 & kcnl-3 & 1.9 \\
\hline apm-1 & 1.78 & crt-1 & 1.82 & K07H8.11 & 1.86 & F09G2.1 & 1.9 \\
\hline unc-63 & 1.78 & droe-4 & 1.82 & acr-23 & 1.86 & C01C10.2 & 1.9 \\
\hline aldo-2 & 1.78 & clec-63 & 1.82 & ZK930.2 & 1.86 & tig-2 & 1.9 \\
\hline fbxa-115 & 1.78 & frpr-8 & 1.82 & Y22D7AR.6 & 1.86 & eif-3.E & 1.9 \\
\hline col-160 & 1.78 & tat- 6 & 1.82 & ZC395.5 & 1.86 & $\mathrm{R} 01 \mathrm{H} 2.7$ & 1.9 \\
\hline
\end{tabular}




\begin{tabular}{|c|c|c|c|c|c|c|c|}
\hline Gene & $\log 2 \mathrm{FC}$ & Gene & $\log 2 \mathrm{FC}$ & Gene & $\log 2 \mathrm{FC}$ & Gene & $\log 2 \mathrm{FC}$ \\
\hline$m t x-2$ & 1.9 & Y47A7.2 & 1.95 & M163.1 & 2 & Y54F10AR.2 & 2.06 \\
\hline clec-198 & 1.9 & T06E6.1 & 1.95 & twk-12 & 2 & clec-17 & 2.06 \\
\hline H10D12.2 & 1.9 & pbo-6 & 1.95 & C35C5.9 & 2 & nlp-19 & 2.06 \\
\hline K09E4.4 & 1.91 & Y59C2A.1 & 1.95 & ZC8.6 & 2 & del-5 & 2.07 \\
\hline T25B9.1 & 1.91 & xpo-1 & 1.95 & R02F2.1 & 2 & C45G9.14 & 2.07 \\
\hline ugt-1 & 1.91 & $\mathrm{nhl}-2$ & 1.95 & C35B8.3 & 2.01 & F07E5.5 & 2.07 \\
\hline F32H5.1 & 1.91 & Y95B8A.6 & 1.95 & prmt-1 & 2.01 & twk-8 & 2.07 \\
\hline ugt-23 & 1.91 & ZK1128.7 & 1.95 & nrf-5 & 2.01 & $\mathrm{mtq}-2$ & 2.07 \\
\hline hgo-1 & 1.91 & ssu-1 & 1.95 & F02A9.7 & 2.01 & adss-1 & 2.07 \\
\hline F19C6.12 & 1.91 & pals-18 & 1.96 & des-2 & 2.01 & endu- 2 & 2.07 \\
\hline aex-5 & 1.91 & C25G4.2 & 1.96 & bath-43 & 2.01 & flp-9 & 2.07 \\
\hline aakb-2 & 1.91 & C54D10.10 & 1.96 & F41C3.7 & 2.02 & ubc-26 & 2.07 \\
\hline col-142 & 1.91 & pde-4 & 1.96 & F11E6.11 & 2.02 & W03D8.8 & 2.08 \\
\hline pry-1 & 1.91 & F31A3.3 & 1.96 & W07E6.2 & 2.02 & cdc- 48.3 & 2.08 \\
\hline gра-17 & 1.92 & cars-1 & 1.96 & ZK993.5 & 2.02 & sca-1 & 2.08 \\
\hline F54D11.4 & 1.92 & ida-1 & 1.96 & ptr-24 & 2.02 & clec-75 & 2.08 \\
\hline akap-1 & 1.92 & F13G3.6 & 1.96 & F11C7.2 & 2.02 & D1054.3 & 2.08 \\
\hline scl-24 & 1.92 & fbxa-175 & 1.97 & C11D2.3 & 2.03 & R09E10.13 & 2.08 \\
\hline К09H9.8 & 1.92 & F46A8.13 & 1.97 & irld-2 & 2.03 & $\mathrm{~F} 28 \mathrm{H} 7.2$ & 2.08 \\
\hline Y48С 3 A. 3 & 1.92 & C07B5.3 & 1.97 & $\mathrm{CO3H} 5.3$ & 2.03 & $\operatorname{tag}-229$ & 2.08 \\
\hline cnnm-3 & 1.92 & сур-33С3 & 1.97 & nst-1 & 2.03 & gsa-1 & 2.08 \\
\hline T22B7.3 & 1.92 & fbxa-163 & 1.97 & dhs-24 & 2.03 & coel-1 & 2.08 \\
\hline C38C3.4 & 1.93 & fbxa-10 & 1.97 & asp-14 & 2.03 & Y32B12C.5 & 2.08 \\
\hline cest-5.1 & 1.93 & ZK180.8 & 1.97 & math-27 & 2.03 & acs-16 & 2.09 \\
\hline memb-2 & 1.93 & fubl-1 & 1.97 & adt-3 & 2.03 & mop-25.1 & 2.09 \\
\hline fkh-9 & 1.93 & nape-2 & 1.97 & $\mathrm{E} 02 \mathrm{H} 4.4$ & 2.03 & cest-13 & 2.09 \\
\hline nhr-108 & 1.93 & gcy-11 & 1.97 & fnip-2 & 2.04 & ztf-7 & 2.09 \\
\hline ril-2 & 1.93 & R186.1 & 1.97 & nhr-221 & 2.04 & pals-32 & 2.09 \\
\hline mrps-11 & 1.93 & F08F3.4 & 1.97 & $\operatorname{tax}-6$ & 2.04 & ptp-1 & 2.09 \\
\hline eif-3.G & 1.93 & Y51H4A.7 & 1.98 & faah-6 & 2.04 & $\mathrm{C} 18 \mathrm{H} 7.1$ & 2.09 \\
\hline Y82E9BR.5 & 1.94 & F40G12.5 & 1.98 & nhr-55 & 2.04 & vps-33.1 & 2.09 \\
\hline Y51H4A.24 & 1.94 & D1044.7 & 1.98 & plc-2 & 2.04 & Y50D7A.8 & 2.1 \\
\hline srh-210 & 1.94 & M01E5.3 & 1.98 & Y56A3A.31 & 2.05 & dhs-7 & 2.1 \\
\hline$n c x-2$ & 1.94 & iff-2 & 1.99 & sqst-2 & 2.05 & nhr-219 & 2.1 \\
\hline ptr-21 & 1.94 & $\mathrm{R} 10 \mathrm{H} 1.1$ & 1.99 & smg-3 & 2.05 & rpom-1 & 2.1 \\
\hline C01C4.3 & 1.94 & ist-1 & 1.99 & chil-22 & 2.05 & F09G2.2 & 2.1 \\
\hline C33H5.13 & 1.94 & R02F11.2 & 1.99 & T02G5.14 & 2.06 & K09C8.7 & 2.11 \\
\hline nsun-2 & 1.94 & Y66H1A.5 & 1.99 & pgp-9 & 2.06 & nca-2 & 2.11 \\
\hline Y105C5A.17 & 1.94 & сур-35B1 & 1.99 & Y40B10B.1 & 2.06 & ZC443.3 & 2.11 \\
\hline Y39G8B.10 & 1.94 & aakg-4 & 2 & timm-23 & 2.06 & ZK512.2 & 2.11 \\
\hline ugt-2 & 1.94 & F08G12.11 & 2 & lipl-5 & 2.06 & F59A7.5 & 2.11 \\
\hline col-42 & 1.94 & W09C5.1 & 2 & M176.4 & 2.06 & F41E7.2 & 2.11 \\
\hline
\end{tabular}




\begin{tabular}{|c|c|c|c|c|c|c|c|}
\hline Gene & $\log 2 \mathrm{FC}$ & Gene & $\log 2 \mathrm{FC}$ & Gene & $\log 2 \mathrm{FC}$ & Gene & $\log 2 \mathrm{FC}$ \\
\hline F41E7.20 & 2.11 & ipla-7 & 2.18 & nhr-94 & 2.26 & R07C12.2 & 2.33 \\
\hline M01H9.4 & 2.11 & T24E12.10 & 2.18 & cps-6 & 2.26 & scl-5 & 2.33 \\
\hline afmd-2 & 2.12 & C35E7.6 & 2.19 & C14A11.2 & 2.26 & fbxa-69 & 2.33 \\
\hline ZK250.13 & 2.12 & fshr-1 & 2.19 & F10E7.5 & 2.26 & F36F2.2 & 2.33 \\
\hline $\mathrm{C} 25 \mathrm{H} 3.18$ & 2.12 & lite-1 & 2.19 & clec- 8 & 2.26 & T01C8.3 & 2.33 \\
\hline cat-4 & 2.12 & B0432.14 & 2.19 & catp-7 & 2.26 & C10E2.2 & 2.34 \\
\hline T09F5.12 & 2.12 & F41G4.7 & 2.2 & asp-5 & 2.26 & Y82E9BR.22 & 2.34 \\
\hline $\mathrm{F} 14 \mathrm{H} 8.4$ & 2.12 & Y54F10BM.12 & 2.2 & F37A8.5 & 2.26 & siah-1 & 2.34 \\
\hline glb-17 & 2.12 & moc-2 & 2.2 & nhr-210 & 2.27 & Y47G6A.7 & 2.34 \\
\hline Y113G7A.13 & 2.12 & exc-14 & 2.2 & Y6E2A.4 & 2.27 & tag-151 & 2.34 \\
\hline lips-15 & 2.12 & clec-82 & 2.2 & B0252.1 & 2.27 & $\mathrm{C} 39 \mathrm{H} 7.4$ & 2.34 \\
\hline peb-1 & 2.13 & Y7A9C.1 & 2.2 & ctn-1 & 2.27 & Y34F4.5 & 2.35 \\
\hline D2045.8 & 2.13 & R05A10.1 & 2.21 & $\mathrm{imb}-3$ & 2.27 & M04D8.7 & 2.35 \\
\hline Y49E10.16 & 2.13 & F26G1.10 & 2.21 & F56C4.4 & 2.27 & ZC239.16 & 2.35 \\
\hline F35B12.10 & 2.13 & chil-23 & 2.21 & F08A7.1 & 2.27 & $\mathrm{cpz}-2$ & 2.35 \\
\hline lact-2 & 2.14 & ZK813.4 & 2.21 & T28F3.8 & 2.27 & F21H7.2 & 2.35 \\
\hline Y43F8C.3 & 2.14 & Y106G6G.2 & 2.21 & elpc-4 & 2.27 & rrbs-1 & 2.35 \\
\hline K10G4.3 & 2.14 & R09B5.11 & 2.21 & math-37 & 2.27 & C02F5.3 & 2.36 \\
\hline F22B7.1 & 2.14 & unc-31 & 2.21 & gtl-1 & 2.27 & linc-39 & 2.36 \\
\hline skr-19 & 2.14 & dao-6 & 2.22 & tpra-1 & 2.28 & rrp-8 & 2.36 \\
\hline T01D3.6 & 2.14 & hil-1 & 2.22 & F44A2.3 & 2.28 & brp-1 & 2.37 \\
\hline $\mathrm{crm}-1$ & 2.15 & rars-1 & 2.22 & wrb-1 & 2.28 & F26A3.1 & 2.37 \\
\hline mrps-17 & 2.15 & $\mathrm{C} 06 \mathrm{H} 5.8$ & 2.22 & wars-1 & 2.29 & cct-3 & 2.37 \\
\hline ZK1307.1 & 2.15 & T10B5.3 & 2.22 & $\mathrm{C} 28 \mathrm{H} 8.3$ & 2.29 & slc-28.1 & 2.37 \\
\hline epg-5 & 2.15 & K02A11.4 & 2.22 & nlp-7 & 2.29 & Н35В03.1 & 2.37 \\
\hline irg-3 & 2.15 & Y42A5A.1 & 2.22 & ins-29 & 2.29 & ncx-7 & 2.37 \\
\hline Y56АЗА.18 & 2.15 & nhr-35 & 2.22 & F56D5.6 & 2.29 & Y71H2AM.1 & 2.38 \\
\hline clec-76 & 2.16 & nspc-19 & 2.22 & kin-3 & 2.29 & ttr-34 & 2.38 \\
\hline ZK971.1 & 2.17 & lin-14 & 2.22 & cnnm-2 & 2.29 & T08B1.4 & 2.38 \\
\hline him-4 & 2.17 & F33D11.16 & 2.23 & alh-8 & 2.3 & T20D3.6 & 2.38 \\
\hline F26F12.3 & 2.17 & F13D11.4 & 2.23 & D1022.4 & 2.3 & $\mathrm{~F} 14 \mathrm{H} 3.12$ & 2.39 \\
\hline F54C8.1 & 2.17 & R119.2 & 2.23 & Y54F10AM.8 & 2.3 & dohh-1 & 2.39 \\
\hline C09E7.6 & 2.17 & alh-12 & 2.23 & Y7A5A.6 & 2.3 & F29C6.1 & 2.4 \\
\hline exos-9 & 2.17 & M01F1.3 & 2.24 & pho-4 & 2.31 & nhr-146 & 2.4 \\
\hline riok-2 & 2.18 & sma-2 & 2.24 & Y6B3B.4 & 2.31 & nap-1 & 2.4 \\
\hline pno-1 & 2.18 & F20D6.5 & 2.24 & W03D8.2 & 2.31 & ZK822.4 & 2.4 \\
\hline F45E1.5 & 2.18 & F41G4.8 & 2.24 & nhr-119 & 2.31 & gst-23 & 2.4 \\
\hline pals-31 & 2.18 & nmad-1 & 2.25 & Imd-4 & 2.31 & Y92H12BL.1 & 2.41 \\
\hline $\mathrm{mgl}-2$ & 2.18 & mak-2 & 2.25 & C49A9.6 & 2.32 & bath-47 & 2.41 \\
\hline H23N18.6 & 2.18 & nhr-80 & 2.25 & C16A3.6 & 2.32 & уар-1 & 2.41 \\
\hline$d d x-17$ & 2.18 & Ipr-2 & 2.25 & riok-3 & 2.32 & gра-8 & 2.41 \\
\hline C08F11.3 & 2.18 & Y51H4A.15 & 2.26 & gdh-1 & 2.33 & R09F10.1 & 2.42 \\
\hline
\end{tabular}




\begin{tabular}{|c|c|c|c|c|c|c|c|}
\hline Gene & $\log 2 \mathrm{FC}$ & Gene & $\log 2 \mathrm{FC}$ & Gene & $\log 2 \mathrm{FC}$ & Gene & $\log 2 \mathrm{FC}$ \\
\hline gap-1 & 2.42 & F53F10.1 & 2.52 & T01G1.4 & 2.64 & R10E8.6 & 2.8 \\
\hline ZK550.2 & 2.42 & ins-35 & 2.52 & $o b r-2$ & 2.65 & dph-3 & 2.8 \\
\hline nhr-170 & 2.42 & C50F4.1 & 2.52 & C15B12.4 & 2.65 & T22F3.2 & 2.8 \\
\hline Y37E11B.5 & 2.43 & clec-146 & 2.52 & F56H9.9 & 2.66 & clec-62 & 2.8 \\
\hline rsd-3 & 2.43 & F35D11.3 & 2.53 & upb-1 & 2.66 & K02C4.5 & 2.8 \\
\hline $\mathrm{T} 10 \mathrm{H} 4.13$ & 2.43 & gst-22 & 2.53 & T19C3.6 & 2.66 & $\mathrm{C} 53 \mathrm{H} 9.2$ & 2.81 \\
\hline ace-2 & 2.43 & $\mathrm{cpz}-1$ & 2.53 & daf-2 & 2.67 & C52E2.4 & 2.81 \\
\hline H34I24.2 & 2.43 & W07A12.4 & 2.54 & ZK430.7 & 2.67 & lep-5 & 2.82 \\
\hline elpc-3 & 2.43 & F30A10.9 & 2.54 & B0507.3 & 2.67 & nhr-103 & 2.82 \\
\hline capa-1 & 2.44 & T19D12.2 & 2.54 & Y47H10A.3 & 2.68 & nlp-52 & 2.83 \\
\hline btb-17 & 2.45 & ulp-3 & 2.54 & lido-10 & 2.68 & Y37Н9A.5 & 2.83 \\
\hline F31E9.3 & 2.45 & R02E4.2 & 2.54 & lec-2 & 2.68 & F53F1.14 & 2.83 \\
\hline C49F5.7 & 2.45 & nlp-47 & 2.54 & gsp-2 & 2.69 & B0511.6 & 2.84 \\
\hline T28A11.2 & 2.45 & twk-7 & 2.54 & csp-2 & 2.69 & sek-1 & 2.84 \\
\hline ugt- 6 & 2.46 & K02D10.8 & 2.54 & asp-6 & 2.7 & ZK1290.13 & 2.84 \\
\hline flp-20 & 2.46 & acl-9 & 2.54 & T07D4.2 & 2.7 & mnk-1 & 2.85 \\
\hline R11D1.13 & 2.46 & C23F12.4 & 2.55 & F59A7.8 & 2.7 & nlp-2 & 2.85 \\
\hline $\mathrm{JC} 8.2$ & 2.46 & C53C11.2 & 2.56 & T20F7.5 & 2.71 & C49C8.8 & 2.85 \\
\hline ads-1 & 2.46 & dph-1 & 2.56 & $\mathrm{mfb}-1$ & 2.71 & clec-186 & 2.85 \\
\hline exos-7 & 2.46 & K11D12.12 & 2.57 & cgr-1 & 2.71 & W07G4.5 & 2.85 \\
\hline Y46G5A.34 & 2.46 & $\mathrm{C} 24 \mathrm{H} 12.4$ & 2.57 & Y54G2A.41 & 2.71 & Y40C5A.4 & 2.86 \\
\hline C16D9.9 & 2.47 & F53F4.11 & 2.57 & W02D7.11 & 2.72 & F21A3.11 & 2.86 \\
\hline C47D12.5 & 2.47 & gst-3 & 2.58 & nhr-133 & 2.72 & К09H9.5 & 2.86 \\
\hline col-92 & 2.47 & hpo-11 & 2.59 & T22H9.1 & 2.72 & Y54H5A.2 & 2.87 \\
\hline asah-1 & 2.47 & T01B7.5 & 2.59 & ZC443.4 & 2.72 & Y54H5A.1 & 2.88 \\
\hline nspc-17 & 2.47 & Y34F4.2 & 2.59 & C34E11.4 & 2.72 & F08F8.7 & 2.88 \\
\hline Y106G6E.4 & 2.48 & pho-5 & 2.6 & Y6G8.2 & 2.73 & F16A11.5 & 2.88 \\
\hline T23D8.3 & 2.49 & nog-1 & 2.61 & nhr-92 & 2.74 & gcy-15 & 2.88 \\
\hline mrpl-55 & 2.49 & F45D11.1 & 2.61 & tag-89 & 2.75 & $\mathrm{~T} 28 \mathrm{H} 10.1$ & 2.88 \\
\hline eif-3.B & 2.49 & Inp-1 & 2.61 & Y53C12B.7 & 2.75 & F36A2.10 & 2.88 \\
\hline Y43C5A.3 & 2.5 & F44G4.1 & 2.61 & nhr-129 & 2.75 & puf-12 & 2.89 \\
\hline smp-2 & 2.5 & F31B12.3 & 2.61 & btb-16 & 2.75 & F48E8.3 & 2.89 \\
\hline aak-1 & 2.5 & $\mathrm{mtl}-2$ & 2.61 & fbxc-7 & 2.76 & ZK688.11 & 2.89 \\
\hline nlp-69 & 2.51 & Т10H9.8 & 2.62 & pud-4 & 2.76 & C53A5.17 & 2.9 \\
\hline nhl-1 & 2.51 & F55A4.7 & 2.62 & nnt-1 & 2.76 & $\mathrm{R} 01 \mathrm{H} 10.4$ & 2.91 \\
\hline C18A11.1 & 2.51 & C36E8.1 & 2.62 & arch-1 & 2.77 & Y51B9A.9 & 2.91 \\
\hline asp-3 & 2.51 & B0563.5 & 2.62 & clec-65 & 2.77 & Y43B11AR.3 & 2.92 \\
\hline $\mathrm{rab}-8$ & 2.51 & Ipd-7 & 2.62 & Y71G10AR.4 & 2.77 & F49C12.6 & 2.92 \\
\hline unc-83 & 2.51 & mps-1 & 2.63 & Y57G11C.1135 & 2.77 & $\mathrm{~F} 47 \mathrm{H} 4.12$ & 2.92 \\
\hline F58A3.3 & 2.51 & T10G3.1 & 2.63 & E02H1.1 & 2.78 & srp-2 & 2.93 \\
\hline C16C10.8 & 2.51 & clec-61 & 2.63 & nlp-59 & 2.78 & nhr-109 & 2.93 \\
\hline paqr-1 & 2.52 & C27F2.4 & 2.63 & smf-3 & 2.79 & C05E11.6 & 2.96 \\
\hline
\end{tabular}




\begin{tabular}{|c|c|c|c|c|c|c|c|}
\hline Gene & $\log 2 \mathrm{FC}$ & Gene & $\log 2 \mathrm{FC}$ & Gene & $\log 2 \mathrm{FC}$ & Gene & $\log 2 \mathrm{FC}$ \\
\hline asm-2 & 2.96 & Y54E10BR.4 & 3.16 & tut-1 & 3.46 & chil-13 & 3.86 \\
\hline clec-85 & 2.97 & metl-18 & 3.19 & C09D4.2 & 3.47 & W08E12.8 & 3.89 \\
\hline C15C7.4 & 2.97 & fbxa-173 & 3.19 & ctps-1 & 3.48 & faah-3 & 3.89 \\
\hline сур-34A9 & 2.98 & nhr-88 & 3.21 & Y15E3A.4 & 3.49 & ttr-49 & 3.93 \\
\hline flp-10 & 2.98 & F36A2.2 & 3.22 & B0513.4 & 3.49 & F35C8.5 & 3.93 \\
\hline M60.7 & 2.99 & Y71F9AL.2 & 3.23 & B0546.3 & 3.49 & T26H5.4 & 3.97 \\
\hline T25G3.3 & 2.99 & nspc-18 & 3.23 & asah-2 & 3.5 & arrd-11 & 4.01 \\
\hline ulp-4 & 3 & F54D5.3 & 3.23 & Y48G8AL.13 & 3.5 & C45E5.4 & 4.04 \\
\hline T10B5.7 & 3 & C25A8.5 & 3.24 & hpo-15 & 3.5 & D1086.2 & 4.07 \\
\hline odr-10 & 3 & Y22D7AL.15 & 3.24 & Y47D3A.32 & 3.5 & $\mathrm{C} 18 \mathrm{H} 9.5$ & 4.08 \\
\hline F42A9.18 & 3.02 & Y39B6А.33 & 3.25 & $\mathrm{H} 06 \mathrm{H} 21.8$ & 3.52 & ZK593.2 & 4.13 \\
\hline zig-9 & 3.02 & F10G7.5 & 3.26 & C25F9.12 & 3.52 & exc-13 & 4.13 \\
\hline metl-2 & 3.02 & nhr-18 & 3.27 & W09C3.7 & 3.54 & сур-37B1 & 4.16 \\
\hline cpr-6 & 3.03 & linc-43 & 3.28 & nas-9 & 3.56 & C05D9.9 & 4.18 \\
\hline T18D3.6 & 3.04 & srap-1 & 3.28 & C45E1.4 & 3.56 & cest-1.2 & 4.2 \\
\hline Y18D10A.3 & 3.05 & F53A2.9 & 3.29 & C39D10.11 & 3.58 & T10C6.15 & 4.24 \\
\hline $\mathrm{C} 23 \mathrm{H} 5.15$ & 3.05 & Y65B4BL.7 & 3.3 & F47B8.4 & 3.58 & F56H9.2 & 4.26 \\
\hline $\mathrm{C} 32 \mathrm{H} 11.4$ & 3.05 & ZC239.17 & 3.3 & ncr-2 & 3.59 & hda-5 & 4.3 \\
\hline nspc-20 & 3.06 & fbxa-6 & 3.31 & tts- 1 & 3.6 & B0379.7 & 4.34 \\
\hline C28G1.6 & 3.07 & adah-1 & 3.31 & T06C12.14 & 3.6 & F47B8.8 & 4.37 \\
\hline gst-20 & 3.07 & Y56АЗА.33 & 3.32 & cup-16 & 3.6 & C32F10.4 & 4.37 \\
\hline ngp-1 & 3.07 & pmt-1 & 3.32 & col-181 & 3.61 & ZC395.4 & 4.47 \\
\hline $\operatorname{lgc}-28$ & 3.07 & R10E8.1 & 3.33 & twk-5 & 3.61 & nspc-14 & 4.52 \\
\hline M01B2.13 & 3.07 & nspc-16 & 3.34 & clec-84 & 3.62 & K05F6.10 & 4.63 \\
\hline$u b c-1$ & 3.07 & C10A4.3 & 3.35 & hrg-2 & 3.67 & spp-8 & 4.68 \\
\hline $\mathrm{C} 28 \mathrm{H} 8.2$ & 3.08 & fbxa-162 & 3.35 & acp-5 & 3.67 & ZK185.5 & 4.77 \\
\hline Y97E10AL.1 & 3.11 & Y52B11A.10 & 3.36 & C17G10.6 & 3.68 & W10G11.1 & 4.79 \\
\hline Y42G9A.1 & 3.11 & pqn-59 & 3.37 & spp-6 & 3.7 & $\mathrm{~F} 21 \mathrm{H} 7.12$ & 4.87 \\
\hline col-118 & 3.11 & Y47G6A.5 & 3.37 & ZK470.14 & 3.71 & srv-4 & 5 \\
\hline T12D8.5 & 3.12 & pho-6 & 3.38 & F18G5.6 & 3.71 & сур-32B1 & 5.16 \\
\hline F40F8.5 & 3.12 & hecw-1 & 3.38 & T26E3.4 & 3.71 & $\mathrm{~F} 43 \mathrm{C} 11.7$ & 5.2 \\
\hline txt-3 & 3.13 & ZK1240.2 & 3.39 & chil-16 & 3.72 & F56F11.1 & 5.37 \\
\hline W04E12.7 & 3.13 & $\mathrm{fbxa}-80$ & 3.4 & F43D2.7 & 3.74 & dpy-5 & 5.53 \\
\hline riok-1 & 3.13 & C08F11.13 & 3.41 & clec-264 & 3.74 & hsp-12.6 & 6.52 \\
\hline tag-243 & 3.13 & F56A8.5 & 3.41 & F21C10.11 & 3.74 & & \\
\hline W06H8.6 & 3.14 & F23F12.3 & 3.41 & col-39 & 3.78 & & \\
\hline T26C11.9 & 3.14 & R07E3.1 & 3.41 & T08A9.13 & 3.79 & & \\
\hline C03G6.17 & 3.14 & pqn-62 & 3.41 & F16B4.5 & 3.81 & & \\
\hline C16A3.4 & 3.15 & T09B4.6 & 3.42 & C29G2.2 & 3.82 & & \\
\hline F52E1.14 & 3.15 & fil-1 & 3.44 & F40E3.5 & 3.83 & & \\
\hline del-9 & 3.16 & B0524.6 & 3.44 & W03D2.6 & 3.83 & & \\
\hline fat- 6 & 3.16 & fard-1 & 3.45 & cpr-2 & 3.85 & & \\
\hline
\end{tabular}




\subsection{Proteome (TMT) - 4h Fed vs $48 \mathrm{~h}$ Starved - N2 (wild-type)}

\begin{tabular}{|c|c|c|c|c|c|c|c|}
\hline Protein & $\log 2 \mathrm{FC}$ & Protein & $\log 2 \mathrm{FC}$ & Protein & $\log 2 \mathrm{FC}$ & Protein & $\log 2 \mathrm{FC}$ \\
\hline H39E23.3 & -10.04 & Y51H4A.7 & -1.98 & spr-4 & -1.49 & Y67H2A.10 & -1.2 \\
\hline B0379.6 & -8.28 & C08E8.4 & -1.97 & T08A11.1 & -1.49 & zyg-8 & -1.19 \\
\hline E02C12.9 & -6.57 & athp-2 & -1.95 & hsb-1 & -1.49 & F31E3.12 & -1.19 \\
\hline Y53F4B.21 & -5.41 & ppw-2 & -1.91 & cWc-15 & -1.48 & ZK353.9 & -1.19 \\
\hline ipla-2 & -5.24 & M03C11.3 & -1.9 & grd-13 & -1.48 & F26E4.3 & -1.18 \\
\hline col-39 & -5.15 & Y65B4A.2 & -1.9 & Y44A6D.2 & -1.48 & W05H12.1 & -1.18 \\
\hline dpy-14 & -4.84 & cut-3 & -1.89 & sec-11 & -1.47 & B0035.3 & -1.18 \\
\hline dpy-17 & -4.59 & col-121 & -1.87 & C14A4.9 & -1.45 & gst-29 & -1.17 \\
\hline fipr-22 & -4.58 & hsp-16.48 & -1.82 & vps-2 & -1.43 & attf-4 & -1.17 \\
\hline col-158 & -4.31 & unc-52 & -1.81 & cth-2 & -1.42 & F13B12.4 & -1.17 \\
\hline сур-33E2 & -4.04 & leo-1 & -1.81 & lars-2 & -1.4 & cox $-5 B$ & -1.16 \\
\hline F55F8.3 & -3.95 & lec-9 & -1.8 & B0495.7 & -1.4 & gst-15 & -1.16 \\
\hline abts-4 & -3.85 & rpi-2 & -1.8 & capg-1 & -1.39 & F07C3.2 & -1.16 \\
\hline grl-16 & -3.83 & sucl-2 & -1.79 & nep-1 & -1.39 & C24G6.8 & -1.15 \\
\hline dpy-10 & -3.67 & tps-1 & -1.77 & $\mathrm{C} 24 \mathrm{H} 10.1$ & -1.38 & $\mathrm{kcc}-2$ & -1.15 \\
\hline spin-1 & -3.3 & sph-1 & -1.75 & fbn-1 & -1.35 & psf-2 & -1.15 \\
\hline hgap-2 & -3.29 & T03F1.11 & -1.75 & T04C9.1 & -1.34 & gst-20 & -1.14 \\
\hline C44C10.9 & -3.12 & $\operatorname{lin}-10$ & -1.74 & vps-60 & -1.34 & sptf-3 & -1.12 \\
\hline nex-4 & -3.1 & Y37A1B.7 & -1.73 & ten-1 & -1.34 & C11D2.4 & -1.12 \\
\hline hsp-16.2 & -2.96 & T10B10.3 & -1.72 & mlt-7 & -1.33 & $\mathrm{~T} 19 \mathrm{H} 12.2$ & -1.11 \\
\hline rnp-6 & -2.87 & nbet-1 & -1.71 & K02F3.9 & -1.33 & $\mathrm{C} 30 \mathrm{H} 6.8$ & -1.11 \\
\hline hda-10 & -2.81 & cht-1 & -1.71 & shc-1 & -1.33 & num-1 & -1.1 \\
\hline $\mathrm{R} 02 \mathrm{C} 2.7$ & -2.8 & clec-266 & -1.68 & cubn-1 & -1.32 & T10H9.8 & -1.1 \\
\hline hum- 6 & -2.78 & acn-1 & -1.68 & fubl-1 & -1.32 & T10B5.4 & -1.1 \\
\hline F54F7.6 & -2.77 & smr-1 & -1.67 & F22D6.2 & -1.32 & lea-1 & -1.09 \\
\hline iscu-1 & -2.75 & $\mathrm{dsl}-3$ & -1.67 & F36H9.7 & -1.31 & Y38C1AA.9 & -1.09 \\
\hline F10D11.6 & -2.7 & egl-27 & -1.67 & tep-1 & -1.3 & ttc-36 & -1.08 \\
\hline F14B4.1 & -2.65 & ensa-1 & -1.65 & F43C9.2 & -1.3 & $\operatorname{lin}-36$ & -1.08 \\
\hline ZK863.8 & -2.5 & Y48G1C.12 & -1.63 & gtf-2F1 & -1.29 & slc-36.5 & -1.08 \\
\hline pde-5 & -2.43 & hmg-3 & -1.61 & F44E5.4 & -1.29 & $\mathrm{ttr}-23$ & -1.07 \\
\hline dve-1 & -2.34 & mlst-8 & -1.6 & W10C8.4 & -1.29 & inx-6 & -1.07 \\
\hline C53B7.3 & -2.3 & C47A4.1 & -1.6 & lex-1 & -1.27 & C09F9.2 & -1.06 \\
\hline Y39B6A.5 & -2.26 & scl-12 & -1.58 & ttr-5 & -1.25 & cav-2 & -1.06 \\
\hline T21B6.3 & -2.26 & F59F3.6 & -1.57 & pdl-1 & -1.24 & Y92H12BL.4 & -1.06 \\
\hline Y39A1A.21 & -2.25 & T18D3.1 & -1.56 & F29B9.5 & -1.24 & F36G3.2 & -1.06 \\
\hline sod-5 & -2.24 & sec-10 & -1.55 & hmg-1.2 & -1.23 & viro-2 & -1.06 \\
\hline C01G6.3 & -2.19 & dgk-1 & -1.54 & K01A2.10 & -1.22 & F59B10.3 & -1.06 \\
\hline C50D2.8 & -2.13 & ints-7 & -1.54 & eif-3.K & -1.22 & C16C10.4 & -1.06 \\
\hline elpc-2 & -2.12 & pqn-94 & -1.54 & Iron-9 & -1.21 & ncam-1 & -1.06 \\
\hline C15C7.7 & -2.09 & F53E10.1 & -1.51 & dpy-27 & -1.2 & ech-8 & -1.05 \\
\hline Y53F4B.23 & -1.99 & pfas-1 & -1.51 & hil-2 & -1.2 & $\operatorname{cox}-6 \mathrm{~B}$ & -1.05 \\
\hline
\end{tabular}




\begin{tabular}{|c|c|c|c|c|c|c|c|}
\hline Protein & $\log 2 \mathrm{FC}$ & Protein & $\log 2 \mathrm{FC}$ & Protein & $\log 2 \mathrm{FC}$ & Protein & $\log 2 \mathrm{FC}$ \\
\hline tut-1 & -1.05 & Ibp-2 & 1.06 & cdr-6 & 1.17 & Y54E10A.10 & 1.33 \\
\hline daf-16 & -1.04 & $w d r-46$ & 1.06 & glh-2 & 1.18 & ugt-22 & 1.33 \\
\hline Y37E11B.5 & -1.04 & pod-2 & 1.06 & iff-2 & 1.18 & nola-3 & 1.34 \\
\hline lin-15B & -1.03 & C05C10.3 & 1.06 & mrpl-37 & 1.18 & fpn-1.1 & 1.34 \\
\hline ttr-33 & -1.03 & hmt-1 & 1.06 & fmo-5 & 1.18 & rnp-1 & 1.35 \\
\hline T05F1.11 & -1.03 & gob-1 & 1.06 & AC3.5 & 1.19 & alh-8 & 1.35 \\
\hline gst-24 & -1.03 & taap-1 & 1.07 & rbm-34 & 1.19 & rps-10 & 1.35 \\
\hline F37F2.2 & -1.02 & lec-4 & 1.07 & acp-6 & 1.2 & $c d r-4$ & 1.36 \\
\hline C50F4.16 & -1.01 & Y104H12D.2 & 1.07 & fbxa-156 & 1.2 & pqn-22 & 1.37 \\
\hline $\mathrm{F} 23 \mathrm{H} 11.5$ & -1.01 & txdc-12.2 & 1.08 & scav-3 & 1.21 & ttr-51 & 1.38 \\
\hline hmg-1.1 & -1.01 & mct-4 & 1.09 & cpn-1 & 1.21 & rme-2 & 1.38 \\
\hline $\operatorname{cox}-6 \mathrm{~A}$ & -1.01 & $\mathrm{mpc}-2$ & 1.09 & Y15E3A.4 & 1.21 & gldc-1 & 1.39 \\
\hline hsp-16.11 & -1.01 & acdh-9 & 1.09 & T10G3.3 & 1.21 & pud-2.1 & 1.39 \\
\hline emb-9 & -1 & mrps-23 & 1.1 & T20B12.3 & 1.21 & cle-1 & 1.39 \\
\hline cept-2 & -1 & sptl-3 & 1.1 & nuc-1 & 1.22 & cps-6 & 1.39 \\
\hline hrpa-1 & -1 & Y16B4A.2 & 1.1 & glh-4 & 1.22 & dod-19 & 1.4 \\
\hline Y106G6H.8 & 1 & C39D10.8 & 1.11 & T21H3.1 & 1.22 & mrpl-13 & 1.4 \\
\hline dut-1 & 1 & cpr-4 & 1.11 & msra-1 & 1.22 & Y10G11A.1 & 1.41 \\
\hline сур-34A9 & 1 & T07D3.9 & 1.11 & Y48A6C.4 & 1.22 & msi-1 & 1.41 \\
\hline F41D9.2 & 1.01 & $\operatorname{csq}-1$ & 1.11 & oxy-4 & 1.22 & lec- 6 & 1.42 \\
\hline otub-1 & 1.01 & pmt-2 & 1.11 & C10G11.8 & 1.23 & ger-1 & 1.42 \\
\hline W01A11.1 & 1.01 & LLC1.2 & 1.11 & rps-25 & 1.23 & pept-1 & 1.43 \\
\hline rps-23 & 1.01 & ogdh-2 & 1.12 & Y34B4A.6 & 1.23 & F53F4.16 & 1.43 \\
\hline arf-6 & 1.01 & $\mathrm{~T} 13 \mathrm{C} 2.6$ & 1.12 & F37C12.3 & 1.24 & comt-4 & 1.43 \\
\hline cka-2 & 1.01 & ubc-14 & 1.12 & eef-1B.1 & 1.24 & bca-2 & 1.44 \\
\hline spp-2 & 1.01 & tag-18 & 1.12 & F33A8.4 & 1.25 & rbm-3.2 & 1.44 \\
\hline Y54E2A.4 & 1.02 & Y67D2.7 & 1.12 & F53B1.8 & 1.25 & mrpl-45 & 1.44 \\
\hline F57F5.1 & 1.02 & F32D8.12 & 1.13 & paic-1 & 1.26 & vha-1 & 1.44 \\
\hline $\mathrm{F} 33 \mathrm{H} 2.6$ & 1.02 & ceh-20 & 1.13 & lact-3 & 1.26 & C17G1.2 & 1.45 \\
\hline sur-5 & 1.03 & D1086.3 & 1.13 & F54D5.12 & 1.26 & ech-6 & 1.45 \\
\hline col-159 & 1.04 & aps-2 & 1.13 & R08B4.3 & 1.27 & gst-27 & 1.45 \\
\hline spp-5 & 1.04 & C01B10.6 & 1.13 & coq-6 & 1.27 & eif-2Balpha & 1.45 \\
\hline hda-5 & 1.04 & vha-6 & 1.13 & lec-10 & 1.28 & $w d r-12$ & 1.46 \\
\hline Y43F4B.7 & 1.04 & R10F2.4 & 1.14 & gst-35 & 1.28 & oxa-1 & 1.46 \\
\hline T21C9.6 & 1.04 & ima-2 & 1.14 & ugt-26 & 1.29 & rhr-1 & 1.47 \\
\hline nex-3 & 1.05 & cest-4 & 1.15 & T01D3.6 & 1.29 & Iron-7 & 1.48 \\
\hline rpl-25.1 & 1.05 & F39H11.1 & 1.16 & acp-5 & 1.29 & pgp-3 & 1.5 \\
\hline $\mathrm{T} 20 \mathrm{H} 4.5$ & 1.05 & ZK1320.9 & 1.16 & coq-3 & 1.31 & pde-3 & 1.52 \\
\hline nas-11 & 1.05 & T25C12.3 & 1.17 & mig-2 & 1.32 & nhr-130 & 1.53 \\
\hline R119.3 & 1.05 & Y54E5A.5 & 1.17 & gly-5 & 1.32 & gfi-1 & 1.53 \\
\hline $\mathrm{cpz}-1$ & 1.06 & W10C8.5 & 1.17 & C45G9.5 & 1.33 & F09C8.1 & 1.53 \\
\hline
\end{tabular}




\begin{tabular}{|c|c|c|c|c|c|}
\hline Protein & $\log 2 \mathrm{FC}$ & Protein & $\log 2 \mathrm{FC}$ & Protein & $\log 2 \mathrm{FC}$ \\
\hline madd-3 & 1.53 & Y7A5A.1 & 1.86 & bigr-1 & 2.63 \\
\hline K08D8.6 & 1.54 & сур-13А5 & 1.86 & T12D8.5 & 2.63 \\
\hline F09F7.6 & 1.54 & pсp-4 & 1.86 & ZK6.11 & 2.63 \\
\hline $\operatorname{csn}-5$ & 1.55 & C09G9.1 & 1.86 & lys-4 & 2.7 \\
\hline nol-10 & 1.55 & R09H10.5 & 1.87 & grl-23 & 2.75 \\
\hline F28B4.3 & 1.56 & elo-1 & 1.89 & D1054.10 & 2.81 \\
\hline asp-5 & 1.57 & Y54F10AM.8 & 1.9 & dct-16 & 2.93 \\
\hline trpa-2 & 1.57 & col-92 & 1.93 & acdh-1 & 2.94 \\
\hline R193.2 & 1.58 & F21C10.9 & 1.93 & F17E9.5 & 2.96 \\
\hline dhs-25 & 1.58 & $\mathrm{C} 34 \mathrm{H} 4.2$ & 1.94 & T28F3.8 & 3 \\
\hline $\mathrm{mpc}-1$ & 1.59 & F35C8.5 & 1.95 & vit-3 & 3 \\
\hline F20G2.1 & 1.59 & timm-23 & 1.97 & Y62H9A.5 & 3.14 \\
\hline hpo-40 & 1.6 & C14F11.4 & 1.97 & rpc-25 & 3.22 \\
\hline $\mathrm{M} 02 \mathrm{H} 5.8$ & 1.6 & pud-1.1 & 1.98 & perm-2 & 3.22 \\
\hline F33H1.4 & 1.6 & pmp-5 & 1.99 & cey-3 & 3.24 \\
\hline F44G4.1 & 1.61 & vps-36 & 2 & fip-5 & 3.24 \\
\hline far- 6 & 1.62 & C03A3.2 & 2.01 & ilys-5 & 3.32 \\
\hline F59B1.2 & 1.62 & argk-1 & 2.02 & glh-1 & 3.36 \\
\hline asps-1 & 1.63 & spp-3 & 2.03 & cey-2 & 3.5 \\
\hline C53D5.5 & 1.64 & B0432.7 & 2.08 & F54E2.1 & 3.63 \\
\hline Y34B4A.9 & 1.65 & $\mathrm{R} 10 \mathrm{H} 10.3$ & 2.09 & $\mathrm{cbd}-1$ & 3.65 \\
\hline hpo-34 & 1.67 & vit-1 & 2.09 & far-2 & 3.67 \\
\hline hach-1 & 1.68 & hphd-1 & 2.11 & ule-1 & 3.69 \\
\hline aagr-2 & 1.69 & ugt-62 & 2.11 & Y39H10A.6 & 3.7 \\
\hline asp-6 & 1.71 & pgp-1 & 2.11 & perm-4 & 3.74 \\
\hline F19C7.1 & 1.71 & asp-13 & 2.19 & $\mathrm{~K} 12 \mathrm{H} 4.7$ & 3.83 \\
\hline tomm-22 & 1.71 & F56C9.7 & 2.19 & folt-2 & 4.38 \\
\hline cdf-1 & 1.72 & lec-8 & 2.24 & $\mathrm{C} 01 \mathrm{H} 6.8$ & 4.77 \\
\hline F58B4.5 & 1.72 & clec-63 & 2.27 & ule-5 & 5.11 \\
\hline Idp-1 & 1.75 & ZK813.3 & 2.27 & Y59E9AR.1 & 5.28 \\
\hline ctsa-1.2 & 1.75 & tba-4 & 2.28 & & \\
\hline sodh-1 & 1.76 & asp-1 & 2.28 & & \\
\hline egg-1 & 1.76 & F17E9.4 & 2.31 & & \\
\hline vit-5 & 1.77 & Y51F10.7 & 2.35 & & \\
\hline Y37H2A.14 & 1.78 & $\mathrm{C} 23 \mathrm{H} 5.8$ & 2.37 & & \\
\hline sft-1 & 1.79 & $\mathrm{C} 23 \mathrm{H} 3.2$ & 2.4 & & \\
\hline mct-2 & 1.8 & T26G10.1 & 2.45 & & \\
\hline W04B5.3 & 1.81 & anp-1 & 2.46 & & \\
\hline let-767 & 1.81 & hrg-7 & 2.47 & & \\
\hline vit-4 & 1.85 & clec-50 & 2.49 & & \\
\hline $\operatorname{trpp}-8$ & 1.85 & W05F2.3 & 2.59 & & \\
\hline
\end{tabular}


6.19. Proteome (TMT) - 4h Fed vs 48 h Starved - HBR227 (aptf-1)

\begin{tabular}{|c|c|c|c|c|c|c|c|}
\hline Protein & $\log 2 \mathrm{FC}$ & Protein & $\log 2 \mathrm{FC}$ & Protein & $\log 2 \mathrm{FC}$ & Protein & $\log 2 \mathrm{FC}$ \\
\hline B0379.6 & -8.69 & vit-6 & -1.72 & C35A5.11 & -1.31 & tut-1 & -1.09 \\
\hline dpy-10 & -5.79 & Ipr-3 & -1.71 & mec-1 & -1.3 & ifc-2 & -1.08 \\
\hline H39E23.3 & -5.21 & Y47H9C.1 & -1.7 & dnc-3 & -1.3 & shc-1 & -1.08 \\
\hline Y53F4B.21 & -5.1 & C14A4.9 & -1.7 & Y55D9A.2 & -1.29 & ttr-35 & -1.07 \\
\hline fipr-22 & -4.92 & vit-2 & -1.67 & C15A11.7 & -1.28 & T05F1.11 & -1.06 \\
\hline E02C12.9 & -4.76 & sec-10 & -1.67 & Y39B6A.5 & -1.28 & $\mathrm{R} 02 \mathrm{C} 2.7$ & -1.04 \\
\hline hum-6 & -4.4 & $\operatorname{lin}-10$ & -1.63 & grd-13 & -1.26 & memo-1 & -1.04 \\
\hline dpy-17 & -4.12 & acn-1 & -1.62 & T10G3.3 & -1.25 & sor-1 & -1.04 \\
\hline ipla-2 & -3.95 & hcp-1 & -1.61 & hsp-16.48 & -1.25 & oig-3 & -1.04 \\
\hline dpy-14 & -3.94 & cth-2 & -1.6 & vit-3 & -1.24 & hch-1 & -1.03 \\
\hline spin-1 & -3.87 & vit-5 & -1.59 & cht-1 & -1.24 & tpst-1 & -1.03 \\
\hline abts-4 & -3.74 & ifc-1 & -1.58 & $\mathrm{~T} 24 \mathrm{H} 10.1$ & -1.24 & mlst-8 & -1.02 \\
\hline leo-1 & -3.5 & ifp-1 & -1.57 & grl-7 & -1.24 & F53E10.1 & -1.02 \\
\hline grl-16 & -3.17 & Y51H4A.7 & -1.56 & R06F6.8 & -1.23 & pch-2 & -1.02 \\
\hline сур-33E2 & -3.04 & asns-2 & -1.55 & psf-2 & -1.2 & mctp-1 & -1.02 \\
\hline F54F7.6 & -3.01 & $\mathrm{mcm}-5$ & -1.53 & F43G9.12 & -1.2 & C49A9.9 & -1.02 \\
\hline hgap-2 & -2.92 & ost-1 & -1.52 & M02H5.8 & -1.2 & noah-1 & -1.01 \\
\hline F14B4.1 & -2.69 & ZK287.1 & -1.51 & lex-1 & -1.19 & T24B8.7 & -1.01 \\
\hline C11D2.4 & -2.61 & lin-14 & -1.51 & madd-3 & -1.19 & natc- 2 & -1 \\
\hline hda-10 & -2.56 & $\mathrm{rnp}-6$ & -1.51 & mlt-11 & -1.19 & Y43F4B.7 & 1 \\
\hline Y39A1A.21 & -2.47 & elpc-2 & -1.5 & fdps-1 & -1.19 & C46G7.2 & 1.01 \\
\hline iscu-1 & -2.38 & ints-7 & -1.49 & M03C11.3 & -1.18 & R07B7.9 & 1.01 \\
\hline grl-21 & -2.32 & Y48G1C.12 & -1.47 & $g \ln -6$ & -1.18 & ZK1058.9 & 1.01 \\
\hline nex-4 & -2.31 & rnr-1 & -1.44 & K01A2.5 & -1.17 & clec-13 & 1.02 \\
\hline Y69E1A.5 & -2.26 & F25B4.7 & -1.43 & lars-2 & -1.17 & drr-1 & 1.02 \\
\hline H2OJ04.1 & -2.17 & R74.8 & -1.43 & $\mathrm{fbn}-1$ & -1.16 & gpd-3 & 1.02 \\
\hline F55F8.3 & -2.12 & rme-2 & -1.42 & W02D3.12 & -1.16 & ifa-4 & 1.03 \\
\hline pfas-1 & -2.1 & sip-1 & -1.42 & C25A1.1 & -1.16 & haao-1 & 1.03 \\
\hline sucl-2 & -2.04 & F20A1.1 & -1.42 & set-18 & -1.15 & $\operatorname{tag}-18$ & 1.03 \\
\hline T05H10.3 & -2.04 & clec-266 & -1.4 & far-3 & -1.15 & pqn-22 & 1.03 \\
\hline dsl-3 & -2.02 & rde-12 & -1.39 & C39D10.8 & -1.15 & T23E7.2 & 1.03 \\
\hline hsp-16.2 & -2.02 & dut-1 & -1.38 & sec- 6 & -1.14 & ZK185.5 & 1.03 \\
\hline col-39 & -2.01 & nasp-2 & -1.38 & hsp-16.11 & -1.13 & gst-26 & 1.04 \\
\hline C01G6.3 & -2 & $\mathrm{hmp}-2$ & -1.37 & eif-3.K & -1.12 & gsnl-1 & 1.05 \\
\hline C44C10.9 & -1.98 & dve-1 & -1.36 & C08E8.4 & -1.12 & $\mathrm{C} 01 \mathrm{H} 6.8$ & 1.05 \\
\hline egl-27 & -1.98 & ZK970.7 & -1.34 & $\operatorname{lin}-13$ & -1.11 & F46H5.7 & 1.05 \\
\hline pde-5 & -1.93 & vit-4 & -1.33 & Y14H12B.1 & -1.11 & C03A3.2 & 1.05 \\
\hline F53F8.4 & -1.76 & hsb-1 & -1.32 & nbet-1 & -1.1 & C25H3.9 & 1.06 \\
\hline rnr-2 & -1.76 & ints-13 & -1.32 & ttc-1 & -1.09 & unc- 7 & 1.06 \\
\hline pad-2 & -1.75 & csr-1 & -1.32 & F22D6.2 & -1.09 & far- 8 & 1.06 \\
\hline T21B6.3 & -1.73 & ppw-2 & -1.32 & $\mathrm{C} 24 \mathrm{H} 10.1$ & -1.09 & F17H10.2 & 1.06 \\
\hline $\mathrm{T} 19 \mathrm{H} 12.2$ & -1.72 & capg-1 & -1.31 & C47A4.1 & -1.09 & gpx-5 & 1.07 \\
\hline
\end{tabular}




\begin{tabular}{|c|c|c|c|c|c|c|c|}
\hline Protein & $\log 2 \mathrm{FC}$ & Protein & $\log 2 \mathrm{FC}$ & Protein & $\log 2 \mathrm{FC}$ & Protein & $\log 2 \mathrm{FC}$ \\
\hline tric-1B.1 & 1.07 & asg-2 & 1.22 & T01D1.4 & 1.5 & Y37H2A.14 & 2.9 \\
\hline C33G3.4 & 1.07 & F13D12.5 & 1.23 & tnt-2 & 1.51 & Y54G2A.57 & 2.99 \\
\hline $\mathrm{cpz}-2$ & 1.08 & Ifi-1 & 1.23 & Y55F3BR.6 & 1.52 & $\mathrm{~K} 12 \mathrm{H} 4.7$ & 3.59 \\
\hline C14B9.10 & 1.08 & C01B10.6 & 1.23 & srw-20 & 1.53 & scl-12 & 3.72 \\
\hline T03G6.3 & 1.08 & gst-28 & 1.24 & paic-1 & 1.54 & folt-2 & 3.75 \\
\hline F53B1.8 & 1.08 & unc-98 & 1.26 & ilys-5 & 1.55 & T12D8.5 & 3.89 \\
\hline droe-4 & 1.09 & D1086.3 & 1.26 & Y106G6H.8 & 1.56 & asp-9 & 4.1 \\
\hline gcp-2.1 & 1.09 & W10C8.5 & 1.27 & F17E9.5 & 1.57 & pud-2.1 & 4.31 \\
\hline srp-1 & 1.09 & D1054.10 & 1.27 & Y54F10AM.8 & 1.6 & cey-3 & 4.43 \\
\hline hsp-17 & 1.09 & argk-1 & 1.27 & msi-1 & 1.62 & cut-1 & 4.81 \\
\hline tomm-22 & 1.09 & F56D5.6 & 1.28 & F40A3.2 & 1.64 & pud-1.1 & 5.25 \\
\hline plpp-1.1 & 1.09 & hex-2 & 1.28 & imp-1 & 1.67 & scl-11 & 5.52 \\
\hline R07B1.9 & 1.1 & cht-4 & 1.28 & fip-5 & 1.67 & grl-23 & 5.84 \\
\hline mec-9 & 1.1 & rhr-1 & 1.28 & F58B4.5 & 1.7 & & \\
\hline acp-6 & 1.1 & Y12A6A.1 & 1.29 & F09B12.3 & 1.71 & & \\
\hline pud-3 & 1.1 & gly-8 & 1.29 & csq-1 & 1.73 & & \\
\hline T27C4.1 & 1.1 & rps-30 & 1.31 & C53D6.7 & 1.74 & & \\
\hline M01H9.3 & 1.13 & R13D11.4 & 1.31 & grd-3 & 1.74 & & \\
\hline mrp-3 & 1.13 & T28F3.8 & 1.32 & timm-23 & 1.75 & & \\
\hline C18H7.1 & 1.14 & E04F6.9 & 1.33 & hil-1 & 1.8 & & \\
\hline cah-5 & 1.14 & K09G1.1 & 1.34 & dct-16 & 1.85 & & \\
\hline bcl-7 & 1.15 & D2092.4 & 1.34 & F59B1.2 & 1.85 & & \\
\hline far- 6 & 1.15 & poml-3 & 1.34 & F45D11.15 & 1.91 & & \\
\hline C53D5.5 & 1.15 & K12C11.1 & 1.34 & AC3.5 & 2 & & \\
\hline glt-1 & 1.15 & catp-2 & 1.35 & $\mathrm{C} 23 \mathrm{H} 3.2$ & 2.05 & & \\
\hline $\mathrm{mpc}-1$ & 1.15 & spp-13 & 1.36 & ZK6.11 & 2.05 & & \\
\hline elo-1 & 1.16 & gst-27 & 1.36 & sod-3 & 2.08 & & \\
\hline Y7A5A.1 & 1.16 & srap-1 & 1.36 & F54E2.1 & 2.09 & & \\
\hline zig-11 & 1.16 & ugt- 62 & 1.38 & $\mathrm{cbd}-1$ & 2.19 & & \\
\hline fld-1 & 1.16 & lec-10 & 1.38 & perm-4 & 2.32 & & \\
\hline fipr-21 & 1.16 & acp-5 & 1.39 & pqn-94 & 2.32 & & \\
\hline mua-6 & 1.17 & ftn-1 & 1.41 & Y59E9AR.1 & 2.34 & & \\
\hline Y73F4A.1 & 1.17 & unc-27 & 1.42 & C14F11.4 & 2.4 & & \\
\hline pat-10 & 1.18 & endu-1 & 1.42 & cey-2 & 2.44 & & \\
\hline msa-1 & 1.19 & hmt-1 & 1.42 & glh-1 & 2.49 & & \\
\hline Y43С5A.2 & 1.19 & $\mathrm{R} 13 \mathrm{H} 4.2$ & 1.44 & Y62H9A.5 & 2.61 & & \\
\hline ZK154.6 & 1.2 & F53B3.6 & 1.45 & сур-34A9 & 2.61 & & \\
\hline T10E9.3 & 1.2 & atic-1 & 1.45 & lec-4 & 2.7 & & \\
\hline tres- 1 & 1.2 & Ibp-2 & 1.47 & lec-8 & 2.7 & & \\
\hline R53.5 & 1.2 & cest-4 & 1.48 & far-2 & 2.7 & & \\
\hline pqn-68 & 1.21 & cal-5 & 1.48 & ule- 5 & 2.84 & & \\
\hline rpl-25.1 & 1.21 & lec- 6 & 1.5 & perm-2 & 2.87 & & \\
\hline
\end{tabular}




\subsection{Proteome (TMT) - 4h Fed vs 48h Starved - HBR1777 (flp-11::EGL-1)}

\begin{tabular}{|c|c|c|c|c|c|c|c|}
\hline Protein & $\log 2 \mathrm{FC}$ & Protein & $\log 2 \mathrm{FC}$ & Protein & $\log 2 \mathrm{FC}$ & Protein & $\log 2 \mathrm{FC}$ \\
\hline B0379.6 & -6.8 & rnp-6 & -1.47 & F25B4.7 & -1.02 & exp-2 & 1.76 \\
\hline Y53F4B.21 & -4.68 & T21B6.3 & -1.47 & csr-1 & -1.01 & C06A5.3 & 1.8 \\
\hline spin-1 & -3.81 & grl-21 & -1.45 & tep-1 & -1.01 & oxa-1 & 1.95 \\
\hline E02C12.9 & -3.72 & ilys-5 & -1.44 & sph-1 & -1 & egg-1 & 1.99 \\
\hline сур-33E2 & -2.98 & Y62H9A.5 & -1.43 & cka-2 & 1.02 & M01H9.3 & 2.07 \\
\hline hgap-2 & -2.98 & C39D10.8 & -1.36 & gstk-1 & 1.02 & scl-11 & 2.16 \\
\hline ipla-2 & -2.92 & Y51H4A.7 & -1.36 & ent-1 & 1.03 & hil-1 & 2.52 \\
\hline Y67D2.7 & -2.67 & egl-27 & -1.35 & tag-65 & 1.03 & mec-9 & 2.68 \\
\hline leo-1 & -2.46 & ost-1 & -1.33 & Ibp-2 & 1.03 & T12D8.5 & 2.75 \\
\hline dpy-14 & -2.31 & athp-2 & -1.32 & C53B7.3 & 1.04 & & \\
\hline $\mathrm{R} 10 \mathrm{H} 10.3$ & -2.23 & trpa-2 & -1.31 & unc-51 & 1.04 & & \\
\hline abts-4 & -2.2 & elpc-2 & -1.28 & $\mathrm{~F} 17 \mathrm{H} 10.2$ & 1.05 & & \\
\hline F55F8.3 & -2.19 & soap-1 & -1.27 & pde- 6 & 1.05 & & \\
\hline pfas-1 & -2.17 & sucl-2 & -1.27 & srap-1 & 1.08 & & \\
\hline F54F7.6 & -2.15 & pfs-2 & -1.26 & sodh-1 & 1.09 & & \\
\hline nex-4 & -2.15 & T05H10.3 & -1.26 & clec-87 & 1.09 & & \\
\hline hum-6 & -2.12 & $\mathrm{fkb}-5$ & -1.24 & tomm-22 & 1.1 & & \\
\hline dpy-10 & -2.11 & vms-1 & -1.22 & $\mathrm{mtl}-1$ & 1.11 & & \\
\hline $\mathrm{mlt}-7$ & -2.02 & ifc-2 & -1.22 & bli-4 & 1.11 & & \\
\hline Y39A1A.21 & -1.97 & unc-52 & -1.21 & T04C9.1 & 1.12 & & \\
\hline hda-10 & -1.96 & sec-10 & -1.21 & cid-1 & 1.13 & & \\
\hline dpy-17 & -1.93 & nhl-1 & -1.2 & $z w l-1$ & 1.13 & & \\
\hline C44C10.9 & -1.89 & set-18 & -1.2 & asp-9 & 1.13 & & \\
\hline H39E23.3 & -1.85 & C32E8.5 & -1.19 & F55G1.9 & 1.14 & & \\
\hline col-92 & -1.8 & cth-2 & -1.18 & Y52E8A.3 & 1.19 & & \\
\hline col-39 & -1.8 & far-8 & -1.17 & far-6 & 1.2 & & \\
\hline T08A11.1 & -1.77 & T10B10.3 & -1.17 & C18H7.1 & 1.22 & & \\
\hline C11D2.4 & -1.73 & ints-7 & -1.17 & clec-91 & 1.24 & & \\
\hline far-3 & -1.71 & lec-9 & -1.13 & srp-2 & 1.26 & & \\
\hline ifc-1 & -1.65 & C15A11.7 & -1.13 & gst-19 & 1.26 & & \\
\hline F10D11.6 & -1.65 & acn-1 & -1.13 & F59B1.2 & 1.31 & & \\
\hline Y75B8A.14 & -1.64 & dve-1 & -1.12 & sir-2.4 & 1.39 & & \\
\hline ifp-1 & -1.64 & Y48A6B.7 & -1.11 & cey-3 & 1.43 & & \\
\hline iscu-1 & -1.64 & C08E8.4 & -1.11 & pqbp-1.1 & 1.45 & & \\
\hline cpr-4 & -1.62 & C47A4.1 & -1.1 & scl-3 & 1.47 & & \\
\hline grl-16 & -1.61 & nbet-1 & -1.1 & vit-1 & 1.49 & & \\
\hline sod-5 & -1.6 & tut-1 & -1.08 & cnnm-1 & 1.53 & & \\
\hline immp-1 & -1.56 & rde-12 & -1.06 & vit-3 & 1.54 & & \\
\hline F47B8.2 & -1.56 & atg-16.2 & -1.04 & ttr-35 & 1.6 & & \\
\hline sor-1 & -1.51 & col-121 & -1.04 & sod-4 & 1.63 & & \\
\hline grd-13 & -1.49 & C14A4.9 & -1.04 & hmg-3 & 1.68 & & \\
\hline fmo-5 & -1.49 & hsp-16.2 & -1.04 & clec-13 & 1.7 & & \\
\hline
\end{tabular}


6.21. RNA-seq (MPI-MG) - N2 (wild-type) vs HBR227 (aptf-1) - 48h

Starved

\begin{tabular}{|c|c|c|c|c|c|c|c|}
\hline Gene & $\log 2 \mathrm{FC}$ & Gene & $\log 2 \mathrm{FC}$ & Gene & $\log 2 \mathrm{FC}$ & Gene & $\log 2 \mathrm{FC}$ \\
\hline Imd-4 & -2.53 & K01A6.7 & -1.24 & ttr-50 & -1.11 & F41G3.3 & -1.03 \\
\hline F14F9.2 & -2.35 & col-131 & -1.23 & fbxc-51 & -1.11 & $\mathrm{~K} 08 \mathrm{H} 2.4$ & -1.03 \\
\hline ZK354.3 & -2.1 & F19B2.12 & -1.23 & fbxc-24 & -1.1 & nas-15 & -1.03 \\
\hline aptf-1 & -1.79 & F36H5.13 & -1.22 & Y75B7AR.1 & -1.09 & Y39D8A.1 & -1.03 \\
\hline flp-11 & -1.77 & C09B8.4 & -1.22 & B0207.5 & -1.09 & R12A1.3 & -1.02 \\
\hline F32A7.8 & -1.73 & W03A5.1 & -1.21 & wrt-10 & -1.09 & $\mathrm{fbxb}-33$ & -1.02 \\
\hline acd-2 & -1.68 & M02G9.1 & -1.21 & Y17D7C. 4 & -1.09 & F40G9.9 & -1.02 \\
\hline acdh-2 & -1.66 & $\mathrm{fbxb}-116$ & -1.21 & F21C10.11 & -1.09 & C07G3.10 & -1.02 \\
\hline argk-1 & -1.58 & C10C5.3 & -1.2 & K02E7.6 & -1.09 & F35A5.4 & -1.02 \\
\hline clec-180 & -1.58 & F45D11.16 & -1.2 & $a b f-2$ & -1.08 & $\mathrm{~K} 08 \mathrm{H} 2.2$ & -1.02 \\
\hline arrd-11 & -1.57 & F45D11.15 & -1.2 & dpy-3 & -1.08 & clec-174 & -1.02 \\
\hline W03F9.4 & -1.53 & F45D11.14 & -1.2 & Y105C5A.2 & -1.08 & mltn-9 & -1.02 \\
\hline F40G12.5 & -1.5 & pqn-26 & -1.19 & sams-5 & -1.07 & C08F1.6 & -1.02 \\
\hline F23A7.8 & -1.46 & Y71A12B.11 & -1.19 & C08F1.10 & -1.07 & F12A10.1 & -1.02 \\
\hline Y39G10AR.15 & -1.46 & Y38E10A.3 & -1.19 & Y82E9BR.17 & -1.07 & K08E3.2 & -1.01 \\
\hline snet-1 & -1.45 & ZK675.4 & -1.18 & Y19D2B.2 & -1.07 & fbxb-94 & -1.01 \\
\hline T14B4.19 & -1.44 & C38D9.8 & -1.18 & $\lg x-1$ & -1.07 & his-16 & -1.01 \\
\hline txdc-12.2 & -1.44 & F48E3.6 & -1.17 & C47E12.9 & -1.07 & skr-8 & -1.01 \\
\hline hsp-12.6 & -1.43 & col-176 & -1.17 & fbxb-62 & -1.06 & W10D9.2 & -1.01 \\
\hline F01D4.8 & -1.42 & $\mathrm{fbxb}-78$ & -1.17 & M153.3 & -1.06 & Y57A10B.6 & -1.01 \\
\hline F59C12.4 & -1.41 & H39E23.3 & -1.17 & W02H5.8 & -1.06 & $\operatorname{lgc}-27$ & -1.01 \\
\hline atz-1 & -1.38 & K03H6.2 & -1.16 & fbxb-57 & -1.06 & C45E5.1 & -1.01 \\
\hline Y6E2A.4 & -1.37 & F33D4.6 & -1.16 & F22E5.20 & -1.06 & C24B5.4 & -1.01 \\
\hline aman-1 & -1.36 & R05H5.7 & -1.16 & F43D9.1 & -1.06 & R12E2.8 & -1 \\
\hline C35E7.5 & -1.34 & cutl-2 & -1.15 & W04A8.9 & -1.06 & T26E3.8 & -1 \\
\hline qua-1 & -1.33 & Y17G9B.2 & -1.15 & bath-31 & -1.06 & $f b x b-18$ & -1 \\
\hline dsl-3 & -1.33 & col-172 & -1.14 & F55C9.5 & -1.06 & Y47G6A.31 & -1 \\
\hline asns-2 & -1.32 & ZC334.7 & -1.13 & R01E6.2 & -1.05 & fipr-7 & 1 \\
\hline ugt-53 & -1.31 & F39G3.2 & -1.13 & C10C5.5 & -1.05 & F49H6.5 & 1 \\
\hline F21E9.2 & -1.31 & F55G11.8 & -1.13 & Y41D4B.26 & -1.05 & D1054.18 & 1 \\
\hline M02G9.2 & -1.3 & Y65A5A.1 & -1.13 & sym-1 & -1.05 & F58F12.12 & 1.01 \\
\hline C46C11.4 & -1.29 & dpy-7 & -1.12 & pqn-13 & -1.05 & F10C1.9 & 1.01 \\
\hline F45B8.3 & -1.29 & R04A9.7 & -1.12 & $\mathrm{fbxb}-7$ & -1.04 & mif-4 & 1.02 \\
\hline ZK105.13 & -1.29 & F16B3.2 & -1.12 & hch-1 & -1.04 & pgp-9 & 1.02 \\
\hline K02E11.15 & -1.29 & $\mathrm{fbxb}-35$ & -1.12 & C40A11.2 & -1.04 & clec-57 & 1.03 \\
\hline C35E7.9 & -1.29 & F56F4.8 & -1.12 & T01D1.8 & -1.04 & K12D9.1 & 1.03 \\
\hline $\mathrm{mtl}-1$ & -1.28 & F40E10.5 & -1.12 & skr-15 & -1.04 & $\mathrm{E} 02 \mathrm{H} 4.4$ & 1.04 \\
\hline Y82E9BR.1 & -1.27 & zipt-17 & -1.11 & nas-12 & -1.04 & C25F9.4 & 1.04 \\
\hline sdz-30 & -1.26 & Y57G7A.2 & -1.11 & C06C3.7 & -1.04 & col-153 & 1.05 \\
\hline$f k b-5$ & -1.26 & C55B7.3 & -1.11 & $f b x b-96$ & -1.03 & F57H12.6 & 1.06 \\
\hline
\end{tabular}




\begin{tabular}{|c|c|c|c|}
\hline Gene & $\log 2 \mathrm{FC}$ & Gene & $\log 2 \mathrm{FC}$ \\
\hline F18E3.13 & 1.06 & slc-17.3 & 1.26 \\
\hline nspc-19 & 1.07 & K08D10.14 & 1.27 \\
\hline cpr-1 & 1.07 & C05D12.3 & 1.28 \\
\hline F18E3.12 & 1.07 & clec- 61 & 1.29 \\
\hline irg-5 & 1.07 & $\mathrm{C} 34 \mathrm{H} 4.2$ & 1.29 \\
\hline Iron-4 & 1.07 & ilys-2 & 1.29 \\
\hline scl-14 & 1.08 & fbxa-166 & 1.3 \\
\hline capa-1 & 1.08 & fipr-9 & 1.34 \\
\hline F53B3.6 & 1.08 & dct-5 & 1.35 \\
\hline $\mathrm{mfb}-1$ & 1.08 & srd-14 & 1.36 \\
\hline fipr-8 & 1.08 & cpr-4 & 1.36 \\
\hline T04C12.32 & 1.09 & F28E10.5 & 1.37 \\
\hline Y69A2AR.19 & 1.1 & clec- 80 & 1.4 \\
\hline clec- 230 & 1.1 & R07C12.1 & 1.42 \\
\hline nspc-17 & 1.1 & str-112 & 1.44 \\
\hline K01D12.9 & 1.11 & nspc-15 & 1.46 \\
\hline F20B6.5 & 1.11 & nspc-12 & 1.46 \\
\hline math-18 & 1.11 & nspc-14 & 1.53 \\
\hline Y46H3A.5 & 1.12 & F46A8.1 & 1.53 \\
\hline T16G1.6 & 1.12 & C23G10.11 & 1.54 \\
\hline wrt-8 & 1.14 & asp-16 & 1.57 \\
\hline F53F4.1 & 1.16 & nspc-18 & 1.58 \\
\hline T04C12.7 & 1.16 & lipl-3 & 1.6 \\
\hline pсp-3 & 1.17 & fbxa-136 & 1.62 \\
\hline nspe-6 & 1.17 & F10A3.4 & 1.7 \\
\hline nspc-20 & 1.17 & hsp-16.48 & 1.77 \\
\hline T20F5.5 & 1.17 & col-106 & 1.81 \\
\hline T16G1.4 & 1.18 & hsp-16.49 & 1.89 \\
\hline clec- 42 & 1.19 & ZK218.1 & 2.57 \\
\hline dnj-20 & 1.19 & & \\
\hline gtf-2H1 & 1.2 & & \\
\hline H02F09.3 & 1.2 & & \\
\hline gba-2 & 1.21 & & \\
\hline tag-244 & 1.23 & & \\
\hline nspc-16 & 1.23 & & \\
\hline clec- 25 & 1.23 & & \\
\hline chil-22 & 1.23 & & \\
\hline C50F7.5 & 1.24 & & \\
\hline ZC204.1 & 1.25 & & \\
\hline C45E1.4 & 1.25 & & \\
\hline
\end{tabular}


6.22. RNA-seq (MPI-MG) - N2 (wild-type) vs HBR1777 (flp-11::EGL-1) 48h Starved

\begin{tabular}{|c|c|c|c|c|c|c|c|}
\hline Gene & $\log 2 \mathrm{FC}$ & Gene & $\log 2 \mathrm{FC}$ & Gene & $\log 2 \mathrm{FC}$ & Gene & $\log 2 \mathrm{FC}$ \\
\hline dmsr-2 & -3.81 & T23F6.1 & -1.89 & $\mathrm{~T} 13 \mathrm{C} 2.2$ & -1.63 & wht-3 & -1.43 \\
\hline F12E12.12 & -2.73 & gst-12 & -1.87 & T19D12.3 & -1.62 & abu-6 & -1.42 \\
\hline M03E7.4 & -2.57 & gst-39 & -1.85 & сур-13A3 & -1.62 & F42A8.1 & -1.42 \\
\hline C04G6.7 & -2.41 & gsto-2 & -1.85 & F33H12.7 & -1.62 & Y102A11A.7 & -1.42 \\
\hline R05D8.7 & -2.4 & ZK1010.4 & -1.85 & $c d r-4$ & -1.61 & F20B10.3 & -1.41 \\
\hline pqn-26 & -2.37 & pqn-29 & -1.84 & C03F11.2 & -1.6 & R04B3.3 & -1.41 \\
\hline F35A5.4 & -2.34 & B0205.13 & -1.84 & plpr-1 & -1.59 & K08D12.4 & -1.4 \\
\hline M153.3 & -2.34 & pqn-63 & -1.82 & $\lg x-1$ & -1.59 & nas-13 & -1.4 \\
\hline T05H4.7 & -2.28 & W08E12.6 & -1.82 & T06D8.10 & -1.59 & col-113 & -1.4 \\
\hline D2096.6 & -2.28 & sri-39 & -1.82 & gst-33 & -1.58 & W08E12.3 & -1.4 \\
\hline F41G3.3 & -2.28 & C35A5.10 & -1.82 & W06H8.2 & -1.58 & F59B10.3 & -1.39 \\
\hline gst-37 & -2.26 & gst-30 & -1.81 & T28C12.6 & -1.58 & pqn-67 & -1.39 \\
\hline R12A1.3 & -2.26 & R03C1.1 & -1.8 & C25F6.8 & -1.56 & numr-2 & -1.38 \\
\hline F12E12.11 & -2.23 & gst-25 & -1.78 & drd-1 & -1.56 & numr-1 & -1.38 \\
\hline abu-14 & -2.22 & T06E4.8 & -1.77 & C34E11.2 & -1.55 & col-89 & -1.38 \\
\hline T24C12.4 & -2.14 & Y43F8B.3 & -1.77 & K01A6.7 & -1.55 & W08E12.4 & -1.37 \\
\hline F07H5.8 & -2.13 & W08E12.2 & -1.77 & W03F9.4 & -1.55 & W08E12.5 & -1.37 \\
\hline T20B6.3 & -2.12 & сур-13A10 & -1.76 & C18E9.7 & -1.52 & grl-6 & -1.37 \\
\hline M195.2 & -2.1 & ZC334.7 & -1.75 & C54D2.1 & -1.52 & F55G1.1 & -1.37 \\
\hline K08D12.8 & -2.08 & C36B7.5 & -1.75 & lact-7 & -1.52 & C08E8.10 & -1.37 \\
\hline ZK354.3 & -2.07 & C26B2.8 & -1.73 & ora-1 & -1.5 & abu-7 & -1.36 \\
\hline ZK662.2 & -2.06 & R09E12.9 & -1.72 & F20G2.1 & -1.5 & twk-11 & -1.35 \\
\hline M02G9.1 & -2.03 & phat-2 & -1.72 & col-172 & -1.5 & abu-8 & -1.34 \\
\hline gst-31 & -2.02 & pqn-74 & -1.72 & K09B3.1 & -1.49 & cpt-5 & -1.34 \\
\hline C06E4.6 & -1.99 & C56C10.4 & -1.71 & F52A8.3 & -1.49 & F55F8.8 & -1.33 \\
\hline nas-15 & -1.96 & F25D1.5 & -1.71 & F32A7.8 & -1.49 & D1054.8 & -1.33 \\
\hline Y50D4B.2 & -1.96 & E01G6.1 & -1.71 & gpdh-1 & -1.48 & R07E3.2 & -1.32 \\
\hline Y53F4B.36 & -1.95 & C06E4.3 & -1.7 & asns-1 & -1.47 & F40F11.4 & -1.32 \\
\hline $\mathrm{K} 10 \mathrm{H} 10.6$ & -1.95 & T25E4.1 & -1.7 & Iron-2 & -1.47 & T06E4.14 & -1.32 \\
\hline gst-8 & -1.95 & K01D12.5 & -1.7 & phg-1 & -1.46 & sri-36 & -1.32 \\
\hline R02F11.1 & -1.93 & M02G9.2 & -1.69 & C06G1.2 & -1.45 & T22F3.11 & -1.32 \\
\hline gst-5 & -1.93 & Y47D3B.6 & -1.68 & F59B1.8 & -1.45 & clec-163 & -1.32 \\
\hline R13H4.8 & -1.92 & abu-11 & -1.68 & gst-21 & -1.45 & F57H12.6 & -1.31 \\
\hline grl-1 & -1.92 & chs-2 & -1.67 & abu-15 & -1.45 & stdh-2 & -1.31 \\
\hline nas-14 & -1.9 & T01G6.1 & -1.66 & Y75B7AR.1 & -1.45 & ZK742.4 & -1.31 \\
\hline sdz-8 & -1.9 & cutl-12 & -1.65 & col-69 & -1.44 & T13F3.8 & -1.3 \\
\hline acd-2 & -1.9 & Y65B4BL.6 & -1.65 & gst-16 & -1.44 & asic-2 & -1.3 \\
\hline B0205.14 & -1.9 & F41E6.11 & -1.65 & F07B7.8 & -1.43 & comt-3 & -1.29 \\
\hline D2092.8 & -1.89 & C29G2.3 & -1.65 & nhr-237 & -1.43 & C56C10.6 & -1.29 \\
\hline T10E10.4 & -1.89 & F40E10.5 & -1.65 & K06C4.1 & -1.43 & pqn-13 & -1.29 \\
\hline
\end{tabular}




\begin{tabular}{|c|c|c|c|c|c|c|c|}
\hline Gene & $\log 2 \mathrm{FC}$ & Gene & $\log 2 \mathrm{FC}$ & Gene & $\log 2 \mathrm{FC}$ & Gene & $\log 2 \mathrm{FC}$ \\
\hline gpx-2 & -1.28 & cpg-9 & -1.14 & nep-12 & -1.06 & F45D11.15 & 1.02 \\
\hline gst-40 & -1.28 & C30A5.10 & -1.13 & Y48G1BM.8 & -1.06 & F45D11.16 & 1.02 \\
\hline gst-26 & -1.28 & grd-12 & -1.13 & R08A2.5 & -1.06 & mex-1 & 1.02 \\
\hline aip-1 & -1.27 & C55A6.6 & -1.13 & Y39G10AR.15 & -1.06 & F45D11.14 & 1.02 \\
\hline gst-35 & -1.27 & col-51 & -1.13 & gba-2 & -1.05 & clec-13 & 1.02 \\
\hline dhs-23 & -1.26 & ugt-25 & -1.13 & abu-12 & -1.05 & W05F2.3 & 1.02 \\
\hline hmit-1.2 & -1.26 & Y53F4B.27 & -1.13 & Y52E8A.3 & -1.05 & T02G5.14 & 1.02 \\
\hline fmo-5 & -1.25 & Y73C8B.1 & -1.13 & his-18 & -1.04 & B0024.4 & 1.02 \\
\hline irg-3 & -1.25 & clec-174 & -1.12 & C15F1.5 & -1.04 & F15D4.5 & 1.03 \\
\hline $\mathrm{H} 12 \mathrm{D} 21.2$ & -1.24 & $\mathrm{fbxb}-36$ & -1.12 & otpl-1 & -1.04 & W09C3.7 & 1.03 \\
\hline folt-2 & -1.24 & col-176 & -1.12 & rpr-1 & -1.04 & C45B11.7 & 1.03 \\
\hline fpn-1.2 & -1.24 & sri-38 & -1.12 & Y113G7B.15 & -1.04 & C17E7.4 & 1.04 \\
\hline ZC412.9 & -1.24 & spl-2 & -1.12 & F23F12.8 & -1.04 & R11D1.3 & 1.04 \\
\hline arf-1.1 & -1.23 & F23A7.8 & -1.12 & ZC21.10 & -1.04 & F07B7.2 & 1.05 \\
\hline cutl-27 & -1.23 & сур-13A2 & -1.11 & H10E21.4 & -1.03 & W09B7.2 & 1.05 \\
\hline slc-17.8 & -1.23 & nas-1 & -1.11 & comt-5 & -1.03 & irg-5 & 1.05 \\
\hline gst-7 & -1.23 & K02D10.2 & -1.11 & R08F11.4 & -1.03 & sip-1 & 1.05 \\
\hline zipt-17 & -1.22 & lagr-1 & -1.11 & ptr-19 & -1.03 & M60.7 & 1.06 \\
\hline K08E3.2 & -1.21 & pqn-36 & -1.11 & dhs- 8 & -1.03 & Y43F8B.24 & 1.06 \\
\hline gst-4 & -1.21 & exc- 6 & -1.11 & npax-2 & -1.02 & K01D12.3 & 1.06 \\
\hline D1007.13 & -1.21 & ram-5 & -1.1 & gst-38 & -1.02 & asm-3 & 1.07 \\
\hline B0554.5 & -1.21 & gpx-1 & -1.1 & qua-1 & -1.02 & scl-14 & 1.07 \\
\hline cat-2 & -1.21 & F59C12.4 & -1.1 & K02E11.15 & -1.01 & D1054.18 & 1.08 \\
\hline dhs-9 & -1.2 & D2005.6 & -1.09 & F53B3.3 & -1.01 & F26D11.12 & 1.08 \\
\hline T06E4.9 & -1.19 & F56F4.8 & -1.09 & T17H7.7 & -1.01 & C35A5.5 & 1.08 \\
\hline T07A5.1 & -1.19 & M88.4 & -1.09 & tts-2 & -1.01 & T10G3.4 & 1.08 \\
\hline Igc- 27 & -1.19 & F53C3.3 & -1.09 & K01A12.4 & -1.01 & M02E1.4 & 1.09 \\
\hline Irx-1 & -1.19 & F08D12.2 & -1.08 & C09B8.4 & -1.01 & clec- 87 & 1.09 \\
\hline jud-4 & -1.18 & T06A4.1 & -1.08 & nas-8 & -1.01 & srap-1 & 1.1 \\
\hline T16A9.3 & -1.18 & $\mathrm{R} 02 \mathrm{C} 2.7$ & -1.08 & col-111 & -1.01 & meg-1 & 1.11 \\
\hline phat-1 & -1.17 & acox-1.2 & -1.08 & Y57G7A.2 & -1.01 & $\mathrm{~F} 15 \mathrm{H} 10.8$ & 1.12 \\
\hline gst-9 & -1.17 & F26D2.15 & -1.07 & K08D8.4 & -1 & F29C6.1 & 1.13 \\
\hline his-5 & -1.17 & twk-34 & -1.07 & acox-1.1 & -1 & C06E8.5 & 1.13 \\
\hline grd-15 & -1.16 & F53H4.3 & -1.07 & C42D4.13 & 1 & F21H7.12 & 1.14 \\
\hline Y57G11B.4 & -1.16 & Y32G9B.1 & -1.07 & Y68A4B.3 & 1.01 & sri-31 & 1.14 \\
\hline D2063.1 & -1.16 & col-54 & -1.06 & C45E5.4 & 1.01 & meg-2 & 1.14 \\
\hline Y76B12C.4 & -1.16 & her-1 & -1.06 & gln-6 & 1.01 & srd-14 & 1.15 \\
\hline pqn-71 & -1.15 & F07A11.1 & -1.06 & Imd-4 & 1.01 & cpg-2 & 1.16 \\
\hline col-48 & -1.15 & Y37F4.1 & -1.06 & clec-15 & 1.01 & fipr-6 & 1.16 \\
\hline F23D12.11 & -1.14 & col-186 & -1.06 & nhr-219 & 1.02 & T19D7.6 & 1.17 \\
\hline
\end{tabular}




\begin{tabular}{|c|c|c|c|}
\hline Gene & $\log 2 \mathrm{FC}$ & Gene & $\log 2 \mathrm{FC}$ \\
\hline $\mathrm{E} 02 \mathrm{H} 4.4$ & 1.17 & dct-5 & 1.66 \\
\hline $\mathrm{C} 24 \mathrm{H} 10.3$ & 1.17 & nspc-19 & 1.71 \\
\hline lipl-3 & 1.18 & unc-119 & 1.72 \\
\hline skr-5 & 1.18 & nspc-20 & 2.03 \\
\hline F08H9.3 & 1.19 & hsp-16.48 & 2.05 \\
\hline col-138 & 1.2 & nspc-16 & 2.07 \\
\hline fipr-8 & 1.2 & nspc-15 & 2.15 \\
\hline fipr-7 & 1.21 & nspc-12 & 2.15 \\
\hline acbp-4 & 1.21 & hsp-16.49 & 2.18 \\
\hline K03D3.2 & 1.22 & nspc-18 & 2.54 \\
\hline T28A11.19 & 1.22 & nspc-14 & 2.89 \\
\hline math-38 & 1.23 & col-108 & 3.1 \\
\hline ZK381.2 & 1.23 & ZK218.1 & 3.43 \\
\hline T28A11.5 & 1.24 & egl-1 & 4.59 \\
\hline capa-1 & 1.24 & & \\
\hline fbxa-136 & 1.26 & & \\
\hline C05D9.9 & 1.26 & & \\
\hline tyr-3 & 1.27 & & \\
\hline cey-2 & 1.27 & & \\
\hline fipr-5 & 1.3 & & \\
\hline cpg-1 & 1.3 & & \\
\hline fipr-10 & 1.31 & & \\
\hline C04E6.8 & 1.34 & & \\
\hline ugt-15 & 1.34 & & \\
\hline C27A7.2 & 1.36 & & \\
\hline Iron-4 & 1.37 & & \\
\hline Y46H3A.5 & 1.38 & & \\
\hline ZC204.1 & 1.4 & & \\
\hline fbxa-185 & 1.4 & & \\
\hline F35G2.5 & 1.42 & & \\
\hline far-5 & 1.44 & & \\
\hline gmn-1 & 1.45 & & \\
\hline fipr-9 & 1.45 & & \\
\hline sre-6 & 1.45 & & \\
\hline F41E7.20 & 1.52 & & \\
\hline asp-16 & 1.54 & & \\
\hline pud-4 & 1.56 & & \\
\hline col-106 & 1.58 & & \\
\hline F07H5.13 & 1.59 & & \\
\hline nspc-17 & 1.6 & & \\
\hline
\end{tabular}


6.23. Proteome (LFQ) - N2 (wild-type) vs HBR227 (aptf-1) - 48h Starved

\begin{tabular}{|c|c|}
\hline Protein & log2FC \\
\hline grd-5 & -1.82 \\
\hline hsp-12.6 & -1.41 \\
\hline ttr-47 & -1.3 \\
\hline pud-1.1 & -1.21 \\
\hline ttr-16 & -1.21 \\
\hline unc-44 & -1.05 \\
\hline F45D11.15 & -1.03 \\
\hline misc-1 & -1.02 \\
\hline acox-3 & -1.01 \\
\hline lim-8 & 1.03 \\
\hline dpy-28 & 1.22 \\
\hline sfxn-1.5 & 1.22 \\
\hline K08D12.3 & 1.26 \\
\hline dao-5 & 1.29 \\
\hline gck-1 & 1.37 \\
\hline
\end{tabular}

6.24. Proteome (LFQ) - N2 (wild-type) vs HBR1777 (flp-11::EGL-1) - 48h Starved

\begin{tabular}{|c|c|c|c|c|c|c|c|}
\hline Protein & $\log 2 \mathrm{FC}$ & Protein & $\log 2 \mathrm{FC}$ & Protein & $\log 2 \mathrm{FC}$ & Protein & $\log 2 \mathrm{FC}$ \\
\hline $\mathrm{rbm}-3.1$ & -2.21 & rho-1 & -1.12 & ifet-1 & 1.03 & T16G1.9 & 1.17 \\
\hline hsp-12.6 & -2.12 & T03F1.11 & -1.1 & K09F5.6 & 1.04 & lim-8 & 1.18 \\
\hline hsp-16.48 & -1.7 & phat-5 & -1.1 & swsn-7 & 1.05 & gck-1 & 1.19 \\
\hline hsp-16.11 & -1.6 & dnj-13 & -1.09 & vha-14 & 1.05 & mrps-2 & 1.25 \\
\hline vpr-1 & -1.38 & Ism-3 & -1.09 & K09G1.1 & 1.06 & vit-3 & 1.26 \\
\hline $\mathrm{T} 20 \mathrm{H} 4.5$ & -1.33 & rps-21 & -1.06 & eif-3.I & 1.06 & rab-8 & 1.36 \\
\hline ostd-1 & -1.32 & ufm-1 & -1.04 & eif-3.F & 1.07 & taap-1 & 1.37 \\
\hline zfp-1 & -1.27 & ttr-16 & -1.03 & $\operatorname{cox}-6 \mathrm{C}$ & 1.09 & acox-1.5 & 1.37 \\
\hline msa-1 & -1.26 & F40A3.3 & -1.03 & swan-1 & 1.1 & vit-5 & 1.39 \\
\hline C32D5.8 & -1.26 & tli-1 & -1.02 & $\mathrm{xrn}-1$ & 1.11 & $\mathrm{~K} 11 \mathrm{H} 12.8$ & 1.5 \\
\hline tni-4 & -1.24 & tdo-2 & 1 & wago-1 & 1.11 & rab-10 & 1.67 \\
\hline ctsa-3.1 & -1.2 & pqn-87 & 1.01 & in $x-3$ & 1.12 & & \\
\hline unc-44 & -1.18 & csnk-1 & 1.02 & dod-19 & 1.12 & & \\
\hline mif-1 & -1.16 & C49A1.10 & 1.02 & T18D3.1 & 1.14 & & \\
\hline kdp-1 & -1.16 & cku-80 & 1.02 & vit-4 & 1.15 & & \\
\hline zig-12 & -1.12 & vrk-1 & 1.03 & F56F11.4 & 1.17 & & \\
\hline
\end{tabular}


6.25. Metabolome - N2 (wild-type) vs HBR227 (aptf-1) - 48h Starved

\begin{tabular}{|c|c|}
\hline Metabolite & $\log 2 \mathrm{FC}$ \\
\hline Sphingomyelin (d18:0/16:1(9Z)) & -1.89 \\
\hline Glycerol 3-phosphate & -1.51 \\
\hline Sphingomyelin (d18:1/26:1(17Z)), Sphingomyelin (d18:0/26:1(17Z)) & -1.44 \\
\hline Sphingomyelin (d18:0/18:0) & -1.43 \\
\hline L-Methionine & -1.37 \\
\hline Sphingomyelin (d18:0/12:0) & -1.35 \\
\hline L-Proline & -1.29 \\
\hline Sphingomyelin (d18:0/22:3(10Z,13Z,16Z)) & -1.11 \\
\hline Sphingomyelin (d18:0/14:0) & -1.09 \\
\hline Uracil & -0.87 \\
\hline Sphingomyelin (d18:0/22:1(13Z)(OH)) & -0.84 \\
\hline Sphingomyelin (d17:1/24:0) & -0.79 \\
\hline Sphingomyelin (d19:1/24:1(15Z)) & -0.78 \\
\hline Sphingomyelin (d18:1/18:1(9Z)), Sphingomyelin (d18:1/18:1(11Z)) & -0.76 \\
\hline Sphingomyelin (d18:1/22:1(13Z)) & -0.76 \\
\hline Sphingomyelin (d18:1/22:0), Sphingomyelin (d18:0/22:1(13Z)) & -0.64 \\
\hline Sphingomyelin (d18:0/26:0) & -0.58 \\
\hline Sphingomyelin (d18:1/24:1(15Z)) & -0.53 \\
\hline Sphingomyelin (d18:1/12:0) & 0.62 \\
\hline Adenosine monophosphate & 0.64 \\
\hline Choline & 0.78 \\
\hline L-Arginine & 1.01 \\
\hline Butyrylcarnitine, Isobutyryl-L-carnitine & 1.09 \\
\hline Propionylcarnitine & 1.1 \\
\hline L-Phenylalanine & 1.42 \\
\hline Uric acid & 1.43 \\
\hline Allantoin & 1.46 \\
\hline Succinic acid & 1.47 \\
\hline Sphingomyelin (d18:1/26:0) & 1.93 \\
\hline 5'-Methylthioadenosine & 1.95 \\
\hline Glutathione & 2.23 \\
\hline Methylmalonylcarnitine & 2.23 \\
\hline L-Tryptophan & 2.53 \\
\hline
\end{tabular}


6.26. Metabolome - N2 (wild-type) vs HBR1777 (flp-11::EGL-1) - 48h

Starved

\begin{tabular}{|c|c|}
\hline Metabolite & $\log 2 \mathrm{FC}$ \\
\hline L-Methionine & -3.16 \\
\hline
\end{tabular}

\subsection{Metabolome - N2 (wild-type) vs HBR227 (aptf-1) - 6 days Starved}

\begin{tabular}{|c|c|}
\hline Metabolite & $\log 2 \mathrm{FC}$ \\
\hline Uridine diphosphate- $\mathrm{N}$-acetylglucosamine & -1.24 \\
\hline Trehalose & -1.18 \\
\hline Methylmalonylcarnitine & -1 \\
\hline L-Tyrosine & -0.82 \\
\hline Sphingomyelin (d18:0/16:1(9Z)) & -0.78 \\
\hline L-Valine & -0.73 \\
\hline Sphingomyelin (d18:0/22:2(13Z,16Z)(OH)) & -0.58 \\
\hline Sphingomyelin (d18:0/24:1(15Z)(OH)), Sphingomyelin (d18:1/25:0) & -0.55 \\
\hline FAD & -0.55 \\
\hline Nicotinic acid & 0.64 \\
\hline L-Tryptophan & 0.67 \\
\hline Cytidine & 0.78 \\
\hline Niacinamide & 0.88 \\
\hline 3-Dehydroxycarnitine & 0.89 \\
\hline CDP-choline & 1.07 \\
\hline Hypoxanthine & 1.09 \\
\hline L-Serine & 1.2 \\
\hline Flavin Mononucleotide & 1.3 \\
\hline Deoxyguanosine & 1.39 \\
\hline Guanosine & 1.73 \\
\hline Kynurenic acid & 1.78 \\
\hline Guanine & 2.38 \\
\hline Inosine & 2.51 \\
\hline
\end{tabular}


6.28. Metabolome - N2 (wild-type) vs HBR1777 (flp-11::EGL-1) - 6 days

Starved

\begin{tabular}{|c|c|}
\hline Metabolite & $\log 2 \mathrm{FC}$ \\
\hline Methylmalonylcarnitine & -4.27 \\
\hline Tyramine & -4.2 \\
\hline Adenylsuccinic acid & -3.34 \\
\hline Pyroglutamic acid & -3.24 \\
\hline Kynurenic acid & -3.12 \\
\hline Thiamine & -2.92 \\
\hline L-Cystathionine & -2.34 \\
\hline Fumaric acid & -2.17 \\
\hline 3-Aminoisobutanoic acid & -2.02 \\
\hline Ophthalmic acid & -2 \\
\hline NADP & -1.96 \\
\hline Methylmalonic acid & -1.79 \\
\hline L-Tyrosine & -1.4 \\
\hline Hydroxypyruvic acid & -1.21 \\
\hline L-Proline & -1.17 \\
\hline L-Valine & -1.1 \\
\hline Riboflavin & -1.09 \\
\hline Hypoxanthine & -1.05 \\
\hline Sphingomyelin (d18:0/20:2(11Z,14Z)) & -0.85 \\
\hline Sphingomyelin (d18:0/24:1(15Z)(OH)), Sphingomyelin (d18:1/25:0) & -0.84 \\
\hline L-Phenylalanine & -0.76 \\
\hline Sphingomyelin (d18:1/23:0) & -0.74 \\
\hline Sphingomyelin (d18:0/20:0) & -0.72 \\
\hline Sphingomyelin (d18:0/22:3(10Z,13Z,16Z)) & -0.68 \\
\hline Sphingomyelin (d18:0/22:1(13Z)(OH)) & -0.68 \\
\hline Sphingomyelin (d18:1/18:0) & -0.67 \\
\hline Sphingomyelin (d17:1/24:1(15Z)) & -0.64 \\
\hline Sphingomyelin (d18:1/22:0), Sphingomyelin (d18:0/22:1(13Z)) & -0.64 \\
\hline Sphingomyelin (d18:1/22:1(13Z)) & -0.62 \\
\hline Pyridoxal & 1.25 \\
\hline Butyrylcarnitine, Isobutyryl-L-carnitine & 1.28 \\
\hline Pantothenic acid & 1.42 \\
\hline L-Carnitine & 1.46 \\
\hline Nicotinic acid & 1.65 \\
\hline Uracil & 1.73 \\
\hline L-Serine & 1.73 \\
\hline L-Targinine & 1.78 \\
\hline L-Acetylcarnitine & 2.05 \\
\hline ADP & 2.36 \\
\hline L-Glutamine & 2.45 \\
\hline
\end{tabular}




\begin{tabular}{|c|c|}
\hline Metabolite & $\log 2 \mathrm{FC}$ \\
\hline Glycerol 3-phosphate & 2.52 \\
\hline Guanine & 2.55 \\
\hline Flavin Mononucleotide & 2.81 \\
\hline L-Tryptophan & 3.1 \\
\hline 2-Aminobenzoic acid & 3.21 \\
\hline CDP-choline & 3.53 \\
\hline L-Glycine & 3.59 \\
\hline
\end{tabular}


6.29. Genetic screen for quiescence phenotypes

\begin{tabular}{|c|c|c|c|}
\hline Strain & Gene & Allele & Effect size \\
\hline HBR2315 & aptf-1; tdc-1 & gk794;n3420 & -3.08 \\
\hline HBR2050 & dgk-1 & ok1462 & -2.94 \\
\hline HBR2269 & flp-11; tdc-1 & $\operatorname{tm} 2706 ; \mathrm{n} 3420$ & -2.8 \\
\hline HBR227 & aptf-1 & gk794 & -2.75 \\
\hline HBR2286 & aptf-1; ser-2 & gk794;pk1357 & -2.73 \\
\hline HBR1777 & flp-11::EGL-1 & goels384 & -2.7 \\
\hline RB1690 & ser-2 & ok2103 & -2.64 \\
\hline VC1339 & efhc-1 & ok1856 & -2.62 \\
\hline HBR1010 & nas-38 & ok3407 & -2.53 \\
\hline HBR2242 & aptf-1; elpc-1 & tm2149; gk794 & -2.45 \\
\hline HBR507 & flp-11 & tm2706 & -2.45 \\
\hline HBR2122 & dgk-1 & sy 428 & -2.37 \\
\hline RB737 & snt-4 & ok503 & -2.34 \\
\hline SCL1 & mcp-1 & $\operatorname{tm} 2679$ & -2.23 \\
\hline HBR2233 & aptf-1; tut-1 & gk794; tm1297 & -2.23 \\
\hline RB1400 & tre-3 & ok394 & -2.2 \\
\hline VC225 & tps-1 & ok373 & -2.18 \\
\hline CB156 & unc-25 & e156 & -2.18 \\
\hline VM487 & $\mathrm{nmr}-1$ & ak4 & -2.18 \\
\hline CF1038 & daf-16 & mu86 & -2.17 \\
\hline DA609 & npr-1 & ad609 & -2.16 \\
\hline RB2105 & ntr-1 & ok2780 & -2.16 \\
\hline HBR2312 & aptf-1; glp-1 & gk794;ar202 & -2.15 \\
\hline RB1073 & dgk-4 & ok1031 & -2.14 \\
\hline HBR2374 & aptf-1; ztf-20 & gk263563;gk794 & -2.09 \\
\hline RB1932 & tofu-7 & ok2535 & -2.04 \\
\hline RB2277 & ser-5 & ok3087 & -2.04 \\
\hline VC1876 & pcyt-2.2 & ok2179 & -2.04 \\
\hline RB1942 & ace-2 & ok2545 & -2.01 \\
\hline RB608 & tag-96 & ok336 & -2 \\
\hline NL1832 & ucr-2.3 & $\mathrm{pk} 732$ & -1.98 \\
\hline RB1974 & smd-1 & ok2602 & -1.94 \\
\hline RB1326 & unc-129 & ok1443 & -1.93 \\
\hline HBR1403 & aptf-1; daf-2 & e1370; gk794 & -1.92 \\
\hline RB1036 & hyl-1 & ok976 & -1.92 \\
\hline VC1198 & Y39G8B.1 & ok1682 & -1.91 \\
\hline VC912 & jmjd-3.1 & gk387 & -1.91 \\
\hline ZG31 & hif-1 & ia4 & -1.9 \\
\hline VC2210 & cbl-1 & ok2954 & -1.9 \\
\hline HBR2372 & aptf-1; xbp-1 & zc12;gk794 & -1.9 \\
\hline RB1482 & gpdh-2 & ok1733 & -1.89 \\
\hline VC2171 & tkr-1 & ok2886 & -1.89 \\
\hline
\end{tabular}




\begin{tabular}{|c|c|c|c|}
\hline Strain & Gene & Allele & Effect size \\
\hline RB1053 & sgt-1 & ok1000 & -1.86 \\
\hline RB1160 & ckb-4 & ok1195 & -1.86 \\
\hline RB775 & plpp-1.2 & ok555 & -1.83 \\
\hline MT8347 & ced-3 & $\mathrm{n} 2452$ & -1.83 \\
\hline VC138 & elo-1 & gk48 & -1.81 \\
\hline RB2061 & ora-1 & ok2724 & -1.8 \\
\hline RB1083 & F27C8.5 & ok1050 & -1.79 \\
\hline RB938 & vha-12 & ok281 & -1.79 \\
\hline RB1588 & $\mathrm{mxl}-3$ & ok1947 & -1.79 \\
\hline RB2135 & F33D4.4 & ok2843 & -1.79 \\
\hline KG2730 & clu-1 & ok2 & -1.79 \\
\hline RB748 & gta-1 & ok517 & -1.79 \\
\hline HS184 & swsn-4 & os13 & -1.74 \\
\hline RB2071 & ced-3 & ok2734 & -1.73 \\
\hline VB674 & nnt-1 & sv34 & -1.72 \\
\hline $\mathrm{OH} 313$ & ser-2 & pk1357 & -1.72 \\
\hline $\mathrm{MH} 1090$ & pnc-1 & ku212 & -1.71 \\
\hline MQ989 & isp-1; ctb-1 & qm150; qm189 & -1.71 \\
\hline VC2520 & aagr-2 & ok3193 & -1.7 \\
\hline RB2128 & idh-1 & ok2832 & -1.7 \\
\hline RB1404 & lec-1 & ok1597 & -1.69 \\
\hline CB5602 & vhl-1 & ok161 & -1.69 \\
\hline RB2550 & ugt-23 & ok3541 & -1.67 \\
\hline RB1795 & fat-1 & ok2323 & -1.67 \\
\hline RB1704 & acp-2 & ok2129 & -1.66 \\
\hline RB2549 & sms-3 & ok3540 & -1.66 \\
\hline RB1134 & hpo-13 & ok1157 & -1.66 \\
\hline HBR12 & goa-1 & sa734 & -1.65 \\
\hline RB2625 & F40F12.7 & ok3684 & -1.65 \\
\hline RB512 & mce-1 & ok243 & -1.64 \\
\hline RB1373 & gpdh-1 & ok1558 & -1.64 \\
\hline FX06710 & pho-5 & $\operatorname{tm} 6710$ & -1.63 \\
\hline RB910 & elo-3 & ok777 & -1.62 \\
\hline VC1896 & ckb-3 & ok2310 & -1.58 \\
\hline RB1498 & hyl-2 & ok1766 & -1.58 \\
\hline JT307 & egl-9 & sa307 & -1.56 \\
\hline RB1031 & fat-4 & ok958 & -1.54 \\
\hline VC26 & pgp-12 & gk19 & -1.53 \\
\hline GR1307 & daf-16 & mgDf50 & -1.52 \\
\hline RB1170 & C04B4.2 & ok1212 & -1.51 \\
\hline TU55 & mec-14 & u55 & -1.51 \\
\hline BC119 & blmp-1 & s71 & -1.5 \\
\hline
\end{tabular}




\begin{tabular}{|c|c|c|}
\hline Gene & Allele & Effect size \\
\hline $\mathrm{mmcm}-1$ & ok1637 & -1.49 \\
\hline dot-1.1 & ok2154 & -1.48 \\
\hline ugt-1 & ok2718 & -1.48 \\
\hline sptl-3 & ok1927 & -1.46 \\
\hline gba-1 & gk399608 & -1.45 \\
\hline gpd-3 & ok2870 & -1.44 \\
\hline fasn-1 & g43 & -1.42 \\
\hline$a m x-2$ & ok1235 & -1.42 \\
\hline met-2 & ok2307 & -1.42 \\
\hline C27A7.3 & tm6393 & -1.41 \\
\hline dgk-5 & ok2366 & -1.41 \\
\hline acs-19 & $\operatorname{tm} 4853$ & -1.39 \\
\hline trpa-1 & ok999 & -1.39 \\
\hline pcbd-1 & tm5924 & -1.38 \\
\hline rabn-5 & ok1555 & -1.37 \\
\hline lin-42 & n1089 & -1.36 \\
\hline tkt-1 & ok3624 & -1.34 \\
\hline ced-3 & $\mathrm{n} 717$ & -1.34 \\
\hline pnk-4 & ok1832 & -1.33 \\
\hline egl-9 & $\mathrm{n} 586$ & -1.33 \\
\hline aldo-2 & gk944084 & -1.33 \\
\hline nnt-1 & $\mathrm{tm} 358$ & -1.31 \\
\hline elo-4 & ok1346 & -1.3 \\
\hline vab-3 & ju468 & -1.29 \\
\hline hosl-1 & gk278589 & -1.28 \\
\hline ZC155.4; hxk-3 & gk961143; gk961144 & -1.28 \\
\hline egl-9; hif-1 & sa307; ia4 & -1.27 \\
\hline haf-9 & gk23 & -1.27 \\
\hline asns-2 & ok3108 & -1.27 \\
\hline acly-2 & tm5123 & -1.26 \\
\hline lagr-1 & gk327 & -1.26 \\
\hline capa-1 & ok3065 & -1.24 \\
\hline glod-4 & gk189 & -1.23 \\
\hline set-21 & ok2327 & -1.22 \\
\hline gpd-1 & gk152785 & -1.22 \\
\hline ace-1 & ok663 & -1.21 \\
\hline idh-2 & ok3184 & -1.21 \\
\hline Y94H6A.7 & gk925915 & 1.22 \\
\hline gln-5 & gk875298 & 1.28 \\
\hline elo-5 & gk208 & 1.31 \\
\hline ace-4 & gk942942 & 1.31 \\
\hline aqp-2 & ok2159 & 1.44 \\
\hline
\end{tabular}




\begin{tabular}{|c|c|c|c|}
\hline Strain & Gene & Allele & Effect size \\
\hline CB1370 & daf-2 & e1370 & 1.47 \\
\hline EG9631 & unc-13 & s69 & 1.58 \\
\hline VL1176 & alh-8 & ww48 & 1.63 \\
\hline
\end{tabular}

\subsection{Representative mobility traces and quiescence bouts}
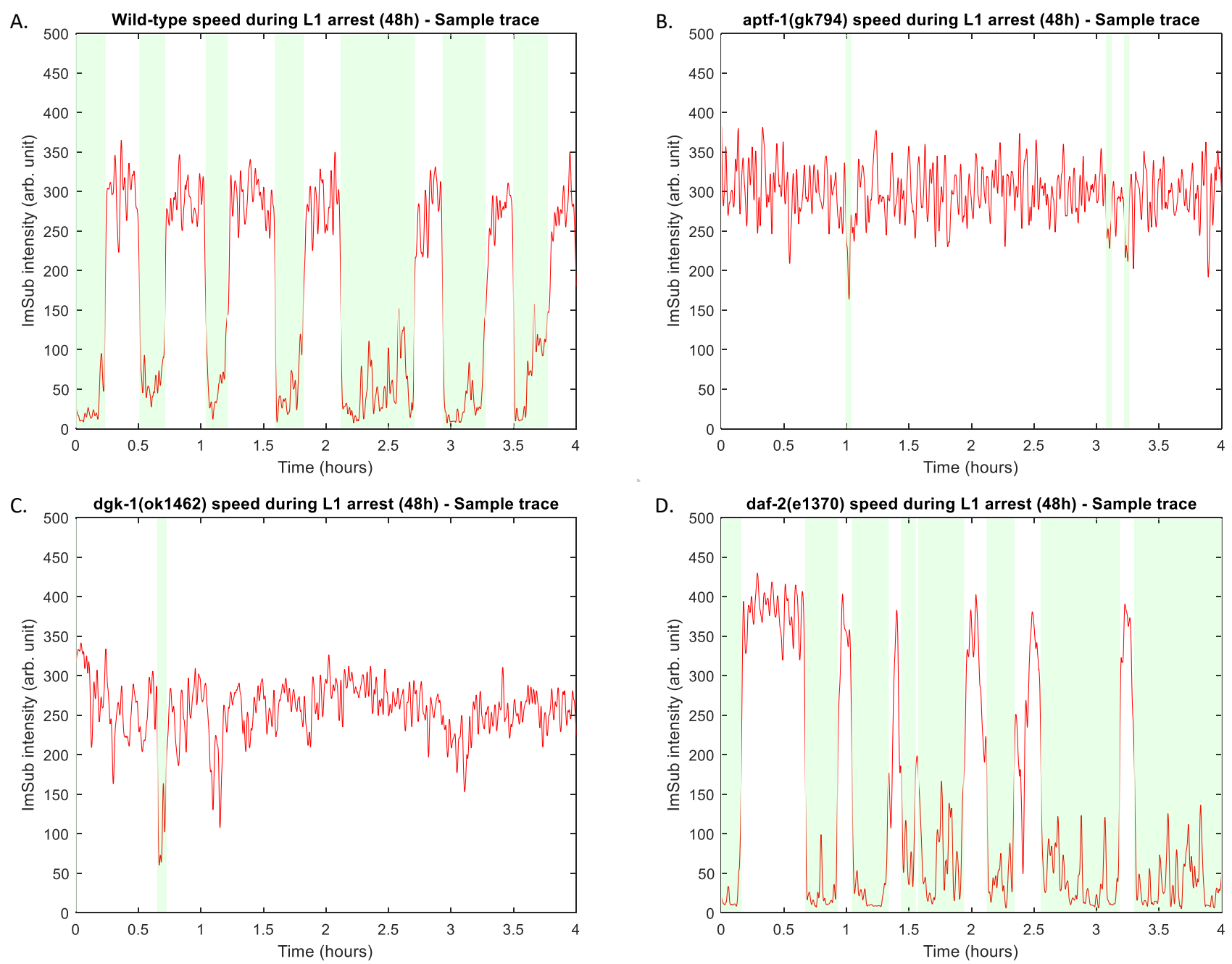

(A-D) Mobility traces of wild-type, aptf-1(gk794), $d g k-1$ (ok1462) and daf-2(e1370) worms. Movement (shown in red) is reduced when the worm goes in a quiescence bout (shown in green). Loss of function of the AP2 transcription factor aptf- 1 and diacylglycerol kinase DGK $\theta$ homolog $d g k-1$ ablates sleep, while loss of function of the insulin receptor $d a f-2$ increases the amount of sleep. 
6.31. Quiescence assessment of wild-type animals after supplementation with methionine, glycine and glutamate

\begin{tabular}{|c|c|}
\hline Treatment & Effect size \\
\hline Methionine $10 \mathrm{mM}$ & -1.19 \\
\hline Methionine $12.5 \mathrm{mM}$ & -1.17 \\
\hline Glycine $10 \mathrm{mM}$ & -0.66 \\
\hline Methionine $25 \mathrm{mM}$ & -0.64 \\
\hline Glutamate $10 \mathrm{mM}$ & -0.63 \\
\hline Glycine $1 \mathrm{mM}$ & -0.52 \\
\hline Glycine $0.1 \mathrm{mM}$ & -0.45 \\
\hline Methionine $1 \mathrm{mM}$ & 0.48 \\
\hline
\end{tabular}

6.32. Impact of maternal growth conditions in the quiescence fraction of the offspring in the wild-type background

\begin{tabular}{|c|c|c|}
\hline Temperature $\left({ }^{\circ} \mathrm{C}\right)$ & Food & Effect size \\
\hline 15 & OP50 & 0.43 \\
\hline 25 & OP50 & -0.41 \\
\hline 20 & Na22 & -0.43 \\
\hline 20 & DL41 & 0.41 \\
\hline
\end{tabular}




\subsection{Representative RIS intensity GCaMP traces}
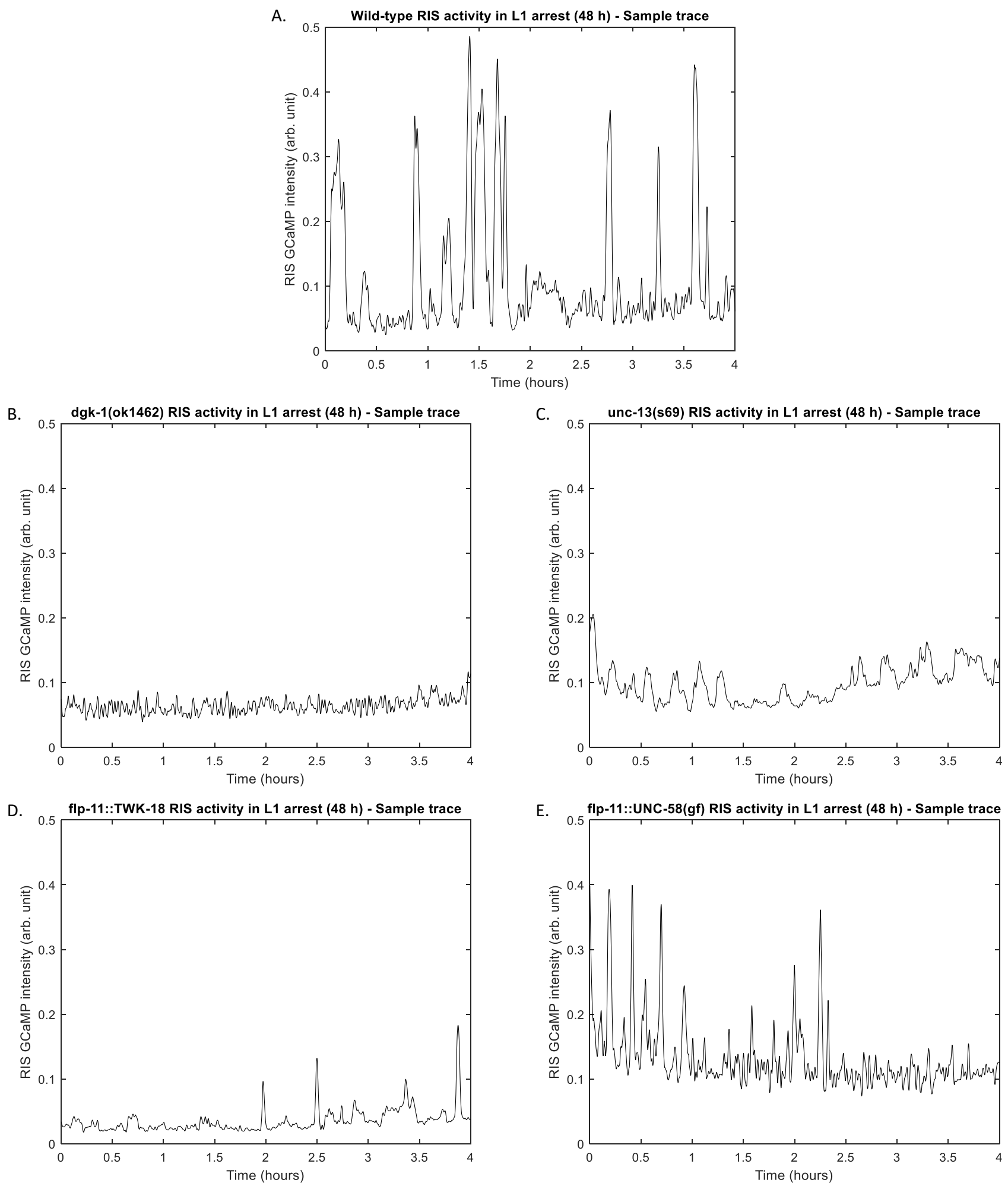

(A) In the wild-type worm characteristic peaks in RIS activity indicate sleep bouts.

(B-C) RIS peaks are absent after loss of function of $d g k-1$ and $u n c-13$.

(D-E) RIS peaks are also absent in the engineered flp-11::TWK-18 strain as expected. In the RIS-hyperactive flp-11::UNC-58(gf) strain RIS does not activate as strong as the wild-type. 


\subsection{HSP-12.6 expression screening in L1 arrest}

A.

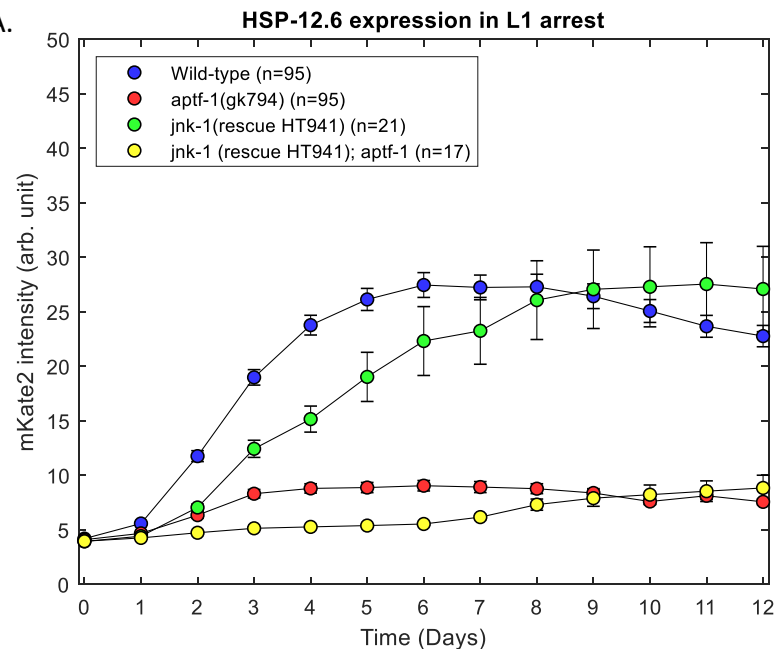

C.

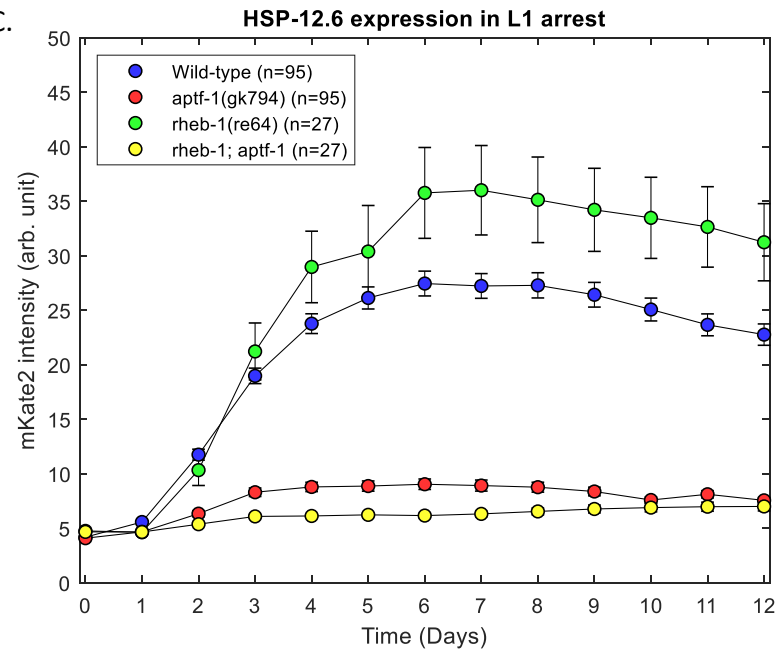

B.

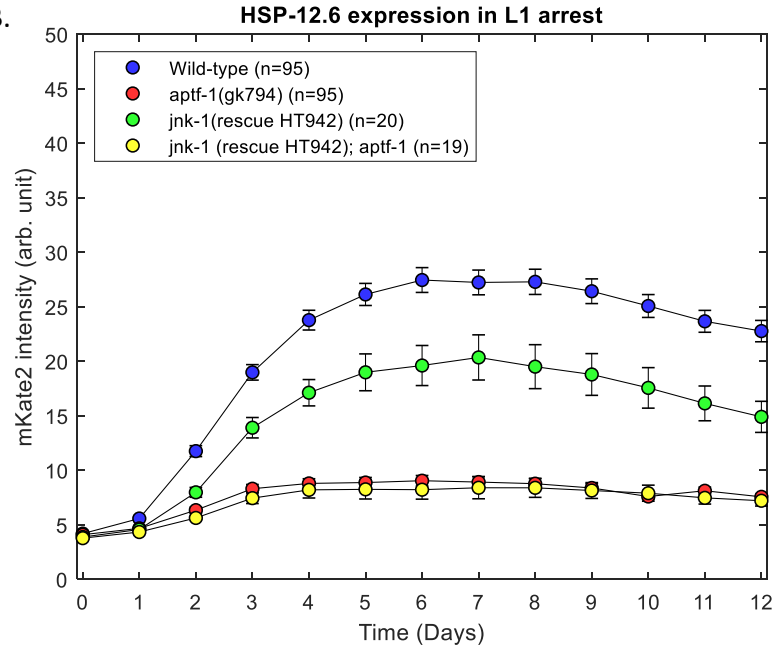

D.

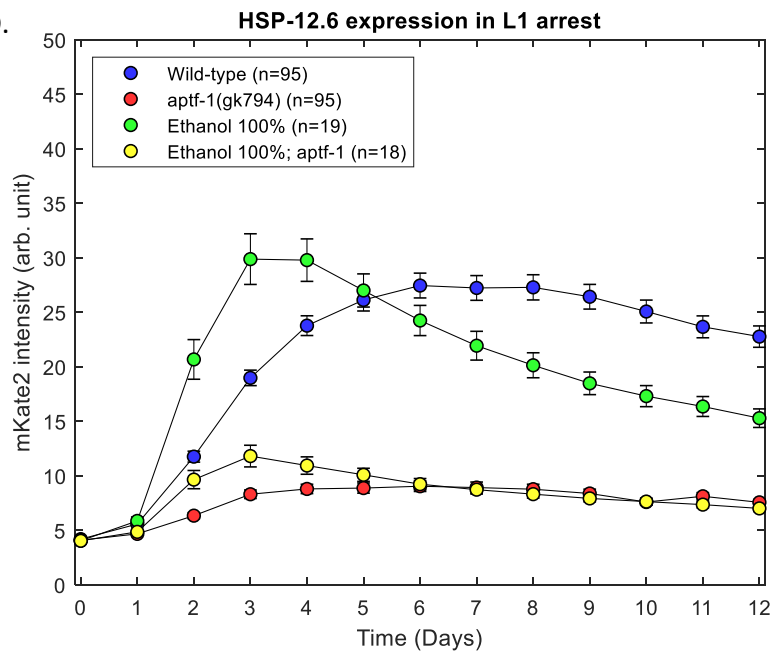

(A) Overexpression of $j n k-1$, only causes significant downregulation of HSP-12.6 expression from day 2 to day 7 (FDR <0.05) in both wild-type and aptf-1 (gk794) backgrounds.

(B) Overexpression of $j n k-1$ with a different strain, causes significant downregulation of HSP12.6 expression only in the wild-type (FDR $<0.05$, except for days 0 and 1 ).

(C) rheb-1 loss of function upregulates HSP-12.6 expression in the wild-type (FDR $<0.05$ from day 6 to day 12) but not in the aptf-1(gk794).

(D) Ethanol (100\%) treatment changes the kinetics of HSP-12.6 expression in a similar way in wild-type and aptf-1(gk794). 
E.

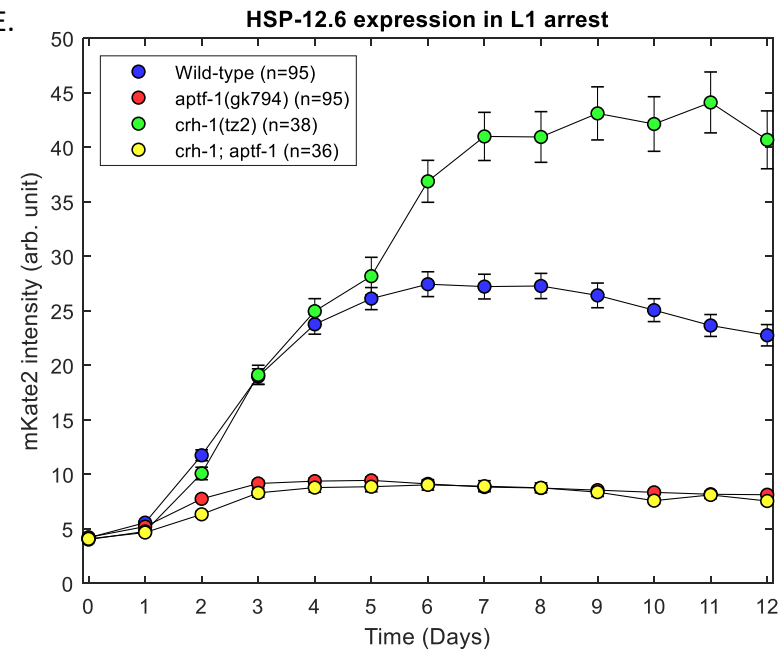

G.

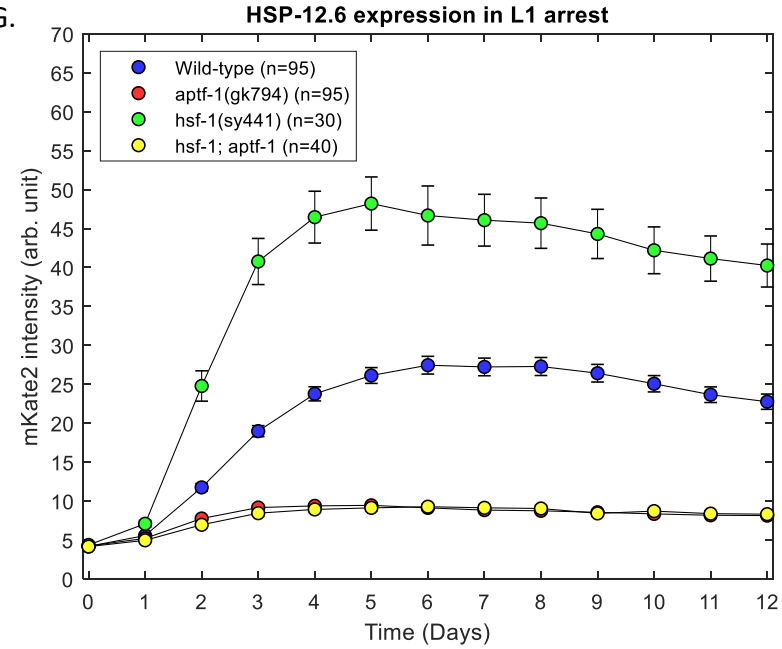

I.

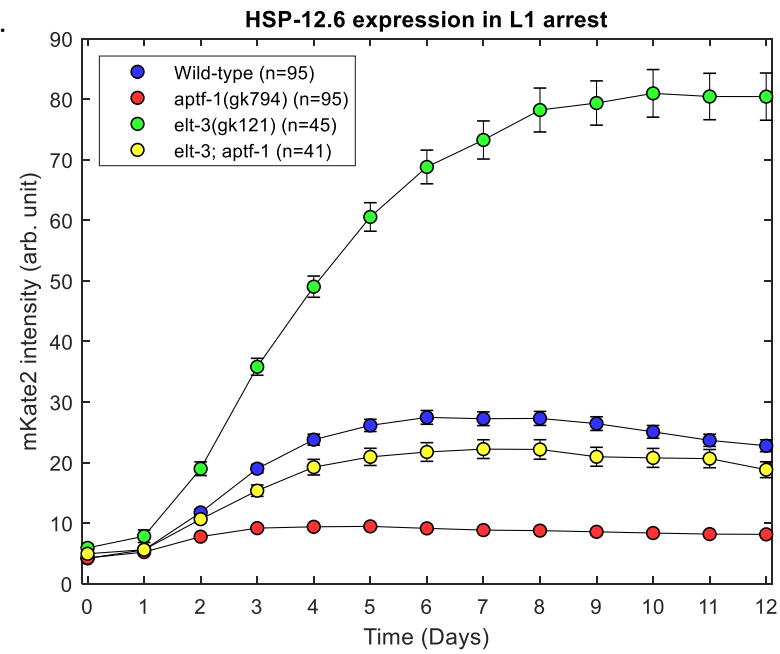

F. HSP-12.6 expression in L1 arrest

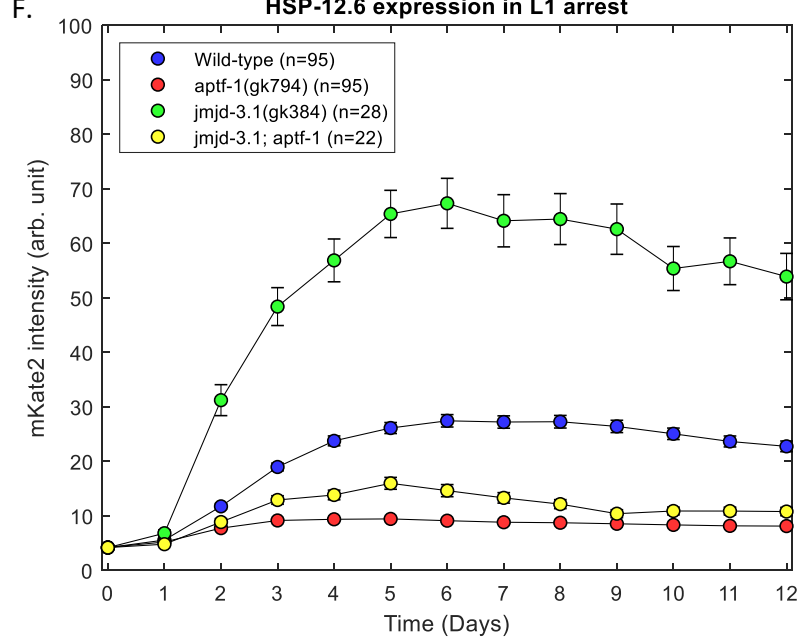

H.

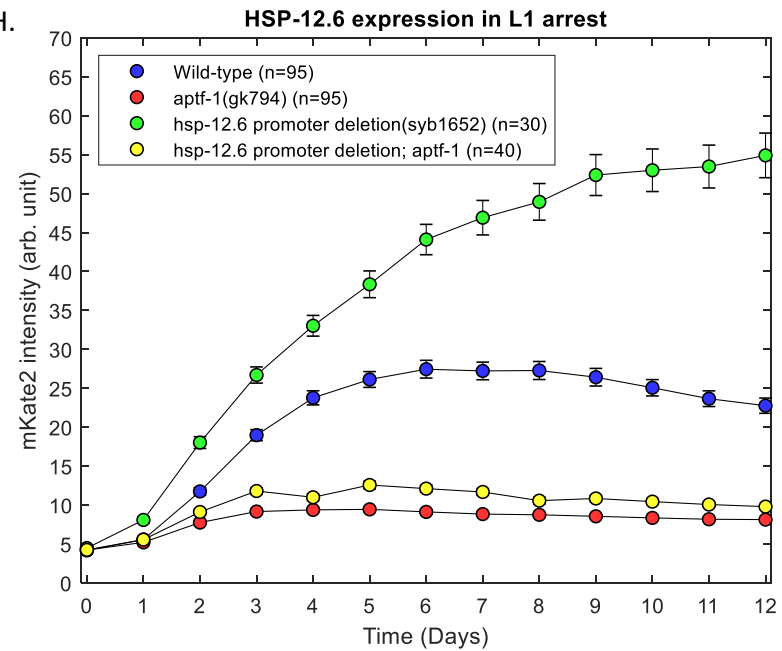

J.

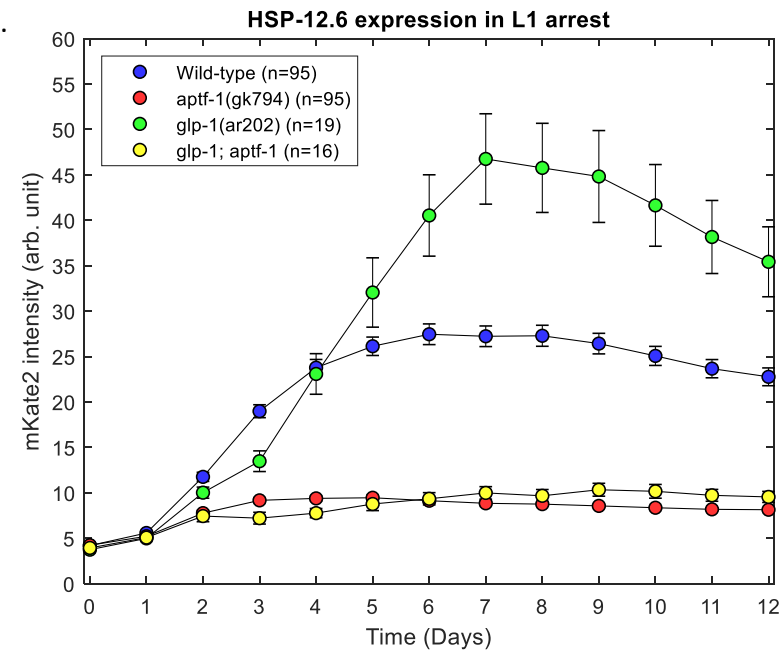

(E) $c r h-1$ loss of function only affects the expression of HSP-12.6 in the wild type after 6 days (FDR $<0.05$ from day 6 to day 12 )

(F) jmjd-3.1 loss of function increases the expression of HSP-12.6 in the wild type (FDR < 0.05 , except for days 0 and 1) and less in the aptf-1(gk794) (FDR < 0.05 from day 3 to day 7) (G-H) Loss of function of $h s f-1$ or a $4 \mathrm{~kb}$ deletion of the promoter region of HSP-12.6 increase HSP-12.6 expression in the wild-type (FDR < 0.05, except for days 0 and 1)

(I) elt-3 loss of function causes upregulation of HSP-12.6 expression in both the wild-type and the aptf-1 (gk794) backgrounds (FDR < 0.05, except for days 0 and 1) 
(J) Gain of function of $g l p-1$ increases HSP-12.6 expression only in the wild type starting from day 6 (FDR $<0.05$, except for days 0 and 1 )

K.

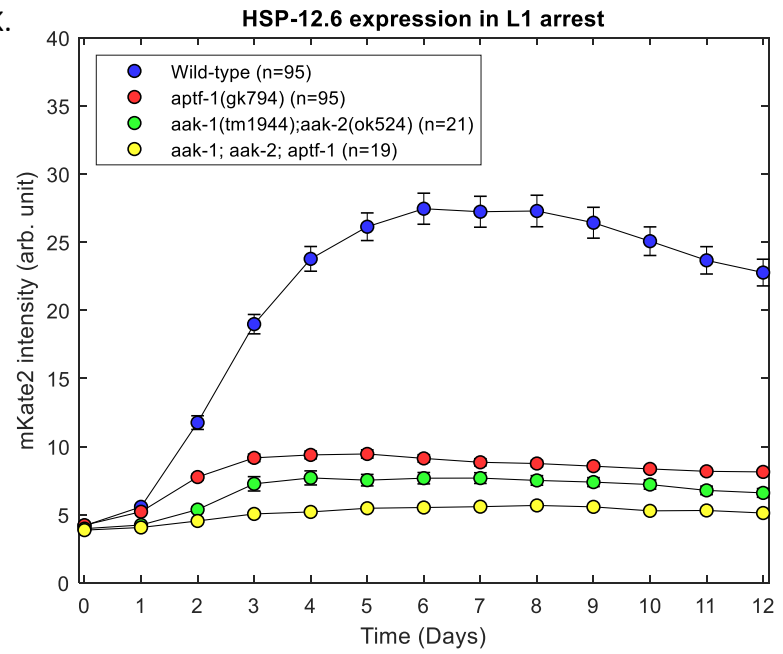

M.

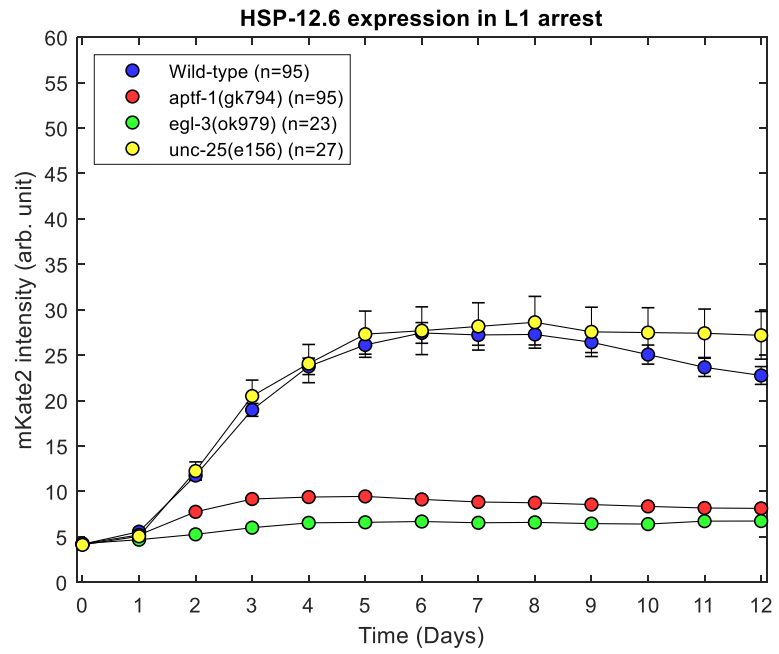

O.

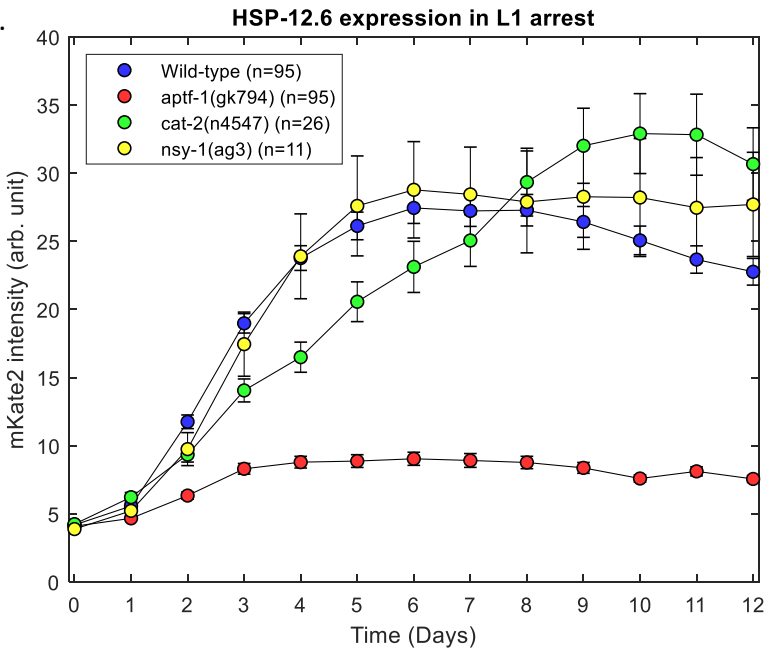

L.

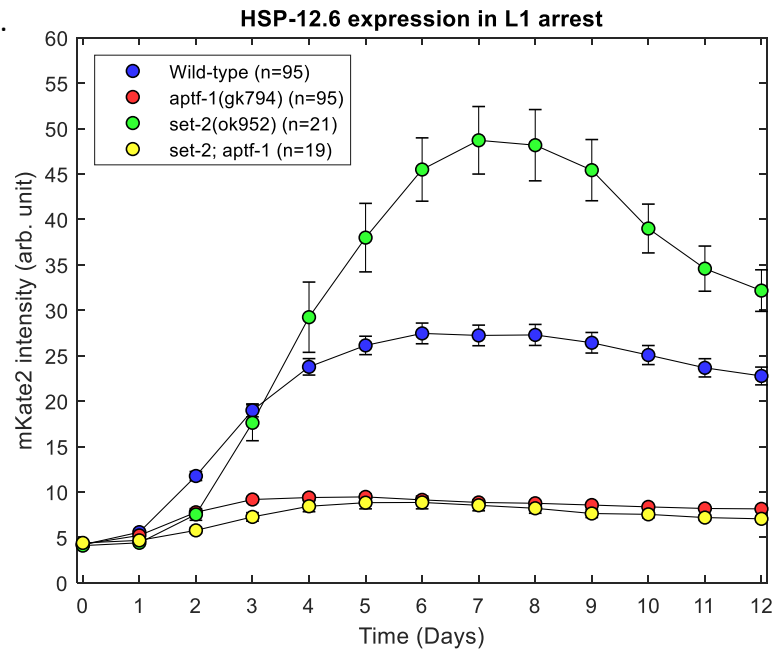

N.

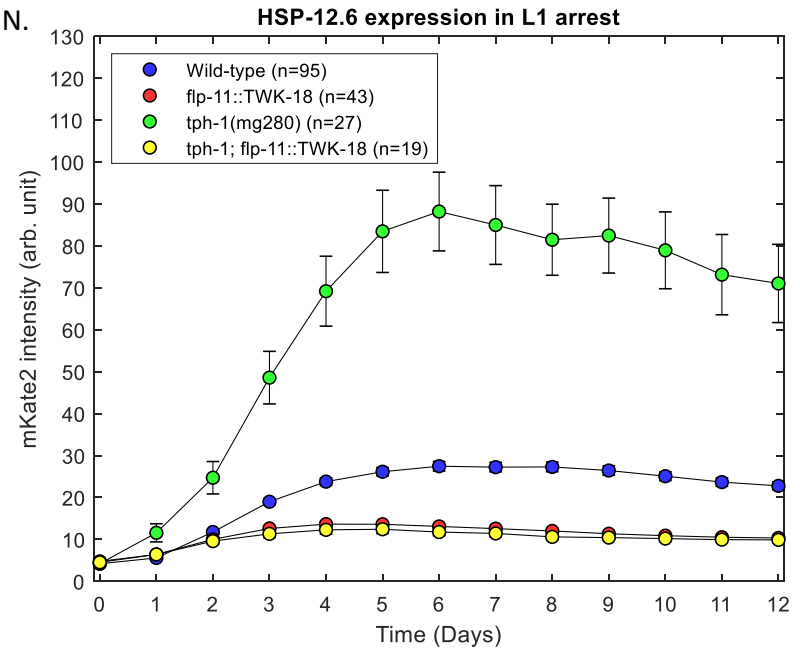

P.

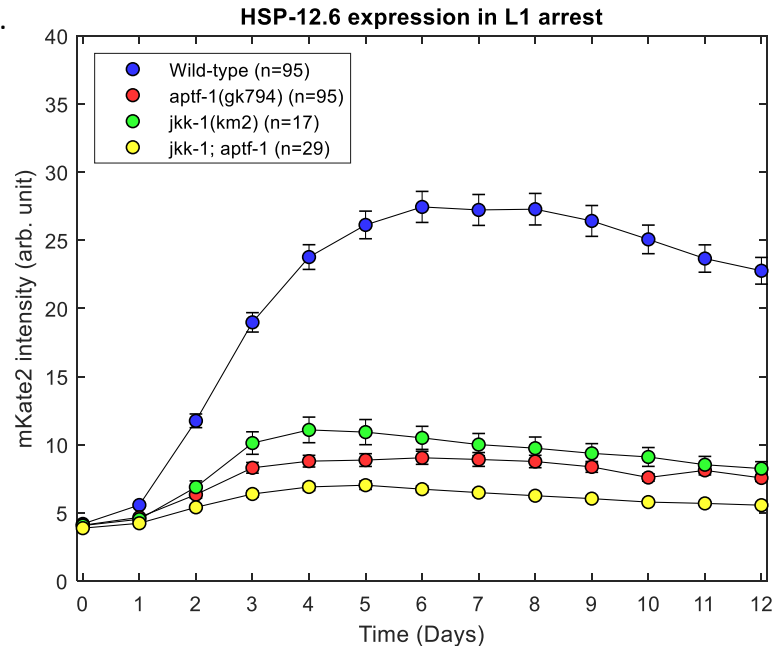

(K) Loss of function of $a a k-1$ and $a a k-2$ reduce HSP-12.6 expression in both wild type and aptf-1(gk794) (FDR < 0.05, except days 0 and 1)

(L) set-2 loss of function increases the expression of HSP-12.6 only in the wild type (FDR < 0.05 from day 5 to day 12 ) 
(M) egl-3 loss of function downregulates the expression of HSP-12.6 (FDR < 0.05, except for day 0 and 1), while $u n c-25$ loss of function does not affect it.

(N) tph-1 loss of function decreases HSP-12.6 expression only in the wild type (FDR $<0.05$, except day 0 )

(O) cat-2 loss of function decreases HSP-12.6 expression from day 2 to day 6 (FDR < 0.05) and increases it from day 10 to day 12 (FDR < 0.05). nsy- 1 loss of function does not affect the expression of HSP-12.6

(P) $j k k-1$ loss of function decreases the expression of HSP-12.6 in the wild-type and aptf1(gk794) (FDR < 0.05, except days 0 and 1)

\subsection{Lifespan screening in $L 1$ arrest}

A.
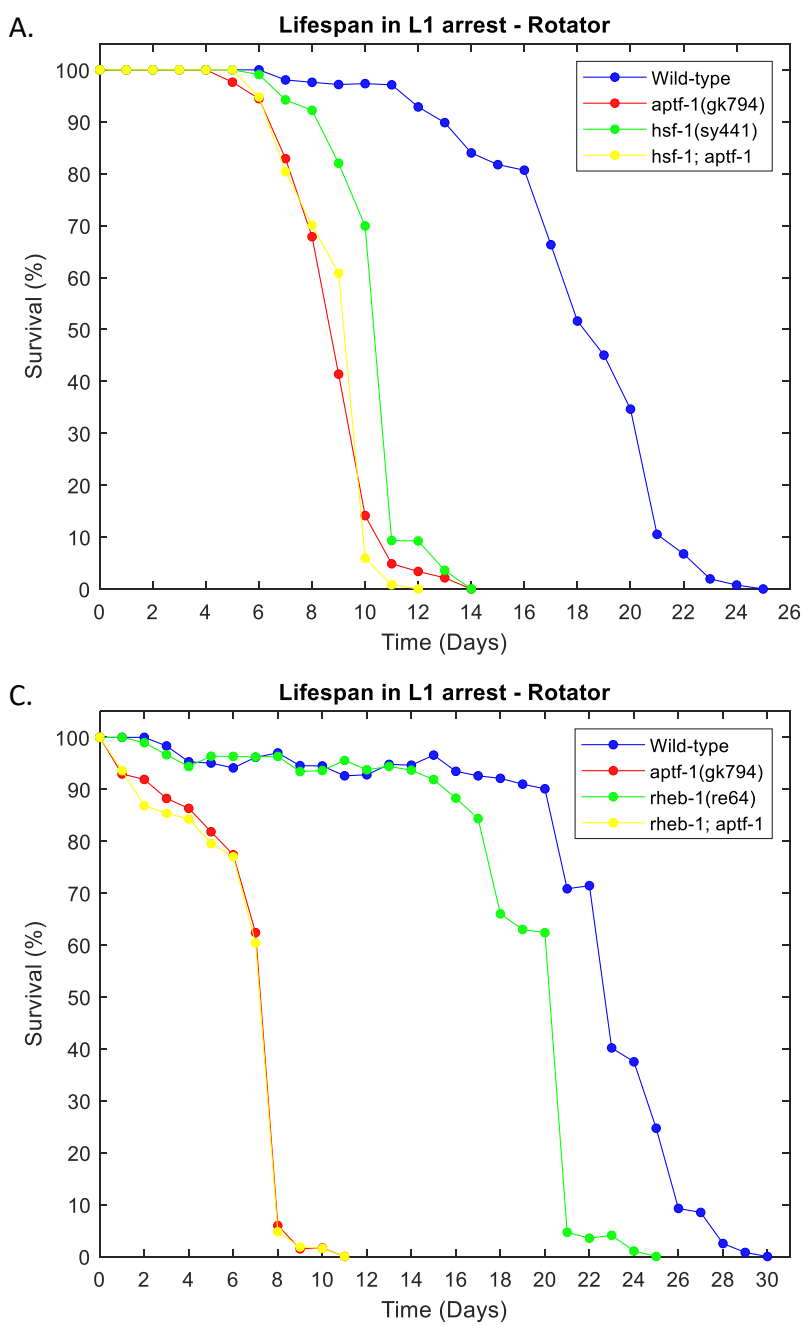

B.

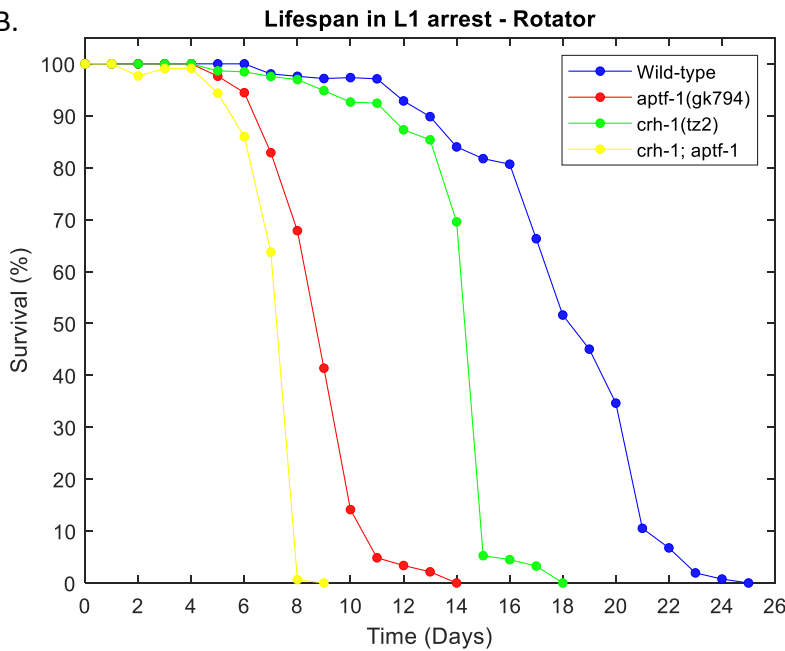

D.

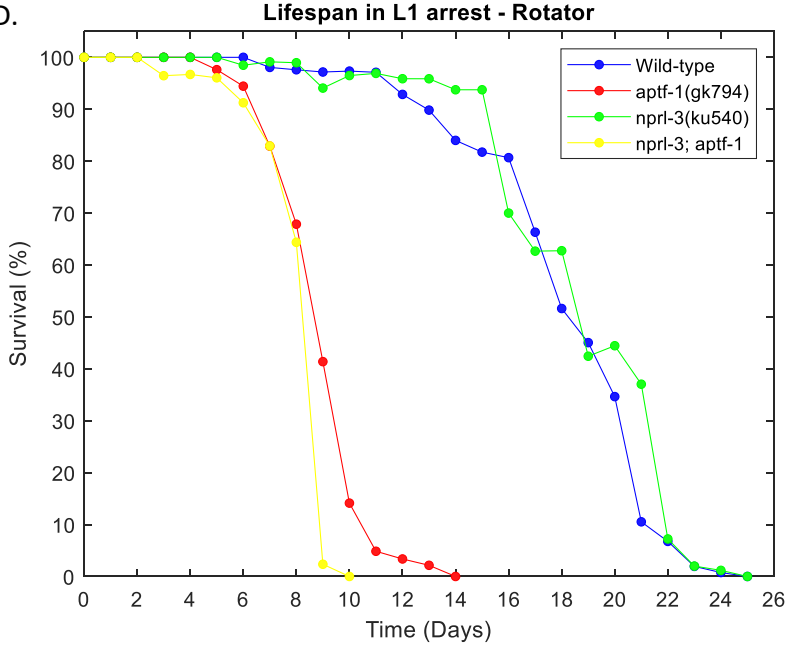

(A) $h s f-1$ loss of function decreases the lifespan of the wild type (FDR $<0.05$ at day 12)

(B) crh-1 loss of function decreases the lifespan of both the wild-type and the aptf-1(gk794) strains (FDR $<0.05$ at day 16 and 7 respectively)

(C) rheb-1 loss of function decreases the lifespan of the wild-type (FDR $<0.05$ at day 23)

(D) $n p r l-3$ loss of function does not alter the lifespan of either the wild type or the aptf1 (gk794) 


\section{References}

1. Irwin M, Why Sleep Is Important for Health: A Psychoneuroimmunology Perspective Annu Rev Psychol. 2015 January 3; 66: 143-172. doi:10.1146/annurev-psych-010213-115205

2. Drake C. et al, Insomnia causes, consequences, and therapeutics: An overview Depression and Anxiety 2003 doi.org/10.1002/da.10151

3. Miyazaki S. et al, Sleep in vertebrate and invertebrate animals, and insights into the function and evolution of sleep Neuroscience Research 118 (2017) 3-12 dx.doi.org/10.1016/j.neures.2017.04.017

4. Campbell and Tobler, Animal Sleep: A Review of Sleep Duration Across Phylogeny Neuroscience \& Biobehavioral Reviews, Vol. 8, pp. 269--300, 1984 doi.org/10.1016/01497634(84)90054-X

5. Nath R. et al, The jellyfish Cassiopea exhibits a sleep-like state Curr Biol. 2017 October 09; 27(19): 2984-2990.e3. doi:10.1016/j.cub.2017.08.014.

6. Bringmann $\mathrm{H}$, Genetic sleep deprivation: using sleep mutants to study sleep functions $E M B O$ Reports (2019) e46807 DOI 10.15252/embr.201846807

7. Saper C. et al, Hypothalamic regulation of sleep and circadian rhythms NATURE Vol 437 2005 doi:10.1038/nature04284

8. Saper C. et al, The sleep switch: hypothalamic control of sleep and wakefulness Trends in Neurosciences Volume 24, Issue 12, 1 December 2001, Pages 726-731 doi.org/10.1016/S01662236(00)02002-6

9. Gaus S. et al, Ventrolateral preoptic nucleus contains sleep-active, galaninergic neurons in multiple mammalian species Neuroscience Volume 115, Issue 1, 15 November 2002, Pages 285-294 doi.org/10.1016/S0306-4522(02)00308-1

10. Weber, F., Dan, Y. Circuit-based interrogation of sleep control. Nature 538, 51-59 (2016). https://doi.org/10.1038/nature19773

11. Inutsuka and Yamanaka, THE REGULATION OF SLEEP AND WAKEFULNESS BY THE HYPOTHALAMIC NEUROPEPTIDE OREXIN/HYPOCRETIN Nagoya J Med Sci. 2013 Feb; 75(1-2): 29-36.

12. Sutcliffe, J.G. and de Lecea, L. (2000), The hypocretins: Excitatory neuromodulatory peptides for multiple homeostatic systems, including sleep and feeding. J. Neurosci. Res., 62: 161-168. doi:10.1002/1097-4547(20001015)62:2<161::AID-JNR1>3.0.CO;2-1

13. Franken P, A role for clock genes in sleep homeostasis Current Opinion in Neurobiology Volume 23, Issue 5, October 2013, Pages 864-872 doi.org/10.1016/j.conb.2013.05.002

14. Dubocovich M, Melatonin receptors: Role on sleep and circadian rhythm regulation Sleep Medicine Volume 8, Supplement 3, December 2007, Pages 34-42 doi.org/10.1016/j.sleep.2007.10.007 
15. Sharma, R, Sahota, P, Thakkar, MM. Melatonin promotes sleep in mice by inhibiting orexin neurons in the perifornical lateral hypothalamus. J Pineal Res. 2018; 65:e12498. https://doi.org/10.1111/jpi.12498

16. Cirelli C, Tononi G (2008) Is Sleep Essential?. PLOS Biology 6(8): e216. https://doi.org/10.1371/journal.pbio.0060216

17. Schmidt M, The energy allocation function of sleep: A unifying theory of sleep, torpor, and continuous wakefulness Neuroscience \& Biobehavioral Reviews Volume 47, November 2014, Pages 122-153 doi.org/10.1016/j.neubiorev.2014.08.001

18. Diering G. et al, Homer1a drives homeostatic scaling-down of excitatory synapses during sleep Science. 2017 Feb 3; 355(6324): 511-515. Published online 2017 Feb 2. doi: $10.1126 /$ science.aai8355

19. Albrecht P. Vorster, Jan Born, Sleep and memory in mammals, birds and invertebrates, Neuroscience \& Biobehavioral Reviews, Volume 50, 2015, Pages 103-119, ISSN 0149-7634, https://doi.org/10.1016/j.neubiorev.2014.09.020.

20. Kang, Jae-Eun et al. "Amyloid-beta dynamics are regulated by orexin and the sleep-wake cycle.” Science (New York, N.Y.) vol. 326,5955 (2009): 1005-7. doi:10.1126/science.1180962

21. Bringmann H, Sleep-Active Neurons: Conserved Motors of Sleep Genetics April 1, 2018 vol. 208 no. 4 1279-1289; https://doi.org/10.1534/genetics.117.300521

22. Meneely, P. M., Dahlberg, C. L., \& Rose, J. K. (2019). Working with worms: Caenorhabditis elegans as a model organism. Current Protocols Essential Laboratory Techniques, 19, e35. doi: 10.1002/cpet.35

23. Raizen, D., Zimmerman, J., Maycock, M. et al. Lethargus is a Caenorhabditis elegans sleep-like state. Nature 451, 569-572 (2008). https://doi.org/10.1038/nature06535

24. Wu Y, Masurat F, Preis J, Bringmann H. Sleep Counteracts Aging Phenotypes to Survive Starvation-Induced Developmental Arrest in C. elegans. Curr Biol. 2018;28(22):3610-3624.e8. doi:10.1016/j.cub.2018.10.009

25. Hill AJ, Mansfield R, Lopez JM, Raizen DM, Van Buskirk C. Cellular stress induces a protective sleep-like state in C. elegans. Curr Biol. 2014;24(20):2399-2405. doi:10.1016/j.cub.2014.08.040

26. Jan Konietzka, Maximilian Fritz, Silvan Spiri, Rebecca McWhirter, Andreas Leha, Sierra Palumbos, Wagner Steuer Costa, Alexandra Oranth, Alexander Gottschalk, David M. Miller, Alex Hajnal, Henrik Bringmann, Epidermal Growth Factor Signaling Promotes Sleep through a Combined Series and Parallel Neural Circuit, Current Biology, Volume 30, Issue 1, 2020, Pages 1-16.e13, ISSN 0960-9822, https://doi.org/10.1016/j.cub.2019.10.048.

27. Turek M. et al, Sleep-active neuron specification and sleep induction require FLP-11 neuropeptides to systemically induce sleep eLife 2016;5:e12499 doi: 10.7554/eLife.12499 
28. Maluck E, Busack I, Besseling J, Masurat F, Turek M, et al. (2020) A wake-active locomotion circuit depolarizes a sleep-active neuron to switch on sleep. PLOS Biology 18(2): e3000361. https://doi.org/10.1371/journal.pbio.3000361

29. MacFadyen UM, Oswald I, Lewis SA. Starvation and human slow-wave sleep. J Appl Physiol. 1973;35(3):391-394. doi:10.1152/jappl.1973.35.3.391

30. Miura, M, Takahashi, A. Starvation tolerance associated with prolonged sleep bouts upon starvation in a single natural population of Drosophila melanogaster. J Evol Biol. 2019; 32: 1117-1123. https://doi.org/10.1111/jeb.13514

31. Baugh LR. To grow or not to grow: nutritional control of development during Caenorhabditis elegans L1 arrest. Genetics. 2013;194(3):539-555. doi:10.1534/genetics.113.150847

32. Kaplan RE, Baugh LR. L1 arrest, daf-16/FoxO and nonautonomous control of postembryonic development. Worm. 2016;5(2):e1175196. Published 2016 Apr 6. doi:10.1080/21624054.2016.1175196

33. Roux AE, Langhans K, Huynh W, Kenyon C. Reversible Age-Related Phenotypes Induced during Larval Quiescence in C. elegans. Cell Metab. 2016;23(6):1113-1126. doi:10.1016/j.cmet.2016.05.024

34. Grant, L.K., Ftouni, S., Nijagal, B. et al. Circadian and wake-dependent changes in human plasma polar metabolites during prolonged wakefulness: A preliminary analysis. Sci Rep 9, 4428 (2019). https://doi.org/10.1038/s41598-019-40353-8

35. Laing E. et al, Blood transcriptome based biomarkers for human circadian phase eLife 2017;6:e20214 doi: 10.7554/eLife.20214

36. Davies S. et al, Effect of sleep deprivation on the human metabolome PNAS July 22, 2014 111 (29) 10761-10766; first published July 7, 2014 https://doi.org/10.1073/pnas.1402663111

37. Hu Y, Korovaichuk A, Astiz M, et al. Functional Divergence of Mammalian TFAP2a and TFAP2b Transcription Factors for Bidirectional Sleep Control [published online ahead of print, 2020 Aug 7]. Genetics. 2020;genetics.303533.2020. doi:10.1534/genetics.120.303533

38. Brenner S. The genetics of Caenorhabditis elegans. Genetics. 1974;77(1):71-94.

39. Fay D.S. Classical genetic methods (December 30, 2013), WormBook, ed. The C. elegans Research Community, WormBook, doi/10.1895/wormbook.1.165.1, http://www .wormbook.org.

40. Ahringer J. (2006) Reverse genetics. WormBook: The online review of C. elegans biology.

41. Ye S. (2001) An efficient procedure for genotyping single nucleotide polymorphisms. Nucleic acids res 29(17):E88-8.

42. Porta-de-la-Riva M, Fontrodona L, Villanueva A, Cerón J. Basic Caenorhabditis elegans methods: synchronization and observation. J Vis Exp. 2012;(64):e4019. Published 2012 Jun 10. doi:10.3791/4019 
43. Bringmann H. (2011) Agarose hydrogel microcompartments for imaging sleep- and wakelike behavior and nervous system development in Caenorhabditis elegans larvae. J Neurosci Methods 201(1):78-88. doi: 10.1016

44. Nagy S, Raizen DM, Biron D. Measurements of behavioral quiescence in Caenorhabditis elegans. Methods. 2014;68(3):500-507. doi:10.1016/j.ymeth.2014.03.009

45. Sawilowsky, Shlomo S. (2009) "New Effect Size Rules of Thumb" Journal of Modern Applied Statistical Methods: Vol. 8 : Iss. 2 , Article26.DOI: 10.22237/jmasm/1257035100

46. Uku Raudvere, Liis Kolberg, Ivan Kuzmin, Tambet Arak, Priit Adler, Hedi Peterson, Jaak Vilo, g:Profiler: a web server for functional enrichment analysis and conversions of gene lists (2019 update), Nucleic Acids Research, Volume 47, Issue W1, 02 July 2019, Pages W191W198, https://doi.org/10.1093/nar/gkz369

47. Bantscheff, M., Lemeer, S., Savitski, M.M. et al. Quantitative mass spectrometry in proteomics: critical review update from 2007 to the present. Anal Bioanal Chem 404, 939-965 (2012). https://doi.org/10.1007/s00216-012-6203-4

48. Gielisch I, Meierhofer D. Metabolome and proteome profiling of complex I deficiency induced by rotenone. J Proteome Res. 2015;14(1):224-235. doi:10.1021/pr500894v

49. Inka Busack, Florian Jordan, Peleg Sapir \& Henrik Bringmann (2020) The OptoGenBox a device for long-term optogenetics in C. elegans, Journal of Neurogenetics, DOI: 10.1080/01677063.2020.1776709

50. Schwarz J, Bringmann H (2013) Reduced Sleep-Like Quiescence in Both Hyperactive and Hypoactive Mutants of the Galphaq Gene egl-30 during lethargus in Caenorhabditis elegans. PLOS ONE 8(9): e75853. https://doi.org/10.1371/journal.pone.0075853

51. Zhang L. et al, The auxin-inducible degradation (AID) system enables versatile conditional protein depletion in C. elegans Development 2015 142: 4374-4384; doi: 10.1242/dev.129635

52. Duong T, Rasmussen NR, Ballato E, Mote FS, Reiner DJ. The Rheb-TORC1 signaling axis functions as a developmental checkpoint. Development. 2020;147(5):dev181727. Published 2020 Mar 2. doi:10.1242/dev.181727

53. Honjoh, S., Yamamoto, T., Uno, M. et al. Signalling through RHEB-1 mediates intermittent fasting-induced longevity in $C$. elegans. Nature 457, 726-730 (2009). https://doi.org/10.1038/nature07583

54. Cao, J., J. S. Packer, V. Ramani, D. A. Cusanovich, C. Huynh et al., 2017 Comprehensive single-cell transcriptional profiling of a multicellular organism. Science 357: 661-667. doi:10.1126/science.aam8940

55. Li, C. and Kim, K. Neuropeptides (September 25, 2008), WormBook, ed. The C. elegans Research Community, WormBook, doi/10.1895/wormbook.1.142.1, http://www .wormbook.org.

56. Altun, Z.F. and Hall, D.H. 2009. Epithelial system, hypodermis. In WormAtlas. doi:10.3908/wormatlas.1.13 
57. Kaletsky R, Yao V, Williams A, Runnels AM, Tadych A, et al. (2018) Transcriptome analysis of adult Caenorhabditis elegans cells reveals tissue-specific gene and isoform expression. PLOS Genetics 14(8): e1007559. https://doi.org/10.1371/journal.pgen.1007559

58. Gumienny T.L., Savage-Dunn C. TGF- $\beta$ signaling in C. elegans (July 10, 2013), WormBook, ed. The C. elegans Research Community, WormBook, doi/10.1895/wormbook.1.22.2, http://www.wormbook.org.

59. Altun, Z.F. and Hall, D.H. 2009. Muscle system, introduction. In WormAtlas. doi:10.3908/wormatlas.1.6

60. Altun, Z.F. and Hall, D.H. 2009. Alimentary system, intestine. In WormAtlas. doi:10.3908/wormatlas.1.4

61. Kaplan RE, Baugh LR. L1 arrest, daf-16/FoxO and nonautonomous control of postembryonic development. Worm. 2016;5(2):e1175196. Published 2016 Apr 6. doi:10.1080/21624054.2016.1175196

62. Murphy, C., McCarroll, S., Bargmann, C. et al. Genes that act downstream of DAF-16 to influence the lifespan of Caenorhabditis elegans. Nature 424, 277-283 (2003). https://doi.org/10.1038/nature01789

63. Sun X, Chen W-D and Wang Y-D (2017) DAF-16/FOXO Transcription Factor in Aging and Longevity. Front. Pharmacol. 8:548. doi: 10.3389/fphar.2017.00548

64. Baugh LR. To grow or not to grow: nutritional control of development during Caenorhabditis elegans L1 arrest. Genetics. 2013;194(3):539-555. doi:10.1534/genetics.113.150847

65. Han, M., Wei, G., McManus, C.E. et al. Isolated C. elegans germ nuclei exhibit distinct genomic profiles of histone modification and gene expression. BMC Genomics 20, 500 (2019). https://doi.org/10.1186/s12864-019-5893-9

66. Kimble, J. and Crittenden, S.L. Germline proliferation and its control (August 15, 2005), WormBook, ed. The C. elegans Research Community, WormBook, doi/10.1895/wormbook.1.13.1, http://www.wormbook.org.

67. Greer EL, Maures TJ, Hauswirth AG, et al. Members of the H3K4 trimethylation complex regulate lifespan in a germline-dependent manner in C. elegans. Nature. 2010;466(7304):383387. doi:10.1038/nature09195

68. Strome, S. Specification of the germ line (July 28, 2005), WormBook, ed. The C. elegans Research Community, WormBook, doi/10.1895/wormbook.1.9.1, http://www.wormbook.org.

69. Altun, Z.F. and Hall, D.H. 2011. Nervous system, general description. In WormAtlas. doi:10.3908/wormatlas.1.18

70. Hobert O. The neuronal genome of Caenorhabditis elegans (August 13, 2013), WormBook, ed. The C. elegans Research Community, WormBook, doi/10.1895/wormbook.1.161.1, http://www.wormbook.org. 
71. Dabbish NS, Raizen DM. GABAergic synaptic plasticity during a developmentally regulated sleep-like state in C. elegans. $J$ Neurosci. 2011;31(44):15932-15943. doi:10.1523/JNEUROSCI.0742-11.2011

72. Dittman JS, Kaplan JM. Behavioral impact of neurotransmitter-activated G-protein-coupled receptors: muscarinic and GABAB receptors regulate Caenorhabditis elegans locomotion. $J$ Neurosci. 2008;28(28):7104-7112. doi:10.1523/JNEUROSCI.0378-08.2008

73. Schaffrath R, Leidel SA. Wobble uridine modifications-a reason to live, a reason to die?!. RNA Biol. 2017;14(9):1209-1222. doi:10.1080/15476286.2017.1295204

74. Meléndez, A. and Levine, B. Autophagy in C. elegans (August 24, 2009), WormBook, ed. The C. elegans Research Community, WormBook, doi/10.1895/wormbook.1.147.1, http://www.wormbook.org.

75. Van Assche R, Temmerman L, Dias DA, Boughton B, Boonen K, Braeckman BP, Schoofs L, Roessner U. Metabolic profiling of a transgenic Caenorhabditis elegans Alzheimer model. Metabolomics. 2015;11(2):477-486. doi: 10.1007/s11306-014-0711-5. Epub 2014 Jul 30. PMID: 25750603; PMCID: PMC4342517.

76. Vohra M, Lemieux GA, Lin L, Ashrafi K. Kynurenic acid accumulation underlies learning and memory impairment associated with aging. Genes Dev. 2018;32(1):14-19. doi:10.1101/gad.307918.117

77. Bastiani, C. and Mendel, J. Heterotrimeric G proteins in C. elegans (October 13, 2006), WormBook, ed. The C. elegans Research Community, WormBook, doi/10.1895/wormbook.1.75.1, http://www.wormbook.org.

78. Trojanowski NF, Nelson MD, Flavell SW, Fang-Yen C, Raizen DM. Distinct Mechanisms Underlie Quiescence during Two Caenorhabditis elegans Sleep-Like States. J Neurosci. 2015;35(43):14571-14584. doi:10.1523/JNEUROSCI.1369-15.2015

79. Kesić S. and Spasić S. Application of Higuchi's fractal dimension from basic to clinical neurophysiology: a review Comput. Methods Progr. Biomed., 133 (September 2016), pp. 5570 doi.org/10.1016/j.cmpb.2016.05.014

80. Sanders J, Scholz M, Merutka I, Biron D. Distinct unfolded protein responses mitigate or mediate effects of nonlethal deprivation of C. elegans sleep in different tissues. BMC Biol. 2017 Aug 28;15(1):67. doi: 10.1186/s 12915-017-0407-1. PMID: 28844202; PMCID: PMC5572162.

81. Kaletsky, R., Lakhina, V., Arey, R. et al. The C. elegans adult neuronal IIS/FOXO transcriptome reveals adult phenotype regulators. Nature 529, 92-96 (2016). https://doi.org/10.1038/nature16483

82. Shao Z, Zhang Y, Powell-Coffman JA. Two distinct roles for EGL-9 in the regulation of HIF-1-mediated gene expression in Caenorhabditis elegans. Genetics. 2009 Nov;183(3):821-9. doi: 10.1534/genetics.109.107284. Epub 2009 Sep 7. PMID: 19737748; PMCID: PMC2778979. 
83. Merkwirth C, Jovaisaite V, Durieux J, et al. Two Conserved Histone Demethylases Regulate Mitochondrial Stress-Induced Longevity. Cell. 2016;165(5):1209-1223. doi:10.1016/j.cell.2016.04.012

84. Goodman, M.B. Mechanosensation (January 06, 2006), WormBook, ed. The C. elegans Research Community, WormBook, doi/10.1895/wormbook.1.62.1

85. L. Ryan Baugh, Paul W. Sternberg, DAF-16/FOXO Regulates Transcription of cki1/Cip/Kip and Repression of lin-4 during C. elegans L1 Arrest, Current Biology, Volume 16, Issue 8, 2006, Pages 780-785, ISSN 0960-9822, https://doi.org/10.1016/j.cub.2006.03.021.

86. Francisca Padez-Vieira \& Pedro Afonso (2016) Sleep disturbances in anorexia nervosa, Advances in Eating Disorders, 4:2, 176-188, DOI: 10.1080/21662630.2016.1175958

87. Cinosi E, Di Iorio G, Acciavatti T, Cornelio M, Vellante F, De Risio L, Martinotti G. Sleep disturbances in eating disorders: a review. Clin Ter. 2011;162(6):e195-202. PMID: 22262340.

88. Michalsen A, Schlegel F, Rodenbeck A, Lüdtke R, Huether G, Teschler H, Dobos GJ. Effects of short-term modified fasting on sleep patterns and daytime vigilance in non-obese subjects: results of a pilot study. Ann Nutr Metab. 2003;47(5):194-200. doi: 10.1159/000070485. PMID: 12748412.

89. Almeneessier AS, BaHammam AS. How does diurnal intermittent fasting impact sleep, daytime sleepiness, and markers of the biological clock? Current insights. Nat Sci Sleep. 2018;10:439-452. Published 2018 Dec 7. doi:10.2147/NSS.S165637

90. Houthoofd, K., Fidalgo, M.A., Hoogewijs, D., Braeckman, B.P., Lenaerts, I., Brys, K., Matthijssens, F., De Vreese, A., Van Eygen, S., Muñoz, M.J. and Vanfleteren, J.R. (2005), Metabolism, physiology and stress defense in three aging Ins/IGF-1 mutants of the nematode Caenorhabditis elegans. Aging Cell, 4: 87-95. doi:10.1111/j.1474-9726.2005.00150.x

91. Sharma S, Kavuru M. Sleep and metabolism: an overview. Int $J$ Endocrinol. 2010;2010:270832. doi:10.1155/2010/270832

92. Laranjeiro R, Harinath G, Burke D, Braeckman BP, Driscoll M. Single swim sessions in C. elegans induce key features of mammalian exercise. BMC Biol. 2017;15(1):30. Published 2017 Apr 10. doi:10.1186/s12915-017-0368-4

93. Pires, GN, Benedetto, L, Cortese, R, et al. Effects of sleep modulation during pregnancy in the mother and offspring: Evidences from preclinical research. J Sleep Res. 2020; 00:e13135. https://doi.org/10.1111/jsr.13135

94. Margreet W Harskamp-van Ginkel, Despo Ierodiakonou, Katerina Margetaki, Marina Vafeiadi, Marianna Karachaliou, Manolis Kogevinas, Tanja G M Vrijkotte, Leda Chatzi, Gestational sleep deprivation is associated with higher offspring body mass index and blood pressure, Sleep, , zsaa110, https://doi.org/10.1093/sleep/zsaa110

95. Zhao Q, Peng C, Wu X, Chen Y, Wang C, You Z. Maternal sleep deprivation inhibits hippocampal neurogenesis associated with inflammatory response in young offspring rats. Neurobiol Dis. 2014 Aug;68:57-65. doi: 10.1016/j.nbd.2014.04.008. Epub 2014 Apr 24. PMID: 24769004. 
96. Keene A. and Duboue E. The origins and evolution of sleep Journal of Experimental Biology 2018 221: jeb159533 doi: 10.1242/jeb.159533

97. Jenkins TA, Nguyen JC, Polglaze KE, Bertrand PP. Influence of Tryptophan and Serotonin on Mood and Cognition with a Possible Role of the Gut-Brain Axis. Nutrients. 2016;8(1):56. Published 2016 Jan 20. doi:10.3390/nu8010056

98. Yamashita M, Yamamoto T. Tryptophan and kynurenic Acid may produce an amplified effect in central fatigue induced by chronic sleep disorder. Int J Tryptophan Res. 2014;7:9-14. Published 2014 May 14. doi:10.4137/IJTR.S14084

99. Vaccaro A. et al, Sleep Loss Can Cause Death through Accumulation of Reactive Oxygen Species in the Gut Cell Volume 181, Issue 6, 11 June 2020, Pages 1307-1328.e15 https://doi.org/10.1016/j.cell.2020.04.049

100. Ribas V, García-Ruiz C, Fernández-Checa JC. Glutathione and mitochondria. Front Pharmacol. 2014;5:151. Published 2014 Jul 1. doi:10.3389/fphar.2014.00151

101. Chikahisa S, Séi H. The role of ATP in sleep regulation. Front Neurol. 2011;2:87. Published 2011 Dec 27. doi:10.3389/fneur.2011.00087

102. Quijano S. et al, Interplay between oxidant species and energy metabolism Redox Biology Volume 8, August 2016, Pages 28-42 https://doi.org/10.1016/j.redox.2015.11.010

103. Monsalve GC, Van Buskirk C, Frand AR. LIN-42/PERIOD controls cyclical and developmental progression of C. elegans molts. Curr Biol. 2011 Dec 20;21(24):2033-45. doi: 10.1016/j.cub.2011.10.054. Epub 2011 Dec 1. PMID: 22137474.

104. Hafycz JM, Naidoo NN. Sleep, Aging, and Cellular Health: Aged-Related Changes in Sleep and Protein Homeostasis Converge in Neurodegenerative Diseases. Front Aging Neurosci. 2019;11:140. Published 2019 Jun 11. doi:10.3389/fnagi.2019.00140

105. Pettus B. et al, Ceramide in apoptosis: an overview and current perspectives Biochimica et Biophysica Acta (BBA) - Molecular and Cell Biology of Lipids

Volume 1585, Issues 2-3, 30 December 2002, Pages 114-125 https://doi.org/10.1016/S13881981(02)00331-1

106. Lucki NC, Sewer MB. Nuclear sphingolipid metabolism. Annu Rev Physiol. 2012;74:131151. doi:10.1146/annurev-physiol-020911-153321

107. Schousboe A, Bak LK, Waagepetersen HS. Astrocytic Control of Biosynthesis and Turnover of the Neurotransmitters Glutamate and GABA. Front Endocrinol (Lausanne). 2013;4:102. Published 2013 Aug 15. doi:10.3389/fendo.2013.00102

108. Chun, L., Gong, J., Yuan, F. et al. Metabotropic GABA signalling modulates longevity in C. elegans. Nat Commun 6, 8828 (2015). https://doi.org/10.1038/ncomms9828

109. Tao L, Xie Q, Ding YH, et al. CAMKII and calcineurin regulate the lifespan of Caenorhabditis elegans through the FOXO transcription factor DAF-16. Elife. 2013;2:e00518. Published 2013 Jun 25. doi:10.7554/eLife.0051 


\section{Anastasios Koutsoumparis}

Sex: Male

Date of birth: 18/01/1989

Current address: Erlenring 13, 35037, Marburg, Germany

Contact: a.koutsoumparis@gmail.com

\section{EDUCATION}

$10 / 2016-9 / 2020$

Ph.D. degree in Systems Neuroscience (4-year program, full-time)

Max Planck Institute for Biophysical Chemistry, Göttingen, Germany

University of Marburg, Marburg, Germany

10/2012 - 9/2015

Master's Degree in Pharmacology and Therapeutics (2-year program, full-time)

Department of Pharmacology, School of Pharmacy

Aristotle University of Thessaloniki (AUTH), Thessaloniki, Greece

10/2007 - 7/2012

Bachelor Degree in Pharmacy (5-year program, full-time)

School of Pharmacy

Aristotle University of Thessaloniki (AUTH), Thessaloniki, Greece

\section{PUBLICATIONS}

Koutsoumparis A. et al. (2018) Erythropoietin (rhEPOa) promotes endothelial transdifferentiation of stem cells of the apical papilla (SCAP). Arch Oral Biol PMID: 30205239 DOI: 10.1016/j.archoralbio.2018.09.001

\section{CONFERENCES - PRESENTATIONS - SCHOLARSHIPS}

$1^{\text {st }}$ prize - poster presentation: "Metabolic regulation of sleep" $(11 / 2018) 10^{\text {th }}$ anniversary of GGNB (Göttingen Graduate center for Neurosciences, Biophysics and Molecular

Biosciences)

$\mathbf{1}^{\text {st }}$ prize - oral presentation: "In culture Differentiation of Human Mesenchymal Stem Cells into pro-Endothelial (Angiogenic) Phenotypes" (11/2014) $2^{\text {nd }}$ Young Scientists Forum Hellenic society of Biochemistry and Molecular Pharmacology

Scholarship from the "Department of Studies of Aristotle University of Thessaloniki", for excellent performance and conduct, as a graduate student (2013-2014)

Scholarship of excellence from the "State Scholarships Foundation of Greece (IKY)", for achieving the highest average grade, during my 4th year in the school of pharmacy (20102011) 
